\title{
Syntactic Theory: A Formal Introduction
}

\author{
Ivan A. Sag Thomas Wasow
}

January 20, 1999

CENTER FOR THE STUDY

OF LANGUAGE

AND INFORMATION 

In memory of our fathers

William E. Sag (1908-1977)

Wolfgang R. Wasow (1909-1993) 



\title{
Contents
}

\author{
Preface vii \\ 1 Introduction 1 \\ 2 Some Simple Theories of Grammar 23 \\ 3 Analyzing Features of Grammatical Categories $\quad 47$ \\ 4 Complex Feature Values $\quad 77$ \\ 5 Semantics 103 \\ 6 How the Grammar Works 129 \\ 7 Binding Theory 149 \\ 8 The Structure of the Lexicon 173 \\ 9 Realistic Grammar 203 \\ 10 The Passive Construction 235 \\ 11 Nominal Types: Dummies and Idioms 253 \\ 12 Infinitival Complements 273 \\ 13 Auxiliary Verbs 297 \\ 14 Variation in the English Auxiliary System 321 \\ 15 Long-Distance Dependencies 341 \\ 16 From Rule to Sign 363 \\ Appendix A: \\ Summary of the Grammar 395 \\ Appendix B: Generative Grammar 423 \\ Glossary $\quad 441$ \\ References $\quad 461$
}




\section{Preface}

This textbook grew out of our efforts to develop teaching material for the undergraduate-level Introduction to Syntax course that has been taught at Stanford University for over twenty years. We used earlier versions of this text for three years; the final version was revised and expanded many times to satisfy the needs of our students, whose feedback has been of immense value in shaping the present volume. We consider ourselves fortunate to have extremely talented and demanding students, whose influence shines through our prose on every page.

In addition, more than one colleague has used our text successfully in first-year graduate classes. We feel it is particularly well suited to general readers or those who work in disciplines related to linguistics, such as psychology, philosophy, mathematics, or computer science. All that is required is an interest in rigorous approaches to the analysis of the grammatical structure of natural languages.

We have tried to strike a balance between linguistic analysis (centered on the development of increasingly broader grammar fragments) and data-oriented problem solving. In addition, we have tried to place the proposals presented here into historical perspective (Chapter 1 and Appendix B). A Glossary is included both to aid the reader unfamiliar with traditional grammatical terminology and to provide easy access to more technical jargon that we have employed.

Chapters 1 through 8 develop most of the technical machinery used in the rest of the book. This section of the text is self-contained and can be used as the basis of an abbreviated introductory course. Chapter 9 contains a summary of the grammar develped in Chapters 1 through 8 , along with some general discussion of language processing by humans and computers.

Chapters 10 through 13 apply the tools developed in the earlier chapters to some well-studied grammatical phenomena. Chapter 14 in- 
troduces a topic not normally included in theoretical syntax courses, namely language variation. Chapter 15 deals with the basics of the complex topic of long-distance dependencies, and in the process introduces some new analytic devices. Chapter 16 has a special status, introducing a streamlined reformulation of the overall theory in terms of an architecture based on 'signs', not rules. Appendix A summarizes the grammar of English developed in Chapters 1-13 and 15 (but includes neither the material on the dialects discussed in Chapter 14, nor the innovations proposed in Chapter 16).

We have many people to thank for their part in helping us bring this book to completion. First and foremost, we would like to thank three people: Emily Bender (who, as a course assistant and close colleague, commented on many drafts and made contributions throughout the text), Georgia Green (whose painstakingly detailed and helpful comments emerged from her experiences using our text at the University of Illinois at Urbana-Champaign), and Bob Carpenter (whose detailed comments on a near-final draft led us to overhaul the 'squiggly bits', as one of our British colleagues likes to put it). Others who gave us detailed comments on earlier drafts are: Gertraud Benke, Frank van Eynde, Daffyd Gibbon, Adam Przepiorkowski, and Gregory Stump. Frank, Daffyd, and Greg also gave us the benefit of their experiences using our text in their own classes. Special thanks are also due to Dick Hudson and Paul Kay for their help with Appendix B. Others who helped us one way or the other are: Farrell Ackerman, Louise Auerhahn, John Baugh, Renee Blake, Bob Borsley, Amy Brynolfson, Chris Callison-Burch, Myong-hi Chai, Brady Clark, Ann Copestake, Erica Denham, Colin Drake, Penny Eckert, Dan Flickinger, Ryan Ginstrom, Mark Goldenson, Lisa Green, Scott Guffey, Matt Kodoma, Jean-Pierre Koenig, Zakiyyah Langford, Chungmin Lee, Hanjung Lee, Rob Malouf, Michael McDaid, Brian Milch, Toshiaki Nishihara, Susanne Riehemann, Dave McKercher, John Rickford, Rachel Nordlinger, Paul Postal, Geoffrey Pullum, Scott Schwenter, Peter Sells, Stuart Tannock, Shiao Wei Tham, Ida Toivonen, Judith Tonhauser, Louise Vigeant, Rick Warren, and Gert Webelhuth. We would also like to thank Dikran Karagueuzian, Director of CSLI Publications, for his multi-facetted support and patience, as well as Tony Gee and Maureen Burke for their help in matters of production. We also acknowledge the support of an Irvine Multicultural Curriculum grant to Stanford University.

This book was written at Stanford's Center for the Study of Language Information - an ideal environment for thought and writing. Thanks to John Perry for keeping CSLI a perfect work environment and to Emma Pease for keeping the computers humming. Some of the material in 
this text is based on research conducted in part under the auspices of CSLI's LINGuistic Grammars online (LINGO) project. In that connection, we gratefully acknowledge support from the Bundesministerium für Bildung, Wissenschaft, Forschung, und Technologie (BMBF), who support LINGO's participation in the VerbmoBIL project under Grant FKZ:01IV7024. 


\section{Introduction}

\subsection{Two Conceptions of Grammar}

The reader may wonder, why would a college offer courses on grammar a topic that is usually thought of as part of junior high school curriculum (or even GRAMMAR school curriculum)? Well, the topic of this book is not the same thing that most people probably think of as grammar.

What is taught as grammar in primary and secondary school is what linguists call 'prescriptive grammar'. It consists of admonitions not to use certain forms or constructions that are common in everyday speech. A prescriptive grammar might contain rules like:

Be sure to never split an infinitive.

Prepositions are bad to end sentences with.

As modern linguists we think that prescriptive grammar is for the most part a pointless activity. We view human language as a phenomenon amenable to scientific investigation, rather than something to be regulated by the decrees of authorities. Your seventh grade math teacher might have discussed the time the Indiana legislature almost passed a bill establishing the value of $\pi$ as 3, and everybody in class no doubt laughed at such foolishness. Linguists regard prescriptive grammar as silly in much the same way: Natural phenomena simply cannot be legislated.

Of course, we do not deny the existence of powerful social and economic reasons for learning the grammatical norms of educated people. ${ }^{1}$ How these norms get established and their influence on the evolution of languages are fascinating questions in sociolinguistics and historical linguistics, but they are beyond the scope of this book. Similarly, we will

\footnotetext{
${ }^{1}$ By the same token, there may well be good economic reasons for standardizing a decimal approximation to $\pi$ (though 3 is almost certainly far too crude an approximation for most purposes).
} 


\section{2 / Syntactic Theory: A Formal Introduction}

not address issues of educational policy, except to say that in dismissing traditional (prescriptive) grammar instruction, we are not denying that attention to linguistic structure in the classroom can turn students into more effective speakers and writers. Indeed, we would welcome more enlightened grammar instruction in the schools. See Nunberg (1983) for an insightful discussion of these issues.

So, if modern grammarians don't worry about split infinitives and the like, then what do they study? It turns out that human languages are amazingly complex systems, whose inner workings can be investigated in large part simply by consulting the intuitions of native speakers. Here are some examples from English.

Example 1 The adjectives unlikely and improbable are virtually synonymous: we talk about unlikely or improbable events or heroes, and we can paraphrase It is improbable that Lee will be elected by saying It is unlikely that Lee will be elected. This last sentence is synonymous with Lee is unlikely to be elected. So why does it sound so strange to say * Lee is improbable to be elected? (In keeping with standard linguistic practice, we will use an asterisk to mark an expression that is not well formed - that is, that doesn't 'sound good' to our ears.)

Example 2 The sentences They saw Pat with Chris and They saw Pat and Chris are near paraphrases. But if you didn't catch the second name, it would be far more natural to ask Who did they see Pat with? than it would be to ask *Who did they see Pat and? Why do these two nearly identical sentences differ with respect to how we can question their parts? Notice, by the way, that the question that sounds well formed (or 'grammatical' in the linguist's sense) is the one that violates a standard prescriptive rule. The other sentence is so blatantly deviant that prescriptivists would never think to comment on the impossibility of such sentences. Prescriptive rules typically arise because human language use is innovative, leading languages to change. If people never use a particular construction - like the bad example above - there's no point in bothering to make up a prescriptive rule to tell people not to use it.

Example 3 The two sentences Something disgusting has slept in this bed and Something disgusting has happened in this bed appear on the surface to be grammatically completely parallel. So why is it that the first has a passive counterpart: This bed has been slept in by something disgusting, whereas the second doesn't: * This bed has been happened in by something disgusting?

These are the sorts of questions contemporary grammarians try to 
answer. The first two will eventually be addressed in this text, but the third will not. ${ }^{2}$ The point of introducing them here is to illustrate a fundamental fact that underlies all modern work in theoretical syntax:

Every normal speaker of any natural language has acquired an immensely rich and systematic body of unconscious knowledge, which can be investigated by consulting speakers' intuitive judgments.

In other words, knowing a language involves mastering an intricate system full of surprising regularities and idiosyncrasies. Languages are phenomena of considerable complexity, which can be studied scientifically. That is, we can formulate general hypotheses about linguistic structure and test them against the facts of particular languages.

The study of grammar on this conception is a field in which hypothesistesting is particularly easy: the linguist can simply ask native speakers whether the predictions regarding well formedness of crucial sentences are correct. ${ }^{3}$ The term 'syntax' is often used instead of 'grammar' in technical work in linguistics. While the two terms are sometimes interchangeable, 'grammar' may also be used more broadly to cover all aspects of language structure; 'syntax', in contrast, refers only to the ways in which words combine into phrases, and phrases into sentences the form or structure of well formed expressions. Although the boundaries are not sharp, 'syntax' contrasts with 'semantics' (the study of linguistic meaning), 'morphology' (the study of word structure), and 'phonology' (the study of the sound patterns of language) in ways that 'grammar' does not.

\This symbol before a problem indicates that it should not be skipped. The problem involves something that will either be elaborated upon or else simply incorporated into subsequent chapters.

\footnotetext{
${ }^{2}$ For extensive discussion of the third question, see Postal (1986).

${ }^{3}$ This methodology is not without its pitfalls. Judgments of acceptability show considerable variation across speakers. Moreover, they can be heavily influenced by context, both linguistic and non-linguistic. Since linguists rarely make any effort to control for such effects, not all of the data employed in the syntax literature should be accepted without question. On the other hand, many judgments are so unequivocal that they can clearly be relied on. In more delicate cases, many linguists have begun to supplement judgments with data from actual usage, by examining grammatical patterns found in written and spoken corpora. The use of multiple sources and types of evidence is always a good idea in empirical investigations. See Schütze (1996) for a detailed discussion of methodological issues surrounding the use of judgment data in syntactic research.
} 


\section{\) Problem 1: Judging Examples}

Indicate whether each of the following examples is acceptable or unacceptable. If it is unacceptable, give an intuitive explanation of what is wrong with it, i.e. whether it:

a. fails to conform to the rules of English grammar,

b. is grammatically well-formed, but bizarre in meaning (if so, explain why), or

c. contains a feature of grammar that occurs only in a particular variety of English, for example, slang, or a regional dialect; if so, identify the feature. Is it stigmatized in comparison with 'standard' English?

If you are uncertain about any judgments, feel free to consult with others. Nonnative speakers of English, in particular, are encouraged to compare their judgments with others.

(i) Kim and Sandy is looking for a new bicycle.

(ii) Have you the time?

(iii) I've never put the book.

(iv) The boat floated down the river sank.

(v) It ain't nobody goin to miss nobody.

(vi) Terry really likes they.

(vii) Chris must liking syntax.

(viii) Aren't I invited to the party?

(ix) They wondered what each other would do.

(x) There is eager to be fifty students in this class.

(xi) They persuaded me to defend themselves.

(xii) Strings have been pulled many times to get people into Harvard.

(xiii) This is the kind of problem that my doctor is easy to talk to about.

(xiv) A long list of everyone's indiscretions were published in the newspaper.

(xv) Which chemical did you mix the hydrogen peroxide and?

(xvi) There seem to be a good feeling developing among the students.

\subsection{An Extended Example}

To get a feel for the sort of research syntacticians conduct, consider the following question: 
In which linguistic environments do English speakers normally use reflexive pronouns (i.e. forms like herself or ourselves), and where does it sound better to use a nonreflexive pronoun (e.g. her, she, us, or $w e)$ ?

To see how to approach an answer to this question, consider, first, some basic examples:

(1) a. ${ }^{*}$ We like us.

b. We like ourselves.

c. She likes her. [where, she $\neq$ her]

d. She likes herself.

e. Nobody likes us.

f. * Nobody likes ourselves.

g. * Ourselves like us.

h. ${ }^{*}$ Ourselves like ourselves.

These examples suggest a generalization along the following lines:

Hypothesis I: A reflexive pronoun can appear in a sentence only if that sentence also contains a preceding expression that has the same reference (i.e. a preceding COREFERENTIAL expression); a nonreflexive pronoun cannot appear in a sentence that contains such an expression.

The following examples are different from the previous ones in various ways, so they provide a first test of our hypothesis:

(2) a. She voted for her. [she $\neq$ her]

b. She voted for herself.

c. We voted for her.

d. ${ }^{*}$ We voted for herself.

e. ${ }^{*}$ We gave us presents.

f. We gave ourselves presents.

g. ${ }^{*}$ We gave presents to us.

h. We gave presents to ourselves.

i. ${ }^{*}$ We gave us to the cause.

j. We gave ourselves to the cause.

k. *Nobody told us about us.

l. Nobody told us about ourselves.

m. * Nobody told ourselves about us.

n. *Nobody told ourselves about ourselves.

These examples are all predicted by Hypothesis I, lending it some initial plausibility. But here are some counterexamples:

(3) a. We think that nobody likes us.

b. ${ }^{*}$ We think that nobody likes ourselves. 


\section{6 / Syntactic Theory: A Formal Introduction}

According to our hypothesis, our judgments in $(3 \mathrm{a}, \mathrm{b})$ should be reversed. Intuitively, the difference between these examples and the earlier ones is that the sentences in (3) contain subordinate clauses, whereas (2) and (1) contain only simple sentences.

\section{Problem 2: Applying Hypothesis I}

But it isn't actually the mere presence of the subordinate clauses in (3) that makes the difference. To see why, consider the following, which contain subordinate clauses but are covered by Hypothesis I.

(i) We think that she voted for her. [she $\neq$ her]

(ii) We think that she voted for herself.

(iii) ${ }^{*}$ We think that herself voted for her.

(iv)* We think that herself voted for herself.

a. Explain how Hypothesis I accounts for the data in (i)-(iv).

b. What is it about the subordinate clauses in (3) that makes them different from those in (i)-(iv) with respect to Hypothesis I?

Given our investigation so far, then, we might revise Hypothesis I to the following:

Hypothesis II: A reflexive pronoun can appear in a clause only if that clause also contains a preceding, coreferential expression; a nonreflexive pronoun cannot appear in any clause that contains such an expression.

For sentences with only one clause (such as (1)-(2)), Hypothesis II makes the same predictions as Hypothesis I. But it correctly permits (3a) because we and us are in different clauses, and it rules out (3b) because we and ourselves are in different clauses.

However, Hypothesis II as stated won't work either:

(4) a. Our friends like us.

b. ${ }^{*}$ Our friends like ourselves.

c. Those pictures of us offended us.

d. ${ }^{*}$ Those pictures of us offended ourselves.

e. We found a letter to us in the trash.

f. ${ }^{*}$ We found a letter to ourselves in the trash.

What's going on here? The acceptable examples of reflexive pronouns have been cases (i) where the reflexive pronoun is functioning as an object of a verb (or the object of a preposition that goes with the verb) and (ii) where the antecedent - that is, the expression it is coreferential 
with - is the subject or a preceding object of the same verb. If we think of a verb as denoting some sort of action or state, then the subject and objects (or prepositional objects) normally denote the participants in that action or state. These are often referred to as the ARGUMENTS of the verb. In the examples in (4), unlike many of the earlier examples, the reflexive pronouns and their antecedents are not arguments of the same verb (or, in other words, they are not COARGUMENTs). For example in (4b), our is just part of the subject of the verb like, and hence not itself an argument of the verb; rather, it is our friends that denotes participants in the liking relation. Similarly, in (4e) the arguments of found are we and a letter to us; us is only part of an argument of found.

So to account for these differences, we can consider the following:

Hypothesis III: A reflexive pronoun must be an argument of a verb that has another preceding argument with the same reference. A nonreflexive pronoun cannot appear as an argument of a verb that has a preceding coreferential argument.

Each of the examples in (4) contains two coreferential expressions (we, us, our, or ourselves), but none of them contains two coreferential expressions that are arguments of the same verb. Hypothesis III correctly rules out just those sentences in (4) in which the second of the two coreferential expressions is the reflexive pronoun ourselves.

Now consider the following cases:

(5) a. Vote for us!

b. ${ }^{*}$ Vote for ourselves!

c. ${ }^{*}$ Vote for you!

d. Vote for yourself!

In (5d), for the first time, we find a well formed reflexive with no antecedent. If we don't want to append an ad hoc codicil to Hypothesis III ${ }^{4}$ we will need to posit a hidden subject (namely, you) in imperative sentences.

Similar arguments can be made with respect to the following sentences.

(6) a. We appealed to him 1 to vote for $\operatorname{him}_{2} .\left[\operatorname{him}_{1} \neq \operatorname{him}_{2}\right]$

b. We appealed to him to vote for himself.

c. We appealed to him to vote for us.

(7) a. We appeared to him to vote for him.

\footnotetext{
${ }^{4}$ For example, an extra clause that says: 'unless the sentence is imperative, in which case a second person reflexive is well formed and a second person nonreflexive pronoun is not.' This would rule out the offending case but not in any illuminating way that would generalize to other cases.
} 


\section{8 / Syntactic Theory: A Formal Introduction}

b. ${ }^{*}$ We appeared to him to vote for himself.

c. We appeared to him to vote for ourselves.

In (6), the pronouns indicate that him is functioning as the subject of vote, but it looks like it is the object of the preposition to, not an argument of vote. Likewise, in (7), the pronouns suggest that we should be analyzed as an argument of vote, but its position suggests that it is an argument of appeared. So, on the face of it, such examples are problematical for Hypothesis III, unless we posit arguments that are in some sense missing. We will return to the analysis of such cases in later chapters.

\section{@) Problem 3: Reciprocals}

English has a 'reciprocal' expression each other (think of it as a single word for present purposes), which behaves in some ways like a reflexive

pronoun. For example, a direct object each other must refer to the subject, and a subject each other cannot refer to the direct object:

(i) They like each other.

(ii)*Each other like(s) them.

A. Construct examples parallel to those in (1)-(3), replacing the reflexives with reciprocals. Is the basic behavior of reciprocals similar to that of reflexives?

B. Construct examples parallel to those in (5)-(7), replacing the reflexives with reciprocals. Is the behavior of reciprocals similar to that of reflexives in imperative sentences and in sentences containing appeal and appear?

C. Are there any constraints that the reciprocal imposes on its antecedent that reflexives don't impose? [Hint: what change to (1d) and (6b) did you have to make in order to construct the corresponding well formed reciprocal sentence?]

D. Consider the following contrast:

They lost each other's books.

* They lost themselves' books.

Discuss how such examples bear on the applicability of Hypothesis III to reciprocals. [Hint: before you answer the question, think about what the verbal arguments are in the above sentences.]

You can see that things get quite complex quite fast, requiring abstract notions like 'coreference', being 'arguments of the same verb', and allowing arguments to be missing from the sentence but 'understood', 
for purposes of the rules for pronoun type. And we've only scratched the surface of this problem. For example, all the versions of the rules we have come up with so far predict that nonreflexive forms of a pronoun should appear only in positions where their reflexive counterparts are impossible. But this is not quite true, as the following examples illustrate:

(8) a. We wrapped the blankets around us.

b. We wrapped the blankets around ourselves.

c. We admired the pictures of us in the album.

d. We admired the pictures of ourselves in the album.

It should be evident by now that formulating precise rules characterizing where English speakers use reflexive pronouns and where they use nonreflexive pronouns will be a difficult task. We will return to this task in Chapter 7. Our reason for discussing it here was to emphasize the following points:

- Normal use of language involves the mastery of a complex system, which is not directly accessible to consciousness.

- Speakers' tacit knowledge of language can be studied by formulating hypotheses and testing their predictions against intuitive judgments of well formedness.

- The theoretical machinery required for a viable grammatical analysis could be quite abstract.

\subsection{Remarks on the History of the Study of Grammar}

The conception of grammar we've just presented is quite a recent development. Until about 1800 , almost all linguistics was primarily prescriptive. Traditional grammar (going back hundreds, even thousands of years, to ancient India and ancient Greece) was developed largely in response to the inevitable changing of language, which is always (even today) seen by most people as its deterioration. Prescriptive grammars have always been attempts to codify the 'correct' way of talking. Hence, they have concentrated on relatively peripheral aspects of language structure. On the other hand, they have also provided many useful concepts for the sort of grammar we'll be doing. For example, our notion

of parts of speech, as well as the most familiar examples (such as noun and verb) come from the ancient Greeks.

A critical turning point in the history of linguistics took place at the end of the eighteenth century. It was discovered at that time that there was a historical connection among most of the languages of Europe, as well as Sanskrit and other languages of India (plus some lan- 


\section{0 / Syntactic Theory: A Formal Introduction}

guages in between). ${ }^{5}$ This led to a tremendous flowering of the field of historical linguistics, centered on reconstructing the family tree of the Indo-European languages by comparing the modern languages with each other and with older texts. Most of this effort concerned the correspondences between individual words and the sounds within those words. But syntactic comparison and reconstruction was also initiated during this period.

In the early twentieth century, many linguists, following the lead of the Swiss scholar Ferdinand de Saussure, turned their attention from the historical (or 'diachronic' ${ }^{6}$ ) study to the 'synchronic'7 analysis of languages - that is, to the characterization of languages at a given point in time. The attention to synchronic studies encouraged the investigation of languages that had no writing systems, which are much harder to study diachronically since there is no record of their earlier forms.

In the United States, these developments led linguists to pay far more attention to the indigenous languages of the Americas. Beginning with the work of the anthropological linguist Franz Boas, American linguistics for the first half of the twentieth century was very much concerned with the immense diversity of languages. The Indo-European languages, which were the focus of most nineteenth-century linguistic research, constitute only a tiny fraction of the approximately five thousand known languages. In broadening this perspective, American linguists put great stress on developing ways to describe languages that would not forcibly impose the structure of a familiar language (such as Latin or English) on something very different; most, though by no means all, of this work emphasized the differences among languages. Some linguists, notably Edward Sapir and Benjamin Lee Whorf, talked about how language could provide insights into how people think. They tended to emphasize alleged differences among the thought patterns of speakers of different languages. For our purposes, their most important claim is that the structure of language can provide insight into human cognitive processes. This idea has wide currency today, and, as we shall see below, it constitutes one of the most interesting motivations for studying syntax.

In the period around World War II, a number of things happened to set the stage for a revolutionary change in the study of syntax. One was that great advances in mathematical logic provided formal tools that seemed well suited for application to studying natural languages.

\footnotetext{
${ }^{5}$ The discovery is often attributed to Sir William Jones who announced such a relationship in a 1786 address, but others had noted affinities among these languages before him.

${ }^{6}$ From the Greek: dia 'across' plus chronos 'time'

${ }^{7}$ syn 'same, together' plus chronos.
} 
A related development was the invention of the computer. Though early computers were unbelievably slow and expensive by today's standards, some people immediately saw their potential for natural language applications, such as machine translation or voice typewriters.

A third relevant development around mid-century was the decline of behaviorism in the social sciences. Like many other disciplines, linguistics in America at that time was dominated by behaviorist thinking. That is, it was considered unscientific to posit mental entities or states to account for human behaviors; everything was supposed to be described in terms of correlations between stimuli and responses. Abstract models of what might be going on inside people's minds were taboo. Around 1950 , some psychologists began to question these methodological restrictions, and arguing they made it impossible to explain certain kinds of facts. This set the stage for a serious rethinking of the goals and methods of linguistic research.

In the early 1950s, a young man named Noam Chomsky entered the field of linguistics. In the late ' 50 s, he published three things that revolutionized the study of syntax. One was a set of mathematical results, establishing the foundations of what is now called 'formal language theory'. These results have been seminal in theoretical computer science, and they are crucial underpinnings for computational work on natural language. The second was a book called Syntactic Structures that presented a new formalism for grammatical description and analyzed a substantial fragment of English in terms of that formalism. The third was a review of B. F. Skinner's (1957) book Verbal Behavior. Skinner was one of the most influential psychologists of the time, an extreme behaviorist. Chomsky's scathing and devastating review marks, in many people's minds, the end of behaviorism's dominance in American social science.

Since about 1960, Chomsky has been the dominant figure in linguistics. As it happens, the 1960s were a period of unprecedented growth in American academia. Most linguistics departments in the United States were established in the period between 1960 and 1980. This helped solidify Chomsky's dominant position.

One of the central tenets of the Chomskyan approach to syntax, known as 'generative grammar', has already been introduced: hypotheses about linguistic structure should be made precise enough to be testable. A second somewhat more controversial one is that the object of study should be the unconscious knowledge underlying ordinary language use. A third fundamental claim of Chomsky's concerns the biological basis of human linguistic abilities. We will return to this claim in the next section. 


\section{2 / Syntactic Theory: A Formal Introduction}

Within these general guidelines there is room for many different theories of grammar. Since the 1950s, generative grammarians have explored a wide variety of choices of formalism and theoretical vocabulary. We present a brief summary of these in Appendix B, to help situate the approach presented here within a broader intellectual landscape.

\subsection{Why Study Syntax?}

Students in syntax courses often ask about the point of such classes: why should one study syntax?

Of course, one has to distinguish this question from a closely related one: why DO people study syntax? The answer to that question is perhaps simpler: exploring the structure of language is an intellectually challenging and, for many people, intrinsically fascinating activity. It is like working on a gigantic puzzle - one so large that it could occupy many lifetimes. Thus, as in any scientific discipline, many researchers are simply captivated by the complex mysteries presented by the data themselves - in this case a seemingly endless, diverse array of languages past, present and future.

This reason is, of course, similar to the reason scholars in any scientific field pursue their research: natural curiosity and fascination with some domain of study. Basic research is not typically driven by the possibility of applications. Although looking for results that will be useful in the short term might be the best strategy for someone seeking personal fortune, it wouldn't be the best strategy for a society looking for long-term benefit from the scientific research it supports. Basic scientific investigation has proven over the centuries to have long-term payoffs, even when the applications were not evident at the time the research was carried out. For example, work in logic and the foundations of mathematics in the first decades of the twentieth century laid the theoretical foundations for the development of the digital computer, but the scholars who did this work were not concerned with its possible applications. Likewise, we don't believe there is any need for linguistic research to be justified on the basis of its foreseeable uses. Nonethe-

less, we will mention three interrelated reasons that one might have for studying the syntax of human languages.

\subsubsection{A Window on the Structure of the Mind}

One intellectually important rationale for the study of syntax has been offered by Chomsky. In essence, it is that language - and particularly, 
its grammatical organization - can provide an especially clear window on the structure of the human mind. ${ }^{8}$

Chomsky claims that the most remarkable fact about human language is the discrepancy between its apparent complexity and the ease with which children acquire it. The structure of any natural language is far more complicated than those of artificial languages or of even the most sophisticated mathematical systems. Yet learning computer languages or mathematics requires intensive instruction (and many students still never master them), whereas every normal child learns at least one natural language merely through exposure. This amazing fact cries out for explanation. ${ }^{9}$

Chomsky's proposed explanation is that most of the complexity of languages does not have to be learned, because much of our knowledge of it is innate: we are born knowing about it. That is, our brains are 'hardwired' to learn languages of certain types.

More generally, Chomsky has argued that the human mind is highly modular. That is, we have special-purpose 'mental organs' that are designed to do particular sorts of tasks in particular sorts of ways. The language organ (which, in Chomsky's view, has several largely autonomous submodules) is of particular interest because language is such a pervasive and unique part of human nature. All people use language, and (he claims) no other species is capable of learning anything much like human language. Hence, in studying the structure of human languages, we are investigating a central aspect of human nature.

This idea has drawn enormous attention not only from linguists but also from people outside linguistics, especially psychologists and philosophers. Scholars in these fields have been highly divided about Chomsky's innateness claims. Many cognitive psychologists see Chomsky's work as a model for how other mental faculties should be studied, while others argue that the mind (or brain) should be regarded as a general-purpose thinking device, without specialized modules. In philosophy, Chomsky provoked much comment by claiming that his work constitutes a modern version of Descartes' doctrine of innate ideas.

Chomsky's innateness thesis and the interdisciplinary dialogue it stimulated were major factors in the birth of the new interdisciplinary field of cognitive science in the 1970s. (An even more important factor was the rapid evolution of computers, with the concomitant growth

\footnotetext{
${ }^{8}$ See Katz and Postal (1991) for arguments against the dominant Chomskyan conception of linguistics as essentially concerned with psychological facts.

${ }^{9}$ Chomsky was certainly not the first person to remark on the extraordinary facility with which children learn language, but, by giving it a central place in his work, he has focused considerable attention on it.
} 


\section{4 / Syntactic Theory: A Formal Introduction}

of artificial intelligence and the idea that the computer could be used as a model of the mind.) Chomsky and his followers have been major contributors to cognitive science in the subsequent decades.

One theoretical consequence of Chomsky's innateness claim is that all languages must share most of their structure. This is because all children learn the languages spoken around them, irrespective of where their ancestors came from. Hence, the innate knowledge that Chomsky claims makes language acquisition possible must be common to all human beings. If this knowledge also determines most aspects of grammatical structure, as Chomsky says it does, then all languages must be essentially alike. This is a very strong universal claim.

In fact, Chomsky tends to use the term 'Universal Grammar' to mean the innate endowment that permits language acquisition. A great deal of the syntactic research since the late 1960s has been concerned with identifying linguistic universals, especially those that could plausibly be claimed to reflect innate mental structures operative in language acquisition. As we proceed to develop the grammar in this text, we will ask which aspects of our grammar are peculiar to English and which might plausibly be considered universal.

If Chomsky is right about the innateness of the language faculty, it has a number of practical consequences, especially in fields like language instruction and therapy for language disorders. For example, since there is evidence that people's innate ability to learn languages is far more powerful very early in life (specifically, before puberty) than later, it seems most sensible that elementary education should have a heavy emphasis on language, and that foreign language instruction should not be left until secondary school, as it is in most American schools today.

\subsubsection{A Window on the Mind's Activity}

If you stop and think about it, it's really quite amazing that people succeed in communicating by using language. Language seems to have a number of design properties that get in the way of efficient and accurate communication of the kind that routinely takes place.

First, it is massively ambiguous. Individual words, for example, often have not just one but a number of meanings, as illustrated by the English examples in (9).

(9) a. Leslie used a pen. ('a writing implement')

b. We put the pigs in a pen. ('a fenced enclosure')

c. They should pen the letter quickly. ('to write')

d. The judge sent them to the pen for a decade. ('a penitentiary')

(10) a. The cheetah will run down the hill. ('to move fast') 
b. The president will run. ('to be a political candidate')

c. The car won't run. ('to function properly')

d. This trail should run over the hill. ('to lead')

e. This dye will run. ('to dissolve and spread')

f. This room will run $\$ 200$ or more. ('to cost')

g. She can run an accelerator. ('to operate')

h. They will run the risk. ('to incur')

i. These stockings will run. ('to tear')

j. There is a run in that stocking. ('a tear')

k. We need another run to win. ('a score in baseball')

1. Fats won with a run of 20. ('a sequence of successful shots in a game of pool')

To make matters worse, many sentences are ambiguous not because they contain ambiguous words, but rather because the words they contain can be related to one another in more than one way, as illustrated in (11).

(11) a. Lee saw the student with a telescope.

b. I forgot how good beer tastes.

(11a) can be interpreted as providing information about which student Lee saw (the one with a telescope) or about what instrument Lee used (the telescope) to see the student. Similarly, (11b) can convey either that the speaker forgot how GOOD beer (as opposed to bad or mediocre beer) tastes, or else that the speaker forgot that beer (in general) tastes good. These differences are often discussed in terms of which element a word like with or good is modifying (the verb or the noun).

Lexical and modificational ambiguity interact to produce a bewildering array of (often comical) ambiguities, like these:

(12) a. Visiting relatives can be boring.

b. If only Superman would stop flying planes!

c. That's a new car dealership.

d. I know you like the back of my hand.

e. An earthquake in Romania moved buildings as far away as Moscow and Rome.

f. The German shepherd turned on its master.

g. I saw that gas can explode.

h. Max is on the phone now.

i. The only thing capable of consuming this food has four legs and flies.

j. I saw her duck. 


\section{Problem 4: Ambiguity}

Give a brief description of each ambiguity illustrated in (12). We will return to many of these examples - or closely related ones - later in the book.

This is not the end of the worrisome design properties of human language. Many words are used to refer to different things on different occasions of utterance. Pronouns like them, (s)he, this, and that pick out different referents almost every time they are used. Even seemingly determinate pronouns like we don't pin down exactly which set of people the speaker is referring to (compare We have two kids/a city council/a lieutenant governor/50 states/oxygen-based life here). Moreover, although certain proper names like Sally Ride or Sandra Day O'Connor might reliably pick out the same person almost every time they are used, most conversations are full of uses of names like Chris, Pat, Leslie, Sandy, Bo, etc. that vary wildly in their reference, depending on who's talking to whom and what they're talking about.

Add to this the observation that some expressions seem to make reference to 'covert elements' that don't exactly correspond to any one word. So expressions like in charge and afterwards make reference to missing elements of some kind - bits of the meaning that have to be supplied from context. Otherwise, discourses like the following wouldn't make sense, or would at best be incomplete.

(13) a. I'm creating a committee. Kim - you're in charge. [in charge of what? - the committee]

b. Lights go out at ten. There will be no talking afterwards. [after what? - after ten]

The way something is said can also have a significant effect on the meaning expressed. A rising intonation, for example, on a one word utterance like Coffee? would very naturally convey 'Do you want some

coffee?' Alternatively, it might be used to convey that 'coffee' is being offered as a tentative answer to some question (say, What was Columbia's former number-one cash crop?). Or even, in the right context, the same utterance might be used in seeking confirmation that a given liquid was in fact coffee. Intonational meaning can be vivified in striking ways.

Finally, note that communication using language leaves a great deal unsaid. If I say to you Can you give me a hand here? I'm not just requesting information about your abilities, I'm asking you to help me out. This is the unmistakable communicative intent, but it wasn't lit- 
erally said. Other examples of such inference are similar, but perhaps more subtle. A famous example ${ }^{10}$ is the letter of recommendation saying that the candidate in question has outstanding penmanship (and saying nothing more than that!).

Summing all this up, what we have just seen is that the messages conveyed by utterances of sentences are multiply ambiguous, vague, and uncertain. Yet somehow, in spite of this, those of us who know the language are able to use it to trasmit messages to one another with considerable accuracy - far more accuracy than the language itself would seem to permit. Those readers who have any experience with computer programming or with mathematical logic will appreciate this dilemma instantly. The very idea of designing a programming language or a logical language whose predicates are ambiguous or whose variables are left without assigned values is unthinkable. No computer can process linguistic expressions unless it 'knows' precisely what the expressions mean and what to do with them.

The fact of the matter is that human language-users are able to do something that modern science doesn't understand well enough to replicate via computer. Somehow, people are able to use nonlinguistic information in such a way that they are never even aware of most of the unwanted intepretations of words, phrases, and sentences. Consider again the various senses of the word pen. The 'writing implement' sense is more common - that is, more frequent in the language you've been exposed to (unless you're a farmer or a prisoner) - and so there is an inherent bias toward that sense. You can think of this in terms of 'weighting' or 'degrees of activation' of word senses. In a context where farm animals are being discussed, though, the weights shift - the senses more closely associated with the subject matter of the discourse become stronger in this case. As people direct their attention to and through a given dialogue, these sense preferences can fluctuate considerably. The human sense selection capability is incredibly robust, yet we have only minimal understanding of the cognitive mechanisms that are at work. How exactly does context facilitate our ability to locate the correct sense?

In other cases, it's hard to explain disambiguation so easily in terms of affinity to the domain of discourse. Consider the following contrast:

(14) a. They found the book on the table.

b. They found the book on the atom.

${ }^{10}$ This example is one of many due to the late H. Paul Grice, the philosopher whose work forms the starting point for much work in linguistics on problems of PRAGMATICS, how people 'read between the lines' in natural conversation; see Grice (1989). 
The preposition on modifies the verb in (14a) and the noun in (14b), yet it seems that nothing short of rather complex reasoning about the relative size of objects would enable someone to choose which meaning (i.e. which modification) made sense. And we do this kind of thing very quickly, as you can see from (15).

(15) After finding the book on the atom, Sandy went into class, confident that there would be no further obstacles to getting that term paper done.

When you read this sentence, there's no strong feeling that you were 'garden pathed', that is, derailed by an incorrect interpretation midsentence. The decision about how to construe on the atom is made well before the words class or confident are even encountered.

When we process language, we integrate encyclopedic knowledge, plausibility information, frequency biases, discourse information, and perhaps more. Although we don't yet know exactly how we do it, it's clear that we do it very quickly and reasonably accurately. Trying to model this integration is probably the most important research task facing the study of language in the coming millenium.

Syntax plays a crucial role in all this. It imposes constraints on how sentences can or cannot be construed. So the discourse context may provide a bias for the 'fenced enclosure' sense of pen, but it is the syntactic context that determines whether pen occurs as a noun or a verb. Syntax is also of particular importance to the development of language-processing models, because it is a domain of knowledge that can be characterized perhaps more precisely than some of the other kinds of knowledge that are involved.

When we understand how language processing works, we probably will also understand quite a bit more about how cognitive processes work in general. This in turn will no doubt enable us to develop better ways of teaching language. We should also be better able to help people who have communicative impairments (and more general cognitive disorders). The study of human language-processing is an important sub-area of the study of human cognition, and it is one that can benefit immensely from precise characterization of linguistic knowledge of the sort that syntacticians seek to provide.

\subsubsection{Natural Language Technologies}

Grammar has more utilitarian applications, as well. One of the most promising areas for applying syntactic research is in the development of useful and robust natural language technologies. What do we mean by 'natural language technologies'? Roughly, what we have in mind is any 
sort of computer application that involves natural languages like English, Japanese, or Swahili in essential ways. These include devices that translate from one language into another (or perhaps more realistically, that provide translation assistance to someone with less than perfect command of a language), that understand spoken language (to varying degrees), that automatically retrieve information from large bodies of text stored on-line, or that help the disabled to communicate.

There is one application that obviously must incorporate a great deal of grammatical information, namely, grammar checkers for word processing. Most modern word processing systems include a grammar checking facility, along with a spell-checker. These tend to focus on the concerns of prescriptive grammar, which may be appropriate for the sorts of documents they are generally used on, but which often leads to spurious 'corrections'. Moreover, they typically depend on superficial pattern-matching for finding likely grammatical errors, rather than employing in-depth grammatical analysis. In short, grammar checkers can benefit from incorporating the results of research in syntax.

Other computer applications in which grammatical knowledge is clearly essential include those in which well formed natural language output must be generated. For example, reliable software for translating one language into another must incorporate some representation of the grammar of the target language. If it did not, it would either produce ill-formed output, or it would be limited to some fixed repertoire of sentence templates.

Even where usable natural language technologies can be developed that are not informed by grammatical research, it is often the case that they can be made more robust by including a principled syntactic component. For example, Stanford University's Center for the Study of Language and Information is developing software to reduce the number of keystrokes needed to input text. This has many potential uses, including facilitating the use of computers by individuals with motor disabilities or temporary impairments such as carpal tunnel syndrome. It is clear that knowledge of the grammar of English can help in predicting what words are likely to come next at an arbitrary point in a sentence. Software that makes such predictions and offers the user a set of choices for the next word or the remainder of an entire sentence - each of which can be inserted with a single keystroke - can be of great value in a wide variety of situations. Word prediction can likewise facilitate the disambiguation of noisy signals in continuous speech recognition and handwriting recognition.

But it's not obvious that all types of natural language technologies need to be sensitive to grammatical information. Say, for example, 


\section{0 / Syntactic Theory: A Formal Introduction}

we were trying to design a system to extract information from an online database by typing in English questions (rather than requiring use of a special database query language, as is the case with most existing database systems). Some computer scientists have argued that full grammatical analysis of the queries is not necessary. Instead, they claim, all that is needed is a program that can extract the essential semantic information out of the queries. Many grammatical details don't seem necessary in order to understand the queries, so it has been argued that they can be ignored for the purpose of this application. Even here, however, a strong case can be made for the value of including a syntactic component in the software.

To see why, imagine that we are using a database in a law office, containing information about the firm's past and present cases, including records of witnesses' testimony. Without designing the query system to pay careful attention to certain details of English grammar, there are questions we might want to ask of this database that could be misanalyzed and hence answered incorrectly. For example, consider our old friend, the rule for reflexive and nonreflexive pronouns. Since formal database query languages don't make any such distinction, one might think it wouldn't be necessary for an English interface to do so either. But suppose we asked one of the following questions:

(16) a. Which witnesses work with defendants who supervise them?

b. Which witnesses work with defendants who supervise themselves?

Obviously, these two questions will have different answers, so an English language 'front end' that didn't incorporate some rules for distinguishing reflexive and nonreflexive pronouns would sometimes give wrong answers.

In fact, it isn't enough to tell reflexive from nonreflexive pronouns: a database system would need to be able to tell different reflexive pronouns

apart. The next two sentences, for example, are identical except for the plurality of the reflexive pronouns:

(17) a. List all witnesses for the defendant who represented himself.

b. List all witnesses for the defendant who represented themselves.

Again, the appropriate answers would be different. So a system that didn't pay attention to whether pronouns are singular or plural couldn't be trusted to answer correctly.

Even features of English grammar that seem useless - things that appear to be entirely redundant - are needed for the analysis of some sentences that might well be used in a human-computer interaction. 
Consider, for example, English subject-verb agreement (a topic we will return to in some detail in Chapters 2-4). Since subjects are marked as singular or plural - the dog vs. the dogs - marking verbs for the same thing - barks vs. bark - seems to add nothing. We would have little trouble understanding someone who always left subject agreement off of verbs. In fact, English doesn't even mark past-tense verbs (other than forms of be) for subject agreement. But we don't miss agreement in the past tense, because it is semantically redundant. One might conjecture, therefore, that an English database querying system might be able simply to ignore agreement.

However, once again, examples can be constructed in which the agreement marking on the verb is the only indicator of a crucial semantic distinction. This is the case with the following pair:

(18) a. List associates of each witness who speaks Spanish.

b. List associates of each witness who speak Spanish.

In the first sentence, it is the witnesses in question who are the Spanishspeakers; in the second, it is their associates. These will, in general, not lead to the same answer.

Such examples could be multiplied, but these should be enough to make the point: Building truly robust natural language technologies that is, software that will allow you to interact with your computer in YOUR language, rather than in ITS language - requires careful and detailed analysis of grammatical structure and how it influences meaning. Shortcuts that rely on semantic heuristics, guesses, or simple patternmatching will inevitably make mistakes.

Of course, this is not to deny the value of clever engineering and statistical approximation. Indeed, the rapid emergence of natural language technology that is taking place in the world today owes at least as much to this as it does to the insights of linguistic research. Our point is rather that in the long run, especially when the tasks to be performed take on more linguistic subtlety and the accuracy of the performance becomes more critical, the need for more subtle linguistic analysis will likewise become more acute.

In short, although most linguists may be motivated primarily by simple intellectual curiosity, the study of grammar has some fairly obvious uses, even in the relatively short term.

\subsection{Conclusion}

In this chapter, we have drawn an important distinction between prescriptive and descriptive grammar. In addition, we provided an illustration of the kind of syntactic puzzles we will focus on later in the 
text. ${ }^{11}$ Finally, we provided an overview of some of the reasons people have found the study of syntax inherently interesting or useful. In the next chapter, we look at some simple formal models that might be proposed for the grammars of natural languages and discuss some of their shortcomings.

\subsection{Further Reading}

An entertaining and knowledgeable exposition of modern linguistics and its implications is provided by Pinker (1994). A somewhat more scholarly survey with a slightly different focus is presented by Jackendoff (1994). For discussion of prescriptive grammar, see Nunberg (1983) and Chapter 12 of Pinker's book (an edited version of which was published in The New Republic, January 31, 1994). For an overview of linguistic science in the nineteenth century, see Pedersen (1959). A succinct survey of the history of linguistics is provided by Robins (1967).

Among Chomsky's many writings on the implications of language acquisition for the study of the mind, we would especially recommend Chomsky (1959) and Chomsky (1972); a more recent, but much more difficult work is Chomsky (1986a). There have been few recent attempts at surveying work in (human or machine) sentence processing. J. A. Fodor, Bever, and Garrett (1974) is a comprehensive review of early psycholinguistic work within the Chomskyan paradigm, but it is now quite dated. Garrett (1990) and J. D. Fodor (1995) are more recent, but much more limited in scope.

\footnotetext{
${ }^{11}$ Our discussion of reflexive and nonreflexive pronouns borrows heavily from the presentation in Perlmutter and Soames (1979: chapters 2 and 3).
} 


\section{Some Simple Theories of Grammar}

\section{$2.1 \quad$ Introduction}

Among the key points in the previous chapter were the following:

- Language is rule-governed.

- The rules aren't the ones we were taught in school.

- Much of our linguistic knowledge is unconscious, so we have to get at it indirectly; one way of doing this is to consult intuitions of what sounds good.

In this text, we have a number of objectives. First, we will work toward developing a set of rules that will correctly predict the acceptability of (a large subset of) English sentences. The ultimate goal is a grammar that can tell us for any arbitrary string of English words whether or not it is a well-formed sentence. Thus we will again and again be engaged in the exercise of formulating a grammar that generates a certain set of word strings - the sentences predicted to be grammatical according to that grammar. We will then examine particular members of that set and ask ourselves: 'Is this example acceptable?' The goal of this enterprise is to make the set of sentences generated by our grammar match the set of sentences that we intuitively judge to be acceptable. ${ }^{1}$

A second of our objectives is to consider how the grammar of English differs from the grammar of other languages (or how the grammar of standard American English differs from those of other varieties of English). The conception of grammar we develop will involve general principles that are just as applicable (as we will see in various exercises)

\footnotetext{
${ }^{1}$ Of course there may be other interacting factors that cause grammatical sentences to sound less than fully acceptable - see Chapter 9 for further discussion. In addition, we don't all speak exactly the same variety of English, though we will assume that existing varieties are sufficiently similar for us to engage in a meaningful discussion of quite a bit of English grammar.
} 


\section{4 / Syntactic Theory: A Formal Introduction}

to superficially different languages as they are to English. Ultimately, as we will see, much of the outward differences among languages can be viewed as lexical in nature.

Finally, as we develop grammars that include principles of considerable generality, we will begin to see constructs that may have universal applicability to human language. So one of our goals will be to consider what our findings might tell us about human linguistic abilities in general.

In developing the informal rules for reflexive and nonreflexive pronouns in Chapter 1, we assumed that we already knew a lot about the structure of the sentences we were looking at - that is, we talked about subjects, objects, clauses, etc. In fact, a fully worked out theory of reflexive and nonreflexive pronouns is going to require that many other aspects of syntactic theory get worked out first. We begin this grammar development process in the present chapter.

We will consider several candidates for theories of English grammar. We begin by quickly dismissing certain simple-minded approaches. We spend more time on a formalism known as 'context-free grammar', which serves as a starting point for most modern theories of syntax. A brief overview of some of the most important schools of thought within the paradigm of generative grammar, situating the approach developed in this text with respect to some alternatives, is included in Appendix B.

\subsection{Two Simplistic Syntactic Theories}

\subsubsection{Lists as Grammars}

The simplest imaginable syntactic theory is that a grammar consists of a list of all the well-formed sentences in the language. The most obvious problem with such a proposal is that the list would have to be too long. There is no fixed finite bound on the length of English sentences, as can be seen from the following sequence:

(1) Some sentences go on and on.

Some sentences go on and on and on.

Some sentences go on and on and on and on.

Some sentences go on and on and on and on and on. ...

Every sentence in this sequence is acceptable English. Since there is no bound on their size, it follows that the number of sentences in the list must be infinite. Hence there are infinitely many sentences of English. Since human brains are finite, they cannot store infinite lists. Consequently, there must be some more compact way of encoding the grammatical knowledge that speakers of English possess. 
Moreover, there are generalizations about the structure of English that an adequate grammar should express. For example, consider a hypothetical language consisting of infinitely many sentences similar to those in (1), except that every other sentence reversed the order of the words some and sentences: ${ }^{2}$

Some sentences go on and on.

* Sentences some go on and on.

* Some sentences go on and on and on.

Sentences some go on and on and on.

Some sentences go on and on and on and on.

* Sentences some go on and on and on and on.

* Some sentences go on and on and on and on and on.

Sentences some go on and on and on and on and on. ...

Of course, none of these sentences ${ }^{3}$ where the word sentences precedes the word some is a well-formed English sentence. Moreover, no natural language exhibits patterns of that sort - in this case, having word order depend on whether the length of the sentence is divisible by 4 . A syntactic theory that sheds light on human linguistic abilities ought to explain why such patterns do not occur in human languages. But a theory that said grammars consisted of lists of sentences could not do that. If grammars were just lists, then there would be no patterns that would be excluded - and none that would be expected, either.

This form of argument - that a certain theory of grammar fails to 'capture a linguistically significant generalization' is very common in generative grammar. It takes for granted the idea that language is 'rule governed', that is, that language is a combinatoric system whose operations are 'out there' to be discovered by empirical investigation. If a particular characterization of the way a language works leads to excessive redundancy and complications, it's assumed to be the wrong characterization of the grammar of that language. We will see this kind of argumentation again, in connection with more plausible proposals than the idea that grammars simply list sentences. In Chapter 9, we will argue that (perhaps surprisingly), a grammar motivated largely on the basis of such parsimony considerations seems to be a good candidate for a psychological model of the knowledge of language that is employed in speaking and understanding.

\footnotetext{
${ }^{2}$ The asterisks in (2) are intended to indicate the ungrammaticality of the strings in the hypothetical language under discussion, not in normal English.

${ }^{3}$ Note that we are already slipping into a common, but imprecise, way of talking about unacceptable strings of words as 'sentences'.
} 


\subsubsection{Regular Expressions}

A natural first step toward allowing grammars to capture generalizations is to classify words into what are often called 'parts of speech' or 'grammatical categories'. There are large numbers of words that behave similarly syntactically. For example, the words apple, book, color, and dog all can appear in roughly the same contexts, such as the following:

(3) a. That _ surprised me.

b. I noticed the

c. They were interested in his _

d. This is my favorite

Moreover, they all have plural forms that can be constructed in similar ways (orthographically, simply by adding an $-s$ ).

Traditionally, the vocabulary of a language is sorted into nouns, verbs, etc. based on loose semantic characterizations (e.g. 'a noun is a word that refers to a person, place, or thing'). While there is undoubtedly a grain of insight at the heart of such definitions, we can make use of this division into grammatical categories without committing ourselves to any semantic basis for them. For our purposes, it is sufficient that there are classes of words that may occur grammatically in the same environments. Our theory of grammar can capture their common behavior by formulating patterns or rules in terms of categories, not individual words.

Someone might, then, propose that the grammar of English is a list of patterns, stated in terms of grammatical categories, together with a lexicon - that is, a list of words and their categories. For example, the patterns could include (among many others):

(4) a. ARTICLE NOUN VERB

b. ARTICLE NOUN VERB ARTICLE NOUN

And the lexicon could include (likewise, among many others):

(5) a. Articles: a, the

b. Nouns: cat, dog

c. Verbs: attacked, scratched

This mini-grammar licenses forty well-formed English sentences, and captures a few generalizations. However, a grammar that consists of a list of patterns still suffers from the first drawback of the theory of grammars as lists of sentences: it can only account for a finite number of sentences, while a natural language is an infinite set of sentences. For example, such a grammar will still be incapable of dealing with all of the sentences in the infinite sequence illustrated in (1). 
We can enhance our theory of grammar so as to permit infinite numbers of sentences by introducing a device that extends its descriptive power. In particular, the problem associated with (2) can be handled using what is known as the 'Kleene star'. ${ }^{4}$ Notated as a superscripted asterisk, the Kleene star is interpreted to mean that the expression it is attached to can be repeated any finite number of times (including zero). Thus, the examples in (1) could be abbreviated as follows:

(6) Some sentences go on and on [and on]*.

A closely related notation is a superscripted plus sign (called Kleene plus), meaning that one or more occurrences of the expression it is attached to are permissible. Hence, another way of expressing the same pattern would be:

(7) Some sentences go on [and on] $]^{+}$.

We shall employ these, as well as two common abbreviatory devices. The first is simply to put parentheses around material that is optional. For example, the two sentence patterns in (4) could be collapsed into: ARTICLE NOUN VERB (ARTICLE NOUN). The second abbreviatory device is a vertical bar, which is used to separate alternatives. ${ }^{5}$ For example, if we wished to expand the mini-grammar in (4) to include sentences like The dog looked angry, we could add the pattern ARTICLE NOUN VERB ADJECTIVE and collapse it with the previous patterns as: ARTICLE NOUN VERB (ARTICLE NOUN)|ADJECTIVE. Of course, we would also have to add the verb looked and the adjective angry to the lexicon. ${ }^{6}$

Patterns making use of the devices just described - Kleene star, Kleene plus, parentheses for optionality, and the vertical bar for alternatives - are known as 'regular expressions'. ${ }^{7}$ A great deal is known about what sorts of patterns can and cannot be represented with regular expressions (see, for example, Hopcroft and Ullman (1979; chaps. 2 and 3)), and a number of scholars have argued that natural lan-

\footnotetext{
${ }^{4}$ Named after the logician Stephen Kleene.

${ }^{5}$ This is the notation standardly used in computer science and in the study of mathematical properties of grammatical systems. Descriptive linguists tend to use curly brackets to annotate alternatives.

${ }^{6}$ This extension of the grammar would license some unacceptables strings, e.g., ${ }^{*}$ The cat scratched angry. This sort of overgeneration is always a danger when extending a grammar, as we will see in subsequent chapters.

${ }^{7}$ This is not intended as a rigorous definition of regular expressions. A precise definition would include the requirement that the empty string is a regular expression, and would probably omit some of the devices mentioned in the text (because they can be defined in terms of others). Incidentally, readers who use computers with the UNIX operating system may be familiar with the command 'grep'. This stands for 'Global Regular Expression Printer'.
} 


\section{8 / Syntactic Theory: A Formal Introduction}

guages in fact exhibit patterns that are beyond the descriptive capacity of regular expressions (see Bar-Hillel and Shamir (1960; secs. 5 and 6)). The most convincing arguments for employing a grammatical formalism richer than regular expressions, however, have to do with the need to capture generalizations.

In (4), the string ARTICLE NOUN occurs twice, once before the verb and once after it. Notice that there are other options possible in both of these positions:

(8) a. Dogs chase cats.

b. A large dog chased a small cat.

c. A dog with brown spots chased a cat with no tail.

Moreover, these are not the only positions in which the same strings can occur:

(9) a. Some people yell at (the) (noisy) dogs (in my neighborhood).

b. Some people consider (the) (noisy) dogs (in my neighborhood) dangerous.

Even with the abbreviatory devices available in regular expressions, the same lengthy string of symbols - something like (ARTICLE) (ADJECTIVE) NOUN (PREPOSITION ARTICLE NOUN) - will have to appear over and over again in the patterns that constitute the grammar. Moreover, the recurring patterns are in fact considerably more complicated than those illustrated so far. Strings of other forms, such as the noisy annoying dogs, the dogs that live in my neighborhood, or Rover, Fido, and Lassie can all occur in just the same positions. It would clearly simplify the grammar if we could give this apparently infinite set of strings a name and say that any string from the set can appear in certain positions in a sentence.

Furthermore, as we have already seen, an adequate theory of syntax must somehow account for the fact that a given string of words can sometimes be put together in more than one way. If there is no more to grammar than lists of recurring patterns, where these are defined in terms of parts of speech, then there is no apparent way to talk about the ambiguity of sentences like those in (10).

(10) a. We enjoyed the movie with Cher.

b. The room was filled with noisy children and animals.

c. People with children who use drugs should be locked up.

d. I saw the astronomer with a telescope.

In the first sentence, it can be us or the movie that is 'with Cher'; in the second, it can be either just the children or both the children and the animals that are noisy; in the third, it can be the children or their 
parents who use drugs, and so forth. None of these ambiguities can be plausibly attributed to a lexical ambiguity. Rather, they seem to result from different ways of grouping the words into phrases.

In short, the fundamental defect of regular expressions as a theory of grammar is that they provide no means for representing the fact that a string of several words may constitute a unit. The same holds true of several other formalisms that are provably equivalent to regular expressions (including what is known as 'finite-state grammar').

The recurrent strings we have been seeing are usually called 'phrases' or '(syntactic) constituents'. ${ }^{8}$ Phrases, like words, come in different types. All of the italicized phrases in (8)-(9) above obligatorily include a noun, so they are called 'Noun Phrases'. The next natural enrichment of our theory of grammar is to permit our regular expressions to include not only words and parts of speech, but also phrase types. Then we also need to provide (similarly enriched) regular expressions to provide the patterns for each type of phrase. The technical name for this theory of grammar is 'Context-free Phrase Structure Grammar' or simply 'Context-free Grammar', sometimes abbreviated as CFG. CFGs, which will also let us begin to talk about structural ambiguity like that illustrated in (10), form the starting point for most serious attempts to develop formal grammars for natural languages.

\subsection{Context-free Phrase Structure Grammar}

The term 'grammatical category' now covers not only the parts of speech, but also types of phrases, such as noun phrase and prepositional phrase. To distinguish the two types, we will sometimes use the terms 'lexical category' (for parts of speech) and 'nonlexical category' or 'phrasal category' to mean types of phrases. For convenience, we will abbreviate them, so that 'NOUN' becomes 'N', 'NOUN PHRASE' becomes 'NP', etc.

A context-free phrase structure grammar has two parts:

- A LEXICON, consisting of a list of words, with their associated grammatical categories. ${ }^{9}$

- A set of RULES of the form A $\rightarrow \varphi$ where A is a nonlexical category,

\footnotetext{
${ }^{8}$ There is a minor difference in the way these terms are used: linguists often use 'phrase' in contrast to 'word' to mean something longer, whereas words are always treated as a species of constituent.

${ }^{9}$ This conception of a lexicon is rather impoverished. In particular, it leaves out information about the meanings and uses of words, except what might be generally associated with the grammatical categories. While this impoverished conception is standard in the formal theory of CFG, attempts to use CFG to describe actual natural languages have had lexicons that also included semantic information. The lexicon we develop later will be much richer in structure.
} 


\section{0 / Syntactic Theory: A Formal Introduction}

and ' $\varphi$ ' stands for a regular expression formed from lexical and/or non-lexical categories; the arrow is to be interpreted as meaning, roughly, 'can consist of'. These rules are called 'phrase structure rules'.

The left-hand side of each rule specifies a phrase type (including the sentence as a type of phrase), and the right-hand side gives a possible pattern for that type of phrase. Because phrasal categories can appear on the right-hand sides of rules, it is possible to have phrases embedded within other phrases. This permits CFGs to express regularities that seem like accidents when only simple regular expressions are permitted.

A CFG normally has one or more phrasal categories that are designated as 'initial symbols'. These are the types of phrases that can stand alone as sentences in the language. Most simple CFGs have just one initial symbol, namely 'S'. Any string of words that can be derived from one of the initial symbols by means of a sequence of applications of the rules of the grammar is licensed (or, as linguists like to say, 'generated') by the grammar. The language a grammar generates is simply the collection of all of the sentences it generates.

\subsubsection{An Example}

Consider the following CFG. (We use 'D' for 'Determiner', which includes what we have up to now been calling 'articles'.)

(11) a. Rules:

$$
\begin{aligned}
& \mathrm{S} \rightarrow \mathrm{NP} \mathrm{VP} \\
& \mathrm{NP} \rightarrow(\mathrm{D}) \mathrm{A}^{*} \mathrm{~N} \mathrm{PP}^{*} \\
& \mathrm{VP} \rightarrow \mathrm{V}(\mathrm{NP})(\mathrm{PP}) \\
& \mathrm{PP} \rightarrow \mathrm{P} \mathrm{NP}
\end{aligned}
$$

b. Lexicon:

D: the, some

A: big, brown, old

$\mathrm{N}$ : birds, fleas, dog, hunter

$\mathrm{V}$ : attack, ate, watched

P: for, beside, with

This grammar generates infinitely many English sentences. Let us look in detail at how it generates one sentence: The big brown dog with fleas watched the birds beside the hunter. We start with the symbol S, for 'Sentence'. This must consist of the sequence NP VP, since the first rule is the only one with $\mathrm{S}$ on the left-hand side. The second rule allows a wide range of possibilities for the NP, one of which is D A A N PP. This PP must consist of a $\mathrm{P}$ followed by an NP, by the fourth rule, and the NP so introduced may consist of just an N. The third rule allows VP 
to consist of V NP PP, and this NP can consist of a D followed by an $\mathrm{N}$. Lastly, the final PP again consists of a $\mathrm{P}$ followed by an NP, and this NP also consists of a D followed by an N. Putting these steps together the S may consist of the string D A A N P N V D N P D N, which can be converted into the desired sentence by inserting appropriate words in place of their lexical categories. All of this can be summarized in the following figure (called a 'tree diagram'):

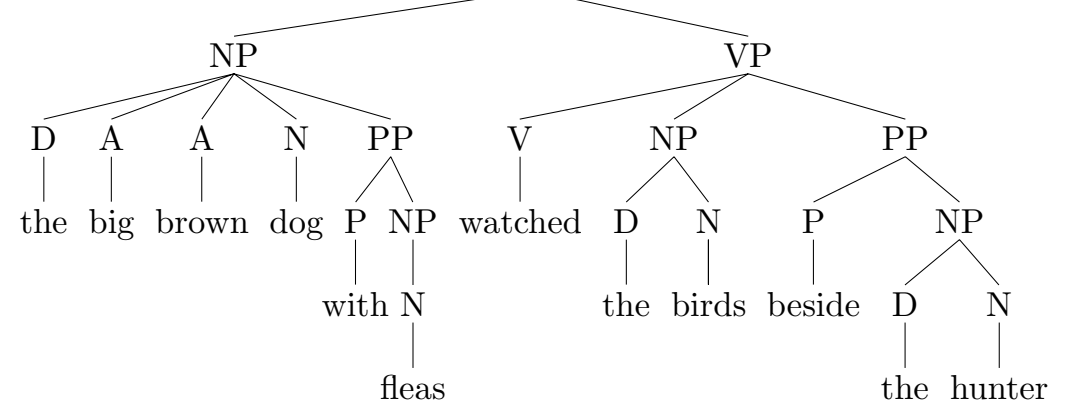

Note that certain sentences generated by this grammar can be associated with more than one tree. (Indeed, the example just given is one such sentence, but finding the other tree will be left as an exercise.) This illustrates how CFGs can overcome the second defect of regular expressions pointed out at the end of the previous section. Recall the ambiguity of (13):

(13) I saw the astronomer with a telescope.

The distinct interpretations of this sentence ('I used the telescope to see the astronomer'; 'What I saw was the astronomer who had a telescope') correspond to distinct tree structures that our grammar will assign to this string of words. The first interpretation corresponds to the tree where the PP with a telescope hangs from the VP; the latter is the meaning associated with the tree structure where that PP is part of the NP constituent: the astronomer with a telescope. CFG thus provides us with a straightforward mechanism for expressing such ambiguities, whereas grammars that use only regular expressions don't.

The normal way of talking about words and phrases is to say that certain word strings 'form a constituent'. What this means is that these strings function as units for some purpose (for example, the interpretation of modifiers) within the sentences in which they appear. So in (12), the sequence with fleas forms a PP constituent, the big brown dog with fleas forms an NP, and the sequence dog with fleas forms no con- 
stituent. Structural ambiguity arises whenever a string of words can form constituents in more than one way.

\section{Problem 1: Practice with CFG}

Assume the CFG grammar given in (11). Draw the tree structure for the other interpretation (i.e. not the one shown in (12)) of The big brown dog with fleas watched the birds beside the hunter.

\section{\. Problem 2: More Practice with CFG}

Assume the grammar rules given in (11), but with the following lexicon:

A: big, unusual, young

$\mathrm{D}$ : $a$, the

$\mathrm{N}$ : cat, dog, hat, man, woman, roof

$\mathrm{P}:$ in, on, with

$\mathrm{V}$ : admired, disappeared, put, relied

A. Give three sanctioned by this grammar that are well-formed English sentences. Draw the tree structures that the grammar assigns to them.

B. Give a well-formed English sentence that is structurally ambiguous according to this grammar. Draw the two distinct tree structures.

C. Give three sentences (using only the words from this grammar) that are not covered by this grammar but which are nonetheless well-formed in English. The examples should differ in their trees, not just in the lexical entries they contain.

D. Explain what prevents each of the examples in (C) from being covered.

E. Give three sentences sanctioned by this grammar that are not wellformed English sentences. Again, make them interestingly different.

F. Discuss how the grammar might be revised to correctly exclude your examples in (E), without simultaneously excluding good sentences.

G. How many sentences does this grammar admit?

H. How many would it admit if the NP rule were replaced by the following rule?

$\mathrm{NP} \rightarrow(\mathrm{D}) \mathrm{A} \mathrm{N}(\mathrm{PP})$ Explain your answer. 


\subsubsection{CFG as a Theory of Natural Language Grammar}

As was the case with regular expressions, the formal properties of CFG are extremely well studied (see Hopcroft and Ullman (1979; chaps. 46 ) for a summary). In the early 1960s, several scholars published arguments purporting to show that natural languages exhibit properties beyond the descriptive capacity of CFGs. The pioneering work in the first two decades of generative grammar was based on the assumption that these arguments were sound. Most of that work can be viewed as the development of extensions to CFG designed to deal with the richness and complexity of natural languages.

The most celebrated proposed extension was a kind of rule called a 'transformation', as introduced into the field of generative grammar by Noam Chomsky. ${ }^{10}$ Transformations are mappings from phrase structure representations to phrase structure representations (trees to trees, in our terms) that can copy, delete, and permute parts of trees, as well as insert specified new material into them. For example, in early work on transformations, it was claimed that declarative and interrogative sentence pairs (such as The sun is shining and Is the sun shining?) were to be derived from the same underlying phrase structure by a transformation that moved certain verbs to the front of the sentence. Likewise, passive sentences (such as The cat was chased by the dog) were derived from the same underlying structures as their active counterparts (The dog chased the cat) by means of a passivization transformation. The initial trees were to be generated by a CFG. The name 'transformational grammar' is sometimes used for theories positing rules of this sort. ${ }^{11}$

In 1982, the earlier arguments against the adequacy of CFG as a theory of natural language structure were called into question by Geoffrey Pullum and Gerald Gazdar. This led to a flurry of new work on the issue, culminating in new arguments that natural languages were not describable by CFGs. The mathematical and empirical work that resulted from this controversy substantially influenced the theory of grammar presented in this text. Many of the central papers in this debate were collected together by Savitch et al. (1987); of particular interest are Pullum and Gazdar's paper and Shieber's paper.

While the question of whether natural languages are in principle beyond the generative capacity of CFGs is of some intellectual interest, working linguists tend to be more concerned with determining what sort

${ }^{10}$ The original conception of a transformation, as developed in the early 1950 s by Zellig Harris, was intended somewhat differently - as a way of regularizing the information content of texts, rather than as a system for generating sentences.

${ }^{11}$ See Appendix B for more discussion of varieties of transformational grammar. 


\section{4 / Syntactic Theory: A Formal Introduction}

of formalisms can provide elegant and enlightening accounts of linguistic phenomena in practice. Hence the arguments that tend to carry the most weight are ones about what formal devices are needed to capture linguistically significant generalizations. In the next chapter, we will consider some phenomena in English that suggest that the simple version of CFG introduced above should be extended.

\subsubsection{Modern Phrase Structure Grammar}

Accompanying the 1980s revival of interest in the mathematical properties of natural languages, considerable attention was given to the idea that, with an appropriately designed theory of syntactic features and general principles, context-free phrase structure grammar could serve as an empirically adequate theory of natural language syntax. This proposition was explored in great detail by Gazdar et al. (1985), who developed the theory known as 'Generalized Phrase Structure Grammar' (or GPSG). Work in phrase structure grammar advanced rapidly, and GPSG quickly evolved into a new framework, now known as 'Headdriven Phrase Structure Grammar' (HPSG), whose name reflects the increased importance of information encoded in the lexical heads ${ }^{12}$ of syntactic phrases. The theory of grammar developed in this text is most closely related to current HPSG.

\subsection{Applying Context-Free Grammar}

In the previous sections, we introduced the formalism of context-free grammar and showed how it allows us to generate infinite collections of English sentences with simple rules. We also showed how it can provide a rather natural representation of certain ambiguities we find in natural languages. But the grammar we presented was just a teaching tool, designed to illustrate certain properties of the formalism; it was not intended to be taken seriously as an attempt to analyze the structure of English. In this section, we begin by motivating some phrase structure rules for English. In the course of doing this, we develop a new test for determining which strings of words are constituents. We also introduce a new abbreviatory convention that permits us to collapse many of our phrase structure rules into rule schemas.

\subsubsection{Some Phrase Structure Rules for English}

For the most part, we will use the traditional parts of speech, such as noun, verb, adjective, and preposition. In some cases, we will find it useful to introduce grammatical categories that might be new to readers, and we may apply the traditional labels somewhat differently than in

\footnotetext{
${ }^{12}$ The notion of 'head' will be discussed in section 2.7 below.
} 
traditional grammar books. But the traditional classification of words into types has proved to be an extremely useful categorization over the past two millenia, and we see no reason to abandon it wholesale.

We turn now to phrases, beginning with noun phrases.

\section{Noun Phrases}

Nouns can appear in a number of positions, such as the positions of the three nouns in Dogs give people fleas. These same positions also allow sequences of an article followed by a noun, as in The boy gave the dog a bath. Since the position of the article can also be filled by demonstratives (e.g. this, these), possessives (e.g. my, their), or quantifiers (e.g. each, some, many), we use the more general term 'determiner' (abbreviated D) for this category. We can capture these facts by positing a type of phrase we'll call NP (for 'noun phrase'), and the rule NP $\rightarrow$ (D) N. As we saw earlier in this chapter, this rule will need to be elaborated later to include adjectives and other modifiers. First, however, we should consider a type of construction we have not yet discussed.

\section{Coordination}

To account for examples like $A \operatorname{dog}$, a cat, and a wombat fought, we want a rule that allows sequences of NPs, with and before the last one, to appear where simple NPs can occur. A rule that does this is $\mathrm{NP} \rightarrow \mathrm{NP}^{+}$CONJ NP.

Whole sentences can also be conjoined, as in The dog barked, the donkey brayed, and the pig squealed. Again, we could posit a rule like $\mathrm{S} \rightarrow \mathrm{S}^{+}$CONJ S. But now we have two rules that look an awful lot alike. We can collapse them into one rule schema as follows, where the variable ' $\mathrm{X}$ ' can be replaced by any grammatical category name:

(14) $\mathrm{X} \rightarrow \mathrm{X}^{+}$CONJ X.

Now we have made a claim that goes well beyond the data that motivated the rule, namely, that elements of any category can be conjoined in the same way. If this is correct, then we can use it as a test to see whether a particular string of words should be treated as a phrase. In fact, coordinate conjunction is widely used as a test for constituency. Though it is not an infallible diagnostic, we will use it as one of our sources of evidence for constituent structure.

\section{Verb Phrases}

Consider (15):

(15) The man yelled, chased the cat, and gave the dog a bone. 


\section{6 / Syntactic Theory: A Formal Introduction}

(15) contains the coordination of strings consisting of $\mathrm{V}, \mathrm{V} \mathrm{NP}$, and V NP NP. According to (14), this means that all three strings are constituents of the same type. Hence, we posit a constituent which we'll call VP, described by the rule VP $\rightarrow \mathrm{V}$ (NP) (NP). VP is introduced by the rule $\mathrm{S} \rightarrow \mathrm{NP}$ VP. ${ }^{13}$

\section{Prepositional Phrases}

Expressions like in Rome or at noon that denote places or times ('locative' and 'temporal' expressions, as linguists would say) can be added to almost any sentence, and to NPs, too. For example:

(16) a. The fool yelled at noon.

b. This disease gave Leslie a fever in Rome.

c. A man in Rome laughed.

These are constituents, as indicated by examples like The fool yelled at noon and at midnight, in Rome and in Paris. We can get lots of them in one sentence, for example, A man laughed on the street in Rome at noon on Tuesday. These facts can be incorporated into the grammar in terms of the phrasal category PP (for 'prepositional phrase'), and the rules:

(17) a. $\mathrm{PP} \rightarrow \mathrm{P}$ NP

b. $\mathrm{VP} \rightarrow \mathrm{VP} \mathrm{PP}$

Since the second rule has VP on both the right and left sides of the arrow, it can apply to its own output. (Such a rule is known as a RECURSIVE rule). ${ }^{14}$ Each time it applies, it adds a PP to the derivation. Thus, this recursive rule permits arbitrary numbers of PPs within a VP.

As mentioned earlier, locative and temporal PPs can also occur in NPs, for example, A painter on the street in Rome on Tuesday at noon laughed. The most obvious analysis to try for this would be a rule that said: NP $\rightarrow$ NP PP. However, we're going to adopt a slightly more complex analysis. We posit a new nonlexical category, which we'll call $\mathrm{NOM}$, and we replace our old rule: $\mathrm{NP} \rightarrow(\mathrm{D}) \mathrm{N}$ with the following:

(18) a. $\mathrm{NP} \rightarrow$ (D) NOM

\footnotetext{
${ }^{13}$ There are other kinds of coordinate sentences that we are leaving aside here - in particular, elliptical sentences that involve coordination of non-constituent sequences:

(i) Chris likes blue and Pat green.

(ii) Leslie wants to go home tomorrow, and Terry, too.

Notice that this kind of sentence, which will not be treated by the coordination rule discussed in the text, has a characteristic intonation pattern - the elements after the conjunction form separate intonational units separated by pauses.

${ }^{14}$ More generally, we use the term RECURSION whenever rules permit a constituent to occur within a larger constituent of the same type.
} 
b. $\mathrm{NOM} \rightarrow \mathrm{N}$

c. $\mathrm{NOM} \rightarrow \mathrm{NOM} \mathrm{PP}$

The category NOM will be very useful later in the text. For now, we will justify it with the following sentences:

(19) a. The love of my life and mother of my children would never do such a thing.

b. The museum displayed no painting by Miro or drawing by Klee.

(19b) means that the museum displayed neither paintings by Miro nor drawings by Klee. That is, the determiner no must be understood as 'having scope' over both painting by Miro and drawing by Klee - it applies to both phrases. The most natural noun phrase structure to associate with this interpretation is:

(20) no [painting by Miro or drawing by Klee]

This, in turn, is possible with our current rules if the bracketed string is a conjoined NOM. It would not be possible without NOM.

Similarly, for (19a), the has scope over both love of my life and mother of my children and hence provides motivation for an analysis involving coordination of NOM constituents.

\subsubsection{Summary of Grammar Rules}

Our grammar now has the following rules:

$$
\begin{aligned}
& \mathrm{S} \rightarrow \mathrm{NP} \mathrm{VP} \\
& \mathrm{NP} \rightarrow(\mathrm{D}) \mathrm{NOM} \\
& \mathrm{VP} \rightarrow \mathrm{V}(\mathrm{NP})(\mathrm{NP}) \\
& \mathrm{NOM} \rightarrow \mathrm{N} \\
& \mathrm{NOM} \rightarrow \mathrm{NOM} \mathrm{PP} \\
& \mathrm{VP} \rightarrow \mathrm{VP} \mathrm{PP} \\
& \mathrm{PP} \rightarrow \mathrm{P} \mathrm{NP} \\
& \mathrm{X} \rightarrow \mathrm{X}^{+} \mathrm{CONJ} \mathrm{X}
\end{aligned}
$$

In motivating this grammar, we encountered three types of arguments for saying a given string is a constituent:

- It exemplifies a pattern that shows up in multiple environments.

- Calling it a constituent helps us account for structural ambiguity.

- It can be a coordinate conjunct.

We will make use of all three of these types of arguments in the coming chapters. 


\subsection{Trees Revisited}

In grouping words into phrases and smaller phrases into larger ones, we are assigning internal structure to sentences. As noted earlier, this structure can be represented in a tree diagram. For example, our grammar so far generates the following tree:

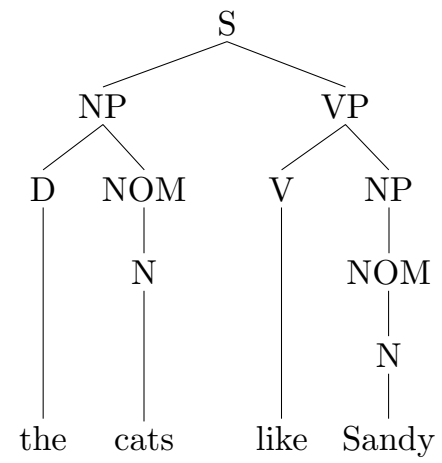

A tree is said to consist of NODES, connected by BRANCHES. A node above another on a branch is said to DOMINATE it. The nodes at the bottom of the tree - that is, those that do not dominate anything else - are referred to as TERMINAL nodes (or occasionally, as 'leaves'). A node right above another node on a tree is said to be its MOTHER and to IMMEDIATELY DOMINATE it. A node right below another on a branch is said to be its DAUGhTER. Two daughters of the same mother node are, naturally, referred to as SISTERS.

One way to think of the way in which a grammar of this kind defines (or generates) trees is as follows. First, we appeal to the lexicon (still conceived of as just a list of words paired with their grammatical categories) to tell us which lexical trees are well formed. (By 'lexical tree', we simply mean a tree consisting of a single nonterminal node dominating a single terminal node). So if cats is listed in the lexicon as belonging to the category $\mathrm{N}$, and like is listed as a $\mathrm{V}$, and so forth, then lexical structures like the following are well formed.

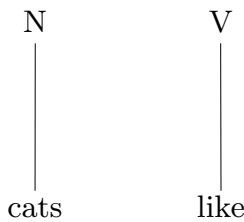

And the grammar rules are equally straightforward. They simply tell us how well-formed trees (some of which may be lexical) can be combined into bigger ones: 
Some Simple Theories of Grammar / 39

(24)

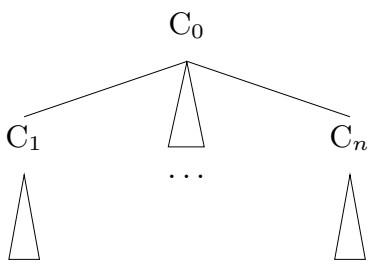

is a well-formed nonlexical tree just in case

$\mathrm{C}_{1} \ldots \quad \mathrm{C}_{n}$ are well-formed trees, and

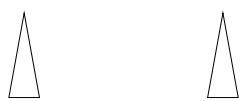

$\mathrm{C}_{0} \rightarrow \mathrm{C}_{1} \ldots \mathrm{C}_{n}$ is a grammar rule.

So we can think of our grammar as generating sentences in a 'bottomup' fashion - starting with lexical trees, and then using these to build bigger and bigger phrasal trees, until we build one whose top node is S. The set of all sentences that can be built that have $\mathrm{S}$ as their top node is the set of sentences the grammar generates. But note that our grammar could just as well have been used to generate sentences in a 'top-down' manner, starting with S. The set of sentences generated in this way is exactly the same. In fact, the definition of well-formed nonlexical tree could also be given in static, constraint-based terms as in (25):

(25) A tree is well formed just in case each local subtree (that is, a mother node with its daughters) within it either

1. is a well-formed lexical tree (see above), or

2. is in one-to-one (tree-to-rule) correspondence with some rule of the grammar.

A CFG is completely neutral with respect to top-down and bottom-up perspectives on analyzing sentence structure. There is also no particular bias toward thinking of the grammar in terms of generating sentences or in terms of parsing.

These design properties - direction neutrality and process neutrality - stem from the fact that the rules and lexical entries simply provide constraints on well-formed structure. As we will suggest in Chapter 9, these are in fact important design features of this theory (and of those we will develop that are based on it), as they facilitate the direct embedding of the abstract grammar within a model of language processing.

The lexicon and grammar rules together thus constitute a system for defining not only well-formed word strings (i.e. sentences), but also well-formed tree structures. Our statement of the relationship between 
the grammar rules and the well-formedness of trees is at present rather trivial, and our lexical entries still consist simply of pairings of words with parts of speech. As we modify our theory of grammar and enrich our lexicon, however, our attention will increasingly turn to a more refined characterization of which trees are well formed.

\subsection{Worksection on Phrase Structure Grammar}

Two of our arguments against overly simple theories of grammar at the beginning of this chapter were that we wanted to be able to account for the infinity of language, and that we wanted to be able to account for structural ambiguity. The purpose of this section is to explore how our grammar handles these so far.

\section{Problem 3: Structural Ambiguity}

Show that our grammar can account for the ambiguity of each of the following sentences by providing at least two trees licensed for each one, and explain briefly which interpretation goes with which tree:

(i) Bo saw the group with the telescope.

(ii) Most dogs and cats with fleas live in this neighborhood.

(iii) The pictures show Superman and Lois Lane and Wonder Woman.

[Note: We haven't provided a lexicon, so technically, our grammar doesn't generate any of these. You can assume, however, that all the words in them are in the lexicon, with the obvious category assignments.]

\section{Problem 4: Infinity}

Our grammar has two mechanisms, each of which permits us to have infinitely many sentences: the Kleene operators (plus and star), and recursion (categories that can dominate themselves). Construct arguments for why we need both of them. That is, why not use recursion to account for the unboundedness of coordination or use Kleene star to account for the possibility of arbitrary numbers of PPs? [Hint: Consider the different groupings into phrases - that is, the different tree structures - provided by the two mechanisms. Then look for English data supporting one choice of structure over another.]

\subsection{Heads}

As we have seen, CFGs can provide successful analyses of quite a bit of natural language. But if our theory of natural language syntax were 
nothing more than CFG, our theory would fail to predict the fact that certain kinds of $\mathrm{CF}$ rules are much more natural than others. For example, consider the rules in (26):

(26) $\mathrm{VP} \rightarrow \mathrm{P}$ NP

$\mathrm{NP} \rightarrow \mathrm{PP} \mathrm{S}$

As far as we are aware, no linguist has ever wanted to write rules like these for any human language. However, nothing in the formalism of CFG indicates that there is anything wrong - or even unusual - about such rules.

What is it that we don't like about the rules in (26)? An intuitive answer is that the categories on the left of the rules don't seem appropriate for the sequences on the right. For example, a VP should have a verb in it. This then leads us to consider why we named NP, VP, and PP after the lexical categories N, V, and P. In each case, the phrasal category was named after a lexical category that is an obligatory part of that kind of phrase. At least in the case of NP and VP, all other parts of the phrase may sometimes be missing (e.g. Dogs bark).

The lexical category that a phrasal category derives its name from is called the HEAD of the phrase. This notion of 'headedness' plays a crucial role in all human languages and this fact points out a way in which natural language grammars differ from some kinds of CFG. The formalism of CFG, in and of itself, treats category names as arbitrary: our choice of pairs like ' $\mathrm{N}$ ' and 'NP', etc., serves only a mnemonic function in simple CFGs. But we want our theory to do more. Many phrase structures of natural languages are headed structures, a fact we will build into the architecture of our grammatical theory. To do this, we will enrich how we represent grammatical categories, so that we can express directly what a phrase and its head have in common. This will lead eventually to a dramatic reduction in the number of grammar rules required.

\subsection{Subcategorization}

The few grammar rules we have so far cover only a small fragment of English. What might not be so obvious, however, is that they also overgenerate - that is, they generate strings that are not well-formed English sentences. Both denied and disappeared would be listed in the lexicon as members of the category $\mathrm{V}$. This classification is necessary to account for sentences like (27):

(27) a. The defendant denied the accusation.

b. The problem disappeared. 
But this would also permit the generation of the ungrammmatical sentences in (28).

(28) a. * The defendant denied.

b. ${ }^{*}$ The teacher disappeared the problem.

Similarly, the verb handed must be followed by two NPs, but our rules allow a VP to be expanded in such a way that V can be followed by only one NP, or no NPs at all. That is, our current grammar fails to distinguish among the following:

(29) a. The teacher handed the student a book.

b. * The teacher handed the student.

c. ${ }^{*}$ The teacher handed a book.

d. ${ }^{*}$ The teacher handed.

To rule out the ungrammatical examples in (29), we need to distinguish among verbs that cannot be followed by an NP, those that must be followed by one NP, and those that must be followed by two NPs. These are often referred to as INTRANSITIVE, TRANSITIVE, and DITRANSITIVE verbs, respectively. In short, we need to distinguish subcategories of the category V.

One possible approach to this problem is simply to conclude that the traditional category of 'verb' is too coarse-grained for generative grammar, and that it must be replaced by at least three distinct categories, which we can call IV, TV, and DTV. We can then replace our earlier phrase structure rule

$$
\mathrm{VP} \rightarrow \mathrm{V}(\mathrm{NP})(\mathrm{NP})
$$

with the following three rules:

(30) a. $\mathrm{VP} \rightarrow \mathrm{IV}$

$$
\begin{aligned}
& \text { b. } \mathrm{VP} \rightarrow \mathrm{TV} \mathrm{NP} \\
& \text { c. } \mathrm{VP} \rightarrow \mathrm{DTV} \mathrm{NP} \mathrm{NP}
\end{aligned}
$$

\section{\1 Problem 5: Properties Common to Verbs}

This grammar embodies the claim that IVs, TVs, and DTVs are entirely different categories. Hence, it provides no reason to expect that they would have more in common than any other collection of three lexical categories, say, N, P, and D. But these three types of verbs do behave alike in a number of ways. For example, they all exhibit agreement with the subject of the sentence; this is discussed in the next section. List as many other properties as you can think of that are shared by intransitive, transitive, and ditransitive verbs. 


\subsection{Transitivity and Agreement}

Most nouns and verbs in English have both singular and plural forms. In the case of nouns, the distinction between, say, bird and birds indicates whether the word is being used to refer to just one fowl or a multiplicity of them. In the case of verbs, distinctions like the one between sing and sings indicate whether the verb's subject denotes one or many individuals. In present tense English sentences, the plurality marking on the head noun of the subject NP and that on the verb must be consistent with each other. This is referred to as SUBJECT-VERB AGREEMENT (or sometimes just 'agreement' for short). It is illustrated in (31).

(31) a. The bird sings.

b. Birds sing.

c. ${ }^{*}$ The bird sing. ${ }^{15}$

d. * Birds sings.

Perhaps the most obvious strategy for dealing with agreement is the one considered in the previous section. That is, we could divide our grammatical categories into smaller categories, distinguishing singular and plural forms. We could then replace the relevant phrase structure rules with more specific ones. In examples like (31), we could distinguish lexical categories of N-SING and N-PLU, as well as IV-SING and IV-PLU. Then we could replace the rule

$$
\mathrm{S} \rightarrow \mathrm{NP} \mathrm{VP}
$$

with two rules:

$$
\begin{aligned}
& \mathrm{S} \rightarrow \text { NP-SING VP-SING } \\
& \text { and } \\
& \mathrm{S} \rightarrow \text { NP-PLU VP-PLU. }
\end{aligned}
$$

Since the marking for number appears on the head noun and head verb, other rules would also have to be changed. Specifically, the rules expanding NP and VP all would have to be divided into pairs of rules expanding NP-SING, NP-PLU, VP-SING, and VP-PLU. Hence, we would need all of the following:
a. NP-SING $\rightarrow$ (D) NOM-SING
b. NP-PLU $\rightarrow$ (D) NOM-PLU
c. NOM-SING $\rightarrow$ NOM-SING PP
d. NOM-PLU $\rightarrow$ NOM-PLU PP
e. NOM-SING $\rightarrow$ N-SING

${ }^{15}$ There are dialects of English in which this is grammatical, but we will be analyzing the more standard dialect in which this kind of agreement marking is obligatory. 
44 / Syntactic Theory: A Formal Introduction

$$
\begin{array}{ll}
\text { f. } & \text { NOM-PLU } \rightarrow \text { N-PLU } \\
\text { g. } & \text { VP-SING } \rightarrow \text { IV-SING } \\
\text { h. } & \text { VP-PLU } \rightarrow \text { IV-PLU }
\end{array}
$$

This set of rules is cumbersome, and clearly misses linguistically significant generalizations. The rules in this set come in pairs, differing only in whether the category names end in '-SING' or '-PLU'. Nothing in the formalism or in the theory predicts this pairing. The rules would look no less natural if, for example, the rules expanding -PLU categories had their right-hand sides in the reverse order from those expanding SING categories. But languages exhibiting this sort of variation in word order do not seem to exist.

Things get even messier when we consider transitive and ditransitive verbs. Agreement is required irrespective of whether the verb is intransitive, transitive, or ditransitive. Thus, along with (31), we have (33) and (34).

(33) a. The bird devours the worm.

b. The birds devour the worm.

c. ${ }^{*}$ The bird devour the worm.

d. ${ }^{*}$ The birds devours the worm.

(34) a. The bird gives the worm a tug.

b. The birds give the worm a tug.

c. ${ }^{*}$ The bird give the worm a tug.

d. ${ }^{*}$ The birds gives the worm a tug.

If agreement is to be handled by the rules in (35):

(35) a. $\mathrm{S} \rightarrow$ NP-SING VP-SING

b. $\mathrm{S} \rightarrow$ NP-PLU VP-PLU

then we will now need to introduce lexical categories TV-SING, TV-PLU, DTV-SING, and DTV-PLU, along with the necessary VP-SING and VP-PLU expansion rules (as well as the two rules in (35)). What are the rules for VP-SING and VP-PLU when the verb is transitive or ditransitive? For simplicity, we will look only at the case of VP-SING with a transitive verb. Since the object of the verb can be either singular or plural, we need two rules:

(36) a. VP-SING $\rightarrow$ TV-SING NP-SING

b. VP-SING $\rightarrow$ TV-SING NP-PLU

Similarly, we need two rules for expanding VP-PLU when the verb is transitive, and four rules each for expanding VP-SING and VP-PLU when the verb is ditransitive (since each object can be either singular or plural). Alternatively, we could make all objects of category NP and introduce the following two rules: 
(37) a. NP $\rightarrow$ NP-SING

b. $\mathrm{NP} \rightarrow \mathrm{NP}-\mathrm{PLU}$

This would keep the number of VP-SING and VP-PLU rules down to three each (rather than seven each), but it introduces extra noun phrase categories. Either way, the rules are full of undesirable redundancy.

\section{\\ Problem 6: Pronoun Case}

There are some differences between the noun phrases that can appear in different positions. In particular, pronouns in subject position have one form (referred to as NOMINATIVE, and including the pronouns $I$, he, she, $w e$, and they), whereas pronouns in other positions take another form (called ACCUSATIVE, and including me, him, her, us, and them). So, for example, we say He saw her, not ${ }^{*}$ Him saw she.

a. How would the category of NP have to be further subdivided in order to account for the difference between nominative and accusative pronouns?

b. How would the rules for $\mathrm{S}$ and the various kinds of VPs have to be modified in order to account for the distributional differences between nominative and accusative pronouns?

It should be clear by now that as additional coverage is incorporated - such as adjectives modifying nouns - the redundancies will proliferate. The problem is that we want to be able to talk about nouns and verbs as general classes, but we have now divided nouns into (at least) two categories (N-SING and N-PLU) and verbs into six categories (IV-SING, IV-PLU, TV-SING, TV-PLU, DTV-SING, and DTV-PLU). To make agreement work, this multiplication of categories has to be propagated up through at least some of the phrasal categories. The result is a very long and repetitive list of phrase structure rules.

What we need is a way to talk about subclasses of categories, without giving up the commonality of the original categories. That is, we need a formalism that permits us to refer straightforwardly to, for example, all verbs, all singular verbs, all ditransitive verbs, or all singular ditransitive verbs. In the next chapter, we introduce a device that will permit us to do this.

\subsection{Conclusion}

In this chapter, we began our search for an adequate model of the grammar of one natural language: English. We considered and rejected 
two simple approaches to grammar, including a theory based on regular expressions ('finite-state grammar'). The theory of Context-Free Grammars, by contrast, solves the obvious defects of these simple approaches and provides an appropriate starting point for the grammatical description of natural language. However, we isolated two ways in which Context-Free Grammars are inadequate as a theory of natural language:

- CFGs are arbitrary. They fail to predict the 'headedness' that is characteristic of many types of phrase in natural language.

- CFGs are redundant. Without some way to refer to kinds of categories rather than just individual categories, there is no way to eliminate the massive redundancy that will be required in order to analyze the agreement patterns of natural languages.

For these reasons, we cannot accept CFG alone as a theory of grammar. As we will show in the next few chapters, however, it is possible to retain much of the character of CFG as we seek to remedy its defects.

\subsection{Further Reading}

The standard reference work for the basic mathematical results on formal languages (including regular expressions and context-free languages) is Hopcroft and Ullman (1979). Partee et al. (1990) covers much of the same material from a more linguistic perspective. Classic works arguing against the use of context-free grammars for natural languages include Chomsky (1963) and Postal (1967). Papers questioning these arguments, and other papers presenting new arguments for the same conclusion are collected in Savitch et al. (1987). For (somewhat dated) surveys of theories of grammar, see Sells (1985) and Wasow (1989). A more detailed presentation of GPSG is Gazdar et al. (1985). The history of generative grammar is presented from different perspectives by Matthews (1993), Newmeyer (1986), Harris (1993), and Huck and Goldsmith (1995).

Perhaps the best discussions of the basic phrase structures of English are to be found in good descriptive grammars, such as Quirk, et al. $(1972,1985)$ or Greenbaum (1996). Important discussions of the notion of 'head' and its role in phrase structure can be found in Chomsky (1970) and Gazdar and Pullum (1981). A detailed taxonomy of the subcategories of English verbs is provided by Levin (1993). 


\section{Analyzing Features of Grammatical Categories}

\subsection{Introduction}

In the last chapter, we saw that there are constraints on which words can go together (what linguists call 'co-occurrence restrictions') that are not efficiently described using the standard formalism of contextfree grammar. Some verbs must take an object; others can never take an object; still others (e.g. put, inform) require both an object and another phrase of a particular kind. These co-occurrence restrictions, as we have seen, give rise to a great deal of redundancy in CFGs. In addition, different forms of a given verb impose different conditions on what kind of NP can precede them (i.e. on what kind of subject they co-occur with). For example, walks requires a third-person singular NP as its subject; walk requires a plural subject, or else one that is firstor second-person singular. As we saw in the last chapter, if we try to deal with this complex array of data by dividing the category $\mathrm{V}$ into more specific categories, each with its unique co-occurrence restrictions, we end up with a massively redundant grammar that fails to capture linguistically significant generalizations.

We also isolated a second defect of CFGs, namely that they allow rules that are arbitrary. Nothing in the theory of CFG reflects the fact that the phrases of human language usually share certain key properties (nounhood, verbhood, prepositionhood, etc.) with a particular daughter within them - their head. We must somehow modify the theory of CFG to allow us to express the property of headedness.

Our solution to the problem of redundancy is to make grammatical categories decomposable into component parts. CFG as presented so far treats each grammatical category symbol as atomic - that is, without

internal structure. Two categories are either identical or different; there 


\section{8 / Syntactic Theory: A Formal Introduction}

is no mechanism for saying that two categories are alike in some ways, but different in others. However, words and phrases in natural languages typically behave alike in certain respects, but not in others. For example, the two words deny and denies are alike in requiring an NP object (both being forms of a transitive verb). But they differ in terms of the kind of subject NP they take: denies requires a third-person-singular subject like Kim or she, while deny accepts most any NP subject except the third-person-singular kind. On the other hand, denies and disappears both take a singular subject NP, but only the former can co-occur with a following object NP. An adequate formalism needs to be able to characterize the fact that words are organized into classes defined in terms of cross-cutting properties.

To accommodate this intuition, we will develop the view that grammatical categories are not atomic, but rather are complexes with internal structure. This innovation, much like the decomposition of molecules into atoms, or of atoms into subatomic particles, will allow us to talk precisely about how categories are the same in certain respects, yet different in others.

\subsection{Feature Structures}

Informally, we speak of verbs differing in their transitivity. More generally, linguists talk about elements that have different combinatoric potential in terms of differing 'valence'. ${ }^{1}$ Likewise, we talk of the number (singular or plural) of a noun, the part of speech of a word (whether it's a noun, verb, etc.), or a verb's form (e.g. whether it is a present participle, an infinitive, etc.). Instead of associating words in the lexicon with a single atomic category, we can treat a lexical category as a complex of grammatical properties. To model such complexes, we use the notion standardly referred to as FEATURE STRUCTURE.

A feature structure is a way of representing grammatical information. Formally, a feature structure consists of a specification of a set of features (which we will write in upper case), each of which is paired with a particular value. Feature structures can be thought of in at least two more or less equivalent ways. For example, they may be conceived of as functions (in the mathematicians' sense of the word) ${ }^{2}$ specifying a value for each of a set of features, or else as directed graphs where feature names label arcs that point to appropriately labelled nodes. For

\footnotetext{
${ }^{1}$ This term, borrowed from chemistry, refers to the capacity to combine with atoms, ions, and the like.

${ }^{2} \mathrm{~A}$ function in this sense is a set of ordered pairs such that no two ordered pairs in the set share the same first element. What this means for feature structures is that each feature in a feature structure must have a unique value.
} 
grammatical purposes, however, it will be most useful for us to focus on DESCRIPTIONS of feature structures, which we will write in a square bracket notation, as shown in (1):

$\left[\begin{array}{cc}\text { FEATURE }_{1} & \text { VALUE }_{1} \\ \text { FEATURE }_{2} & \text { VALUE }_{2} \\ \ldots & \\ \text { FEATURE }_{n} & \text { VALUE }_{n}\end{array}\right]$

For example, we might treat the category of the word bird in terms of a feature structure that specifies both part of speech and number. We may assume such a category includes appropriate specifications for two appropriately named features: its part of speech (POS) is noun, and its number (NUM) is singular (sg). The lexical entry for bird, then, would be a pair consisting of a form and a feature structure description, roughly as shown in $(2):^{3}$

$$
\left\langle\text { bird, }\left[\begin{array}{ll}
\text { POS } & \text { noun } \\
\text { NUM } & \text { sg }
\end{array}\right]\right\rangle
$$

Implicit in our use of feature structures is a commitment to developing a theory of what kinds of features go together, what values are appropriate for each particular feature, etc. - that is, a commitment to specifying which feature structure categories are well formed and which are not. Note that this enterprise is also naturally viewed as providing a theory of what kind of linguistic entities exist in a given domain, and what properties those entities exhibit. Much of our grammar development will be concerned with formulating a natural theory of linguistic generalizations in terms of the constraints that govern the feature structure categories we are led to posit.

One of the first things we will want to do in developing a theory of grammar is to classify linguistic entities in various ways. To this end, it is particularly useful to introduce the notion of TYPE. This concept is really quite simple: if we think of a language as a system of linguistic entities (words, phrases, categories, sounds, and other more abstract entities that we will introduce as we go along), then types are just classes of those entities. We assign entities to these classes on the basis of certain properties that they share. Naturally, the properties we employ in our type classification will be those that we wish to refer to

\footnotetext{
${ }^{3}$ Throughout this book, we will describe linguistic forms in terms of standard English orthography. In fact, a lexical entry such as this should contain a phonological description that will play a role in the word's phonological realization, a topic we will not consider in detail here.
} 


\section{0 / Syntactic Theory: A Formal Introduction}

in our descriptions of the entities. Thus each grammatical type will be associated with particular features and sometimes with particular values for those features.

Let us make this very abstract discussion more concrete by considering the use of feature structures to describe a simple nonlinguistic domain. Imagine that we used feature structures to model universities and the people who are associated with them. We'll start from the assumption that the people and the other entities are really 'out there' in the real world. Our first step then in constructing a theory of this part of the world is to develop a model. A simple model will be a set of mathematical entities that we assume to correspond to the real ones. Our theory will be successful to the extent that we can show that the properties that our theory ascribes to our modeling entities (through stipulation or deduction from the stipulations) also hold of the real world entities they are assumed to correspond to.

The most general kind of entity in the domain at hand should include universities, departments, and individuals (people). We might want to talk about certain properties of these entities, for example their name or telephone number. In this case, we would declare the existence of a general type called entity and say that the features NAME and TEL(EPHONE) are appropriate features for all entities (tokens) of this type. So for each university, department, or person in this university world, we would hypothesize a distinct feature structure model that we could describe as follows:

$\begin{aligned}(3) \text { a. } & {\left[\begin{array}{ll}\text { entity } & \\ \text { NAME } & \text { Stanford University } \\ \text { TEL } & 650-723-2300\end{array}\right] } \\ \text { b. } & {\left[\begin{array}{ll}\text { entity } & \\ \text { NAME } & \text { Gerhard Casper } \\ \text { TEL } & 650-723-2481\end{array}\right] } \\ \text { c. } & {\left[\begin{array}{ll}\text { entity } & \\ \text { NAME } & \text { Stanford Linguistics } \\ \text { TEL } & 650-723-4284\end{array}\right] }\end{aligned}$

Note that we use type names, written in italics, as labels on the top line within feature structures.

Of course 'entity' is a very general classification - our theory would not have progressed far if it recognized no more specific kinds of things. So in fact, we would want our theory to include the fact that there are different subtypes of the type entity. Let's call these new types university, 
department, and individual. Entities belonging to each of these types have their own special properties. For example, individual people have birthdays, but universities and departments don't (or not in the same sense). Similarly, departments have chairs (or 'heads of department'), but neither universities nor individuals do. Finally, only universities have presidents. We can accommodate these facts by declaring each of the relevant features (BIRTHDAY, CHAIR, PRESIDENT) to be appropriate for the right one of our new subtypes. This formal declaration is just a precise way of saying that the members of the relevant subclasses have certain properties that distinguish them from other entitites in the system. The resulting descriptions that we write will be appropriately more specific, as in (4):

$\begin{aligned} \text { (4) a. } & {\left[\begin{array}{ll}\text { university } & \\ \text { NAME } & \text { Stanford University } \\ \text { PRESIDENT } & \text { Gerhard Casper } \\ \text { TEL } & 650-723-2300\end{array}\right] } \\ \text { b. } & {\left[\begin{array}{ll}\text { individual } & \\ \text { NAME } & \text { Gerhard Casper } \\ \text { BIRTHDAY } & 12-25-1937 \\ \text { TEL } & 650-723-2481\end{array}\right] } \\ \text { c. } & {\left[\begin{array}{ll}\text { department } & \\ \text { NAME } & \text { Stanford Linguistics } \\ \text { CHAIR } & \text { Stanley Peters } \\ \text { TEL } & 650-723-4284\end{array}\right] }\end{aligned}$

Note that each of these descriptions reflects the hierarchical organization of types. Each type of entity has its own constellation of features - some of them were declared appropriate for the indicated subtype; others were sanctioned by the supertype entity. This is a simple illustration of how a hierarchical classification system works. A given feature structure contains only those features that are declared appropriate by one of its types, that is, by its LEAF type ${ }^{4}$ or one of its supertypes. As we will see, a feature structure also inherits any type constraints, (that is, constraints on feature values) that are associated with its supertypes. Articulating a type hierarchy and the feature structures associated with each type is an important component of a theory that uses typed feature structures as models.

\footnotetext{
${ }^{4}$ The leaf types are the basic or bottom-level types in a hierarchy, i.e. the types that have no subtypes. These are often referred to in the literature (somewhat counterintuitively) as 'maximal' types.
} 


\section{2 / Syntactic Theory: A Formal Introduction}

To talk about merging information from more than one source, that is, when we know that two feature structure descriptions describe the same entity, we're going to need a precise method for combining these descriptions. A standard method for doing this is called UNIFICATION. Unification corresponds intuitively to the notion of identity that we have already used, indicated by two occurrences of a single variable (e.g. the category variable ' $\mathrm{X}$ ' used in our coordination rule) to refer to a single entity (e.g. a particular grammatical category like NP or S in particular instantiations of the coordination schema). Up to now, we have used these identities only for atomic values. Once we recognize feature structures as entities, however, we can think of each description as being satisfied by some set of feature structures. A feature structure description can thus be partial (satisfied by many distinct feature structures) or total (being satisified by only one). Any such description must be consistent, precisely because it specifies a set of objects (those that satisfy the description).

Unification, then, is just a general method for allowing two compatible descriptions to amalgamate the information they contain into a single (usually larger) description. Since all descriptions must be consistent, it follows that two feature structure descriptions can unify only if they are consistent - that is, unless they specify conflicting types or different atomic values for the same feature. The unification of two feature structure descriptions is just the description obtained by combining all of the information from both of them. If $\mathrm{D}_{1}$ is satisfied by a set (of feature structures) $\sigma_{1}$ and $\mathrm{D}_{2}$ is satisfied by a set $\sigma_{2}$, then the unification of $\mathrm{D}_{1}$ and $\mathrm{D}_{2}$ (written $\mathrm{D}_{1} \sqcup D_{2}$ ) is satisifed by the intersection of $\sigma_{1}$ and $\sigma_{2}$.

For example, the feature structure descriptions in (5) cannot unify

a. $\left[\begin{array}{ll}\text { university } & \\ \text { NAME } & \text { Stanford University }\end{array}\right]$
b. $\left[\begin{array}{ll}\text { university } & \\ \text { NAME } & \text { Harvard University }\end{array}\right]$

because they differ in the value they assign to the feature NAME. Intuitively, these two descriptions cannot describe the same entity. Similarly, the descriptions in (6) cannot unify

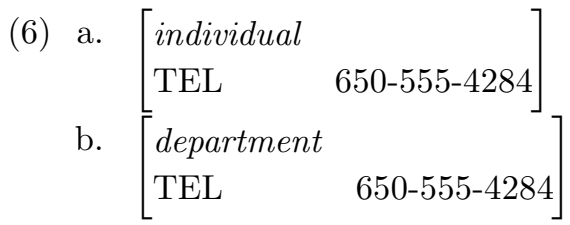

because they specify incompatible types, namely, individual and depart- 
ment, and hence cannot describe the same entity. But the feature structure description in (7) unifies with any of those in (8a)-(8c).

$$
\begin{aligned}
& \text { [TEL 910-234-5789] } \\
& \text { [university] } \\
& \text { b. individual } \\
& \text { NAME Xena: Warrior Princess } \\
& \text { c. } \\
& \text { department } \\
& \text { NAME Metaphysics } \\
& \text { CHAIR Alexius Meinong, Jr. }
\end{aligned}
$$

In each case, the result of unification is the description containing the information from each of the two descriptions unified, and nothing more. Thus the unification of (7) and (8b) is (9):

$$
\left[\begin{array}{ll}
\text { individual } & \\
\text { NAME } & \text { Xena: Warrior Princess } \\
\text { TEL } & 910-234-5789
\end{array}\right]
$$

We will often use unification to indicate that two different feature structures in fact have the same value for a given feature. To take a simple example, we might want to indicate that two different departments have the same phone number in the world we are describing. We will indicate this by two identical occurrences of a given boxed integer, or 'tag', as illustrated in (10).

$$
\left[\begin{array}{ll}
\text { department } & \\
\text { NAME } & \text { Metaphysics } \\
\text { TEL } & 2
\end{array}\right] \quad\left[\begin{array}{ll}
\text { department } & \\
\text { NAME } & \text { Philosophy } \\
\text { TEL } & 2
\end{array}\right]
$$

And of course we might want to simultaneously indicate a feature identity and the value of the feature, as shown in (11):

$$
\left[\begin{array}{ll}
\text { department } & \\
\text { NAME } & \text { Metaphysics } \\
\text { TEL } & \text { 2800-374-6786 }
\end{array}\right]\left[\begin{array}{ll}
\text { department } & \\
\text { NAME } & \text { Philosophy } \\
\text { TEL } & 2
\end{array}\right]
$$

Note that it would make no difference if we wrote the phone number after the other occurrence of 2 in (11). The intended interpretation would be exactly the same. It also makes no difference what order we write the features in. So (11) is equivalent, for example, to the following: 
54 / Syntactic Theory: A Formal Introduction

$\left[\begin{array}{ll}\text { department } & \\ \text { TEL } & \text { 2 } 800-374-6786 \\ \text { NAME } & \text { Philosophy }\end{array}\right]\left[\begin{array}{ll}\text { department } & \\ \text { NAME } & \text { Metaphysics } \\ \text { TEL } & 2\end{array}\right]$

Since the value of the feature TEL is atomic (i.e. it can bear no feature specifications of its own), the unification of values of this feature is much like the simple identity we expressed in the previous chapter by means of multiple occurrences of a variable (e.g. the variable $\mathrm{X}$ in the coordination schema). But in the chapters that follow, we will have occasion to unify feature structure descriptions that are embedded as the value of some feature.

\subsection{The Linguistic Application of Feature Structures 3.3.1 Feature Structure Categories}

So how do feature structures help us with our linguistic deliberations? Instead of saying that there is just one kind of linguistic entity, which must bear a value for every feature we recognize in our feature structures, we will often want to say that a given entity is of a certain type and that certain features are appropriate only for things of that type. We will use typing in many ways, to ensure that [NUM sg] (or [NUM pl]) can only be specified for certain kinds of words (nouns and pronouns, for example), but not, for instance, for prepositions or adjectives. ${ }^{5}$ Likewise, we will eventually want to distinguish auxiliaries (helping verbs like will and have) from other kinds of verbs in terms of a feature AUX. However, we will not want to say that nouns are all redundantly specified as [AUX -], but rather that the feature AUX just isn't appropriate for nouns. We can use types as a basis for classifying the feature structures we assume and the constraints we use to describe them. In so doing, we can then simply state that particular features only go with certain types of feature structures. This amounts to the beginnings of a linguistic ontology - that is, the types tell us what kind of linguistic entities exist, according to our theory, and the features associated with those types tell us what general properties those entitites exhibit. In this way, we also make feature structures compact, that is, we make them reflect only information that is appropriate for the kind of linguistic entity they are associated with.

The hierarchical organization of linguistic types in our theory is significant. As illustrated above, this enables us to classify feature structures in more subtle ways that will allow intermediate level categories

\footnotetext{
${ }^{5}$ Many such restrictions are language-particular. For example, adjectives are distinguished according to number (agreeing with the noun they modify) in many languages. Even prepositions exhibit agreement inflection in some languages (e.g. modern Irish) and need to be classified in similar terms.
} 
of various sorts. For example, verbs may be classified as intransitive or transitive; and transitive verbs may then be further subclassified as strict transitive (those taking a direct object and nothing else) or ditransitive. A hierarchical type system will let us talk about the properties shared by two distinct types (e.g. strict transitive and ditransitive) by associating a feature or a constraint with their common supertype (transitive).

To start, let us draw a very intuitive type distinction - between word and phrase. Our grammar rules (i.e. our phrase structure rules) all specify the properties of phrases; the lexicon provides a theory of words. Continuing our practice of indicating types as labels on feature structures, we will replace (2) above with (13):

$$
\left\langle\text { bird, }\left[\begin{array}{ll}
\text { word } & \\
\text { POS } & \text { noun } \\
\text { NUM } & \text { sg }
\end{array}\right]\right\rangle
$$

We will now reformulate our grammar in terms of typed feature structures. We do so in two stages: first, we introduce the feature VAL (for 'valence'), without worrying about agreement. Then we present our first feature-based treatment of subject-verb agreement. Both of these analyses will be refined in subsequent chapters.

\subsubsection{Representing Valence with a Feature}

We can identify our earlier categories IV, TV, and DTV with the following three feature structures:

$$
\begin{aligned}
\mathrm{IV} & =\left[\begin{array}{ll}
\text { word } & \\
\text { POS } & \text { verb } \\
\text { VAL } & \text { itr }
\end{array}\right] \quad \mathrm{TV}=\left[\begin{array}{ll}
\text { word } & \\
\text { POS } & \text { verb } \\
\text { VAL } & \text { tr }
\end{array}\right] \\
\mathrm{DTV} & =\left[\begin{array}{ll}
\text { word } & \\
\text { POS } & \text { verb } \\
\text { VAL } & \text { dtr }
\end{array}\right]
\end{aligned}
$$

These three categories all share the type word and the feature specification [POS verb]. This is just the combination of types and features that we would naturally identify with the category $\mathrm{V}$. That is, by analyzing categories in terms of types and features, we can distinguish between the different valence possibilities for verbs, while still recognizing that all verbs fall under a general category. We can represent the general category by leaving the value of the VAL feature unspecified, as follows: 
56 / Syntactic Theory: A Formal Introduction

$$
\mathrm{V}=\left[\begin{array}{cc}
\text { word } & \\
\mathrm{POS} & \text { verb }
\end{array}\right]
$$

The commonly used term in linguistics for leaving out feature specifications in order to specify a larger class of linguistic entities is UNDERSPECIFICATION.

The category VP differs from the category V only with respect to its type assignment. So the feature structure for VP is the following:

$$
\mathrm{VP}=\left[\begin{array}{ll}
\text { phrase } & \\
\mathrm{POS} & \text { verb }
\end{array}\right]
$$

And if we wanted to refer to the class that includes just verbs and verb phrases, we would refer to it as the underspecification in (17):

$$
\left[\begin{array}{ll}
\text { POS } & \text { verb }
\end{array}\right]
$$

Similarly, we can analyze the categories N and NP as follows:

$$
\mathrm{N}=\left[\begin{array}{ll}
\text { word } & \\
\text { POS } & \text { noun }
\end{array}\right] \quad \mathrm{NP}=\left[\begin{array}{ll}
\text { phrase } & \\
\mathrm{POS} & \text { noun }
\end{array}\right]
$$

Our method of analysis in fact allows underspecification of various kinds. Our goal is to provide compact descriptions for those categories that our grammar will actually need to refer to, what linguists usually call 'natural classes'.

We will continue to use labels like V, N, VP, and NP, but they should now be regarded as abbreviations for the typed feature structures just described. Notice that the feature analysis we have just sketched does not accommodate the category NOM: since there are only two syntactic types, this system does not permit the sort of three-level phrases we posited for NPs in the previous chapter. In the next chapter, we will revise our treatment of the internal structure of categories in such a way that NOM can be distinguished both from $\mathrm{N}$ and from NP.

Turning now to the phrase structure rules, we can reformulate our VP rules in terms of our new feature structure categories. Consider the following way of stating these rules:

$$
\begin{aligned}
& {\left[\begin{array}{ll}
\text { phrase } & \\
\mathrm{POS} & \square
\end{array}\right] \rightarrow\left[\begin{array}{ll}
\text { word } & \\
\mathrm{POS} & 0 \\
\mathrm{VAL} & \mathrm{itr}
\end{array}\right]} \\
& {\left[\begin{array}{ll}
\text { phrase } & \\
\mathrm{POS} & 1
\end{array}\right] \rightarrow\left[\begin{array}{ll}
\text { word } & \\
\mathrm{POS} & 1 \\
\mathrm{VAL} & \mathrm{tr}
\end{array}\right] \mathrm{NP}}
\end{aligned}
$$


Analyzing Features of Grammatical Categories / 57

$$
\left[\begin{array}{ll}
\text { phrase } & \\
\text { POS } & 1
\end{array}\right] \rightarrow\left[\begin{array}{ll}
\text { word } & \\
\text { POS } & 1 \\
\text { VAL } & \text { dtr }
\end{array}\right] \text { NP } \quad \text { NP }
$$

The two occurrences of 1 in each of these rules tell us that the POS value of the mother and that of the first daughter must be unified (which amounts to simple identity of atomic values in this case). Since the rules in (19) were introduced as VP rules, the obvious value to assign to 1 is 'verb'. But by stating the rules in this underspecified way, we can use them to cover some other structures as well. The first rule, for intransitives, can be used to introduce nouns, which can never be sisters to NPs. This is done simply by instantiating 1 as 'noun', which will in turn cause the mother to be an NP. To make this work right, we will have to specify that lexical nouns, like intransitive verbs, must be [VAL itr]. Similarly, the second rule can subsume our PP expansion rule, if 1 is instantiated as 'prep' and prepositions are lexically marked [VAL tr], thus allowing PPs to be built from a preposition and an NP object.

We would also like to use underspecification to collapse into one the two recursive rules introducing $\mathrm{PPs}$, that is, the rules $\mathrm{VP} \rightarrow \mathrm{VP} \mathrm{PP}$ and $\mathrm{NOM} \rightarrow \mathrm{NOM}$ PP. At this point, we can't do so, because we haven't yet integrated NOM into our system of types and features. But we would like eventually to be able to replace these two rules with something like the following: ${ }^{6}$

$$
\left[\begin{array}{ll}
\text { phrase } & \\
\mathrm{POS} & 2
\end{array}\right] \rightarrow\left[\begin{array}{ll}
\text { phrase } & \\
\mathrm{POS} & 2
\end{array}\right] \mathrm{PP}[\ldots]
$$

\section{1! Problem 1: Other Uses for Rule (20)}

The rule for PP modifiers given in (20) interacts with the coordination rule presented in the previous chapter. The coordination rule, as you may recall, is the following (with the ' $\mathrm{X}$ 's of Chapter 2 now replaced by the more general tag notation):

$$
1 \rightarrow 1^{+} \text {CONJ } 1
$$

In particular, the interaction of these rules predicts that a PP can modify a coordinate structure, as in examples like:

(i) Kim walks and reads books without difficulty

(ii) the poetry and the music on the program.

\footnotetext{
${ }^{6}$ Note that the choice of a particular tag here is entirely arbitrary - all that is crucial is that there be two occurrences of the same tag.
} 
58 / Syntactic Theory: A Formal Introduction

For the purposes of this problem, assume the following grammar, which we will refer to as $\mathbf{G}_{1}$.

Rules of $\mathbf{G}_{1}$ :

$$
\begin{aligned}
& {\left[\begin{array}{ll}
\text { phrase } & \\
\text { POS } & \text { 2 }
\end{array}\right] \rightarrow\left[\begin{array}{ll}
\text { phrase } & \\
\text { POS } & \text { 2 }
\end{array}\right] \mathrm{PP}[\ldots]} \\
& 1 \rightarrow \square^{+} \text {CONJ } 1 \\
& \mathrm{~S} \rightarrow \mathrm{NP} \text { VP } \\
& \mathrm{NP} \rightarrow(\mathrm{D}) \mathrm{N} \\
& {\left[\begin{array}{ll}
\text { phrase } & \\
\text { POS } & 1
\end{array}\right] \rightarrow\left[\begin{array}{ll}
\text { word } & \\
\text { POS } & 1 \\
\text { VAL } & \text { itr }
\end{array}\right]} \\
& {\left[\begin{array}{ll}
\text { phrase } & \\
\text { POS } & \square
\end{array}\right] \rightarrow\left[\begin{array}{ll}
\text { word } & \\
\text { POS } & 1 \\
\text { VAL } & \text { tr }
\end{array}\right] \mathrm{NP}} \\
& {\left[\begin{array}{ll}
\text { phrase } & \\
\text { POS } & \square
\end{array}\right] \rightarrow\left[\begin{array}{ll}
\text { word } & \\
\text { POS } & 1 \\
\text { VAL } & \text { dtr }
\end{array}\right] \text { NP } \quad \mathrm{NP}}
\end{aligned}
$$

Sample Lexical Entries of $\mathbf{G}_{1}$ :

$$
\begin{aligned}
& \left\langle\text { bird, }\left[\begin{array}{ll}
\text { word } & \\
\text { POS } & \text { noun } \\
\text { NUM } & \text { sg } \\
\text { VAL } & \text { itr }
\end{array}\right]\right\rangle \\
& \left\langle\text { flies, },\left[\begin{array}{ll}
\text { word } & \\
\text { POS } & \text { verb } \\
\text { VAL } & \text { itr }
\end{array}\right]\right\rangle
\end{aligned}
$$

A. Draw the tree structure defined by $\mathbf{G}_{1}$ for (i) and (ii). Make sure the PP modifies the entire coordinate structure.

B. Draw a distinct tree structure defined by $\mathbf{G}_{1}$ for each of these same examples, but make sure that the PP does not modify the entire coordinate structure.

The new version of the PP modifier rule also predicts that other categories besides VP and NP can occur with PP modifiers.

C. Using coordination as part of your argument, construct crucial examples demonstrating that $\mathrm{S}$ can also co-occur with PP modifiers. 
D. Find at least one more category that can be modified by a PP, providing examples to support your claim. (Note: You may consider types of phrases we have not yet discussed, as well as ones that have appeared in our rules.)

In the next chapter, we will carry even further the collapsing of phrase structure rules across POS values, when we revise our analysis of the valence feature. First, however, let us examine how features might be employed in the analysis of subject-verb agreement.

\subsubsection{Representing Agreement with Features}

One device introduced in the last section lends itself well to dealing with the problem of agreement. This is the use of a tag linking two feature values, the effect of which is to force two distinct nodes in a tree admitted by a rule to have identical values for a given feature. We can also employ this device to handle agreement by tagging values for the feature NUM. In the rule expanding $\mathrm{S}$, we could require that the NP and the VP both have the same value of NUM, and we could use the same technique to ensure that the NUM value of a given phrase is identical to the NUM value of the lexical head of that phrase. With these revisions, the rules in question look like (21).

$$
\begin{aligned}
& \mathrm{S} \rightarrow\left[\begin{array}{ll}
\text { phrase } & \\
\mathrm{POS} & \text { noun } \\
\text { NUM } & 1
\end{array}\right]\left[\begin{array}{ll}
\text { phrase } & \\
\mathrm{POS} & \text { verb } \\
\text { NUM } & 1
\end{array}\right] \\
& {\left[\begin{array}{ll}
\text { phrase } & \\
\mathrm{POS} & 1 \\
\mathrm{NUM} & 2
\end{array}\right] \rightarrow\left[\begin{array}{ll}
\text { word } & \\
\mathrm{POS} & 1 \\
\mathrm{NUM} & 2 \\
\mathrm{VAL} & \mathrm{itr}
\end{array}\right]} \\
& {\left[\begin{array}{ll}
\text { phrase } & \\
\mathrm{POS} & 1 \\
\mathrm{NUM} & 2
\end{array}\right] \rightarrow\left[\begin{array}{ll}
\text { word } & \\
\mathrm{POS} & 1 \\
\mathrm{NUM} & 2 \\
\mathrm{VAL} & \mathrm{tr}
\end{array}\right] \mathrm{NP}} \\
& {\left[\begin{array}{ll}
\text { phrase } & \\
\text { POS } & 1 \\
\text { NUM } & 2
\end{array}\right] \rightarrow\left[\begin{array}{ll}
\text { word } & \\
\text { POS } & 1 \\
\text { NUM } & 2 \\
\text { VAL } & \text { dtr }
\end{array}\right] \quad \mathrm{NP} \quad \mathrm{NP}}
\end{aligned}
$$

b.

c.

d. 
60 / Syntactic Theory: A Formal Introduction

\subsection{The Head Feature Principle}

The last three rules in (21) require that the mother and one of the daughters bear identical (unified) values both for POS and for NUM. In fact, the constituent on the right-hand side that carries the matching feature values is always the head daughter. As we will see in later chapters, there are a number of properties of phrases that are also properties of their lexical heads. ${ }^{7}$ In this section, we will develop general mechanisms for capturing the generalization that certain properties are characteristically shared by phrases and their heads.

Rather than stipulating identity of features in an ad hoc manner on both sides of the rules (as in (21)), our analysis will recognize that in a certain type of phrase - a HEADED PHRASE - one daughter is assigned special status as the HEAD DAUGHTER. Once such a notion is incorporated into our theory (thus providing a remedy for the second defect of standard CFGs noted in the last chapter), we can factor out the identity constraint that we need for all the headed phrases, making it a general principle. We will call this generalization the Head Feature Principle (HFP).

Certain rules, such as those in (21), introduce an element that functions as the head of the phrase characterized by the rule. We will call such rules HEADED RULES. To indicate which element introduced in a headed rule is the head daughter, we will label one element on the right hand side of the rule with the letter ' $H$ '. So a headed rule will have the following general form:

$$
[\text { phrase }] \rightarrow \ldots \mathrm{H}[] \ldots
$$

Our goal is to formulate a general theory of what features the head daughter shares with its mother in a headed phrase, that is, what features will always be the same for the element labeled ' $\mathrm{H}$ ' in a headed rule and the phrase licensed by the rule. ${ }^{8}$

Before proceeding, we need to reflect for a moment on parts of speech. As we noted above, there are certain features that are appropriate for certain parts of speech, but not others. For example, CASE is appropri-

\footnotetext{
${ }^{7}$ Alternative formulations might be that some properties of lexical heads are inherited by the phrases they 'project' or that properties of phrases are marked on the lexical heads. While it is often helpful to think of information as propagating up or down through a tree, this is just a metaphor. Our formulation of the generalization in the text avoids attributing directionality of causation in the sharing of properties between phrases and their heads.

${ }^{8}$ Note that ' $H$ ', unlike the other shorthand symbols we will use occasionally (e.g. ' $\mathrm{V}$ ', 'NP'), does not abbreviate a feature structure in a grammar rule. Rather, it merely indicates which feature structure in the rule corresponds to the phrase's head daughter.
} 
ate only for nouns (in English), while the feature AUX is specifiable only for verbs (to distinguish helping verbs from all others). Likewise, here we will use the features PER(SON) and NUM(BER) only for nouns, verbs, and determiners. ${ }^{9}$ To guarantee that only the right features go with the right parts of speech, we will treat parts of speech not as atomic values of the POS feature, but rather in terms of a set of types. ${ }^{10}$ Then we can declare which features are appropriate for each part of speech type.

We therefore introduce the types noun, verb, adj, prep, det, and conj for the six lexical categories we have so far considered. We then make all of these subtypes of a type called part-of-speech (pos). Our grammar must also specify the appropriate values for each feature it employs. We will make the traditional assumption throughout this book that NUM takes either 'sg' (singular) or 'pl' (plural) as its value and that the values of PER are '1st', '2nd', or '3rd'.

Having eliminated the old feature POS, we now introduce a new one called HEAD. HEAD will always take as its value a part of speech, that is, a feature structure assigned to some subtype of the type pos. In this way, HEAD does the work formerly assigned to POS; but it also does more, namely, it provides a way for us to begin to provide an account of which features are appropriate for which parts of speech.

In making this change, it should be noted that we have also introduced a significant innovation into our theory of feature structures. Previously, all of our features' values were atoms (e.g. 'itr' or 'sg') with no internal structure. By introducing complexes of type noun, verb, etc. as values of HEAD, we have introduced complex values for features: feature structures within feature structures. This is a technique that will serve us well in the chapters to come. Moreover, it will be of immediate use in providing us with a simple way to express the relation between a headed phrase and its head daughter. That is, the Head Feature Principle (given below) can be stated simply in terms of the unification of HEAD specifications.

In a similar vein, we can now improve our treatment of agreement by introducing a new feature called AGR, whose value will be a feature structure containing the features NUM and PER. That is, AGR contains

\footnotetext{
${ }^{9}$ This analysis will in fact be revised in the next chapter, where these features are used only for nouns and determiners.

${ }^{10}$ We might instead introduce some mechanism for directly stipulating dependencies between values of different features - such as a statement that the existence of a value for AUX implies that the value for POS is 'verb'. (For a theory that incorporates just such a mechanism, see Gazdar et al. (1985).) But such a mechanism is unnecessary, given the availability of types in our theory.
} 
just the information that matters for agreement. ${ }^{11}$ Since we need to have agreement features specified on phrases as well as on their heads, AGR must be a head feature. Hence, it shows up in feature structures like (23).

$$
\left[\operatorname{HEAD}\left[\begin{array}{lll}
\text { noun } & & \\
\text { AGR } & \left.\left[\begin{array}{ll}
\mathrm{PER} & 3 \mathrm{rd} \\
\mathrm{NUM} & \mathrm{pl}
\end{array}\right]\right]
\end{array}\right]\right.
$$

Now we have a more compact way to say that two elements agree with respect to all agreement features: we say that their AGR specifications are unified.

The type hierarchy for the parts of speech introduced in this section is summarized in (24), which also indicates the features declared to be appropriate for each individual type.

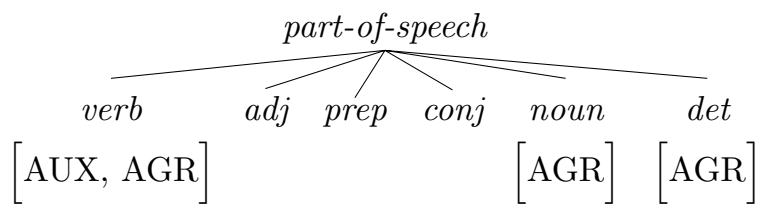

So far, we have done two things: (i) we have identified the head daughter in a headed rule and (ii) we have bundled together (within the HEAD value) all the feature specifications that the head daughter must share with its mother. With these two adjustments in place, we are now in a position to simplify the grammar of headed phrases.

First we simplify all the headed rules: they no longer mention anything about number or the part of speech - the information specified within the HEAD value.

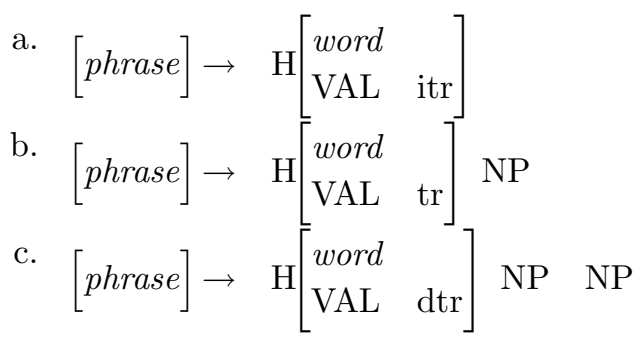

Recall that the element labeled ' $\mathrm{H}$ ' in the above rules is the head daughter.

\footnotetext{
${ }^{11}$ Formally, this will be specified by defining a type agreement-category and saying that the features NUM and PER are appropriate only for entities of this type.
} 
Second, we state the Head Feature Principle as a general constraint governing all trees built by headed rules.

(26) Head Feature Principle (HFP)

In any headed phrase, the HEAD value of the mother and the HEAD value of the head daughter must be unified.

The HFP makes our rules simpler by factoring out those properties common to all headed phrases, and making them conditions that will quite generally be part of the trees defined by our grammar. By formulating the HFP in terms of unification of HEAD values, we allow information specified by the rule, information present on the daughter or the mother, or information required by some other constraint all to be amalgamated, as long as that information is compatible.

\subsection{Trees and Phrase Structure}

\subsubsection{The Formal System: an informal account}

At this point, we must address the general question of how rules, lexical entries and principles like the HFP interact to define linguistic structures. Our earlier discussion of the question in Chapter 2 requires some revision, now that we have introduced feature structures and types. In the case of simple context-free grammars, descriptions and structures are in simple correspondence: in CFG, each local subtree (that is, a mother node with its daughters) corresponds in a straightforward fashion to a rule of the grammar. All of the information in that local subtree comes directly from the rule. There is no reason to draw a distinction between the linguistic objects and the grammar's descriptions of them. But now that rules, lexical entries and principles like the HFP all contribute partial information about linguistic tokens, we must take care to specify how these partial descriptions are amalgamated and how the grammar specifies which expressions are grammatical.

The distinction between descriptions and the structures they describe is fundamental. We use feature structures as our models of linguistic entities. Consider what this meant for the feature structures we used to model universities, departments and individuals. Each such model is assumed to have all the properties relevant to understanding the university system; this includes (for individuals) a name, a birthday, a telephone number (let's assume), and so forth. The objects we take as models are thus complete in relevant respects. ${ }^{12}$ Contrast this with descriptions of

\footnotetext{
${ }^{12}$ Of course, a model and the thing it is a model of differ with respect to certain irrelevant properties. Our models of university individuals should omit any irrelevant properties that all such individuals presumably have, ranging from hair color to grandmothers' middle names to disposition with respect to Indian food.
} 


\section{4 / Syntactic Theory: A Formal Introduction}

university individuals. These come in various degrees of completeness. A description may be partial in not specifying values for every feature, in specifying only part of the (complex) value of a feature, in failing to specify a type, or in specifying nothing at all. A complete description of some entity will presumably be satisfied by only one thing - the entity in question. An empty description is satisfied by all the entities in the modeling domain. Any nonempty partial description is satisfied by some things in the modeling domain, and not by others.

Our theory of language works the same way. We use feature structures and trees to model expressions like words and phrases; we also use feature structures to model other things like grammatical categories. Since the structures we use are models, they too are complete (or RESOLVED) with respect to all linguistically relevant properties. So a given word (token), say a pronoun, is a feature structure that has a determinate value for number - it is either singular or plural. Likewise, it is determinately 1st, 2 nd or 3 rd person. The only kind of thing that could be indeterminate - underspecified for this kind of information - is a feature structure description.

Grammars are linguistic descriptions; resolved feature structures and tree structures are the kinds of things that these descriptions describe. The grammar is successful to the extent that it can be shown that these structures - its models - have properties that are in correspondence with our observations about how the language (out there in the world, in society, or in people's heads) really is.

As we start to get more precise about our grammar, it will be important not to confuse linguistic structures and their descriptions. With this goal in mind, let us introduce a notational distinction. We will continue to use square bracket matrices (or ATTRIBUTE VALUE MATRICES) for feature structure descriptions, and whenever we want to make reference to a resolved feature structure model, we will use box diagrams. Thus the lexical entry for the noun fish will include a category description like the one shown in (27).

$$
\left\langle\text { fish , }\left[\begin{array}{lll}
\text { word } & & \\
\text { HEAD } & {\left[\begin{array}{lll}
\text { noun } & & \\
\text { AGR } & {[\text { PER }} & 3 \mathrm{rd}
\end{array}\right]}
\end{array}\right]\right)
$$

We will interpret this lexical entry as a word description that has two distinct word structure models, corresponding to the category of the singular word fish and that of the plural word fish. We will take these 
word structures, illustrated in (28), to be non-branching trees whose mother is a resolved feature structure satisfying the description in (27).
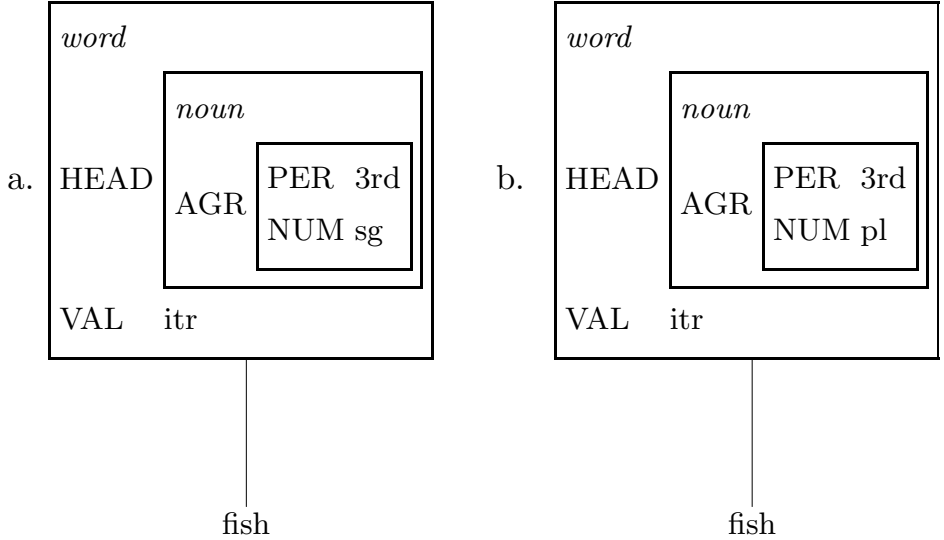

As our grammar becomes more fully developed, there will in fact be more than two models for this description, each exhibiting a unique determination for such properties as mass vs. count, nominative vs. accusative case, and so forth. It is the goal of a grammar to enumerate all these linguistic entities (structures assumed to correspond to bits of reality) and to correctly describe their grammatical properties.

Phrase structures are not different in kind from word structures, except that they are licensed by grammar rules, rather than lexical entries. In our theory, a grammar rule is in fact viewed as a very general description of a certain kind of phrase, one that is satisfied by infinitely many resolved phrase structures. The purpose of principles like the Head Feature Principle then is to constrain the set of phrase structure models. So if we reconsider the grammar rule in (25a), repeated here as (29),

$$
[\text { phrase }] \rightarrow \mathrm{H}\left[\begin{array}{ll}
\text { word } \\
\text { VAL } & \text { itr }
\end{array}\right]
$$

we see that there are four conceivable phrase structure models containing the word structures illustrated in (28), namely those in (30). 
66 / Syntactic Theory: A Formal Introduction

(30) Four Phrase Structures

a.

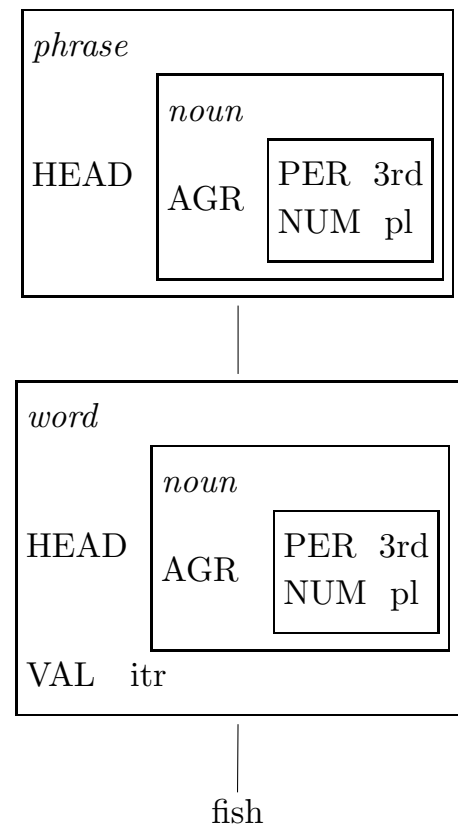

b.

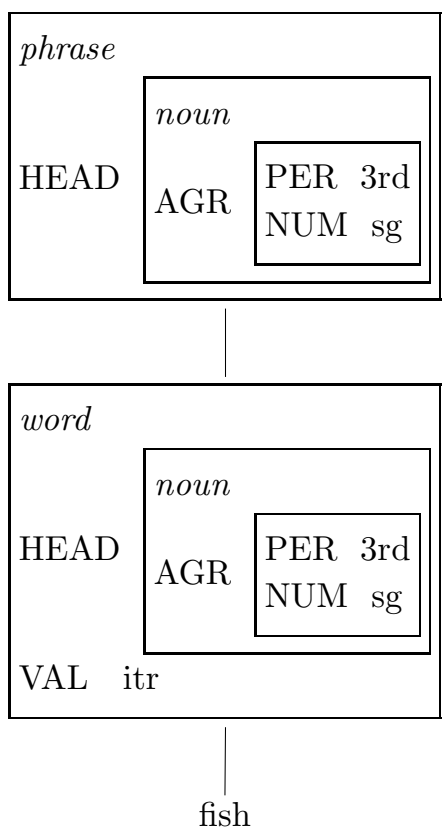


c.

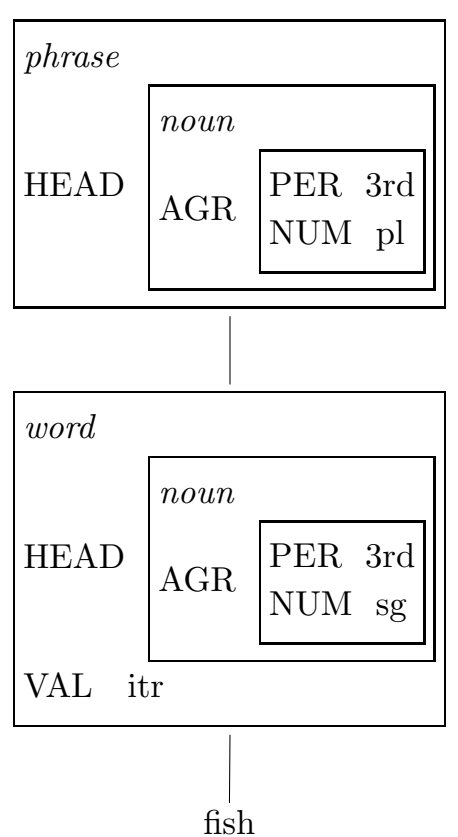

d.

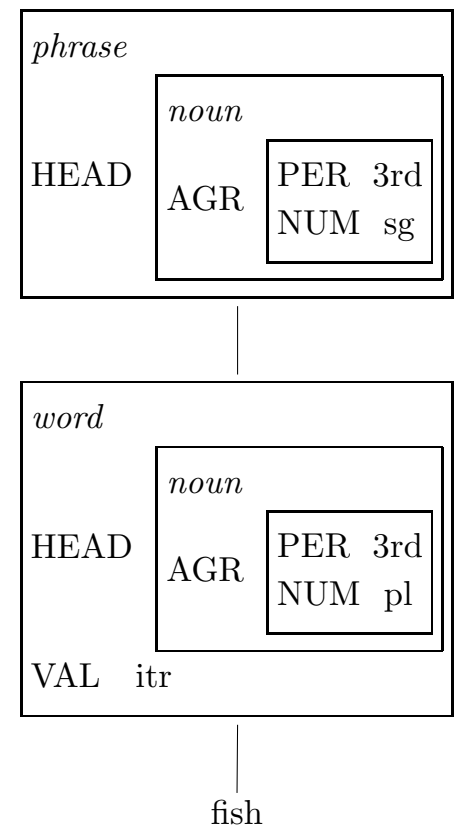

Without further principles, all of these resolved phrase structures would satisfy the rule in (29). By adding the HFP to our grammar, we rule out the phrase structures in $(30 \mathrm{c}, \mathrm{d})$ as models of $(29)$, because in each of these structures the HEAD value of the highest node fails to agree with that of its head daughter.

So the way our theory works is in terms of a notion of constraint satisfaction:

(31) A phrase structure is well-formed just in case each local subtree within it either:

1. satisfies a lexical entry, or

2. satisfies some grammar rule and all grammatical principles (e.g. the $\mathrm{HFP}$ ).

This is quite similar to the way theories and models of them are presented in other sciences. ${ }^{13}$

As we develop our grammar to deal with more intricate phenomena, we will have occasion to introduce more grammatical features into our feature structures. For this reason, it would quickly become cumbersome to illustrate details of our analyses in terms of the resolved word

\footnotetext{
${ }^{13} \mathrm{We}$ will leave the intuitive notion of 'satisfaction' unexplicated for the moment,
} postponing a more formal presentation of our theory until Chapter 6 . 
and phrase structures that satisfy them. To avoid this problem, in the rest of this book we will present analyses in terms of descriptions of these structures, or STRUCTURAL DESCRIPTIONs (SDs), which are the amalgamation of constraints from lexical entries, grammar rules, and relevant grammatical principles. This will allow us to continue to use familiar, tree-like objects throughout our discussions, but without having to specify values for irrelevant features.

Descriptions of word structures will be called LEXICAL SDs and descriptions of phrase structures will be called PHRASAL SDs. A lexical $\mathrm{SD}$ is a tree diagram with two nodes: the lower node is a form that appears as the first member of a lexical entry, and the upper node is any word description that is an EXTENSION of the one that is the lexical entry's second member. ${ }^{14} \mathrm{~A}$ well-formed description $\mathrm{A}$ is an extension of a description B (or alternatively 'B subsumes A') just in case A contains all the information in $\mathrm{B}$, and possibly some more.

For phrases, the matter is slightly more complex. There are a number of constraints that we want to include in a well-formed phrasal SD. First of all, the mother and daughters of each local subtree of a phrasal SD must include all the information provided by the grammar rule that licensed that subtree. In addition, these local subtrees need to 'unify in' the information that comes from constraints like the HFP.

\subsubsection{An Example}

Consider the sentence Fish swim. Let's suppose that the lexical entry for fish is underspecified for number, as shown above (repeated in (32)), and that the lexical entry for the plural form swim is underspecified for person as shown in $(32 \mathrm{~b})$ :

(32) a.

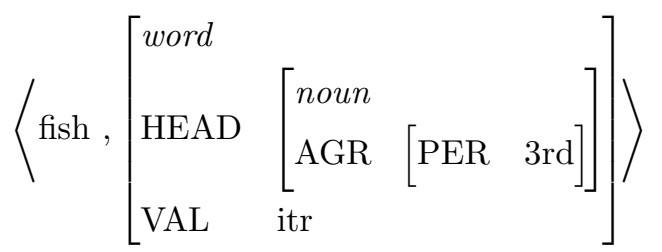

b.

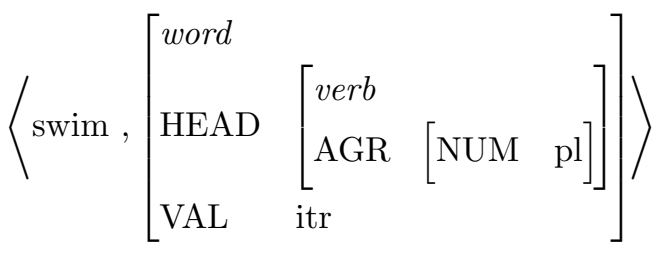

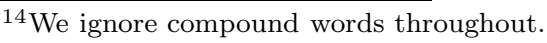


Given these two lexical entries, the following are both lexical SDs, according to our theory:

(33) a.

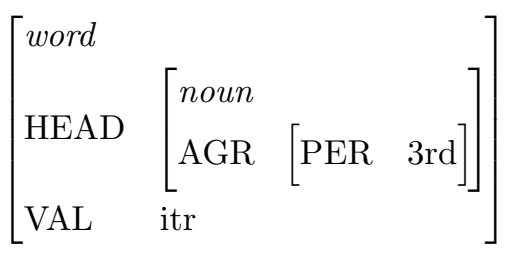

b.

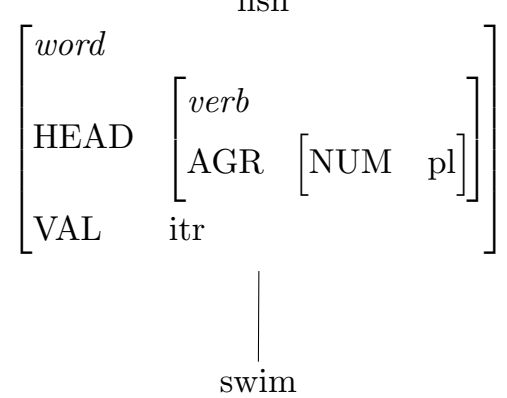

Note that the lexical SDs in (33) are MINIMAL, that is, they specify exactly the same information that we find in the lexical entries $(32 \mathrm{a}, \mathrm{b})$.

These SDs can now be embedded within larger SDs sanctioned by the rule in $(29)$, as illustrated in $(34 \mathrm{a}, \mathrm{b})$.

(34) a.

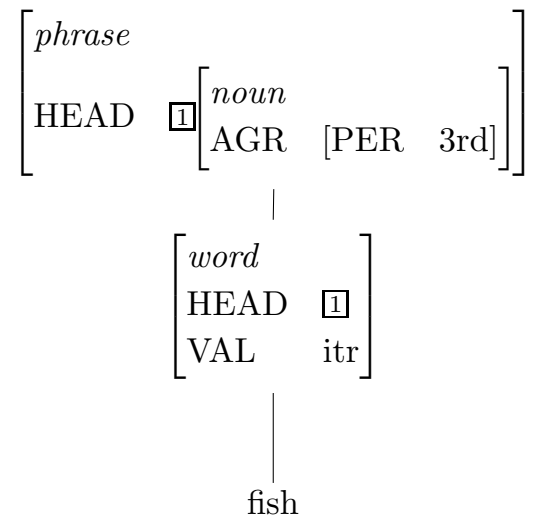


70 / Syntactic Theory: A Formal Introduction

b.

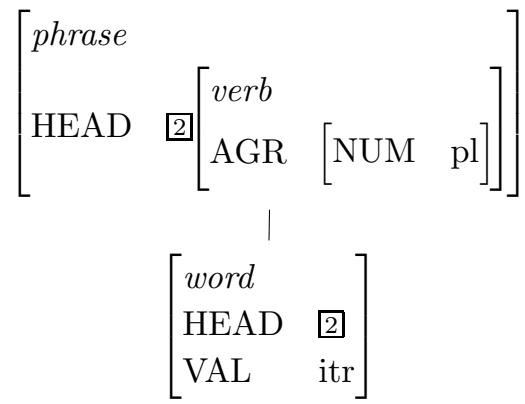

Both of these phrasal SDs obey the HFP, as the values of HEAD (and hence the values for all features within HEAD, e.g. AGR) on the mother are identified with those on the head daughter. Note further that the AGR values of (34a) and (34b) are distinct, but compatible.

And finally, assuming we enforce agreement as before, by constraining our S rule as shown in (35), then we will build sentence descriptions like the one shown in (36).

$$
\mathrm{S} \rightarrow\left[\begin{array}{lll}
\text { phrase } & & \\
\text { HEAD } & {\left[\begin{array}{ll}
\text { noun } & \\
\text { AGR } & 1
\end{array}\right]}
\end{array}\right]\left[\begin{array}{lll}
\text { phrase } & \\
\text { HEAD } & {\left[\begin{array}{ll}
\text { verb } & \\
\text { AGR } & 1
\end{array}\right]}
\end{array}\right]
$$

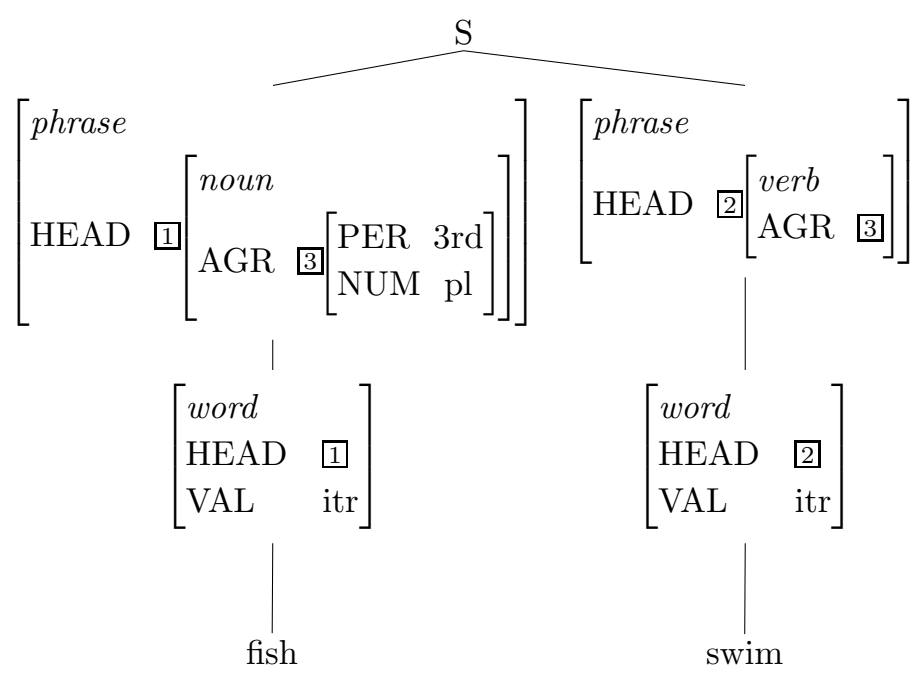


The symbol ' $\mathrm{S}$ ' here is of course just an abbreviation for a feature structure description of a sort yet to be determined.

It is at this level that the real effect of unification is seen. The AGR values of the two daughters of $\mathrm{S}$ in (36) are unified, as they must be because the values for these features are unified in the rule (35) and our theory therefore requires that these values be identified in any tree constructed in accordance with that rule. The unified AGR value contains the two different pieces of compatible information, one coming from each of the phrase's daughters. Note that the lexical SDs contained in this phrasal SD are not minimal. Because the AGR values are identified at the highest level of structure in (36), the AGR information in each lexical subtree includes the information from both the noun fish and the verb swim.

Crucially, given our theory, the rule in (35) cannot give rise to an SD like the following, where the NP and VP daughters contain conflicting AGR information:

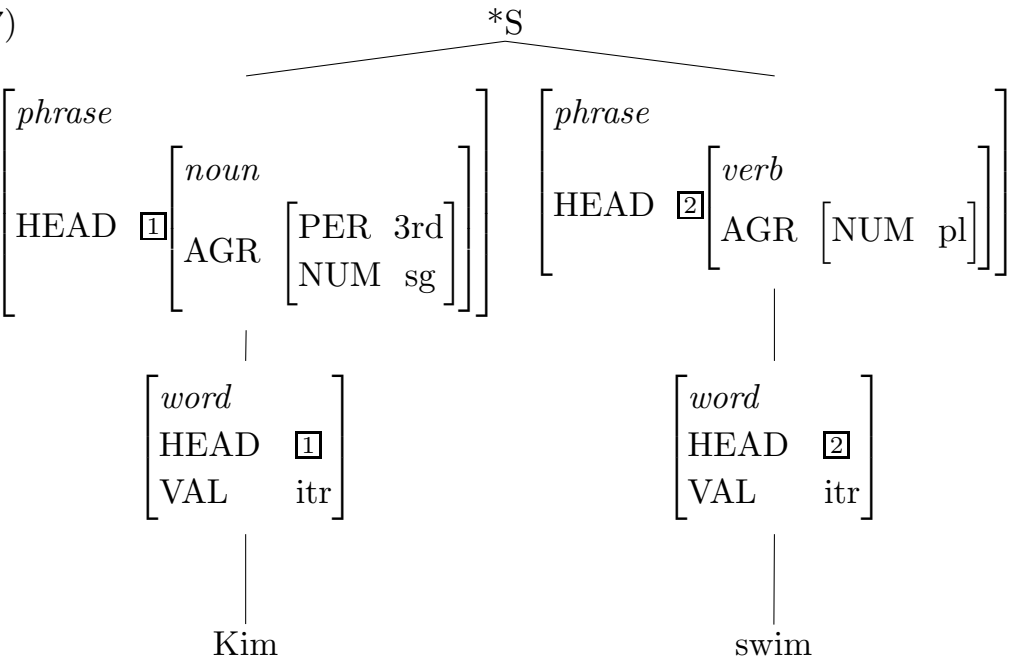

In this way, the various constructs of our theory work together to rule out certain sentences as inconsistent with the simultaneous satisfaction of all relevant constraints. The only resolved feature structure that can be built from the lexical entries and grammar rules we have been discussing is the following one, of which (36) is a minimal description: 
72 / Syntactic Theory: A Formal Introduction

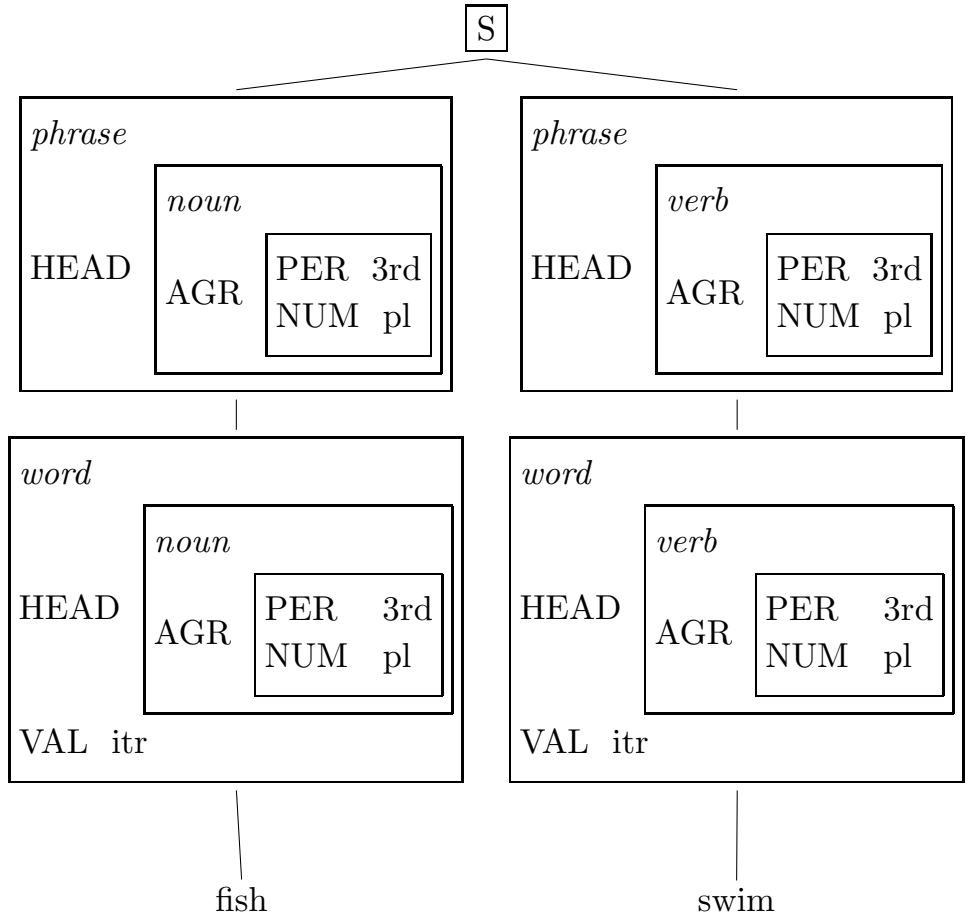

In short, the theory we have begun to sketch provides a precise MODEL-THEORETIC account of which expressions of a language are grammatical, which potential expressions are ungrammatical, which expressions are ambiguous, and so forth. The account proceeds by expressing generalizations in terms of a CFG-like conception of grammar rules, underspecified descriptions, and general constraints like the Head Feature Principle.

Somewhere along the line, of course, an adequate grammar will need to specify the well-formed 'stand alone' utterances of the language: the phrases that can be used in isolation to express a complete message are those whose mother is of the category S. ${ }^{15}$ But we are not quite ready yet to define the notion ' $\mathrm{S}$ '. This will emerge clearly only after we consider the feature structures of phrases a bit more carefully, as we do in the next chapter.

\footnotetext{
${ }^{15}$ This matter is actually more complex than it might seem, given that single word exclamations (e.g. Help!) can also express complete messages in isolation.
} 


\section{Problem 2: Applying the Rules}

The grammar just illustrated in the text, which we may refer to as $\mathbf{G}_{2}$ may be summarized as follows:

Rules of $\mathbf{G}_{2}$ :

$$
\begin{aligned}
& \left.\mathrm{S} \rightarrow\left[\begin{array}{lll}
\text { phrase } & & \\
\text { HEAD } & {\left[\begin{array}{ll}
\text { noun } & \\
\text { AGR } & 1
\end{array}\right]}
\end{array}\right]\left[\begin{array}{lll}
\text { phrase } & \\
\text { HEAD } & \text { verb } & \\
\text { AGR } & 1
\end{array}\right]\right] \\
& {[\text { phrase }] \rightarrow \mathrm{H}\left[\begin{array}{ll}
\text { word } & \\
\text { VAL } & \text { itr }
\end{array}\right]} \\
& {[\text { phrase }] \rightarrow \mathrm{H}\left[\begin{array}{ll}
\text { word } \\
\text { VAL } & \text { tr }
\end{array}\right] \mathrm{NP}} \\
& {[\text { phrase }] \rightarrow \mathrm{H}\left[\begin{array}{ll}
\text { word } & \\
\text { VAL } & \mathrm{dtr}
\end{array}\right] \mathrm{NP} \quad \mathrm{NP}}
\end{aligned}
$$

Sample Lexicon of $\mathbf{G}_{2}$ :

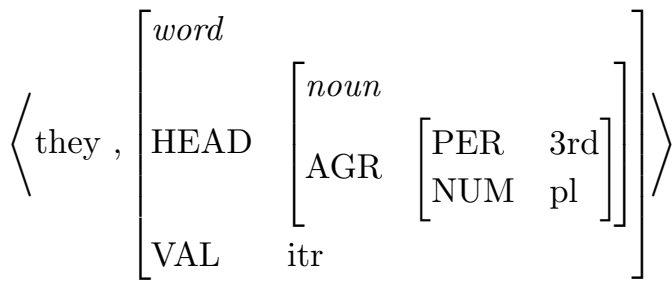

$$
\begin{aligned}
& \left\langle\operatorname{sing},\left[\begin{array}{lll}
\text { word } & & \\
\text { HEAD } & {\left[\begin{array}{lll}
\text { verb } & & \\
\text { AGR } & \text { NUM } & \text { pl }
\end{array}\right]}
\end{array}\right]\right\rangle
\end{aligned}
$$

A. Formulate a lexical entry for the word sings and explain how this entry interacts with other aspects of $\mathbf{G}_{2}$ so as to rule out unacceptable examples like ${ }^{*}$ They sings.

B. Formulate a feature structure version of the following rule (note that the head daughter is a word): $\mathrm{NP} \rightarrow(\mathrm{D}) \mathrm{N}$

Make sure that your rule is compatible with $\mathbf{G}_{2}$ and that it specifies all identities of feature values required to answer the remaining parts of this problem. 
C. Give precise lexical entries for the determiners ([HEAD det] $) a$ and many.

D. Demonstrate precisely how your answers to (B) and (C) work together to account for the following data:

(i) *a birds

(ii) *many bird

E. Explain (by constructing lexical entries and illustrating which rules cannot be applied) precisely how your analysis of NPs interacts with the rule for expanding $\mathrm{S}$ to explain the following examples.

(iii) *The bird sing.

(iv) *Many birds sings.

\subsection{Conclusion}

The introduction of features has given us a formal mechanism for talking about ways in which sets of words (and phrases) behave both alike and differently. By allowing embedded feature structures, underspecifying categories, and formulating general constraints stating identities that must hold in well-formed trees, we have been able to generalize our phrase structure rules and reduce their number. This in turn has led us to carefully distinguish between our grammar rules, the resolved structures that satisfy them, and the (partial) descriptions of those structures that we use throughout.

The theory we are developing is still closely related to standard CFG, yet it is somewhat more abstract. We no longer think of our phrase structure rules as specifying all the information that labels the nodes of trees. Rather, the rules, the lexicon, and some general principles - of which the HFP is the first example - all place certain constraints on trees, and any imaginable tree is well formed so long as it conforms to these constraints. In this way, our grammar continues to be constraint-based, with the rules, lexical entries, and general principles all contributing to linguistic descriptions and hence placing partial constraints on the well-formed structures of the language.

But the changes introduced in this chapter are not yet sufficient. They still leave us with three rules that have too much in common and could be collapsed. Moreover, as we will see in the next chapter, we have simplified the facts of agreement too much. And, as we mentioned above, our new feature-based system of rules doesn't allow us to reconstruct the category NOM. These problems will be dealt with in the next chapter, 
where we enrich our conception of features and of the lexicon, to allow still further simplification of the phrase structure rules.

\subsection{Further Reading}

One of the earliest (but often ignored) demonstrations of the descriptive power of feature structures is Harman (1963). Chomsky (1965) provides one of the earliest explicit discussions of syntactic features in generative grammar. The modern tradition of using complex feature structures (that is, features with feature structures as their values) begins with Kay (1979), Bear (1981), Bresnan (1982a), and Gazdar (1981) (see also Kaplan (1975) and Gazdar et al. (1985)). For an elementary discussion of the formal properties of unification and its use in grammatical description, see Shieber (1986). For differing and more detailed technical presentations of the logic of typed feature structures, see King (1989) and Carpenter (1992). The mathematics of tree structures is discussed by Partee et al. (1990). 



\section{Complex Feature Values}

\subsection{Introduction}

By analyzing grammatical categories into features, we were able to generalize some of our phrase structure rules and eliminate others. This is not only a more compact way to represent syntactic information, it is also a way to encode systematically the fact that phrases of different types exhibit parallel structures. In addition, the analysis predicts that different phrase types generally will be similarly structured. In particular, the rules we gave in the previous chapter suggest that lexical head daughters in English uniformly occur at the left edge of their phrases. ${ }^{1}$

Of course, VPs and PPs are consistently head-initial, but it's not completely clear that NPs exhibit the same head-first pattern. For example, NPs may take determiners and adjectives before the lexical head, as in the noisy dogs. Recall that in our earlier treatment, NPs had an intermediate level of structure - every NP contained a NOM consituent; and every NOM contained a lexical head - a noun. This mode of analysis allowed us to maintain the generalization that the lexical head daughter is always the leftmost element under its mother node. It seems desirable, then, to find a way of reconstructing the NOM analysis within our new, feature-based theory.

Another motivation for revising our current analysis is that our rules are still not maximally general. We have three distinct rules introducing lexical heads, one for each of the three valences. We would like to consolidate these. Moreover, these three valences are far from the only possible environments lexical heads may require. Consider the examples in (1).

\footnotetext{
${ }^{1}$ This is not true in some other languages, e.g. in Japanese, the lexical head daughters are phrase-final, resulting in SOV (Subject-Object-Verb) ordering, as well as noun-final NPs.
} 
(1) a. Pat relies on Kim.

b. ${ }^{*}$ Pat relies.

c. The child put the toy on the table.

d. ${ }^{*}$ The child put the toy.

e. The teacher became angry with the students.

f. *The teacher became.

g. The jury believed the witness lied.

Examples $(1 \mathrm{a}, \mathrm{b})$ show that some verbs require following PPs; $(1 \mathrm{c}, \mathrm{d})$ show that some verbs must be followed by both an NP and a PP; (1e,f) show a verb that can be followed by a kind of phrase we have not yet discussed, called an adjective phrase (AP); and (1g) shows a verb that can be followed by an S. We say only that became CAN be followed by an AP and that believed CAN be followed by an S, because they can also appear in sentences like Pat became an astronaut and Pat believed the story, in which they are followed by NPs. In fact, it is extremely common for verbs to be able to appear in multiple environments. Similarly, (2) shows that ate, like many other English verbs, can be used either transitively or intransitively.

(2) The guests ate (the cheese).

Facts like these show that the number of values of VAL must be far greater than three. This in itself would not be problematic, except that our current formalism, as developed in the previous chapter, requires a separate phrase structure rule for each value of VAL. This is an unwanted redundancy, for the lexical distinctions would be encoded twice: once in the phrase structure rules and once in the (many) new values of VAL that would be required.

\subsection{Complements}

Intuitively, we would like to have our rule simply say that a phrase (a $\mathrm{VP}$, in the cases above) may consist of a lexical head (a V, in these cases) followed by whatever other phrases the lexical head requires. We could then relegate to the lexicon the task of specifying for each word what elements must appear together with (or co-occur with) that word. In this section, we develop a way to do just this. It involves enriching our conception of valence features in a way somewhat analogous to what we did with grammatical categories in the previous chapter.

Before we begin the discussion of this analysis, however, let us consider briefly the status of the kinds of co-occurrence restrictions we have been talking about. For this discussion (and much else that follows), it will be convenient to have a term for the elements that characteristically co-occur with a lexical head - that is, for things like the phrases that 
occur after the verbs in (1). The term COMPLEMENT (of the head) is more or less standard and will be used throughout this text.

It has sometimes been argued that the number and type of complements a verb takes is fully determined by its meaning. For example, the verb disappear is used to describe events involving a single entity (expressed by its subject); deny's semantics involves events with two participants, one typically human and the other a proposition; and an event described by hand must include three participants: the person who does the handing, the thing handed, and the recipient of the transaction. Correspondingly, disappear takes no complements, only a subject; deny takes a subject and a complement, which may be either an NP (as in The defendant denied the charges) or an $\mathrm{S}$ (as in The defendant denied he was guilty); and hand takes a subject and two NP complements (or one NP and one PP complement).

It is undeniable that the semantics of a verb is intimately related to its valence. There is, however, a certain amount of syntactic arbitrariness to it, as well. For example, the words eat, dine, and devour all denote activities necessarily involving both a consumer of food and the food itself. Hence, if a word's valence were fully determined by its meanings, one might expect that all three would be simple transitives, requiring a subject and an NP complement (that is, a direct object). But this expectation would be wrong: dine is intransitive, devour is obligatorily transitive, and (as noted above), eat can be used intransitively or transitively.

(3) a. The guests devoured the meal.

b. ${ }^{*}$ The guests devoured.

c. ${ }^{*}$ The guests dined the meal.

d. The guests dined.

e. The guests ate the meal.

f. The guests ate.

Thus, though we recognize that there is an important link between meaning and valence, we will continue to specify valence syntactically. We will say more about the connection between meaning and valence - and more generally about the syntax-semantics interface - in later chapters.

\subsection{The COMPS Feature}

In our current grammar, the lexical entry for a verb like deny would specify that it is [VAL tr]. This ensures that it can only appear in word structures that are specified as [VAL tr], and such word structures can be used to build larger structures only by using the rule of our grammar 


\section{0 / Syntactic Theory: A Formal Introduction}

that introduces an immediately following NP. Hence, deny has to be followed by an NP. ${ }^{2}$

In effect, the value of the VAL feature in this system functions as a pointer to the relevant phrase structure rule for licensing the verb in question. In fact, the values of VAL correspond one-to-one with the phrase structure rules that assign the appropriate kinds of complements to the lexical head daughters. This redundancy is another we would like to eliminate from our grammar.

An alternative approach to complement selection is to use features directly in licensing complements - that is, to have a feature whose value specifies what the complements must be. We will now make this intuitive idea explicit. First, recall that in the last chapter we allowed some features (e.g. HEAD, AGR) to take values that are feature structures themselves. If we replace VAL with such a feature, we can allow its value to state directly what the word's complement must be. So we propose to replace VAL with a feature-valued feature, which we call COMPLEMENTS (COMPS). The value of COMPS for deny can simply be NP - the feature structure description in (4):

$$
\left[\begin{array}{ll}
\text { phrase } & \\
\text { HEAD } & \text { noun }
\end{array}\right]
$$

Similarly, we can indicate that a verb takes another type of complement: rely, become, and believe, for example, can take COMPS values of $\mathrm{PP}, \mathrm{AP}$, and S, respectively. ${ }^{3}$ Optional complements, such as the object of eat can be indicated using parentheses; that is, the lexical entry for eat can specify [COMPS (NP)]. Likewise, we can indicate alternative choices for complements using the vertical bar notation introduced in the discussion of regular expressions in Chapter 2. So the entry for deny or believe includes the specification: [COMPS NP | S].

Of course there is a problem with this proposal: it does not cover verbs like hand and put that require more than one complement. But it's not hard to invent a straightforward way of modifying the COMPS analysis to let it encompass multiple complements. Instead of treating the value of COMPS as a single feature structure, we will let it be a LIST of feature structures. Intuitively, the list specifies a sequence of

\footnotetext{
${ }^{2}$ As noted earlier, we have not dealt with the other possible environment for deny, namely the one where it is followed by a clause. We ignore this problem for the moment, but the analysis developed in this chapter provides a way of dealing with it.

${ }^{3}$ We have not yet said anything about how $\mathrm{S}$ is analyzed in terms of feature structures. Later in this chapter, however, we will present a treatment of S as an abbreviation for a feature structure, just like other category names.
} 
categories corresponding to the complements that the word combines with. So, for example, the COMPS values for deny, become, and eat will be lists of length one. For hand, the COMPS value will be a list of length two, namely $\langle\mathrm{NP}, \mathrm{NP}\rangle .^{4}$ For verbs taking no complements, like disappear, the value of COMPS will be \langle\rangle (a list of length zero). We interpret this to mean that trees containing the verb in question will be well formed only if the sisters of the $\mathrm{V}$-node are compatible with the categories specified on the list. For example, rely will only be allowed in trees where the VP dominates a V and a PP.

Now we can collapse all the different rules for expanding a phrase into a lexical head $(\mathrm{H})$ and other material. We can just say:

(5) Head-Complement Rule

$$
\left[\begin{array}{ll}
\text { phrase } & \\
\text { COMPS } & \langle\rangle
\end{array}\right] \rightarrow \mathrm{H}\left[\begin{array}{ll}
\text { word } & \\
\text { COMPS } & \langle\square, \ldots, n\rangle
\end{array}\right] \square \ldots \square
$$

Thus, if a word is specified lexically as [COMPS $\langle\mathrm{AP}\rangle]$, it must cooccur with exactly one AP complement; if it is [COMPS $\langle\mathrm{NP}, \mathrm{NP}\rangle]$, it must co-occur with exactly two NP complements, and so forth. Note that we also want (5) to allow for a phrase that contains a head daughter but no complements (letting $n=0$ ). So if the head daughter is lexically specified as [COMPS \langle\rangle$]$, it must appear as the only daughter in a phrase structure licensed by (5). Finally, the mother of any structure licensed by (5), which we will term a HEAD-COMPLEMENT PHRASE, must be specified as [COMPS \langle\rangle ], because that mother must satisfy the description on the left-hand side of the rule. ${ }^{5}$

In short, the COMPS list of a lexical entry specifies a word's cooccurrence requirements; and the COMPS list of a phrasal node is empty. So, in particular, a V must have sisters that match all the feature structures in its COMPS value, and the VP that it heads has the empty list as its COMPS value and hence cannot combine with complements. The Head-Complement Rule, as stated, requires all complements to be realized as sisters to the lexical head. ${ }^{6}$

If you think in terms of building the tree bottom-up, starting with

\footnotetext{
${ }^{4}$ We use angle brackets to designate lists.

${ }^{5}$ Note that by underspecifying the complements introduced by this rule - not even requiring them to be phrases, for example - we are implicitly leaving open the possibility that some complements will be nonphrasal. This will become important in the analysis of negation presented in Chapter 13.

${ }^{6}$ This appears well motivated for English, but our general theory allows us to write a Head-Complement Rule for some other language that allows some of the complements to be introduced higher in the tree structure. For example, structures like the one in (i) would be allowed by a version of the Head-Complement Rule that required
} 
the verb as head, then the verb has certain demands that have to be satisfied before a complete, or 'saturated', constituent is formed. On this conception, the complements can be thought of as being 'cancelled off' of the head daughter's COMPS list in the process of building a headed phrase. We illustrate this with the VP put flowers in a vase: the verb put requires both a direct object $\mathrm{NP}$ and a $\mathrm{PP}$ complement, so its COMPS value is $\langle\mathrm{NP}, \mathrm{PP}\rangle$. The requisite NP and $\mathrm{PP}$ will both be sisters to the $\mathrm{V}$, as in (6), as all three combine to form a VP, i.e. a verbal phrase whose complement requirements have been fulfilled.

(6)

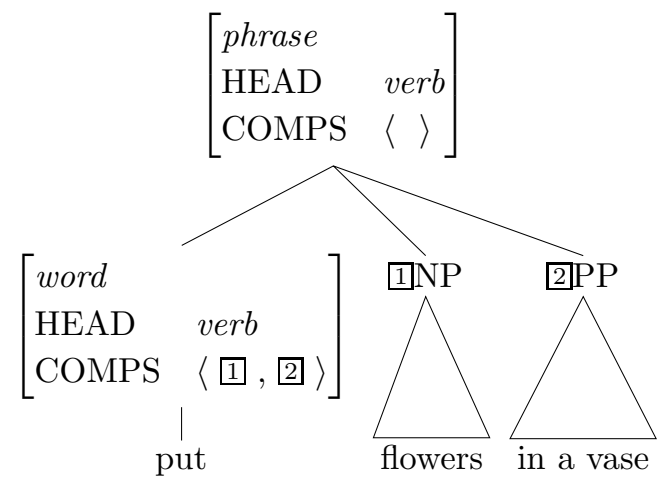

As is evident from this example, we assume that the elements in the

neither that the head daughter be of type word nor that the mother have an empty COMPS list:

(i)

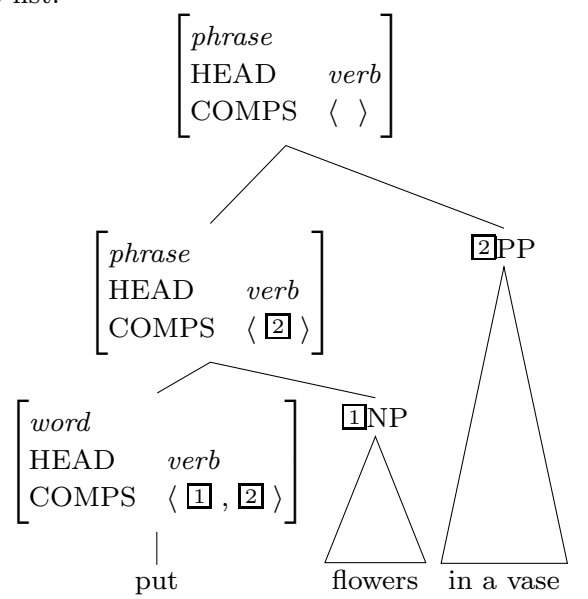

Such grammatical variations might be regarded as 'parameters' that are set differently in particular languages. That is, it may be that all languages manifest the HeadComplement Rule, but there are minor differences in the way languages incorporate the rule into their grammar. The order of the head and the complements is another such parameter of variation. 
value of COMPS occur in the same order as they appear in the sentence. We will continue to make this assumption, though ultimately a more sophisticated treatment of linear ordering of phrases in sentences may be necessary.

A common source of confusion is that some kinds of constituents, notably PPs, can function either as complements or as modifiers. This often raises the question of how to analyze a particular PP: should it be treated as a complement, licensed by a PP on the COMPS list of a nearby word, or should it be analyzed as a modifier, introduced by a different grammar rule? Some cases are clear. For example, we know that a $\mathrm{PP}$ is a complement when the choice of preposition is idiosyncratically restricted by another word, such as the verb rely, which requires a PP headed by on or upon. In fact, PPs that are obligatorily required by a head (e.g. the directional PP required by put) can fairly safely be treated as complements. Conversely, there are certain kinds of PPs that seem to be able to co-occur with almost any kind of verb, such as temporal or locative PPs, and these are almost always analyzed as modifiers. PPs of a kind that can iterate, as in examples like We celebrated in the streets in the rain on Tuesday in the morning, are generally treated as modifiers. The underlying intuition here is that complements refer to the essential participants in the situation denoted, whereas modifiers serve to further refine the description of the situation. This is not a precisely defined distinction, and there are problems with trying to make it into a formal criterion. Consequently, there are difficult borderline cases that syntacticians disagree about. Nevertheless, there is considerable agreement that the distinction between complements and modifiers is a real one that should be reflected in a formal theory of grammar.

Returning to our analysis of complements, notice that although we have motivated our treatment of complements entirely in terms of verbs and verb phrases, we have formulated our analysis to be more general. In particular, our grammar of head-complement structures allows adjectives, nouns, and prepositions to take complements of various types. The following examples suggest that, like verbs, these kinds of words exhibit a range of valence possibilities.

(7) Adjectives

a. The children are happy (with the ice cream).

b. The children are fond of ice cream.

c.* The children are fond.

d. The children are happy (that they have ice cream).

e. ${ }^{*}$ The children are fond that they have ice cream. 
(8) Nouns

a. A magazine (about crime) appeared on the newsstands.

b.* Newsweek about crime appeared on the newsstands.

c. Newsweek appeared on the newsstands.

d. The report (that crime was declining) surprised many people.

e.* The book that crime was declining surprised many people.

f. The book surprised many people.

(9) Prepositions

a. The storm arrived after the picnic.

b. The storm arrived after we ate lunch.

c. The storm arrived during the picnic.

d. ${ }^{*}$ The storm arrived during we ate lunch.

e. ${ }^{*}$ The storm arrived while the picnic.

f. The storm arrived while we ate lunch.

\section{\. Problem 1: Valence Variations}

In this problem, you may use NP, VP, etc. as abbreviations for the feature structures on COMPS lists.

A. What does the grammaticality of sentences like Kim put the book here/there suggest about the COMPS and HEAD values of the words here and there?

B. What is the COMPS value for the adjective fond?

C. Assume that motion verbs like jump, move, etc. take an optional PP complement. Given that, what do the following examples tell you about the COMPS values of the lexical entries of the prepositions out, from and of:

(i) Kim jumped out of the bushes.

(ii) Bo jumped out from the bushes.

(iii) Lee moved from under the bushes.

(iv) Leslie jumped out from under the bushes.

(v) Dana jumped from the bushes.

(vi) Chris ran out the door.

(vii)*Kim jumped out of from the bushes.

(viii) Kim jumped out.

(ix)* Kim jumped from.

D. Based on the following data, sketch the lexical entries for the words grew (in the 'become' sense, not the 'cultivate' sense), seemed, happy, and close.

(i) They seemed happy (to me). 
(ii) Lee seemed an excellent choice (to me).

(iii) They grew happy.

(iv)* They grew a monster (to me).

(v)* They grew happy to me.

(vi) They grew close to me.

(vii) They seemed close to me to Sandy.

[Note: APs have an internal structure analogous to that of VPs. Though no adjectives select NP complements (in English), there are some adjectives that select PP complements (e.g. to me), and some that do not.]

\subsection{Specifiers}

Co-occurrence restrictions are not limited to complements. As we have noted in earlier chapters, certain verb forms appear with only certain types of subjects. In particular, in the present tense, English subjects and verbs must agree in number. Likewise, certain determiners co-occur only with nouns of a particular number.

(10) a. This dog barked.

b. ${ }^{*}$ This dogs barked.

c. ${ }^{*}$ These dog barked.

d. These dogs barked.

Moreover, some determiners are restricted to occur only with 'mass' nouns (e.g. furniture, footwear, information), and others only with 'count' nouns (e.g. chair, shoe, fact):

(11) a. Much furniture was broken.

b. ${ }^{*}$ A furniture was broken.

c. ${ }^{*}$ Much chair was broken.

d. A chair was broken.

We can handle such co-occurrence restrictions in much the same way that we dealt with the requirements that heads impose on their complements. First, we introduce the term SPECIFIER to refer to both subjects and determiners. We then introduce the feature SPECIFIER (SPR), with which we can state the co-occurrence restrictions for heads and the specifiers they select. On analogy with COMPS, we make the value of SPR a list. This decision may strike some readers as odd, since sentences only have a single subject and NPs never have more than one determiner. But making SPR list-valued provides a uniform way of formulating the idea that a particular valence requirement is unfulfilled 
(the valence feature - that is, COMPS or SPR - has a nonempty value) or else is fulfilled (the value of the valence feature is the empty list).

We can now define the category NOM in terms of the following feature structure descriptions:

$$
\mathrm{NOM}=\left[\begin{array}{lll}
\text { phrase } & & \\
\text { HEAD } & \text { noun } \\
\text { COMPS } & \langle\rangle \\
\text { SPR } & \langle[\text { HEAD } & \text { det }]\rangle
\end{array}\right]
$$

Notice the similarity between (12) and (what is now) the feature specification for VP:

$$
\mathrm{VP}=\left[\begin{array}{ll}
\text { phrase } & \\
\text { HEAD } & \text { verb } \\
\text { COMPS } & \langle\rangle \\
\mathrm{SPR} & \langle\mathrm{NP}\rangle
\end{array}\right]
$$

Both (12) and (13) are of type phrase with empty COMPS lists and a single element in their SPR lists. Both are intermediate between lexical categories (type word, with possibly nonempty COMPS lists) and SATURATED phrases - that is, phrases with both their COMPS and SPR lists empty.

Similarly, we can now introduce a verbal category that is analogous in all relevant respects to the saturated category NP. It is just the feature structure analogue of the familiar category S.

$$
\mathrm{NP}=\left[\begin{array}{ll}
\text { phrase } & \\
\text { HEAD } & \text { noun } \\
\text { COMPS } & \langle\rangle \\
\text { SPR } & \langle\rangle
\end{array}\right] \quad \mathrm{S}=\left[\begin{array}{ll}
\text { phrase } & \\
\text { HEAD } & \text { verb } \\
\text { COMPS } & \langle\rangle \\
\text { SPR } & \langle\rangle
\end{array}\right]
$$

Because NP and S now have a parallel formulation in terms of feature structures and parallel constituent structures, we may collapse our old rules for expanding these categories (given in (15)) into a single rule, shown in (16), that sanctions all HEAD-SPECIFIER PHRASES.

(15) a. $\mathrm{S} \rightarrow \mathrm{NP}$ VP

b. $\mathrm{NP} \rightarrow(\mathrm{D}) \mathrm{NOM}$

Head-Specifier Rule

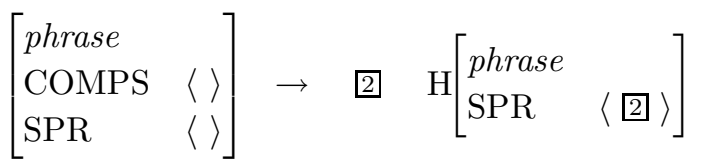


Having consolidated the rules in this way, we need to explain more precisely how our treatment of the SPR feature can account for the various co-occurrence restrictions between heads and specifiers. These include the fact that the specifier daughter of a sentence (i.e. its subject) is an $\mathrm{NP}$, whereas the specifier daughter of an NP is a D. They also include facts like those in (10)-(11), and the agreement between subjects and verbs, which will be dealt with in sections 4.6 and 4.7 below.

The first of these is relatively simple: the value for SPR in the lexical entries for nouns is the list $\langle\mathrm{D}\rangle$, and in the lexical entries for verbs, it is the list $\langle\mathrm{NP}\rangle$. Notice that this analysis entails that the lexical head's value for the SPR feature must be available at the nonlexical level where the specifier phrase is attached. Thus for the new rule in (16) to work, we will have to modify our Head-Complement Rule so that it 'passes' the SPR value of the lexical head 'up' to its mother. ${ }^{7}$ We might thus add a stipulation to this effect, as shown in (17):

(17) Head-Complement Rule

$$
\left[\begin{array}{ll}
\text { phrase } & \\
\mathrm{SPR} & \mathrm{a} \\
\mathrm{COMPS} & \langle\rangle
\end{array}\right] \rightarrow \mathrm{H}\left[\begin{array}{ll}
\text { word } & \\
\operatorname{SPR} & \square \\
\mathrm{COMPS} & \langle 1, \ldots, n\rangle
\end{array}\right] \text { 1... } n
$$

(Note that here we are using the tag $a$ to designate neither an atomic value nor a feature structure, but rather a list of feature structures. ${ }^{8}$ )

The modification in (17) solves the problem of getting the SPR selection information from the lexical head up to VP or NOM - the phrase that will combine directly with the specifier (via our new rule (16)), but it does so at the cost of adding a stipulation to our rules. Moreover, more stipulations are needed if we consider additional rules. In particular, recall the rule for introducing PP modifiers, discussed in the previous chapter. Because no complements or specifiers are added by this rule, we do not want any cancellation from either of the head daughter's valence features. Hence, we would need to complicate the rule so as to transmit values for both valence features up from the head daughter to the mother, as shown in (18):

\footnotetext{
${ }^{7}$ At first glance, one might be tempted to accomplish this by making SPR a head feature, but in that case the statement of the HFP would have to be complicated, to allow rule (16) to introduce a discrepancy between the HEAD value of a mother and its head daughter.

${ }^{8}$ We will henceforth adopt the convention of using numbers to tag feature structures and letters to tag lists of feature structures.
} 
88 / Syntactic Theory: A Formal Introduction

$$
\left[\begin{array}{ll}
\text { phrase } & \\
\mathrm{SPR} & \mathrm{a} \\
\text { COMPS } & \mathrm{b}
\end{array}\right] \rightarrow \mathrm{H}\left[\begin{array}{ll}
\text { phrase } & \\
\mathrm{SPR} & \mathrm{a} \\
\text { COMPS } & \mathrm{b}
\end{array}\right] \mathrm{PP}
$$

Without some such constraint, our modifiers cannot combine with a VP head daughter to build another VP. It is time to contemplate a more general theory of how the valence features behave in headed phrases.

\subsection{The Valence Principle}

The intuitive idea in the previous section is quite straightforward: certain lexical entries specify what they can co-occur with by listing (as the value of the features COMPS and SPR) the particular kinds of dependents they select. And we formulated general rules, stating that all the head's COMPS members are 'discharged' in a head-complement phrase, and the item in the SPR value is discharged in a head-specifier phrase. But to make these rules work, we had to add constraints preserving valence specifications in all other instances: the mother in the Head-Specifier Rule preserves the head's COMPS value (the empty list); the mother in the Head-Complement Rule preserves the head's SPR value, and the mother in the Head-Modifier Rule must preserve both the COMPS value and the SPR value of the head. The operant generalization that can be factored out of our rules can be expressed as the following principle which, like the HFP, constrains the set of phrase structure models that satisfy our grammar rules:

(19) The Valence Principle

Unless the rule says otherwise, the mother's SPR and COMPS

values are identical to those of the head daughter.

By 'unless the rule says otherwise', we mean simply that the Valence Principle is enforced unless a particular grammar rule specifies both the mother's and the head daughter's value for some valence feature.

The effect of the Valence Principle is that (1) the appropriate elements mentioned in particular rules are canceled from the relevant valence specifications of the head daughter in head-complement or headspecifier phrases, and (2) all other valence specifications are simply passed up from head daughter to mother. Once we factor these constraints out of our headed rules and put them into a single principle, it again becomes possible to simplify our grammar rules. This is illustrated in $(20)$. 
(20) a. Head-Specifier Rule

$$
\left[\begin{array}{ll}
\text { phrase } & \\
\mathrm{SPR} & \langle\rangle
\end{array}\right] \rightarrow \square \quad \mathrm{H}\left[\begin{array}{ll}
\text { phrase } & \\
\mathrm{SPR} & \langle\square\rangle
\end{array}\right]
$$

b. Head-Complement Rule

$$
\left[\begin{array}{ll}
\text { phrase } & \\
\text { COMPS } & \langle\rangle
\end{array}\right] \rightarrow \mathrm{H}\left[\begin{array}{ll}
\text { word } & \\
\text { COMPS } & \langle\square, \ldots, n\rangle
\end{array}\right] \text { 目 } \ldots
$$

c. Head-Modifier Rule

$$
[\text { phrase }] \rightarrow \mathrm{H}[\text { phrase }] \mathrm{PP}[\ldots]
$$

Our work is not yet done. We will modify the Head-Modifier Rule in the next chapter; in addition there are modifications of (20a,b) yet to be introduced. Nevertheless, the simplicity of the rules as formulated in (20) is striking.

Note that since head-specifier structures require a phrasal head and the Head-Complement Rule requires a lexical head, it follows that headcomplement phrases must be embedded within head-specifier phrases (rather than vice versa), as shown in (21):

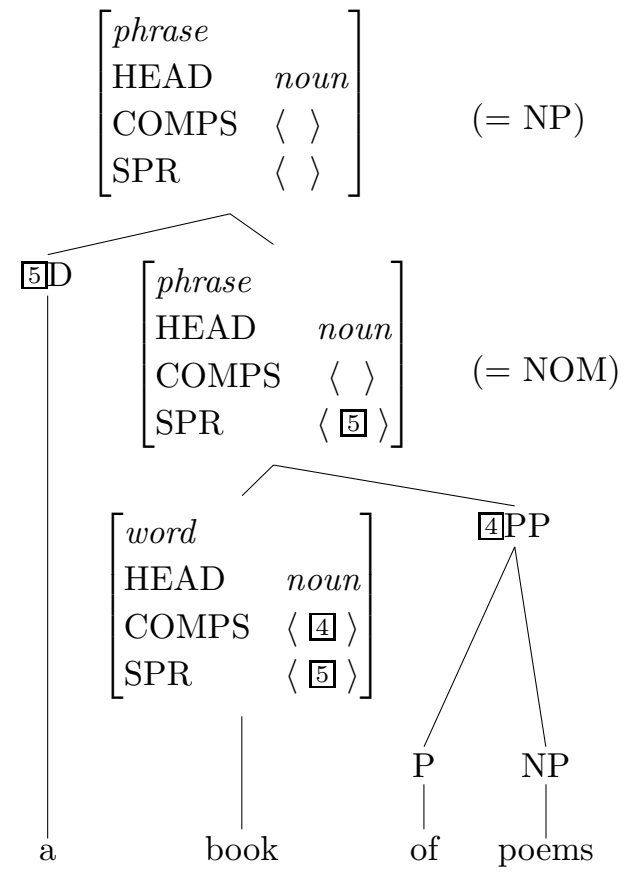

Thus COMPS cancellation happens at the NOM level and SPR cancellation happens at the NP level. The same is true of verbal structures: 
complements must be introduced lower (within a VP) than specifiers (which appear as sisters to a VP head within S).

\subsection{Subject-Verb Agreement Revisited}

Let us now return to the problem of subject-verb agreement. Our earlier analysis assigned the feature AGR to both nouns and verbs, and one of our grammar rules stipulated that the AGR values of VPs and their subjects had to match. But our new analysis has no need to specify AGR values for VPs. Since VPs all select their subject via the SPR feature, what are traditionally referred to as 'plural verbs' can be treated simply in terms of the specification [SPR $\langle\mathrm{NP}[\mathrm{AGR}$ [NUM pl]]〉]; 'singular verbs' can similarly be specified just as [SPR $\langle\mathrm{NP}$ [AGR [NUM sg]] $\rangle]$. Additional agreement features of verbs, VPs, or even S are simply unnecessary.

However, subject-verb agreement is a bit more complex than this, because it does not depend only on number. More specifically, English agreement also depends on PERSON. As noted earlier, we analyze person in terms of varying specifications for the feature PER. [PER 1st] is our notation for first person, that is, the pronouns $I$ and we. [PER 2nd] denotes second person, which in English is always you. [PER 3rd] covers all nonpronominal NPs, as well as he, she, it, and they. Most present tense English verbs have one form when their subjects are third-person singular (namely a form ending in $-s$ ) and another form covering all other persons and numbers. The only verb whose present tense system makes finer distinctions than this is be, which has a special first-person singular form, am and an additional form are (appropriate wherever am and $i s$ are not).

We can couch our analysis of these verb forms in terms of a distinction between two kinds of values of the feature AGR. Suppose we call these two types 3sing and non-3sing. The distinction among the third-person singular pronouns he, she, and it is attributed to a feature GEND(ER), with values masc, fem, and neut. GEND also differentiates among him, her, it, as well as among himself, herself, and itself. There is no motivation in English for assigning GEND to anything other than words that are third-person and singular. ${ }^{9}$

What this means is that we can set up our system so that the values of the feature AGR obey restrictions on the combinations of values of the

\footnotetext{
${ }^{9}$ This can be taken as independent evidence for the existence of $3 s i n g$ as a separate type - we can express this restriction by declaring the feature GEND as appropriate only for feature structures of the type 3sing.
} 
features PER and NUM. Instances of the type 3sing obey the constraint shown in (22):

$$
\text { 3sing : }\left[\begin{array}{ll}
\mathrm{PER} & 3 \mathrm{rd} \\
\mathrm{NUM} & \mathrm{sg}
\end{array}\right]
$$

And we enumerate the subtypes of non-3sing so as to include only firstperson, second-person, or third-person plural specifications. ${ }^{10}$ As a consequence of this analysis, the only possible values for the feature AGR (in English) are those described in (23).

$$
\begin{aligned}
& \text { Possible AGR Values } \\
& {\left[\begin{array}{ll}
\text { 3sing } & \\
\text { PER } & \text { 3rd } \\
\text { NUM } & \text { sg } \\
\text { GEND } & \text { fem }
\end{array}\right]\left[\begin{array}{ll}
\text { 3sing } & \\
\text { PER } & \text { 3rd } \\
\text { NUM } & \text { sg } \\
\text { GEND } & \text { masc }
\end{array}\right]\left[\begin{array}{ll}
\text { 3sing } & \\
\text { PER } & \text { 3rd } \\
\text { NUM } & \text { sg } \\
\text { GEND } & \text { neut }
\end{array}\right]} \\
& {\left[\begin{array}{ll}
\text { non-3sing } & \\
\text { PER } & \text { 3rd } \\
\text { NUM } & \mathrm{pl}
\end{array}\right]\left[\begin{array}{ll}
\text { non-3sing } & \\
\text { PER } & 1 \mathrm{st} \\
\text { NUM } & \text { sg }
\end{array}\right]\left[\begin{array}{ll}
\text { non-3sing } & \\
\text { PER } & 1 \text { st } \\
\text { NUM } & \text { pl }
\end{array}\right]} \\
& {\left[\begin{array}{ll}
\text { non-3sing } & \\
\text { PER } & 2 \text { nd } \\
\text { NUM } & \text { sg }
\end{array}\right]\left[\begin{array}{ll}
\text { non-3sing } \\
\text { PER } & 2 n d \\
\text { NUM } & \mathrm{pl}
\end{array}\right]}
\end{aligned}
$$

A pronoun may specify one of these possibilities in its lexical entry; other elements, for example proper names, will have lexical entries whose AGR value is specified only as 3sing, making these elements compatible in principle with any gender specification.

This treatment of the AGR values of nouns and NPs enables us to streamline our analysis of verbs. We can require that the lexical entries

\footnotetext{
${ }^{10}$ One organization of these subtypes is as in (i), with constraints defined as in (ii):

(i)

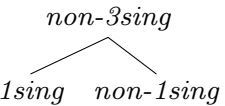$$
\widehat{2 \operatorname{sing} p l}
$$

(ii) 1 sing : $\left[\begin{array}{ll}\text { PER } & 1 \text { st } \\ \text { NUM } & \text { sg }\end{array}\right]$

2sing : $\left[\begin{array}{ll}\text { PER } & 2 \mathrm{nd} \\ \mathrm{NUM} & \mathrm{sg}\end{array}\right]$

$p l:\left[\begin{array}{ll}\mathrm{NUM} & \mathrm{pl}\end{array}\right]$
} 
for words like walks, runs, or is (all derived in later chapters by lexical rules) include the following specification:

$$
\left[\operatorname{SPR}\left\langle\left[\begin{array}{lll}
\operatorname{HEAD} & {\left[\begin{array}{ll}
\text { noun } & \\
\text { AGR } & 3 \text { sing }
\end{array}\right]} \\
\operatorname{COMPS} & \langle\rangle \\
\operatorname{SPR} & \langle\rangle &
\end{array}\right]\right\rangle\right]
$$

The third-person singular proper noun Kim and the present-tense verb form walks thus have lexical entries like the following:

(25) a.

$$
\left\langle\operatorname{Kim},\left[\begin{array}{lll}
\operatorname{HEAD} & {\left[\begin{array}{ll}
\text { noun } & \\
\text { AGR } & \text { 3sing }
\end{array}\right]} \\
\operatorname{COMPS} & \langle\rangle \\
\operatorname{SPR} & \langle\rangle &
\end{array}\right]\right\rangle
$$

b.

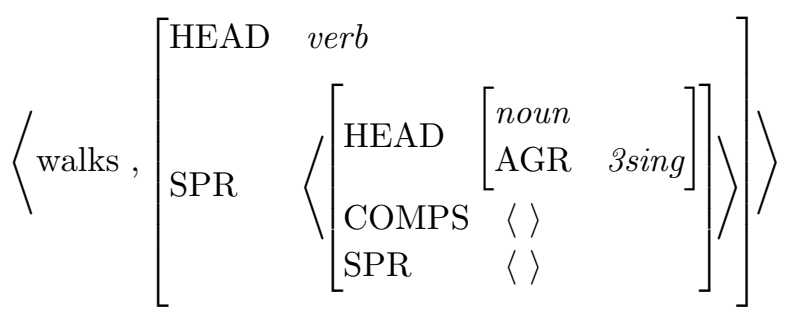

Because AGR is a head feature, any NP that serves as the subject of a verb like walks will have to contain a lexical head noun (for example a proper noun like Kim) that is also specified as [AGR 3sing]. This is a consequence of the HFP, which identifies the HEAD value of an NP with that of its head daughter. Thus verbs whose lexical entries include (24) will occur with NPs headed by third-person singular nouns, but never with plural nouns, or with first-person or second-person pronouns.

It is often assumed that it is necessary to posit separate lexical entries for present tense verb forms that take plural subjects and those that take singular, non-third person subjects, as sketched in (26a,b):

(26) a.

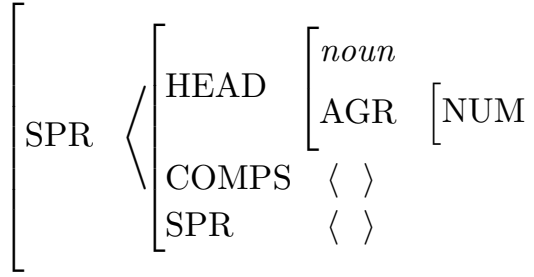

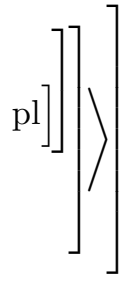


b.

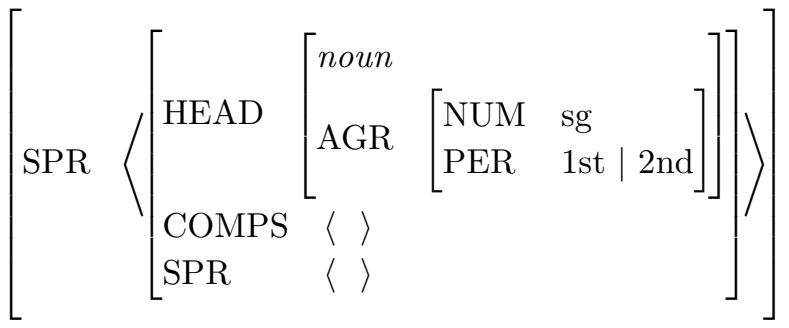

But such an analysis would fail to explain the fact that the former type of verb would always be identical in form to the latter: again, a suspicious loss of generalization in the lexicon.

Once we bifurcate the types of AGR values, as described above, this problem disappears. We need only a single kind of verb, one that includes the following lexical information:

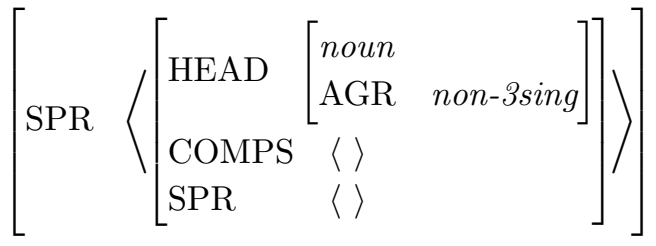

Verbs so specified project VPs that take subjects whose head nouns must bear non-3sing AGR values, and these, as described above, must either be first-person singular, second-person singular, or plural. ${ }^{11}$

Problem 2: The SPR Value of am

What would the SPR value in the lexical entry for am be?

\subsection{Determiner-Noun Agreement}

We have just seen how our new analysis of specifiers, taken together with the Head Feature Principle, provides an account of the fact that third-person singular verb forms (e.g. walks) take subject NPs headed by third-person singular nouns. But, as we saw in section 4.4, the specifiers of the phrases projected from these nouns also agree in number. In

\footnotetext{
${ }^{11}$ The disjunctions needed for describing classes of verbs are thus given by the type hierarchy, not by writing arbitrarily disjunctive lexical entries. One of the goals for a type-based grammar is to predict in this manner which disjunctions will be required for the grammatical analysis of a given language (or of language in general). A grammar that specifies a family of types consequently makes predictions about which classes of linguistic entities pattern together. A theory that simply specifies disjunctions wherever necessary makes no such predictions and is hence of considerably less explanatory value.
} 
fact, the relevant data are slightly more complicated here, too. English has determiners like this and $a$, which only appear with singular nouns, plural determiners like these and few, which only appear with plural nouns, and other determiners like the, which go either way. These facts are illustrated in (28)-(30).

(28) a. This dog barked.

b. ${ }^{*}$ This dogs barked.

c. A dog barked.

d. ${ }^{*}$ A dogs barked.

(29) a. ${ }^{*}$ These dog barked.

b. These dogs barked.

c. *Few dog barked.

d. Few dogs barked.

(30) a. The dog barked.

b. The dogs barked.

In addition to the 'feature passing' that is guaranteed by the HFP, there is systematic agreement of person and number between heads and specifiers within the NP. To express this fact generally, we add the following constraint on all nouns: ${ }^{12}$

$$
\begin{aligned}
& \text { Nominal SPR Agreement (NSA): } \\
& \text { Nouns must be specified as: }\left[\begin{array}{ll}
\text { HEAD } & {\left[\begin{array}{ll}
\text { AGR } & 1
\end{array}\right]} \\
\operatorname{SPR} & \left\langle\left(\left[\begin{array}{ll}
\text { AGR } & 1
\end{array}\right]\right)\right\rangle
\end{array}\right]
\end{aligned}
$$

The NSA requires that the AGR value of a noun be identified (unified) with that of its determiner, if the latter is realized. It thus makes determiner-noun agreement a lexical fact about nouns. This account presupposes that determiners and nouns both bear AGR specifications, as illustrated in (32).

$$
\begin{aligned}
& \text { person, boat, a, this: }\left[\text { AGR }\left[\begin{array}{ll}
\text { PER } & 3 \mathrm{rd} \\
\text { NUM } & \text { sg }
\end{array}\right]\right] \\
& \text { people, boats, few, these: }\left[\text { AGR }\left[\begin{array}{ll}
\text { PER } & 3 \mathrm{rd} \\
\text { NUM } & \text { pl }
\end{array}\right]\right]
\end{aligned}
$$

${ }^{12}$ Since we have not yet developed our account of word types, we do not yet have a subtype of word that only words specified as [HEAD noun] belong to. In Chapter 8 , the NSA will be stated as a constraint on the type noun-lexeme. 


$$
\text { fish, the: }\left[\text { AGR }\left[\begin{array}{ll}
\text { PER } & 3 \mathrm{rd}
\end{array}\right]\right]
$$

These lexical specifications, taken together with the NSA and the HFP, provide a complete account of the agreement data in (28) - (30) above.

In section 4.4 above, we also observed that some determiners are restricted to occur only with 'mass' nouns (e.g. furniture), and others only with 'count' nouns (e.g. chair):

(33) a. Much furniture was broken.

b. ${ }^{*}$ A furniture was broken.

c. ${ }^{*}$ Much chair was broken.

d. A chair was broken.

The co-occurrence restriction illustrated in (33) - that is, the count noun/mass noun distinction - might, of course, be solely a semantic matter. The contrasting judgments in (33) are particularly striking, however, and may well warrant our treating the distinction as a matter of syntax. Assuming the syntactic account to be desirable (and such matters are often difficult to decide), we could analyze the data in (33) by introducing a feature COUNT. Certain determiners (e.g. $a$ and few) will be lexically specified as [COUNT +$]$ and others (e.g. much) will be lexically treated as [COUNT -]. Still others, such as the, will be lexically unmarked for this feature.

Once these lexical entries are in place, we can account for the phenomenon by treating nouns as follows. The SPR value of a count noun like chair would be $\langle\mathrm{D}[\mathrm{COUNT}+]\rangle$, forcing such nouns to co-occur with a count determiner. And the SPR value of a mass noun like furniture would be $\langle(\mathrm{D}[\mathrm{COUNT}-])\rangle$. As usual, the parentheses here designate optionality, and they appear only with mass nouns, because singular count nouns require determiners: ${ }^{13}$

(34) a. (The) furniture is expensive.

b. The chair is expensive.

c. ${ }^{*}$ Chair is expensive.

Notice that under this analysis the feature COUNT is marked only on the determiners, not on the nouns themselves. (Hence COUNT is not an AGR feature, but rather a HEAD feature on a par with AGR.) Count and mass nouns are distinguished only by whether their SPR lists contain a [COUNT + ] or [COUNT - ] element. The matching of count nouns and count determiners is thus handled entirely by the Valence Principle and the Head-Specifier Rule, which will identify the SPR value

\footnotetext{
${ }^{13}$ This analysis of mass and plural nouns will be compared with an alternative in
} Problem 3 of Chapter 8. 
of NOM (the head of the NP) and the category of its specifier. No new machinery need be introduced. Notice that this analysis also predicts that the COUNT specification is never passed up to the NP, and hence can never be selected by the VP. Thus there should be no verbs in English that require a count or mass subject or object.

\section{Problem 3: COUNT and NUM}

Is this last prediction right? That is, are there any verbs in English that require a count subject or object NP or a mass subject or object NP? Provide examples in support of your answer.

\section{\1 Problem 4: COUNT and NUM}

An alternative to the analyses just presented would eliminate the feature COUNT and assign three values to the feature NUM: sg, pl, and mass. That is, mass nouns like furniture would be given the value [NUM mass]. Use the following data to provide an argument favoring the analysis given in the text over this alternative:

$$
\begin{aligned}
& \text { (i) We don't have much }\left\{\begin{array}{l}
\text { rice } \\
\text { oats }
\end{array}\right\} \text {. } \\
& (\mathrm{ii})_{*} \text { We don't have many }\left\{\begin{array}{l}
\text { rice } \\
\text { oats }
\end{array}\right\} \text {. }
\end{aligned}
$$

(iii) The rice is in the bowl.

(iv)* The rice are in the bowl.

(v) The oats are in the bowl.

(vi) ${ }^{*}$ The oats is in the bowl.

[Note: You may speak a variety of English that accepts many oats as a well-formed NP. There are some other nouns that are like oats in the relevant respects in at least some dialects, including grits (as a kind of cereal), mashed potatoes, and (somewhat distastefully, but grammatically more clearly) feces. If you can find a noun that patterns as we claim oats does in examples (i)-(vi), work the problem using that noun. If your dialect has no such nouns, then work the problem for the dialect described here, putting aside your own judgments.]

The picture we now have of head-specifier structures is summarized in (35). 


$$
\left[\begin{array}{ll}
\text { phrase } & \\
\text { HEAD } & \text { 0verb } \\
\text { SPR } & \langle\rangle \\
\text { COMPS } & \langle\rangle
\end{array}\right]
$$

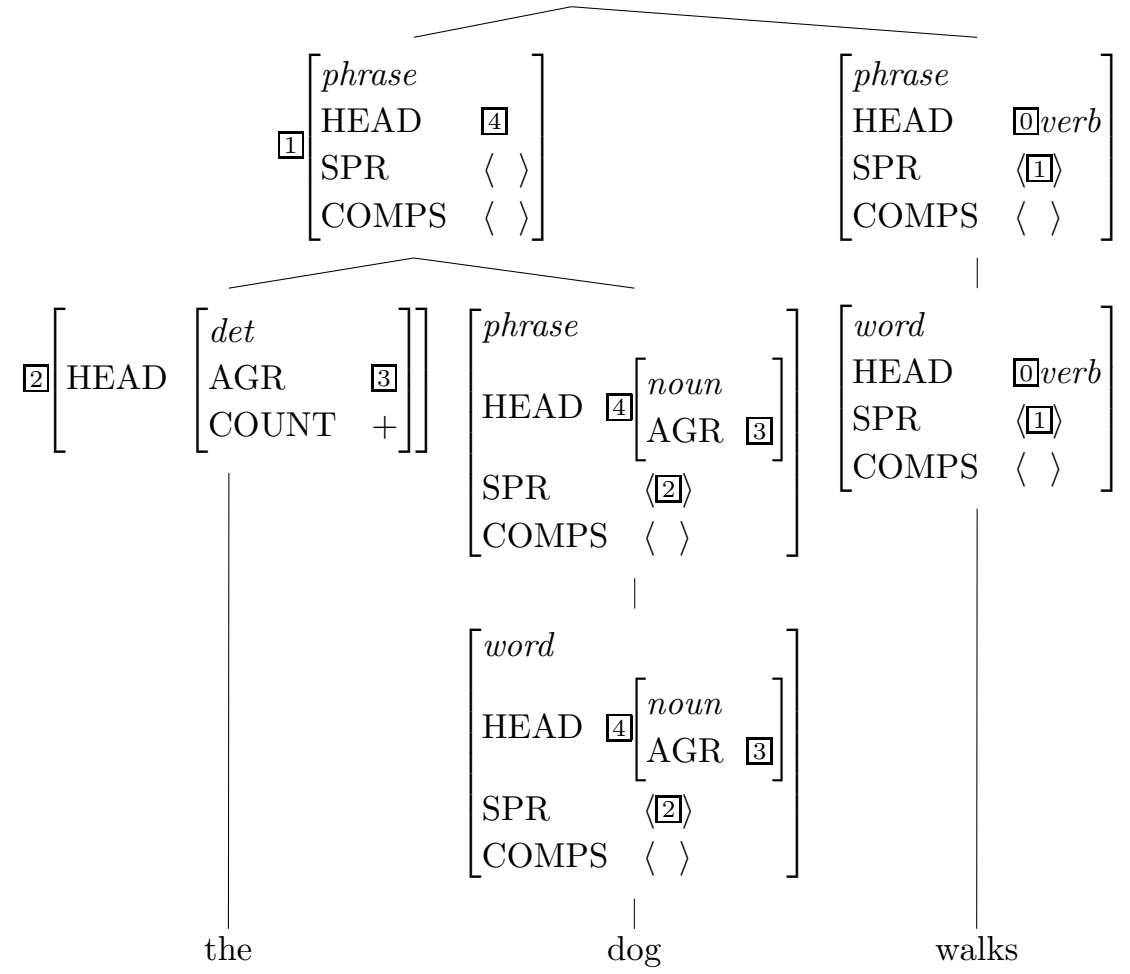

Note that the HEAD value of the noun $\operatorname{dog}(4)$ and those of the two phrases above it are unified in virtue of the HFP. Similarly, the HFP guarantees that the HEAD value of the verb walks (0) and those of the two phrases above it are unified. The NSA guarantees that the AGR value of the noun (3) is unified with that of the determiner it selects as a specifier (2), and since the AGR specification is within the HEAD value 4 , it follows from the interaction of the NSA and the HFP that the AGR values of the NP, NOM, N, and D in (35) are all unified. This means in turn that whenever a verb selects a certain kind of subject NP (an [AGR 3sing] NP in the case of the verb walks in (35), that selection will restrict what kind of noun and determiner can occur within the subject NP, as desired. 
98 / Syntactic Theory: A Formal Introduction

\subsection{Worksection on Case Marking}

We close this chapter with a series of problems dealing with grammatical case. The tools developed in this chapter should suffice to provide an analysis of the phenomenon of case, in a manner analogous to the treatment of agreement.

\subsubsection{Case Marking in English}

Consider the following data:

(36) a. Dogs like him.

b. ${ }^{*}$ Dogs like he.

c. ${ }^{*}$ Him likes dogs.

d. He likes dogs.

These sentences exemplify what linguists call CASE MARKING: him is what is called an ACCUSATIVE (or OBJECTIVE) case pronoun, and he is in what is called the NOMINATIVE (or SUBJECTIVE) case.

For the following problems, assume that there is a feature CASE with the values 'acc' and 'nom', and assume that English pronouns have CASE values specified in their lexical entries.

\section{\1 Problem 5: Assessing the Facts}

As noted in Chapter 2, NPs appear in a variety of positions in English, including subject of a sentence, direct object of a verb, second object of a ditransitive verb like give, and object of a preposition. For each of these NP positions, determine which case the pronouns in that position must have. Give examples to support your claims.

\section{\1 Problem 6: A Lexical Analysis}

In Chapter 2, problem 6 asked you to write phrase structure rules that would account for the different case markings associated with different positions in English. This kind of analysis of case marking no longer makes much sense, because we have replaced the very specific phrase structure rules of earlier chapters with more general rule schemas. Instead, we can now handle case entirely in the lexicon, without changing our grammar rules. Show how this could be done.

[Hint: It will involve modifying the lexical entries of elements that combine with NPs.] 


\section{Problem 7: Case and Coordination}

There is considerable variation among English speakers about case marking in coordinate NPs. Consult your own intuitions (or those of a friend, if you are not a native English speaker) to determine what rule you use to assign case to pronouns in coordinate structures. State the rule informally - that is, give a succinct statement, in English, of a generalization that covers case in coordinate NPs in your dialect. Provide both grammatical and ungrammatical examples in support of your rule.

\subsubsection{Case Marking in Icelandic}

Icelandic is closely related to English, but it has a much more elaborate and interesting case system. For one thing, it has four cases: nominative, accusative, genitive, and dative. Second, case is marked not just on pronouns, but also on nouns. A third difference is illustrated in the following examples: ${ }^{14}$

(37) a. Drengurinn kyssti stúlkuna.

the-boy-NOM kissed the-girl-ACC

'The boy kissed the girl'

b. Drengina vantar mat.

the-boys-ACC lacks food-ACC

'The boys lack food'

c. Verkjanna gatir ekki.

the-pains-GEN is-noticeable not

'The pains are not noticeable'

d. Barninu batnaði veikin

the-child-DAT recovered-from the-disease-NOM

'The child recovered from the disease'

The case markings indicated in these examples are obligatory. Thus, for example, the following is ungrammatical because the subject should be accusative:

(38) *Drengurinn vantar mat

the-boy-NOM lacks food-ACC

\section{Problem 8: Choosing Analyses in Icelandic}

\footnotetext{
${ }^{14}$ In the glosses, NOM stands for 'nominative', ACC for 'accusative', GEN for 'genitive', and DAT for 'dative'. Although it may not be obvious from these examples, there is in fact ample evidence (which we cannot present here) that the initial NPs in these examples are the subjects of the verbs that follow them.
} 


\section{0 / Syntactic Theory: A Formal Introduction}

Explain how the examples in (37) bear on the analysis of case marking in Icelandic. In particular, explain how they provide direct empirical evidence for treating case marking as a lexical phenomenon, rather than one associated with particular phrase structure positions. Be sure to sketch the lexical entry for at least one of these verbs.

\subsubsection{Agreement and Case Marking in Wambaya}

In Wambaya, a language of Northern Australia, nouns are divided into four genders: masculine (m), feminine (f), vegetable (v), and neuter (n). They are also inflected for case, such as ergative (E) and accusative (A). Consider the following Wambaya sentences, paying attention only to the agreement between the determiners and the nouns (you do not have to worry about accounting for, or understanding, the internal structure of these words or anything else in the sentence). ${ }^{15}$

(i) Ngankiyaga bungmanyani ngiya-ngajbi yaniyaga darranggu that.f.e woman.f.e she-saw that.n.A tree.n.A 'That woman saw that tree'

(ii) Ngankiyaga bungmanyani ngiya-ngajbi mamiyaga jigama that.f.e woman.f.e she-saw that.v.A yam.v.A 'That woman saw that yam'

(iii) Ngankiyaga bungmanyani ngiya-ngajbi iniyaga bungmaji that.f.e woman.f.e she-saw that.m.A man.m.A 'That woman saw that man'

(iv) Ninkiyaga bungmanyini gina-ngajbi naniyaga bungmanya that.m.E man.m.E he-saw that.f.A woman.f.A 'That man saw that woman'

(v) Ninkiyaga bungmanyini gina-ngajbi yaniyaga darranggu that.m.E man.m.E he-saw that.n.A tree.n.A 'That man saw that tree'

(vi) Ninkiyaga bungmanyini gina-ngajbi mamiyaga jigama that.m.E man.m.E he-saw that.v.A yam.v.A 'That man saw that yam'

Ergative is the standard name for the case of the subject of a transitive verb in languages like Wambaya, where intransitive and transitive sub-

\footnotetext{
${ }^{15}$ In fact, the Wambaya data presented here are simplified in various ways: only one of the numerous word-order patterns is illustrated and the auxiliary plus verb sequences (e.g. ngiya-ngajbi) are here presented as a single word, when in fact the auxiliary is an independent verb in 'second' position. We are grateful to Rachel Nordlinger, who constructed this problem, in addition to conducting the field work upon which it is based.
} 
jects show different inflection patterns. Nothing crucial in this problem hinges on the distinction between nominative and ergative case. Note that the agreement patterns in (39) are the only ones possible; for example, changing mamiyaga to yaniyaga in (vi) would be ungrammatical. Note also that the verbs are selecting for the case of the subject and object NPs, so, for example, gina-ngajbi must take an ergative subject and accusative object.

\section{Problem 9: Analyzing Case in Wambaya}

A. Since verbs select subjects and objects of a particular case and this case shows up in terms of the inflection of the head noun, what does this illustrate (minimally) about the feature CASE (i.e. where must it go in our feature geometry)?

B. Explain how our analysis of English determiner-noun agreement would have to be modified in order to account for Wambaya determiner-noun agreement.

C. Illustrate your analysis with lexical entries for bungmanyani, ngankiyaga, bungmaji, and iniyaga.

\subsection{Summary}

In the previous chapter, we had already seen that cross-categorial generalizations about phrase structure can be expressed in terms of schematic phrase structure rules and categories specified in terms of feature structures. In this chapter, the real power of feature structure grammars has begun to emerge. We have begun the process of providing a unified account of the generalizations about complementation and specifier selection, in terms of the list-valued features COMPS and SPR. These features, together with the Valence Principle, have enabled us to eliminate further redundancy from our grammar rules. In fact, our grammar has now been reduced to the following four very general rules:

(40) a. Head-Specifier Rule

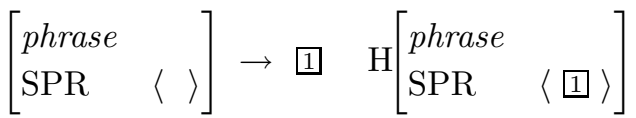

b. Head-Complement Rule

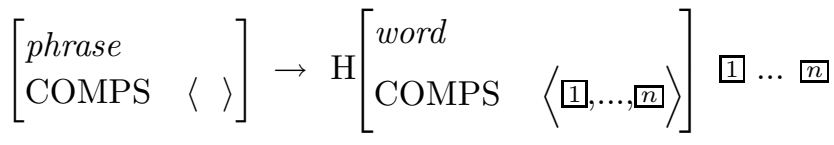


102 / Syntactic Theory: A Formal Introduction

c. Head-Modifier Rule

$$
\text { [phrase }] \rightarrow \mathrm{H}[\text { phrase }] \mathrm{PP}[\ldots]
$$

d. Coordination Rule

$$
1 \rightarrow 1^{+} \text {CONJ }
$$

Moreover, we have seen in this chapter that key generalizations about agreement and case marking can also be expressed in terms of this highly compact rule system, once we rely on categories modeled as feature structures.

\subsection{Further Reading}

The idea of schematizing phrase structure rules across parts of speech was introduced into generative grammar by Chomsky (1970). For a variety of perspectives on grammatical agreement, see Barlow and Ferguson (1988). A helpful discussion of Icelandic case is provided by Andrews (1982). 


\section{Semantics}

\subsection{Introduction}

Before we can return to the distribution of reflexive and nonreflexive pronouns, which will be the topic of Chapter 7 , we need to consider the nature of reference and coreference - topics that are fundamentally semantic in nature (i.e. that have to do in large part with meaning). And before we can do that, we need to discuss meaning more generally and to sketch how meaning will be represented in our grammar.

Reflexive pronouns provide perhaps the clearest case in which a semantic factor - coreference, in this case - plays an essential role in the grammatical distribution of particular words. But there are many other syntactic phenomena that are closely linked to meaning. Consider, for example, subject-verb agreement, which we have discussed extensively in the past two chapters. Singular nouns generally refer to individual objects, and plural nouns normally refer to collections of objects. Mass nouns usually refer to substances - that is, entities that are not naturally packaged into discrete objects. Of course, how objects, collections, and substances are individuated is not fully determined by the structure of the world, so there may be differences between languages, or even between individuals in how things are referred to. Hence the German word Hose means essentially the same thing as English pants or trousers, but the German is singular while the English is plural. Likewise, individual English speakers differ as to whether they can use lettuce as a count noun. Syntactic properties (including such basic ones as the part-ofspeech distinctions) are often closely linked to semantic characteristics, though the correspondences are usually imperfect. Trying to do syntax without acknowledging the associated semantic regularities would lead to missing many fundamental generalizations about linguistic structure.

The study of meaning is even older than the study of grammar, and there is little hope of doing justice to problems of semantics in a textbook 


\section{4 / Syntactic Theory: A Formal Introduction}

whose primary concern is grammatical structure. However, if the study of grammar is going to play any role in modelling real language use, then grammar minimally has to include an analysis of the meaning of individual words and a treatment of how these combine with each other - that is, an account of how meanings of phrases and sentences are built up from the meanings of their parts. So let us begin by contemplating the nature of sentence meaning.

\subsection{Semantics and Pragmatics}

Meaning is inextricably bound up with actions - people use language intentionally for many kinds of communicative purposes. Some sentences are used to convey questions; others simple assertions; still others conventionally convey commands (or 'directives', as they are sometimes called). Even a piece of a sentence, say an NP like the student sitting behind Leslie, can be used in isolation to perform a simple act of referring to an individual.

The kind of meaning that (a particular use of) a sentence conventionally conveys depends crucially on its syntactic form. For example, a simple 'inverted' sentence like (1), with an auxiliary verb before the subject NP, conventionally conveys a question.

(1) Is Sandy tall?

And the question posed by (1) is closely related to the proposition that is asserted by an utterance of the noninverted sentence in (2).

(2) Sandy is tall.

In fact, uttering (2) is a perfectly good way of answering (1).

To even begin to deal with semantic problems such as these, we first have to clarify what the units of meaning are and how particular kinds of sentences or smaller phrases are tied to particular types of meaning by linguistic conventions. We will make what is a standard assumption, that communication has two components: linguistic meaning and reasoning about communicative goals. On this view, when a linguistic expression is uttered, its linguistic meaning makes a significant contribution to, but does not fully determine, the communicative function of the utterance. Consider, for example, an utterance of (3).

(3) Do you have a quarter?

The linguistic meaning of this sentence is a familiar kind of semantic object: a question. And a question of this form has a determinate answer: yes or no. However, an utterance of (3) might serve to communicate much more than such a simple factual inquiry. In particular, in addition to posing a financial question to a given hearer, an utterance of (3) is 
likely to convey a further message - that the speaker was making the following request of the addressee.

(4) Please give me a quarter!

The question conveyed by an utterance of (3) is generally referred to as its Literal or CONVEntional meaning. A request like (4) is communicated as a further message above and beyond the message expressed by the literal meaning of the question in (3). We will leave the account of such embellished communication (even the routine 'reading between the lines' that occurs more or less effortlessly in cases like this) to a more fully developed theory of language use, that is, to a theory of linguistic PRAGMATICS. The inference from question to request is a pragmatic one.

By contrast, the fact that a sentence like (3) must express a question as its literal meaning is semantic in nature. Semantics is the study of linguistic meaning, that is, the contribution to communication that derives directly from the conventions of the language. Pragmatics is a more general study, of how linguistic meaning interacts with situational factors and the plans and goals of conversational participants to achieve more subtle, often elaborate communicative effects.

The semantic analysis that a grammar provides serves as input for a theory of pragmatics or language use. Such a theory sets as its goal to explain what actually gets communicated via pragmatic inferences derived from the linguistic meaning of an utterance. For example, pragmatic theory might include a principle like $(5):^{1}$

Quantity Principle (simplified)

If $X$ is weaker than $Y$, then asserting $X$ implies the denial of $Y$.

This principle leads to pragmatic inference via 'proofs' of the following kind (justifications for steps of the proof are given in parentheses):

- A says to B: Two things bother Pat.

- A uttered something whose linguistic meaning is: 'Two things bother Pat'. (semantic analysis)

- 'Two things bother Pat'. is weaker than 'Three things bother Pat'. (This is true in the context; possibly true more generally)

\footnotetext{
${ }^{1}$ The principle in (5), due to Grice (1989), relies on the undefined term 'weaker'. In some cases (such as the example that follows), it is intuitively obvious what 'weaker' means. But a full-fledged pragmatic theory that included (5) would have to provide a precise definition of this term.
} 
- B assumes that A also meant to communicate: 'It's not the case that three things bother Pat'. (Quantity Principle)

Note that exactly the same pragmatic inference would arise from an utterance by A of any semantically equivalent sentence, such as There are two things that bother Pat or Pat is bothered by two things. This is because pragmatic theory works from the linguistic meaning of an utterance (as characterized by our semantic analysis) and hence is indifferent to the form by which such meanings are expressed. ${ }^{2}$

There is much more that could be said about the fascinating topic of pragmatic inference. Here, the only purpose has been to show that the semantic analysis that must be included in any adequate grammar plays an essential role, albeit an indirect one, in explaining the communicative function of language in context.

\subsection{Linguistic Meaning}

\subsubsection{Kinds of Meaning}

When we ask a question, make an assertion, or even issue a command, we are also making reference to something that is often called a siTUATION or EVENT. ${ }^{3}$ If you utter a sentence like Kim is running, for example, you assert that there is some running situation in the world that involves something (usually a person) named Kim. The proposition that you assert is either true or false depending on a number of things, for example, whether this situation is a running event (maybe Kim is moving too slowly for it to really qualify as running), or whether the runner is someone named Kim (maybe the person you have in mind is really Leslie), whether the running situation is really happening now (maybe Kim has already run the race but my watch stopped several hours ago). If any of these 'maybes' turns out to be the case, then what you have said is false - the situation you are describing as specified by the linguistic meaning of the sentence is not part of the real world.

\footnotetext{
${ }^{2}$ This is not quite true. Sometimes the manner in which something is said (the form of an utterance) can make some pragmatic contribution to an utterance, but a discussion of such cases would take us too far afield.

${ }^{3}$ Although the term 'event' is often used in a general sense in semantic discussions, this terminology can be misleading, especially in connection with circumstances like the following, where nothing very event-like is happening:

(i) Bo knows baseball.

(ii) Dana is aggressive.

(iii) Sydney resembles Terry.

(iv) Chris is tall.

(v) 37 is a prime number.

We find it much more intuitive to discuss such sentences in terms of 'situations' and hence have adopted this as our official terminology for the semantics of sentences.
} 
An important part of the business of semantics is specifying truth conditions such as these, that is, specifying restrictions which must be satisfied by particular situations in order for assertions about them to be true. And since truth conditions are determined in large part by linguistic meaning, our grammar will be incomplete unless we introduce (i) some way of representing the linguistic meanings of words and (ii) a set of constraints that allows us to correctly predict the linguistic meanings of phrase structures in terms of the meanings of their parts (their subconstituents).

Consider what this means in the case of Kim is running. What we need to guarantee is that this sentence gets a semantics that is a proposition (not a question or a directive, for example) specified in terms of the following conditions: ${ }^{4}$

(7) a. there is a situation $s$

b. $s$ is a running situation

c. the runner is some individual $i$

d. $i$ is named Kim

e. $s$ is temporally located around the time of utterance

If there is some situation $s$ and some individual $i$ such that all the conditions in (7) are satisfied, then the proposition expressed by Kim is running is true. If not, then that proposition is false.

So to take care of semantic business, we will need a way to ensure that the various pieces of this sentence - the noun Kim, the verb $i s$, and the verb running - each make its appropriate contribution to the set of constraints summarized in (7) and that the grammar specifies how such propositions are built up from the substructures of the sentence. Our account must also be sufficiently general so as to assign the correct semantic description to all sentences of the language. So for example, a sentence like Is Kim running? should be assigned a semantics of a different type - that of a question - but a question about whether or not there is a situation $s$ and an individual $i$ such that all the conditions in (7) are satisfied.

In this book, we will construct meaning descriptions by providing constraints that specify how a phrase's semantics is built up from the semantics of its immediate constituents. Our method for predicting the semantics of a given phrase will thus be the same as our method of constraining the syntactic feature specifications of the mother node

\footnotetext{
${ }^{4}$ The exact meaning of the progressive (be...-ing) construction is a fascinating semantic topic with a considerable literature that we cannot do justice to here. We have adopted clause (7e) as a convenient first approximation of the truth conditional contribution of the present progressive in English.
} 
in a phrase structure: in terms of general constraints that determine how the mother's value for a given feature is related to the daughters' specifications for the same feature.

The semantic objects of our grammar will be classified in terms of four SEMANTIC MODES - that is, the four basic kinds of meanings that are enumerated and illustrated in (8).

(8)

\begin{tabular}{lll}
\hline SEMANTIC MODE & KIND OF PHRASE & EXAMPLE \\
\hline proposition & noninverted sentence & Kim is happy. \\
\hline question & inverted sentence & Is Kim happy? \\
\hline directive & imperative phrase & Be happy! \\
\hline reference & NP & Palo Alto \\
\hline
\end{tabular}

To achieve this classification, we will represent the meanings of all kinds of linguistic expressions in terms of feature structures that specify three things: a semantic mode, an index corresponding to the situation or individual referred to, and a restriction (abbreviated 'RESTR') specifying a list of conditions that the situation or individual has to satisfy for the expression to be applicable to it. Semantic structures then will look like (9):

$$
\left[\begin{array}{l}
\text { MODE } \\
\text { INDEX } \\
\text { RESTR }
\end{array}\right]
$$

The feature INDEX differs from other features we have encountered, in that it can take an unlimited number of different atomic values. This is because there is no limit to the number of different individuals or situations which can be referred to in a single sentence. Consequently, we must have an unbounded number of values of INDEX available. These atomic values of INDEX will conventionally be written with lower-case Roman letters; instead of tagging two occurrences of the same INDEX value, we will simply write the same index (that is, the same lower-case Roman letter) in both places.

Propositions are analyzed in terms of feature structures like the one in (10) (where 'prop' is short for 'proposition').

$$
\left[\begin{array}{cc}
\text { MODE } & \text { prop } \\
\text { INDEX } & s \\
\text { RESTR } & \langle\ldots\rangle
\end{array}\right]
$$

A proposition like (10) will be true just in case there is some actual situation $s$ (and there exist appropriate other individuals corresponding to whatever variables are present in (10)) such that the constraints spec- 
ified in the RESTR value of (10) are all satisfied. These restrictions, the nature of which will be explained in the next section, must include all those that are relevant to the meaning of the sentence, for example, all the constraints just mentioned in conjunction with the truth or falsity of Kim is running. Our grammatical analysis must ensure that we end up with exactly the right constraints in the RESTR list of a sentence's semantics, so that we associate exactly the right meaning with any sentence sanctioned by our grammar.

Questions and directives have a similar analysis, though the intuition behind their meaning is somewhat different. A question like Is Kim running? must be assigned a semantics just like the one assigned to Kim is running, except that the MODE value must be 'question' ('ques' for short), rather than 'prop':

$$
\left[\begin{array}{cc}
\text { MODE } & \text { ques } \\
\text { INDEX } & s \\
\text { RESTR } & \langle\ldots\rangle
\end{array}\right]
$$

The question of whether a situation $s$ satisfies a set of restrictions is the kind of semantic object that can be resolved positively or negatively in a given context, though it is not itself true or false. We can thus talk of true or false answers to questions, but questions themselves are neither true nor false.

Neither are directives ('dir' for short), which are represented as in (12).

$$
\left[\begin{array}{lc}
\text { MODE } & \text { dir } \\
\text { INDEX } & s \\
\text { RESTR } & \langle\ldots\rangle
\end{array}\right]
$$

A directive - for example, Shut the door - is rather something that can be fulfilled or not; and what the RESTR list does in the case of a directive is to specify what conditions have to be satisfied in order for a directive to be fulfilled.

A reference ('ref' for short) is similar to the kinds of meanings just illustrated, except that it can be used to pick out (or DENOTE) all kinds of entities - not just situations. We use INDICES notated with the letters $i, j, k$, etc. as the INDEX values for the semantics of nominal expressions. These function much in the same way as variables in algebra or in mathematical logic. The INDEX values written with the letters $s, t, u$, etc. are for indices that refer only to situations. Other indices are free 
to be associated with any kind of entity found in a discourse context. ${ }^{5}$ So the semantics we assign to a referring NP has the following form:

$\left[\begin{array}{lc}\text { MODE } & \text { ref } \\ \text { INDEX } & i \\ \text { RESTR } & \langle\ldots\rangle\end{array}\right]$

As we have just seen, there are a number of differences among the various semantic modes we have assumed. Despite these differences, the modes have something in common. Every kind of linguistic expression we have considered, irrespective of its semantic mode, refers to something that must satisfy an indicated list of restrictions for the expression to be correctly applicable. Our approach to semantics expresses this general fact by treating all expressions in terms of a single type of semantic object that includes a referential index of one kind or another. The semantic work of distinguishing the ways in which the individuals and situations referred to contribute to linguistic meaning is left to the differing values of the feature MODE. Many words and phrases that cannot be used by themselves to express a proposition, ask a question, refer to an individual, etc. (e.g. determiners, prepositions, PPs, and conjunctions) will be treated here in terms of the specification [MODE none].

\subsubsection{Predications}

The goal of semantics is to account for the systematic role of linguistic meaning in communication. As we have just seen, we can approach this goal in terms of a semantic analysis that recognizes diverse semantic modes and reference to both individuals and situations. Much of the interesting work in linguistic semantics is done by the conditions associated with particular linguistic expressions, conditions which situations and individuals must meet in order for those expressions to be applicable to them. In terms of our analysis, this means that it will be particularly important how individual words contribute to the values of the feature RESTR, and how the RESTR values of phrases are built up from those of their parts.

Semantic restrictions associated with expressions come in many varieties, which concern what properties some individual has, who did what to whom in some situation, when, where, or why some situation occurred, and so forth. That is, semantically relevant restrictions specify which of the properties must hold of individuals and situations, and

\footnotetext{
${ }^{5}$ There are any number of intriguing referential puzzles that are the subject of ongoing inquiry by semanticists. For example, what does an NP like a page refer to in the sentence: A page is missing from this book? Or the unicorn that Chris is looking for in the sentence: The unicorn that Chris is looking for doesn't exist?
} 
which relations must hold among them, for an expression to be applicable.

To represent this sort of information, we must introduce into our semantics some way of specifying relations among entities quite generally. We do this by introducing a type of feature structure called predication (pred). The features of a predication specify (i) what kind of relation is involved and (ii) who or what is participating in the relation. Examples of feature structures of type pred are given in (14). ${ }^{6}$

$\left[\begin{array}{l}\text { RELN } \\ \text { SIT(UATION) } \\ \text { LOVER } \\ \text { LOVED }\end{array}\right.$

c.

$\left[\begin{array}{lc}\text { RELN } & \text { give } \\ \text { SIT } & s \\ \text { GIVER } & i \\ \text { RECIPIENT } & j \\ \text { GIFT } & k\end{array}\right]$
$\left[\begin{array}{lc}\text { RELN } & \text { happy } \\ \text { SIT } & s \\ \text { INSTANCE } & i\end{array}\right]$

b.

$\left[\begin{array}{lc}\text { RELN } & \text { walk } \\ \text { SIT } & s \\ \text { WALKER } & i\end{array}\right]$

d.

$\left[\begin{array}{lc}\text { RELN } & \text { book } \\ \text { SIT } & s \\ \text { INSTANCE } & k\end{array}\right]$

f. $\left[\begin{array}{lc}\text { RELN } & \text { under } \\ \text { SIT } & s \\ \text { LOWER } & i \\ \text { HIGHER } & j\end{array}\right]$

The predications in (14) are meant to correspond to conditions such as: ' $s$ is a situation wherein $i$ loves $j$ ', 's is a situation wherein $i$ walks', 's is a situation wherein $i$ gives $k$ to $j$ ', 's is a situation wherein $k$ is a book', ' $s$ is a situation wherein $i$ is happy', and ' $s$ is a situation wherein $i$ is under $j$, respectively. We will henceforth make frequent use of predications like these, without taking the time to present a proper theory of relations, predications, and the features that go with them.

\footnotetext{
${ }^{6}$ The kind of event-based semantic analysis we employ was pioneered by the philosopher Donald Davidson in a number of papers. (See, for example, Davidson (1980).) Our simplified representations differ from certain popular formulations where all talk of existence is represented via explicit existential quantification, i.e. in terms of representations like (i):

(i) there is an event $s$ and an individual $i$ such that: $s$ is a running event, the runner of $s$ is $i, i$ is named Kim, and $s$ is temporally located around the time of utterance

We will treat all such existential quantification as implicit in our semantic descriptions.
} 


\section{2 / Syntactic Theory: A Formal Introduction}

Note that the restriction associated with many nouns and adjectives (book, happy, etc.) includes a predication of only one (nonsituation) argument. In such cases - for example, $(14 \mathrm{~d}, \mathrm{e})$ - we use the feature INST(ANCE).

Almost all words specify restrictions that involve predications of one kind or another, including verbs, adjectives, prepositions, and nouns. In order for phrases containing such words to inherit these restrictions, there must be constraints that (minimally) guarantee that the RESTR values of a phrase's daughters are part of that phrase's RESTR value. Only in this way will we end up with a sentence whose RESTR value includes all the necessary restrictions on the relevant event participants.

For example, for a simple sentence like (15), we will want a semantic description like the one sketched in (16).

(15) A girl saved a boy.

$$
\left[\begin{array}{lcc}
\text { MODE } & \text { prop } \\
\text { INDEX } & s & \langle \\
\text { RESTR } & \left\langle\left[\begin{array}{ll}
\text { RELN } & \text { save } \\
\text { SIT } & s \\
\text { SAVER } & i \\
\text { SAVED } & j
\end{array}\right],\left[\begin{array}{ll}
\text { RELN } & \text { girl } \\
\text { SIT } & t \\
\text { INST } & i
\end{array}\right],\left[\begin{array}{ll}
\text { RELN boy } \\
\text { SIT } & u \\
\text { INST } & j
\end{array}\right]\right\rangle
\end{array}\right]
$$

The conditions on $s$ come from the lexical entry for the verb save, the constraint that $i$ - the saver - must be a girl comes from the noun girl, and the constraint that $j$ - the saved (person) - must be a boy comes from the lexical entry for the noun boy. By associating (15) with the feature structure in (16), our semantic analysis says that the linguistic meaning of (15) is the proposition that will be true just in case there is an actual situation that involves the saving of a boy by a girl. But in order to produce the right set of conditions in the sentence's semantic description, the conditions of the parts of the sentence have to be amalgamated into a single list of conditions. Note in addition that the main situation of the sentence is derived from that introduced by the verb. It is true in general that the semantics of a phrase will crucially involve the semantics of its head daughter.

\subsection{How Semantics Fits In}

In earlier chapters, we considered only the syntactic properties of linguistic expressions. To accommodate the basic analysis of linguistic meaning just sketched, we need some way of introducing semantic structures into the feature structures we use to analyze words and phrases. We do this 
by adding two new features - SYN(TAX) and SEM(ANTICS) - and adding a level of embedding within our feature structures, as illustrated in (17):

$$
\begin{aligned}
\text { SYN } & {\left[\begin{array}{ll}
\text { HEAD } & {[. .} \\
\text { SPR } & \ldots \\
\text { COMPS } & \ldots
\end{array}\right] } \\
\text { SEM } & {\left[\begin{array}{ll}
\text { MODE } & \\
\operatorname{INDEX} & \\
\operatorname{RESTR} & \langle\ldots\rangle
\end{array}\right] }
\end{aligned}
$$

There is now a syntactic side and a semantic side to all feature structures like (17), which we will assign to the type called synsem-struc(ture). Although we will add a few more features as we progress, this is in essence the feature geometry that we will adopt in the remainder of the book. We will frequently have occasion in what follows to refer to the synsem-struc of a phrase. What we mean by this is the full feature structure that is the top node of the SD of that phrase, including both its SYN and SEM values.

This changes the way lexical entries look, of course; and this is illustrated in (18):

(18) a.

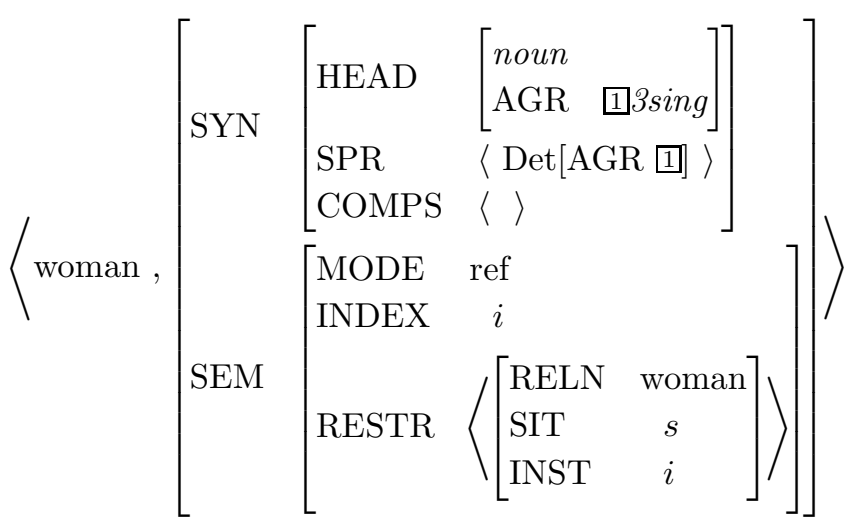


114 / Syntactic Theory: A Formal Introduction

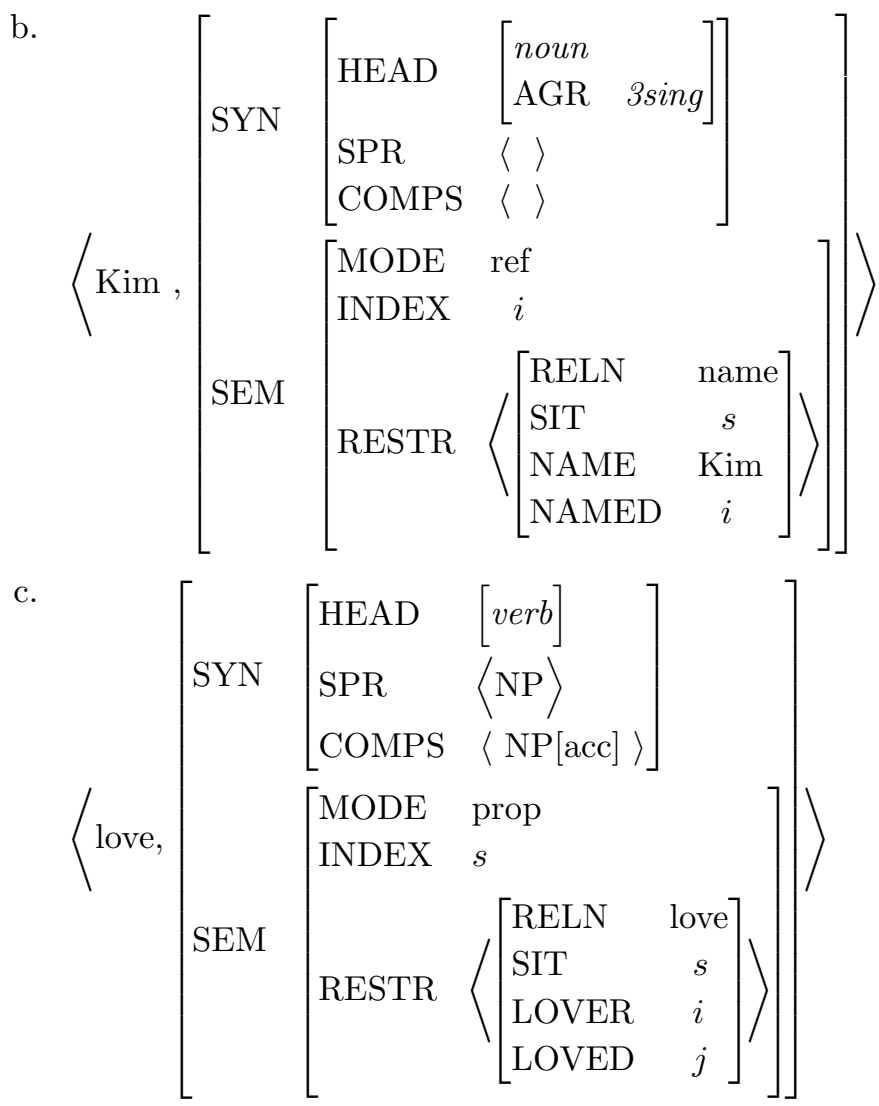

It should be noted that our semantic analysis of proper names (one of many that have been proposed over the centuries) treats them as simple referring expressions whose referent must be appropriately named. ${ }^{7}$ Under our assumption that all $\mathrm{NPs}^{8}$ have an index as their INDEX value, it is straightforward to associate the semantics of NPs with the particular roles they play in a verb's predication. We shall assume that the role arguments within predications are indices, and hence we can achieve the desired result by letting a verb (and other elements with predicational semantics) specify links between the indices of its dependents (specifiers and complements) and the role arguments in the predication on the RE-

\footnotetext{
${ }^{7}$ In a more precise account, we might add the further condition that the speaker intend to refer to the referent. Under this analysis, a sentence like Kim walks would be regarded as true just in case there is a walking event involving a certain individual that the speaker intends to refer to and who is named 'Kim'.

${ }^{8}$ Except 'dummy' NPs like there, which we will turn to in Chapter 11.
} 
STR list of its own semantics. This is illustrated in (19) for the verb love.

NB: Here and throughout, we use $N P_{i}$ as a shorthand for an NP whose $S E M$ value's INDEX is $i$.

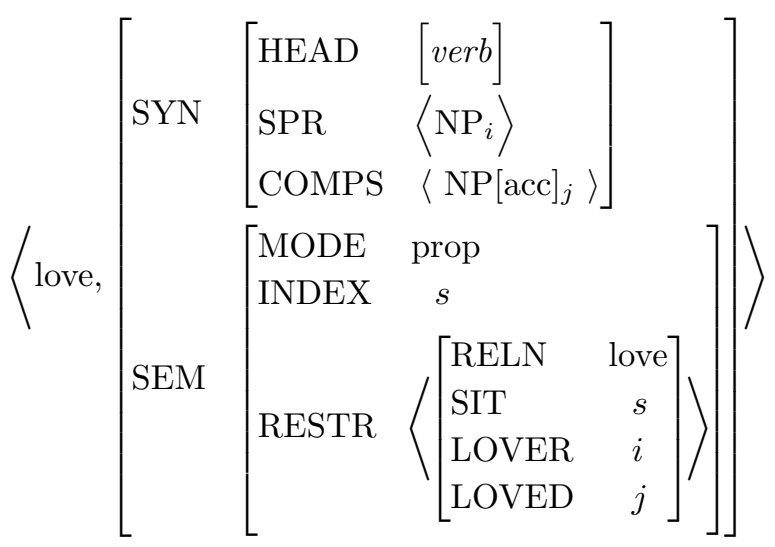

In this way, as the verb combines with a particular NP object, the index of that NP is identified with the value of the feature LOVED in the verb's semantics. The verb's INDEX value is identified with that of the VP it heads, and the verb's restrictions are included in the VP's RESTR value, so that when that VP combines with the subject, the identities specified in (19) will also guarantee that the index of the actual subject of the sentence will be the LOVER in the loving predication on the VP's RESTR list. This will in turn be included in the RESTR value of the S, by the Semantic Compositionality Principle (introduced later in this chapter) which requires that the mother's RESTR list include all the RESTR lists of its daughters. Hence the unifications specified in (19) ensure that the conditions specified by the sentence's subject and object are those conditions about the lover and loved participants in the predication that involves the situation that the sentence as a whole is about.

We will illustrate how the SEM value of a complex expression relates to its parts in more detail in section 5.6. Before concluding this section, however, we must point out that we are glossing over one important issue (among others) in our cursory presentation of semantic analysis. This is the matter of quantification. Sentences like those in (20) require a semantic treatment that goes beyond simple reference to individuals.

(20) a. Everyone liked Sense and Sensibility. 
b. Most people are reading a book by Austen.

c. Few people who have met you say there is nothing unusual about you.

The approach to NP semantics sketched here can easily be extended to deal with sentences containing quantified NPs (everyone, most people, etc.), by augmenting our feature structures to allow more complex propositions (as well as questions and directives) that represent quantification over individuals explicitly (in terms of 'binding' of indices). We will leave this entire topic unexplored here, however, noting only that it is possible to introduce explicit quantification over situations as well.

\subsection{Modification}

Suppose that we introduce a HEAD feature called MOD and that the MOD value of a word specifies the kind of thing the word modifies. Then we could make it a lexical property of adjectives that they were [MOD NOM] (or [MOD NP]) and a lexical property of adverbs that they were [MOD VP] (or [MOD S]). Since these modifiers would then 'pass up' their MOD value, courtesy of the Head Feature Principle, to any phrase that they projected (i.e. that they were the head daughter of), it would then be possible to use a single Head-Modifier Rule, as in (21), to account for both nominal and verbal modification.

(21) Head-Modifier Rule

$$
[\text { phrase }] \rightarrow \mathrm{H}[1 \text { phrase }]\left[\begin{array}{l}
\text { phrase } \\
\text { HEAD [MOD }
\end{array}\right]
$$

That is, rule (21) will license a NOM just in case the head daughter is a phrase of category NOM and the modifier daughter's MOD value is also of category NOM:

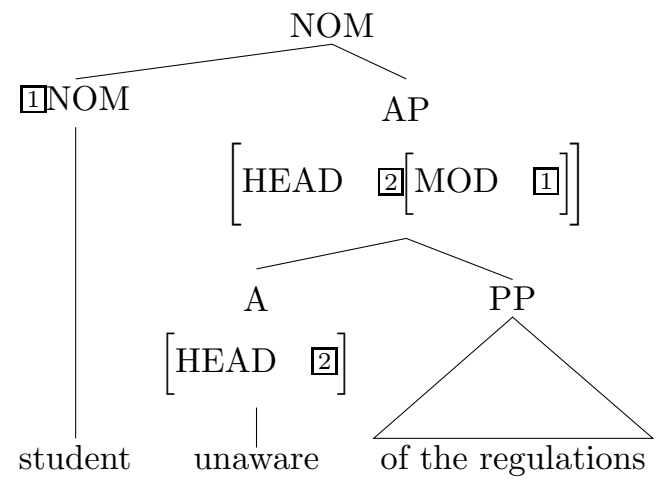

This NOM can combine with a D as specifier to build an NP like (23): 
(23) a student unaware of the regulations

The Head-Modifier Rule in (21) will also license the verb phrase in (24), under the assumption that adverbs are lexically specified as $[\mathrm{MOD} \quad \mathrm{VP}] .^{9}$

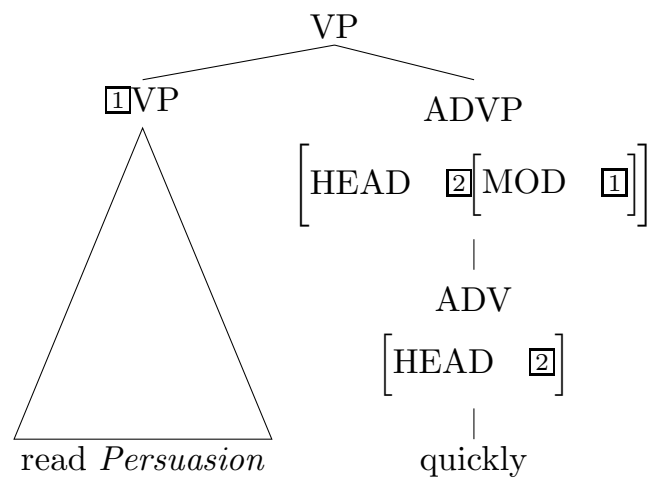

And a VP satisfying this description can combine with a subject like the one in (23) to build sentence (25).

(25) A student unaware of the regulations read Persuasion quickly.

Note further that many prepositional phrase modifiers can modify either nominal or verbal constituents:

(26) a. The [reporter [in Rome]]...

b. We [went shopping [in Rome]]

(27) a. The [weather [on Sunday]]...

b. We [went shopping [on Sunday]]

The bare bones treatment of modification just sketched can easily account for these examples by allowing underspecified or disjunctively specified MOD values for such prepositions.

\subsection{The Semantic Principles}

We are now not only able to analyze the form of sentences of considerable complexity using our grammar, but in addition we can analyze the meanings of complex sentences by adding semantic constraints on the structures defined by our rules. The most general of these semantic constraints is given in (28):

\footnotetext{
${ }^{9} \mathrm{We}$ assume here that there is a lexical category (a subtype of part-of-speech) for adverbs (ADV). The Head Complement Rule then licenses adverb phrases (ADVP). We do not examine the syntax of adverb phrases in any detail in this text.
} 
118 / Syntactic Theory: A Formal Introduction

(28) Semantic Compositionality Principle

In any well-formed phrase structure, the mother's RESTR value is the sum of the RESTR values of the daughters.

In other words, all restrictions from all the daughters in a phrase are collected into the RESTR value of the mother. The term 'sum' has a straightforward meaning here: the sum of the RESTR values of the daughters is the list whose members are those values, taken in order. ${ }^{10}$ We will use the symbol ' $\oplus$ ' to designate the sum operator. ${ }^{11}$

In addition to the Semantic Compositionality Principle, we introduce the following constraint on the MODE and INDEX values of headed phrases:

(29) Semantic Inheritance Principle

In any headed phrase, the mother's MODE and INDEX values are identical to those of the head daughter.

The Semantic Inheritance Principle guarantees that the semantic MODE and INDEX of a phrase are identified with those of the head daughter, giving the semantics, like the syntax, a 'head-driven' character.

The effect of these two semantic principles is illustrated in the following simple example:

${ }^{10}$ That is, the sum of lists $\langle A\rangle,\langle\mathrm{B}, \mathrm{C}\rangle$, and $\langle\mathrm{D}\rangle$ is the list $\langle\mathrm{A}, \mathrm{B}, \mathrm{C}, \mathrm{D}\rangle$.

${ }^{11}$ Notice that, unlike the familiar arithmetic sum operator, $\oplus$ is not commutative: $\langle\mathrm{A}\rangle \oplus\langle\mathrm{B}\rangle=\langle\mathrm{A}, \mathrm{B}\rangle$, but $\langle\mathrm{B}\rangle \oplus\langle\mathrm{A}\rangle=\langle\mathrm{B}, \mathrm{A}\rangle$. And $\langle\mathrm{A}, \mathrm{B}\rangle \neq\langle\mathrm{B}, \mathrm{A}\rangle$, because the order of the elements matters. Although the order of elements in RESTR lists has no semantic significance, the relative ordering of elements in the ARG-ST lists that we construct using $\oplus$ in Chapter 7 is crucial to our account of reflexive binding. 


\begin{tabular}{|c|c|}
\hline phrase & \\
\hline SPR & \langle\rangle \\
\hline MODE & prop \\
\hline INDEX & $s$ \\
\hline RESTR & 10 \\
\hline
\end{tabular}
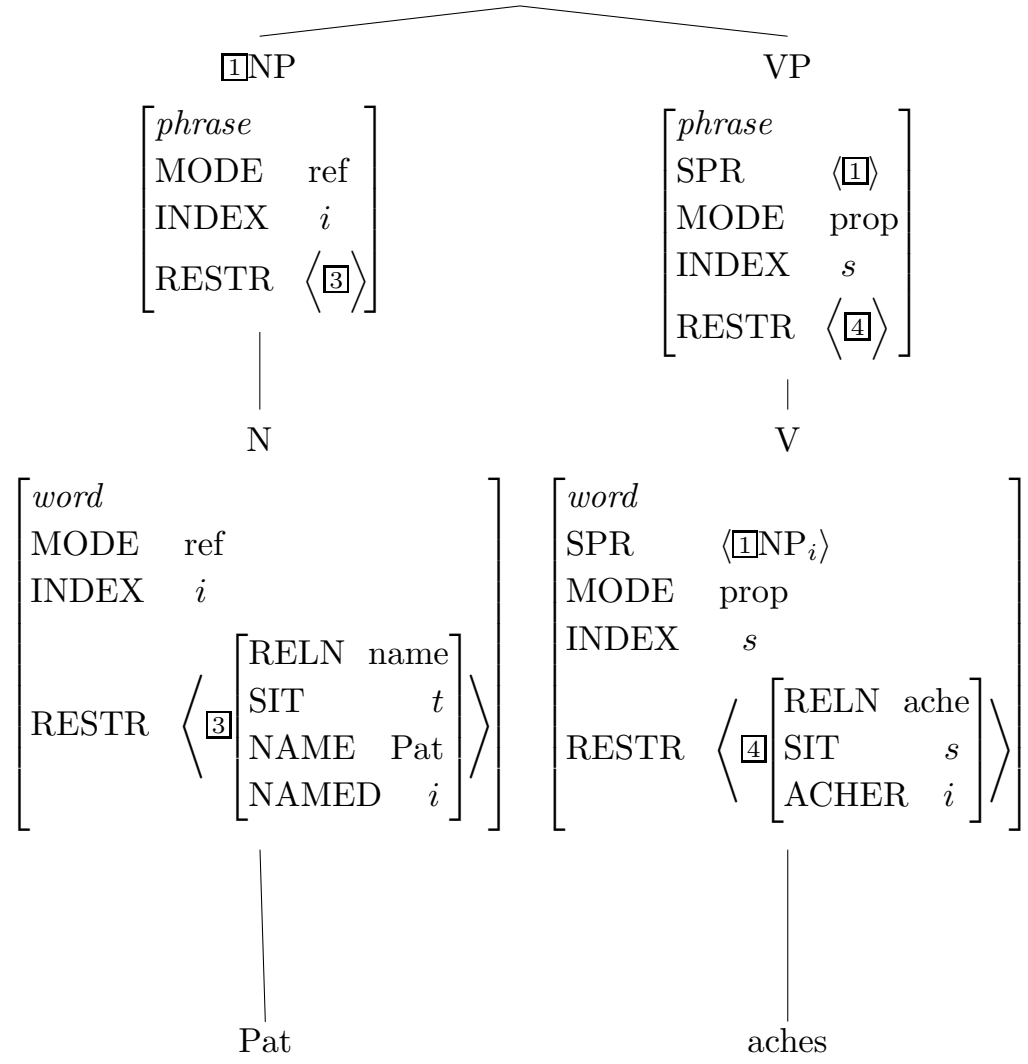

The effect of both semantic principles can be clearly observed in the $\mathrm{S}$ node at the top of this SD. The MODE is 'prop', inherited from its head daughter, the VP node, (and ultimately from the head verb, aches) by the Semantic Inheritance Principle. In this same way, the INDEX value 's' comes from the verb, through the VP. The RESTR value of the S node, [RESTR $\langle$ 3, 田〉], is the sum of the RESTR values of the NP and VP nodes, as specified by the Semantic Compositionality Principle. Note that here, as before, we use abbreviations like 'NP', 'S', and 'V' 
to abbreviate feature structure descriptions specifying purely syntactic information. Since the labels of nodes in phrasal SDs will now include semantic information, the notational abbreviations should henceforth be reinterpreted accordingly (as standing for entire synsem-strucs).

With these semantic principles in place, we can now complete our account of modification. Let us assume that an adverb like today has a lexical entry like the one in (31).

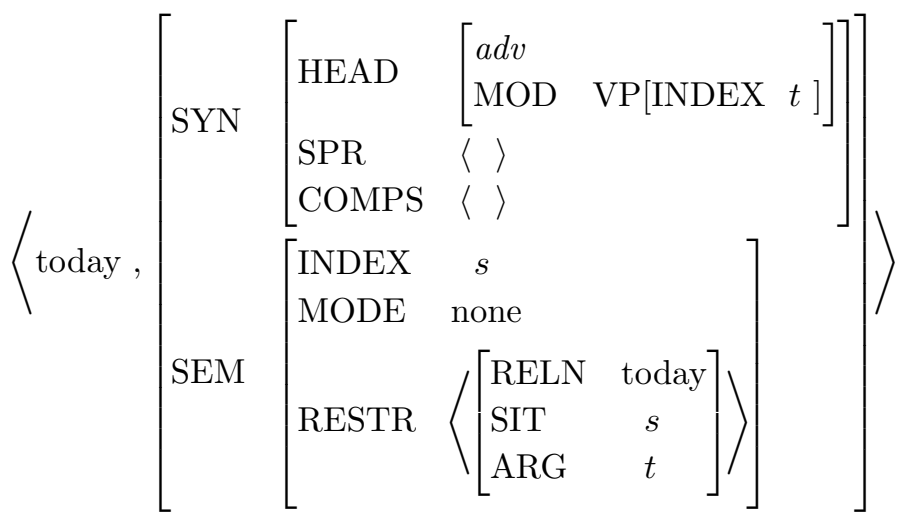

The key point here is that the MOD value identifies the index of the VP to be modified as ' $t$ ', the same situation that is the argument of the relation 'today' in the semantic restriction. This means that once the adverb combines with a VP, the (situational) index of that VP is the argument of 'today'. ${ }^{12}$

Our two semantic principles, the Head-Modifier Rule, and the lexical entry in (31) thus interact to define SDs like the following (only SEM values are indicated):

\footnotetext{
${ }^{12}$ It would be equivalent to use tags when identifying indices, e.g. as in (i):

(i) $\left[\begin{array}{ll}\operatorname{INDEX} & 1\end{array} \mathrm{~s}\right] \ldots[\operatorname{INDEX} \quad 1]$

Following standard mathematical practice, however, we will continue to use multiple occurrences of a given index to indicate that two features have the same index as their value.
} 


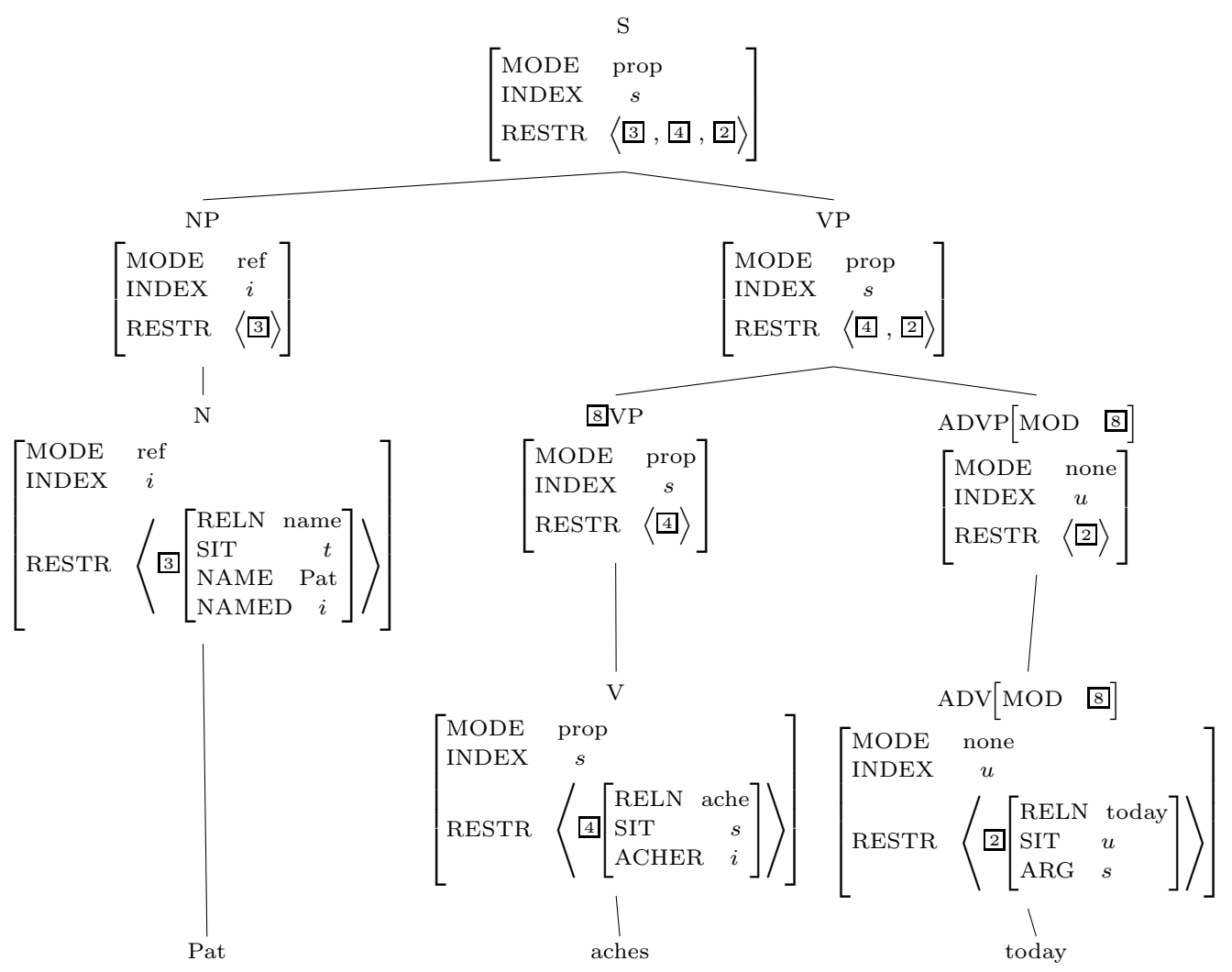


In this way, our analysis provides a general account of how meanings are constructed. The Semantic Compositionality Principle and the Semantic Inheritance Principle together embody a simple yet powerful theory of the relation between the structures of our grammar and the meanings they convey.

\subsection{Coordination Revisited}

The analysis of the previous section specifies how meanings are associated with the headed structures of our grammar, by placing appropriate constraints on those phrasal SDs that result from our headed rules. It also covers the composition of the RESTR values in nonheaded rules. But nothing in the previous discussion specifies the MODE or INDEX values of coordinate phrases - the one kind of phrase licensed by the Coordination Rule, a nonheaded rule.

In the previous chapter, we wrote this rule as follows:

$$
\text { 1 } \rightarrow \square^{+} \mathrm{CONJ}
$$

This is equivalent to the following formulation, where the Kleene plus has been replaced by a schematic enumeration of the conjunct daughters:

$$
\square \rightarrow \square_{1} \ldots \square_{n-1} \text { CONJ } \square_{n}
$$

We will employ this notation for now because it lets us enumerate schematically the arguments that the semantic analysis of conjunctions requires.

Unlike the other relations we have used for semantic analysis, where each predication specifies a fixed (and small) number of roles, the relations that express the meanings of conjunctions like and and or allow any number of arguments. Thus each conjunct of coordinate structures like the following is a semantic argument of the conjunction:

(35) a. Chris $\left[[\text { walks }]_{1} \text {, [eats broccoli }\right]_{2}$, and [plays squash $\left.]_{3}\right]$.

b. $\left.\left[[\text { Chris walks }]_{1},[\text { Pat eats broccoli }]_{2} \text {, and [Sandy plays squash }\right]_{3}\right]$.

Because the number of arguments is not fixed, the predications for conjunctions allow not just indices as arguments, but lists of indices. Consequently, the sentences in (35) may be represented in terms of a semantic structure like the following: 


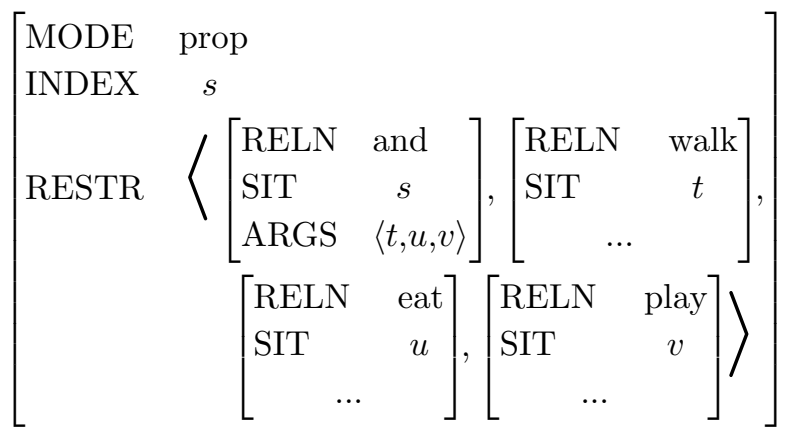

Note that it is the conjunction's index (' $s$ ' in (36)) that is the index of the entire coordinate phrase. Otherwise, the work of combining the RESTR values of all the daughters in a coordinate structure to form the RESTR value of the mother is taken care of by the Semantic Compositionality Principle.

Let us assume then that the lexical entry for a conjunction looks roughly as shown in (37).

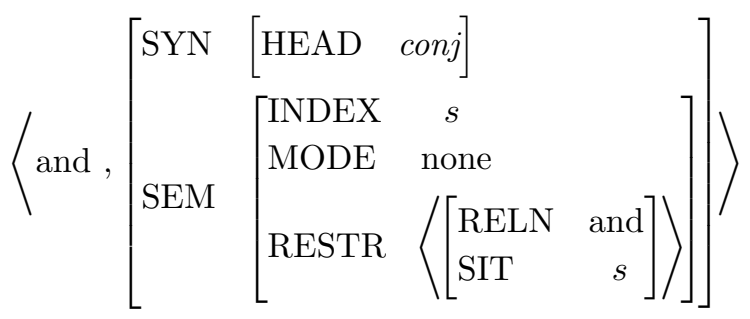

The Coordination Rule must then be modified to identify the indices of the conjuncts with the ARGS value of the conjunction.

Before doing this, however, we must consider the syntactic matching of the conjuncts. In our earlier formulation, we identified the entire grammatical category of all conjuncts (and their mother). But, as we have just seen, the semantics of conjuncts is not shared. (In fact, it would be pragmatically quite strange to conjoin multiple expressions that mean the same thing). Rather, the conjuncts become semantic arguments of the conjunction, and the combined list of predications must become the RESTR value of the mother of the coordinate structure. Given the need to treat the semantics separately, a reasonable hypothesis about the sharing in a coordinate structure is that the SYNTAX value of all conjuncts and of their mother is the same. This leads us to the following reformulation of our Coordination Rule: ${ }^{13}$

\footnotetext{
${ }^{13}$ The careful reader will note that we have omitted the feature name 'SEM' to the left of 'IND' in each of the terms of (38). In the remainder of the book, we will
} 
124 / Syntactic Theory: A Formal Introduction

(38) Coordination Rule

$\left[\begin{array}{ll}\mathrm{SYN} & 0 \\ \mathrm{IND} & s_{0}\end{array}\right] \rightarrow$

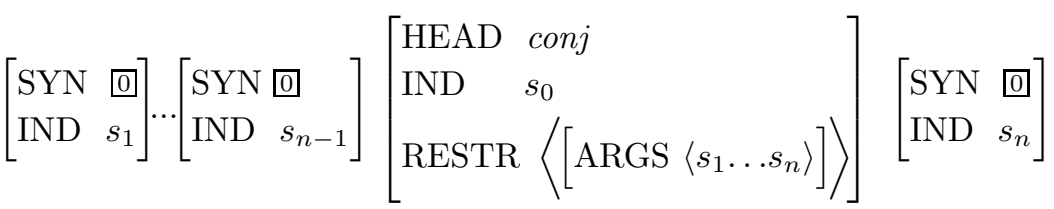

This rule accomplishes a number of goals, including

- requiring that all conjuncts of a coordinate structure have identical values for HEAD, SPR, and COMPS,

- collecting the RESTR values of all daughters into the RESTR list of the mother (guaranteed because the structures built in accordance with this rule must satisfy the Semantic Compositionality Principle),

- identifying the indices of the conjuncts with the semantic arguments of the conjunction, and

- identifying the index of the conjunction with that of the coordinate structure.

These effects are illustrated in the following SD, which describes a (coordinate) phrase structure satisfying the Coordination Rule.
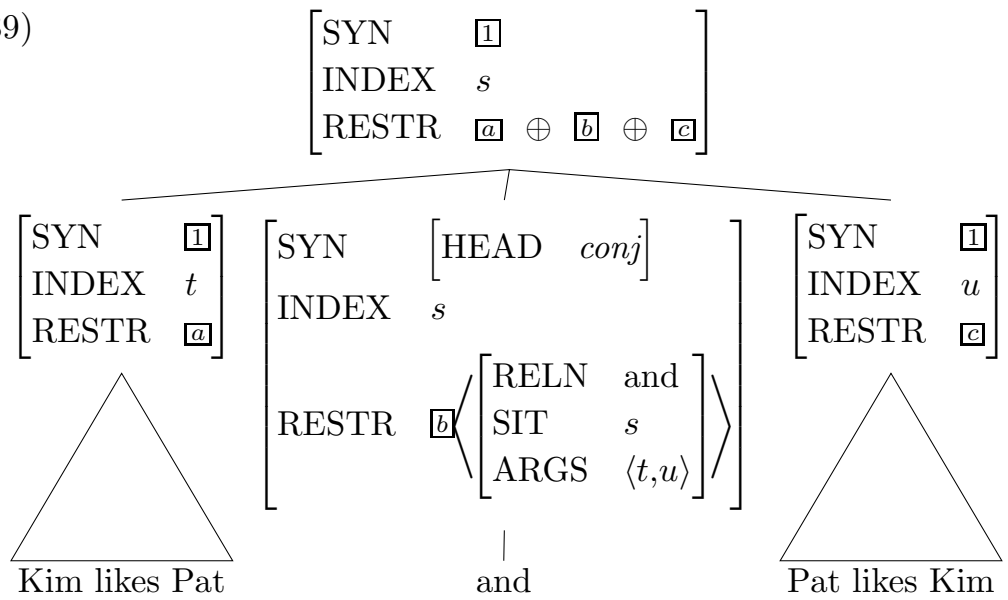

often simplify our feature structure descriptions in this way, leaving out some of the outer layers of feature names when the information of interest is embedded within the feature structure description. We will only simplify in this way when no ambiguity about our intended meaning can result. 
Our revised Coordination Rule thus goes a long way toward accounting for sentences containing coordinate structures and associating them with appropriate meanings.

\section{Problem 1: NP Coordination}

Consider the following data.

(i) $\operatorname{Kim}\left\{\begin{array}{c}\text { walks } \\ { }^{\text {walk }}\end{array}\right\}$.

(ii) Sandy $\left\{\begin{array}{c}\text { walks } \\ { }^{*} \text { walk }\end{array}\right\}$.

(iii) Kim and Sandy $\left\{\begin{array}{c}{ }^{*} \text { walks } \\ \text { walk }\end{array}\right\}$.

A. What conclusion can you draw about the NUMBER value of coordinate NPs?

Now consider the question of what the PERSON value of coordinate NPs is. Choice of verb form does not usually help very much in determining the person of the subject, because those whose AGR value is non-3sing are compatible with a subject of any person (except those whose AGR is 3sing).

However, there is another way to detect the person of the subject NP. If the VP contains a direct object reflexive pronoun, then (as we saw in Chapter 1) the reflexive must agree in person and number with the subject. This co-occurrence pattern is shown by the following examples.

(iv) $\left\{\begin{array}{l}\text { You } \\ * \mathrm{I} \\ * \text { She } \\ * \text { They } \\ * \text { We }\end{array}\right\}$ distinguished yourself. (2nd person singular)
(v) $\left\{\begin{array}{c}\text { She } \\ * \text { You } \\ * \mathrm{I} \\ * \text { They } \\ * \text { We }\end{array}\right\}$ distinguished herself. (3rd person singular) 
126 / Syntactic Theory: A Formal Introduction

(vi)

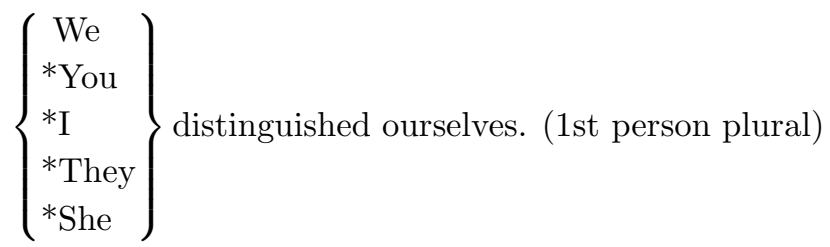

(vii)

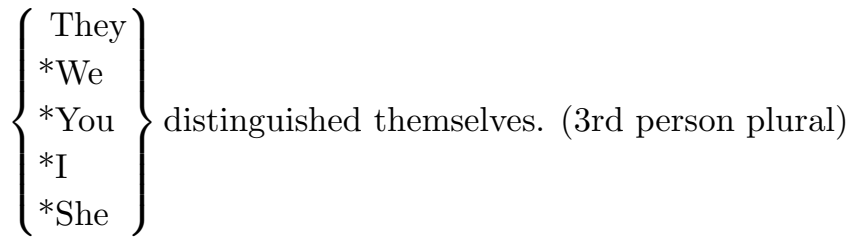

In light of this patterning, we can now consider the person of coordinate NPs by examining examples like the following:

$$
\text { You and she distinguished }\left\{\begin{array}{c}
\text { yourselves } \\
* \text { themselves } \\
* \text { ourselves }
\end{array}\right\} \text {. }
$$

$$
\text { You and I distinguished }\left\{\begin{array}{c}
* \text { yourselves } \\
* \text { themselves } \\
\text { ourselves }
\end{array}\right\} \text {. }
$$

B. Construct further examples of sentences with coordinate subjects (stick to the conjunction and) that could help you discover what the person value of the coordinate NP is for every combination of PERSON value on the conjuncts. State the principles for determining the PERSON value of a coordinate NP in as general terms as you can.

C. Our Coordination Rule in (38) cannot easily be weakened to allow conjuncts to have disparate HEAD values. Otherwise we would run the risk of generating examples like the following:

(x) $*$ Kim [walks] and [happy].

(xi) *Pat visited [Chris and sad].

So on the basis of your results in (A) and (B), what conclusion should you draw about the analysis of NP coordination?

\section{(1) Problem 2: Modes of Coordination}

Consider the following data.

(i) Kim left and Sandy left.

(ii) ?*Kim left and did Sandy leave. 
(iii) ?*Did Sandy leave and Kim left.

(iv) Did Sandy leave and did Kim leave?

(v) Go away and leave me alone!

(vi) ?*Kim left and leave me alone!

(vii) ?*Leave me alone and Kim left.

(viii) ?*Leave me alone and did Kim leave?

(ix) ?*Did Kim leave and leave me alone!

A Formulate a generalization about the MODE value of conjuncts (and their mother) that could account for these data.

B Modify the Coordination Rule in (38) so that it enforces the generalization you formulated in (A).

\subsection{Summary}

In this chapter, we introduced fundamental issues in the study of linguistic meaning and extended our grammar to include semantic descriptions. We then provided a systematic account of the relation between syntactic structure and semantic interpretation based on two constraints: the Semantic Compositionality Principle and the Semantic Inheritance Principle. These principles together provide a general account of how the semantics of a phrase is related to the semantics of its daughters. This chapter also presented a rudimentary analysis of modification and extended the treatment of coordinate structures to include an account of their linguistic meaning.

\subsection{Further Reading}

Essentially all work on linguistic pragmatics builds directly on the pioneering work of the philosopher H. Paul Grice (see Grice 1989). A seminal work in modern resarch on natural language semantics is Frege's (1892) essay, 'Über Sinn und Bedeutung' (usually translated as 'On Sense and Reference'), which has been translated and reprinted in many anthologies (e.g. Geach and Black, eds., (1980)). More recently, the papers of Richard Montague (Montague, 1970) had a revolutionary influence, but they are extremely technical and difficult. An elementary presentation of his theory is given by Dowty, Wall, and Peters (1981). General introductory texts in semantics include Chierchia and McConnellGinet (1990), Gamut (1991), and de Swart (1998). Shorter overviews of semantics include Bach (1989), Barwise and Etchemendy (1989), and Partee (1995). 



\section{How the Grammar Works}

\subsection{A Factorization of Grammatical Information}

Four chapters ago, we began modifying the formalism of context-free grammar to better adapt it to the sorts of generalizations we find in natural languages. We broke grammatical categories down into features, and then we broke the values of features down into features, as well. In the process, we moved more and more syntactic information out of the grammar rules and into the lexicon. In effect, we changed our theory of grammar so that the rules give only very general patterns that cut across grammatical categories. Details about which expressions can go with which are specified in lexical entries in terms of valence features.

With the expanded ability of our new feature structure complexes to express cross-categorial generalizations, our four remaining grammar rules cover a wide range of cases. Two of them - the rules introducing complements and specifiers - were discussed extensively in Chapter 4. The third one - a generalization of our old rules introducing PP modifiers to VP and NOM - was illustrated in the previous chapter. The fourth is the coordination schema. The formal statements of these rules are given in (1), along with informal translations (given in italics below the rules). ${ }^{1}$

\footnotetext{
${ }^{1}$ It should be noted that the Head-Modifier Rule does not cover all kinds of modifiers. In particular, some modifiers - such as adjectives inside NPs - precede the heads that they modify. To accommodate such modifiers, we would need an additional grammar rule.
} 
130 / Syntactic Theory: A Formal Introduction

\section{(1) Head-Complement Rule}

$\left[\begin{array}{ll}\text { phrase } & \\ \text { COMPS } & \langle\rangle\end{array}\right] \rightarrow \mathrm{H}\left[\begin{array}{ll}\text { word } & \\ \text { COMPS } & \langle\square, \ldots, n\rangle\end{array}\right]$ 1... $n$

A phrase can consist of a lexical head followed by all its complements.

\section{Head-Specifier Rule}

$\left[\begin{array}{ll}\text { phrase } & \\ \mathrm{SPR} & \langle\rangle\end{array}\right] \rightarrow \quad$ 目 $\quad \mathrm{H}\left[\begin{array}{ll}\text { phrase } & \\ \operatorname{SPR} & \langle\square\rangle\end{array}\right]$

A phrase can consist of a phrasal head preceded by its specifier.

\section{Head-Modifier Rule}

$$
\left[\text { phrase } \rightarrow \mathrm{H} \square[\text { phrase }] \quad\left[\begin{array}{ll}
\text { phrase } & \\
\text { MOD } & 1
\end{array}\right]\right.
$$

A phrase can consist of a phrasal head followed by a compatible modifier phrase.

\section{Coordination Rule}

$\left[\begin{array}{ll}\mathrm{SYN} & 0 \\ \mathrm{IND} & s_{0}\end{array}\right] \rightarrow$

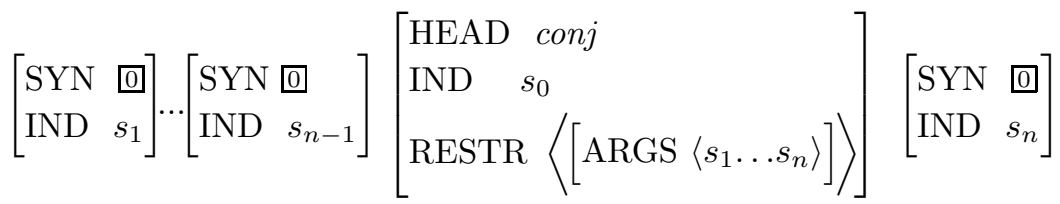

Any number of occurrences of elements of the same syntactic category can be conjoined to make a coordinate element of that category.

In addition to our grammar rules, we must provide (as we did in the case of CFGs) some characterization of the 'initial symbols', corresponding to the types of phrases that can stand alone as sentences of the language. We postpone a characterization of this until Chapter 9, when we will have introduced a method for distinguishing finite (that is, tensed) clauses from others.

The richer feature structures we are now using, together with our highly schematized rules, have required us to refine our notion of how a grammar is related to the fully determinate phrase structures of the 
language and to the informal descriptions of those structures that we will use to explicate those structures. Intuitively, here is how it works: First, each lexical entry licenses a family of word structures - each of which is a nonbranching tree whose mother is a resolved feature structure satsifying the feature structure description of the lexical entry.

Put somewhat differently, given a lexical entry $\langle\omega, \Phi\rangle$, each of these word structures must satisfy the following lexical SD:

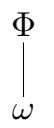

Such lexical SDs form the bottom layer of well-formed phrasal SDs. They can be combined ${ }^{2}$ into larger SDs in the ways permitted by the grammar rules, filling in features as required by our four principles: the Semantic Compositionality Principle, the Head Feature Principle, the Valence Principle, and the Semantic Inheritance Principle. This process can apply to its own output, making ever larger phrasal SDs. So long as each local SD that we construct is licensed by a grammar rule and conforms to these principles, it is well formed. Typically, each node in a well-formed SD will contain some information that was stipulated by a rule and other information that percolated up (metaphorically speaking) from lower nodes (and ultimately from the lexical entries) via the principles.

For most of the material presented in the chapters that follow, an intuitive grasp of how the lexicon, grammar rules, and principles interact to license phrasal SDs will suffice. Nevertheless, for completeness we include an explicit definition of the resolved structures permitted by our grammar as an appendix to this chapter. Our SDs contain exactly as much information as is obtained by unifying the constraints that percolated up from the daughters, the constraints that are specified in each rule, and those constraints that are part of our general theory (the Head Feature Principle, etc.). Hence the relation between our phrasal SDs and the phrase structures that satisfy them should be transparent: a phrasal SD consolidates all relevant conditions that a given phrase structure must satisfy.

We have formulated our theory so that, as successively larger SDs are produced, the descriptions of the (nonterminal) nodes expand through unification. That is, information that is left underspecified in an SD

\footnotetext{
${ }^{2}$ Our informal discussion is worded in terms of a process of building trees up from the bottom. This is a conceptually natural way of thinking about it, but it should not be taken too literally. The formal definition of well-formed tree structure that we give below is deliberately nonprocedural.
} 
often needs to be included if it is to be embedded in a larger SD. This important side effect of unifying SDs can be illustrated simply. Consider the SD in (3).

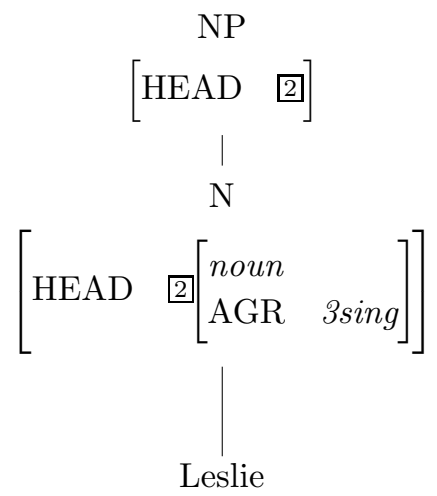

This contains information about the HEAD value of the phrase (unified with the HEAD value of the $\mathrm{N}$ dominating Leslie). But when this SD is embedded within a larger one like (4), licensed by the Head-Complement Rule, the result is as shown in (4).

(4)

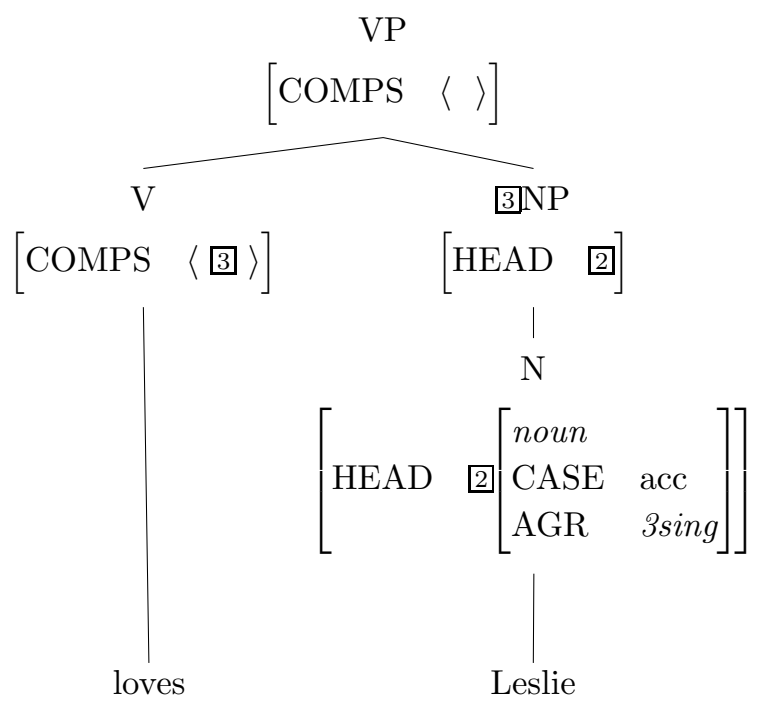

We have assumed here (following the results of Chapter 4, problem 6) that the lexical entry for loves specifies that its complement is [CASE acc]. Because the Head-Complement Rule identifies the head daughter's COMPS list with the list of (the feature structures of the) complement daughters, the accusative case specification is part of the 
SD of the object NP's HEAD value. And since that NP's HEAD specification is identified with the HEAD specification of its head daughter (namely, the $\mathrm{N}$ dominating Leslie), it follows that the accusative case specification is part of the SD of this embedded category as well.

The information specified by our rules and lexical entries is thus partial information. Each rule says, in effect, that subtrees of a certain kind are sanctioned, but the rule only specifies some of the constraints that the SDs that it licenses must obey. Likewise, a lexical entry says that certain trees dominating the phonological form in that entry are sanctioned, but the entry only specifies some of the information relevant at higher levels of structure. The general principles of our theory constrain the ways in which feature values can be distributed in wellformed phrase structure trees. The unification of partial descriptions (or constraints) is the basic method that allows the job of determining well-formedness to be distributed among the pieces of our grammatical system in a parsimonious way.

In short, we have arrived at a particular factorization of the information necessary for a precise account of grammatical descriptions. By far the richest source of information in this factorization is the lexicon. That is, our account embodies the claim that both the problem of determining which strings of words constitute well-formed sentences and the problem of specifying the linguistic meaning of sentences depend mostly on the nature of words. Of course, it must also be recognized that there are many regularities about which words go together (and how they go together). The theoretical constructs summarized here capture a number of such regularities; subsequent chapters will provide ways of capturing more.

\subsection{A Detailed Example}

The components of our grammatical theory interact so as to license certain phrase structure trees as well-formed sentences, but not others. The nature of these interactions can best be understood through careful analysis of linguistic examples. In this subsection, we show in detail how the grammar of English, as we have developed it to this point, would handle one simple sentence of English, namely:

(5) They sent us a letter.

We begin our analysis with the lexical SD for the word letter. 
134 / Syntactic Theory: A Formal Introduction

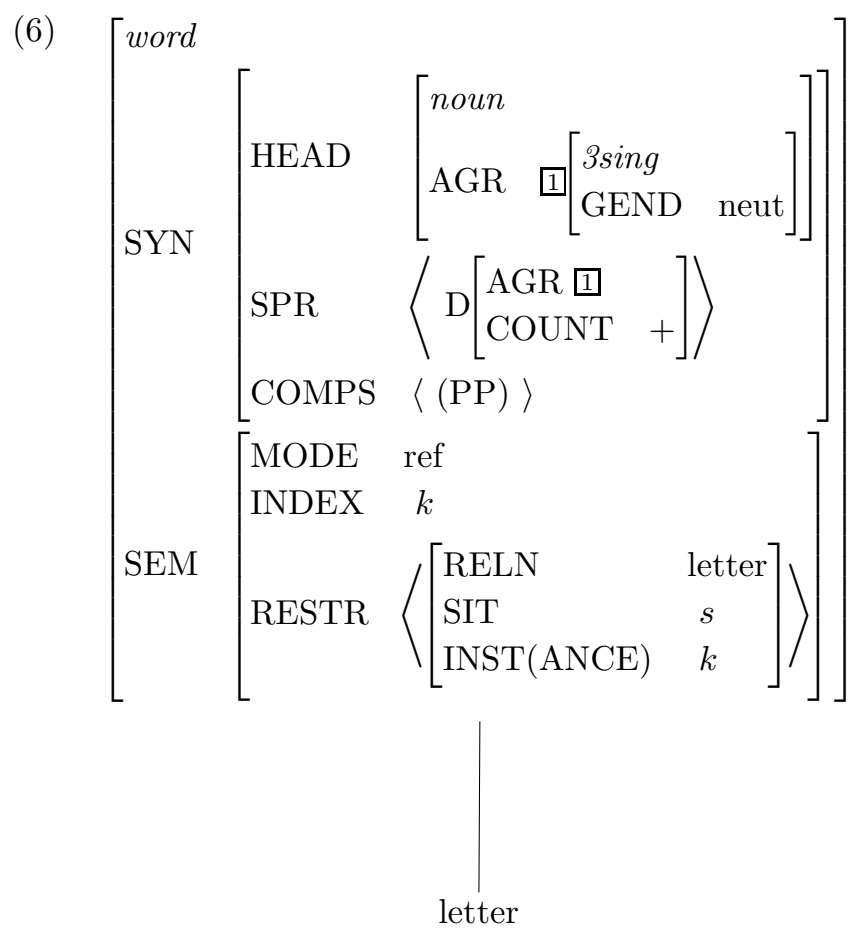

This combines with zero complements via the Head-Complement Rule to give the SD shown in (7). Note that the COMPS value is disambiguated (to the empty list) by the presence of no complements in this SD. The Head Feature Principle (HFP) is obeyed; the SPR specifications obey the Valence Principle; and the COMPS specifications are as dictated by the Head-Complement Rule.

The lexical SD for the word $a$ is (8). Providing semantics for determiners (such as quantifiers) is something we have avoided, since it would take us too far afield. So we simply omit any description of the determiner's SEM value. 
How the Grammar Works / 135

(7)

$\left[\begin{array}{ll}\text { shrase } & \\ \text { SYN } & {\left[\begin{array}{ll}\text { HEAD } & 27 \\ \text { SPR } & \langle 28\rangle \\ \text { COMPS } & \langle\rangle\end{array}\right]} \\ \text { SEM } & {\left[\begin{array}{lc}\text { MODE } & \text { ref } \\ \text { INDEX } & k \\ \text { RESTR } & \square\end{array}\right]}\end{array}\right]$

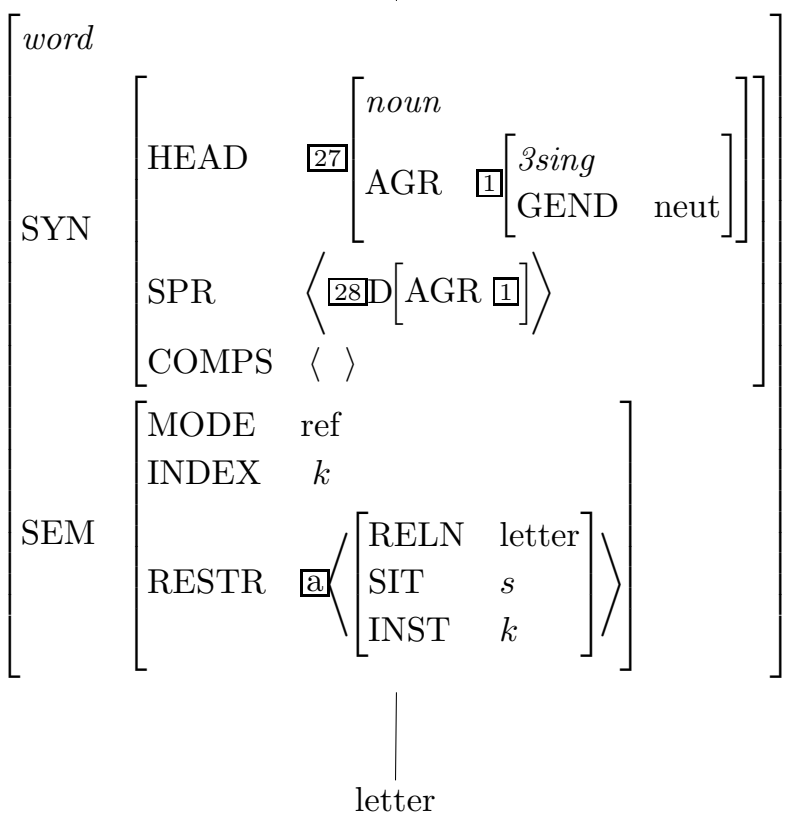

(8)

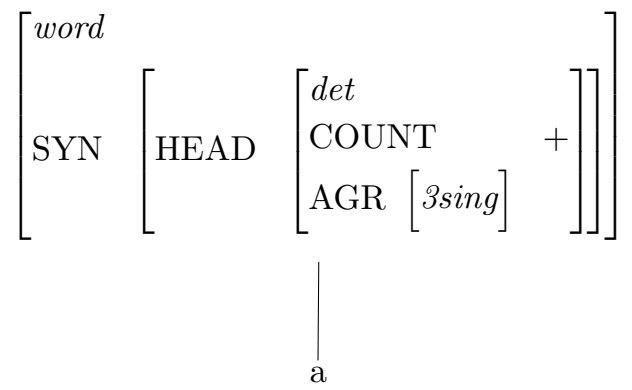


The following SD results from combining (7) and (8) via the HeadSpecifier Rule:

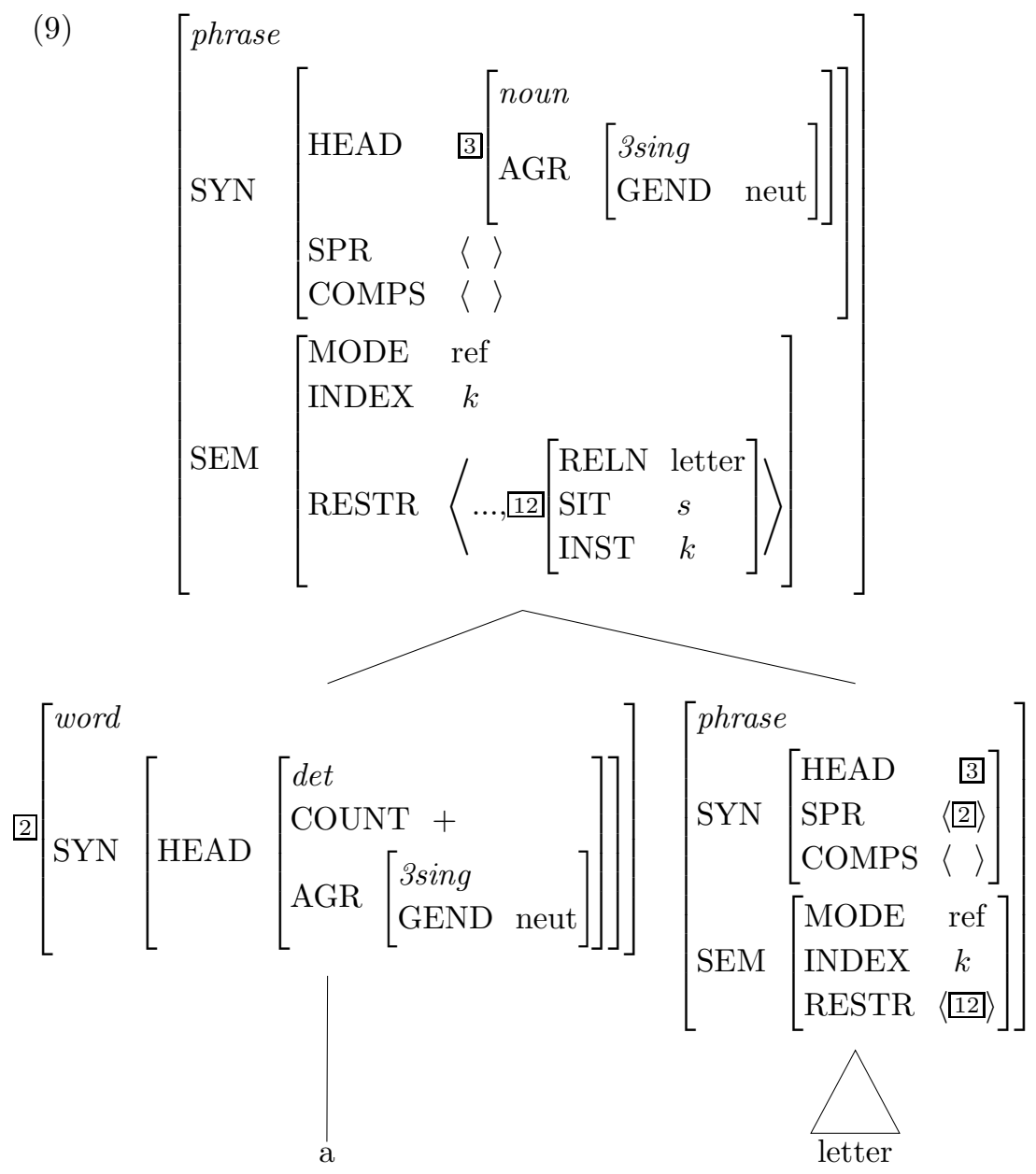

In this $\mathrm{SD}$, the left subtree is exactly the one shown in (8), except that the feature specification [GEND neut] has been added, via unification with the SPR value of the head noun. This unification is required by the Head-Specifier Rule, which licenses the combination of this determiner with this noun. The right subtree in (9) is exactly the one shown in (7) (though abbreviated with a triangle). (9) also obeys the HFP: the HEAD value of the head daughter is identified with that of the mother. And it obeys the Valence Principle: the COMPS value of the phrase is 
the same as that of the head daughter (the empty list). The mother's SPR value is the empty list, as required by the Head-Specifier Rule.

The Semantic Inheritance Principle says that the MODE and INDEX values of the head daughter must be shared by the mother, which is the case in (9). And the Semantic Compositionality Principle requires that the mother's RESTR value be the sum of the two daughters' RESTR lists. Since we waved our hands at the semantics of $a$, we do not have all the information needed to specify the RESTR value of the whole NP. To indicate this, we have written '...' for the contribution to the RESTR of the NP made by $a$.

The lexical entry for the pronoun us is quite straightforward, except for the RESTR list in the semantics. In the following, we have chosen to characterize the meaning of us roughly as reference to a group of which the speaker is a member. We have formalized this as a RESTR list with components, but there are many other possible ways of doing this. Our version gives rise to the following lexical SD:

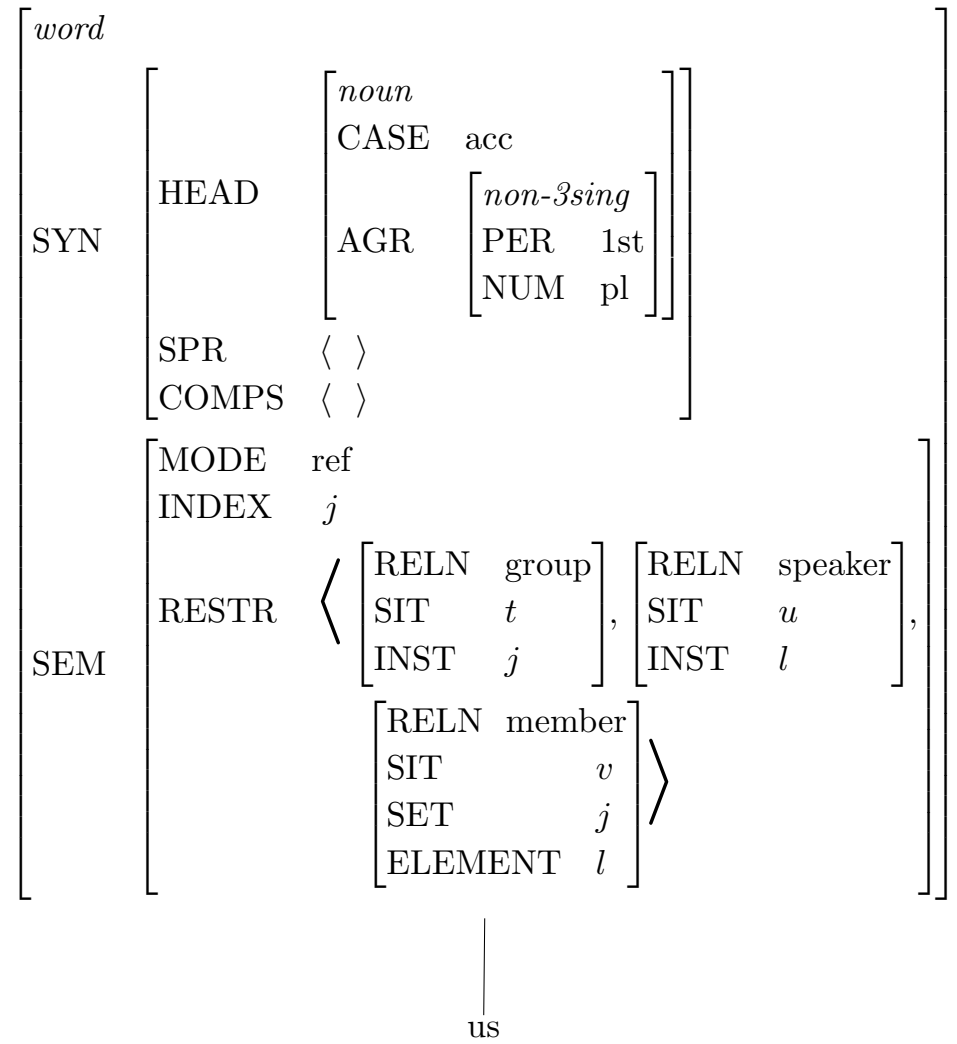


138 / Syntactic Theory: A Formal Introduction

All this information is lexically specified. Note that because the AGR value is of type non-3sing, it contains no GEND specification.

And this lexical SD (like that of the noun letter) combines with zero complements via the Head-Complement Rule to form the following SD:

$$
\left[\begin{array}{ll}
\text { Shrase } & \\
\text { SYN } & {\left[\begin{array}{ll}
\text { HEAD } & 71 \\
\text { SPR } & \langle\rangle \\
\text { COMPS } & \langle\rangle
\end{array}\right]} \\
\text { SEM } & {\left[\begin{array}{lc}
\text { MODE } & \text { ref } \\
\text { INDEX } & \text { j } \\
\text { RESTR } & \text { b }
\end{array}\right]}
\end{array}\right]
$$

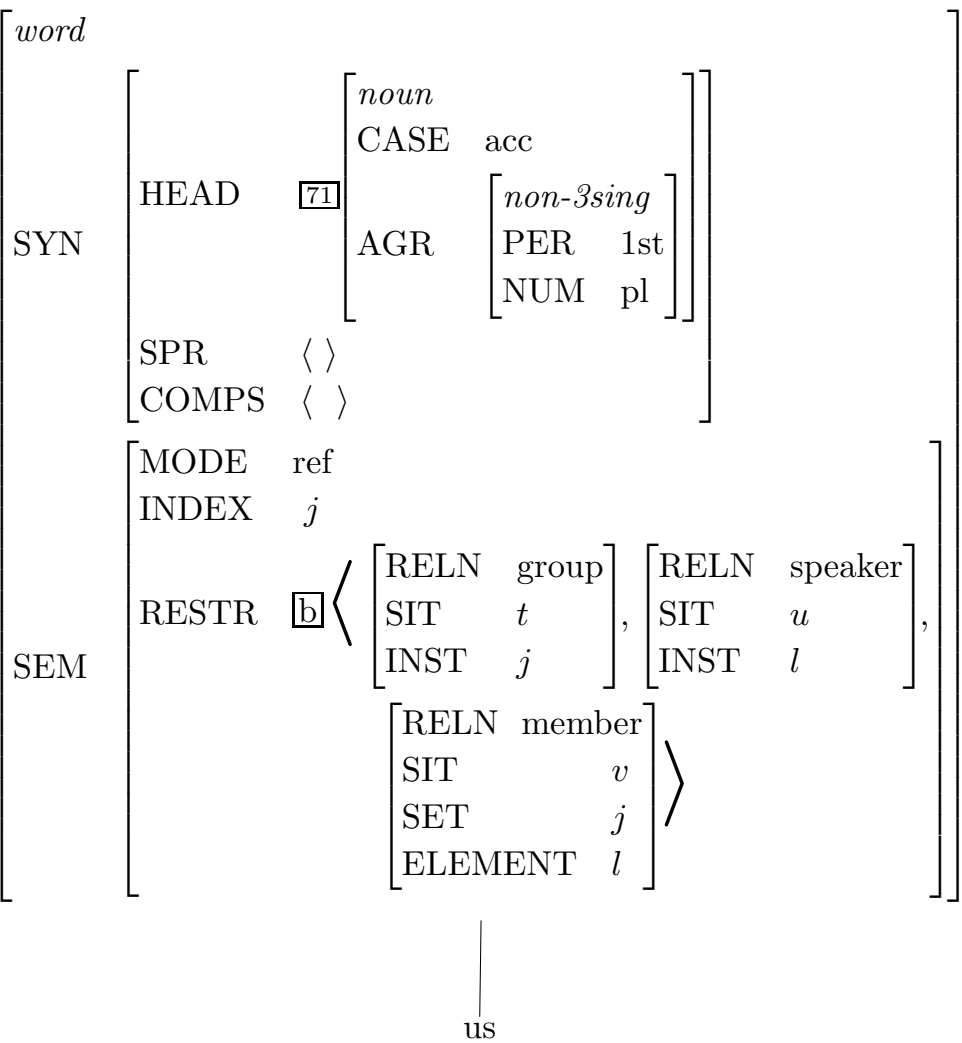


Note the effect of the HFP, the semantic principles, the Valence Principle (SPR only) and the Head-Complement Rule (COMPS value only).

Now consider the lexical SD covering the verb sent:

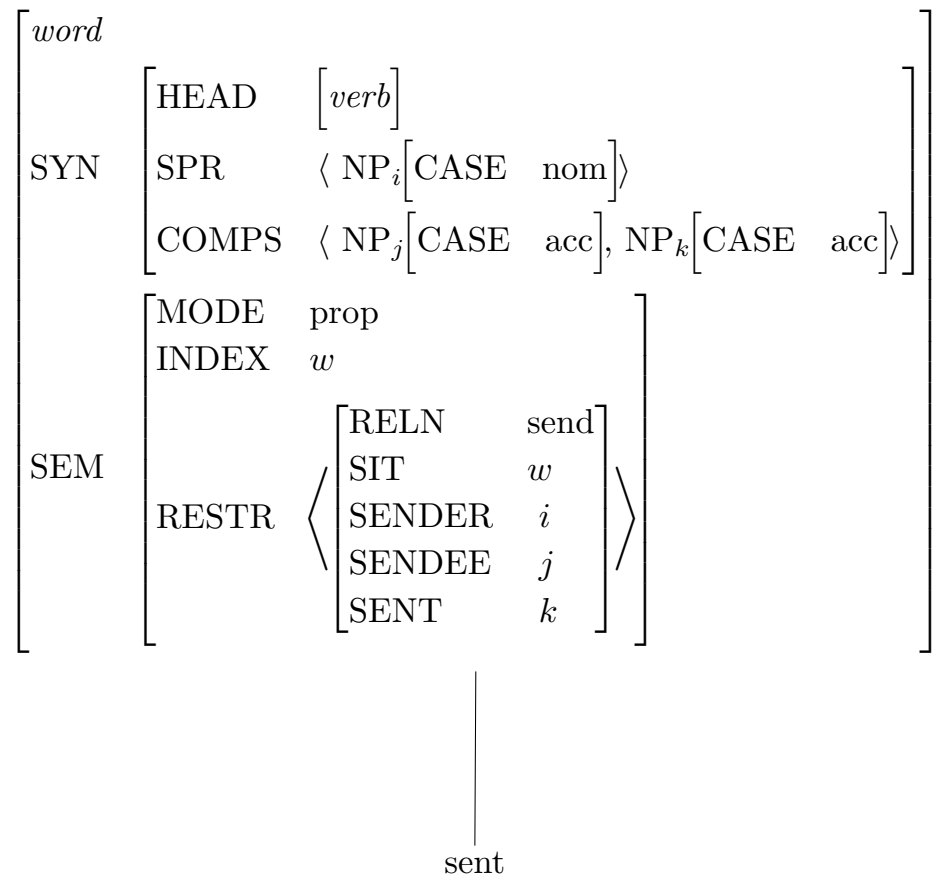

Again, everything here is specified lexically. Note that this past tense form places no restrictions on the AGR values of its subject.

The three trees we have now built up combine via the Head-Complement Rule to give the following phrasal SD: 
140 / Syntactic Theory: A Formal Introduction

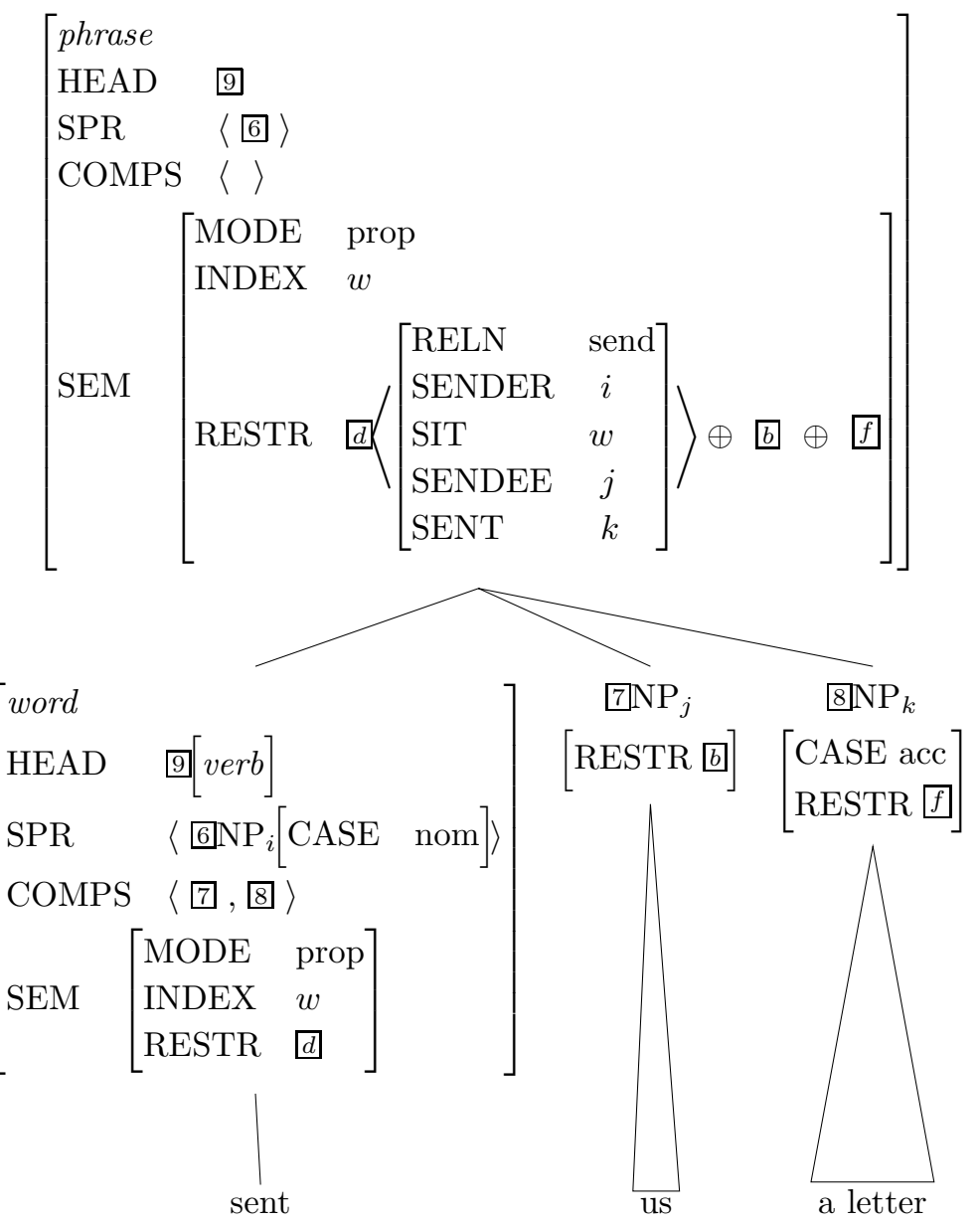

We have done a bit of abbreviating here. The node tagged 7 is identical to the top node in (11). That is, it is just the NP node above the subtree in (11). Likewise, the node tagged 8 is identical to the top node in (10), except that it also contains the feature specification [CASE acc]. This specification comes from the COMPS value of the lexical entry for sent (see (12)), and hence appears on this node, as required by the Head-Complement Rule. The RESTR values in the semantics for the two NP nodes (that is, 7 and 8 ) are the ones shown in (11) and (9), respectively. We abbreviated the former with the tag $\mathrm{b}$; we use the tag $f$ for the latter.

(13) obeys the condition on COMPS values specified in the HeadComplement Rule, that is, the head daughter's complements are 'cancelled' and the mother's COMPS value is empty. (13) obeys the Valence 
Principle, as the SPR value of the head daughter, not mentioned in the rule, is preserved as the mother's SPR value. Likewise, the HEAD value of mother and head daughter are correctly identified here, in accordance with the Head Feature Principle. Finally, the MODE and INDEX values of the mother are those of the head daughter, while the RESTR value of the mother is the sum of those of all the daughters, as specified by the semantic principles.

The last step is to combine the VP in (13) with the SD of its subject NP. The following is the SD built up (via the Head-Complement Rule in accordance with the HFP, the Valence Principle, the Semantic Inheritance Principle and the Semantic Compositionality Principle) from the lexical subtree for they:

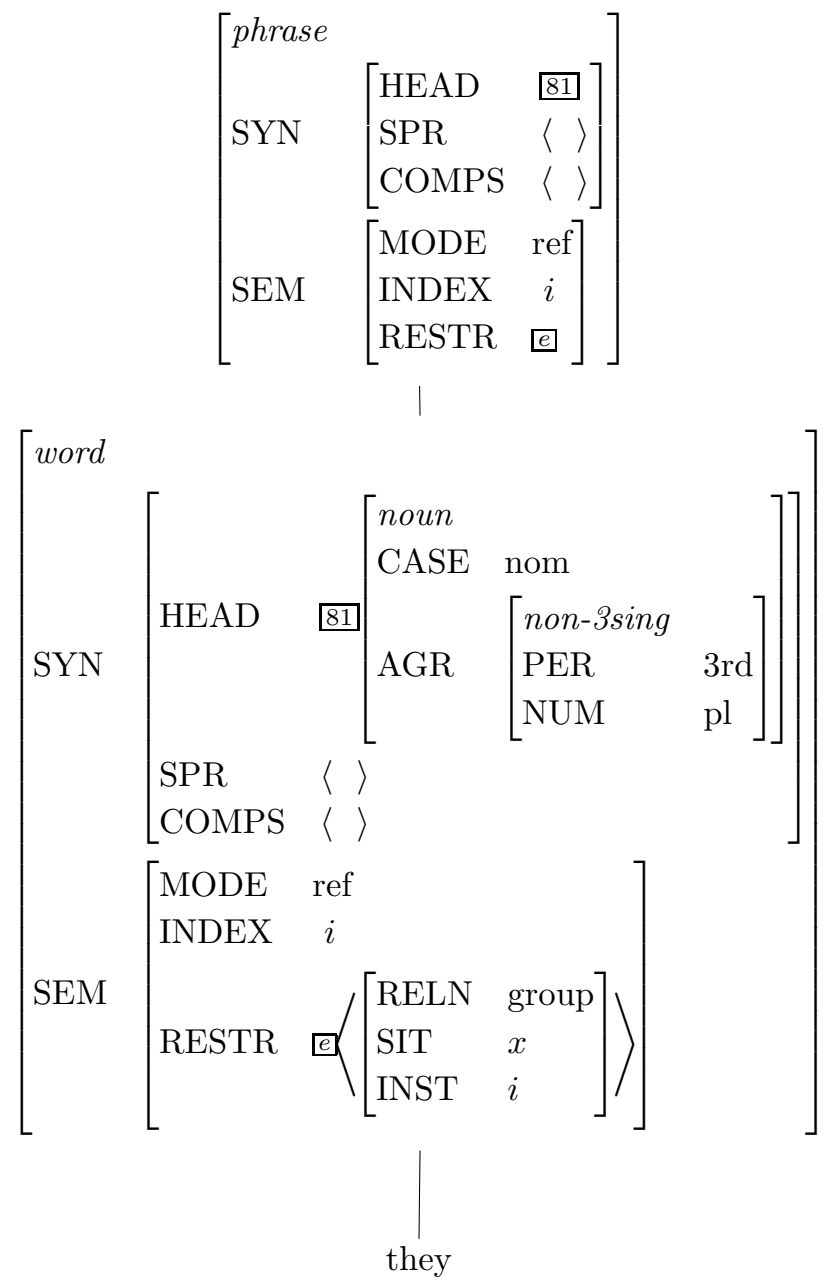


142 / Syntactic Theory: A Formal Introduction

The result is the SD in (15):

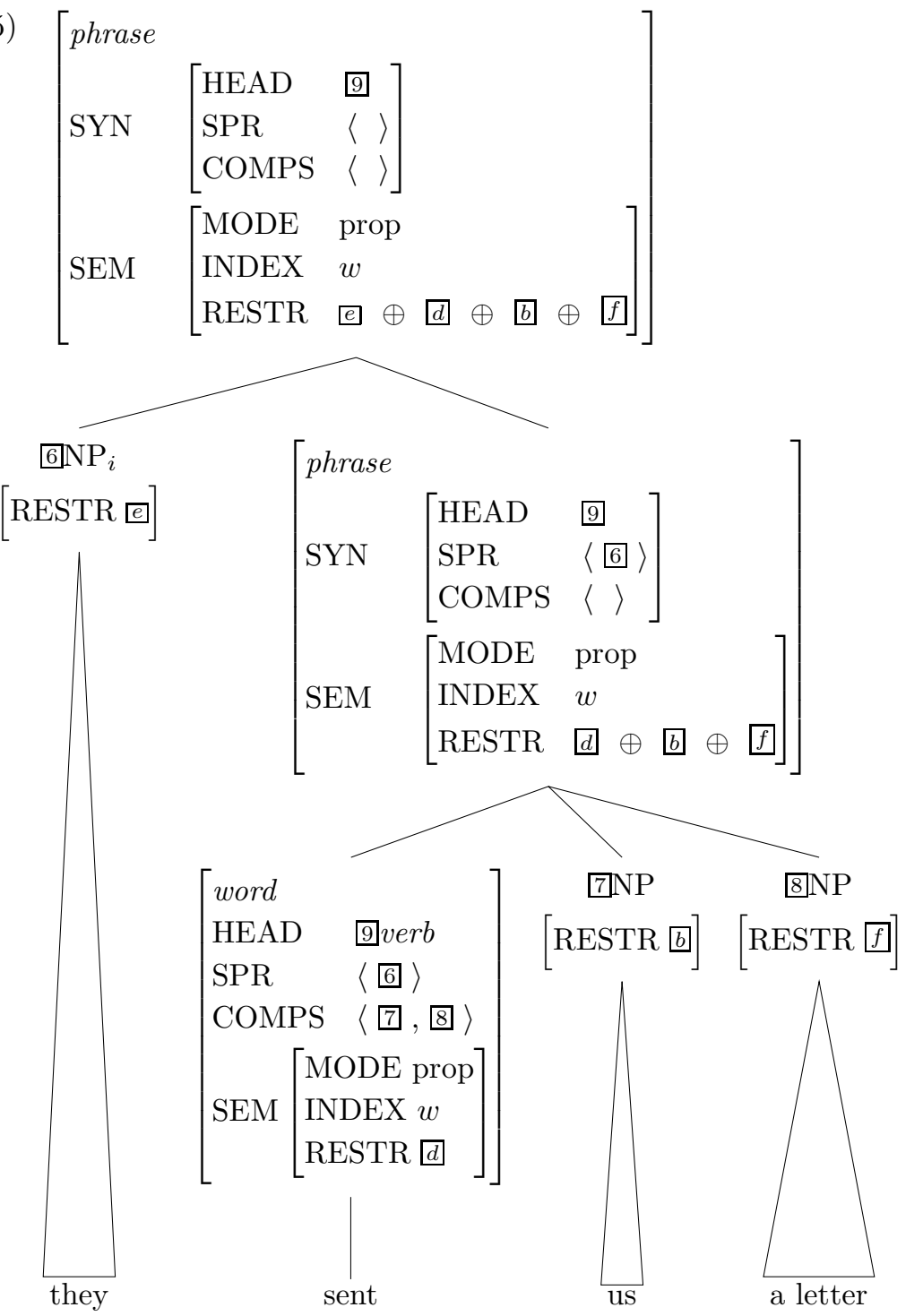

Again, we have abbreviated. The node labeled 6 is just the top node in (14). The nodes labeled 7 and 8 are exactly as they were in (13). The VP node is almost identical to the top node in the SD in (13), except that the SPR value has the additional information that it is 
third-person plural, because it is unified with the subject. Finally, we have abbreviated the RESTR values, simply putting in tags or sums of tags. The RESTR value of the top node, fully spelled out, is the list consisting of the following six SDs in order:

$$
\begin{aligned}
& {\left[\begin{array}{ll}
\text { RELN } & \text { group } \\
\text { SIT } & x \\
\text { INST } & i
\end{array}\right],\left[\begin{array}{ll}
\text { RELN } & \text { send } \\
\text { SIT } & w \\
\text { SENDER } & i \\
\text { SENDEE } & j \\
\text { SENT } & k
\end{array}\right],\left[\begin{array}{ll}
\text { RELN } & \text { group } \\
\text { SIT } & t \\
\text { INST } & j
\end{array}\right],} \\
& {\left[\begin{array}{ll}
\text { RELN } & \text { speaker } \\
\text { SIT } & u \\
\text { INST } & l
\end{array}\right],\left[\begin{array}{lc}
\text { RELN } & \text { member } \\
\text { SIT } & v \\
\text { SET } & j \\
\text { ELEMENT } & l
\end{array}\right], \ldots\left[\begin{array}{ll}
\text { RELN } & \text { letter } \\
\text { SIT } & s \\
\text { INST } & k
\end{array}\right]}
\end{aligned}
$$

(15), licensed by the Head-Specifier Rule, obeys the Head Feature Principle, the Valence Principle, and the two semantic principles.

\1 Problem 1: English Possessives

Consider noun phrases like Kim's brother and the president's salary. One traditional treatment of the possessive marker ('s) in English is to claim it is a case marker. The principal objection to a treatment of ' $s$ as a case marker is that, in conjunction with our analysis of case (in particular our assumption that CASE is a HEAD feature), it makes the wrong predictions about examples like the following:

(i) The Queen of England's crown disappeared.

(ii)*The Queen's of England crown disappeared.

A. Explain what the analysis of possessives as case markers predicts about the grammaticality of examples like (i) and (ii), and why.

An alternative analysis of the possessive is to say that it is a determiner that builds a determiner phrase (abbreviated DetP). On this analysis, 's selects for no complements, but it obligatorily takes an NP specifier. The word 's thus has a lexical category that is similar to that of an intransitive verb.

This analysis is somewhat unintuitive, perhaps, because (1) it requires that we have an independent lexical entry for 's, which seems more like a piece of a word, phonologically, and (2) it makes the nonword 's the head of its phrase! However, this analysis does a surprisingly good job of predicting the facts of English possessives, so we shall adopt it, at least for purposes of this text. 
B. Ignoring semantics for the moment, give the lexical entry for 's assuming its analysis as a determiner, and draw a phrasal SD (that is, a tree description) for the NP Kim's brother (Use abbreviations when necessary, for example: NP[3rd, sg] or [SPR $\langle\mathrm{NP}[\mathrm{nom}]\rangle])$.

C. Explain how your lexical entry gets the facts right in the Queen of England examples above.

D. How does this analysis handle recursion in possessives, for example, Kim's brother's wife, or Kim's brother's wife's parents? Provide at least one tree description to illustrate your explanation (again you can abbreviate complex feature structures if the omitted details are clearly irrelevant).

E. The semantics we want to end up with for Kim's book is this (poss is the name of the general possession relation that we will assume is the right semantics for all possessive constructions):

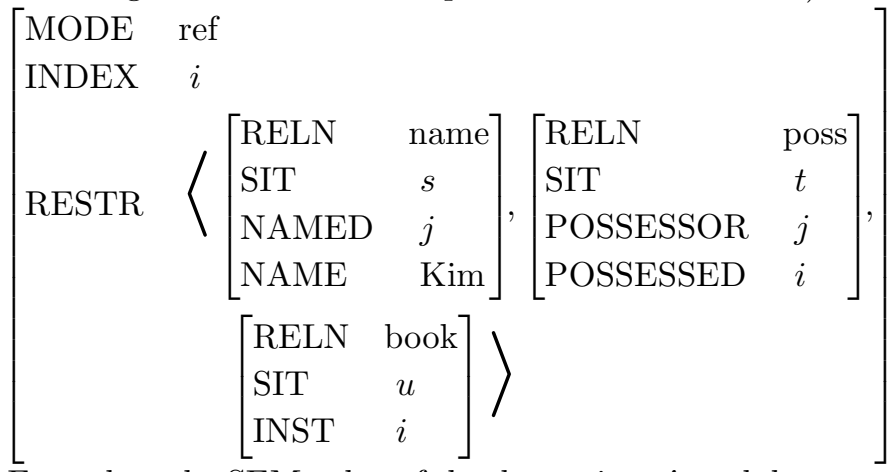

Formulate the SEM value of the determiner 's and draw an explicit SD for the phrase Kim's book, showing exactly how your analysis guarantees the right SEM value for the phrase.

[Hint: Notice that in the RESTR value above, the second RELN, which is the one inherited from 's, contains the index of book as its POSSESSED value. For that to be possible, the lexical entry for the noun book will have to say something about the relationship between its INDEX value and that of its specifier. So, in addition to determining the SEM value of 's, you will also have to figure out how to modify the lexical entry of a common noun like book to get the right relation between its INDEX and that of its specifier.]

F. Possessive pronouns like my, your, etc. function as determiners in NPs like my books and your mother. You might think we should treat possessive pronouns as determiners that have the same AGR value as the corresponding nonpossessive pronoun. That is, you might think that $m y$ should be specified as: 


$$
\left[\operatorname{HEAD}\left[\begin{array}{lll}
\text { det } & & \\
\text { AGR } & \left.\left[\begin{array}{ll}
\text { PER } & 1 \text { st } \\
\text { NUM } & \text { sg }
\end{array}\right]\right]
\end{array}\right]\right.
$$

Explain, providing appropriate examples, why this analysis will fail to provide an adequate account of my books and your mother.

G. The semantics we want to end up with for my book is this:

$$
\begin{gathered}
{\left[\begin{array}{ll}
\text { MODE } & \text { ref } \\
\text { INDEX } & i \\
\text { RESTR } & \left\langle\left[\begin{array}{ll}
\text { RELN } & \text { speaker } \\
\text { SIT } & s \\
\text { INST } & j
\end{array}\right],\left[\begin{array}{ll}
\text { RELN } & \text { poss } \\
\text { SIT } & t \\
\text { POSSESSOR } & j \\
\text { POSSESSED } & i
\end{array}\right],\right. \\
& \left.\left[\begin{array}{ll}
\text { RELN } & \text { book } \\
\text { SIT } & u \\
\text { INST } & i
\end{array}\right]\right\rangle
\end{array}\right]}
\end{gathered}
$$

Formulate the SEM value of the determiner $m y$ and draw an explicit SD for the phrase my book, showing exactly how your analysis guarantees the right SEM value for the phrase.

[Hint: Note our earlier hint in (E).]

\section{\1 Problem 2: A Sentence}

Give an analysis of the following sentence along the lines of the detailed analysis of (5) presented in this section:

\section{I rely on Kim.}

Be sure to discuss the role of relevant lexical entries, grammar rules, and general principles of our grammar. For the purposes of this problem, assume that the preposition on in this example makes no contribution to the semantics other than to pass up the index of its object NP. That is, assume it has the following lexical entry: 
146 / Syntactic Theory: A Formal Introduction

$$
\left\langle\text { on },\left[\begin{array}{ll}
\text { SYN } & {\left[\begin{array}{ll}
\text { HEAD } & {[\text { prep }]} \\
\text { SPR } & \langle\rangle \\
\text { COMPS } & \left\langle\mathrm{NP}_{i}\right\rangle
\end{array}\right]} \\
\text { SEM } & {\left[\begin{array}{ll}
\text { MODE } & \text { none } \\
\text { INDEX } & i \\
\text { RESTR } & \langle\rangle
\end{array}\right]}
\end{array}\right]\right\rangle
$$

\subsection{Appendix: Well-Formed Structures}

In the following definitions, we will take for granted the notions of resolved feature structure and tree structure. A resolved feature structure $\phi$ is a directed graph of a particular kind, where: (i) $\phi$ is assigned to a maximally specific type $\tau$, (ii) $\phi$ is specified for every feature $F$ that is appropriate for $\tau$ and (iii) $\phi$ 's value for each such feature $F$ is maximally specific. A tree structure is a configuration of nodes that satisfies a number of conditions, e.g. (i) that each node have only one mother, (ii) each nonterminal node is a resolved feature structure, and (iii) each terminal node is a lexical form. Recall from Chapter 2 that a local subtree consists of a mother and all its daughters.

We also presuppose the notion of feature structure satisfaction: intuitively, a resolved feature structure $\phi$ satisfies a feature structure description $\delta$ when all the information specified in $\delta$ is true of $\phi$.

(17) Well-Formed Tree Structure

$\Phi$ is a well-formed tree structure just in case every local subtree in $\Phi$ satisfies some lexical entry $\eta$ or some grammar rule $\rho$.

(18) Lexical Satisfaction

A word structure:

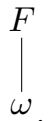

satisfies a lexical entry $\langle\omega, \delta\rangle$ just in case $F$ satisfies $\delta$ (i.e. just in case $\delta$ is true of $F$ ). 
(19) Phrasal Satisfaction

A local subtree $\Phi=$

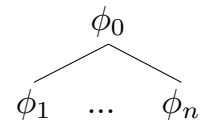

satisfies a grammar rule $\rho=\delta_{0} \rightarrow \delta_{1} \ldots \delta_{n}$ just in case: ${ }^{3}$

1. The sequence $\left\langle\phi_{0}, \phi_{1}, \ldots \phi_{n}\right\rangle$ satisfies the description $\left\langle\delta_{0}, \delta_{1}, \ldots \delta_{n}\right\rangle$

2. $\Phi$ satisfies the Semantic Compositionality Principle,

3. If $\rho$ is a headed rule, then $\Phi$ satisfies the Head Feature Principle and

the Semantic Inheritance Principle,

4. If $\rho$ is a headed rule other than the Head-Complement Rule, then $\Phi$ satisfies Part A of the Valence Principle, and

5. If $\rho$ is a headed rule other than the Head-Specifier Rule, then $\Phi$ satisfies Part B of the Valence Principle.

\footnotetext{
${ }^{3}$ Note that clause (1) here must speak of a sequence of resolved feature structures satisfying a sequence description. This is because of identities that must hold across members of the sequence, e.g. those required by the rules themselves or by the principles given below.
} 
148 / Syntactic Theory: A Formal Introduction

(20) Semantic Compositionality Principle

$$
\begin{aligned}
& {\left[\begin{array}{ll}
\operatorname{RESTR} \quad \square \oplus \ldots \oplus \square]
\end{array}\right.} \\
& {\left[\begin{array}{lll}
\operatorname{RESTR} & \mathbf{1}]
\end{array}\right]}
\end{aligned}
$$

(21) Head Feature Principle

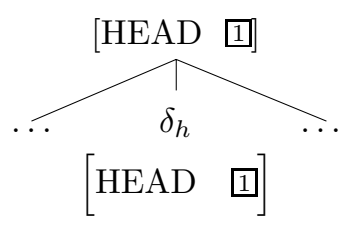

(22) Semantic Inheritance Principle

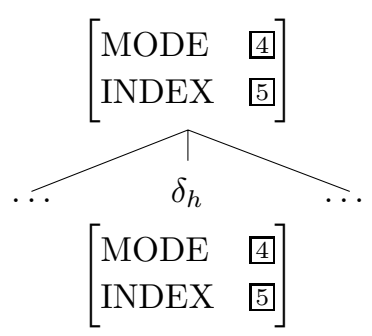

(23) Valence Principle

A

[ [ COMPS 3$]$

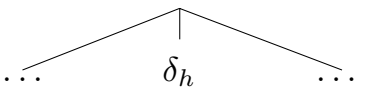

[COMPS 3$]$

B

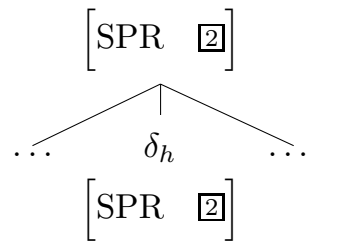




\section{Binding Theory}

\subsection{Introduction}

This chapter revisits a topic introduced very informally in Chapter 1, namely, the distribution of reflexive and nonreflexive pronouns. In that discussion, we noticed that the well-formedness of sentences containing reflexives usually depends crucially on whether there is another expression in the sentence that has the same referent as the reflexive; we called such an expression the 'antecedent' of the reflexive. For nonreflexive pronouns, the issue is typically whether a particular NP could have the same referent as (or, as linguists often put it, be COREFERENTIAL with) a given pronoun - that is, whether that NP could serve as the antecedent for that pronoun.

In discussing these phenomena, we will use the notation of subscripted indices to mark which expressions are intended to have the same referent and which are intended to have distinct referents. Two expressions with the same index are to be taken as coreferential, whereas two expressions with different indices are to be understood as having distinct referents.

Thus the markings in (1) indicate that himself must refer to the same person as John, and that the referent of her must be someone other than Susan.

(1) a. $\mathrm{John}_{i}$ frightens himself .

b. ${ }^{*} \operatorname{Susan}_{i}$ frightens her ${ }_{i}$.

c. $\operatorname{Susan}_{i}$ frightens her ${ }_{j}$.

As mentioned in the previous chapter, the subscript notation is shorthand for the value of the feature INDEX.

In examples like (1a), the reflexive himself is often said to be 'bound' by its antecedent. This terminology derives from an analogy between natural language pronouns and variables in mathematical logic. The 


\section{0 / Syntactic Theory: A Formal Introduction}

principles governing the possible pairings of pronouns and antecedents are often called BINDING PRINCIPLES, and this area of study is commonly referred to as BINDING THEORY. ${ }^{1}$ The term ANAPHORA is also used for expressions (including pronouns) whose interpretation requires them to be associated with other elements in the discourse.

With this notation and terminology in place, we are now ready to develop a more precise and empirically accurate version of the binding theory we introduced in Chapter 1.

\subsection{Binding Theory of Chapter 1 Revisited}

Recall that in Chapter 1, on the basis of examples like (2)-(9), we formulated the hypothesis in (10).

(2) a. $\operatorname{Susan}_{i}$ likes herself . b. ${ }^{*} \operatorname{Susan}_{i}$ likes her $_{i}$.

(3) a. $\operatorname{Susan}_{i}$ told herself $i$ a story. b. ${ }^{*} \operatorname{Susan}_{i}$ told her $i$ a story.

(4) a. $\operatorname{Susan}_{i}$ told a story to herself $f_{i}$. b. ${ }^{*} \operatorname{Susan}_{i}$ told a story to her ${ }_{i}$.

(5) a. $\operatorname{Susan}_{i}$ devoted herself $f_{i}$ to linguistics. b. ${ }^{*} \operatorname{Susan}_{i}$ devoted her ${ }_{i}$ to linguistics.

(6) a. Nobody told $\operatorname{Susan}_{i}$ about herself ${ }_{i}$. b. ${ }^{*}$ Nobody told $\operatorname{Susan}_{i}$ about her ${ }_{i}$.

(7) a. ${ }^{*} \operatorname{Susan}_{i}$ thinks that nobody likes herself $f_{i}$. b. $\operatorname{Susan}_{i}$ thinks that nobody likes her ${ }_{i}$.

(8) a. ${ }^{*} \operatorname{Susan}_{i}$ 's friends like herself ${ }_{i}$. b. $\operatorname{Susan}_{i}$ 's friends like her ${ }_{i}$.

(9) a. ${ }^{*}$ That picture of $\operatorname{Susan}_{i}$ offended herself ${ }_{i}$. b. That picture of $\operatorname{Susan}_{i}$ offended her ${ }_{i}$.

\footnotetext{
${ }^{1}$ Much of the literature on binding theory actually restricts the term 'binding' to elements in certain syntactic configurations. Specifically, an element $A$ is often said to bind an element $B$ if and only if: (i) they have the same index; and (ii) $A$ c-commands $B$. The technical term 'c-command' has been defined in several (nonequivalent) ways in the literature; the most commonly used definition is the following: node $A$ in a tree c-commands node $B$ if and only if every branching node dominating $A$ dominates $B$. Intuitively, this means roughly that $A$ is at least as high in the tree as $B$. Our investigations into binding theory will not impose any such configurational limitation, as we will be deriving a similar, arguably superior characterization of constraints on binding in terms of ARG-ST lists (see below).

Note that we are interested in determining the conditions governing the pairing of pronouns and antecedents in a sentence. We will not, however, consider what possible things outside the sentence (be they linguistic expressions or entities in the world) can serve as antecedents for pronouns.
} 
(10) Reflexive pronouns must be coreferential with a preceding argument of the same verb; nonreflexive pronouns cannot be.

Our task in this chapter is to reformulate something close to the generalization in (10) in terms of the theoretical machinery we have been developing in the last five chapters. We would also like to extend its empirical coverage to deal with examples our informal statement did not adequately handle. Toward this end, let us divide (10) into two principles, one for reflexive pronouns and the other for nonreflexive pronouns. Our first try at formulating them using the new binding terminology is then simply the following:

Principle A (version I)

A reflexive pronoun must be bound by a preceding argument of the same verb.

Principle B (version I)

A nonreflexive pronoun may not be bound by a preceding argument of the same verb.

\subsection{A Feature-Based Formulation of Binding Theory}

Our binding principles make use of several intuitive notions that need to be explicated formally within the theory we have been developing. The terms 'reflexive pronoun' and 'nonreflexive pronoun' have not been defined. Moreover, as we saw in Chapter 1, Problem 3, there could be elements other than reflexive pronouns that share their binding properties. Hence we introduce a new feature, which we will call ANAPHOR (ANA), to distinguish reflexive pronouns from nonreflexive pronouns. Reflexives are [ANA +]; nonreflexive pronouns are [ANA -]. In addition, we will assume (at least for the purposes of this text) that reciprocals (that is, each other and perhaps one another) are [ANA +], but ordinary nonpronominal NPs are [ANA -]. This will allow us to reformulate Principle A in terms of the feature ANA, keeping open the possibility that reflexives and reciprocals might not be the only elements subject to the principle. Since ANA specifications must percolate up from the head noun to the NP it projects, we will treat ANA as a HEAD feature, as shown in the following lexical entries. 
152 / Syntactic Theory: A Formal Introduction

(12) a

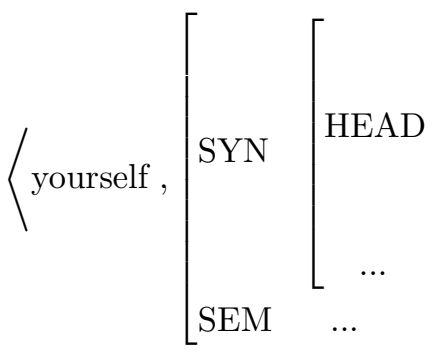

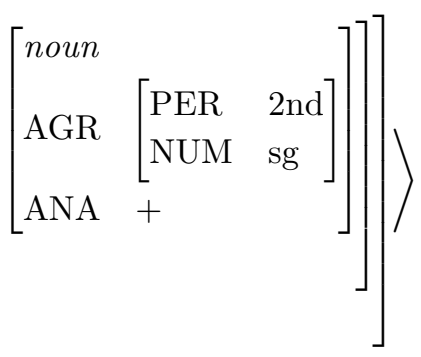

b.

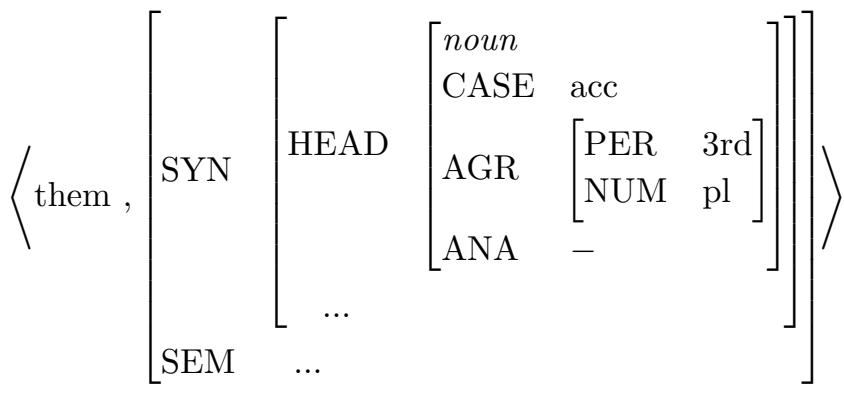

c.

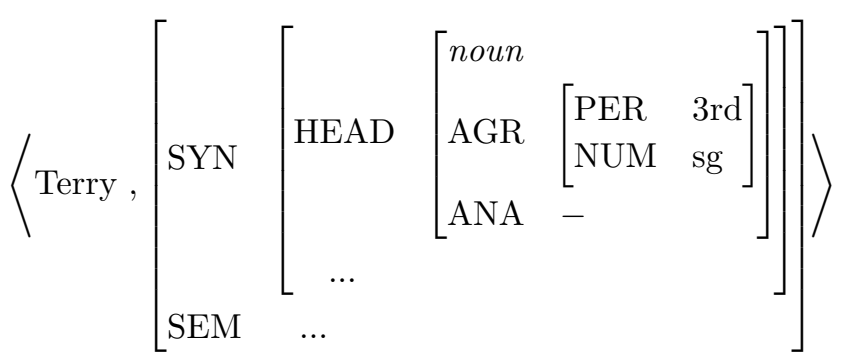

\subsubsection{The Argument Structure List}

Both of our binding principles contain the phrase 'a preceding argument of the same verb'. Formalizing this in terms of our theory will take a bit more work. The features that encode information about what arguments a verb takes are the valence features, SPR and COMPS. Though we have not said much about the linear ordering of arguments, we have placed elements on our COMPS lists in the order in which they appear in the sentence. Hence to the extent that precedence information is encoded in our feature structures, it is encoded in the valence features. So the valence features are a natural place to start trying to formalize the binding principles.

There is a problem, however. For examples like (3)-(6), the binding in question involves the subject NP and one of the nonsubject NPs; but our valence features separate the subject (specifier) and the nonsubject 
(complements) into two different lists. To facilitate talking about all of the arguments of a verb together, we will posit a new list-valued feature, ARGUMENT-STRUCTURE (ARG-ST), consisting of the sum (in the sense introduced in Chapter 5) of the SPR value (the subject) and the COMPS value (the complements).

Words obey the following generalization, where ' $\oplus$ ' again denotes the operation we have called 'sum', appending one list onto another:

(13) Argument Realization Principle (Version I)

A word's value for ARG-ST is $\oplus$, SPR and $b$ is its value for COMPS.

So, if a verb is specified as [SPR $\langle\mathrm{NP}\rangle]$ and [COMPS $\langle\mathrm{NP}\rangle]$, then the verb's argument structure list is $\langle\mathrm{NP}, \mathrm{NP}\rangle$. And if some other verb is specified as [SPR $\langle\mathrm{NP}\rangle]$ and [COMPS $\langle\mathrm{PP}, \mathrm{VP}\rangle]$, then that verb's argument structure list is $\langle\mathrm{NP}, \mathrm{PP}, \mathrm{VP}\rangle$, and so on. Of course we mean real identity between the members of these lists, as shown by the specifications in (14).

(14) a

$$
\begin{aligned}
& {\left[\begin{array}{lll}
\text { SYN } & {\left[\begin{array}{ll}
\text { SPR } & \langle\square\rangle \\
\text { COMPS } & \langle\square\rangle
\end{array}\right]} \\
\text { ARG-ST } & \langle\text { घNP, 2 2NP }\rangle
\end{array}\right]}
\end{aligned}
$$

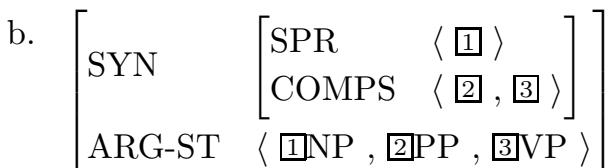

These identities are crucial, as they have the side effect of ensuring that the binding properties of the complements are actually merged into the verb's argument structure, where they are governed by our binding principles. For example, the Head-Specifier Rule identifies a subject's synsem-struc with the synsem-struc of the sole member of the VP's SPR list. This means that the subject's synsem-struc is identical to the feature structure on the VP's SPR list. It follows (by the Valence Principle) that the subject's synsem-struc is also the sole member of the verb's SPR list. This, in turn, entails (by the Argument Realization Principle) that the subject's synsem-struc is the first member of the verb's ARG-ST list. Thus once the distinctions relevant to binding theory are encoded (by whatever means) in the feature structures of reflexive and nonreflexive $\mathrm{NPs}$, this same information will be present in the ARG-ST of the lexical head of the sentence, where the binding principles can be enforced.

The generalization in (13) holds only of words; in fact, only word structures have the feature ARG-ST. Despite its close relationship to 
the valence features, ARG-ST serves a different function and hence has different formal properties. SPR and COMPS, with the help of the Valence Principle, keep track of elements that a given expression needs to combine with. As successively larger phrase structure descriptions are constructed, the list values of these features get shorter. By contrast, the argument structure list exists only as part of a word description embedded within a larger description, and it always contains the same number of elements. But as larger phrases enter the picture, the ARGST list gradually acquires more and more information via unification.

ARG-ST, then, is a feature we only find in lexical heads, and the ordering of the elements in its value imposes a ranking on the phrases in the phrase structure that correspond to those elements. A bit more precisely, we can say:

(15) A phrase $A$ outranks a phrase $B$ just in case $A$ 's synsem-struc precedes B's synsem-struc on some argument structure (ARG-ST) list.

We can now reformulate our binding principles as follows:

Principle A (Version II)

A reflexive pronoun of an ARG-ST list must be outranked by a coindexed element.

Principle B (Version II)

A nonreflexive pronoun of an ARG-ST list must not be outranked by a coindexed element.

\subsubsection{Two Problems for Binding Theory}

These formulations have certain problems, requiring further discussion and refinement.

\subsubsection{Pronominal Agreement}

First, (16) says nothing about agreement between pronouns and antecedents; but we do not want Principle A to license examples like (17).

(17) a. *I enjoy yourself.

b. *He enjoys themselves.

c. *She enjoys himself.

We could rule these out by adding a stipulation to Principle A, requiring a reflexive and its antecedent to agree. But this ad hoc approach wouldn't explain much. It is intuitively clear why coindexed elements should exhibit a form of agreement: coindexation indicates that the expressions denote the same entity, and the properties indicated by agreement features are characteristically properties of the entity referred to (the expression's DENOTATION). Thus, for example, singular 
NPs normally denote single entities, whereas plural NPs denote collections. Hence a singular pronoun cannot normally be coindexed with a plural NP, because they cannot have the same denotation.

We will consequently refrain from any mention of agreement in the binding principles. Instead, we adopt the following general constraint:

(18) Anaphoric Agreement Principle (AAP)

Coindexed elements agree.

By 'agree', we mean have the same values for AGR. Recall that AGR was introduced in Chapter 4 as a feature whose value is a feature structure that specifies values for the features PER (person), NUM (number), and GEND (gender). Only PER and NUM matter for the purposes of subject-verb agreement, but pronouns must also agree with their antecedents in gender, as illustrated in (17c). Since GEND is part of AGR, it is covered by the AAP.

One important advantage of leaving agreement out of the formulation of binding principles themselves is that the AAP also covers agreement between nonreflexive pronouns and their antecedents. Since Principle B only says which expressions must NOT be coindexed with nonreflexive pronouns, it says nothing about cases in which such pronouns ARE legally coindexed with something. The AAP rules out examples like (19), which were not covered by our formulation of Principle B.

(19) ${ }^{*} \mathrm{I}_{i}$ thought that nobody liked $\operatorname{him}_{i}$.

It is important to realize that coindexing is not the same thing as coreference, though the former entails the latter. There are some tricky cases that might seem to be counterexamples to the AAP, and all of these turn on this distinction. One such example is the following:

(20) An interesting couple walked in. He was four foot nine; she was six foot two.

Here, we would say that the NP an interesting couple refers to one entity - the couple, which is a collection of two individuals. As the collection is introduced into the discourse, however, it also makes salient each individual that is in the collection, and it is these individuals that the pronouns in the next sentence refer to. Thus in this discourse, the NP an interesting couple, the pronoun he and the pronoun she all refer to different things. Therefore they have distinct indices and need not agree, according to the AAP.

Similar examples involve collective nouns like family, which can denote a single entity, as shown by the singular verb agreement in (21), but which can, as a 'side effect', introduce a collection of entities that can serve as the antecedent for a subsequent plural pronoun: 
(21) My family hates cornflakes. But they love granola.

Again there are two distinct entities being referred to by distinct indices. ${ }^{2}$

And there are even some cases that we will probably want to treat as two nonagreeing NPs that nonetheless denote the same thing:

(22) The solution to this problem is rest and relaxation.

Here the singular NP the solution to this problem appears to refer to the same thing as the plural NP rest and relaxation. And indeed we would say that the two NPs are coreferential, but they are not coindexed. Thus while coindexing and coreference usually go hand in hand, they don't in this case. The whole point of identity sentences of this kind is to convey the information that two distinct (i.e. distinctly indexed) expressions refer to the same thing. If you are familiar with mathematical logic, this might remind you of situations in which two distinct variables are assigned the same value (making, e.g. ' $x=y$ ' true). Indices are like variables; thus binding theory constrains variable identity, not the assignments of values to variables.

\subsubsection{Binding in Prepositional Phrases}

A second problem with our formulation of the binding principles is that reflexives and their antecedents can be objects of prepositions. A PP that consists of a prepositional head daughter like to or about and a reflexive NP object can then become a complement of the verb; and when this happens, the reflexive NP inside the PP enters into binding relations with the other arguments of the verb. Similarly, when a nonreflexive pronoun functions as a prepositional object, it can behave like an argument of the verb for purposes of binding. Thus we find the pattern of binding illustrated in (23) and (24).

(23) a. They ${ }_{i}$ talk [to themselves ${ }_{i}$.

b. ${ }^{*}$ They $_{i}$ talk [to them $i$.

(24) a. Nobody told $\operatorname{Susan}_{i}\left[\right.$ about herself ${ }_{i}$.

b. ${ }^{*}$ Nobody told $\operatorname{Susan}_{i}[$ about her $i$.

And in similar examples, the prepositional object can serve as the binder of a reflexive, but not of a nonreflexive:

(25) a. Nobody talked [to $\operatorname{Susan}_{i}$ ] [about herself ${ }_{i}$.

b. ${ }^{*}$ Nobody talked [to $\operatorname{Susan}_{i}$ ] [about her ${ }_{i}$.

\footnotetext{
${ }^{2}$ For some speakers, this is even possible in the context of reflexive pronouns, i.e. in examples like (i).

(i) Pat's family is enjoying themselves.

The theory we develop does not allow for examples of this sort.
} 
In examples like these, the binding principles as formulated above make the wrong predictions: the Argument Realization Principle (henceforth $\mathrm{ARP}$ ) requires that the verb's ARG-ST contain the synsem-struc of the $\mathrm{PP}$, not that of the prepositional object NP within the PP. Hence if a reflexive pronoun is inside a $\mathrm{PP}$ that is a complement to a verb, the reflexive's synsem-struc will not appear on the same ARG-ST list with the synsem-strucs of the verb's subject and object NPs. The binding theory, as formulated, thus fails to take into account the fact that certain prepositions seem to be transparent for binding purposes. That is, if prepositions such as these were simply not there and the prepositional object were an object of the verb, then binding theory would then make just the right predictions about (23)-(25) and related examples.

This problem raises both empirical and formal questions. The empirical question is the issue of precisely when objects of prepositions can enter into binding relations with elements of higher ARG-ST domains. As we noted in our initial discussion of binding theory in Chapter 1, there is some variability about the binding possibilities of objects of prepositions. This is illustrated in $(26) .^{3}$

$$
\text { The house } i \text { had a fence around }\left\{\begin{array}{l}
\text { it }_{i} \\
*_{\text {itself }_{i}}
\end{array}\right\} \text {. }
$$

b.

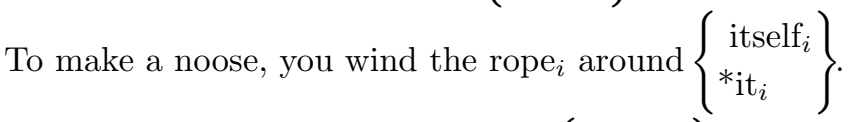

c.

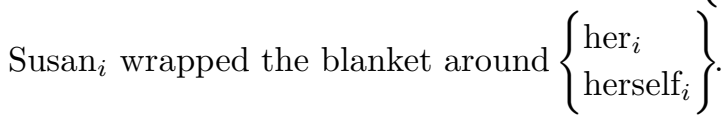

These examples also show that it is not simply the choice of preposition that determines whether a prepositional object can be reflexive.

One possible explanation of such differences is based on the intuitive idea underlying our binding theory, namely, that reflexives and their antecedents are always arguments of the same predicate. It seems plausible to claim that English prepositions have two distinct semantic functions. In some uses, they function much like verbs, introducing new predicates, and having their own argument structures. In other uses, they are simply functioning as argument markers - that is, they indicate what role their object plays in the situation denoted by the verb of the clause they appear in. The clearest examples of this argument-marking use of prepositions are sentences like (4a) above, in which to is used to mark what traditional grammarians called the indirect object. In these cases,

${ }^{3}$ It is likely that there is some cross-speaker variation as to whether examples like (26c) are acceptable or not. 


\section{8 / Syntactic Theory: A Formal Introduction}

the preposition can actually be omitted if the order of the complements is reversed: Susan told herself a story.

It seems plausible to claim that in (26a), the preposition functions as a separate predicate (making the sentence mean roughly, 'The house had a fence, and the fence was around the house'), whereas in (26b), the preposition simply marks one of the arguments of the verb wind. Cases like (26c), then, would have to be treated as having prepositions that are ambiguous between being independent predicates and argument markers.

Let us now formalize this intuition. For the purposes of binding theory, nothing new needs to be said about the prepositions that function as independent predicates. If the object of such a preposition is [ANA +], then Principle A will require it to be coindexed with something that outranks it on the preposition's ARG-ST list. This is not the case in (26a). ${ }^{4}$ If the prepositional object is [ANA - ], it must not be coindexed with anything that outranks it on the preposition's ARG-ST list. Since the subject of the sentence in (26a) does not appear on the ARG-ST list of around, Principle B permits a nonreflexive pronoun it coindexed with the house to appear as the object of around.

For prepositions that function as argument markers, however, we need to provide some way by which they can transmit information about their object NP up to the PP that they project. The higher verb that takes such a PP as its complement will then have the information about the PP's object NP in its ARG-ST, within the PP's synsem-struc. Note that without some method for transmitting this information up to the $\mathrm{PP}$, the information about the preposition's object is invisible to the higher verb selecting the PP as its complement. The COMPS list of the $\mathrm{PP}$, for example, is empty.

The method we use to transmit this information is a HEAD feature we will call P-OBJ. Only argument-marking prepositions like (some uses of) to, about, and of will allow nonempty P-OBJ specifications. And the nonempty values of this feature are constrained to be identical to the feature structure of the preposition's object, as shown in the lexical entry in (27).

\footnotetext{
${ }^{4}$ We leave open for now the question of how many arguments such predicative prepositions have. If around in (26a) has two arguments (as seems intuitive from its relational meaning), then the first argument should be identified with a fence; hence, itself could still not be coindexed with the house. In Chapter 12, we will investigate mechanisms by which different ARG-ST lists can have elements with the same index.
} 


$$
\left\langle\text { to },\left[\begin{array}{ll}
\text { SYN } & {\left[\begin{array}{ll}
\text { HEAD } & \left.\begin{array}{ll}
\text { prep } & \\
\text { P-OBJ } & \square
\end{array}\right] \\
\text { SPR } & \langle\rangle
\end{array}\right]} \\
\text { ARG-ST } & \langle\text { ⿴囗N }[\text { acc }]\rangle \\
\text { SEM } & \ldots
\end{array}\right]\right\rangle
$$

Because P-OBJ is a HEAD feature, it is projected up as shown in (28).

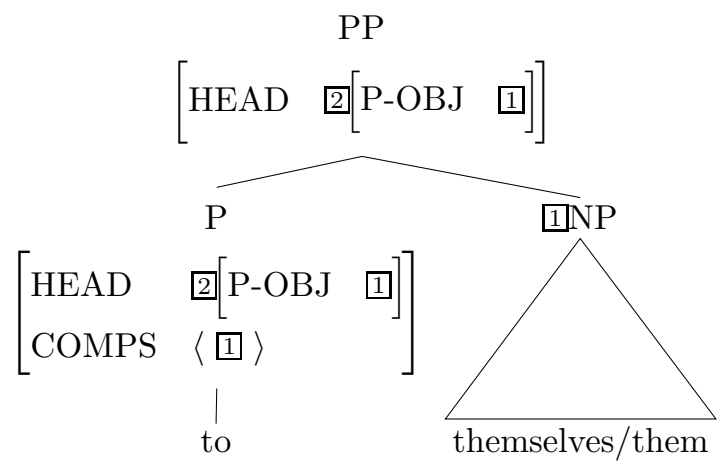

A PP like this can be selected by a verb like tell (in one of its valence manifestations). Hence, the PP on its ARG-ST list will contain the P-OBJ value within it. Put another way, all the information about the object of the preposition is available in the ARG-ST list.

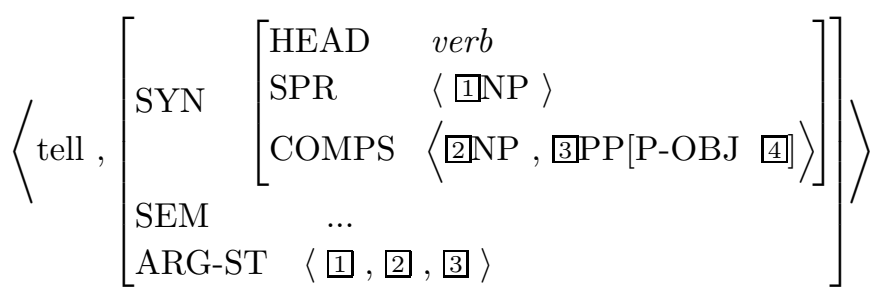

To get the right binding results, we now need to make our binding principles sensitive to the presence of a P-OBJ specification within one (or more) elements of an ARG-ST list. We do this by making a slight modification to our definition of 'outranks'. In particular, we now say that a PP and its P-OBJ are 'of equal rank', and we treat the notion of 'ranking' as a transitive relation in the standard way. ${ }^{5}$ More precisely:

$$
\text { (i) A PP and its P-OBJ value are of equal rank. }
$$

\footnotetext{
${ }^{5}$ That is, if $A$ outranks $B$ and $B$ outranks $C$, then $A$ outranks $C$; and if $D$ and $E$ are of equal rank, then they outrank exactly the same elements and are outranked by exactly the same elements.
} 
(ii) If there is an ARG-ST list on which $A$ precedes $B$, then $A$ has a higher rank than (i.e. outranks) $B$.

With these innovations in place, we may finally reformulate the binding principles in terms of the feature ANA. (We assume our modified definition of 'outrank').

Principle A (Version III)

An $[$ ANA +] synsem-struc must be outranked by a coindexed synsem-struc.

Principle B (Version III)

An [ANA -] synsem-struc must not be outranked by a coindexed synsem-struc.

The formal machinery we have just developed is designed to capture the fact that objects of prepositions in English exhibit different binding properties in different environments. It involves positing two kinds of lexical entries for prepositions, one with a P-OBJ value and the other without one. We attempted to motivate this distinction through an intuition that the two kinds of prepositions serve different semantic functions. But such intuitions vary considerably from speaker to speaker, so it would be dangerous to put too much weight on them. Our analysis provides a more reliable means of classifying prepositions as argument marking or predicative, namely, exploring their binding properties. Prepositions that are transparent for purposes of binding should be analyzed as argument markers; those whose objects cannot be bound by a preceding NP in the clause should be analyzed as predicative.

\subsubsection{Examples}

So far, this section has motivated several technical innovations in our theory (ARG-ST, P-OBJ, and the distinction between the two types of prepositions). In this subsection, we present two examples to illustrate the formal machinery we have been discussing.

Consider first (4a), repeated here for convenience as (32).

(32) $\operatorname{Susan}_{i}$ told a story to herself ${ }_{i}$.

The structure licensed by our grammar must satisfy the following SD (omitting irrelevant details): 
(33)

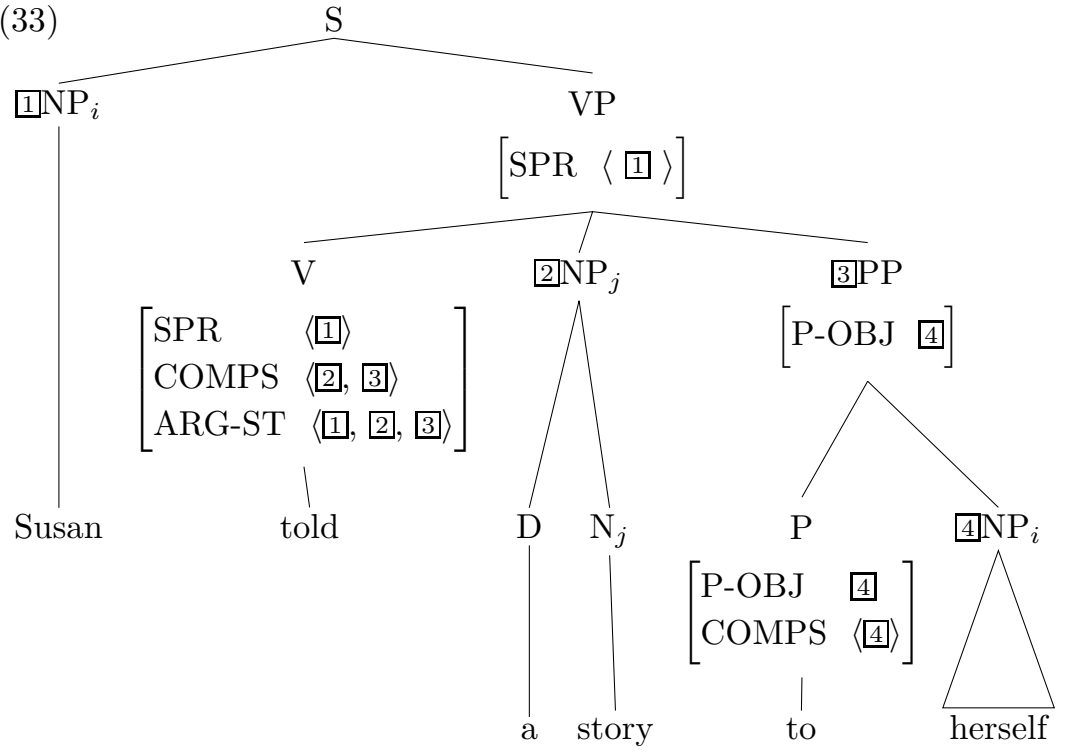

The geometry of this SD is given by our phrase structure rules in ways that are by now familiar. The aspect of the SD we are concerned with here is the coindexing of the nodes, indicated by the subscripted $i$ and the resulting argument structure of the verb told, which is displayed in (34):

$$
\left.\left[\begin{array}{ccc}
\text { ARG-ST }\left\langle\mathrm{NP}_{i}\right. & \mathrm{NP}_{j} & \mathrm{PP} \\
{[\mathrm{ANA}-]^{\prime},[\mathrm{ANA}-]^{\prime},\left[\mathrm{P}-\mathrm{OBJ} \mathrm{NP}[\mathrm{ANA}+]_{i}\right]}
\end{array}\right]\right\rangle
$$

This ARG-ST conforms to the binding theory: the [ANA +] NP and the $\mathrm{PP}$ are of equal rank because the former is the $\mathrm{P}-\mathrm{OBJ}$ value of the latter; both are therefore outranked by the first [ANA - ] NP, which precedes the PP on the ARG-ST list; and since this first NP and the [ANA +] NP are coindexed, Principle A is satisfied. Further, neither [ANA -] NP is outranked by a coindexed element. Notice that the effect of Principle A, though it is a local constraint on the verb's ARG-ST list, is to require coindexing between the subject NP one level higher in the structure and the prepositional object one level lower.

Principle A would also be satisfied if the anaphor were coindexed with the direct object NP: 
162 / Syntactic Theory: A Formal Introduction

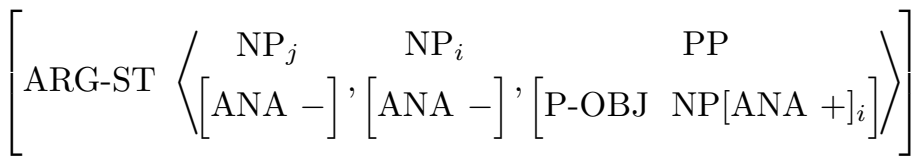

Although this is implausible with told (because of the nonlinguistic fact that people are not the kind of thing that get told to others), it is much easier to contextualize grammatically analogous sentences with the verb compared:

(36) a. We compared $\operatorname{him}_{i}[$ to himself $i]$ (at an earlier age)

b. We compared them $i$ [to each other $i$.

Thus in the ARG-ST lists of both (34) and (35), the anaphor is outranked by some coindexed element. This prediction seems correct, as far as grammar is concerned, though there are orthogonal factors of plausibility that interact to diminish the acceptability of many grammatical examples.

Now consider (4b), repeated here for convenience as (37).

(37) ${ }^{*} \operatorname{Susan}_{i}$ told a story to her .

The tree structure that our grammar must rule out is the following:

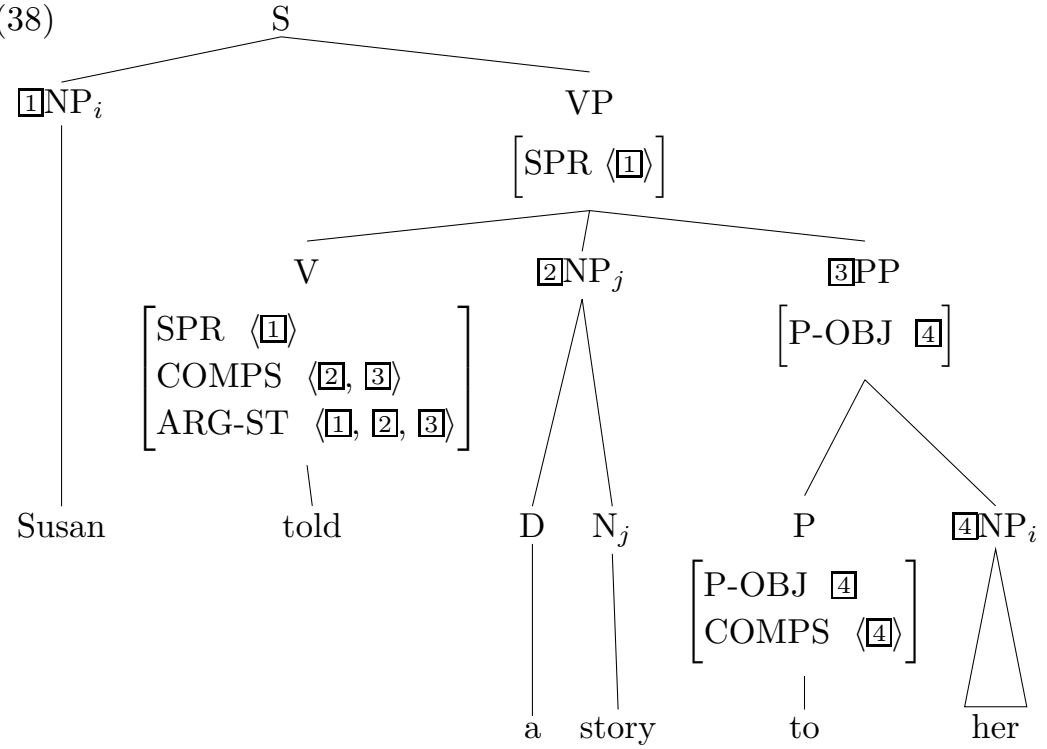

The lexical entry for her specifies that it is [ANA - ], that is, that it is not a reflexive (or reciprocal) pronoun. As in the case of the previous example, the P-OBJ feature passes this information up to the PP, which carries it along into the verb's ARG-ST list, which looks like (39). 
BINDING THEORY / 163

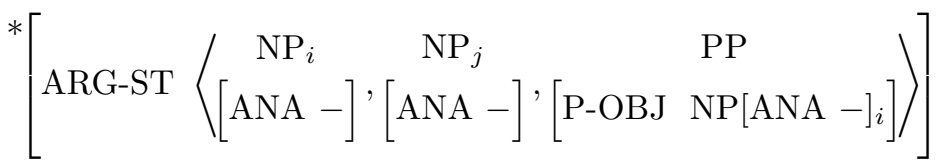

As before, the $\mathrm{PP}$ and the $\mathrm{P}-\mathrm{OBJ}$ value (in this case, $\mathrm{NP}[\mathrm{ANA}-]_{i}$ ) are of equal rank, and the first NP outranks them. But in this case, since we have an [ANA - ] NP that is outranked by a coindexed NP, Principle $\mathrm{B}$ is violated, and there is no legitimate structure that satisfies this SD.

\section{\) Problem 1: Classifying Prepositions}

We have divided prepositions into two sorts: those functioning as predicates and those functioning as argument-markers. Classify the italicized prepositions in each of the following sentences into one of these two sorts (or as being ambiguously both), using facts about the possible occurrence of reflexive and nonreflexive coreferential pronouns to justify your classification.

(i) The dealer dealt an ace to Bo.

(ii) The chemist held the sample away from the flame.

(iii) Alex kept a loaded gun beside the bed.

(iv) We bought flowers for you.

(v) The car has a scratch on the fender.

\subsection{Imperatives}

In Chapter 1 we noted that the behavior of reflexive and nonreflexive pronouns in sentences like (40) is what one would expect if they had second-person subjects.

(40) a. Protect yourself!

$$
\begin{aligned}
& \text { b. } * \text { Protect }\left\{\begin{array}{l}
\text { myself } \\
\text { himself }
\end{array}\right\} \text { ! } \\
& \text { c. }{ }^{*} \text { Protect you! } \\
& \text { d. Protect }\left\{\begin{array}{l}
\text { me } \\
\text { him }
\end{array}\right\} !
\end{aligned}
$$

Sentences like these are known as IMPERATIVE sentences. Their characteristic properties are that they lack an overt subject, employ an uninflected form of the verb, and are used to express directives. Such sentences are sometimes said to have 'understood' second-person subjects. The distribution of reflexives illustrated in (40) shows that imperatives 
do indeed behave in at least one way as if they had second-person subjects.

Our theory provides a straightforward way of capturing the intuition that imperatives have understood subjects. First we need to allow for verb forms that lack the inflections of the verb forms we have been considering thus far. These forms, produced by a lexical rule discussed in the next chapter, have no inflectional endings and are distinguished from other kinds of verbal forms in terms of differing values for the HEAD feature FORM. The infinitival form of a verb has the FORM value 'inf(initive)'.

We introduce a new grammar rule to analyze imperative sentences. This rule allows a sentence to consist of a single daughter: a VP specified as [FORM inf]. In requiring that the daughter be so specified, we ensure that the lexical head of that phrase will be an uninflected verbal form, such as be, get, run, or look. The new rule we need for imperative sentences is a nonheaded rule that says a sentence may consist of a [FORM inf] VP that behaves as though it had a second-person subject and is interpreted as a directive.

(41) Imperative Rule

$$
\left.\left.\left[\begin{array}{ll}
\text { phrase } & \\
\text { HEAD } & {[\text { verb }]} \\
\text { SPR } & \langle\rangle \\
\text { COMPS } & \langle\rangle \\
\text { SEM } & {\left[\begin{array}{ll}
\text { MODE } & \text { dir } \\
\text { INDEX } & s
\end{array}\right]}
\end{array}\right] \rightarrow\left[\begin{array}{lll}
\text { phrase } & \\
\text { HEAD } & {\left[\begin{array}{ll}
\text { verb } & \\
\text { FORM } & \text { inf }
\end{array}\right]} \\
\text { SPR } & \langle\text { NP[PER } & 2 \text { nd }
\end{array}\right]\right\rangle\right]
$$

Recall that imperative sentences require their subject to be second person, a fact that is captured by the constraint on the SPR of the daughter in (41). And though all verbs are lexically specified as [MODE prop] (which is in turn passed up to the [FORM inf] VP that enters into the imperative construction), (41) ensures that any phrase it sanctions is specified as [MODE $\operatorname{dir}]$ - that is, that it has a meaning appropriate for an imperative.

The Imperative Rule will thus sanction structures like the one depicted in (42). 
BINDING THEORY / 165

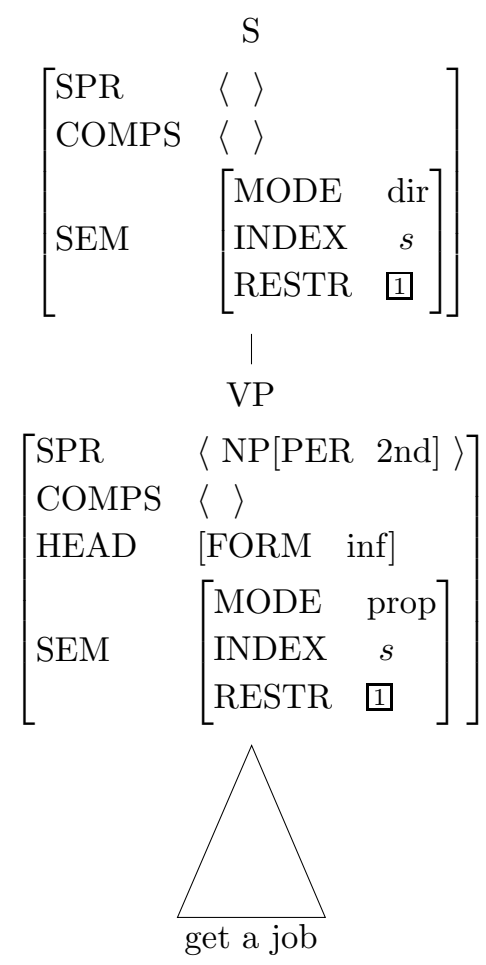

Note that, because the Imperative Rule is a not a headed rule, the Head Feature Principle, the Valence Principle, and the Semantic Inheritance Principle are not relevant to licensing the S node in (42) (though the Semantic Compositionality Principle identifies the RESTR value of the mother in (42) with the RESTR value of the daughter). Instead, the values for the features on the $S$ node are dictated by the rule itself. ${ }^{6}$

The last thing to understand about the rule in (41) is that it explains the observations we have made about anaphor binding in imperative sentences. By requiring the specifier of imperative VPs to be secondperson, we make the first argument of the head verb be second person as well, thanks to the ARP. This, in turn, entails that in a structure like the following, Principle A will require a reflexive object to be coindexed with (and hence, by the AAP, to agree with) the second person subject:

\footnotetext{
${ }^{6}$ There are further constraints on what can be a 'stand alone' clause. In Chapter 9 we will require that the 'initial symbol' of our grammar must include the specification [FORM fin], which will distinguish past and present tense verbs (e.g. went, loves) from all others. FORM values for verbs are discussed in Chapter 8.
} 
166 / Syntactic Theory: A Formal Introduction

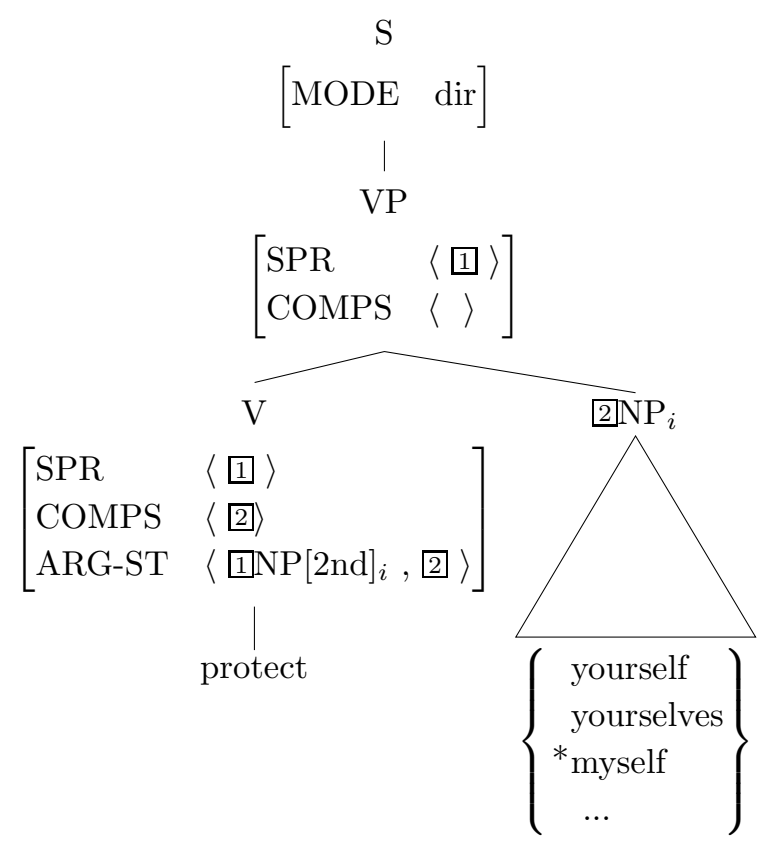

In this way, our treatment of imperatives interacts with our treatment of ARG-ST so as to provide an account of 'understood' arguments. The ARG-ST may include synsem-strucs that are not overtly expressed, that is, which correspond to no overt phrase, and these can play a role in binding relations.

Note that we can use binding theory to confirm whether or not a given subjectless clause should involve an understood subject. For example, it would be a mistake to analyze sentences of the form Damn NP! along the lines just employed for imperatives. If we posited an understood NP in the ARG-ST of damn, it would license a reflexive pronoun (of the appropriate person, number, and gender) in the position after damn. But this is not possible:

*Damn $\left\{\begin{array}{l}\text { myself } \\ \text { yourself } \\ \text { herself } \\ \text { himself } \\ \text { itself } \\ \text { themselves }\end{array}\right\}$ !

Hence, damn in this use will have to be analyzed as being truly subjectless, in the sense that it has only one element in argument structure 
(and an empty SPR list). Examples like (44) are then ruled out because the reflexive element in the ARG-ST is not outranked by any coindexed element.

We have given a preview here of the analysis of verb forms that will be developed in the next chapter. There we will address the question of how the forms are differentiated formally, and how to manage the proliferation of entries for different forms of the same word.

\section{Problem 2: Imperative 'Subjects'}

There are imperative sentences that contain an NP that looks like it is the subject of the infinitival VP:

(i) You get out of here!

(ii) Everybody take out a sheet of paper!

But the initial NPs in these examples don't seem to particpate in the normal agreement pattern with respect to reflexive pronouns. For example, we know that an NP like everybody is third person because of its behavior in (iii). ${ }^{7}$

$$
\text { Everybody found }\left\{\begin{array}{l}
\text { ?himself } \\
* \text { yourself } \\
\text { ?themselves } \\
* \text { myself }
\end{array}\right\} \text { a seat. }
$$

Yet in imperative sentences, we still find the second-person reflexive pattern illustrated in (iv):

$$
\text { Everybody find }\left\{\begin{array}{c}
\text { ??himself } \\
\text { yourself } \\
\text { ??themselves } \\
* \text { myself }
\end{array}\right\} \text { a seat! }
$$

Assuming that we do not want to license examples marked '??', what minimal modification of the Imperative Rule would account for the indicated data? Make sure that your proposal still accounts for all relevant facts illustrated above for imperative sentences with no initial NP. For the purposes of this problem, don't worry about the semantics: concentrate on providing a syntactic analysis that will get the binding facts right.

\footnotetext{
${ }^{7}$ Following standard practice of generative grammarians, we use designations '?', '??', and '?*' to indicate different levels of naturalness between full acceptability and complete unacceptability.
} 


\section{\. Problem 3: Binding in Japanese}

Japanese is a classic example of a 'Subject-Object-Verb' (SOV) language: ${ }^{8}$

(i) Taroo-ga hon-o yonda

Taroo- $\mathrm{N}$ book-A read-past

'Taroo read the book'

But in Japanese, both subjects and objects can in general be omitted. When such an element is omitted, the sentence usually has a meaning that we would express in English by using a pronoun to refer to the element that is left implicit in Japanese.

(ii) Taroo-ga yonda.

Taroo-N read-past

'Taroo read it/them'

(iii) Hon-o yonda.

book-A read-past

'He/she/they read the book'

(iv) Yonda.

read-past

'He/she/they read it/them

A. This phenomenon presents some apparent problems for the ARP as we have formulated it. For purposes of this problem, assume that Japanese will require a somewhat different version of the ARP - possibly by making it a default. With that assumption, show how the phenomenon illustrated in (ii)-(iv) could be handled. You will probably want to have separate lexical entries for those verbs whose arguments are missing. (Don't worry now about the lexical redundancy this appears to cause.) Be explicit about the verbs' values for SPR, COMPS, and ARG-ST. How could you account for the various possible pronominal interpretations for unexpressed elements?

Now consider the following data:

(v) Hanako-ga zibun-o tatai-ta.

Hanako-N pro-self-A hit-PST

'Hanako $i$ hit $\operatorname{herself}_{i} /{ }^{*} \operatorname{him}_{j} /{ }^{*} \operatorname{her}_{j} /{ }^{*}$ them $_{j}{ }^{\prime}$

\footnotetext{
${ }^{8}$ This problem is not concerned with the word-order difference between English and Japanese. It is mentioned only to help the reader understand the glosses of the Japanese sentences. Here we use the symbols $\mathrm{N}$ and $\mathrm{A}$ to designate nominative and accusative case.
} 
(vi) Taroo-ga tatai-ta. Taroo-NOM hit-PST

'Taroo $i$ hit * himself $_{i} / \operatorname{him}_{j} /$ her $_{j} /$ them $_{j}{ }^{\prime}$

B. Does your analysis predict these data? If so, explain how. If not, modify your analysis to take these data into account.

Consider some more data. ${ }^{9}$

(vii) Taroo-ga Hanako-ga zibun-o tatai-ta koto-o hookokusi-ta.

Taroo-N Hanako-N pro-self-A hit-PST that-A tell-PST

' Taroo $_{i}$ said that Hanako $_{j}$ hit

herself $_{j} / \operatorname{him}_{i} /{ }^{*}$ them $_{k} /{ }^{*} \operatorname{him}_{k} /{ }^{*} \operatorname{her}_{k}$ '

(viii) Taroo-ga Hanako-ga tatai-ta koto-o hookokusi-ta.

Taroo-N Hanako-N hit-PST that-A tell-PST

' Taroo $_{i}$ said that Hanako $j$ hit ${ }^{*}$ herself $_{j} /$ him $_{i, k} /$ her $_{k} /$ them $_{k}$ '

Here you may assume that the verb hookokusi-ta takes an S complement, much as the verb say does in English (though the verb in Japanese always follows the complements, no matter what they are).

C. On the basis of these facts, how would you characterize the difference between the binding theory we need for Japanese and the one we have developed for English? Be sure to be explicit in your discussion of both Principle A and Principle B. (You may want to construct analogous examples involving reflexive and nonreflexive pronouns in the $\mathrm{S}$ complements of say in English, to see the difference more clearly.)

\subsection{The Argument Realization Principle Revisited}

ARG-ST lists in general, and the ARP in particular, will play an increasingly important role in the chapters to come. We will place various constraints on the ARG-ST values of particular kinds of words, yet these would be vacuous without the ARP, which relates ARG-ST values to the values of the valence features SPR and COMPS. This connection is central, if the constraints we place on lexical heads are to interact with the elements that heads syntactically combine with. The binding theory presented in this chapter illustrates the importance of both ARG-ST and the ARP in our theory.

\footnotetext{
${ }^{9}$ As the word-by-word glosses indicate, zibun is a reflexive. However, because the Japanese binding principles are not quite the same as those for English, we sometimes have to translate it with a nonreflexive English pronoun.
} 
A further motivation for separating out ARG-ST in our lexical entries is the fact that the syntactic environments in which a verb can appear are determined largely (though not entirely) by its meaning. Attempts to formulate what is sometimes called a LINKING THEORY - that is, a set of principles to explain the syntactic properties of verbs in terms of their meanings - have typically mapped some sort of semantic structure onto a single syntactic argument structure. Our approach is similar, except that our notion of ARG-ST is not purely syntactic, as it is a list of feature structures, each of which contains both syntactic and semantic information.

In Chapter 6, the Head Feature Principle, Valence Principle, and the two semantic principles were all conceptualized (and formalized) as part of the definition of a well-formed tree structure: local trees have to conform not only to the constraints defined by our rules and lexical entries, but also to the constraints embodied in our principles. The ARP can be conceived of in the same way - that is, as a constraint on the relation between lexical entries and word structures that must satisfy them. We would like our lexical entries not to have to specify values for SPR and COMPS. Rather, we want the ARG-ST values in the lexical entries together with the ARP to determine the values of the valence features in word structures. That is, a word structure is well formed only if its SPR and COMPS values add up to its ARG-ST value.

This idea is easy to incorporate into the formal definition of Lexical Satisfaction given in the appendix to Chapter 6 .

(45) Lexical Satisfaction

A word structure:

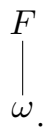

satisfies a lexical entry $\langle\omega, \delta\rangle$ just in case:

1. $F$ satisfies $\delta$, and

2. Argument Realization Principle

$F$ satisfies the following feature structure description:

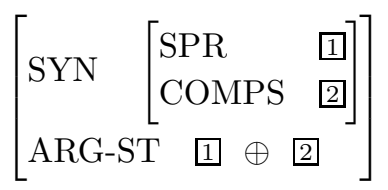

Now suppose a lexical entry specified nothing about SPR and COMPS, as in (46). 


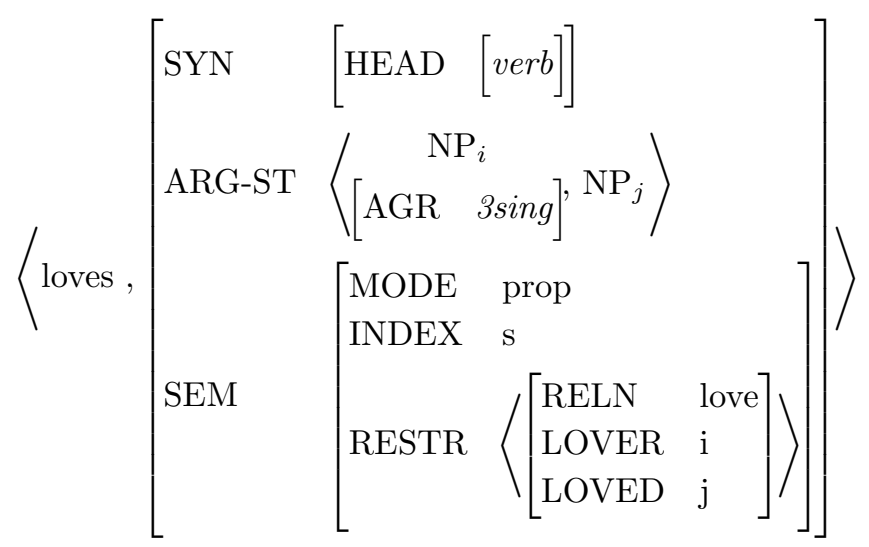

In conjunction with the ARP, this will license word structures only if they satisfy the lexical SD in (47), which includes a value for each valence feature.

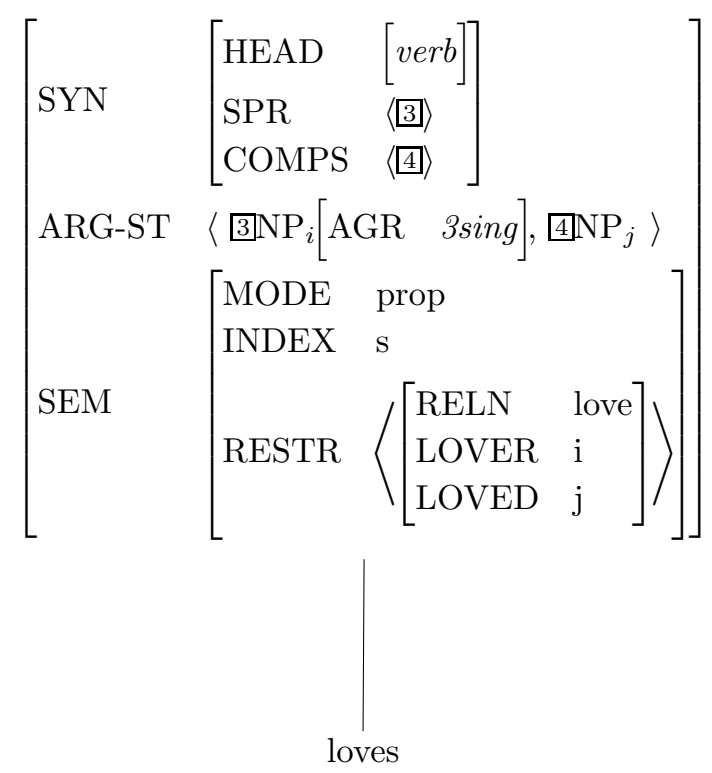

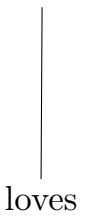

Given what we have said so far, (47) is not the only lexical SD that would be consistent with (46). The ARP would also allow word structures in which both of the elements of the argument structure list in (46) were identified with complements - that is, where both 3 and 4 occur on the COMPS list. Similarly, they could both be on the SPR list. Such lexical SDs will need to be ruled out. In the next chapter, we introduce a constraint requiring verbs to have exactly one element 
on their SPR lists. This will ensure that all relevant word structures in fact satisfy (47).

\subsection{Further Reading}

The binding of anaphors has been the topic of an extensive literature since the late 1960s. A seminal and very readable paper is Lasnik (1976). To our knowledge, the first proposal to treat reflexive binding in terms of a hierarchy of the verb's arguments was made by Johnson (1977). The Binding Theory of Chomsky (1981) distilled many of the insights of the research of the preceding decade into three principles; this theory was developed further in a number of works within the Government and Binding theory of grammar. A detailed account of binding within Lexical Functional Grammar is presented by Dalrymple (1993). The theory of binding presented in this chapter is based on Pollard and Sag (1992, 1994), with terminological revision ('(out)ranking') due to Bresnan (1995). One of the most detailed attempts to date at formulating a linking theory is Davis (1996), whose theory of the alignment of semantics and argument structure allows a further streamlining of all our lexical descriptions. 


\section{The Structure of the Lexicon}

\subsection{Introduction}

Several chapters ago, we began with context-free grammar and motivated numerous modifications to develop a formalism better tailored to the description of natural language phenomena. In the course of doing this, we have put more and more of the descriptive burden of our theory into the lexicon. Lexical entries have evolved from simple pairings of phonological forms with grammatical categories into elaborate information structures, in which phonological forms are now paired with more articulated feature structure descriptions. This has permitted us to reduce our inventory of grammar rules to a few very general schemas, and to account for a range of syntactic phenomena.

Since our theory relies heavily on rich lexical representations, we need to consider what kind of internal organization the lexicon should have. In particular, we do not want to claim that all information contained in lexical entries is simply listed. A great deal of what we are now putting into lexical entries is predictable, so stipulating all of it would miss linguistically significant generalizations. For example, we handle subject-verb agreement by having the lexical entries for certain verbs (basically, those with the suffix -s) specify that their SPR values have to be third person and singular. Aside from that specification, these entries are essentially identical to those for other forms of what is intuitively the same verb: their part of speech is verb; they have the same COMPS values; and their semantics is virtually the same. This is no accident, nor is the fact that the same suffix is used to mark almost all third-person singular present tense verb forms.

Notice, by the way, that capturing such generalizations is motivated not only by general considerations of parsimony, but also by psychological considerations. On encountering a novel English verb (say, a recent coinage such as email or an obscure word like cark), any com- 
petent speaker will add the suffix $-s$ when using it in the present tense with a third person singular subject. In short, speakers know that there are systematic (or, as linguists say, 'productive') relationships among different forms of the same word, and our grammar should reflect this systematicity. The focus of the present chapter is to develop mechanisms for expressing regularities within the lexicon.

\subsection{Lexical Types}

One mechanism for reducing the redundancy of the lexicon has already been introduced: a hierarchy of types. Our initial motivation for introducing types was to define feature appropriateness, to avoid having to specify values for features that are irrelevant to particular classes (such as COUNT for prepositions). Later, we also used types for stating constraints on feature structures. In particular, in Chapter 4, we motivated the 'Nominal SPR Agreement' (NSA) constraint, which required unification of any noun's AGR specification with the AGR specification of the element on its SPR list - that is, nouns have to agree with their specifiers.

We also introduced the idea that some types are subtypes of others, in the following sense:

(1) If $T_{2}$ is a subtype of $T_{1}$, then

a. every feature specified as appropriate for $T_{1}$ is also appropriate for $\mathrm{T}_{2}$, and

b. every constraint associated with $\mathrm{T}_{1}$ affects all instances and subtypes of $\mathrm{T}_{2}$.

Formulated in this way, the inheritance of constraints in our type hierarchy is MONOTONIC: constraints on supertypes affect all instances of subtypes, without exception. An intuitive alternative to this conception is to allow for DEFAULT INHERITANCE of constraints, according to which contradictory information associated with a subtype takes precedence over (or OVERRIDES) constraints that would otherwise be inherited from a supertype. Default inheritance allows a type system to express the idea that language embodies general constraints with exceptions - subclasses with idiosyncratic constraints that violate the general ones.

This intuitive idea is simple to express: we need only allow constraints associated with lexical types to be marked as defaults. Suppose a constraint associated with a lexical type $\mathrm{T}_{i}$ is marked as a default. Then this constraint holds of any lexical entry of type $\mathrm{T}_{i}$ for which it is not explicitly overridden. It could be overridden in one of two ways. First, a subtype of $\mathrm{T}_{i}$ might have a constraint associated with it that contradicts the default. That is, there could be a type $\mathrm{T}_{j}$ that is a sub- 
type of $\mathrm{T}_{i}$ and has a constraint associated with it that is incompatible with the default. Then the constraint on $\mathrm{T}_{j}$ takes precedence. Second, the information stipulated in a particular lexical entry might contradict the default. That is, a particular instance of a maximal ${ }^{1}$ type $\mathrm{T}_{m}\left(\mathrm{~T}_{m}\right.$ a subtype of $T_{i}$ ) could contradict the default. In this case, too, the information associated with the lexical entry takes precedence over the default. But the default constraint is true of all instances of subtypes of $\mathrm{T}_{i}$ in which it is not overridden (as are all nondefault constraints associated with those types).

Natural languages exhibit a great many regularities that have exceptions that are naturally modelled in terms of type hierarchies of this sort. Returning to the example of agreement between nouns and specifiers, recall that NSA was originally motivated by considering what are standardly referred to as COMMON NOUNS, specifically, the noun $d o g$. Names, which are often called PROPER NOUNS, are for the most part irrelevant to NSA because they do not take specifiers. This is illustrated in (2).

(2) a. Cameron skates.

b. $\left\{\begin{array}{l}*^{*} \mathrm{~A} \\ *^{*} \text { The }\end{array}\right\}$ Cameron skates.

Moreover, proper nouns are normally third-person singular.

(3) *Cameron skate.

These generalizations will be captured in our type system by introducing a type for proper nouns with a constraint (stated more formally below) specifying that the value of AGR must be of type 3sing and that the ARG-ST (and hence both SPR and COMPS lists) must be empty. But there are exceptions to this constraint. In particular, there are several proper nouns in English naming mountain ranges that appear only in the plural and only with a determiner.

(4) a. The Andes are magnificent.

b. ${ }^{*}$ The Ande is magnificent.

c. Hannibal crossed the Alps.

d. ${ }^{*}$ Hannibal crossed Alps.

This is a typical situation: many broad and productive generalizations in languages have exceptions, either idiosyncratic lexical items or classes of idiosyncratic expressions. For this reason, we shall adopt the method of default constraint inheritance in our type hierarchy. This will allow us both to restrict the number of types that are required and

${ }^{1}$ Recall that a 'maximal' type is a type that has no subtypes. 
to keep our constraints simple, without precluding the possibility that some instances or subtypes might be exceptions to the constraints.

\section{Problem 1: Mountains and NSA}

Does NSA apply to the lexical entries for proper nouns like Alps, Andes, and Himalayas? Explain your answer and provide data to justify it.

The lexicon itself can be treated in terms of a type hierarchy. By organizing the lexicon in this way, together with the use of default inheritance, as described above, we can minimize the stipulations associated with particular lexical entries and express the shared properties of different word classes, at the same time that we allow for idiosyncrasy of the kind we have been discussing. The overall conception of the lexicon is as shown in (5), where lex-item (short for lexical-item) encompasses all types of lexical items.

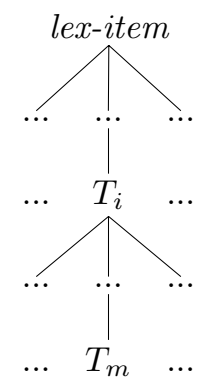

Each lexical item will belong to some maximal type $T_{m}$ and $T_{m}$ will have a family of supertypes $T_{i}$, that are intermediate between lex-item and $T_{m}$. The various intermediate types then correspond to intermediate levels of classification, where type constraints can express linguistic generalizations. Each type in the lexical hierarchy has constraints associated with it - some inviolable, and others that are default in nature. Since this is a default inheritance hierarchy, we can provide a natural account of the fact that lexical items have many properties in common but may differ from one another in terms of particular constraints that override the general constraints governing their supertypes. The idea is that each lexical item inherits constraints associated with the maximal type $T_{m}$ that it belongs to and those associated with supertypes of $T_{m}$. A lexical item inherits all inviolable constraints and all compatible default constraints. Once a lexical hierarchy (with associated constraints) is put into place, the lexical entries that we write become highly streamlined (perhaps indicating no more than which maximal type a given item 
is assigned to). All further grammatically relevant constraints (i.e. the rest of the constraints that need to be included in the lexical entry) are inherited automatically, according to the method just described.

The rest of this section is devoted to the details of the particular lexical hierarchy we are assuming to be part of our grammar. As indicated, we assume that lex-item is the most general type of feature structure. We posit two immediate subtypes of lex-item, which we call word and lexeme. These two types correspond to what, in everyday English, are two different uses of the term 'word'. In some contexts, people informally distinguish, for example, runs and ran as two different words: they are pronounced differently, have (subtly) different meanings, and have slightly different co-occurrence restrictions. But in other contexts, the same people would have no hesitation in referring to runs and ran as two forms of the word run. Clearly, these are two very different conceptions of a word: the first refers to a certain pairing of sounds and meanings, whereas the latter refers to a family of such pairings. In a formal theory of grammar, these two concepts must not be conflated. Our type word corresponds to the first usage (in which runs and ran are distinct words). The lexical entries that give rise to word structures must all be of type word.

But we also want to capture what people have in mind when they use 'word' in the second sense. That is, we want to be able to express the relationship between runs and ran (and run and running). We do this by means of the type lexeme. A lexeme can be thought of as an abstract proto-word, which, by means to be discussed in this chapter, gives rise to genuine words (that is, instances of the type word).

The most basic lexical entries then are pairings of a form and a lexeme, which give rise to a set of word entries. ${ }^{2}$ Lexemes thus serve as the atoms from which all linguistic descriptions are built. In the next section (and much of the remainder of this book) we will discuss lexical rules, the mechanisms we employ to derive words from lexemes.

Many of the constraints we present here are stated solely in terms of ARG-ST specifications. These constraints affect lexemes directly, but they also indirectly affect words derived from those lexemes, as most of the lexical rules we will propose have the effect of preserving ARG-ST specifications. Because of the ARP, developed in the last chapter, the

\footnotetext{
${ }^{2}$ More precisely, basic lexical entries are a pair consisting of a form and a lexeme description; these are used to derive lexical entries that pair a form with a word description. For ease of exposition, we will sometimes blur the distinctions among 'lexeme', 'lexeme description', and 'lexical entry pairing a form with a lexeme description'. A similar blur will sometimes occur among 'word', 'word description', and 'lexical entry pairing a form and a word description'.
} 
SPR and COMPS values in the relevant word structure will be appropriately constrained as well.

We have assumed that lexeme and word are the two immediate subtypes of the type lex-item. Among lexemes, we draw a further distinction between those that give rise to a set of inflected forms and those that do not inflect. That is, we posit inflecting-lexeme (infl-lxm) and constantlexeme (const-lxm) as two subtypes of lexeme. ${ }^{3}$ This gives rise to the type hierarchy sketched in (6).

(6)

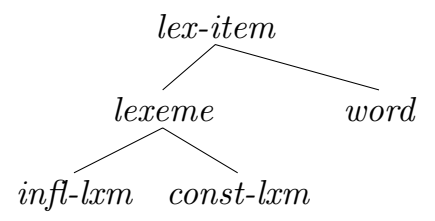

The feature ARG-ST is introduced on the type lex-item, so every lexeme and word has an argument structure. Lexemes also have the MODE value none by default:

$$
\text { lexeme: }[\mathrm{SEM}[\mathrm{MODE} / \mathrm{none}]]
$$

Here we use the symbol '/' to indicate that a certain constraint is a default and hence can be overridden by some conflicting specification. ${ }^{4}$

We further classify inflecting lexemes in terms of the subtypes nounlexeme (noun-lxm) and verb-lexeme (verb-lxm), with the former being further subclassified in terms of the types proper-noun-lexeme ( $p n$ lxm), common-noun-lexeme (cn-lxm), and pronoun-lexeme (pron-lxm), as shown in (8).

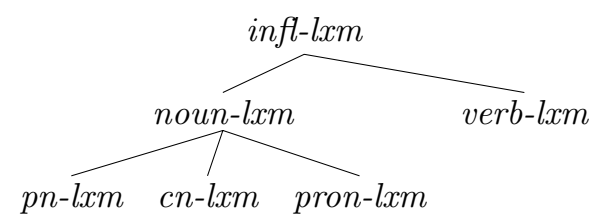

We can see how constraints on lexical types work by considering as an example the constraints associated with some of the types in (8).

First, noun lexemes are governed by the constraint in (9).

\footnotetext{
${ }^{3}$ We assume that even noninflected words are derived from lexemes. Not much hangs on the choice to formalize our lexicon in this way, rather than to enter such words directly into the lexicon with no corresponding lexemes.

${ }^{4}$ This notion of defaults (as well as the '/' notation) is taken from Lascarides et al. (1996). See also Lascarides and Copestake (1998).
} 
The Structure of the Lexicon / 179

(9)

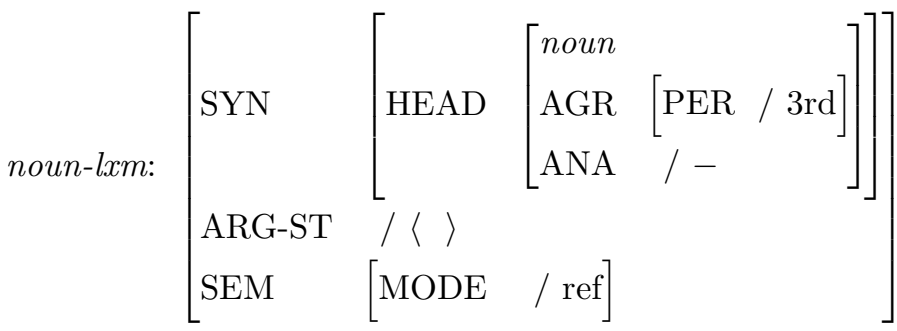

This type constraint thus makes it a general but DEFEASIBLE (that is, overrideable) property of noun lexemes that, among other things, they are third person and not anaphors. These properties are then inherited by all instances of the subtypes of noun-lxm except when it is overridden, as described above. Default properties are also inherited by words derived by some lexical rule (unless the lexical rule in question explicitly changes the default specification).

(9) ensures that all nouns, whether they are proper nouns or common nouns, have a HEAD value of the type noun, and hence bear specifications for CASE (a feature appropriate for noun objects, but not, say, for verb objects). As just noted, the '-' default specification for ANA is inherited by instances of noun-lxm, but this value will be overridden in the entries for reflexive pronouns and reciprocals. The [PER / 3rd] specification will be overridden by certain pronoun lexemes. (9) also states that nouns by default have an empty ARG-ST list, that is, that they take no specifiers or complements. Common nouns will also override this constraint, a matter we turn to directly. ${ }^{5}$ Finally, (9) also specifies the semantic mode of nouns as 'ref'. This SEM specification is a default, for reasons that will become clear in Chapter 11.

The subtypes of noun-lxm are constrained as follows: ${ }^{6}$

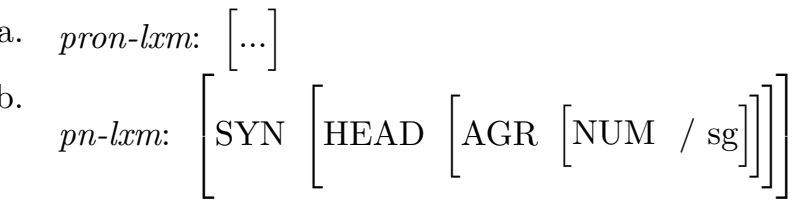

\footnotetext{
${ }^{5}$ This particular constraint is overridden by subordinate types not discussed here (e.g. types we might use for nouns like picture, that select for PPs headed by of) or by nouns with idiosyncratic complement selection properties of one sort or another (e.g. difference, which selects an optional PP argument headed by between or among).

${ }^{6}$ The notation '[ ]' is used to indicate the obligatory presence of a synsem-struc, whose features are left unspecified.
} 
180 / Syntactic Theory: A Formal Introduction

c.

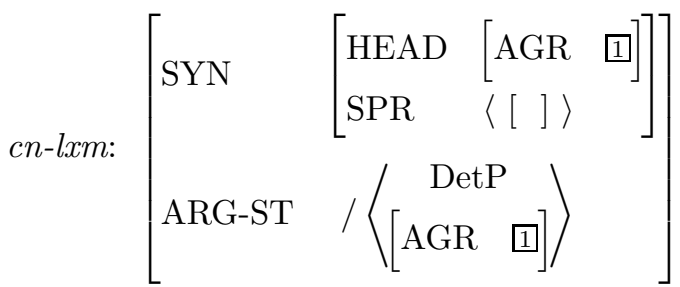

There are no constraints particular to the type pron-lxm (used for personal pronouns and reflexives). However, the type pn-lxm (used for proper names) is specified as singular by default. By default inheritance of constraints from noun-lxm, instances of both these types are third person, and have an empty ARG-ST list. (10c) requires that common nouns by default select agreeing DetPs as their specifier, thus incorporating NSA and overriding the default empty ARG-ST specification. ${ }^{7}$ Further subtypes of $\mathrm{cn}$-lxm might be introduced to distinguish count and mass nouns, ${ }^{8}$ constraining them to select for an appropriate ([COUNT + ] or [COUNT - ]) specifier. Note that (10b) need not identify the member of the SPR list with the sole member of the ARG-ST list, as this unification (in any lexical SDs that lexemes of this type give rise to) is already a consequence of the ARP. ${ }^{9}$

Given the type hierarchy and constraints just outlined, the rather complex set of specifications that we want to associate with a particular lexeme can be largely determined simply by associating the lexeme with the appropriate type in the lexical hierarchy. For example, from the fact that the lexical specification for book says it is assigned to the type cn-lxm, it follows that that the lexeme book is associated with all the constraints shown in (11).

\footnotetext{
${ }^{7}$ Note that if lists are represented in terms of the features FIRST and REST (as they often are in computational work), then the proper formulation of the ARG-ST default constraint in (10) would be as in (i):

(i)

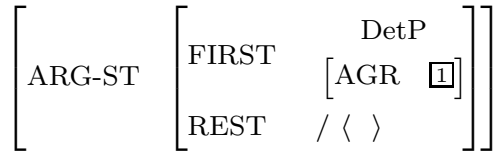

${ }^{8}$ The distinction between count and mass nouns was discussed in Chapter 4 .

${ }^{9}$ The alert reader may have noticed that (10b)'s claim that specifiers are obligatory for common nouns appears to be inconsistent with the existence of plural and mass NPs that lack determiners. The analysis of such NPs is the topic of Problem 3 below.
} 


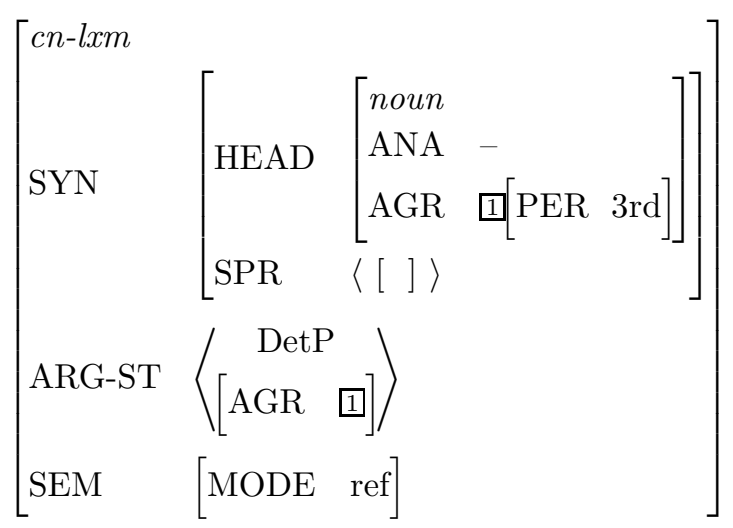

All that remains to be stipulated about this lexeme is its phonological form and the particular predication in its semantic restriction - its form and its idiosyncratic meaning - and perhaps also that it selects for a [COUNT +] determiner. The rest of its grammatical properties follow from the fact that it is assigned to a particular type in our system of grammatical descriptions. This is precisely what lexical stipulation should be reduced to whenever possible.

We require that any instance of the type verb-lxm must have: a HEAD value of type verb, a SPR list that contains one element, and a MODE value of 'prop'. In addition, the argument structure of a lexeme of this type begins by default with an NP. ${ }^{10}$ This information is specified in the following constraint on the type verb-lxm:

$$
\text { verb-lxm: }\left[\begin{array}{ll}
\text { SYN } & {\left[\begin{array}{ll}
\text { HEAD } & \text { verb } \\
\text { SPR } & \langle[]\rangle
\end{array}\right]} \\
\text { ARG-ST } & /\langle\text { NP, }, \ldots\rangle \\
\text { SEM } & {\left[\begin{array}{lll}
\text { MODE } & \text { prop }
\end{array}\right]}
\end{array}\right]
$$

The various subtypes of verb-lxm, distinguished by their ARG-ST specifications, are organized as shown in (13).

\footnotetext{
${ }^{10}$ In Chapter 11, we discuss verbs that take non-NP subjects, and hence override this default, which also lends itself to the formulation in fn. 6 .
} 
182 / Syntactic Theory: A Formal Introduction
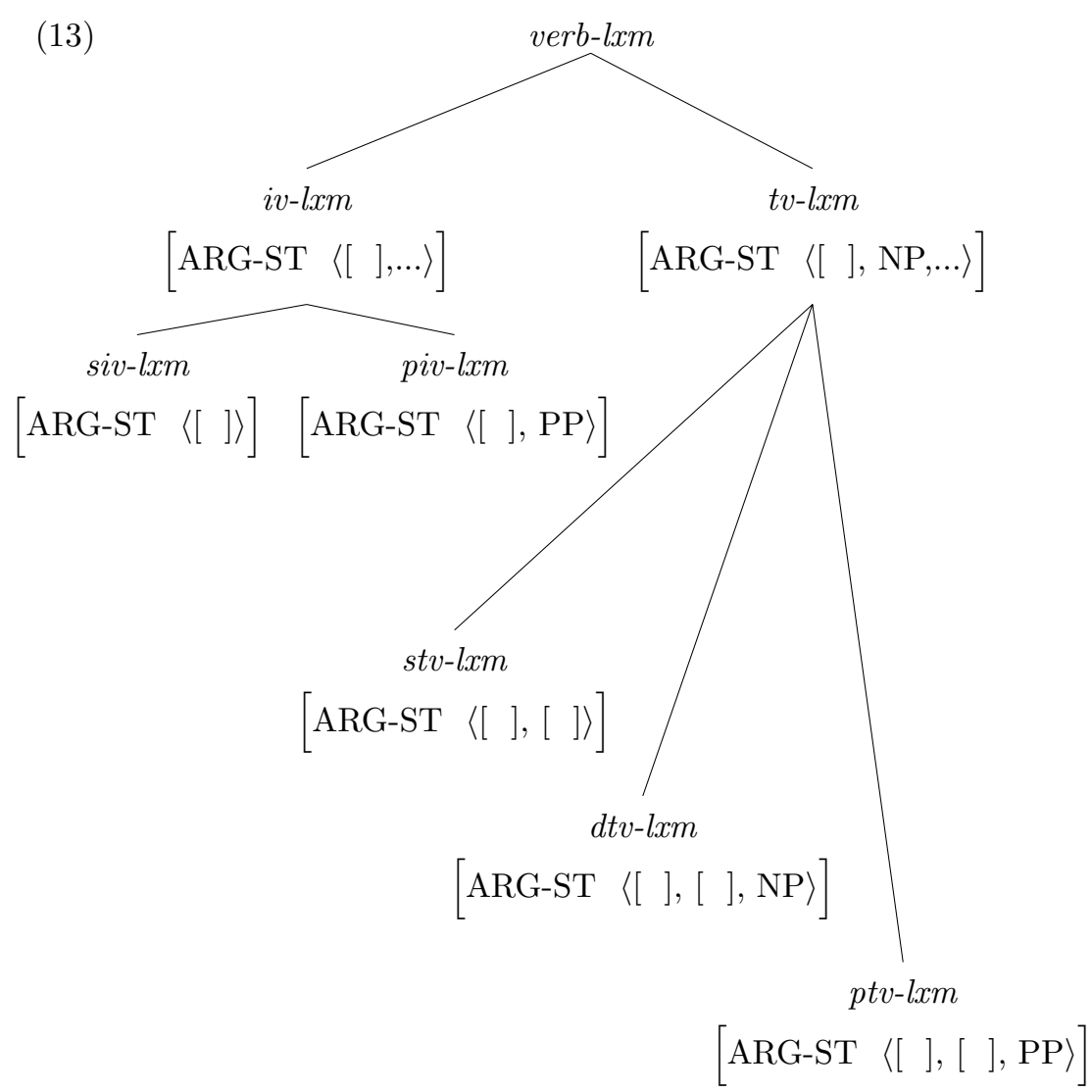

Here we have introduced the types intransitive-verb-lexeme (iv-lxm) and transitive-verb-lexeme (tv-lxm) at the first level of subhierarchy. The intransitive verb lexemes are then further classified in terms of the subtypes strict-intransitive-verb-lexeme (siv-lxm) and prepositionalintransitive-verb-lexeme (piv-lxm). The former type of verb takes no complements at all (e.g. sleep); the latter takes a PP complement (e.g. rely). Similarly, the transitive verb lexemes are subclassified into strict-transitive-verb-lexeme (stv-lxm, e.g. devour), ditransitive-verblexeme (dtv-lxm, e.g. hand), and prepositional-transitive-verb-lexeme (ptvlxm, e.g. put). As before, these types and their associated constraints allow us to eliminate lexical stipulation in favor of type-based inference. Thus from the simple statement that give belongs to the type $d t v$-lxm, we can infer that it has all the properties shown in (14): 
The Structure of the Lexicon / 183

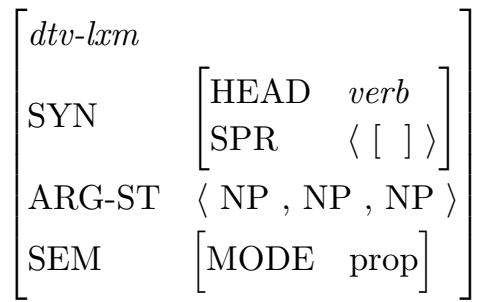

Because all instances of verb-lxm are specified as [SPR $\langle[$ ] $\rangle]$, words formed from these lexemes will also be so specified. This in turn interacts with the ARP to ensure that in verbal word structures, the first member of the ARG-ST list must also be on the SPR list. Without this $[\mathrm{SPR}\langle[]\rangle]$ constraint, there would be another way to satisfy the ARP, namely with an empty SPR list and all the arguments on the COMPS list. As noted in the final section of the previous chapter, this consequence must be avoided (to ensure, for example, that the the verb's first argument is realized as a subject, i.e. before the VP), as it is in the present analysis.

Let us turn now to noninflecting lexemes, that is, the various subtypes of the type const-lxm illustrated in (15).

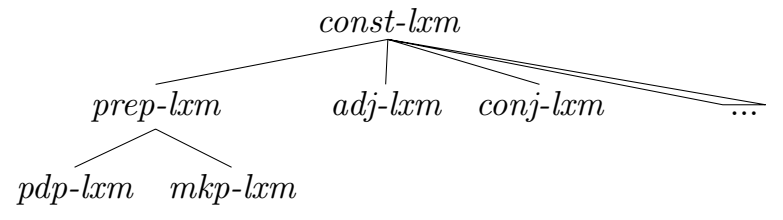

These correspond to the various kinds of lexeme that undergo no inflectional rules in English. ${ }^{11}$ Here the types preposition-lexeme, adjectivelexeme, and conjunction-lexeme have their obvious abbreviations. Once again, we specify general constraints on elements of each subtype of const-lxm. For example, the general properties of prepositions are expressed in terms of the following type constraint:

$$
\text { prep-lxm: }[\mathrm{SYN} \quad[\mathrm{HEAD} \text { prep }]]
$$

In Chapter 7 we distinguished two kinds of prepositions - those that function as predicates and those that serve as argument markers. This distinction corresponds to the two types predicational-preposition-lexeme (pdp-lxm) and marking-preposition-lexeme (mkp-lxm) in (15). Recall that in our earlier discussion we distinguished these prepositions in terms

\footnotetext{
${ }^{11}$ The type $a d j-l x m$ arguably should be classified as a subtype of infl-lxm, rather than as a subtype of const-lxm, in light of the fact that many adjectival lexemes give rise to comparative and superlative forms, e.g. tall, taller, tallest. We will not pursue this matter here.
} 
of the feature $\mathrm{P}-\mathrm{OBJ}$, which is present only in argument-marking prepositions and is unified with the sole member of the ARG-ST list. We may now incorporate this distinction into our lexicon by attaching the following type constraints to the two subtypes of prep-lxm:

(17) a.

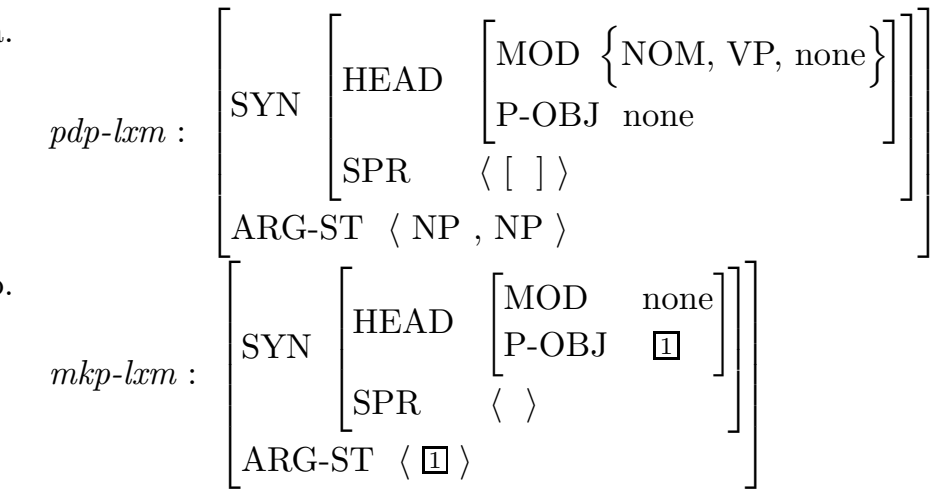

The constraint in (17a) allows predicational prepositions to function as modifiers of nominal phrases or VPs, but it also allows for phrases that modify nothing, as is the case when such a PP occurs as the complement of a verb, as in some of the cases discussed in Chapter $7:{ }^{12}$

(18) I wrapped the blanket [around me].

Note that the first argument of a predicational preposition must be identified with the SPR element, in accordance with the ARP. What plays these roles in (18) is the NP the blanket, which is also an argument of the verb wrapped. This is the first time we have seen one constituent serving as an argument of more than one predicate at the same time. This is a common phenomenon, however, as we will see in subsequent chapters, especially in Chapter 12.

The argument-marking prepositions, because of the constraint in (17b), project a nonmodifying [SPR \langle\rangle$] \mathrm{PP}$ whose P-OBJ value is identified with the category of the preposition's NP object:

(19) He talks [to himself].

As described in Chapter 7, the P-OBJ value of such a PP is of the same rank as the synsem-struc of the whole PP. Hence it can outrank or be outranked by elements of the ARG-ST list of the higher verb (e.g. talks). This allows the objects of argument-marking prepositions

${ }^{12}$ Note in addition that nothing in our analysis blocks the projection of subjectsaturated PPs like [My blanket [around me]]. In English, these occur only in restricted circumstances, e.g. as 'absolutive' clauses:

(i) [My blanket around me], I was ready to curl up for the night. 
to enter into binding relations with other NPs. Finally, recall that some prepositions, for example, around, behave either as predicational or as argument-marking. Hence the following example is also well formed.

(20) I wrapped the blanket [around myself].

This pattern of optional reflexivization is now neatly accounted for by allowing around to live a double life (via two separate lexical entries) as either a predicational or argument-marking preposition.

Some readers may have noticed that our type hierarchy posits two distinct types corresponding roughly to each of the traditional parts of speech. In addition to noun, verb, adjective, preposition, etc. - the subtypes of pos introduced in Chapter 3 - we now have noun-lxm, verb-lxm, adj-lxm, prep-lxm, etc., which are subtypes of lexeme. A careful examination of the way we use these two sets of types reveals that they serve rather different functions in our grammar. The subtypes of pos specify which features are appropriate for particular categories of words and phrases. These distinctions manifest themselves in the HEAD features, so that the pos subtypes show up in our feature structures as specifications of which type of HEAD value we have. The subtypes of lexeme, on the other hand, introduce constraints on what combinations of feature values are possible, for example, the NSA or the constraint that verbs take NP subjects (SPRs). These typically involve argument structure (and/or valence features) as well as HEAD features. Consequently, the pos subtypes (noun, verb, etc.) frequently appear inside of the constraints associated with the lexeme subtypes (noun-lxm, verb-lxm, etc.).

The type hierarchy simplifies our descriptions in two ways: it saves us from having to assign values to features where they would do no work, for example, PER (person) in prepositions; and it allows us to stipulate common combinations of feature values only once, using (default) inheritance to account for their distribution. The hierarchy contains two sets of types corresponding roughly to the traditional parts of speech then, because the hierarchy serves these two separate functions.

\section{Problem 2: Parts of Speech and Types}

What would happen if we tried to eliminate the pos subtypes like noun and verb? To answer this, you will need to consider where the features currently associated with the pos subtypes would have to be declared, and what consequences this would have for our feature structures. Be explicit.

Up to this point, we have made no mention of CASE specifications 
in our lexical type hierarchy. Thus nothing yet guarantees that NPs in English must be accusative except when they are the subject of a finite verb form. One might think this is a constraint on lexemes, but in fact the various lexical rules we will formulate, for example, the Passive Lexical Rule, never have the effect of transferring accusative case to their first argument (the subject), as we will see in Chapter 10. For this reason, we will treat the assignment of accusative case as a fact about words, not about lexemes. The easiest way to do this is to add the following constraint to our definition of lexical satisfaction.

(21) Case Constraint

Any NP in a noninitial position of a word's ARG-ST list is [CASE acc].

This principle allows us to keep our constraints on verbal lexemes just as we formulated them above, with no mention of case. Thus it is unnecessary to specify lexically the accusative case for most objects, providing a significant improvement on the analysis of English case suggested in the worksection in Chapter $4 .{ }^{13}$ Notice, however, that (21) is a one-way implication: it says that certain NPs are accusative, but it says nothing about which NPs are not accusative. The nominative case, characteristic of subjects, will need to be specified in some other way (a point to which we return later in this chapter).

Finally, it must be stressed that the Case Constraint is specific to English. Many other languages exhibit far more complex case systems; see, for example, the problems on Icelandic and Wambaya in Chapter 4.

\subsection{Lexical Rules}

The lexical rule is a mechanism for further reducing redundancy and stipulation in the lexicon by using information in one lexical entry as the basis for generating another lexical entry. The idea is that the lexicon contains two types of entries: basic ones (lexemes) and those that are 'based on' or 'derived from' these. The lexical rules are used for deriving predictably related lexical entries, such as inflected forms of verbs and nouns.

It is traditional to think of words (or at least certain kinds of words) as being built up from smaller units through the addition of affixes. We have followed this tradition by using our notion of type to distinguish lexeme from word. For most nouns and verbs, we will assume that there is only one basic lexical entry, which contains a description of

\footnotetext{
${ }^{13}$ But argument-marking prepositions, since they have only one NP argument, are not covered by the Case Constraint; they must specify lexemically that that argument is [CASE acc].
} 
type lexeme. We then derive all the nominal and verbal word entries by applying lexical rules to these lexeme entries.

Lexical rules have the general form ' $X \Rightarrow Y$ '. The intended interpretation of this is that for any lexical entry that is consistent with the description in $X$ (which we will sometimes refer to as the 'input' to the rule), there is another lexical entry (the 'output' of the rule) that includes the description in $Y .{ }^{14}$ The input and output entries are identical, except in those ways that the rule specifies. This breaks down into several special cases:

- If a feature $F_{1}$ is given conflicting values in $X$ and $Y$, the input and output entries must have different values for $F_{1}$.

- If $X$ specifies a value $v_{2}$ for a feature $F_{2}$, but $Y$ says nothing about $F_{2}$, then the rule applies only to entries whose value for $F_{2}$ is $v_{2}$; in this case, both input and output entries have the same value for $F_{2}$ (namely $v_{2}$ ).

- If $Y$ specifies that feature $F_{3}$ has value $v_{3}$ but $X$ says nothing about $F_{3}$, the situation is slightly more complex. In this case, the output's value for $F_{3}$ is the unification of $v_{3}$ with the input's value for $F_{3}$. Thus,

o if the input's value for $F_{3}$ is compatible with $v_{3}$ (including where the input does not even mention $F_{3}$ ), the output's value for $F_{3}$ consists of both the input's value and $v_{3}$ (more precisely, their unification); but

- if the input's value for $F_{3}$ is incompatible with $v_{3}$, then there is no output (that is, the rule cannot apply).

A lexical rule applies to a lexical entry (of type lexeme) and produces as output a new lexical entry whose (morphological) form, syntactic category and meaning are systematically related to the input. Lexical rules often add morphological endings in English, but not always.

Our first example of a lexical rule in fact does nothing to the phonological form of the base; but it adds a restriction requiring that the specifier's AGR value is [NUM sg].

${ }^{14}$ More precisely, if the left-hand side of a lexical rule specifies a value $v$ for a feature $F$, then the value of $F$ in any input to the rule must unify with $v$. This includes instances in which the input's value for $F$ is underspecified or disjunctive. 
188 / Syntactic Theory: A Formal Introduction

(22) Singular Noun Lexical Rule

$$
\begin{aligned}
& \langle\text { 口, }[\text { noun-lxm }]\rangle \Rightarrow
\end{aligned}
$$

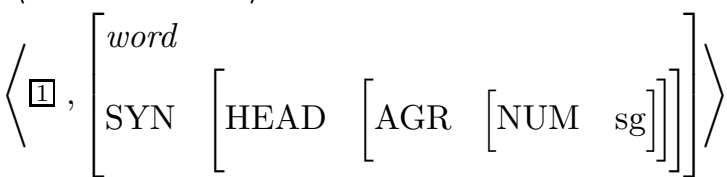

This rule produces lexical entries containing singular forms of both count and mass nouns without mentioning anything about their form or meaning. ${ }^{15}$ Notice that the rule output only specifies the AGR value of the noun itself. But by NSA (which we incorporated into the constraints on the type $c n$-lxm, as discussed in the previous section), this value is identified with the AGR value of the noun's specifier. Thus an output of the rule in (22), such as the one illustrated in (23), must combine with a singular determiner.

${ }^{15}$ The careful reader will have noted that the Singular Noun Lexical Rule as formulated will also apply to proper nouns and pronouns. It may at first seem a bit odd that pronouns undergo the rule, since not all pronouns are singular. But the lexeme entries for pronouns specify their number, so that inherently plural pronouns like them cannot undergo rule (22). And if we were to limit (22) to common nouns, we would have no mechanism allowing pronoun lexemes and proper noun lexemes to give rise to words. 
(23) Example output of the Singular Noun Lexical Rule

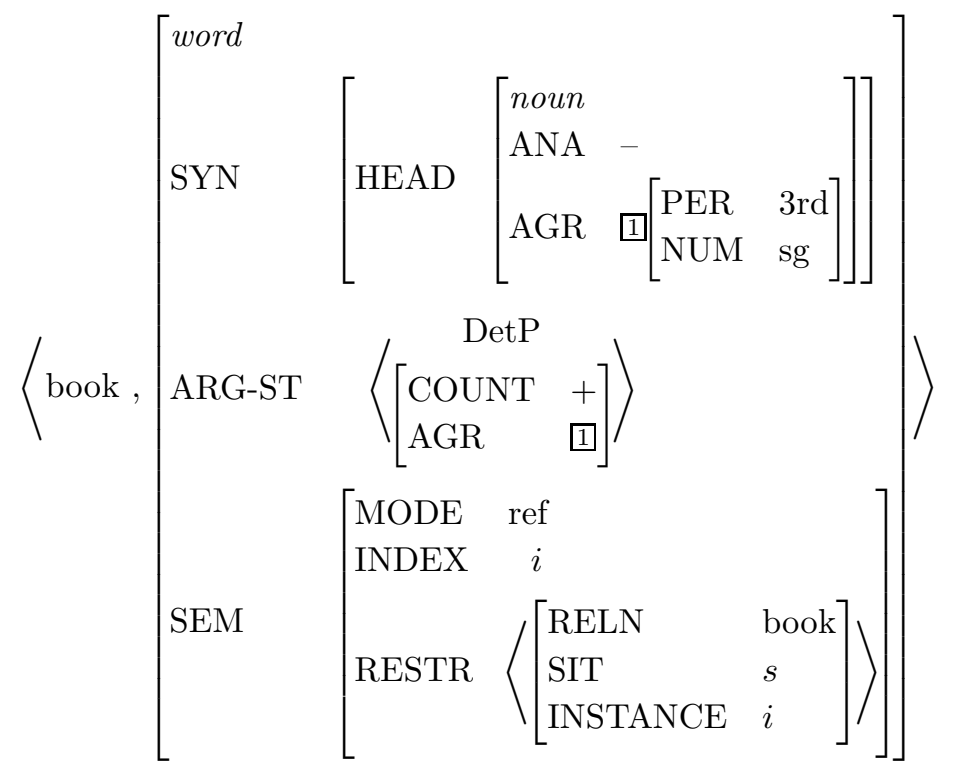

Now consider the closely related rule that maps nominal lexemes into lexical entries for their corresponding plural forms:

(24) Plural Noun Lexical Rule

$$
\begin{aligned}
& \left\langle\square,\left[\begin{array}{ll}
\text { noun-lxm } & \\
\text { ARG-ST } & \langle[\text { COUNT }+]
\end{array}\right]\right\rangle \Rightarrow \\
& \left.\left\langle\mathrm{F}_{N P L}(\mathbb{1}),\left[\begin{array}{l}
\text { word } \\
\mathrm{SYN}
\end{array}\left[\begin{array}{llll}
\mathrm{HEAD} & \text { AGR } & \text { NUM } & \mathrm{pl}
\end{array}\right]\right]\right]\right\rangle
\end{aligned}
$$

Here, $\mathrm{F}_{N P L}$ is a morphological function that applies to nominal bases in English, giving their plural forms. In most cases, it suffixes an $-s$, though its full specification would also include a listing of irregular plurals. Thus, $\mathrm{F}_{N P L}($ cat $)=$ cats, but $\mathrm{F}_{N P L}($ child $)=$ children. The rule says that for every nominal lexeme that takes a [COUNT +] specifier, there is a corresponding lexical entry for a plural noun whose form is dictated by the function $\mathrm{F}_{N P L}$. The requirement that the input take a [COUNT +] specifier keeps the rule from applying to mass nouns like furniture, so that there is no word ${ }^{*}$ furnitures. ${ }^{16}$

\footnotetext{
${ }^{16}$ Note that plural pronouns, e.g. they and us, are listed as belonging to pron-lxm,
} 
A complete formulation of both these lexical rules would require the introduction of a fundamental difference between the semantics of singular and plural nouns. Working out the semantics of singular and plural nouns - which would have to include a semantic analysis of the count/mass distinction - is beyond the scope of this book.

\section{\. Problem 3: Plural and Mass NPs Without Specifiers}

There is a problem with our treatment of common nouns. The type cn-lxm requires common nouns to have nonempty SPR lists, and this requirement is preserved in the Plural Noun Lexical Rule above. But specifiers are optional for plural nouns and mass nouns.

A. Give at least three examples showing the optionality of specifiers for plural and mass nouns.

Two obvious approaches to this problem are the following:

(i) allow empty SPR lists in the lexical entries for plural and mass nouns (as we did in the treatment of mass nouns sketched in Chapter 4, section 7); or

(ii) introduce a new grammar rule to account for NPs with plural or mass heads and no specifiers.

Alternative (i) would involve modifying the Plural Noun Lexical Rule, as well as introducing a new subtype of $c n$-lxm for mass nouns. The rule in alternative (ii) is analogous to the Imperative Rule given in Chapter 7 , in that it would have only one constituent on the right hand side, and its function would be to license a constituent without a specifier, although its daughter has a nonempty SPR list.

B. The ARP does not manage to sufficiently constrain what alternative (i) would allow. Explain why. [Hint: Assume that alternative (i) involves making the determiner an optional member of the noun's ARG-ST. Consider all the ways that an ARG-ST element could be realized on the SPR or COMPS list. You may also want to construct examples of common nouns with complements but no specifiers.]

C. Formulate the rule required for alternative (ii). Be as precise as you can.

[Hint: The trickiest part is stating the disjunction of plural or

and hence can also undergo the Plural Noun Lexical Rule (assuming that pronouns are [COUNT +]). This is as desired, since we need to be able to build words from such lexemes. The lack of an $-s$ inflection on these words can be accounted for simply by defining $\mathrm{F}_{N P L}(u s)$ as us, $\mathrm{F}_{N P L}($ they) as they, etc. 
mass nouns. The place to do this is in the SPR list of the feature structure description on the right hand side of the rule.]

We posit additional lexical rules to generate entries for the various inflected forms of verbs. Since the forms differ in their distributions, it is useful to have a feature whose value distinguishes among them. We have already seen this feature, which we call FORM, in the analysis of imperatives presented in the previous chapter. Later, we will use this same feature (with different values) to distinguish among prepositions and to mark certain fixed expressions. For now, we will identify just the values of FORM that correspond to verbal inflectional categories for which we need to introduce lexical rules. These are: ${ }^{17}$

The bare uninflected form, as in Andy would eat rice, Andy tried to eat rice, or Eat rice!

fin 'Finite', i.e. present or past tense, as in Andy eats rice or Andy ate rice

prp 'Present participle', suffixed with -ing, usually following some form of be, as in Andy is eating rice

psp 'Past participle' (or 'perfect participle'), the form that follows have, as in Andy has eaten rice

pass 'Passive', as in Rice was eaten by Andy (to be discussed in Chapter 10)

Since FORM is a HEAD feature, VP nodes carry the FORM value of their lexical heads. This permits us to specify in the ARG-ST value of certain elements that they take VP complements whose lexical heads have a particular inflection. For example, the to that occurs before verbs

\footnotetext{
${ }^{17}$ Other works that have made use of a feature FORM to distinguish verbal inflectional categories have not always posited precisely these values. In particular, what we call 'inf' has often been called 'base' (with [FORM inf] being reserved for the to of infinitives). In addition, a value 'ger' (for 'gerund') has sometimes been proposed for a kind of word not covered here. Like present participles, gerunds are suffixed with -ing, but unlike present participles, gerunds head phrases that have the distribution of NPs. The occurrences of singing in (i)-(iii) are present participles; those in (iv)-(vi) are gerunds.

(i) The birds are singing.

(ii) Anyone singing in class will be punished.

(iii) Ashley began singing Christmas carols in October.

(iv) Ashley's singing Christmas carols in October annoyed Jordan.

(v) We denied singing during class.

(vi) Don't even think about singing!

The analysis of gerunds is beyond the scope of this text. Hence, we will not consider the question of whether there should be a FORM value for gerunds.
} 
(which we will analyze in more detail in Chapter 12) takes [FORM inf] VP complements, and the auxiliary verb have takes [FORM psp] VP complements. Such co-occurrence restrictions will be discussed in detail in subsequent chapters. We mention them here only to indicate why FORM is needed, and why it is classified as a HEAD feature.

We are now in a position to state several lexical rules for verbal inflections.

(26) 3rd-Singular Verb Lexical Rule

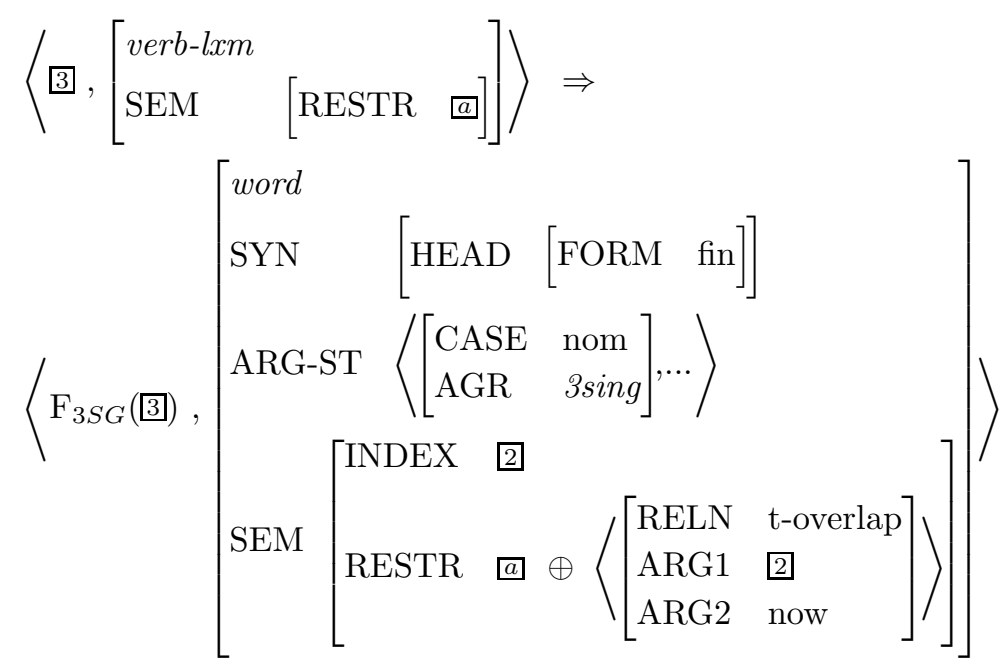

As with the Plural Noun LR, we have glossed over the morphological component of the $3 \mathrm{sg}$-Verb LR by simply giving it a name, $\mathrm{F}_{3 S G}$. The semantic effect of this rule is clear, however: it preserves the semantics of the input, except for the value of the feature RESTRICTION, which is augmented to include a further predication requiring that the INDEX value - the situation described by the verb - be in the temporal-overlap (t-overlap) relation with 'now', an atomic value that will always designate the time of an utterance. Thus, according to this rule, using a 3rd singular form of a verb lexeme imposes the requirement that the situation introduced by the verb be located in some temporal interval that overlaps the time of the utterance. This is perhaps only a first attempt at the semantics of present tense verb forms, but our goal in presenting it is to illustrate how a precise semantic analysis, however refined, can be integrated into lexical rules of the sort outlined here. What the rule in (26) says, then, is that for any verbal lexeme, there is a corresponding finite verb that takes a nominative, third-person singular subject. Fur- 
ther, the morphology and semantics of the latter verb are systematically related to those of the base verb.

Problem 4: $-s$

In most cases, $\mathrm{F}_{3 S G}$ has the same effect as $\mathrm{F}_{N P L}$, namely, that of suffixing -s. In fact, both suffixes have multiple pronunciations, and the conditions under which they are pronounced like $s$, like $z$, or like $i z$ are identical. (They depend on phonological properties of the preceding sound.) Nevertheless, these two morphological functions are not identical. Why?

[Hints: 1. Remember that a function is single-valued, i.e. it specifies only one output for each input. 2. Consider elements that can be used as both nouns and verbs.]

We turn next to the rule that generates the entries for finite verbs with subjects other than third-person singular NPs. Because the type distinction we have drawn between the AGR values 3sing and non-3sing already distinguishes third singular NPs from all others, this rule is almost identical to the last one, as shown in (27).

(27) Non-3rd-Singular Verb Lexical Rule

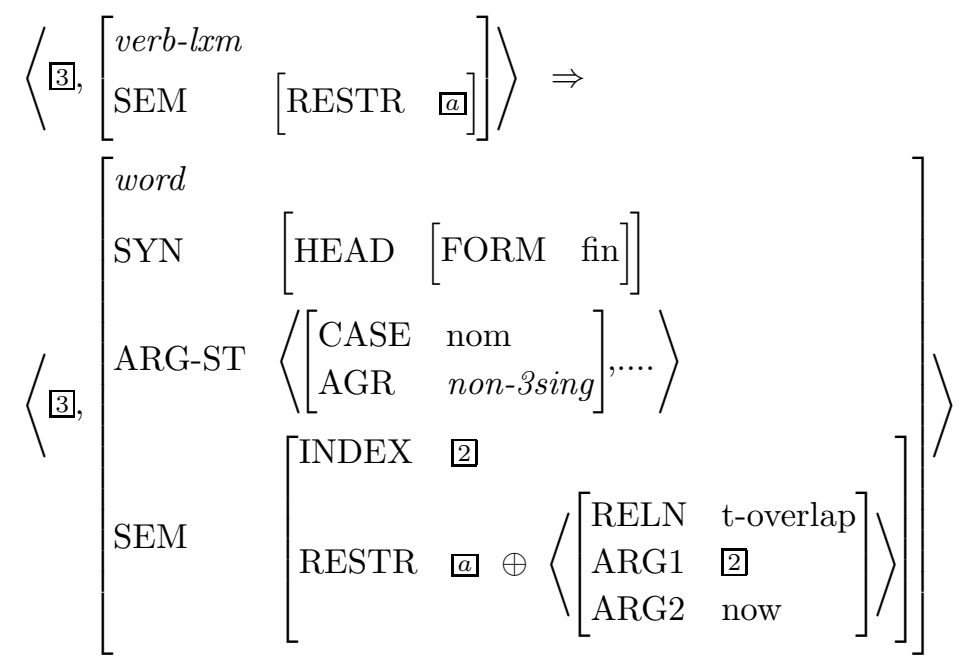

No change in form is introduced, the nominative subject of the output must have a non-3sing AGR value (see Chapter 4 for further discussion), and the situation picked out by the verb must overlap the utterance time. A typical output, illustrated in (28), can never combine with a 
194 / Syntactic Theory: A Formal Introduction

third-person singular subject and will project a sentence whose semantics includes the restrictions illustrated in addition to those that arise from the particular dependents of the verb or from modifiers.

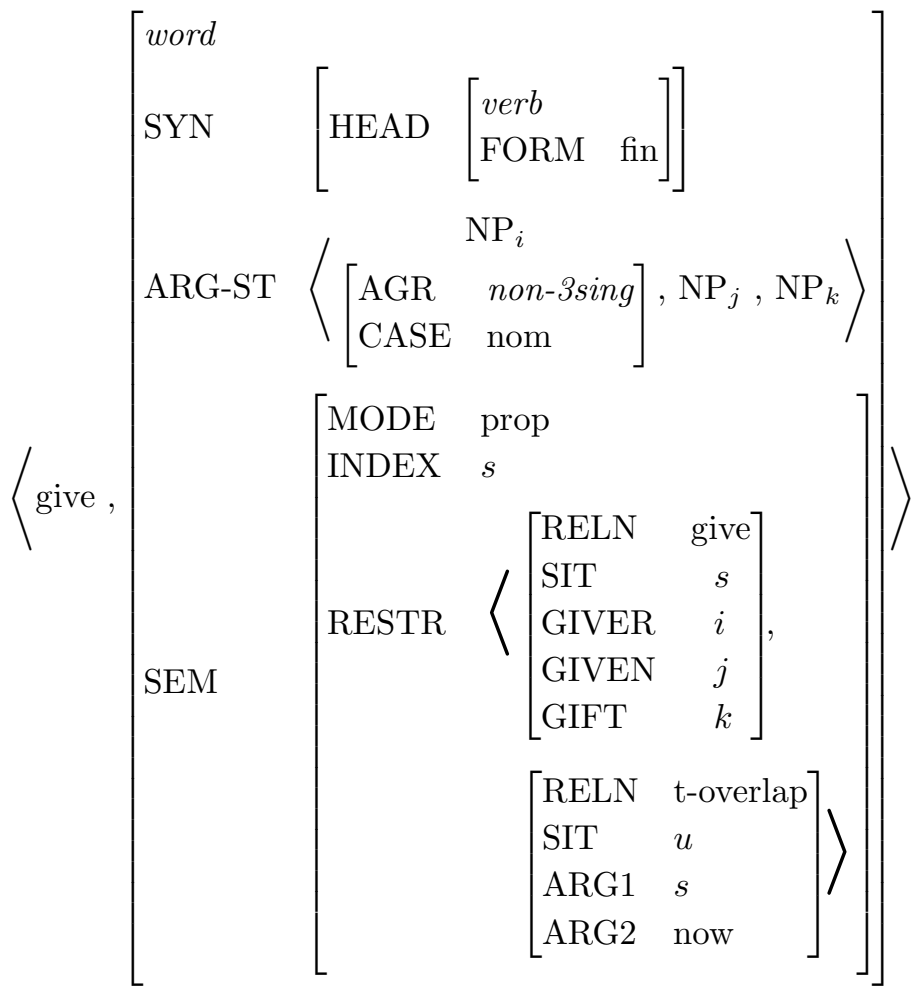

The two rules just discussed create lexical entries that license the present tense forms of verbs. The next rule creates entries for the past tense forms. English makes no distinction between singular and plural 
in past tense forms (aside from was vs. were) $;^{18}$ hence only one rule is needed.

(29) Past-Tense Verb Lexical Rule

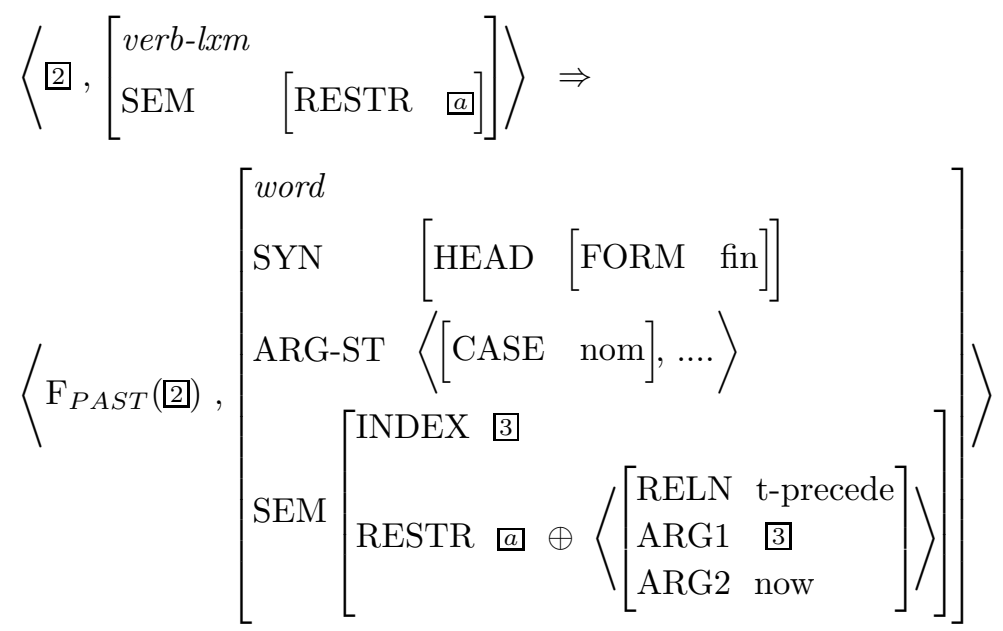

(29) posits a function $\mathrm{F}_{P A S T}$ to account for the morphological relation between verbal lexemes and their past tense forms; in most cases, this consists of suffixing - ed, though there are many exceptions (such as sleep/slept, eat/ate, and put/put). Like the lexical rules for present tense

\footnotetext{
${ }^{18}$ Of course, something must be said about this exception and about the first-person singular form $a m$. The fact that be makes finer distinctions among its verb forms than other verbs does not justify making these distinctions throughout the rest of the verbal system in English. Rather, it is more parsimonious to make be an exception to some of these lexical rules, and to stipulate the individual forms in the lexicon or to posit highly specialized lexical rules for the forms of be. (The latter course may be desirable because, as we shall see in Chapters 10-14, there appear to be several different be lexemes in English). We will not go into the question of what kind of formal machinery to use to specify that certain lexical entries are exceptions to certain lexical rules, though some such mechanism is surely needed irrespective of be.

The inflectional paradigm of be looks quite confusing at first, with one form (am) that goes only with first-person subjects and others (are, were) that go only with subjects that are second person or plural. The situation looks a bit less arbitrary if we make use of the hierarchy of subtypes of non-3sing suggested in footnote 10 of Chapter 4. That hierarchy makes available a type 1 sing that is the AGR value we need for the specifier of am. It also provides a type non-1sing encompassing just second-person and plural AGR values (that is, it excludes just the first-person singular and third-person singular values). This is precisely the AGR value we need for the specifier of are and were. To accommodate was, we would need either two lexical entries (one requiring a 1 sing specifier and another requiring a 3 sing specifier) or a lexical entry with a disjunctive SPR value.
} 
verbs, (29) requires its subject to be nominative (to rule out examples like ${ }^{*}$ Him died); but unlike the present tense rules, it puts no number or person restrictions on the subject, since English past tense verbs exhibit no agreement with their subjects. The semantic effect of the rule is parallel to that of the two present tense rules, except that the relation on the restriction added is 't-precede', rather than 't-overlap'. That is, the situation denoted by the index of the verb temporally precedes the time of utterance if the verb is in the past tense. Again, this is only an approximation of the semantics of the English past tense, but it is good enough for our purposes.

Finally, we need a trivial lexical rule for noninflecting lexemes.

(30) Constant Lexeme Lexical Rule

$$
\langle\square,[\text { const-lxm] }\rangle \Rightarrow\langle\square,[\text { word }]\rangle
$$

This rule does nothing except promote such lexemes to wordhood so that the resulting entries can give rise to word SDs.

\section{\1 Problem 5: More LRs}

Write the lexical rules that generate the infinitival (base), present participle, and past participle entries for verbs in English. You should be able to specify the syntactic effects of these rules quite precisely and (for the most part) straightforwardly. For the semantics, you will need to invent some relation names (that is, some new values for the feature RELN).

\section{(1) Problem 6: Coordination and Verbal Form}

FORM values must be preserved in coordinate structures. That is, if a VP[FORM 1$]$ is a coordinate structure, then each conjunct must also be VP[FORM 1]. Demonstrate that this is true by constructing examples with coordinate VPs of various kinds, for example, the VP head of a finite sentence or the VP complement of verbs like will, have, or (progressive) be.

\section{Problem 7: Coordination and Tense}

Notice that we have not introduced distinct FORM values to distinguish between past and present tense verbs - we treat both as [FORM fin]. Using the result of the preceding problem, explain why this decision is right or wrong. Be sure to consider examples where finite VPs that differ in tense are coordinated. 


\subsection{Derivational Rules}

Each of the lexical rules in the previous section maps entries of type lexeme into lexical entries for inflected words. Such rules are usually called INFLECTIONAL rules. In contrast to these, languages have another kind of lexical rule (called a DERIVATIONAL rule) that relates lexemes to lexemes. This is pretty clearly what we want for certain elements built up by prefixes and suffixes from basic lexemes. So noun lexemes like driver or eater might be derived by the following lexical rule.

(31) Agent Nominalization Lexical Rule

$$
\begin{aligned}
& \left\langle 2,\left[\begin{array}{ll}
\text { verb-lxm } & \\
\mathrm{ARG}^{\mathrm{STT}} & \left\langle\mathrm{NP}_{i}(, \mathbb{\mathrm { NNP }})\right\rangle \\
\mathrm{SEM} & {\left[\begin{array}{ll}
\operatorname{INDEX} & s
\end{array}\right]}
\end{array}\right]\right\rangle \Rightarrow
\end{aligned}
$$

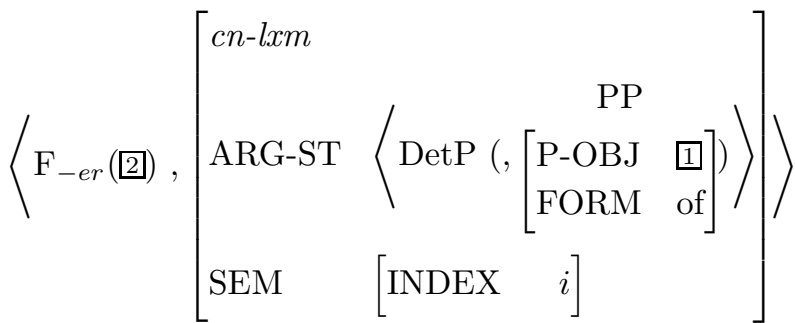

Here the function $\mathrm{F}_{-e r}$ adds the appropriate suffix to the form of the rule output. The input is a verbal lexeme whose subject's index $i$ is identified with the index of the nominal output. Note that the change in type from verb-lxm to $\mathrm{cn}$-lxm has many side effects in terms of values of HEAD features and in terms of the MODE value within the semantics. The RESTR value remains unchanged, however, as the information present in the input is compatible with the type constraints associated with the output type.

The ARG-ST values in (31) deserve some comment. The input must be either a strictly transitive or a strictly intransitive verb. Thus we correctly rule out agent nominals of such verbs as rely or put. The output, like other common nouns, takes a determiner. In addition, if the input is a transitive verb, the agent nominal may take a PP complement whose object is identified with the object of the input verb. This is 
for agent nominals such as the discoverer of oxygen and a builder of bridges. ${ }^{19}$

Consider, for example, the lexical entry for the verbal lexeme drive, the semantics of which is a proposition whose RESTR value contains a driving predication, with the role of driver assigned to the referent of the verb's subject. Applying the Agent Nominalization Lexical Rule to this lexeme yields an entry for the nominal lexeme driver, whose index is restricted to be the driver in a driving predication (since the RESTR value is unchanged). This lexical entry can now undergo both our nominal lexical rules, and so we derive entries for the singular form driver and its plural analog drivers.

There are further semantic constraints that must be placed on our derivational rule, however. For example, the subject in the input verb has to be sufficiently agentive - that is, it must play an active (usually volitional) role in the situation. That's why nominalizations like knower or resembler sound funny. But the formulation in (31) is a reasonable first pass at the problem, and it gives you an idea of how phenomena like this can be analyzed within our framework.

There are many other cross-categorial relations that work this way in English. Noun lexemes, both common and proper, can be converted into verbal lexemes:

(32) a. Sandy porched the newspaper without difficulty.

b. The senator houdinied his way out of the accusations.

c. They have been computering me to death all morning.

This kind of derivation without morphological change, an instance of what is often called ZERO DERIVATION, could be handled by one or more derivational rules.

Finally, note that lexical rules are a traditional way of approaching the problem of valence alternations, that is, the fact that many verbs allow systematically related valence patterns. Among the most famous of these is the dative alternation illustrated in (33) - (34).

(33) a. Birch gave Brooke a book.

b. Birch gave a book to Brooke.

(34) a. Birch handed Brooke a book.

b. Birch handed a book to Brooke.

Rather than list entries for two distinct verbal lexemes for give, hand, and a family of related elements, it makes much more sense to list only one (with one of the two valence patterns fixed) and to derive the other

\footnotetext{
${ }^{19}$ Notice that in formulating this rule, we have used the FORM value 'of' to indicate that the preposition heading this PP must be of. We return to the matter of FORM values for prepositions in Chapter 10.
} 
by a derivational rule. Note however, that there are certain other verbs or particular idiomatic uses that appear in only one of the two valence patterns.

(35) a. Kris donated a book to the library.

b. ${ }^{*}$ Kris donated the library a book.

(36) a. Dale gave Brooke a hard time.

b.??Dale gave a hard time to Brooke.

These underline once again the need for a theory of exceptions to lexical rules and lexical irregularity.

Other famous examples of valence alternation are illustrated in (37)(41).

(37) a. The police sprayed the protesters with water.

b. The police sprayed water on the protesters. ('spray/load' alternations)

(38) a. The students drove cars.

b. These cars drive easily. ('middle' uses)

(39) a. Pat sneezed.

b. Pat sneezed the napkin off the table. ('caused motion' uses)

(40) a. The horse kicked me.

b. The horse kicked me black and blue. ('resultative' uses)

(41) a. Pat yelled.

b. Pat yelled her way into the meeting. (the 'X's way' construction)

All these patterns of valence alternation are governed by both semantic and syntactic constraints of the kind that could be described by finely tuned lexical rules.

\section{\. Problem 8: Arguments in Japanese}

As noted in Chapter 7, Japanese word order differs from English in a number of ways, including the fact that it is a 'Subject-Object-Verb' (SOV) language. Here are a few relevant examples ('nom', 'acc', and 'dat' stand for nominative, accusative, and dative case, respectively). ${ }^{20}$

(i) hitorino otoko-ga sono hon-o yonda

one man-nom that book-acc read-past

'One man read that book'

${ }^{20}$ The examples marked with '*' here are unacceptable with the indicated meanings. Some of these might be well formed with some other meaning of no direct relevance; others might be well formed with special intonation that we will ignore for present purposes. 
[cf. *Yonda hitorino otoko-ga sono hon-o

*Hitorino otoko-ga yonda sono hon-o

*Otoko-ga hitorino sono hon-o yonda

*Hitorino otoko-ga hon-o sono yonda

*Hitorino otoko-ni/-o sono hon-o yonda

*Hitorino otoko-ga sono hon-ga/-ni yonda.]

(ii) Hanako-ga hon-o yonda

Hanako-nom book-acc read-past

'Hanako read the book(s)'

[cf. *Yonda Hanako-ga hon-o

*Hanako-ga yonda hon-o

*Hanako-ni/-o hon-o yonda

*Hanako-ga hon-ni/-ga yonda.]

(iii) sensei-ga Taroo-ni sono hon-o ageta

sensei-nom Taroo-dat that book-acc gave-past

'The teacher(s) gave that book to Taroo'

[cf. *Ageta sensei-ga Taroo-ni sono hon-o

*Sensei-ga ageta Taroo-ni sono hon-o

*Sensei-ga Taroo-ni ageta sono hon-o

*Sensei-o/-ni Taroo-ni sono hon-o ageta

*Sensei-ga Taroo-ga/-o sono hon-o ageta

*Sensei-ga Taroo-ni sono hon-ga/-ni ageta.]

(iv) Sono hon-ga akai-desu

that book-nom red-copula

'That book is red'

As the contrasting ungrammatical examples show, the verb must appear in final position in Japanese. In addition, we see that verbs select for NPs of a particular case, much as in English. In the following tasks, assume that the nouns and verbs of Japanese are inflected words, derived by lexical rule from the appropriate lexemes.

A. Given the data illustrated here, how could the Head-Specifier and Head-Complement rules be revised to deal with Japanese? Explain the effect of the difference(s) you have posited.

B. Give the lexical entry for each of the verbs illustrated in (i)-(iv) (treating akai-desu as a simple intransitive verb). The data given only permit you to specify some features; leave others unspecified. Make sure your entries interact with the rules you formulated in Task 1 to account for the above data.

C. Assume that nouns like Taroo, hon, etc. are entered in the Japanese lexicon as nominal lexemes. Give lexical entries for these lexemes 
(again limiting the features specified to those for which you have data).

D. Formulate three lexical rules for deriving the inflected forms (the words ending in $-g a,-o$, and $-n i$ ) from the nominal lexemes.

E. Explain the role of the Head Feature Principle in accounting for the case-selecting properties of verbs in (i)-(iv).

As you know from Problem 3 of Chapter 7, both subjects and objects in Japanese can in general be omitted. When such an element is omitted, the sentence usually has a meaning that we would express in English by using a pronoun corresponding to the unexpressed element:

(v) Taroo-ga yonda

Taroo-nom read-past

'Taroo read it/them'

(vi) hon-o yonda

book-acc read-past

'He/she/they read the book(s)'

(vii) yonda

read-past

'He/she/it/they read it/them'

(viii) Taroo-ga tatai-ta.

Taroo-NOM hit-PST

' $\operatorname{Taroo}_{i}{\text { hit } \operatorname{him}_{j} / \text { her }_{j} / \text { them }_{j} /{ }^{*} \text { himself }_{i} .}$.

F. Sketch an account of this phenomenon that uses lexical rules. Be explicit about the verbs' values for SPR, COMPS, and ARG-ST. You should assume that all complements can be omitted, not just those illustrated in (v) - (viii); i.e. your lexical rules should apply with maximum generality. Following the suggestion in Chapter 7, Problem 2, you should assume that the ARP is a default principle in Japanese (unlike English).

\subsection{Conclusion}

An important insight, going back at least to Saussure, is that all languages involve arbitrary (that is, unpredictable) information. Most clearly, the association between the forms (sounds) and meanings of words is purely conventional, in the vast majority of cases. A grammar of a language must list these associations somewhere. The original con- 
ception of the lexicon in modern linguistics was simply as the repository of such arbitrary information.

This conception did not last long, however. Beginning in the early years of transformational grammar, linguists began enriching their conception of the lexicon to include information that was not idiosyncratic to individual words. This trend continued in a great deal of research carried out within a variety of grammatical frameworks.

In this text, we have to some extent recapitulated this history. We began with context-free grammar in which the lexicon contained only idiosyncratic information, and we gradually enriched our lexical representations, including more and more information - much of it systematic and predictable - about the grammatical and semantic properties of words. Indeed, most of the information needed to determine the wellformedness of sentences is now encoded into our lexical entries.

With the increased expressiveness and concomitant complexity of lexical entries came a need to express succinctly certain generalizations about words. In this chapter, we have examined two formal mechanisms for capturing such generalizations. Structuring the lexicon in terms of an inheritance hierarchy of types has made it possible to factor out information common to many lexical entries, thereby greatly reducing lexical redundancy. By allowing inherited information to be overridden, we can encode default values for features, while still allowing for lexical idiosyncrasy. The second mechanism, the lexical rule, is an older idea, going back to work in transformational grammar of the 1970s. We will make extensive use of lexical rules in subsequent chapters. In fact, many of the phenomena that provided the motivation for transformations in the 1950s and 1960s can be reanalyzed in our theory using lexical rules. These include the passive construction - the topic of Chapter 10 - and many of the properties of the English auxiliary verb system, which we treat in Chapter 13.

\subsection{Further Reading}

An important early paper on lexical rules is Jackendoff (1975). The idea of combining lexical rules with an inheritance hierarchy was first put forward by Flickinger et al. (1985). See also Pollard and Sag (1987, chap. 8). Briscoe et al. (1993) is a collection of more recent papers about lexical hierarchies, default inheritance, and related issues. 


\section{Realistic Grammar}

\subsection{Introduction}

In the preceding eight chapters, we have laid out the theory that we will apply to more complex data in the remainder of this book. Perhaps surprisingly, only one more grammatical principle and one more grammar rule are required; the other extensions we will present in subsequent chapters (aside from a minor reformulation of the ARP in chapter 15) all concern the lexicon. Before we proceed, however, it is useful to consolidate the components of our treatment of English grammar and to reflect on the strategy we have adopted for solving syntactic problems to reflect on the motivation for the design of grammar.

As we noted briefly in Chapter 2, syntacticians rely heavily on considerations of parsimony: the desirability of 'capturing generalizations' is given great weight in choosing between analyses. This concern with providing elegant descriptions is not unique to this field, though it probably figures more prominently in linguistic argumentation than elsewhere. It is natural to ask, however, whether a grammar whose design has been shaped in large measure by concern for parsimony corresponds straightforwardly to the way linguistic knowledge is represented in the minds of language users. We argue in this chapter that the available psycholinguistic evidence fits rather well with the conception of grammar that we have been developing in this book.

First, however, we turn to a summary of our grammar to date. The next section of this chapter gives a formal presentation of everything we have covered so far, including types, lexical entries, grammar rules, the well-formedness definitions (incorporating various principles), and lexical rules.

Section 9.2.1 presents the type hierarchy in terms of a table that provides details of the features and constraints associated with each type, as well as an indication of each type's immediate supertype (IST) in the 


\section{4 / Syntactic Theory: A Formal Introduction}

hierarchy. Constraints on the possible values of features are introduced by enclosing the list of possible values in curly brackets, following the feature name. Almost all of the types and constraints listed in Section 9.2.1 have been introduced in earlier chapters. We have added almost nothing that is new. Two new types are gram-cat (for 'grammatical category') and sem-struc (for 'semantic structure). The first of these is the type of the value of SYN - that is, feature structures of type gram-cat have the features HEAD, SPR, and COMPS. The second is the type of the value of SEM, which consists of the features MODE, INDEX, and RESTR. Both of these have been implicit in the discussion of the preceding chapters. The type verb lists no features or constraints associated with it, but we have put in the placeholder ' $[\ldots]$ ', because this will change in subsequent chapters.

Section 9.2.2 gives some sample lexical entries. It is worth noting that most of what we have to stipulate in our entries is semantic. By virtue of having a richly structured lexicon, we are able to limit the amount of syntactic information that has to be listed in individual entries, thereby greatly reducing redundant stipulation.

Section 9.2.3 lists our familiar grammar rules from Chapter 6, together with the Imperative Rule introduced in Chapter 7.

Section 9.2.4 gives the formal definitions of well-formed tree structure and lexical and phrasal satisfaction, incorporating all of the general principles of grammar we have adopted so far. This version is slightly modified from the one given in Chapter 6, in that the Argument Realization Principle has been incorporated into the first part (that is, the definition of lexical satisfaction). In addition, our binding theory and the Anaphoric Agreement Principle have been built in. This version of the definition also provides something that was only promised in Chapter 6 : an initial symbol for our grammar. It says, in effect, that a well-formed stand-alone expression in English must be a finite sentence.

Section 9.2.5 lists the lexical rules that were presented in Chapter 8 .

\subsection{The Grammar So Far}

The following pages contain a summary of the type hierarchy developed in the preceding chapters. ${ }^{1}$

\footnotetext{
${ }^{1}$ We use the notation 'list $(\tau)$ ' to indicate a (possibly empty) list, all of whose members are of type $\tau$.
} 
Realistic Grammar / 205

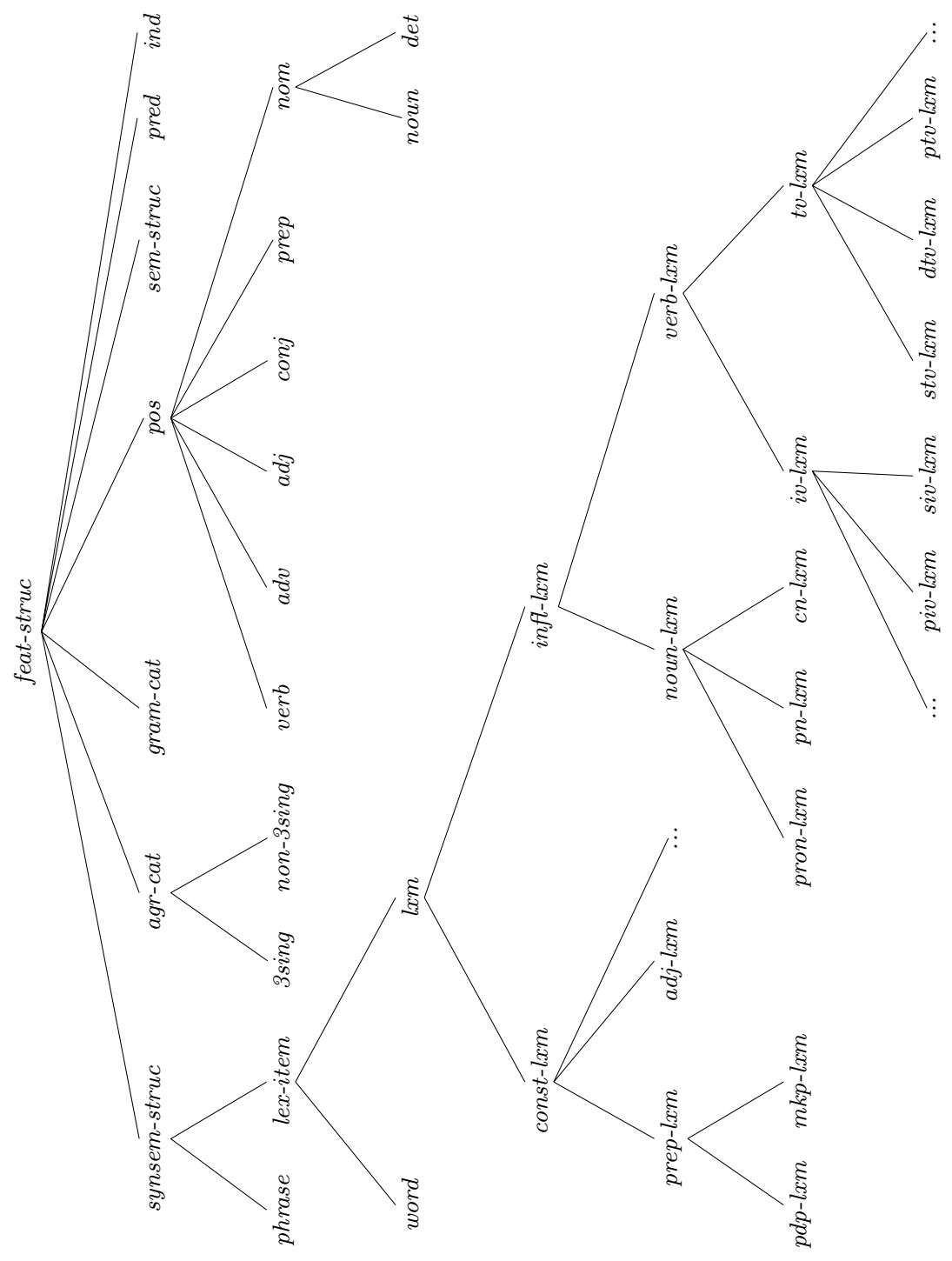


206 / Syntactic Theory: A Formal Introduction

\subsubsection{Feature Declarations and Type Constraints}

\begin{tabular}{|c|c|c|}
\hline \multicolumn{3}{|c|}{ SOME GENERAL TYPES } \\
\hline TYPE & "FEATURES/CONSTRAINTS & IST \\
\hline \multicolumn{3}{|l|}{ feat-struc } \\
\hline synsem-struc & {$\left[\begin{array}{ll}\mathrm{SYN} & \text { gram-cat } \\
\mathrm{SEM} & \text { sem-struc }\end{array}\right]$} & feat-struc \\
\hline phrase & & synsem-struc \\
\hline lex-item & [ARG-ST $\quad$ list(synsem-struc)] & synsem-struc \\
\hline word & & lex-item \\
\hline lexeme & {$[\operatorname{SEM}[$ MODE / none $]]$} & lex-item \\
\hline infl-lxm & & lexeme \\
\hline const-lxm & & lexeme \\
\hline noun-lxm & $\begin{array}{l}{\left[\text { SYN }\left[\text { HEAD }\left[\begin{array}{ll}\text { noun } & \\
\text { AGR } & {[\text { PER } / 3 r d} \\
\text { ANA } & /-\end{array}\right]\right.\right.} \\
\text { ARG-ST / } /\langle\rangle \\
\text { SEM }[\text { MODE } / \text { ref }]\end{array}$ & infl-lxm \\
\hline pron-lxm & & noun-lxm \\
\hline pn-lxm & {$[$ SYN [HEAD [AGR [NUM / sg]]]] } & noun-lxm \\
\hline cn-lxm & 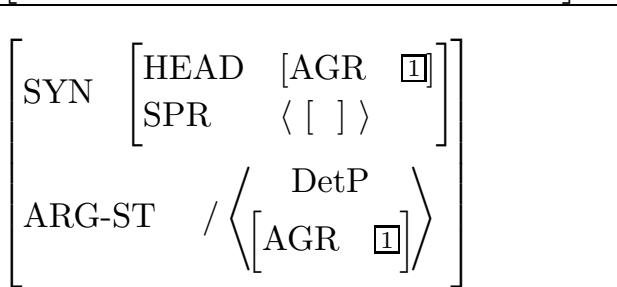 & noun-lxm \\
\hline verb-lxm & {$\left[\begin{array}{lll}\text { SYN } & {\left[\begin{array}{ll}\text { HEAD } & \text { verb } \\
\text { SPR } & \langle[]\rangle\end{array}\right]} \\
\text { ARG-ST } & /\langle\mathrm{NP}, \ldots\rangle \\
\text { SEM } & {[\text { MODE }} & \text { prop }]\end{array}\right]$} & infl-lxm \\
\hline
\end{tabular}




\begin{tabular}{|c|c|c|c|}
\hline \multicolumn{4}{|c|}{ MORE LEXEME TYPES } \\
\hline$\overline{\text { TYPE }}$ & $\overline{\overline{\text { FEATI }}}$ & JRES/CONSTRAINTS & $\overline{\mathrm{IST}}$ \\
\hline$i v-l x m$ & & & verb-lxm \\
\hline siv-lxm & {$[\mathrm{ARG}-$} & $\mathrm{T}\langle[]\rangle]$ & $i v-l x m$ \\
\hline piv-lxm & {$[\mathrm{ARG}-$} & $\mathrm{T}\langle[], \mathrm{PP}\rangle]$ & $i v-l x m$ \\
\hline tv-lxm & {$[\mathrm{ARG}-$} & $\mathrm{T}\langle[], \mathrm{NP}, \ldots\rangle]$ & verb-lxm \\
\hline stv-lxm & ARG- & $\mathrm{T}\langle[],[]\rangle]$ & tv-lxm \\
\hline$d t v-l x m$ & ARG- & $\mathrm{T}\langle[],[], \mathrm{NP}\rangle]$ & $t v-l x m$ \\
\hline ptv-lxm & ARG- & $\mathrm{T}\langle[],[], \mathrm{PP}\rangle]$ & $t v-l x m$ \\
\hline prep-lxm & $\mathrm{SYN}$ & {$\left[\begin{array}{ll}\text { HEAD } & \text { prep }\end{array}\right]$} & const-lxm \\
\hline $\begin{array}{l}\text { adj-lxm, } \\
\text { conj-lxm, } \\
\text { det-lxm,... }\end{array}$ & {$[\ldots]$} & & const-lxm \\
\hline$p d p-l x m$ & {$\left[\begin{array}{l}\text { SYN } \\
\text { ARG- }\end{array}\right.$} & $\begin{array}{l}\left.\left[\begin{array}{l}\text { HEAD } \\
\text { MOD }\end{array} \begin{array}{l}\text { NOM, } \\
\text { VP, } \\
\text { none }\end{array}\right\}\right] \\
\text { SPR }\left\langle\left[\begin{array}{l}\text { none }\end{array}\right]\right\rangle \\
\operatorname{ST}\langle\mathrm{NP}, \mathrm{NP}\rangle\end{array}$ & prep-lxm \\
\hline$m k p-l x m$ & {$\left[\begin{array}{l}\mathrm{SYN} \\
\mathrm{ARG}-\end{array}\right.$} & $\begin{array}{l}{\left[\begin{array}{l}\text { HEAD }\left[\begin{array}{ll}\text { MOD } & \text { none } \\
\text { P-OBJ } & 1\end{array}\right] \\
\operatorname{SPR}\langle\rangle\end{array}\right]} \\
\operatorname{ST}\langle\square\rangle\end{array}$ & prep-lxm \\
\hline
\end{tabular}


208 / Syntactic Theory: A Formal Introduction

\begin{tabular}{|c|c|c|}
\hline \multicolumn{3}{|c|}{ OTHER GRAMMATICAL TYPES } \\
\hline$\overline{\text { TYPE }}$ & FEATURES/CONSTRAINTS & $\overline{\mathrm{IST}}$ \\
\hline gram-cat & {$\left[\begin{array}{ll}\text { HEAD } & \text { pos } \\
\text { COMPS } & \text { list }(\text { synsem-struc }) \\
\text { SPR } & \text { list }(\text { synsem-struc })\end{array}\right]$} & feat-struc \\
\hline pos & {$[$ FORM $\{$ fin, to,$\ldots\}]$} & feat-struc \\
\hline verb & {$[\ldots]$} & pos \\
\hline prep & {$\left[\begin{array}{ll}\text { P-OBJ } & \left\{\begin{array}{l}\text { synsem-struc, none } \\
\text { MOD }\end{array}\right. \\
& \left\{\begin{array}{l}\text { NOM, } \\
\text { VP, } \\
\text { none, } \\
\ldots\end{array}\right\}\end{array}\right.$} & pos \\
\hline nominal & {$[$ AGR $\quad$ agr-cat } & pos \\
\hline noun & $\begin{array}{ll}\text { ANA } & \{+,-\} \\
\text { CASE } & \{\text { nom, acc }\}\end{array}$ & nominal \\
\hline det & {$[\operatorname{COUNT} \quad\{+,-\}]$} & nominal \\
\hline$a d v$ & {$[\operatorname{MOD} \quad\{\mathrm{VP}$, none,...$\}]$} & pos \\
\hline$a d j$ & {$[\operatorname{MOD}\{$ NOM, none $\}]$} & pos \\
\hline conj & & pos \\
\hline$a g r-c a t$ & $\begin{array}{ll}\text { PER } & \{1 \mathrm{st}, 2 \mathrm{nd}, 3 \mathrm{rd}\} \\
\mathrm{NUM} & \{\mathrm{sg}, \mathrm{pl}\}\end{array}$ & feat-struc \\
\hline $3 \operatorname{sing}$ & $\left.\begin{array}{ll}\text { PER } & \text { 3rd } \\
\text { NUM } & \text { sg } \\
\text { GEND } & \{\text { masc, fem, neut }\}\end{array}\right]$ & $a g r-c a t$ \\
\hline non-3sing & & $a g r-c a t$ \\
\hline
\end{tabular}




\begin{tabular}{|l|ll|l|}
\hline \multicolumn{3}{|c|}{ MORE GRAMMATICAL TYPES } \\
\hline \hline TYPE & FEATURES/CONSTRAINTS & IST \\
\hline sem-struc & {$\left[\begin{array}{ll}\text { MODE } & \text { prop, ques, dir, ref, none }\} \\
\text { INDEX } & \text { index } \\
\text { RESTR } & \text { list }(\text { predication })\end{array}\right.$} & feat-struc \\
\hline predication & {$\left[\begin{array}{ll}\text { RELN } & \text { love,walk,... }\} \\
\ldots & \end{array}\right]$} & \\
\hline index & & feat-struc \\
\hline
\end{tabular}

\subsubsection{The Basic Lexicon}

Here are some sample lexical entries that are part of the basic lexicon. We have included only information that is not inherited from higher types. For example, the first entry does not contain a specification for the feature ANA, because it is of type pron-lxm, a subtype of noun$l x m$, which has the specification [ANA / -]. As before, the notation '.. ' indicate things we haven't dealt with but which a complete grammar would have to.

\subsubsection{Nouns}

(1)

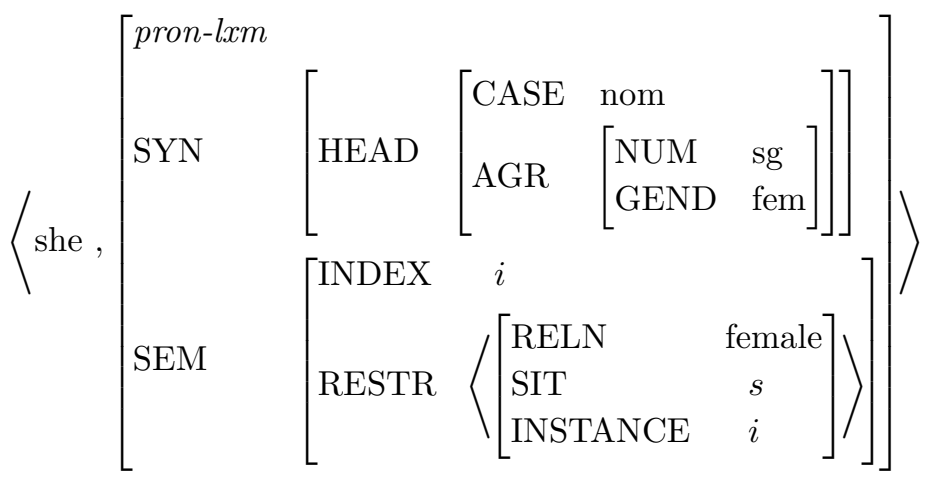


210 / Syntactic Theory: A Formal Introduction

(2)

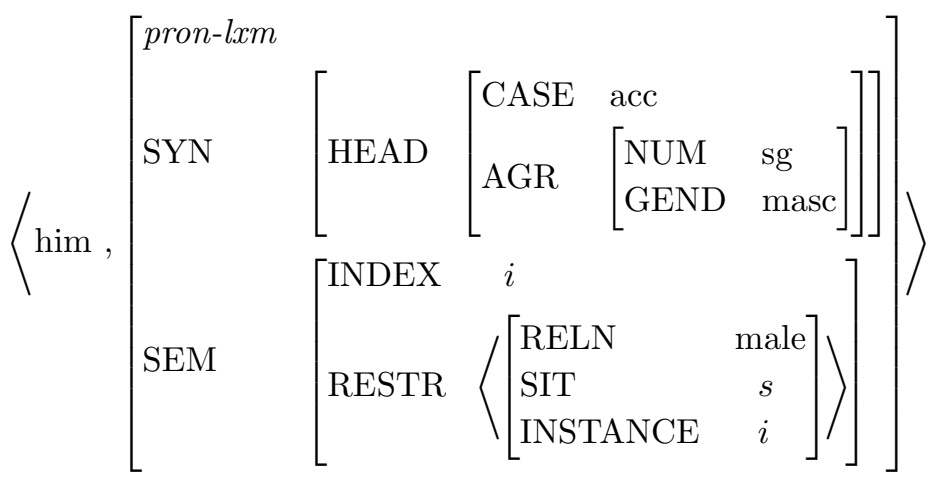

(3)

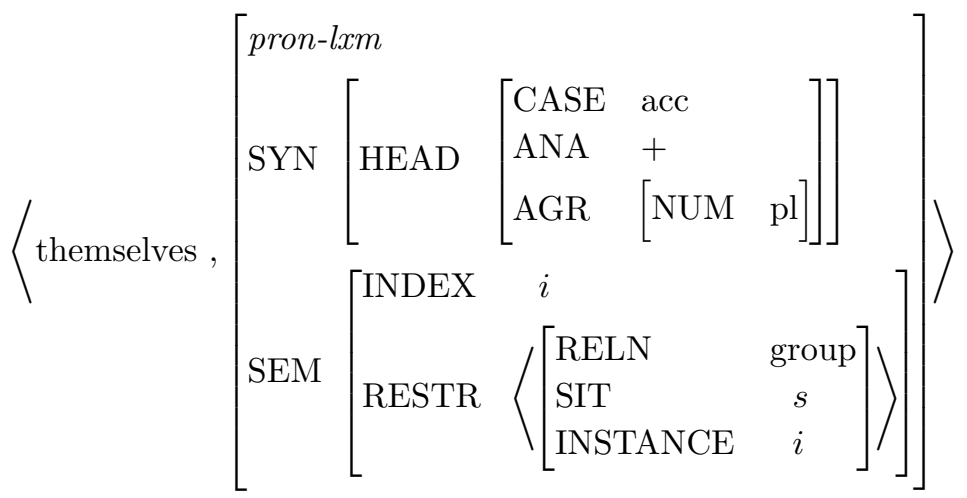

(4)

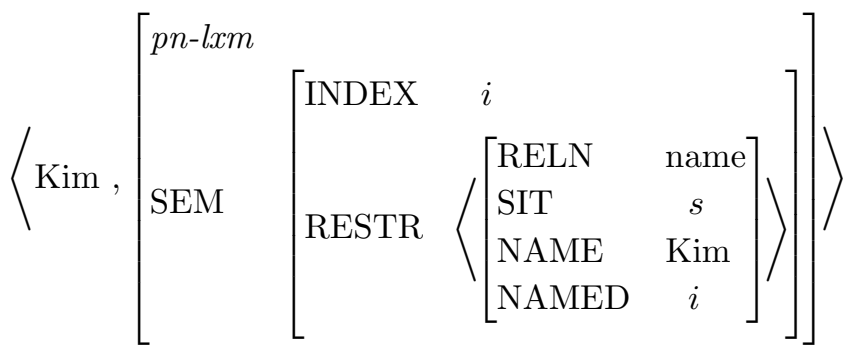

(5)

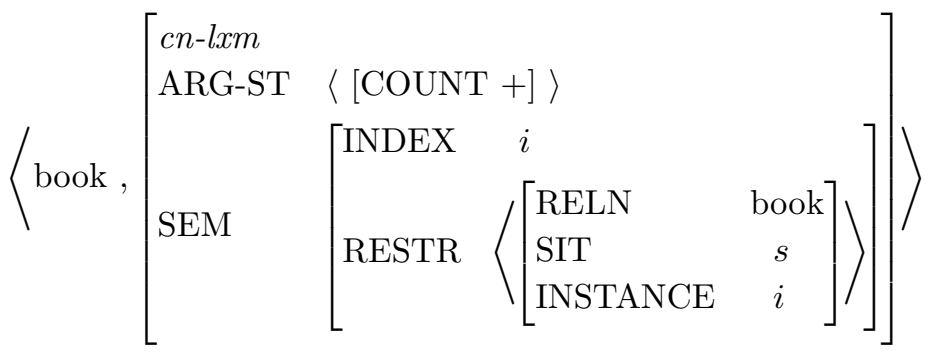




\subsubsection{Verbs}

(6)

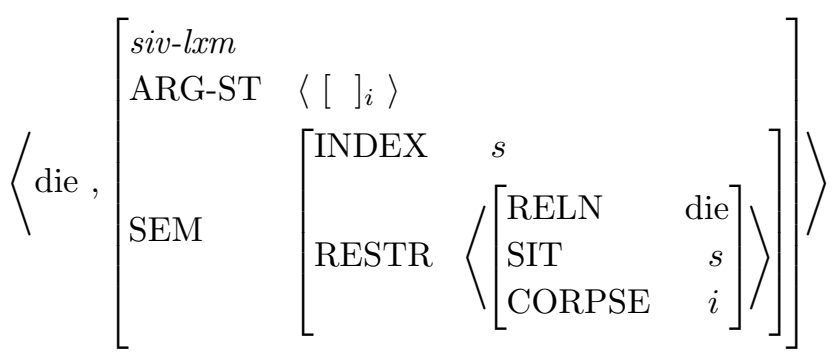

(7)

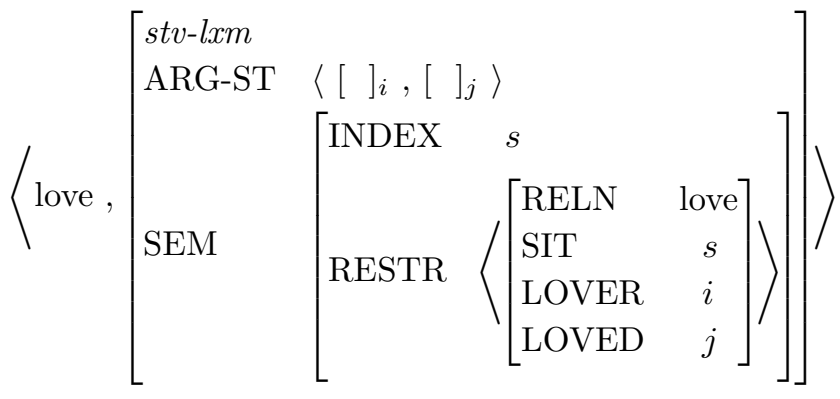

(8)

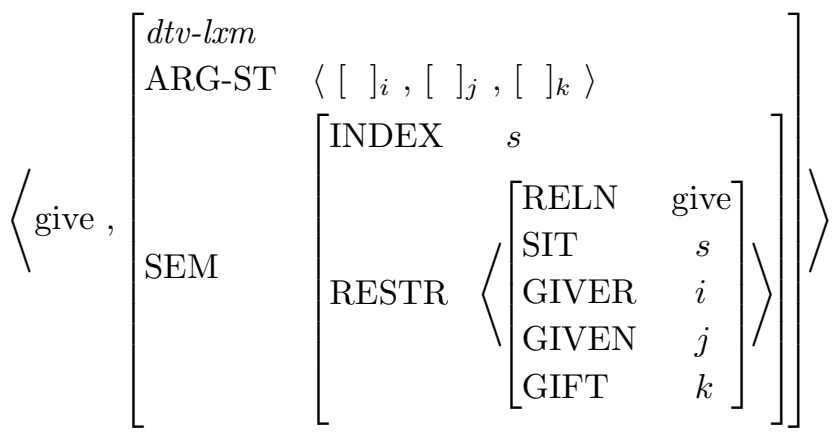

(9)

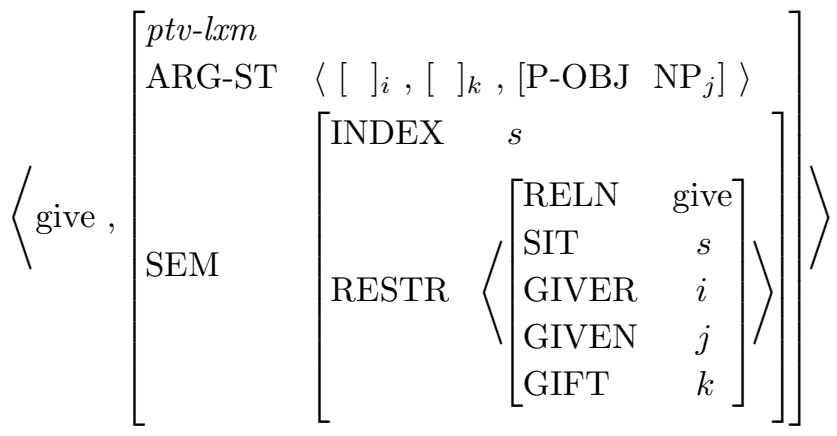


212 / Syntactic Theory: A Formal Introduction

\subsubsection{Miscellaneous}

(10)

$$
\left\langle\text { the },\left[\begin{array}{ll}
\text { det-lxm } & \\
\text { ARG-ST } & \langle\rangle \\
\text { SEM } & {[\ldots]}
\end{array}\right]\right\rangle
$$

(11)

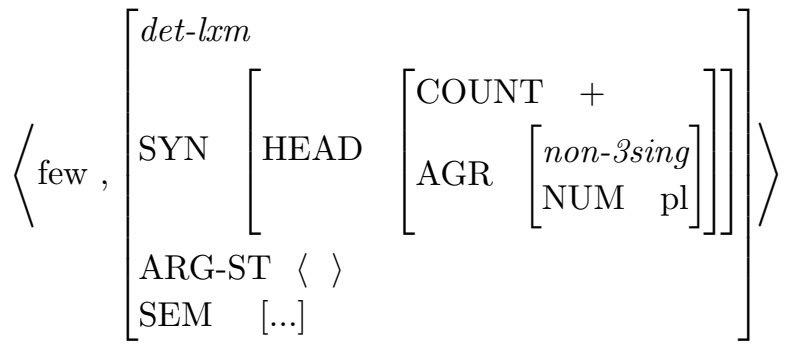

(12)

$$
\left\langle\text { 's },\left[\begin{array}{ll}
\text { det-lxm } & \\
\text { ARG-ST } & \langle\mathrm{NP}\rangle \\
\mathrm{SEM} & {[\ldots]}
\end{array}\right]\right\rangle
$$

(13) $\left\langle\right.$ to,$\left.\left[\begin{array}{c}m k p-l x m \\ \ldots\end{array}\right]\right\rangle$

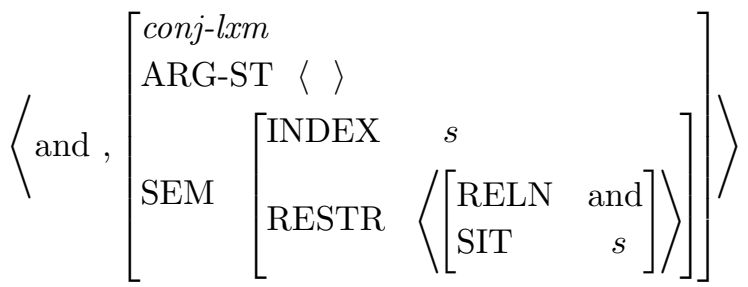




\subsubsection{The Grammar Rules}

- Head-Complement Rule $\left[\begin{array}{ll}\text { phrase } & \\ \text { COMPS } & \langle\rangle\end{array}\right] \rightarrow \mathrm{H}\left[\begin{array}{ll}\text { word } & \\ \text { COMPS } & \langle\square, \ldots, n\rangle\end{array}\right]$ 1... $n$

- Head-Specifier Rule $\left[\begin{array}{ll}\text { phrase } & \\ \mathrm{SPR} & \langle\rangle\end{array}\right] \rightarrow \quad \mathrm{U} \quad \mathrm{H}\left[\begin{array}{ll}\text { phrase } & \\ \mathrm{SPR} & \langle\square\rangle\end{array}\right]$

- Head-Modifier Rule $[$ phrase $] \rightarrow \mathrm{H}\left[\begin{array}{l}\text { phrase }] \\ \text { MOD }\end{array}\right]$

- Coordination Rule $\left[\begin{array}{ll}\mathrm{SYN} & 0 \\ \mathrm{IND} & s_{0}\end{array}\right] \rightarrow$

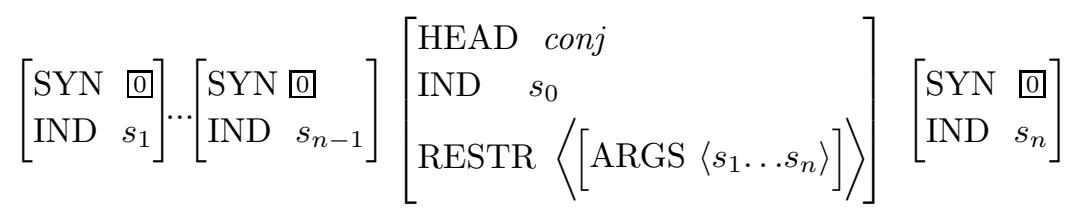

- Imperative Rule

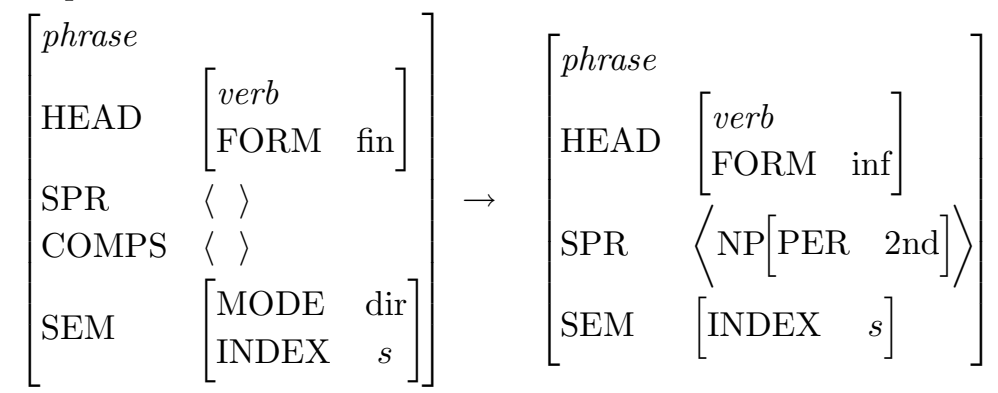


214 / Syntactic Theory: A Formal Introduction

\subsubsection{Well-Formedness Definitions}

In the following definitions, we take for granted the notions of resolved feature structure and tree structure. ${ }^{2}$ We also presuppose the notion of feature structure satisfaction. ${ }^{3}$

(15) Well-Formed Tree Structure

$\Phi$ is a well-formed tree structure just in case every local subtree in $\Phi$ satisfies some lexical entry $\eta$ or some grammar rule $\rho$.

(16) Lexical Satisfaction

A word structure:<smiles>F[Te]</smiles>

satisfies a lexical entry $\langle\omega, \delta\rangle$ just in case:

1. $F$ is of type word and $F$ satisfies $\delta$,

2. Argument Realization Principle

$F$ satisfies the following feature structure description:

$$
\left[\begin{array}{l}
\text { SYN }\left[\begin{array}{ll}
\text { SPR } & \square \\
\text { COMPS } & b
\end{array}\right] \\
\text { ARG-ST } \square \oplus \square
\end{array}\right]
$$

and

3. Case Constraint

Any NP in a noninitial position of F's ARG-ST list is [CASE acc].

\footnotetext{
${ }^{2}$ As noted in Chapter 6, a resolved feature structure $\phi$ is a directed graph of a particular kind, where: (i) $\phi$ is assigned to a maximally specific type $\tau$, (ii) $\phi$ is specified for every feature $F$ that is appropriate for $\tau$ and (iii) $\phi$ 's value for each such feature $F$ is maximally specific. A tree structure is a configuration of nodes that satisfies a number of conditions, e.g. (i) that each node have only one mother, (ii) each nonterminal node is a resolved feature structure, and (iii) each terminal node is a lexical form.

${ }^{3}$ Intuitively, a resolved feature structure $\phi$ satisfies a feature structure description $\delta$ when all the information specified in $\delta$ is true of $\phi$.
} 
(17) Phrasal Satisfaction

A local subtree $\Phi=$

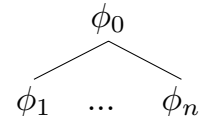

satisfies a grammar rule $\rho=\delta_{0} \rightarrow \delta_{1} \ldots \delta_{n}$ just in case: ${ }^{4}$

1. the sequence $\left\langle\phi_{0}, \phi_{1}, \ldots \phi_{n}\right\rangle$ satisfies the description $\left\langle\delta_{0}, \delta_{1}, \ldots \delta_{n}\right\rangle$,

2. $\Phi$ satisfies the Semantic Compositionality Principle, the Binding Theory, and the Anaphoric Agreement Principle,

3. if $\rho$ is a headed rule, then $\Phi$ satisfies the Head Feature Principle and the Semantic Inheritance Principle,

4. if $\rho$ is a headed rule other than the Head-Complement Rule, then $\Phi$ satisfies Part A of the Valence Principle, and

5. if $\rho$ is a headed rule other than the Head-Specifier Rule, then $\Phi$ satisfies Part B of the Valence Principle.

(18) Semantic Compositionality Principle

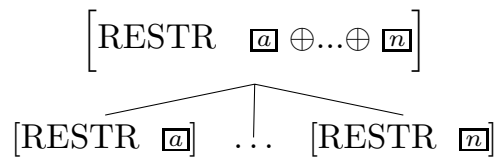

(19) The Binding Theory

Principle A: An [ANA +] synsem-struc must be outranked by a coindexed synsem-struc.

Principle B: An [ANA -] synsem-struc must not be outranked by a coindexed synsem-struc.

(20) Anaphoric Agreement PrincipleCoindexed elements agree (i.e. share a single AGR value).

\footnotetext{
${ }^{4}$ Note that clause (1) here must speak of a sequence of resolved feature structures satisfying a sequence description. This is because of identities that must hold across members of the sequence, e.g. those required by the rules themselves or by the principles given below.
} 
216 / Syntactic Theory: A Formal Introduction

(21) Head Feature Principle

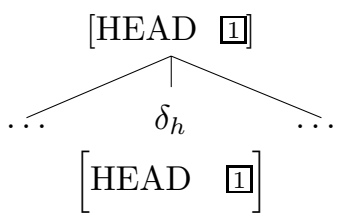

(22) Semantic Inheritance Principle

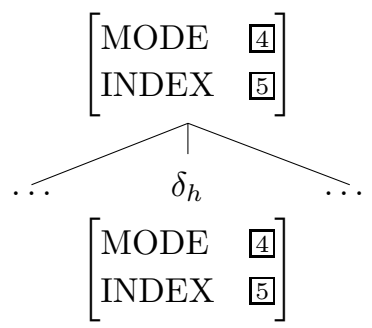

(23) Valence Principle

A

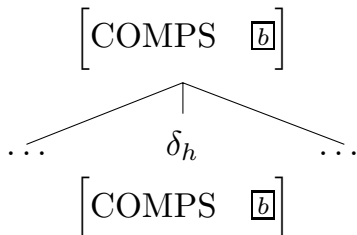

$\mathrm{B}$

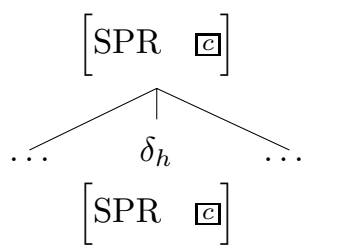

(24) Initial Symbol Definition

A CFG must include a statement of which category (or categories) can start a tree derivation. The analogue of this 'initial symbol' definition in our grammar is a constraint guaranteeing that for a phrase to stand alone, its synsem-struc must satsify the following description: 


$\left[\begin{array}{lll}\text { Shrase } & & \\ \text { SYN } & {\left[\begin{array}{lll}\text { HEAD } & \text { FORM } & \text { fin }\end{array}\right]} \\ \text { SPR } & \langle\rangle & \\ \text { COMPS } & \langle\rangle & \end{array}\right]$

\subsubsection{Lexical Rules}

(25) Singular Noun Lexical Rule

$\langle\square,[$ noun-lxm $]\rangle \Rightarrow$

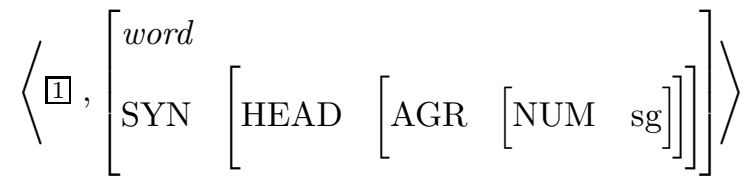

(26) Plural Noun Lexical Rule

$$
\begin{aligned}
& \left\langle\square,\left[\begin{array}{ll}
\text { noun-lxm } \\
\text { ARG-ST } & \langle\text { COUNT }+]\rangle
\end{array}\right]\right\rangle \Rightarrow
\end{aligned}
$$

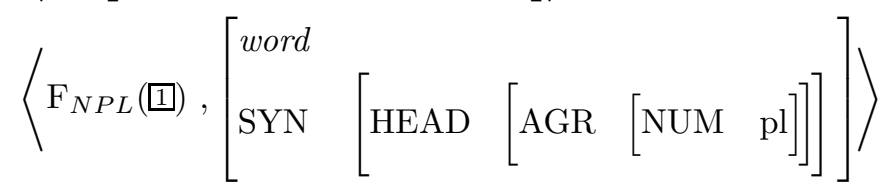


218 / Syntactic Theory: A Formal Introduction

(27) 3rd-Singular Verb Lexical Rule

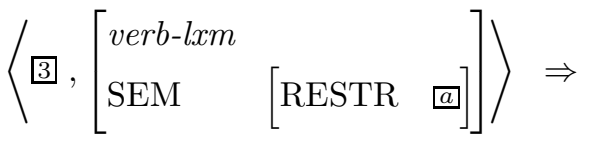

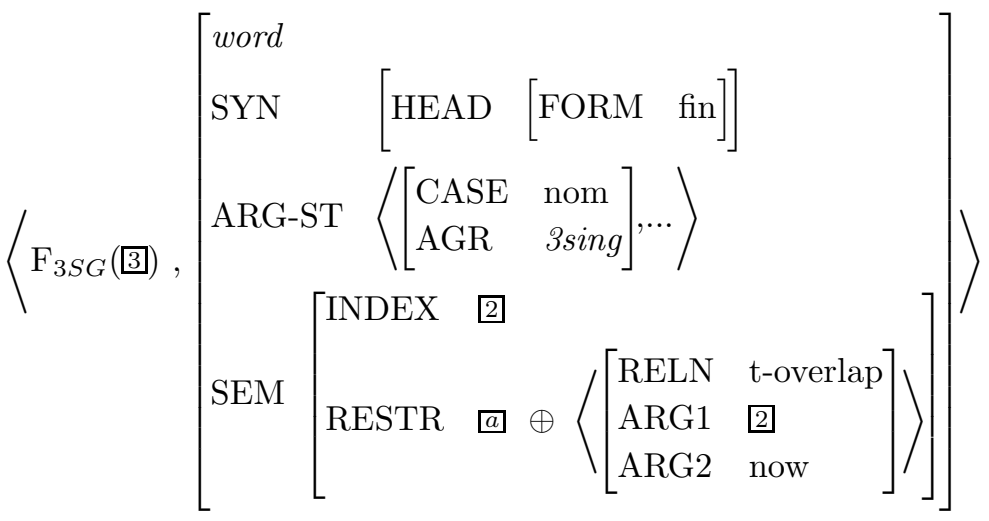

(28) Non-3rd-Singular Verb Lexical Rule

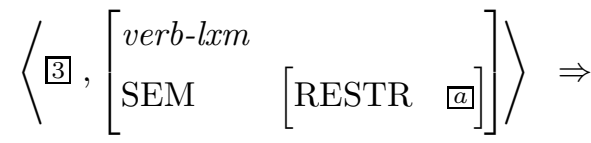

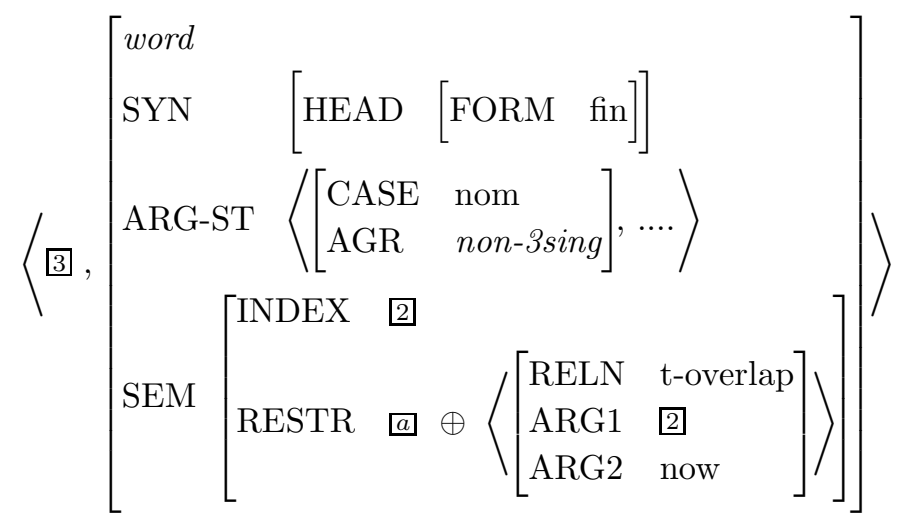


(29) Past-Tense Verb Lexical Rule

$$
\begin{aligned}
& \left\langle\text { 2, }\left[\begin{array}{ll}
\text { verb-lxm } & \\
\operatorname{SEM} & {\left[\begin{array}{ll}
\text { RESTR } & \square
\end{array}\right]}
\end{array}\right]\right\rangle \Rightarrow
\end{aligned}
$$

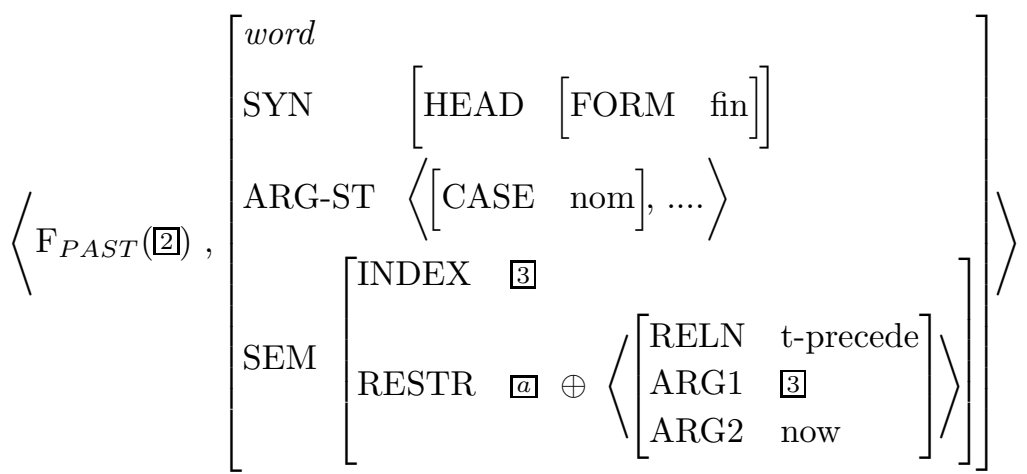

(30) Present Participle Lexical Rule

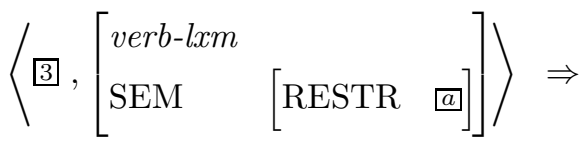

$$
\begin{aligned}
& \left.\left\langle\mathrm{F}_{P R P}(\text { 目 }),\left[\begin{array}{llll}
\text { word } & & & \\
\text { SYN } & {[\text { HEAD }} & {[\text { FORM }} & \text { prp }
\end{array}\right]\right]\right\rangle
\end{aligned}
$$

(31) Past Participle Lexical Rule

$$
\begin{aligned}
& \left\langle 2,\left[\begin{array}{ll}
\text { verb-lxm } & \\
\operatorname{SEM} & {\left[\begin{array}{ll}
\text { RESTR } & \square
\end{array}\right]}
\end{array}\right]\right\rangle \Rightarrow
\end{aligned}
$$

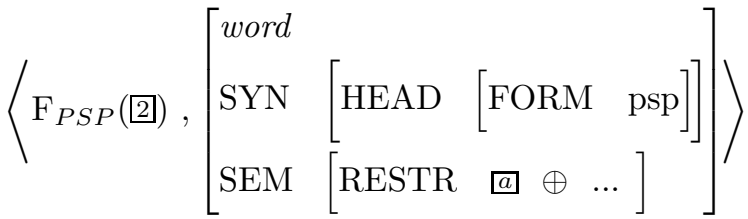


220 / Syntactic Theory: A Formal Introduction

(32) Infinitival Lexical Rule

$$
\begin{aligned}
& \left\langle 2,\left[\begin{array}{ll}
\text { verb-lxm } & \\
\text { SEM } & {\left[\begin{array}{ll}
\text { RESTR } & a
\end{array}\right]}
\end{array}\right]\right\rangle \Rightarrow
\end{aligned}
$$

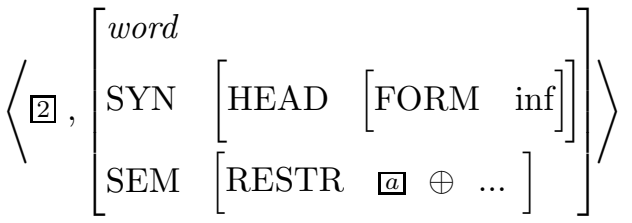

(33) Constant Lexeme Lexical Rule

$$
\langle 1,[\text { const-lxm }]\rangle \Rightarrow\langle 1,[\text { word }]\rangle
$$

(34) Agent Nominalization Lexical Rule

$$
\begin{aligned}
& \left\langle 2,\left[\begin{array}{ll}
\text { verb-lxm } & \\
\text { ARG-ST } & \left\langle\mathrm{NP}_{i}(, \text { 1]NP })\right\rangle \\
\mathrm{SEM} & {\left[\begin{array}{ll}
\operatorname{INDEX} & s
\end{array}\right]}
\end{array}\right]\right\rangle \Rightarrow
\end{aligned}
$$

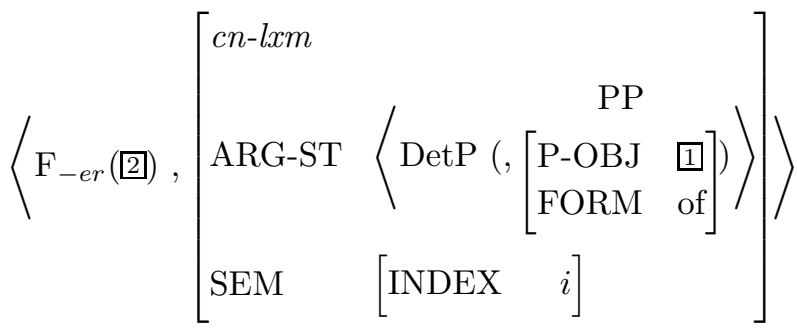




\subsection{Constraint-Based Lexicalism}

We turn now to some reflections on the relationship between the sort of grammatical descriptions in this text and what is known about the mental processes underlying human language comprehension and production. We believe it is possible to draw at least preliminary conclusions about how the flexibility and incrementality of human language processing - language comprehension in particular - should inform the design of grammars that aspire to be models of human linguistic knowledge. Adopting the familiar terminology of Chomsky (1965), we will suggest that competence grammars, if they are ever to be directly embedded within realistic models of how language is used (what Chomsky would call linguistic 'performance'), should embody certain design properties that make them consistent with these basic facts about processing.

Let us start with three basic observations about the grammar we have been developing:

1. It is SURfACE ORIENTED. Our grammar (like standard contextfree grammars) provides a reasonably simple structure that is directly associated with the string of words that constitute each sentence. The ancillary structure that has to be computed to ascertain whether a given sentence is grammatical expresses information that is straightforwardly derivable from properties of the words in the string. No additional abstract structures are posited.

2. It is CONSTRAINT-BASED. There are no operations that destructively modify any representations. The principles of the theory, the grammar rules, and the lexical entries that result once the grammar is 'closed' under lexical rules are all just constraints that interact so as to define a set of phrase structures - those that simultaneously satisfy the relevant constraints of our grammar.

3. It is STRONGLY LEXICALIST. We have localized most grammatical and semantic information within lexical entries. These lexical entries furthermore correspond directly to the words present in the sentence, which can be viewed as the key elements that drive the construction of the syntactic and semantic structure of the sentence.

Each of these three design properties, we believe, plays an important role when we turn to embedding our theory of linguistic competence within a theory of performance - specifically a theory of how language is processed. Any theory that has these design properties exemplifies a viewpoint that we will refer to as CONSTRAINT-BASED LEXICALISM $(\mathrm{CBL})$. 


\subsection{Incremental Processing}

We don't have to venture into a psycholinguistic laboratory to convince ourselves that language processing is highly incremental. We saw this already in Chapter 1, when we considered examples like (35).

(35) After finding the book on the atom, Sandy went into class, confident that there would be no further obstacles to getting that term paper done.

When we hear such a sentence, we process it as it comes - more or less word by word - building structure and partial interpretation incrementally, using what nonlinguistic information we can to make the right decisions at certain points, for example, when we encounter the PP on the atom and have to decide whether it modifies VP or NOM. We make this decision 'on-line' it seems, using a plausibility assessment of the meaning that would result from each structure.

Psycholinguists have shown us that sentence processing sometimes goes astray. GARDEN PATH examples like $(36 \mathrm{a}, \mathrm{b})$ are as remarkable today as they were when they were first brought to the attention of language researchers. ${ }^{5}$

(36) a. The horse raced past the barn fell.

b. The boat floated down the river sank.

On first encountering such sentences, almost all English speakers judge them to be totally ungrammatical. However, after seeing them juxtaposed to fully well-formed examples like (37), speakers recognize that sentences like (36) are grammatical, though very hard to process.

(37) a. The horse that was raced past the barn fell.

b. The horse taken to the hospital died.

c. The boat that was floated down the river sank.

d. The boat seen down the river sank.

Experimental researchers thought at first that these garden paths showed that certain purely linguistic processing strategies (like trying to build an S out of the NP the horse and a VP beginning with raced past) were automatic - virtually impossible to turn off. But modern psycholinguistics has a very different story to tell.

First, note that in the right context, one can eliminate the garden path effect even with the sentences in (36). The right context can even make the NOM-modifying interpretation of raced past the barn the most natural one: ${ }^{6}$

\footnotetext{
${ }^{5}$ By Bever (1970).

${ }^{6}$ This kind of effect is discussed by Crain and Steedman (1985).
} 
(38) The horse that they raced around the track held up fine. The horse that was raced down the road faltered a bit. And the horse raced past the barn fell.

The context here highlights the need to identify one horse among many, which in turn favors the meaning of the NOM-modifying structure of (36a).

Moreover, if we keep the same potential for ambiguity, but change the words, we can eliminate the garden path effect even without an elaborate preceding context. Consider examples like (39a,b).

(39) a. The evidence examined by the judge turned out to be unreliable.

b. The thief arrested by the police turned out to be our nephew.

As shown in a number of studies, ${ }^{7}$ examples like these present no more processing difficulty than their unambiguous counterparts in (40).

(40) a. The evidence that was examined by the judge turned out to be unreliable.

b. The thief who was arrested by the police turned out to be our nephew.

That is, the examples in (39), even in the absence of a prior biasing context, do not cause garden path effects.

The explanation for this difference lies in the relevant nonlinguistic information. Evidence (or, say, a particular piece of evidence) is a pretty implausible examiner and the sentence built out of a subject NP the evidence and a VP headed by examined would require precisely that interpretation. (Similar claims hold for (39b), given that a thief is unlikely to be the one doing the arresting.) That is, it is a fact about the world that examiners are animate, and since evidence is inanimate, that hypothesis about the interpretation of the sentence is implausible. The fact that the decision to reject that interpretation (and hence the associated sentential structure) is made so quickly as to be imperceptible (i.e. so as to produce no noticeable garden path effect) is evidence that language comprehension is working in a highly integrative and incremental fashion. Linguistic and nonlinguistic constraints on the interpretation are interleaved in real time.

Language understanding appears to be a process of constraint satisfaction. Competing interpretations exist in parallel, but are active to varying degrees. A particular alternative interpretation is active to the extent that evidence is available to support it as the correct interpreta-

\footnotetext{
${ }^{7}$ See, for example, Trueswell, Tanenhaus, and Garnsey (1992), Pearlmutter and MacDonald (1992), and Tabossi et al. (1994).
} 


\section{4 / Syntactic Theory: A Formal Introduction}

tion of the utterance being processed. Note, by the way, that frequency has a very important role to play here. The reason the horse raced past the barn example is such a strong garden path is that raced occurs much more frequently as a finite verb form than as the passive participle of the transitive use of race, which is precisely what the NOM-modifying reading requires. Ambiguity resolution is a continuous process, where inherent degrees of activation, for example, those correlating with gross frequency, fluctuate as further evidence for particular interpretations become available. Such evidence may in principle stem from any aspect of the sentence input or the local or discourse context. A garden-path sentence is one that has an interpretation strongly supported by initial evidence that later turns out to be incorrect.

\subsection{A Performance-Plausible Competence Grammar}

\subsubsection{Surface-Orientation}

We know that sentence processing proceeds in a more or less word-byword (or even syllable-by-syllable) fashion. In comprehending utterances, hearers use their knowledge of language to build partial hypotheses about the intended meaning. These hypotheses become more or less active, depending on how plausible they are, that is, depending on how well their meaning squares with the hearers' understanding of what's going on in the discourse.

Sometimes the process even gets short-circuited. We have all had the experience of completing someone else's utterance (a phenomenon that is, incidentally, far more common than one might imagine, as shown, e.g. by Wilkes-Gibbs (1986)) or of having to wait for someone to finish an utterance whose completion had already been made obvious by context. One striking example of this is English 'echo questions', as illustrated in the following kind of dialogue: ${ }^{8}$

[Speaker A:] Senora Maria Consuelo Bustamante y Bacigalupo is coming to dinner tomorrow night.

[Speaker B:]

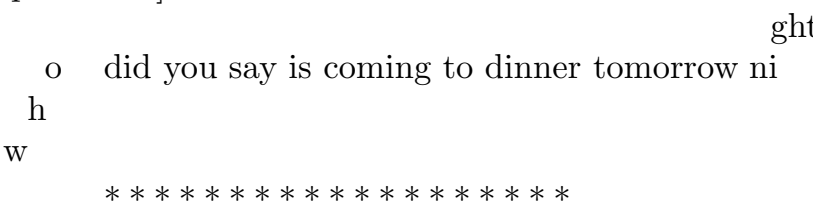

\footnotetext{
${ }^{8}$ The relative height of the type here is meant to indicate variation in fundamental frequency, i.e. pitch.
} 
In a dialogue like this, it is quite likely that Speaker A may comprehend the intent of Speaker B's utterance well before it is complete, somewhere in the region indicated by the asterisks. Presumably, this is possible precisely because Speaker A can recognize that the remainder of B's utterance is a repetition of A's own utterance and can graft that bit of content onto the partial analysis A has performed through word-byword processing of B's utterance. What examples like this show is that a partial linguistic analysis (e.g. the partial linguistic analysis of who did you, who did you say or who did you say is) is constructed incrementally, assigned a (partial) interpretation, and integrated with information from the context to produce an interpretation of a complete utterance even before the utterance is complete. Amazing, if you think about it!

So if a grammar is to be realistic, that is, if it is to be directly embedded in a model of this kind of incremental and integrative language processing, then it needs to characterize linguistic knowledge in a way that allows for the efficient incremental computation of partial analyses. Moreover, the partial grammatical analyses have to be keyed in to partial linguistic meanings, because these are what interacts with other factors in processing.

The kind of grammar we are developing seems quite compatible with these performance-driven design criteria. The lexical information that comes with each word provides information about the structure of the sentence directly, that is, about the phrases that the words are part of and about the neighboring phrases that they combine with syntactically. In addition, the words of our grammar provide partial information about the meaning of those phrases, and hence, since all phrases are built up directly from the component words and phrases in a context-free manner, there is useful partial semantic information that can be constructed incrementally, using our surface-oriented grammar.

Of course this is no rigorous proof that there is a precise performance model based on a grammar like ours, but the context-free-like architecture of the theory and the hybrid syntactic-semantic nature of the lexical data structures are very suggestive. Incremental computation of partial semantic structures, the key to modelling integrative sentence processing, seems to fit in well with our grammar.

\subsubsection{Constraint-Based Grammar}

In addition to the incremental and integrative nature of human language processing, we can also observe that there is no fixed order in which particular kinds of information are considered. For example, it is not the case that syntactic information (e.g. agreement information that might rule out a particular parse) is always consulted before semantic infor- 
mation (e.g. semantic incompatibility that would favor or disfavor some potential interpretation of an utterance). In fact, it is possible to make an even stronger claim. In examples like (42), early accessing of morphological information allows the number of sheep under discussion to be determined incrementally, and well before the nonlinguistic knowledge necessary to select the 'fenced enclosure' sense of pen, rather than its 'writing implement' sense.

(42) The sheep that was sleeping in the pen stood up.

In (43), on the other hand, the relevant information about the world - that sheep might fit inside a fenced enclosure, but not inside a writing implement - seems to be accessed well before the relevant morphological information constraining the number of sheep. ${ }^{9}$

(43) The sheep in the pen had been sleeping and were about to wake up.

So the information accessed in on-line language processing is typically made available in an order determined by the input stream, not by the constructs of any grammatical theory. In comprehending these examples, for example, a hearer accesses morphological information earlier in (42) and later in (43) precisely because the order of access is tied fairly directly to the order of the words being processed. A theory positing a fixed order of access - for example, one that said all strictly linguistic processing must be completed before nonlinguistic knowledge could be brought to bear on utterance interpretation - would not be able to account for the contrast between (42) and (43).

Finally, we know that for the most part linguistic information functions fairly uniformly in many diverse kinds of processing activity, including comprehension, production, translation, playing language games, and the like. By 'fairly uniformly' we mean that the set of sentences reliably producible ${ }^{10}$ by a given speaker-hearer is similar - in fact bears a natural relation (presumably proper inclusion) - to, the set of sentences that that speaker-hearer can comprehend. This might well have been otherwise. That there is so close and predictable a relation between the production activity and the comprehension activity of any given speaker of a natural language militates strongly against any theory on which the production grammar is independent from the comprehension grammar, for instance. This simple observation suggests rather that the differences between, say, comprehension and production should be explained

\footnotetext{
${ }^{9}$ This pair of examples is due to Martin Kay.

${ }^{10}$ That is, sentences short enough to utter in a real language-use situation. We also intend to rule out production errors.
} 
by a theory that posits distinct processing regimes making use of a single linguistic description. And that description should therefore be a process-neutral grammar of the language, which can serve each kind of process that plays a role in on-line linguistic activity. ${ }^{11}$

Observations of this sort, namely, that linguistic descriptions are used in an order-independent fashion, lead naturally to the conclusion that the constructs of linguistic theory should have an order-independent character. That is, the architecture of grammar should be such that linguistic constraints can be independently deployed in different situations. Finally, given that linguistic knowledge is process-independent, there should be no bias within a linguistic theory - whether overt or hidden, intentional or inadvertent - toward one kind of processing, rather than another.

Grammars whose constructs are truly process-neutral, then hold the most promise for the development of processing models. And the best known way to ensure process-neutrality is to formulate a grammar as a declarative system of constraints. Such systems of constraints fit well into models of processing precisely because all the information they provide is in principle on an equal footing. What these observations add up to is a view of grammar as a set of constraints, each expressing partial information about linguistic structures, rather than a system employing destructive operations of any kind. Moreover, we have also seen that these constraints should exhibit certain further properties, such as order-independence, if performance-compatability is to be achieved. The grammar we've been developing has just these design properties all the constructs of the grammar (lexical entries, grammar rules, even our general principles) are nothing more than constraints that produce equivalent results no matter what order they are applied in.

\subsubsection{Strong Lexicalism}

Our theory partitions grammatical information into a number of components whose interaction determines the well-formedness of particular examples. By far the richest locus of such information, however, is the lexicon. Our grammar rules are simple in their formulation and general in their application, as are such aspects of our formal theory as the Head Feature Principle and the Valence Principle. Most of the details we need

\footnotetext{
${ }^{11}$ The fact that comprehension extends beyond systematic production can be explained in terms of differences of process - not differences of grammar. Speakers that stray far from the grammar of their language run a serious risk of not being understood; yet hearers that allow grammatical principles to relax when necessary will understand more than those that don't. There is thus a deep functional motivation for the two kinds of processing to differ as they appear to.
} 
in order to analyze individual sentences are codified in the lexical entries (though much of it need not be stipulated, thanks to lexical rules and inheritance through the type hierarchy).

However, other divisions of grammatical labor are conceivable. Indeed, a number of theories with highly articulated rule systems and relatively impoverished lexicons have been developed in considerable detail (e.g. early transformational grammar and Generalized Phrase Structure Grammar, both of which are described briefly in Appendix B). We have argued for strong lexicalism on the basis of linguistic adequacy (along with general considerations of elegance and parsimony). It turns out, moreover, that the psycholinguistic evidence on language processing points in the same direction. Investigations of syntactic ambiguity resolution and garden path effects have shown that both phenomena are sensitive to a variety of types of information. That is, the difficulty listeners exhibit in resolving such ambiguities and overcoming garden paths is influenced by factors other than syntactic structure. These include semantic compatibility and pragmatic plausibility, type and valence of the words involved, and the frequencies with which individual words occur in particular constructions (see Tanenhaus and Trueswell (1995) for a survey of relevant results).

For example, a sentence beginning with the sequence $\mathrm{NP}_{1}-\mathrm{V}-\mathrm{NP}_{2}$ can be continued in a number of ways. $\mathrm{NP}_{2}$ could be the object of the verb, or it could be the subject of a complement sentence. This is illustrated in (44a), which can be continued as in (44b) or (44c).

(44) a. Lou forgot the umbrella ...

b. Lou forgot the umbrella was broken.

c. Lou forgot the umbrella in the closet.

Hence a listener or reader encountering (44a) must either postpone the decision about whether to attach the NP the umbrella to the VP, or decide prematurely and then have to reanalyze it later. Either way, this places a burden on the parser in at least some cases. Various experimental paradigms have been used to verify the existence of this parsing difficulty, including measuring reading times and tracking the eye movements of readers.

However, not all verbs that could appear in place of forgot in (44a) can appear in both of the contexts in (44b) and (44c). This is illustrated in (45).

(45) a. Lou hoped the umbrella was broken.

b. * Lou hoped the umbrella in the closet.

c. ${ }^{*}$ Lou put the umbrella was broken.

d. Lou put the umbrella in the closet. 
The increased parsing load in (44a) is reduced greatly when the valence of the verb allows for no ambiguity, as in (45). This has been demonstrated using the methods used to establish the complexity of the ambiguity in the first place (see Trueswell et al. (1993)). This provides strong evidence that people use lexical valence information incrementally as they process sentences.

Similarly, listeners use semantic and pragmatic information about the verb and the following NP to choose between possible attachment sites for the NP. For example, though learn may take either an NP object or a sentential complement, illustrated in (46), when the immediately following NP is not the sort of thing one can learn, people do not exhibit the level of complexity effects in parsing that show up in (44).

(46) a. Dana learned the umbrella was broken.

b. Dana learned a new theorem in class.

The same sort of effect of lexical meaning on parsing shows up with PP attachment ambiguities, like those in (47).

(47) a. The artist drew the child with a pencil.

b. Lynn likes the hat on the shelf.

In (47a), the pencil could be either the artist's instrument or something in the child's possession; in (47b), on the shelf could identify either Lynn's preferred location for the hat, or which hat it is that Lynn likes. The structural ambiguity of such sentences causes parsing complexity, but this is substantially mitigated when the semantics or pragmatics of the verb and/or noun strongly favors one interpretation, as in (48).

(48) a. The artist drew the child with a bicycle.

b. Lynn bought the hat on the shelf.

Furthermore, the information that we have been led to posit in our lexical entries has independently been found to play a role in language processing. After reviewing a number of studies on the factors that influence syntactic ambiguity resolution, MacDonald et al. (1994) discuss what information they believe needs to be lexically specified to account for the psycholinguistic results. Their list includes:

- valence;

- 'coarse-grained semantic information' (i.e. the sort of information about who did what to whom that is given in our SEM feature); and

- 'grammatically relevant features' such as 'tense..., finiteness..., voice (active or passive), number..., person..., and gender...'.

They also mention grammatical category, which we represent in our lexical entries by means of types (specifically, the subtypes of pos). In 
short, the elements MacDonald et al. list correspond remarkably well to the information that we list in our lexical entries.

\subsection{Universal Grammar: A Mental Organ?}

In the preceding sections we have argued that the design features of our grammatical theory comport well with existing evidence about how people process language. There is yet another psycholinguistic consideration that has played a central role in much work in generative grammar, namely, learnability. In this section, we briefly address the question of evaluating our theory by this criterion.

As noted in Chapter 1, Chomsky has argued that the most remarkable fact about human language - and the one he thinks linguists should be primarily concerned with explaining - is that virtually all children become fluent speakers of a language, with little apparent effort or instruction. The puzzle, as Chomsky sees it, is how people can come to know so much about language so quickly and easily. His solution in a nutshell is that people's knowledge of language is for the most part innate, not learned. This entails that much linguistic structure - namely, those aspects that are innate - must be common to all languages. Consequently, a central goal of much work in modern syntactic theory has been to develop a conception of universal grammar rich enough to permit the descriptions of particular languages to be as simple as possible.

Chomsky's strong claims about the role of innate knowledge in language acquisition are by no means uncontroversial among developmental psycholinguists. In particular, many scholars disagree with his position that the human language faculty is highly task-specific - that is, that people are born with a 'mental organ' for language which is distinct in its organization and functioning from other cognitive abilities (see, e.g. Bates and MacWhinney (1989) and Tomasello (1992) for arguments against Chomsky's position).

There can be little doubt that biology is crucial to the human capacity for language; if it were not, family pets would acquire the same linguistic competence as the children they are raised with. It is far less clear, however, that the human capacity for language is as specialized as Chomsky says. A range of views on this issue are possible. At one end of the spectrum is the idea that the language faculty is a fully autonomous module, unrelated to general cognitive capacity. At the other end is the idea that there are no specifically linguistic abilities - that our capacity to learn language arises essentially as a side-effect of our general intelligence or of other abililities. Chomsky's view is close to the 
former; Tomasello (1992) argues for something close to the latter. Other scholars have defended views somewhere in between.

The participants in this debate often seem to be talking past one another. Opponents of task-specificity tend to take a simplistic view of linguistic structure, emphasizing basic communicative functions while ignoring the intricacies of syntax that are the bread and butter of generative grammar. On the other hand, proponents of task-specificity have a tendency to leap from the complexity of their analyses to the conclusion that the knowledge involved must be innate and unique to language.

We find much of the argumentation on both sides of this controversy unconvincing, and hence we take no position in this book. Nevertheless, the theory presented here can contribute to its resolution. By making syntactic and semantic analyses explicit, we make it possible to formulate more precisely what is at issue in the debate over task-specificity. Moreover, providing formal representations of our data structures and their interactions permits us to see more clearly where there could be analogues in other cognitive domains. Our position is that the grammatical constructs we have been developing in this text are well suited to a theory of universal grammar, whether or not that theory turns out to be highly task-specific, and that the explicitness of our proposals can be helpful in resolving the task-specificity question.

To justify this claim, we will consider various components of our theory, namely: the phrase structure rules, the features and their values, the type hierarchy with its feature declarations and constraints, the definition of phrasal satisfaction (incorporating the Head Feature Principle, the Valence Principle, and the two semantic principles), the binding theory, and the lexical rules. We will find that most of these have elements that are very likely universal, and that our formulations do not prejudge the issue of task-specificity.

- Our grammar rules (with the exception of the Imperative Rule) are sufficiently general that, aside from their linear ordering of the constituents, they are natural candidates for universality. It would not be hard to factor out the ordering, so that versions of these rules could be posited as part of universal grammar. The sort of hierarchical structure induced by the rules, which we represent with trees, is arguably not unique to language: it also seems appropriate, for example, to aspects of mathematical reasoning. On the other hand, the concepts of 'head', 'complement', 'specifier', and 'modifier', which are crucial to our formulation of the rules, appear to be specialized to language. If it should turn out, however, that they can be shown to be instances of some more generally 
applicable cognitive relations, this would in no way undermine our analysis.

- Most of the features we have posited have obvious cross-linguistic application. It seems at least plausible that a more fully worked out version of the theory presented here could include an inventory of features from which the feature structures of all languages must be constructed. In later chapters, we will identify the values of some features with particular English words, a practice inconsistent with saying that the set of possible feature values is part of universal grammar. It might be possible, however, to restrict feature values to come from either the set of morphological forms of the language or a universally specifiable set. Some features (e.g. PER, GEND, COUNT) clearly reflect properties of the world or of human thought, whereas others (e.g. ANA, P-OBJ, FORM) seem specifically linguistic. Our treatment is neutral on the question of whether grammatical features will ultimately be reducible to more general aspects of cognition.

- The types we have proposed could arguably be drawn as well from a fixed universal inventory. The feature declarations associated with the types are likewise probably quite similar across languages. The constraints introduced by some types (such as NSA), on the other hand, appear to be more specific to the particular language. Our types are arranged in a default inheritance hierarchy, a kind of structure that very likely plays an important role in how people organize many kinds of information. Indeed, the use of such hierarchies in linguistics was inspired by earlier work in artificial intelligence, which suggested this sort of structure for taxonomies of concepts. The particular types we have posited appear taskspecifically linguistic, though again, this is not built into our theory.

- Our definition of Phrasal Satisfaction involves both universal and English-specific elements. As noted earlier, the Argument Realization Principle may well differ across languages. And clearly, the Case Constraint as we have formulated it applies only to English. On the other hand, the Head Feature Principle and the two semantic principles are intended to apply to all languages. Some parts of the Phrasal Satisfaction definition make reference to specifically linguistic constructs (such as grammar rules, heads, and particular features), but the idea of unifying information from diverse sources into a single structure has nonlinguistic applications as well.

- All languages evidently have some binding principles, and they 
are quite similar. Characteristically, there is one type of element that must be bound within a local domain and another type that cannot be locally bound. But there is cross-language variation in just what counts as 'local' and in what can serve as the antecedents for particular elements. Our particular binding theory is thus not part of universal grammar. Ideally, a grammatical theory would delineate the range of possible binding principles, of which the ones presented in Chapter 7 would be instances. While these principles appear to be quite language-specific, it is conceivable that they might be explained in terms of more general cognitive principles governing identity of reference.

- The lexical rules presented in the previous chapter are clearly parochial to English. A general theory of what kinds of lexical rules are possible would be desirable, but is beyond the scope of this book. The contents of these rules are quite specific to language, but their general form is one that one might expect to find in many domains: if a database contains an object of form X, then it also contains one of form $\mathrm{Y}$.

To sum up this superficial survey of the components of our theory: it contains many elements (the grammar rules, the definition of WellFormed Tree Structure, the features and types) that are plausible candidates for playing a role in a theory of universal grammar. Moreover, some elements (the binding principles, some lexical rules) probably have close analogues in many other languages. Although our central purpose in this book is to present a precise framework for the development of descriptively adequate grammars for human languages, rather than to account for the puzzle of language learnability through the development of a highly restrictive theory of universal grammar, the framework we have presented here is nevertheless quite compatible with the latter goal.

Further, our grammatical theory suggests a number of parallels between the kinds of information structures needed to account for linguistic competence and those employed in other cognitive domains. However, we need not commit ourselves on the question of task-specificity; rather, we offer the hope that increasingly precise linguistic descriptions like those that are possible within the framework developed here will help to clarify the nature of this controversy and its resolution.

\subsection{Conclusion}

Chomsky's famous distinction between knowledge of language ('competence') and use of language ('performance') has allowed syntacticians to concentrate on relatively tractable problems, by abstracting away from 
many features of the way people actually speak. But most generative grammarians agree that an optimal theory of competence will play a role in explaining many features of linguistic performance. To the extent that a theory of grammar attains this ideal, we call it 'realistic'.

We have argued in this chapter that the theory we are developing in this book does well by this criterion. Our theory, by virtue of being surface-oriented, constraint-based, and strongly lexicalist, has properties that fit well with what we know about how people process utterances and extract meaning from them. Our understanding of the mechanisms that underlie linguistic performance is incomplete at present, and many of the points discussed in this chapter remain controversial. Nevertheless, a preliminary examination of what is known about processing provides grounds for optimism about our approach to syntactic theory. Considerations of learnability also support such a favorable assessment.

\subsection{Further Reading}

Many of the issues raised in this chapter are discussed at a relatively elementary level in the essays in Gleitman and Liberman (1995). 


\section{The Passive Construction}

\section{$10.1 \quad$ Introduction}

Perhaps the most extensively discussed syntactic phenomenon in generative grammar is the English passive construction. The active/passive alternation provided one of the most intuitive motivations for early transformational grammar, and it has played a role in the development of almost all subsequent theories of grammar.

In this chapter, we present an account of the English passive using the formal mechanisms we have developed in this text. Given the strongly lexical orientation of our theory, it should come as no surprise that we treat the active/passive relationship primarily as a relationship between two verb forms, and we use a lexical rule to capture the generality of that relationship.

We begin with some data to exemplify the phenomenon in question. We then formulate our rule and explain how it works. Finally, we turn to the question of the status of the forms of the verb be that characteristically occur in passive sentences.

\subsection{Basic Data}

Consider sets of sentences (and nonsentences) like the following:

(1) a. The dog bit the cat.

b. The cat was bitten (by the dog).

c. ${ }^{*}$ The cat was bitten the mouse (by the dog).

(2) a. Pat handed Chris a note.

b. Chris was handed a note (by Pat).

c. ${ }^{*}$ Chris was handed Sandy a note (by Pat).

(3) a. TV puts ideas in children's heads.

b. Ideas are put in children's heads (by TV).

c. *Ideas are put notions in children's heads (by TV). 


\section{6 / Syntactic Theory: A Formal Introduction}

The (b)-sentences in (1)-(3) are what are standardly called 'passive'; the (a)-sentences are referred to as their 'active' counterparts. There is clearly a close semantic relationship between active and passive pairs. In particular, the semantic roles of the arguments are the same - in (1), the dog is the biter, and the cat is the one being bitten. To put it informally, in an active sentence and its passive counterpart, 'who does what to whom' is the same. The crucial difference between active and passive sentences is that the subject of the passive corresponds to the object of the active. The participant denoted by the subject of the active, if expressed at all in the passive, is referred to by the object of the preposition by. Consequently, the verb in a passive sentence always has one fewer object (that is, NP complement) than the verb in its active counterpart. This is illustrated in the (c)-sentences of (1)-(3). It follows that sentences with intransitive verbs, like (4a), normally do not have passive counterparts, as in (4b).

(4) a. The patient died.

b. * The patient was died (by the doctor).

c. ${ }^{*}$ The doctor died the patient.

Moreover, aside from this one difference, active sentences and their corresponding passives have identical valence requirements. This is illustrated in (5), where the absence of an obligatory complement renders both the active and passive sentences ungrammatical.

(5) a. *Pat handed Chris.

b. ${ }^{*}$ Chris was handed (by Pat).

c. ${ }^{*} \mathrm{TV}$ puts ideas.

d. *Ideas are put (by TV).

It would not be hard to formulate lexical entries for passive forms of verbs. To capture the generalizations stated informally above, however, we need to formulate a rule that can relate actives and passives. As was the case with the rules discussed in Chapter 8 , our passive rule is motivated by more than just parsimony. Faced with novel transitive verbs either new coinages like email or rare words like cark - English speakers can (and often do) immediately use them correctly in passive sentences. Hence a rule-governed treatment of the active/passive alternation will be psychologically more realistic than a mere listing of the passive forms for all transitive verbs.

Intuitively, then, we want a rule that does the following: 
- turns the first NP complement into the subject;

- allows the subject either to turn into the object of a PP headed by by or to be omitted altogether;

- leaves the valence features otherwise unchanged;

- leaves the semantics unchanged; and

- makes the appropriate morphological change in the form of the verb.

This last item is one we have not mentioned until this point. A moment's reflection should reveal that the morphology of the passive form of a verb (or 'passive participle', as it is commonly called) is always identical to that of the past participle; this is especially clear if we consider verbs with exceptional past participles, such as do (done), sink (sunk) and cut (cut). This generalization is captured easily in our framework by invoking the same morphological function, $\mathrm{F}_{P S P}$, for both the Past Participle Lexical Rule and the Passive Lexical Rule.

The following is a lexical rule that satisfies these desiderata:

(6) Passive Lexical Rule

$$
\begin{aligned}
& \left\langle\square,\left[\begin{array}{llll}
t v-l x m & & & \\
\mathrm{ARG}-\mathrm{ST} & \left\langle\mathrm{NP}_{i}\right\rangle & \oplus
\end{array}\right]\right\rangle \Rightarrow
\end{aligned}
$$

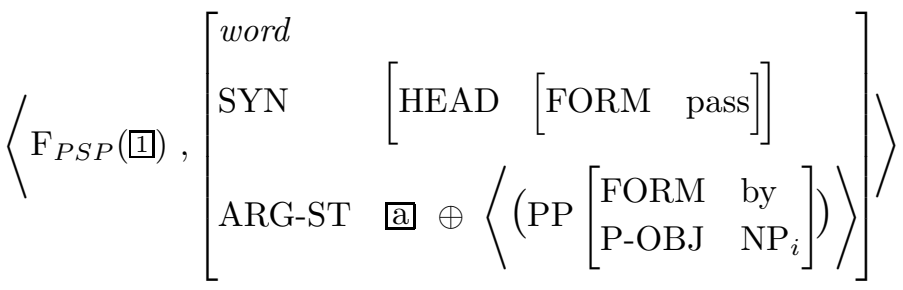

There are several points of explanation that need to be made here.

First, notice that most of the effects of the rule (which applies to any lexeme belonging to a subtype of $t v-l \mathrm{xm}$ ) are in the ARG-ST. At a coarse level of description, what the rule does is rearrange the elements of the ARG-ST list. Because of the Argument Realization Principle (Chapter 7), these rearrangements also affect the values of the valence features. Specifically, (6) eliminates the first element of the input's ARG-ST list, moves up the other elements, and introduces an optional PP at the end of the new list. More precisely, the second element (corresponding to the direct object) of the input becomes the first element (corresponding to the subject) of the output; whatever follows the second element in the input also moves up in the list. Also, an optional ' $\mathrm{PP}$ [FORM by]' is added to the end of the list, to indicate that the PP must be headed by 
the preposition by. We will abbreviate 'PP[FORM by]' as 'PP[by]' (and similarly with other values of FORM). We will return to the question of what this means formally, and discuss closely related matters in the next chapter.

Second, recall that subscripts indicate values of the feature INDEX; so (6) says that the optional PP [by] in the rule output has a P-OBJ value that is coindexed with the subject of the lexical rule input. This means that whatever semantic role the verbal lexeme assigns to its subject will be assigned to the $\mathrm{P}$-OBJ value of the $\mathrm{PP}[\mathrm{by}]$ of the passive word, and hence to the prepositional object within the PP [by] (see below). Likewise, since the verbal lexeme's object - the first element in the list - is identified with the subject of the passive word, it follows that the index of the subject of the passive word is the same as that of the verbal lexeme's direct object. Therefore, since the semantics remains unchanged by this lexical rule (in virtue of not being mentioned), the semantic role of the active object will be the same as that of the passive subject. The overall result of this rule, then, is to shift the role assignments from subject to $\mathrm{PP}[\mathrm{by}]$ and from object to subject.

Third, note that the passive rule does not mention case at all. Verbal lexemes do not specify CASE values for any of their arguments (in English); hence, though the lexeme's object NP becomes its subject, there is no need to 'unassign' an accusative case specification. All nonsubject arguments of verbs must be accusative, but the constraint that guarantees this (namely, the Case Constraint - see Chapter 8, section 8.2) applies to lexical trees (word structures), not to lexemes. (See the definition of lexical satisfaction in Chapter 9, section 9.2.4.) Nor does the passive rule assign nominative case to the first argument of the rule output, as one might expect on the basis of examples like (7).

(7) a. He was arrested by the police.

b. *Him was arrested by the police.

The nominative case of the subject in examples like (7) is determined by the auxiliary verb was, whose SPR value is unified with that of the passive VP, as discussed in the next section. There are in fact instances of passive verbs whose subjects are not nominative, as in (8).

(8)

$$
\left\{\begin{array}{c}
\text { Him } \\
\text { His } \\
* \mathrm{He}
\end{array}\right\} \text { being arrested by the police upset many people. }
$$

Our passive rule achieves the desired effect in such instances by leaving the subject of the passive word unspecified for CASE. Hence, whatever 
case requirements the particular grammatical context imposes will determine the CASE value of a passive verb's subject. ${ }^{1}$

Fourth, the rule says that passive verbs are [FORM pass]. The justification for having a separate value 'pass' for the FORM of passive verbs has not yet been provided; this will be addressed in the next section.

Returning to the use of the FORM feature on the PP in (6), recall that FORM has so far been used primarily for distinguishing among verb forms. But in the Agent Nominalization Lexical Rule presented in Chapter 8, we already made use of the FORM feature on PPs: a PP specified as [FORM of] was meant to be one that could only be headed by the preposition of. In fact, we want to emply the feature FORM more generally, to mark the choice of preposition in other contexts as well. Since the set of prepositions in English is a relatively small, closed set, we might (in the limiting case) have a separate value of FORM for each preposition. Having FORM values for prepositions allows us, for example, to represent the fact that the verb rely requires a PP complement headed by either on or upon. The FORM value of the lexical preposition will be shared by the entire PP (since FORM is a HEAD feature and hence is governed by the Head Feature Principle), and it will therefore allow for by-phrases of the sort sketched in (9).

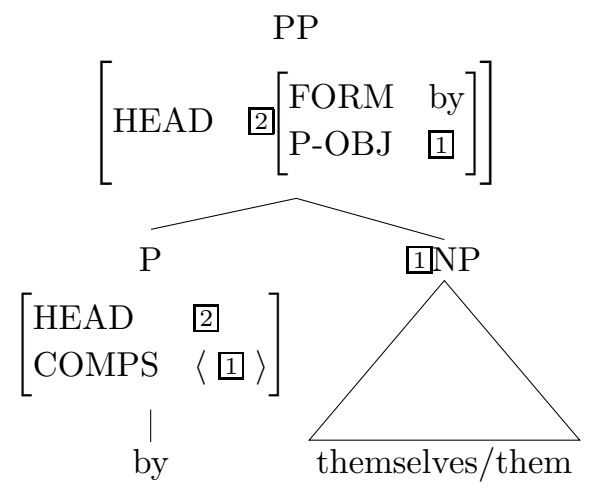

Crucially, we assume by is an argument-marking preposition that bears an NP category as its P-OBJ value. Thus whatever index the passive participle assigns to the $\mathrm{P}$-OBJ value of its $\mathrm{PP}[\mathrm{by}]$ complement will be identified with the index of the NP object within that PP, along with all the other information identified as 1 in (9).

The effect of the Passive Lexical Rule, then, is to map lexical entries like (10) into word entries like (11).

\footnotetext{
${ }^{1}$ Verbal gerunds like being in (8), for example, might lexically specify the case of their subject (which is identified with the subject of the passive participle in (8)).
} 
240 / Syntactic Theory: A Formal Introduction
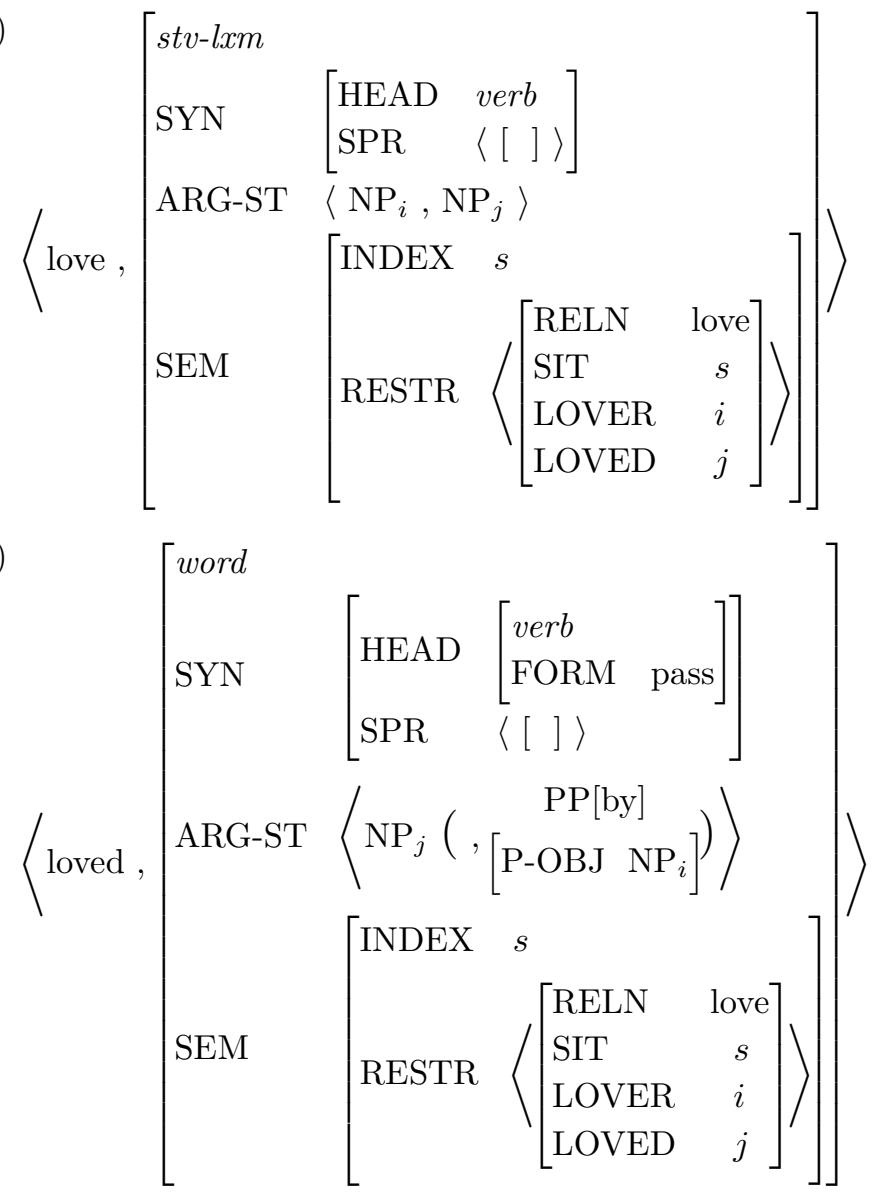

Note that in both (10) and (11), the element on the SPR list is not identified with any member of the ARG-ST list. But in any word structure that satisfies a lexical entry like (11), the element on the SPR list must be identified with the first ARG-ST member, courtesy of the ARP.

\subsection{The Verb $B e$ in Passive Sentences}

What about the forms of be, which in all our examples (so far) immediately precede the passive participle? The first thing to observe is that passive participles can also occur in environments that lack any form of be. Some examples are given in (12). 
(12) a. The cat got bitten (by the dog).

b. Liked by many people but respected by few, Jean will have to run an aggressive reelection campaign.

c. Anyone handed a note will be watched closely.

Hence, though some form of be is typical in passive sentences, it would have been a mistake to try to build it into the rule introducing the passive form of verbs. Rather, we need to provide an analysis of the relevant lexical entry for be that links its occurrence to the presence of a passive participle. ${ }^{2}$

More precisely, our analysis needs to say that the passive be takes a VP[FORM pass] argument - that is, its ARG-ST list contains a VP[FORM pass]. A few points are worth noting here. First, this is the first time we have considered VP arguments/complements in detail, though our Head-Complement Rule permits them. We will see many more soon. Second, since FORM is a HEAD feature, a verb's FORM value will show up on its mother VP node. This allows us to say that this be must have a passive complement.

The trickiest and most important aspect of our analysis of be in passives is how we deal with the subject (i.e. with the value of SPR). In a sentence like (1b), the agreement indicates that the cat should be treated as the subject (that is, the SPR) of was; note the unacceptability of * The cat were bitten. But in our discussion of passive participles in the previous section, the cat was treated as the subject of bitten. This was necessary for semantic reasons (i.e. to ensure that the cat functions semantically as the thing bitten, rather than as the biter), and to capture the correspondence between the valence values of the active and passive forms. It seems we need to treat the cat as the subject of both was and bitten. The data about the case marking on the subjects of passive verbs, illustrated in (7)-(8), point to the same conclusion: The finite forms of be (e.g. was) are the only forms of be that allow a nominative case subject - an instantiation of the more general fact that all finite verb forms take nominative case subjects.

To represent the fact that be and its passive VP complement share the same subject, we use the familiar device of tagging, specifying that two SPR values are identical. We can now formulate the lexical entry for the passive be as follows:

\footnotetext{
${ }^{2}$ We'll return to the issue of whether we can analyze other uses of be in terms of this same lexical entry.
} 
242 / Syntactic Theory: A Formal Introduction

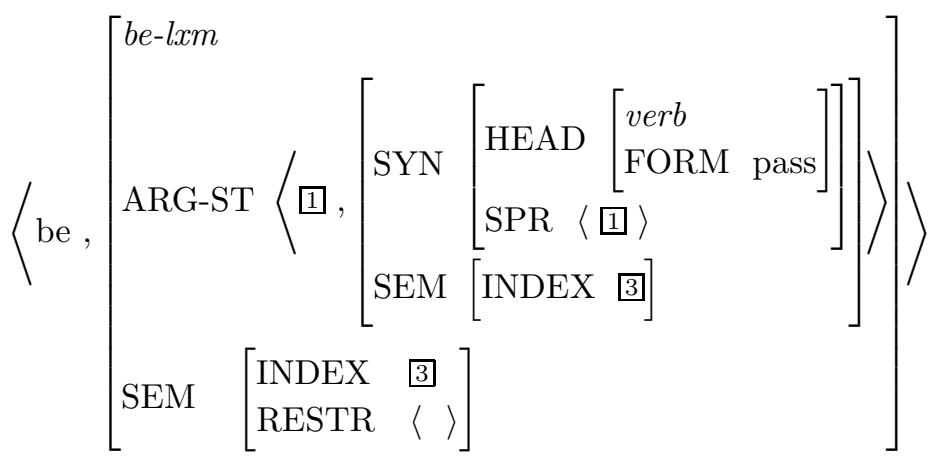

What this entry says is that be belongs to a new type be-lxm (a subtype of verb-lxm whose properties do not yet concern us) and takes a VP argument headed by a passive participle. In addition, the subject of the sentence serves as subject for both this form of be and for its passive argument. And because be adds nothing to the meaning except the information that the complement's INDEX value is the same as that of be (which ensures that that index is also the INDEX value of the VP be heads), (13) also guarantees that the semantics of the verb phrase headed by be is identical to the semantics of be's VP complement.

We will see in the next two chapters that the idea of having a verb and its argument share a subject is extremely useful in describing a number of phenomena. In Chapter 13, we will see in addition how using lexical types can simplify lexical entries such as these.

\subsection{An Example}

We conclude this chapter with a detailed analysis of example $(2 \mathrm{~b})$. The phrasal SD we need to license is the following: 
The Passive Construction / 243

(14)

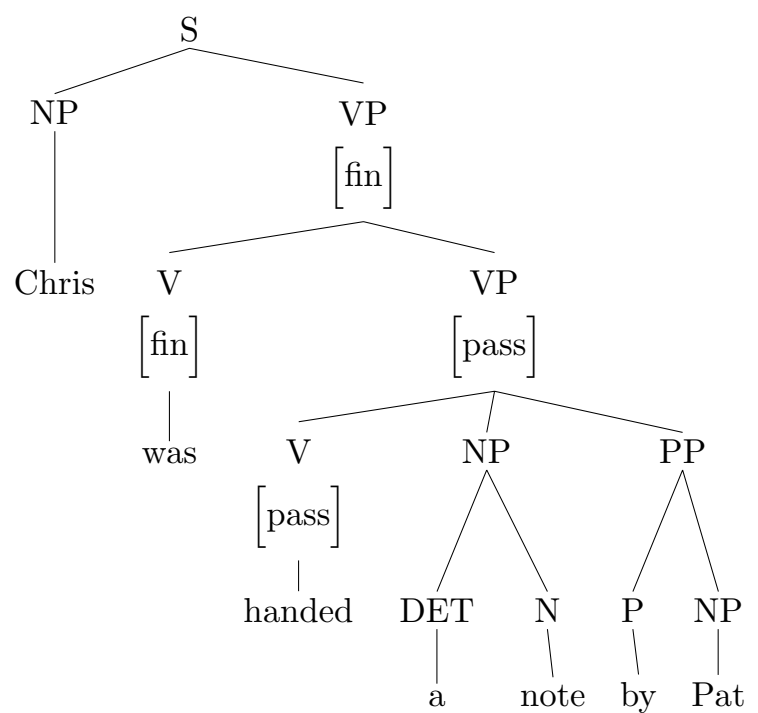

And the entry for was is:

(15)

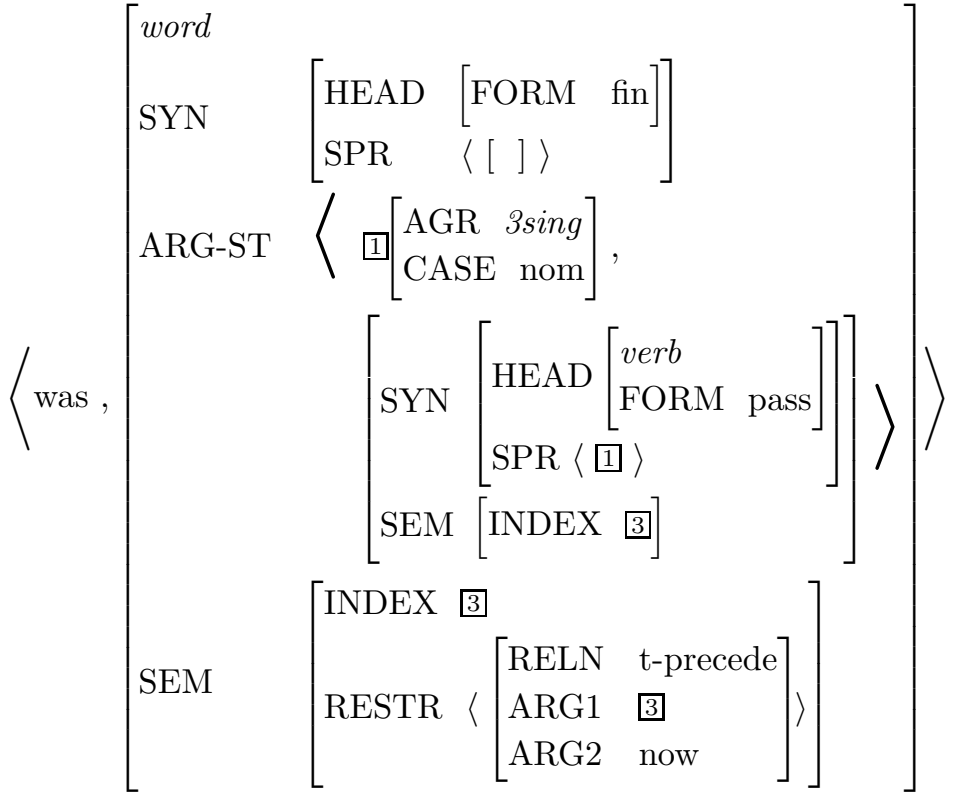


This is the same as (13), except that it is finite (i.e. [FORM fin]), has past tense semantics, and requires a third-person singular subject. ${ }^{3}$ Note that the subject is identical to the complement's subject (as was the case in (13)) and that the verb's SPR value remains underspecified. However, the lexical entry in (15) gives rise to the lexical SD shown in (16), where the SPR and and ARG-ST lists share a member and the VP[pass] is included in the COMPS specification, both courtesy of the ARP.

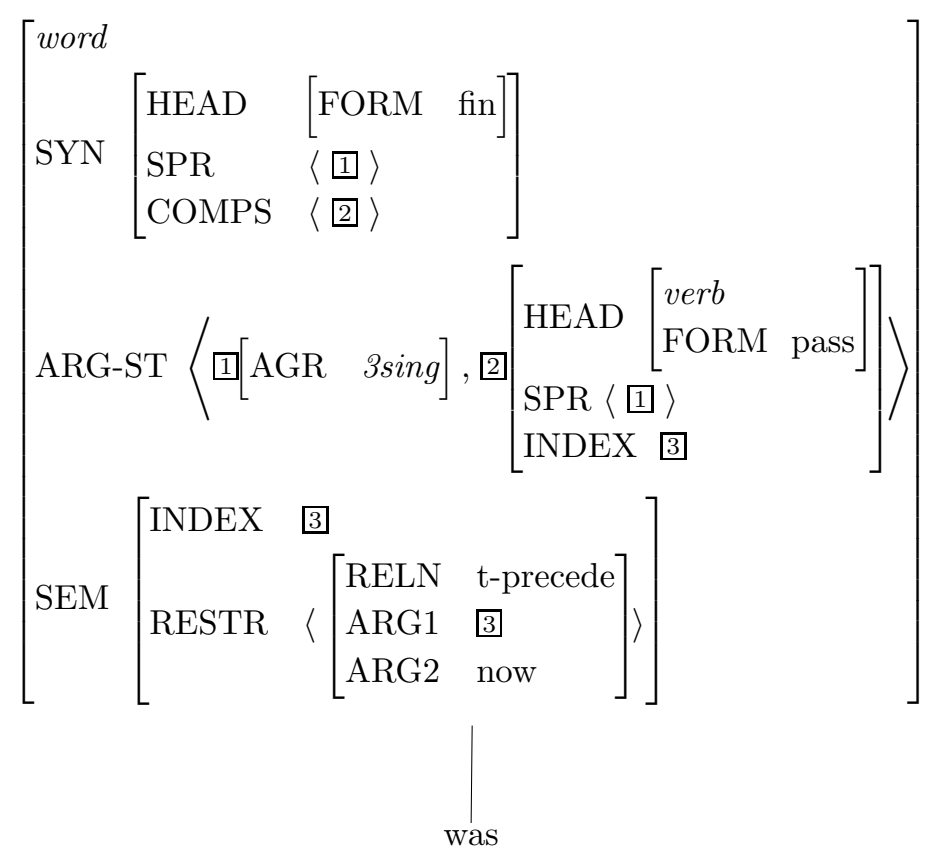

So now let us consider the SD of the VP[pass], whose head is the passive participle handed. The basic lexical entry for hand is something like the following:

\footnotetext{
${ }^{3}$ The first-person singular pronoun $I$ also occurs with was, as in $I$ was handed a note. We are assuming here that was has distinct entries for its uses with firstperson and third-person subjects. Alternatively, the entry could be elaborated to have a disjunction in the AGR value of the first element of the ARG-ST. See Chapter 8 , footnote 18 for a discussion of this.
} 
The Passive Construction / 245

(17)

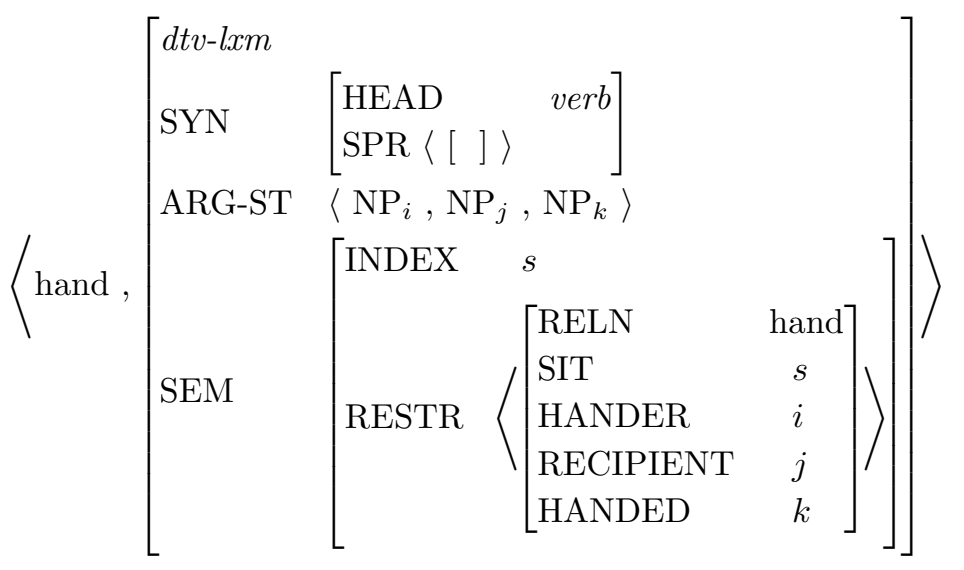

This lexeme undergoes the Passive Lexical Rule, yielding the following lexical entry:

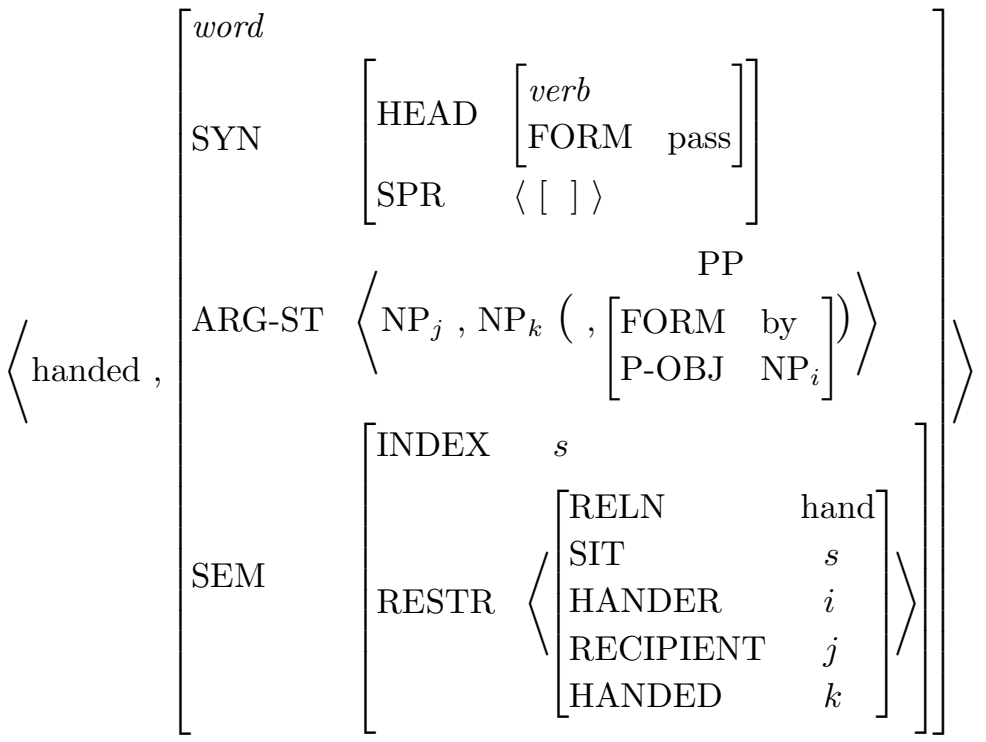

And this forms the basis for lexical SDs like (19): 
246 / Syntactic Theory: A Formal Introduction

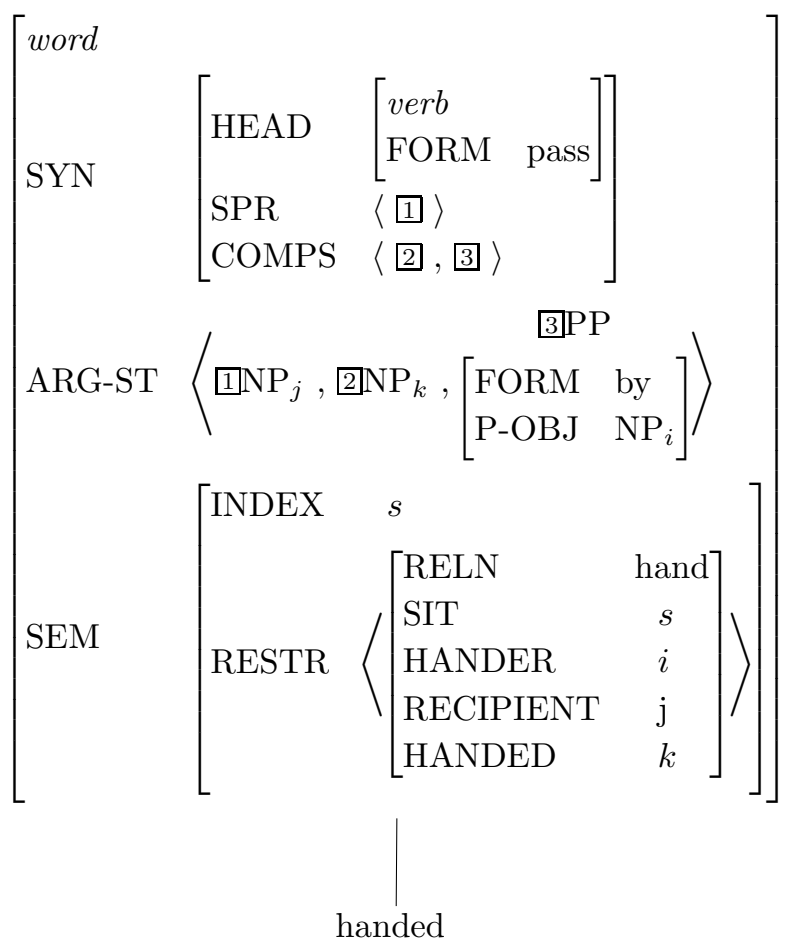

This is consistent with the use of handed in (14). (19) fits into the larger SD corresponding to the VP[pass] shown in (20). 
The Passive Construction / 247

(20)

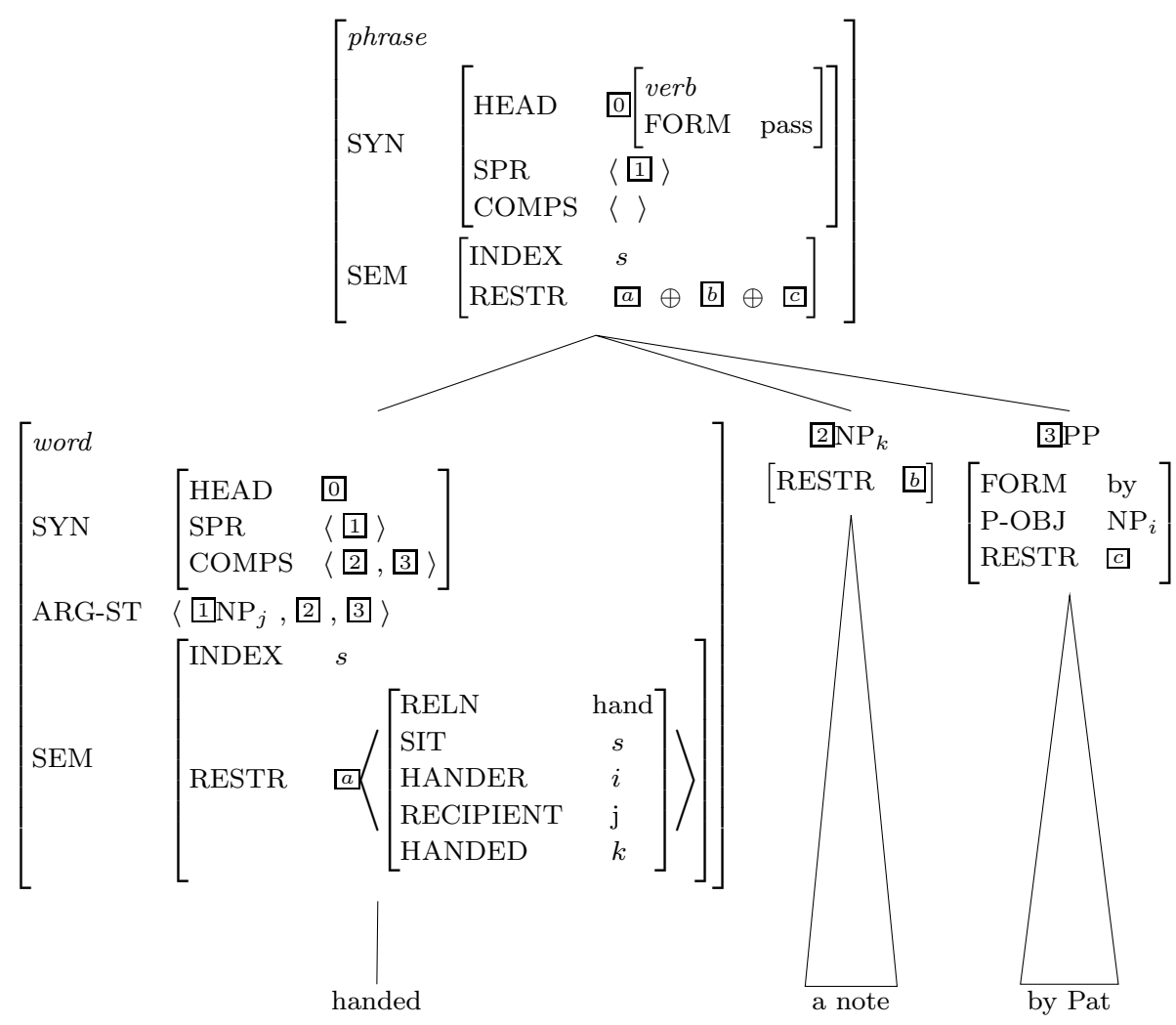


As usual, the HEAD, SPR, and INDEX values of the mother are the same as those of the head daughter (courtesy of the HFP, the Valence Principle, and the Semantic Inheritance Principle, respectively), and the mother's RESTR value is the sum of the daughters' RESTR values (courtesy of the Semantic Compositionality Principle).

This VP[pass] combines with the lexical SD in (16) to form the SD for the VP[fin] in (14), which is shown in more detail in (21).

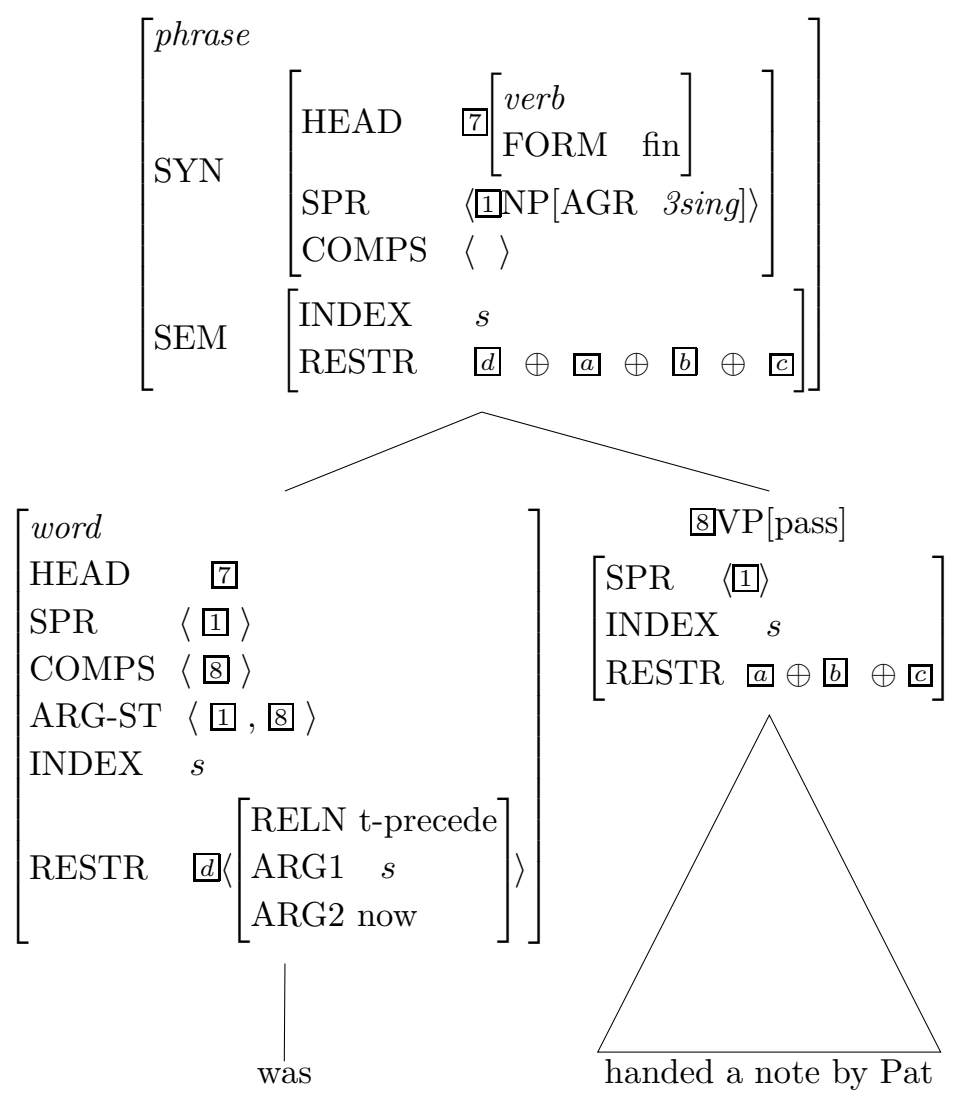

Again note the effect of the HFP, the Valence Principle, the Semantic Compositionality Principle, and the Semantic Inheritance Principle.

And finally, this VP combines with the subject NP in (14). Since the NP dominating Chris is singular, it is consistent with the SPR specification in (21). Hence Chris (more precisely the synsem-struc of the mother of the SD dominating Chris) is the subject of both was and handed. This assigns the correct semantic interpretation to the sentence: Chris 
plays the recipient role of the handing relation. The other two roles are straightforwardly determined by the indexing shown in (20).

\. Problem 1: Passive and Binding Theory

The analysis of passive just sketched makes some predictions about binding possibilities in passive sentences. Consider the following data: ${ }^{4}$

(i) $\mathrm{She}_{i}$ was introduced to herself $f_{i}$ (by the doctor).

(ii) ${ }^{*}$ She $_{i}$ was introduced to her ${ }_{i}$ (by the doctor).

(iii) The barber $_{i}$ was shaved (only) by himself . $_{\text {. }}$

(iv) ${ }^{*}$ The barber $_{i}$ was shaved (only) by $\operatorname{him}_{i}$.

(v) The students $i$ were introduced to each other (by Leslie).

(vi) $^{*}$ The students $i$ were introduced to them ${ }_{i}$ (by Leslie).

(vii) Unfortunately, he ${ }_{i}$ was introduced to the crowd by himself . $_{i}$

(viii) ${ }^{*}$ Unfortunately, he ${ }_{i}$ was introduced to the crowd by himi ${ }_{i}$.

Assuming that to and by in these examples are uniformly treated as argument-marking prepositions, does the treatment of passives sketched in the text correctly predict the judgements in (i)-(viii)? If so, explain why; if not, discuss the inadequacy of the analysis in precise terms. Be sure to provide a detailed justification of your answer.

\section{Problem 2: Pseudopassives}

Consider the following passive sentences:

(i) Dominique was laughed at by the younger kids.

(ii) This bed was slept in by the ambassador to Dalmatia.

(iii) This problem is talked about in every home.

A. Explain why our current passive rule does not allow sentences like (i)-(iii) to be generated.

\footnotetext{
${ }^{4}$ It may require a little imagination to construct contexts where such examples have a plausible meaning, e.g. a doctor dealing with an amnesia victim. Being able to construct such contexts is an essential part of being able to understand what conclusions to draw from the fact that some sentence you are interested in doesn't sound completely acceptable.

We know of cases where grammatical deviance has not been separated with sufficient care from semantic implausibility. For example, sentences like ? I smell funny to myself have on occasion been cited as ungrammatical. However, a bit of reflection will reveal, we think, that what is strange about such examples is the message they convey, not their grammar. If one needed to convey that one's own olfactory self-impression was strange (in whatever odd context such a need might arise), then I smell funny to myself is probably the most straightforward way the grammar of English allows such a meaning to be expressed.
} 
B. Propose an additional lexical rule that will produce appropriate lexical entries for the passive participles in these sentences. [Hints: This is difficult, but feasible if you make a few assumptions. First, don't try to generate the passive lexical entries for (i)-(iii) directly with one rule. Instead, formulate a rule whose outputs will then undergo the Passive Lexical Rule to provide the lexical entries you will need to account for these examples. Second, since the outputs of your lexical rule will always have to undergo the Passive Lexical Rule, you will want to specify this fact in the output of your rule (using the FORM feature). Third, assume that the prepositions involved in examples of this sort are all argument-marking prepositions - that is, they have P-OBJ values. Your rule will need to use these $P-O B J$ values (and the FORM values of the prepositions) in producing the entries that then get passivized, to license examples like (i)-(iii).]

C. Illustrate how your rule works by sketching the input and appropriate output for one of the passive participles in (i)-(iii).

D. Assuming the lexical entry in (iv), does the rule you formulated in (B) predict that both (iii) and (v) are grammatical?

(iv)

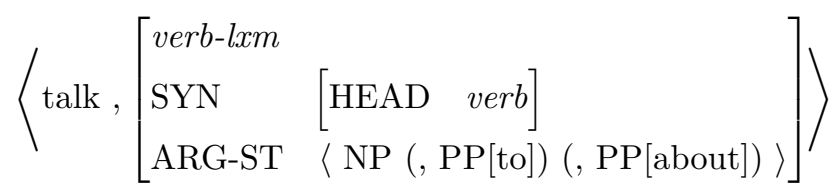

(v) This person was talked to by every teacher.

Explain your answer.

\section{\1 Problem 3: The Dative Alternation}

In Chapter 8, we mentioned the possibility of formulating a lexical rule describing the 'dative alternation' - that is, a class of verbs that appear in both of the valence patterns exemplified in (i) and (ii):

(i)

Birch $\left\{\begin{array}{l}\text { gave } \\ \text { handed } \\ \text { sold } \\ \text { loaned } \\ \text { mailed }\end{array}\right\}$ Brooke a book.


The Passive Construction / 251

(ii)

Birch $\left\{\begin{array}{l}\text { gave } \\ \text { handed } \\ \text { sold } \\ \text { loaned } \\ \text { mailed }\end{array}\right\}$ a book to Brooke.

A. Provide an argument that this alternation is productive enough (that is, systematic enough) to warrant positing a rule of some sort.

B. Formulate a lexical rule for the dative alternation. [Hint: You can choose either of the valences illustrated in (i) and (ii) as the input and the other as the output. It should not be easier one way than the other.]

C. Show how your rule interacts with the Passive Lexical Rule to make possible the generation of both (iii) and (iv)

(iii) Brooke was handed a book by Birch.

(iv) A book was handed to Brooke by Birch.

D. Explain why your rule correctly fails to license (v) (or, more precisely, fails to license (v) with the sensible meaning that the book was the thing handed to Brooke).

(v) ?*A book was handed Brooke by Birch.

\subsection{Further Reading}

The English passive has been analyzed and reanalyzed throughout the history of generative grammar. Among the most influential works on the subject are: Chomsky (1957), Chomsky (1965), Chomsky (1970), Perlmutter and Postal (1977), Wasow (1977), Bresnan (1982b), Burzio (1986) and Postal (1986). 

11

\section{Nominal Types: Dummies and Idioms}

\subsection{Introduction}

In the last chapter, we presented a lexical entry for the verb be as it occurs in passive sentences. We begin this chapter with a consideration of how to generalize the formulation of this lexical entry to cover other uses of be as well. This will lead us to the use of forms of be in combina-

tion with the subject there as a way of presenting an entity or asserting its existence, as in (1).

(1) a. There are storm clouds gathering.

b. There is a monster in Loch Ness.

This, in turn, will take us to an examination of other NPs that seem to have very restricted distributions, and whose semantic contributions cannot readily be isolated from the meanings of the larger constructions in which they occur. Examples are the use of it in sentences like (2a) and advantage in $(2 \mathrm{~b})$.

(2) a. It is obvious that Pat is lying.

b. Pat took advantage of the opportunity.

\subsection{Be Revisited}

The lexical entry for be presented in the last chapter demanded a VP[FORM pass] complement, but of course forms of be occur with a variety of other types of complements:

(3) a. Pat is on the roof.

b. Pat is the captain of the team.

c. Pat is fond of Chris.

d. Pat is singing the blues. 


\section{4 / Syntactic Theory: A Formal Introduction}

Such examples show that the possible complements of be include, besides VP[FORM pass], at least PP, NP, AP, and VP[FORM prp]. At first glance, one might think that this could be handled simply by removing the FORM feature (and hence, implicitly, the part of speech information) from the second element of the ARG-ST list in the lexical entry for passive be - that is, allowing any type of phrase (of the appropriate valence) as a complement. However, the distribution of be is not quite this free.

(4) a. *Pat is likes Chris.

b. *Pat is hate Chris.

c. *Pat is mere.

These examples show that only some verb forms can head a VP complement of be and that not all adjectives can head AP complements of $b e$. The traditional name for the kind of phrase that can appear after $b e$ is 'predicative', so we will introduce a binary feature PRED to encode this distinction. So fond is [PRED +], while mere is [PRED -], though both have HEAD values of type adjective. Likewise, passive and present participles are [PRED +], and all other verb forms are [PRED -]. The type verb-lxm can thus be associated with the constraint: [PRED -].

\section{\) Problem 1: Predicative Verb Forms}

How could our grammar be modified to accommodate (in as general a way as possible) the fact that passive and present participles are $[$ PRED +] but all other verbs are [PRED - ]? Be as precise as you can. [Hint: Lexical rules preserve all information that isn't explicitly contradicted.]

Using the feature PRED, we can reformulate the lexical entry for be to handle not only passive VP complements, but also complements like those in (3). The new formulation ${ }^{1}$ is the following: ${ }^{2}$

\footnotetext{
${ }^{1}$ We will incorporate this entry (in slightly revised form) into our lexical type hierarchy in Chapter 13, sec. 2.1.

${ }^{2}$ The formulation in (5) requires that predicative nominals (that is, NP[PRED +]) have a nonempty SPR - more specifically, that they are [SPR $\langle\mathrm{NP}\rangle]$ to handle examples like (i):

(i) Pat is a scholar.

Since NPs normally have empty SPR values, our account is incomplete. We will not attempt to provide a solution to this problem here. Notice, however, that this syntactic distinction between predicative and nonpredicative NPs reflects a semantic difference between two uses of certain NPs: one involving properties; the other individuals. Thus, the NP a scholar in (i) is used to predicate a property of Pat
} 
Nominal Types: Dummies And Idioms / 255

$(5)$

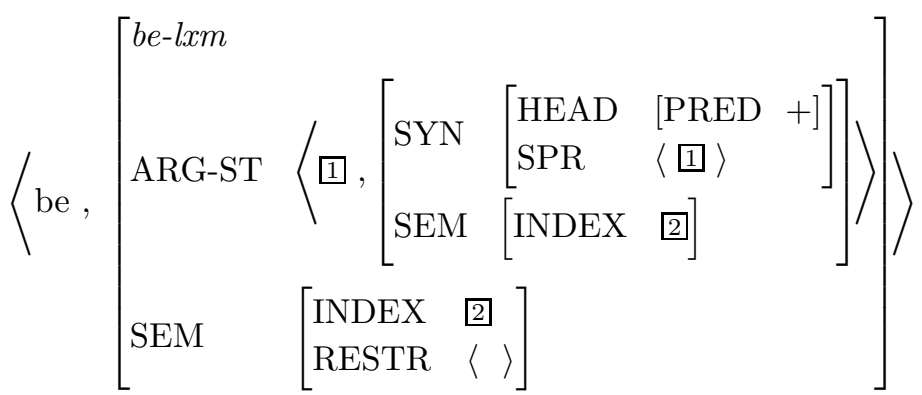

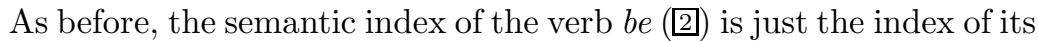
predicative complement - the verb contributes nothing to the semantics of the sentences; it is just a syntactic placeholder. Indeed, in many languages (including some dialects of English) the meanings like those expressed by the sentences in (3) would normally be expressed without any verb at all, as in the following examples:

(6) a. Ona xorošij vrač

she good doctor

'She is a good doctor.'

(Russian)

b. A magyar zászló piros-fehér-zőld.

the Hungarian flag red-white-green

'The Hungarian flag is red, white, and green.'

(Hungarian)

\subsection{The Existential There}

Consider another sort of sentence that involves be:

(7) a. There is a unicorn in the garden.

b. There were many people fond of Pat.

c. There are people looking through the window.

d. There was a felon elected to the city council.

These involve a nonreferential subject, there (often called the 'dummy' there), an NP following be, and a [PRED +] phrase following the NP. We can see that there are in fact two complements and not just one complex

(scholarliness) and hence its semantic mode is actually prop, whereas the same string of words in (ii) is used simply to make reference to an individual, i.e. its semantic mode is ref.

(ii) A scholar arrived. 
one (that is, an NP with some kind of modifying phrase attached to it) on the basis of sentences like (8).

(8) a. There is a seat available.

b. ${ }^{*}$ A seat available was in the last row.

c. ${ }^{*}$ Pat took a seat available.

d. ${ }^{*}$ I looked for a seat available.

If a seat available in (8a) were a single NP, we would expect it to be able to appear in other typical NP positions, such as those in (8b-d). So a seat and available must be two separate arguments of be. But if this use of be takes a subject and two more arguments, then it cannot be subsumed under (5), whose ARG-ST list contains only two elements. Hence, we will need a separate lexical entry for this lexeme, which we will call the 'existential be'.

Stating the restrictions on the existential be's complements is not difficult. ${ }^{3}$ But restricting the subject to the word there is not entirely trivial. This is the first case we have seen in which a verb requires that a particular word be its subject. We have, however, previously encountered an instance in which a verb specified that a specific word must head one of its complements. This was in the passive construction: passive forms of verbs can always take an optional PP headed by by to express the argument of the passive that corresponds semantically to the subject of the active. Similar selections are involved with other verb-preposition pairs, such as rely and on. Indeed, the argument-marking prepositions discussed in Chapter 7 are often selected by verbs, sometimes quite idiosyncratically.

Recall that to deal with the argument-marking prepositions, we extended the feature FORM (previously used only for distinguishing verbal inflections) to prepositions, and introduced new FORM values such as 'by', 'to', etc. This same device can now be used with nouns and noun phrases. We can put the feature specification [FORM there] in the lex-

\footnotetext{
${ }^{3}$ This is an oversimplification (as is almost any claim that some aspect of grammar is easy). Examples like (i) and (ii) are markedly worse than sentences like (7):

(i) ?*There is each unicorn in the garden.

(ii) ?There was the felon elected to the city council.

It is often claimed that the postverbal NP in existential sentences must be indefinite, but this is too strong: examples like (ii) are acceptable if interpreted as part of a listing of exemplars of something, and sentences like There is the cutest puppy outside are commonplace (in certain styles, at least). We will not pursue the problem of characterizing the so-called definiteness restriction on the NPs in existential sentences, on the assumption that the restriction is actually a semantic one.
} 
ical entry for the existential there, and require that the subject of the existential be must be [FORM there]. ${ }^{4}$

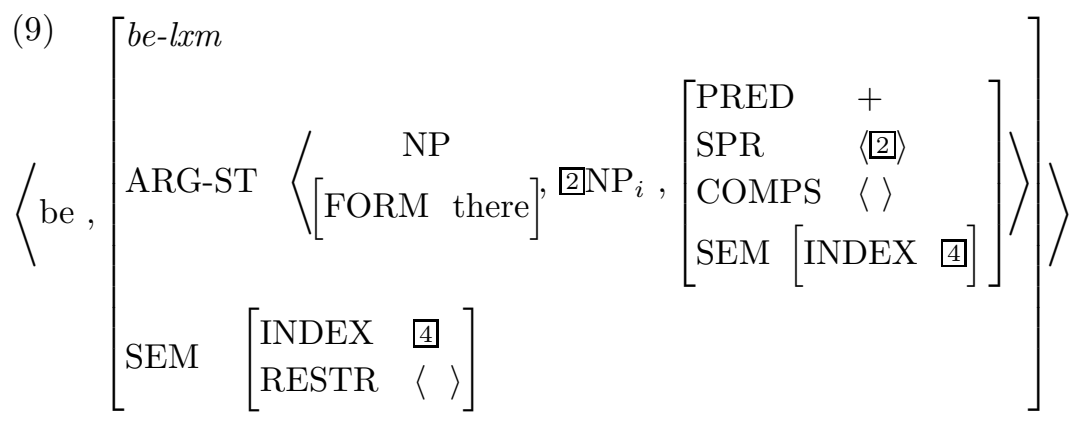

Notice that existential be contributes nothing to the meaning of the sentence, except the identification of its index with that of its predicative complement. This index will be inherited by phrases projected from existential be (and words formed from be). Moreover, since the NP argument is identified with the SPR of the predicative complement, the semantics of these two will be combined within the VP in the same way as they would be in a simple subject-predicate sentence, namely, by merging the RESTR lists of the daughters. ${ }^{5}$ Thus, the sentences in (7) are analyzed as paraphrases of those in (10). ${ }^{6}$

(10) a. A unicorn is in the garden.

b. Many people were fond of Pat.

c. People are looking through the window.

d. A felon was elected to the city council.

We turn now to the lexical entry for the existential there. Its key feature is that it is the only word that is [FORM there]. Hence, the SPR

\footnotetext{
${ }^{4}$ By extending FORM to nouns in this way, we are allowing a potentially unlimited number of possible values for this feature. In actual practice, we believe that the number of words entering into such morphologically-sensitive co-occurrence relations in any language is quite manageable.

${ }^{5}$ The notation ' $\mathrm{NP}_{i}$ ' means that this $\mathrm{NP}$ is required to bear an index; see below.

${ }^{6}$ This account of the semantics of the existential there construction is only a first approximation. For one thing, the use of there involves an explicit assertion of existence not associated with sentences like (10). In addition, it seems that the [PRED +] phrase in the there construction must denote a potentially transient property of the referent of the NP, whereas this is not required in the analogous examples without there. This is illustrated in (i)-(iv):

(i) A vase is blue.

(ii) ${ }^{*}$ There is a vase blue.

(iii) A unicorn was the winner of the Kentucky Derby.

(iv) ${ }^{*}$ There was a unicorn the winner of the Kentucky Derby.

We will not pursue these subtle semantic issues here.
} 
value of (9) uniquely picks out this word as the subject. Non-dummy NPs (proper nouns, pronouns, and phrases headed by common nouns alike) will now be specified as [FORM none], in virtue of a default constraint which we will associate with the type noun-lxm. The existential there is also exceptional in that, although it is a pronoun, it has no referential function, and under our analysis, as noted in the preceding paragraph, it does not contribute to the meaning of the sentences in which it occurs (but see footnote 6).

The lexical entry for existential there, then, is the following:

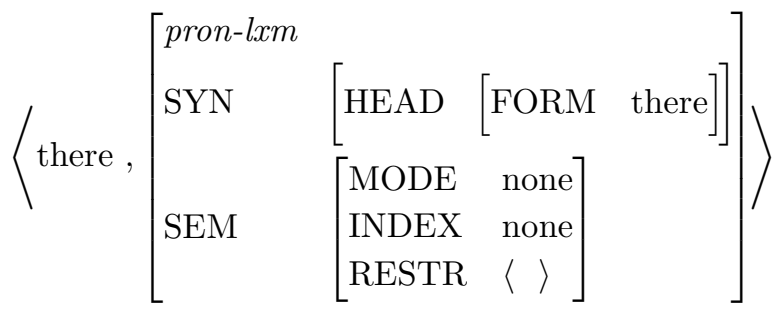

The requirement that the HEAD value in (11) be of the type noun is inherited from the type noun-lxm (a supertype of pron-lxm); and the requirement that the argument structure be empty is inherited (by default) from this type as well. Note that the AGR specification in (11) is underspecified for number; this is because there can be plural, as in $(7 \mathrm{~b}, \mathrm{c})$. Note in addition that the empty list specification for the feature RESTR guarantees that there will not contribute to the RESTR list of phrases that contain it. And finally, the 'none' values that we have introduced for the features MODE and INDEX reflect the fact that there has no referential potential and no referential index. ${ }^{7}$

This last fact is particularly significant, as it allows us to explain the restricted distribution of existential there. The verbs we have considered thus far (except for be) have lexical entries which state that the indices of the NP (if there are any) are assigned semantic roles (e.g. LOVER, GIVEN). But because existential there has no index, it follows that any attempt to combine there with a role-assigning verb will produce a conflicting SD. Thus from the semantic vacuity of existential there, it follows immediately that examples like the following are ungrammatical:

(12) a. *There loved Sandy.

b. *Merle gave there a book.

c. *We talked to them about there.

\footnotetext{
${ }^{7}$ The [INDEX none] specification also guarantees that there can never be the $\mathrm{NP}_{i}$ argument of (9). This ensures the ungrammaticality of examples like (i):
}

(i) *There is [there] [being someone missing]. 
Problem 2: There and Agreement

The analysis of existential there sentences presented so far says nothing about verb agreement.

A. Consult your intuitions (and/or those of your friends, if you wish) to determine what the facts are regarding number agreement of the verb in there sentences. Give an informal statement of a generalization covering these facts, and illustrate it with both grammatical and ungrammatical examples. [Note: Intuitions vary regarding this question, across both individuals and dialects. Hence there is more than one right answer to this question.]

B. How would you elaborate or modify our analysis of the there construction so as to capture the generalization you have discovered? Be as precise as you can.

\subsection{Extraposition}

Consider the following pairs of sentences:

(13) a. That Cleveland lost the series sucks.

b. It sucks that Cleveland lost the series.

(14) a. That dogs bark annoys people.

b. It annoys people that dogs bark.

(15) a. That Chris knew the answer (never) occurred to Pat.

b. It (never) occurred to Pat that Chris knew the answer.

(16) a. That the Cardinal won the game gave Sandy a thrill.

b. It gave Sandy a thrill that the Cardinal won the game.

This seems to be a systematic alternation that we would like to account for. Moreover, it is productive: an English speaker unfamiliar with the verb cark who heard (17a) would know that (17b) is also well formed.

(17) a. That many languages are dying out carks linguists.

b. It carks linguists that many languages are dying out.

And speakers who have acquired the lexical item bite as a synonym for suck (as in 'be bad') should find both the following sentences to be well formed.

(18) a. That Cleveland lost the series (really) bites.

b. It (really) bites that Cleveland lost the series. 
Thus the alternation illustrated in (13)-(16) has some claim to psychological reality.

The (b)-sentences in (13)-(18) all have a nonreferential pronoun it as their subject and a that-clause at the end. In our treatment of another 'dummy' (i.e. non-referential) NP earlier in the chapter, we gave it an empty RESTR list, and the value 'none' for INDEX. It seems natural to do the same with this use of $i t$. As was the case with the existential there, the dummy it is very restricted in its distribution. In examples like (19)-(20), which do not fit the pattern of (13)-(18), the uses of it are referential.

(19) a. * That Pat is innocent proves.

b. It proves that Pat is innocent.

(20) a. ${ }^{*}$ That Sandy had lied suggested.

b. It suggested that Sandy had lied.

Following the treatment of the existential there, then, we are led to posit lexical entries for the verbs in the (b)-sentences of (14)-(18) that specify that their subjects must be the nonreferential $i t$. We can do this as we did with there by positing a FORM value 'it', which uniquely identifies the dummy it.

The lexical entry for the dummy it is therefore the following:

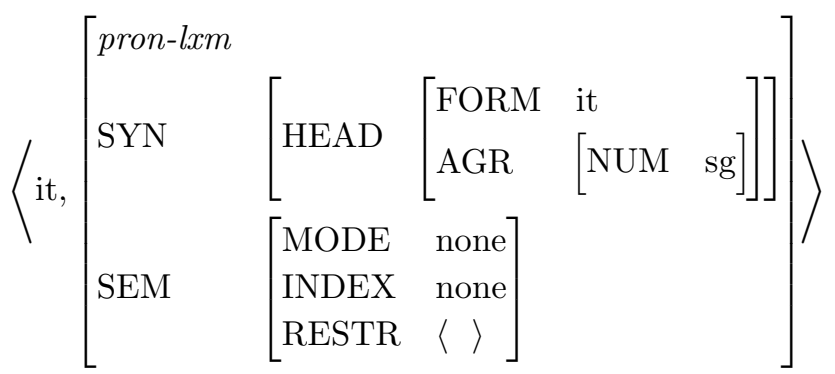

Note that the dummies it and there have slightly different AGR values: it is specified as [NUM sg] and is also [PER 3rd] (by default inheritance from the type noun-lxm), but there is only specified as third person (again by default), with the number left underspecified. This underspecification permits a single lexical entry there to appear with are and were, as well as with is and was.

Like the dummy existential there, and for exactly the same reasons, dummy it can never appear in a role-assigned position:

(22) a. *It loved Sandy.

b. *I gave it to Pat. 
Such examples are fully grammatical, of course, if we interpret it as the personal pronoun it (i.e. as a pronoun referring to something in the context), rather than as the dummy it.

To capture the regularity of the alternation illustrated in (13)-(18), we will want to posit a lexical rule whose output is the version of the verbs taking the dummy subject $i t$. But before we can do this, we need to consider how to analyze the that-clauses that occur in the examples in question.

The part after that is just a finite $\mathrm{S}$ (i.e. a phrase headed by a finite verb, with empty COMPS and SPR specifications - as noted in Chapter 4 , we sometimes call such a phrase 'saturated'). It is less obvious how to deal with that, which might be thought of as simply 'marking' the sentence that follows it. We propose to treat that as a head, taking a finite $\mathrm{S}$ as its only argument (note that in this respect, that is similar to the argument-marking prepositions such as to and of first discussed in Chapter 7). In order to handle words like that, however, we will have to introduce a new part of speech type: comp (for 'complementizer'). That-clauses, then, are complementizer phrases (CPs, for short).

Some verbs (largely verbs of thinking or communicating, such as guess, know, promise, remember, say, and think) can take that-clauses as complements. Most of these can also take clausal complements without that. A clever way of handling this is to say that that is [FORM fin], just like finite verbs. Under this assumption, the lexical entries for verbs such as guess can have an ARG-ST specification like the following:

$$
\left[\text { ARG-ST }\left\langle\text { NP },\left[\begin{array}{ll}
\text { FORM } & \text { fin } \\
\text { SPR } & \langle\rangle \\
\text { COMPS } & \langle\rangle
\end{array}\right]\right\rangle\right]
$$

This specification then allows the second argument to be either a thatclauses or a finite clause without that. Our feature system thus attributes a common property to this class of categories, predicting that they are a 'natural class' - that is, that they should behave alike in some ways. And we just noted that they can appear as complements to many of the same verbs.

Returning to examples with that-clause subjects, notice that the complementizer is obligatory:

(24) *Dogs bark annoys people.

This can be handled by saying that a verb like annoy takes a finite complementizer phrase as its subject - that is, (25) is the ARG-ST specification found in the relevant lexical entries: 
262 / Syntactic Theory: A Formal Introduction

$$
\left.\left.\left.\left[\begin{array}{ll}
\text { ARG-ST }\left\langle\left[\begin{array}{ll}
\text { HEAD } & \text { comp } \\
\text { FORM } & \text { fin }
\end{array}\right]\right. \\
\text { SPR } & \langle\rangle \\
\text { COMPS } & \langle\rangle
\end{array}\right], \ldots\right\rangle\right\rangle\right]
$$

We can now turn to the problem of formulating our lexical rule, which we will call 'extraposition'. ${ }^{8}$ Given our analysis of that-clauses, the crucial step in formulating the rule is ensuring that the that-clause plays the same semantic role in both sentences.

So far, our SEM values have been specifications (in referential or propositional mode) where the RESTR list has contained simple predications, that is, predications where the semantic role features (LOVER, INSTANCE, etc.) take indices as their arguments. These indices in general correspond to individuals that are referred to by NPs within the sentence. One important exception to this had to do with modification. In Chapter 5, we allowed situational indices to be the value of the feature ARG(UMENT) that appeared in certain predications introduced by adverbial modifiers, for example, as in (26).

$$
\left[\begin{array}{ccc}
\text { MODE } & \text { prop } & \\
\text { INDEX } & s & \\
\text { RESTR } & \left\langle\left[\begin{array}{lc}
\text { RELN } & \text { today } \\
\text { SIT } & s \\
\text { ARG } & t
\end{array}\right]\right\rangle
\end{array}\right]
$$

This in fact is the general technique we will use for semantic embedding - for making one semantic complex the argument of another. That is, we will never embed one feature structure within another inside the value of SEM, as is done in (27):

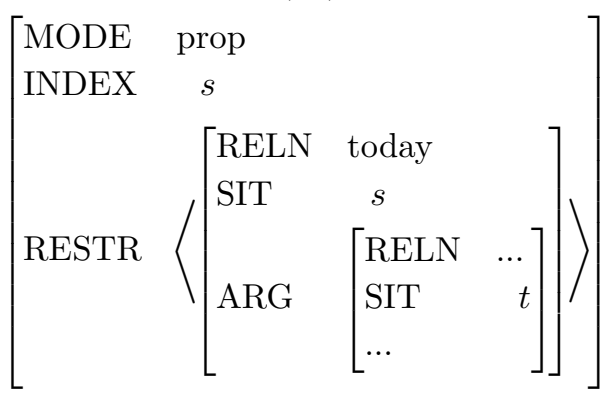

\footnotetext{
${ }^{8}$ In this we follow terminology introduced by the renowned Danish grammarian Otto Jespersen (1860-1943).
} 
Instead, we will use sameness of situational indices to get the same semantic effect. We will use various (hopefully intuitive) feature names to designate the roles whose value is an embedded proposition. In this way, we can express meanings that involve arbitrarily deep semantic embeddings, but we can keep the RESTR lists inside our semantic structures 'flat'. 9

On this view, we will be able to deal with the semantics of subordinate clauses in terms of index identity, using the kind of semantic analysis we have already developed. For example, we can make the reasonable assumption that the semantics of Fido barks in That Fido barks annoys me is the same as that of the stand-alone sentence Fido barks, namely, (28).

$$
\left[\begin{array}{lcc}
\text { MODE } & \text { prop } & \\
\text { INDEX } & s & \\
\text { RESTR } & \left.\left\langle\begin{array}{ll}
\text { RELN } & \text { name } \\
\text { SIT } & t \\
\text { NAME } & \text { Fido } \\
\text { NAMED } & i
\end{array}\right],\left[\begin{array}{ll}
\text { RELN } & \text { bark } \\
\text { SIT } & s \\
\text { BARKER } & i
\end{array}\right]\right\rangle
\end{array}\right]
$$

How do we construct such a semantics?

The complementizer that belongs to a new type of lexeme associated with the constraints in $(29):^{10}$

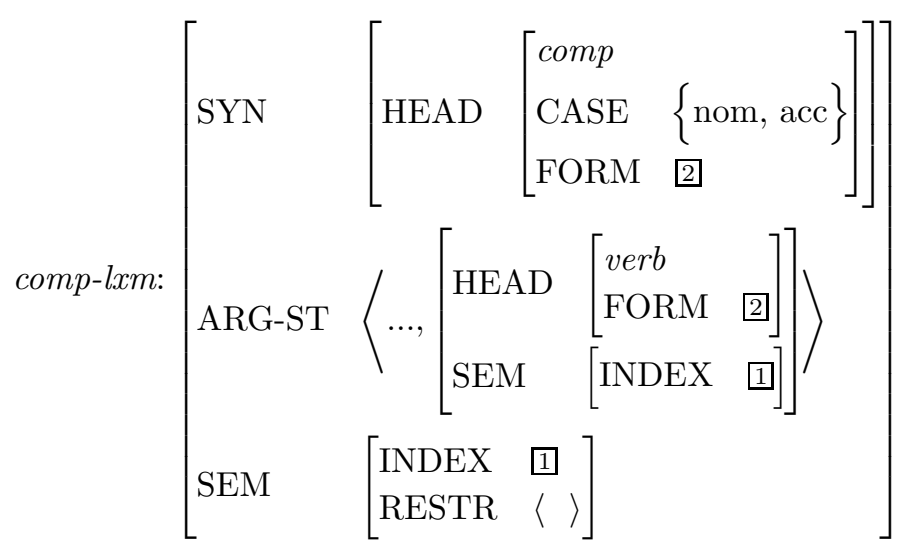

\footnotetext{
${ }^{9}$ We are simplifying here in not providing any apparatus for distinguishing embedded propositions from embedded questions, exclamations, etc.

${ }^{10}$ The type comp-lxm is a subtype of const-lxm (though some varieties of certain Germanic languages show what appear to be inflected forms of complementizers!).
} 
(29) says that all instances of this type of lexeme share the semantic index of their last argument. ${ }^{11}$ In addition, it is required that the complementizer and its last argument share values for the feature FORM. Finally, we have constrained complementizers to take values for CASE. This is necessary in order to permit CPs to occur as subjects (as in the (a)-examples in (13)-(18), since finite verbs require that their SPR values be [CASE NOM]. For uniformity, we will also generalize the Case Constraint (introduced in Chapter 8) so that it requires that CPs, as well as NPs, be [CASE acc] when they are noninitial in an ARG-ST.

With these type constraints in place, the lexical entry for that need say nothing more than what is shown in (30).

$$
\left.\left\langle\text { that, }\left[\begin{array}{ll}
\text { comp-lxm } & \\
\text { SYN } & {\left[\begin{array}{lll}
\text { HEAD } & {[\text { FORM }} & \text { fin }
\end{array}\right]} \\
\text { SPR } & \langle\rangle
\end{array}\right]\right)\right\rangle
$$

The constraints passed on through type inheritance thus interact with those that are lexically specified to ensure that the complementizer that has the INDEX and FORM value of its only argument, which in turn must be a saturated finite clause. Type inheritance thus gives us the fully specified lexical entry in (31).

\footnotetext{
${ }^{11}$ Other complementizers, e.g. the complementizer to discussed in the next chapter, allow more than one argument.
} 


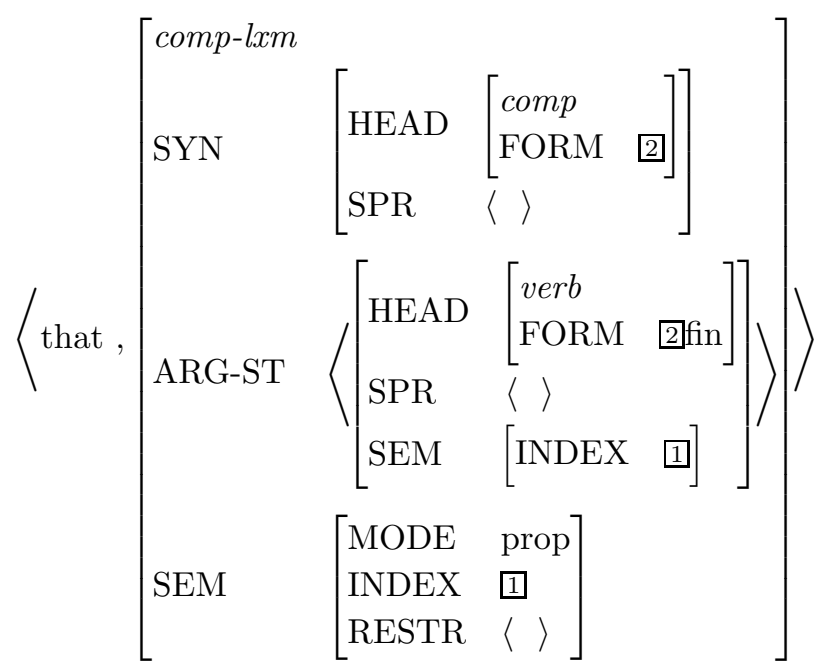

Given this lexical entry and its interaction with the semantics principles of Chapter 5 , it follows that the semantics of that-clauses is identical to the semantics of the clause that that takes as its complement. The meaning of a clause like That Fido barks annoys me will then be represented as follows:

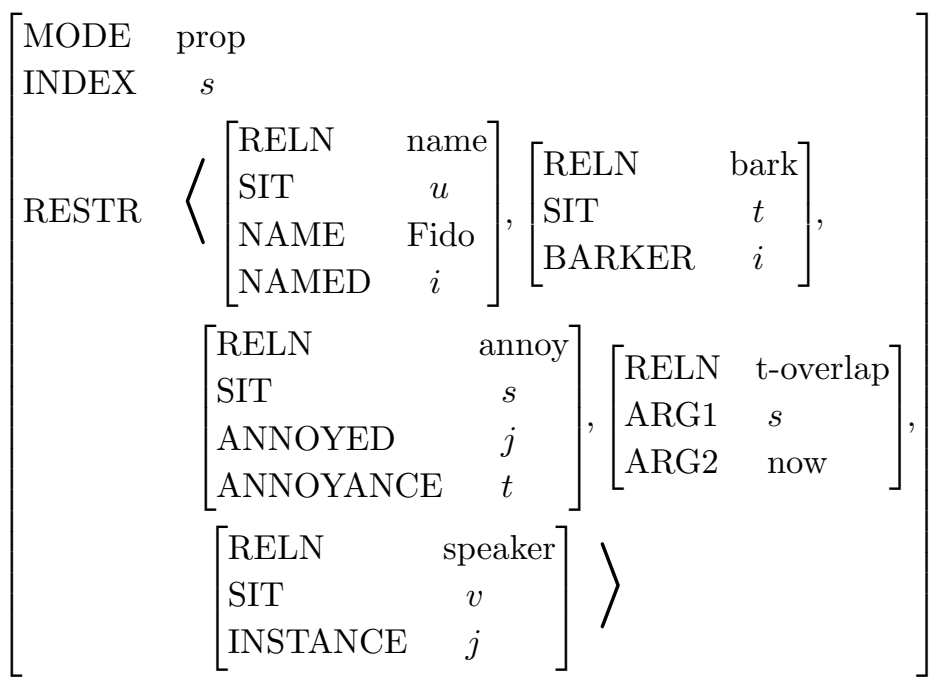

This will result from combining the CP subject that Fido barks with the VP annoys me. As before, we are simply following the Semantic Com- 
positionality Principle and the Semantic Inheritance Principle. Hence, we identify the INDEX of the S with that of its VP head, and we form the S's RESTR list by concatenating the RESTR lists of the subject and the VP.

We are now almost ready to state our extraposition rule. We want the rule to take as input a lexeme whose first argument is a $\mathrm{CP}$ and produce as output a lexeme with the $\mathrm{CP}$ at the end of its ARG-ST list and an NP[FORM it] at the beginning. This change affects the length of the ARG-ST list, since the NP[FORM it] is added and nothing is removed. That means that the output lexeme cannot, in general, be of the same type as the input, for many of our lexeme types (e.g. those for verbs) specify how many items are on the ARG-ST list. Our earlier encounters with lexical rules that changed the order of elements on the ARG-ST list (such as the Passive Lexical Rule) produced outputs of type word, so there was no issue of conflict between constraints on the output's type and those specified for the output by the rule. The type word, for example, imposes no constraint on ARG-ST lists. In the case of extraposition, however, the output needs to undergo further inflectional rules. For instance, both (33a) and (33b) are possible, indicating that the output of the Extraposition Lexical Rule must be able to undergo both the Past Tense Verb LR and the 3rd Singular Verb LR.

(33) a. It annoyed me that Fido barked.

b. It annoys me that Fido barked.

Hence, the output of the Extraposition Rule should belong to some subtype of lexeme.

We handle this problem by introducing a new lexeme type, derived$l x m$, that has no constraints associated with it. We then assign the output of the Extraposition Lexical Rule to this new type. This way, the type of the input lexeme will be changed, allowing for the increased length of the ARG-ST list on the output without contradicting any constraints associated with the input type. All other input information will either be carried forward to the output lexeme or else will be explicitly overridden by the rule itself. The following statement of the rule incorporates this idea:

(34) Extraposition Lexical Rule 
Nominal Types: Dummies And Idioms / 267

$$
\begin{aligned}
& \left\langle 1,\left[\begin{array}{l}
l x m \\
\text { ARG-ST } \left.\mathrm{a},\left\langle\left[\begin{array}{ll}
\mathrm{HEAD} & \text { comp }
\end{array}\right]\right\rangle \oplus \mathrm{b}\right]
\end{array}\right]\right\rangle \Rightarrow \\
& \left\langle 1,\left[\begin{array}{l}
\text { derived-lxm } \\
\text { ARG-ST }\langle\mathrm{NP}[\mathrm{FORM} \text { it }]\rangle \oplus \mathrm{b} \oplus \mathrm{a}]
\end{array}\right]\right\rangle
\end{aligned}
$$

This rule creates new lexemes from any lexeme (not just verb-lxms, as we will see) whose first argument is a CP. The output lexeme always takes a dummy it as its subject and takes as a final argument whatever kind of CP was specified as the input's first argument. Notice that in this analysis we do not need new phrase structure rules to handle extraposition. Any word formed from the lexeme outputs of this rule fits one of the general patterns already provided for by our existing grammar rules. All we have needed to handle this new phenomenon was a new value for the feature FORM. It was then straightforward to formulate a lexical rule that captures the regularity illustrated by the sentence pairs at the beginning of this section. ${ }^{12}$

${ }^{12}$ There remains the question of where the new type derived-lxm fits into our type hierarchy. If we make the simplifying assumption that only verbs undergo the Extraposition Lexical Rule, then derived-lxm can be an immediate subtype of verb-lxm. However, extending the treatment of extraposition to include adjectives as well will lead us to introduce a number of refinements that are beyond the scope of this text. 


\section{\1 Problem 3: An Annoying Exercise}

Assume that the lexical entry for the verb annoy is the following. (Note that the first argument of this lexeme overrides the default condition associated with the type verb-lxm - requiring the first argument to be an NP.)

(i)

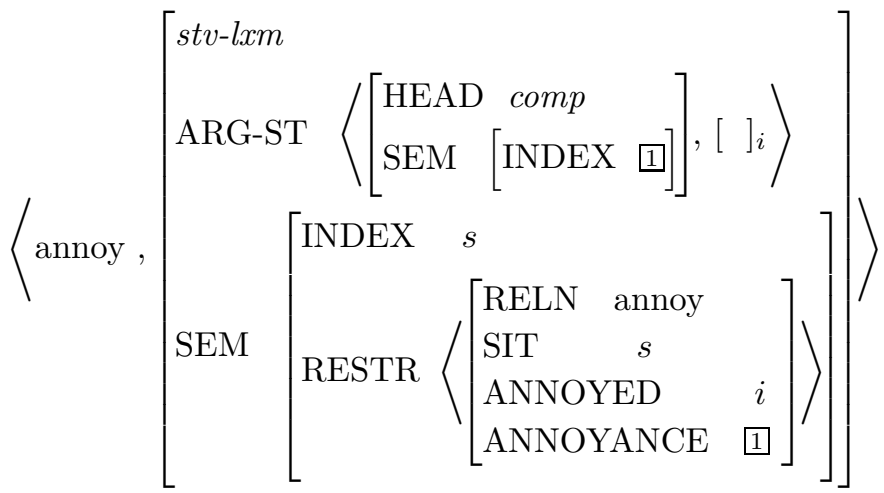

A. Show the lexical entry that results from applying the Extraposition Lexical Rule to (i).

B. Using the lexical entry given in the text for the complementizer that, describe in a few sentences (with an appropriate SD) the analysis of the clause that Fido barks. Make sure you explain exactly how this clause is assigned the semantics shown in (28).

C. Using the lexical entry you gave in (A) and the analysis of that Fido barks you came up with in (B), sketch the analysis of It annoys Lee that Fido barks. Your solution should show the lexical entry for annoys in this sentence (explaining where it came from), the lexical SD it gives rise to, and how it is embedded in the SD for the whole sentence. Be sure that the semantics that results from your analysis is like (32), except for the difference between me and Lee (and the ordering of the items in the RESTR list).

D. Modify the lexical entry in (i) so that it also allows sentences like Sandy annoys me.

\subsection{Idioms}

We have now encountered two nonreferential NPs with highly restricted distributions, namely, the dummies there and it. Other NPs that share the properties of nonreferentiality and restricted distribution can be 
found in idioms - that is, in fixed (or partially fixed) combinations of words that are used to express meanings that aren't determined in the usual way from those words. For example:

(35) a. Carrie kicked the bucket last night. ('Carrie died last night')

b. The FBI kept (close) tabs on Sandy. ('The FBI (carefully) observed Sandy')

c. The candidates take (unfair) advantage of the voters. ('The candidates exploit the voters (unfairly)')

The idioms kick the bucket, keep tabs on, and take advantage of each has an idiosyncratic meaning, which requires that all of its parts cooccur. That is, the words in these idioms take on their idiomatic meanings only when they appear together with the other parts of the idioms. For example, the following sentences do not have interpretations related to those in (35) (if they are acceptable at all):

(36) a. Chris dreads the bucket.

b. The police put tabs on undocumented workers.

c. The candidates bring advantage to the voters.

Since the lexical entries for verbs contain information about the arguments they co-occur with (but not vice versa), one way to capture the idiosyncratic properties of idioms is to encode them in the entries of the verbs. That is, we can treat idiomatic nouns, such as tabs and advantage by:

- giving them their own FORM values;

- marking them as [MODE none] and [INDEX none]; and

- specifying that they are [RESTR \langle\rangle$]$

This amounts to treating idiom parts (or 'idiom chunks', as they are often called) similarly to the dummies just considered.

We can now have entries for keep and take specifying that their objects must be [FORM tabs] and [FORM advantage], respectively. These verbal entries will contain all of the idioms' semantic information. ${ }^{13}$ The detailed entries for idiomatic nouns tabs and advantage and the verbs that go with them are given in (37) and (38). ${ }^{14}$

\footnotetext{
${ }^{13}$ This treatment (like a number of others in this book) is a simplification. For a more thorough discussion of the authors' views on the semantics of idioms, see Nunberg, Sag, and Wasow (1994).

${ }^{14}$ Note that the entries for tabs and advantage override default specifications for both ARG-ST and SPR.
} 
270 / Syntactic Theory: A Formal Introduction

(37) a.

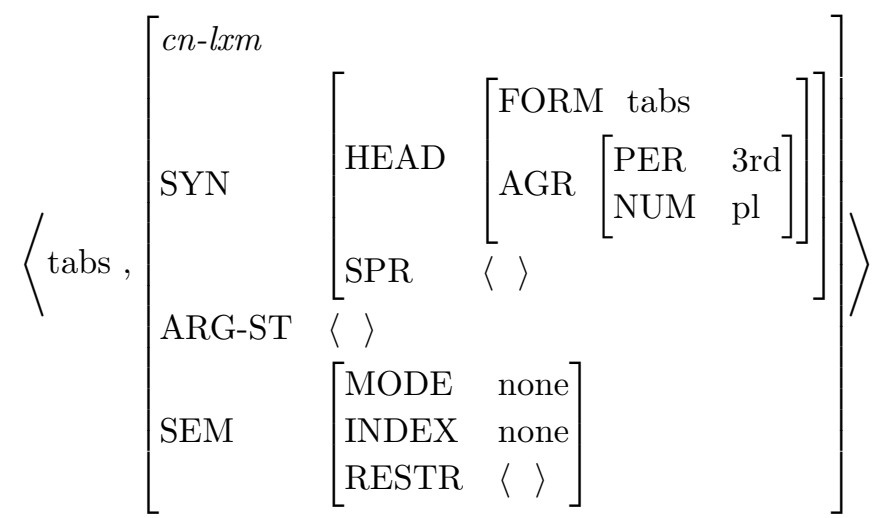

b.

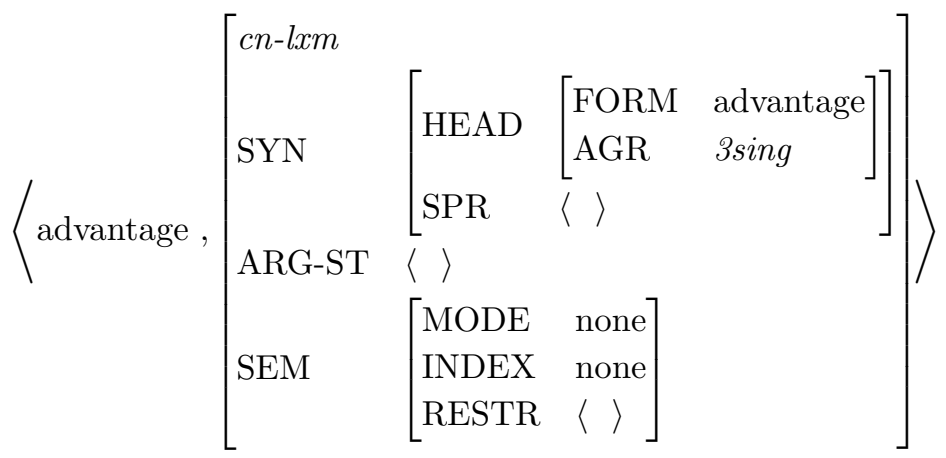

(38) a.

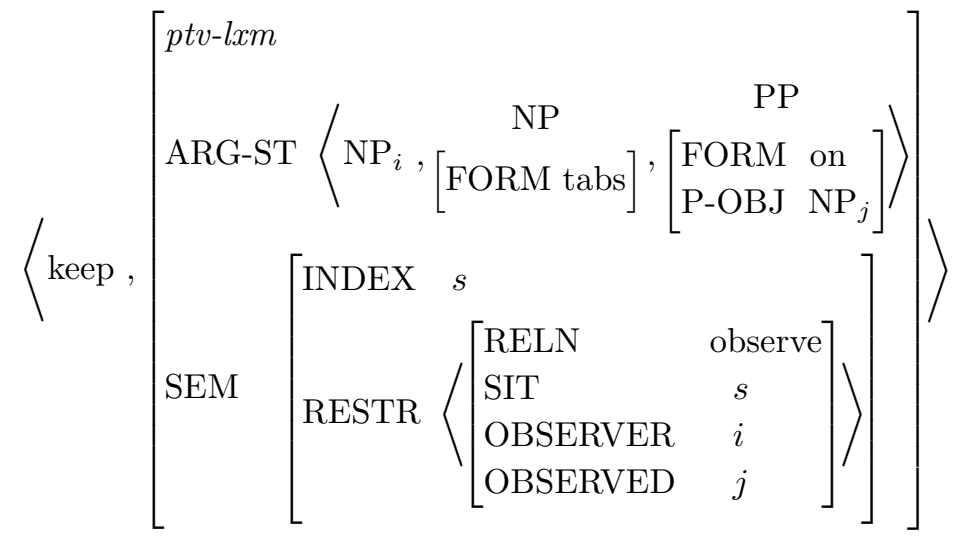


Nominal Types: Dummies And Idioms / 271

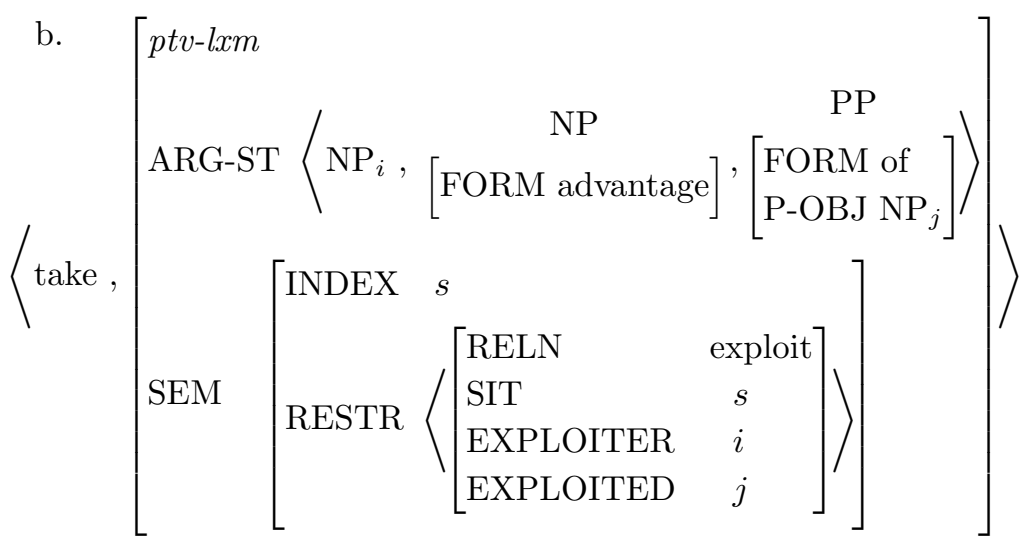

Notice that we have given no entry for kick the bucket. There is a reason for this: different idioms exhibit different syntactic behavior, so not all idioms should be analyzed in the same fashion. In particular, kick the bucket differs from keep tabs on and take advantage of in its lack of a passive form. That is, while $(39 \mathrm{a}, \mathrm{b})$ allow idiomatic interpretations, (39c) can only convey its literal meaning, which entails that Pat's foot made contact with an actual bucket.

(39) a. Tabs are kept on suspected drug dealers by the FBI.

b. Advantage is taken of every opportunity for improvement.

c. The bucket was kicked by Pat.

The analysis of keep tabs on and take advantage of presented above correctly allows them to have passive forms. These idiomatic verb entries meet the input conditions of the Passive Lexical Rule, and so can give rise to passive forms. The FORM restrictions on the NP complements of the active idiomatic verbs are restrictions on the subjects (that is, SPRs) of their passive versions. Hence, idiomatic taken (as a passive) requires that its subject be advantage.

\section{Problem 4: Idiomatic Kept}

A. Give the lexical entry for the passive of the idiomatic kept - that is, the result of applying the Passive Lexical Rule to (38a).

B. Explain precisely how the contrast between (i) and (ii) is explained on our analysis:

(i) Tabs were kept on Chris by the FBI.

(ii)*Advantage was kept on Chris by the FBI.

Be sure to discuss the role of the verb be. 
If kick the bucket were to be analyzed in a parallel fashion, we would incorrectly predict that (39c) had an idiomatic interpretation (namely, 'Pat died'). To avoid this, we need a different analysis of this idiom. The most straightforward treatment is to say that the whole string, kick the bucket, is the verb. ${ }^{15}$ Thus, there is a single lexical entry for the entire idiom kick the bucket, given in (40).

$$
\left\langle\langle\text { kick, the, bucket }\rangle,\left[\begin{array}{l}
\text { ARG-ST }\left\langle\mathrm{NP}_{i}\right\rangle \\
\left.\operatorname{SEM}\left[\begin{array}{cc}
\operatorname{INDEX} & s \\
& \left.\left\langle\left[\begin{array}{ll}
\mathrm{RELN} & \text { die } \\
\text { CORPSE } & i
\end{array}\right]\right\rangle\right]
\end{array}\right]\right)
\end{array}\right.\right.
$$

This entry is an intransitive multi-element verbal lexeme, so it doesn't have a passive form. Or, to put it more formally, entry (40) does not satisfy the conditions necessary to serve as input to the Passive Lexical Rule: since it is not a $t v$-lxm, it does not passivize.

\subsection{Conclusion}

In this chapter, we have extended the use of the FORM feature to NPs and made use of it in the analysis of existential sentences containing the dummy there, the extraposition construction, and idioms. Each of these three constructions involves nonreferential NPs. The distribution of such NPs is more than an idle curiosity, however. In more complex sentences, it plays a crucial role in motivating the analysis of infinitival and other kinds of complements, which is precisely the concern of the next chapter.

\subsection{Further Reading}

Influential early discussions of the existential there and extraposition include Rosenbaum (1967), Milsark (1977), and Emonds (1975). See also Chomsky (1981) and Postal and Pullum (1988). Of the many generative discussions of idioms, see especially Fraser (1970), Chomsky (1980), Ruwet (1991), and Nunberg et al. (1994).

\footnotetext{
${ }^{15}$ In order to ensure that the verbal morphology appears on the first word in this multiword lexical entry, we adopt the general convention that morphological functions apply only to the first element of such entries. This also covers a number of other cases, such as the locations of the plural $-s$ in runs batted in and the comparative suffix -er in harder of hearing.
} 


\section{Infinitival Complements}

\subsection{Introduction}

In our discussion of extraposition in the last chapter, we encountered for the first time sentences expressing complex meanings - those in which one situation functions as a semantic argument of another. ${ }^{1}$ In this chapter, we will investigate additional constructions involving this sort of complex semantics. In particular, we will focus on infinitival complements in sentences such as (1).

(1) a. Pat continues to avoid conflict.

b. Pat tries to avoid conflict.

We will see that, despite their superficial parallelism, examples (1a) and (1b) are quite different in their semantics and in certain associated syntactic properties. These two examples are representative of two basic ways in which propositions can be combined into complex meanings.

\subsection{The Infinitival To}

Before we delve into the distinction illustrated in (1), we need to provide an analysis for the word to that appears in both sentences. Like the lexeme that discussed in the previous chapter, the infinitival to is a complementizer and hence belongs to the type comp-lxm. An important difference between the two complementizers, however, is that while that introduces a subordinate clause, to introduces a subordinate VP. Like that, to does not appear to contribute to the semantics of the sentences in any substantive way. This is evident in those rare cases where it is optional. For example, there is no apparent difference in meaning between (2a) and (2b).

\footnotetext{
${ }^{1}$ As we noted, the semantic analysis we have given for a sentence like That dogs bark annoys people (or its extraposed counterpart) involves not the embedding of one feature structure within another, but rather the unification of the SIT value of one predication with the ARG value of another.
} 
(2) a. Pat helped Chris to solve the problem.

b. Pat helped Chris solve the problem.

We can capture both the similarities and the differences between that and to with the following lexical entry.

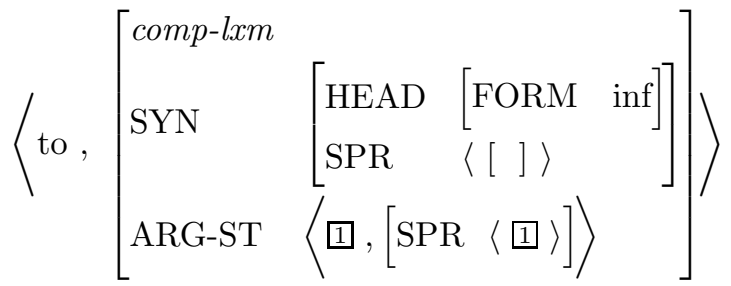

This interacts with the constraints on the type comp-lxm, introduced in the last chapter, to give us, via constraint inheritance, the following fully specified entry for the complementizer $t o$.

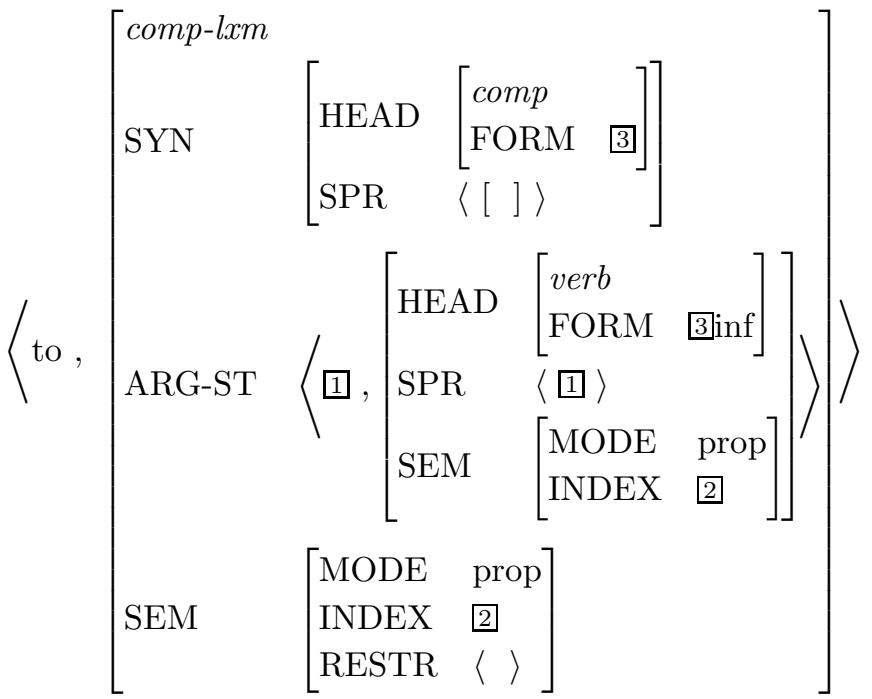

One constraint that the complementizer to inherits from comp-lxm is that its FORM value is unified with that of its last argument. Note that to (unlike that) and its complement are both [FORM inf]. Hence to is a complementizer that selects as its second argument an infinitival VP with an element on its SPR list that is identified with the first argument of to. (To is like be in this respect.) To differs from that in requiring an element on its SPR list. But since to is like that in bearing the semantic specification [RESTR \langle\rangle ], it follows that when to combines with its VP complement, only the latter contributes to the semantic restriction 
of the resulting CP. Moreover, the VP's index is the same as to's index and hence becomes the index of the $\mathrm{CP}$ that to heads.

With this complementizer added to our lexicon, our grammar rules and principles interact to license structures like the following:

(5)
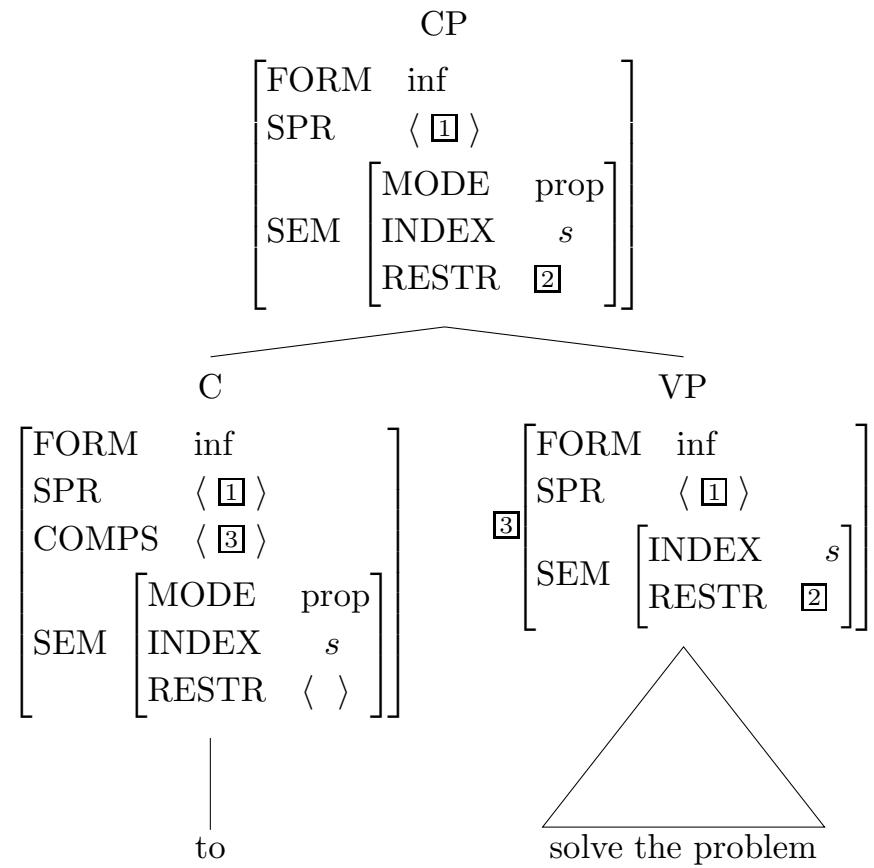

\subsection{The Verb Continue}

Recall that the dummies it and there, as well as idiom chunks like (close) tabs or (unfair) advantage, have a restricted distribution - they occur only as subjects or objects of verbs that select them in those positions. What these NPs all have in common is that they are nonreferential - that is, they take 'none' as their value for MODE and INDEX. They are therefore inherently unsuited to play a role in any predication. Consequently, on semantic grounds, we have already explained the ungrammaticality of (6) and the fact that it must be referential in (7).

(6) a. $*$ I hate $\left\{\begin{array}{l}\text { advantage } \\ \text { tabs } \\ \text { there }\end{array}\right\}$. 
b. $*\left\{\begin{array}{l}\text { Advantage } \\ \text { tabs } \\ \text { there }\end{array}\right\}$ really affected us.

(7) a. I hate it.

b. It really affected us.

It might seem surprising, then, that there are some other verbs that allow subject NPs without referential indices. Continue is one such example:

(8) a. Sandy continues to eat oysters.

b. There continued to be riots in Freedonia.

c. It continues to bother me that Chris lied.

d. (Close) tabs continue to be kept on Bo by the FBI.

e. (Unfair) advantage continues to be taken of the refugees.

A nonreferential subject can occur with continue only if the verb within the infinitival $\mathrm{CP}$ is one that selects it. ${ }^{2}$

(9) a. *There continues to $\left\{\begin{array}{l}\text { eat oysters } \\ \text { bother me that Chris lied } \\ \text { be kept on Bo by the FBI } \\ \text { be taken of the refugees }\end{array}\right\}$.

b. *It continues to $\left\{\begin{array}{l}\text { eat oysters } \\ \text { be riots in Freedonia } \\ \text { be kept on Bo by the FBI } \\ \text { be taken of the refugees }\end{array}\right\}$.

c. $*$ (Close) tabs continue to $\left\{\begin{array}{l}\text { eat oysters } \\ \text { be riots in Freedonia } \\ \text { bother me that Chris lied } \\ \text { be taken of the refugees }\end{array}\right\}$.

d.

$$
\text { *(Unfair) advantage continues to }\left\{\begin{array}{l}
\text { eat oysters } \\
\text { be riots in Freedonia } \\
\text { bother me that Chris lied } \\
\text { be kept on Bo by the FBI }
\end{array}\right\} \text {. }
$$

The contrast between (8) and (9) suggests that the verb continue is in an intuitive sense transparent to the co-occurrence restrictions between the subject and the verb in its complement.

\footnotetext{
${ }^{2}$ The versions with it are, of course, acceptable on the irrelevant reading where it is referential.
} 
We can capture this intuition by specifying that continue and its complement must have the same subject. We do this as we did earlier for the passive be and for the infinitival to above: the first element in continue's ARG-ST list (the subject) will be identical to the SPR value of the second element in the ARG-ST list (the complement). Since the complement is a $\mathrm{CP}$ headed by to, the $\mathrm{SPR}$ value of the $\mathrm{CP}$ will be identical to the SPR value of the embedded VP. Hence the co-occurrence restrictions involving the nonreferential NPs will be transmitted from the verbs heading the infinitival VPs through to up to the subject of the verb continue.

Notice also that if we passivize the complement's verb, making the accompanying change in the subject of continue, the meaning of the sentence does not change. That is, examples (10a) and (10b) are essentially paraphrases. ${ }^{3}$

(10) a. The FBI continues to visit Lee.

b. Lee continues to be visited by the FBI.

In what follows, we will informally describe the relationship between pairs of sentences like (10a) and (10b) by saying that 'passivizing the complement verb' of the former yields the latter. The relationship is of course more complex, but it is convenient to have a simple way of referring to it, since we will use it as a diagnostic test.

The observation that passivizing the complement of continue does not change its meaning suggests that, semantically, continue takes only one argument - the situation of its CP complement - and predicates of it simply that it continues to be the case. Thus, both sentences in (10) mean that it continues to be the case that the FBI visits Lee. Crucially, the subject of continue is not assigned a role in the predication whose RELN is 'continue' (otherwise the nonreferential NPs we looked at above would be impossible as subjects of continue, since they have no index that can be assigned a role). All of this can be formalized by assigning the sentences in (10) a semantics like the following:

\footnotetext{
${ }^{3}$ We say 'essentially' because there are subtle differences in emphasis between the two sentences. The crucial test, for our purposes, is that there are no conceivable conditions under which one of the sentences would be true and the other would be false. This is the operational test we will use throughout to determine whether sentences do or do not mean the same thing.
} 
278 / Syntactic Theory: A Formal Introduction

(11)

$$
\begin{aligned}
{\left[\begin{array}{ccc}
\text { MODE } & \text { prop } \\
\text { INDEX } & s & \\
\text { RESTR } & \left\langle\left[\begin{array}{ll}
\text { RELN } & \text { continue } \\
\text { SIT } & s \\
\text { ARG } & t
\end{array}\right],\left[\begin{array}{lc}
\text { RELN } & \text { visit } \\
\text { SIT } & t \\
\text { VISITOR } & i \\
\text { VISITED } & j
\end{array}\right],\right. \\
& \left.\left[\begin{array}{ll}
\text { RELN } & \text { name } \\
\text { SIT } & u \\
\text { NAME } & \text { The FBI } \\
\text { NAMED } & i
\end{array}\right],\left[\begin{array}{ll}
\text { RELN } & \text { name } \\
\text { SIT } & v \\
\text { NAME } & \text { Lee } \\
\text { NAMED } & j
\end{array}\right]\right\rangle
\end{array}\right] }
\end{aligned}
$$

Putting this together with our observation about the subject-sharing between continue and its complement, we may postulate the following lexical type, which is a kind of (i.e. an immediate subtype of) verb-lxm: ${ }^{4}$

$$
\begin{aligned}
& \text { subject-raising-verb-lxm (srv-lxm): } \\
& \left.\left.\left[\begin{array}{ll}
\text { ARG-ST }\langle\square, ~ & \text { phrase } \\
\text { SPR } & \langle\square\rangle
\end{array}\right]\right\rangle\right]
\end{aligned}
$$

And in terms of this type, we can formulate the lexical entry for continue:

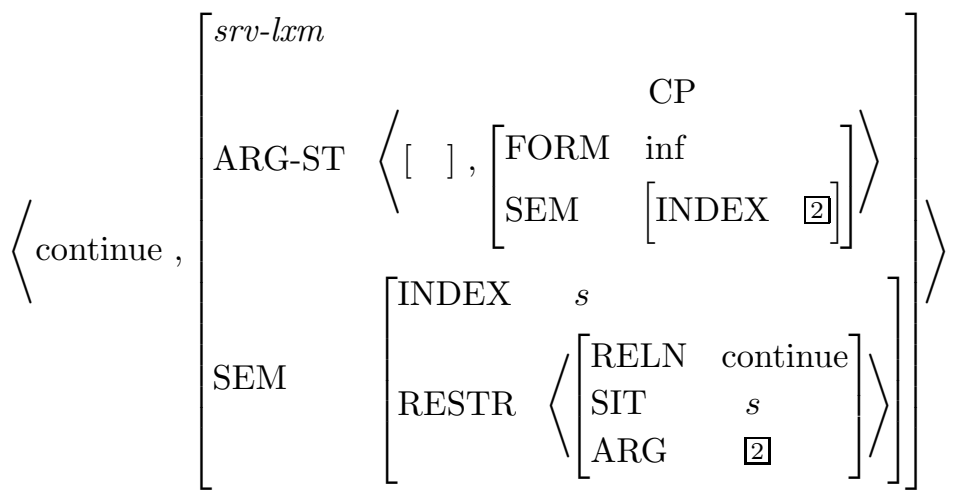

This analysis, where the lexeme continue inherits information not only from the type srv-lxm but also from the supertype verb-lxm, achieves the desired effects:

- the $\mathrm{CP}$ is the semantic argument of continue,

- the subject of continue is the subject of the CP complement.

- the subject of continue plays no role in the continue relation, and

\footnotetext{
${ }^{4}$ See footnote 6 for a brief discussion of the term 'subject raising'.
} 
- as a result of the above points, the sentences in (10) both receive a semantic analysis equivalent to the one sketched in (11).

These properties are illustrated in (14) and (15); note that the tags 10 - 4 refer to the same feature structure descriptions in (14)-(16):

(14)

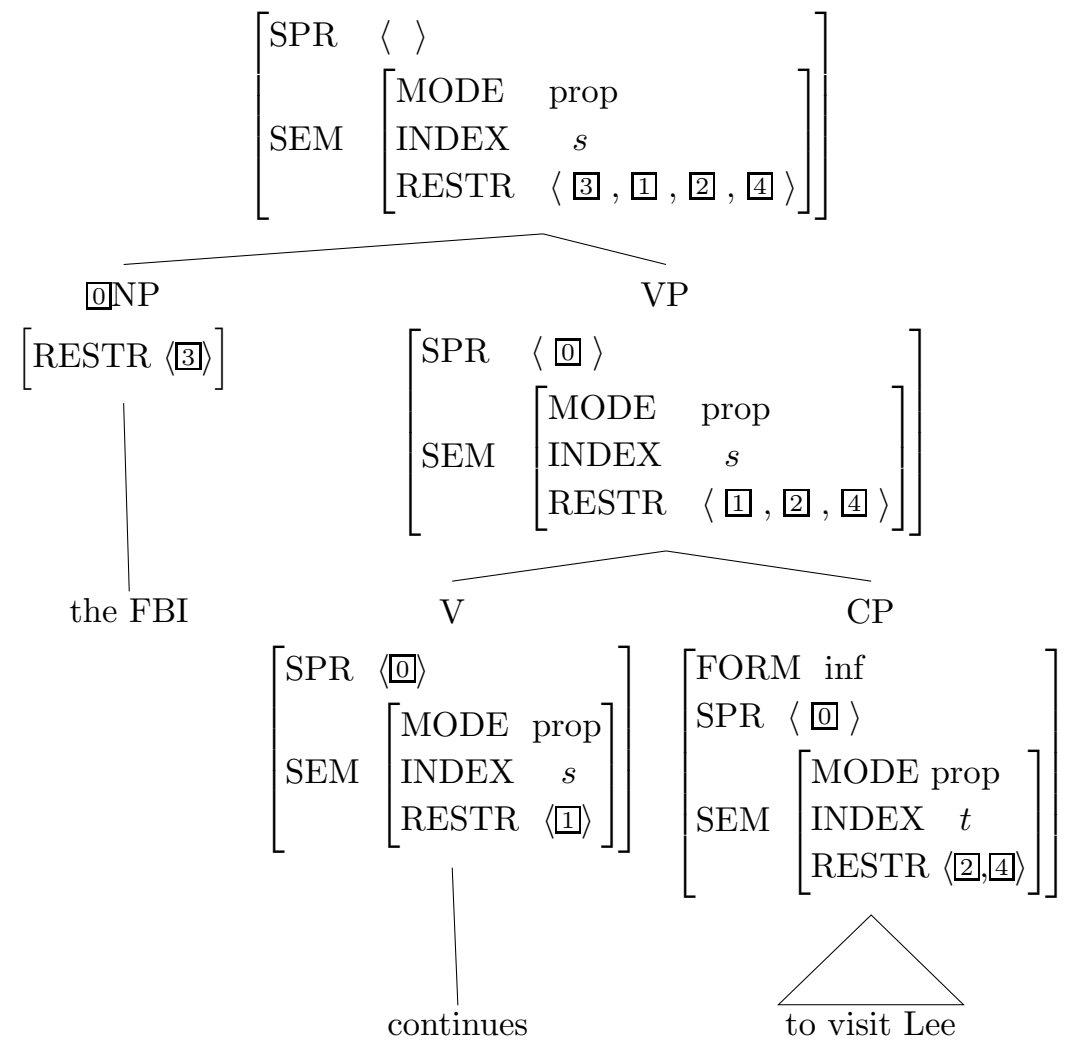


280 / Syntactic Theory: A Formal Introduction

$\mathrm{S}$

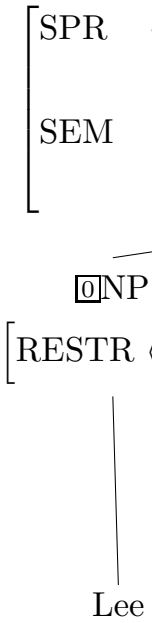

\langle\rangle
$\left[\begin{array}{lc}\text { MODE } & \text { prop } \\ \text { INDEX } & s \\ \operatorname{RESTR} & \langle\text { 4, 1 , 2 , 3 }\rangle\end{array}\right]$

$\widehat{\mathrm{NP}} \quad \mathrm{VP}$

Lee

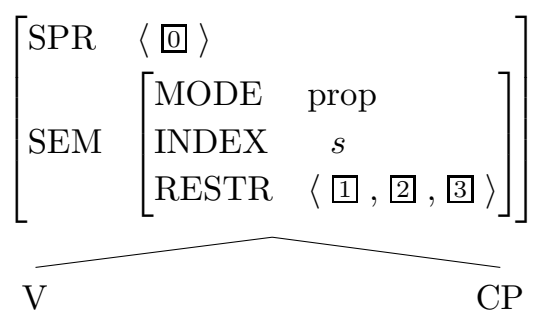

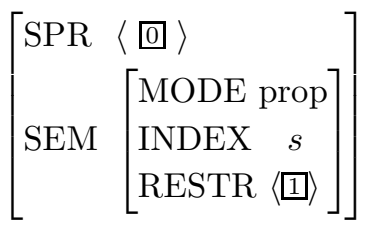
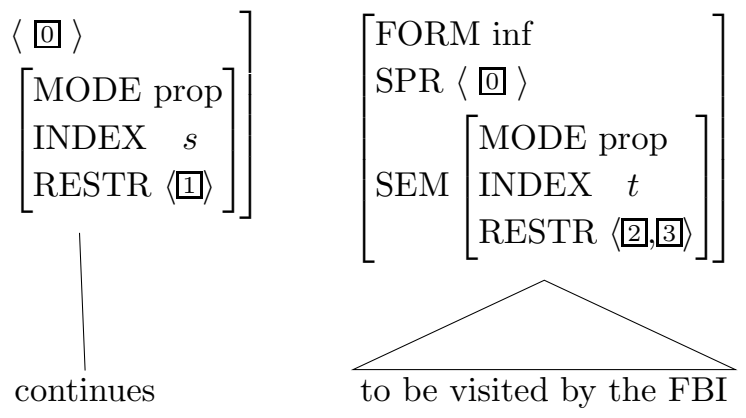

Here the relevant predications are those given earlier and tagged appropriately in (16).

(16) MODE prop

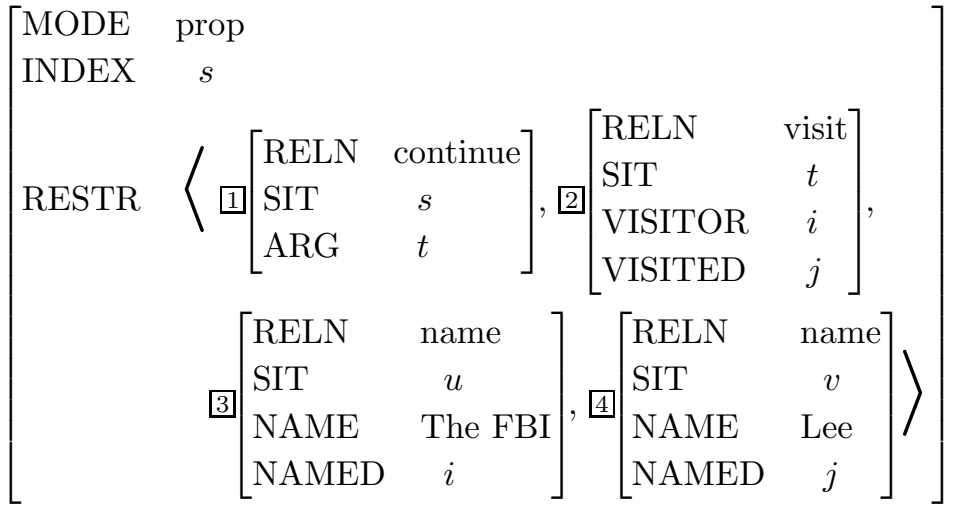


The two semantic values are thus equivalent, differing only in the order of elements on the RESTR list, a matter of no semantic consequence.

\subsection{The Verb Try}

The analysis of the verb continue we just developed was motivated by two observations: (i) that continue is transparent to co-occurrence restrictions between its subject and its complement's verb; and (ii) that passivizing its complement had no essential semantic effect.

Turning to the superficially similar verb try, we see that it differs from continue with respect to both (i) and (ii). Thus the analogues to (8b-e), with nonreferential subjects, are systematically ill formed:

(17) a. Sandy tried to eat oysters.

b. ${ }^{*}$ There tried to be riots in Freedonia.

c. ${ }^{*}$ It tried to bother me that Chris lied.

d. *(Close) tabs try to be kept on Bo by the FBI.

e. ${ }^{*}$ (Unfair) advantage tries to be taken of the refugees.

Likewise, the following two sentences are not synonymous:

(18) a. The FBI tried to find Lee.

b. Lee tried to be found by the FBI.

(18a) could be true under circumstances where (18b) would be false; indeed, it is quite likely that most people whom the FBI is trying to find are not trying to be found by them (or by anybody else!). Since the analysis of continue was designed to account for points (i) and (ii) above, it is clear that we need to analyze try quite differently.

Let us begin with the semantics of try. Unlike the continue relation, which takes only one semantic role (ARG, whose value is a situation), situations of trying involve two things: an individual (the entity that is trying) and some situation or state of affairs that the trier is trying to bring about. This is why the examples in (18) differ in meaning: the two triers are not the same. Notice also what the trier is trying to bring about always involves the trier. That is, it is not possible to express a meaning in which, say, what Kim is trying is for Sandy to visit Bo. ${ }^{5}$ These remarks are synthesized in the following semantic structure for Sandy tries to visit Bo:

\footnotetext{
${ }^{5}$ Maybe you could force an interpretation on this, something like 'Kim tried to bring it about that Sandy visit Bo', but notice that in so doing you are coercing the interpretation of the complement to a meaning that does contain the trier. We will ignore such coercions here.
} 
(19)

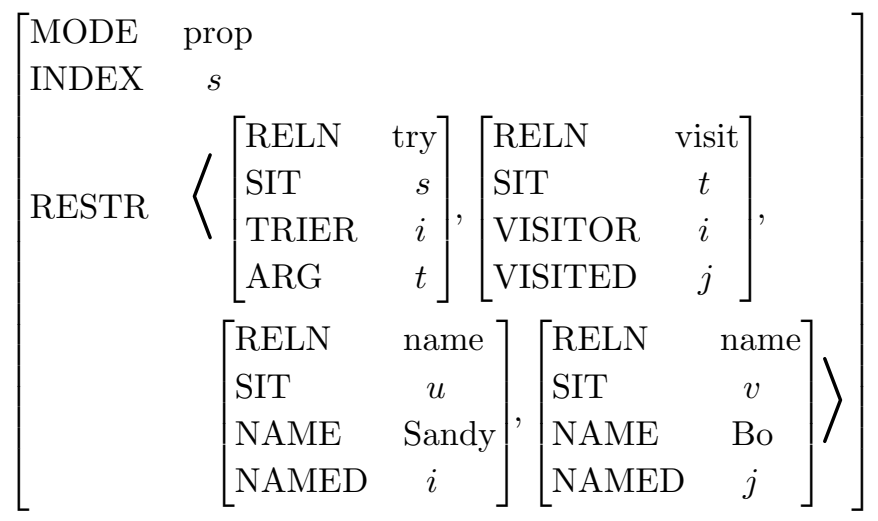

Semantic structures like this immediately rule out the use of nonreferential subjects (i.e. dummies and idiom chunks) with try. This is because the subject position of try always corresponds to a semantic argument slot, namely, TRIER. Since nonreferential NPs have no INDEX feature, it follows that there can be no semantics for examples like $(17 \mathrm{~b}-\mathrm{e})$ - the index of the TRIER cannot be unified with the subject NP's index.

Verbs like continue, on the other hand, can take nonreferential subjects, as we saw in the previous section, because their subjects do not correspond to semantic argument slots. This is the critical difference between what are referred to as 'raising' verbs and 'control' verbs. ${ }^{6}$ We represent this difference formally by assigning the index of the subject to a role in the semantics of control verbs, but not of raising verbs.

As before, we will want to use lexical types to express constraints general to this class of verbs. So we will want to introduce another subtype of verb-lxm like the one shown in (20):

$$
\begin{aligned}
& \text { subject-control-verb-lxm }(\text { scv-lxm }) \text { : } \\
& {\left[\text { ARG-ST }\left\langle\mathrm{NP}_{i},\left[\begin{array}{ll}
\text { phrase } & \\
\text { FORM } & \text { inf } \\
\text { SPR } & \left\langle\mathrm{NP}_{i}\right\rangle
\end{array}\right]\right\rangle\right]}
\end{aligned}
$$

And the following lexical entry for try, taken together with the constraints it inherits from its various types, accounts for the facts we have been considering:

\footnotetext{
${ }^{6}$ These perhaps non-mnemonic terms reflect commonly used terminology in the field. They derive from the analysis of this distinction that was developed in standard theory transformational grammar (see Appendix B).
} 
INFINITIVAL COMPLEMENTS / 283

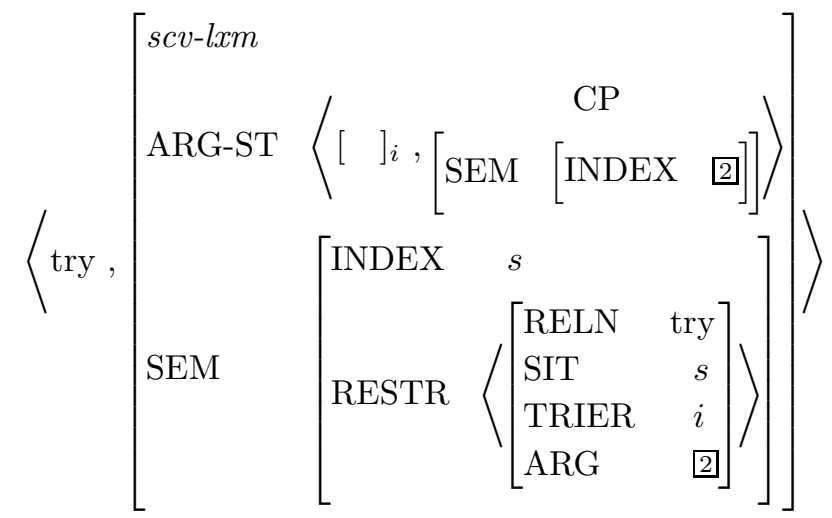

Note that the first argument of try and the subject of the CP are not unified. Rather, only their indices must be identical. This difference in the formal analysis of raising verbs and control verbs has not yet been motivated. The unification analysis is necessary for raising verbs, because continue accepts a nonreferential subject just in case that subject satisfies the co-occurrence restrictions imposed by the $\mathrm{CP}$ complement. But we have given no argument for using coindexing rather than unification to express the relation between the subject and the complement's subject with control verbs. Such an argument is provided in Problem 5 of this chapter.

Our analysis of control verbs like try ensures:

- that the subject of try is assigned to the TRIER role; and hence

- that nonreferential NPs can never be the subject of try;

- that the infinitival complements can never be of a kind that requires a nonreferential subject (because they must have an index identified with the trier); and

- that (18a) and (18b) have different meanings (because in one case the FBI is the trier and in the other, Lee is).

This analysis is illustrated in the following pair of SDs. 
284 / Syntactic Theory: A Formal Introduction

$\left[\begin{array}{ll}\mathrm{SPR} & \langle\rangle \\ \mathrm{MODE} & \text { prop } \\ \mathrm{INDEX} & s \\ \operatorname{RESTR} & \langle\text {, } 3, \text { 1 , 2 , 4 }\rangle\end{array}\right]$

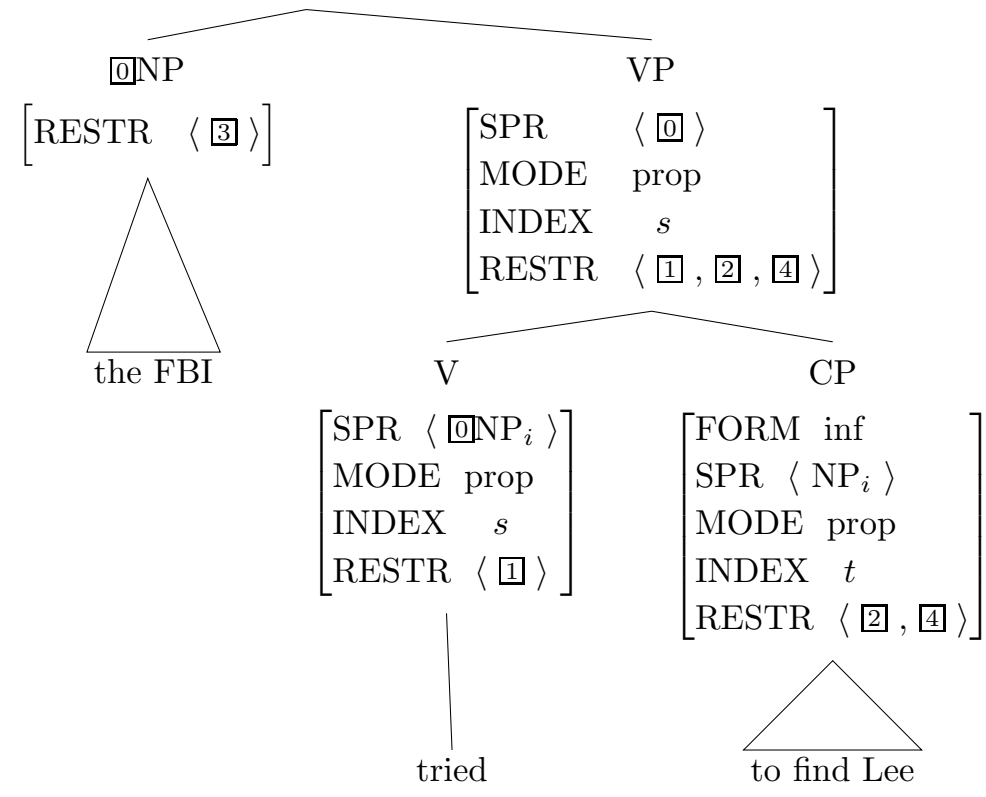


(23)

S

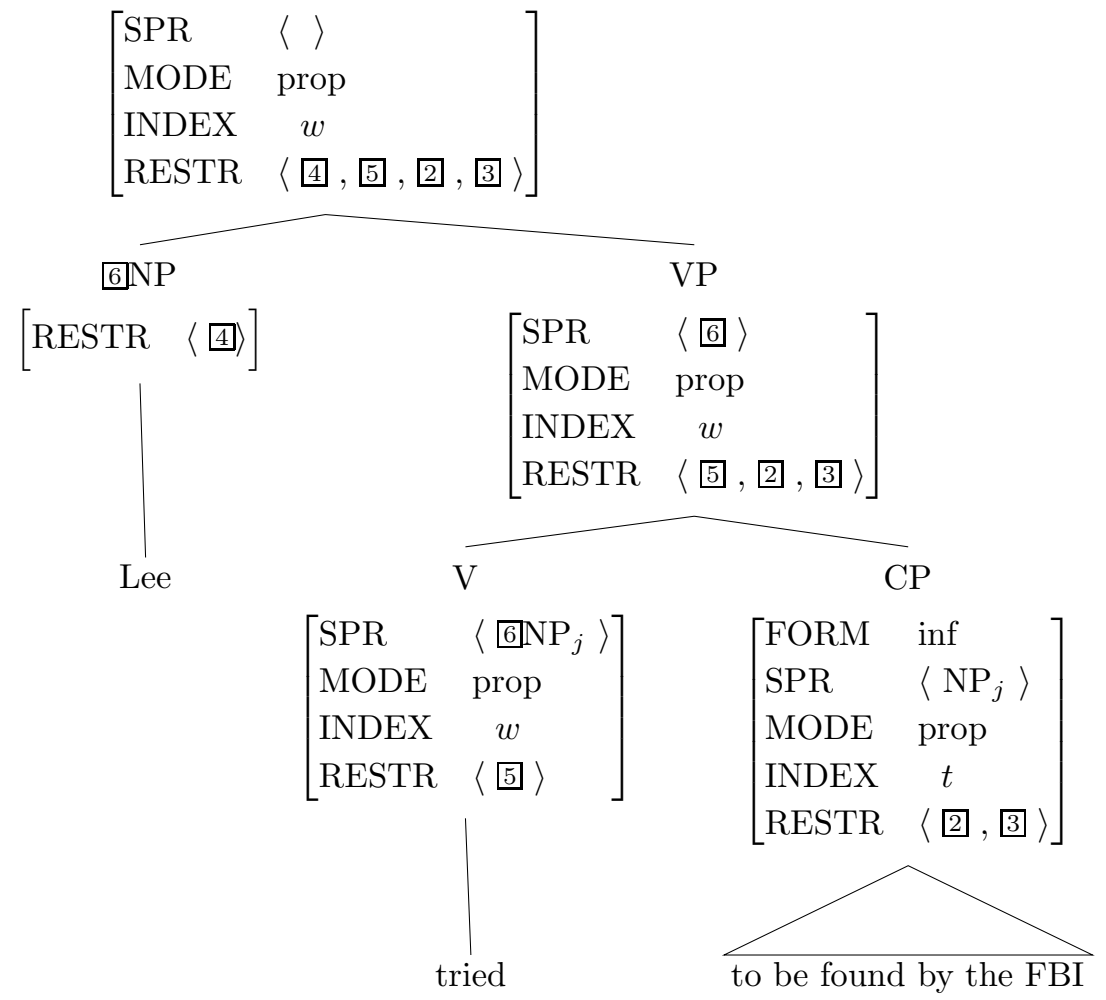

The first of these has the semantics shown in (24).

(24)

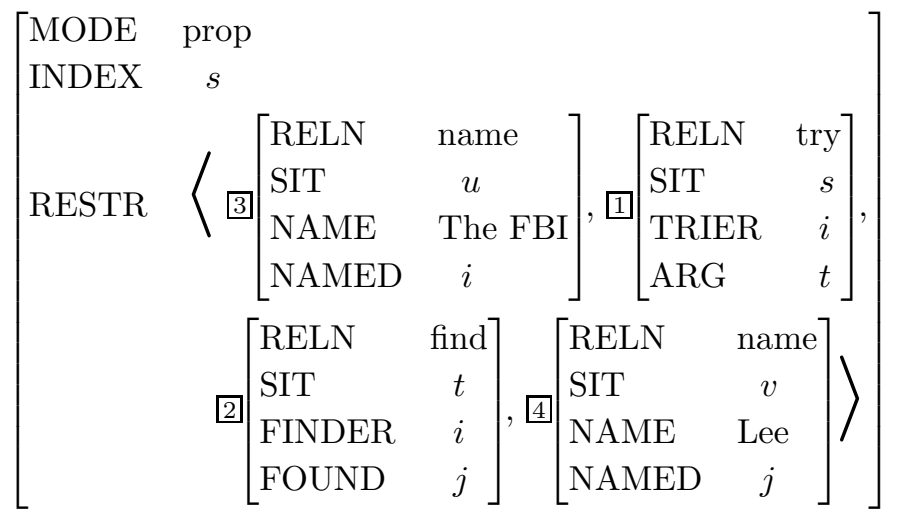

In contrast, the sentence with the passive complement in (23) has the 
semantics given in (25), where the trier is $j$, the index of Lee, not the FBI.

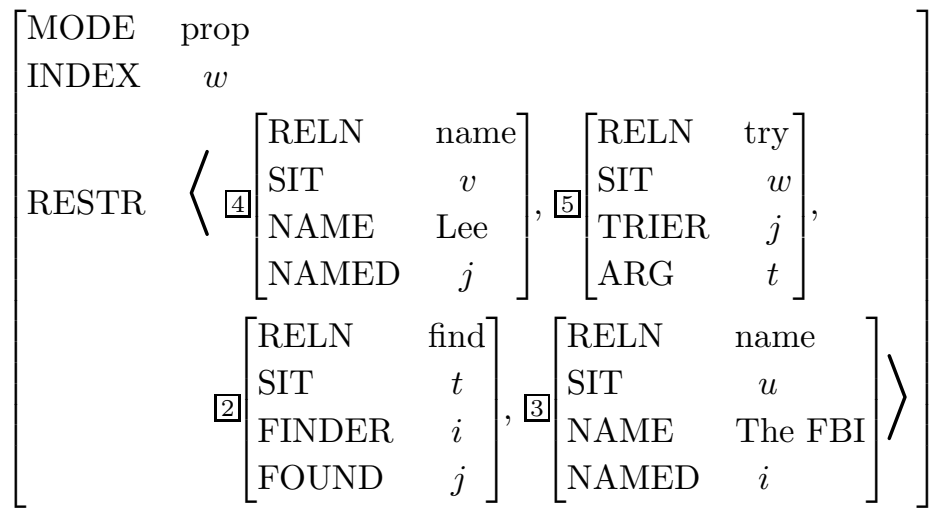

By positing a lexical distinction between raising and control verbs in the hierarchy of lexemes, we thus correctly account for their differing properties without adjusting our grammar rules or any other aspect of our theory.

\subsection{Subject Raising and Subject Control}

The verbs continue and try are not arbitrarily chosen examples: each one is representative of a class of verbs that share the properties we have been discussing. Subject raising verbs (or sometimes just 'raising' verbs) like continue express properties (relations of one argument) of propositions, allow nonreferential subjects (so long as their complements are compatible with them), and don't change meaning when their complements are passivized. Control verbs (sometimes also known as 'subject control' or 'equi' verbs) like try express a relation between an individual and a situation (referred to via its situational index), never take nonreferential subjects, and can induce a change of meaning when their complements are passivized.

In fact, it is not just verbs that can be divided into these two classes; there are also raising adjectives and control adjectives. They are exemplified in (26), with the diagnostic properties illustrated in (27)-(29). ${ }^{7}$

(26) a. Pat is likely to scream.

b. Pat is eager to scream.

(27) a. There is likely to be a letter in the mailbox.

b. It is likely to upset Pat that Chris left.

\footnotetext{
${ }^{7}$ Here we use the symbol ' $\approx$ ' to indicate sameness of truth conditions, and ' $\neq$ ' to indicate difference of truth conditions.
} 
c. Tabs are likely to be kept on participants.

d. Advantage is likely to be taken of unwary customers.

(28) a. The doctor is likely to examine Pat. $\approx$ Pat is likely to be examined by the doctor.

b. The doctor is eager to examine Pat. $\neq$ Pat is eager to be examined by the doctor.

(29) a. ${ }^{*}$ There is eager to be a letter in the mailbox.

b. ${ }^{*}$ It is eager to upset Pat that Chris left.

c. ${ }^{*}$ Tabs are eager to be kept on participants.

d. *Advantage is eager to be taken of unwary customers.

This suggests that our system of lexical types should be somewhat more abstract (perhaps introducing a type like subject-raising-lxm as a supertype of srv-lxm and a similar type of adjectival lexeme), in order to accommodate generalizations that cut across the various part of speech distinctions such as verb vs. adjective. ${ }^{8}$ Even the lexical entry for the infinitival complementizer to given at the beginning of this chapter could be simplified, if to were classified in terms of a cross-categorial type such as subject-raising-lxm.

\section{Problem 1: Lexical Entries}

Provide lexical entries for the raising and control adjectives likely and eager. Be as detailed as you need to be to represent the distinctions that account for the differences in their behavior discussed above. This should include discussing the role of be in mediating the relationship between these adjectives and their subjects. Do not assume the existence of a subject-raising-lxm type, unless you are prepared to give a precise characterization of that type.

\section{Problem 2: Classifying Predicates}

A. Classify the following verbs as raising or control. Give as many distinct tests as you can to justify your classifications, constructing three or four interestingly different examples for each verb.
$\circ$ tend
- condescend
$\circ$ manage
$\circ$ fail
$\circ$ happen

${ }^{8}$ This matter is taken up again in Chapter 16 . 
Make sure you restrict your attention to cases of the form: NP V to VP. That is, ignore cases like Kim manages a store, Carrie failed physics, and any other valence that doesn't resemble the continue vs. try pattern.

B. Classify the following adjectives as raising or control. Justify your classifications, constructing three or four interestingly different relevant examples for each adjective.
$\circ$ anxious
o apt
$\circ$ certain
- lucky

Make sure you restrict your attention to cases of the form: NP be Adj to VP. That is, ignore cases like Kim is anxious about the exam, Carrie is certain of the answer, and any other valence that doesn't resemble the likely vs. eager pattern.

\subsection{Object Raising and Object Control}

Consider now two new verbs: expect and persuade. These two verbs are similar in that both can occur in examples like the following.

(30) a. I expected Leslie to be aggressive.

b. I persuaded Leslie to be aggressive.

There are two possible analyses one could imagine for these verbs. There could be some kind of phrase that includes both the NP and the infinitival $\mathrm{CP}$ to be aggressive, as in:

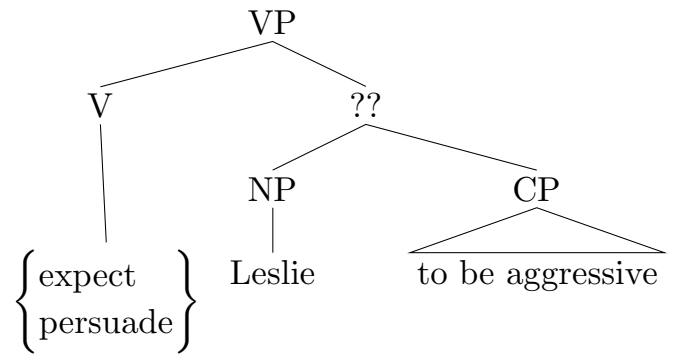

Alternatively, it is possible that the NP is the direct object of the verb and the infinitival $\mathrm{CP}$ is also a complement of the verb: 


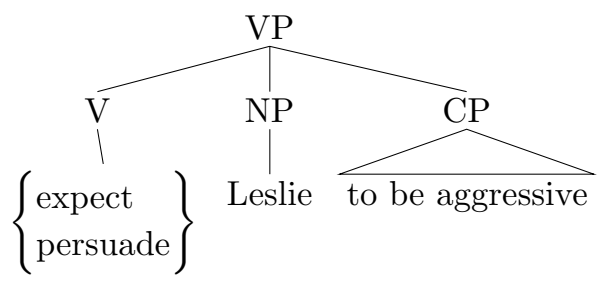

But in fact, only the latter structure is consistent with the analyses of other phenomena presented in earlier chapters.

\section{\. Problem 3: Justifying the Structure}

Use passivization to justify the choice of the structure in (32) over the alternative in (31). ${ }^{9}$

The difference between expect and persuade in structures like (32) is analogous to the distinction we just drew between continue and try. Expect is an example of what is usually called an 'object raising' verb (or 'subject-object raising' verb) and persuade is an 'object control' (or 'object equi') verb. Hence we will want to introduce the two types in (33) with the indicated constraints and then provide lexical entries for expect and persuade like the ones shown in (34).

(33) a. object-raising-verb-lxm (orv-lxm):

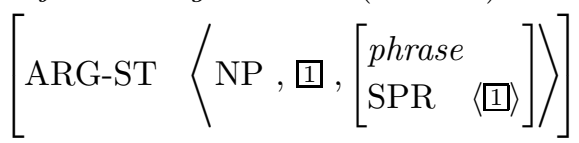

b. object-control-verb-lxm (ocv-lxm):

$$
\left[\text { ARG-ST }\left\langle\mathrm{NP}, \mathrm{NP}_{i},\left[\begin{array}{ll}
\text { phrase } & \\
\operatorname{SPR} & \left\langle\mathrm{NP}_{i}\right\rangle
\end{array}\right]\right\rangle\right]
$$

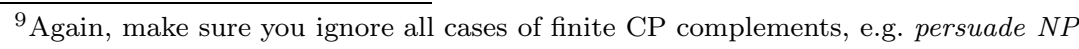
that ... or expect that ... and anything else not directly relevant. So ignore sentences like I expect to go, I am expecting Kim, and She is expecting.
} 
290 / Syntactic Theory: A Formal Introduction

(34) a

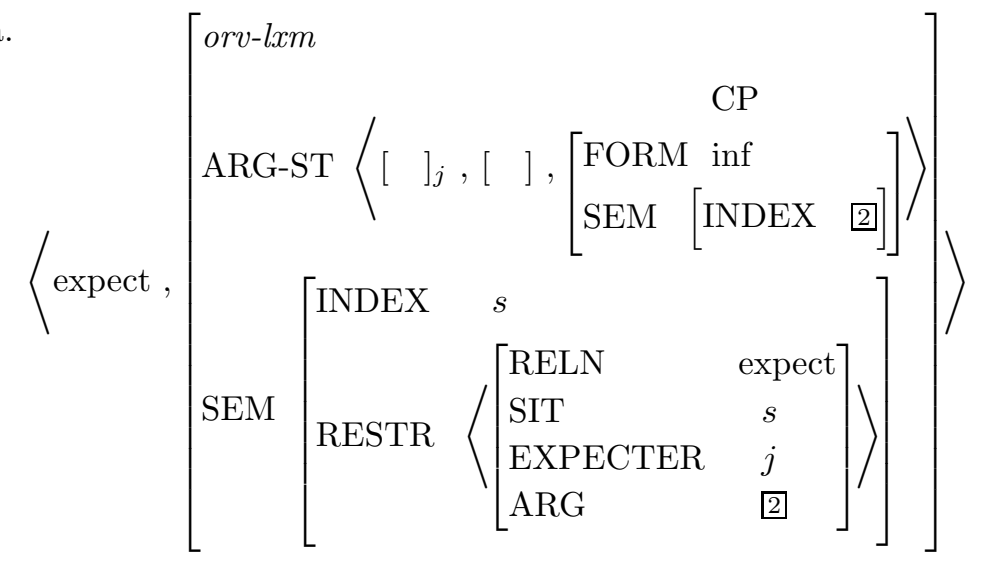

b.

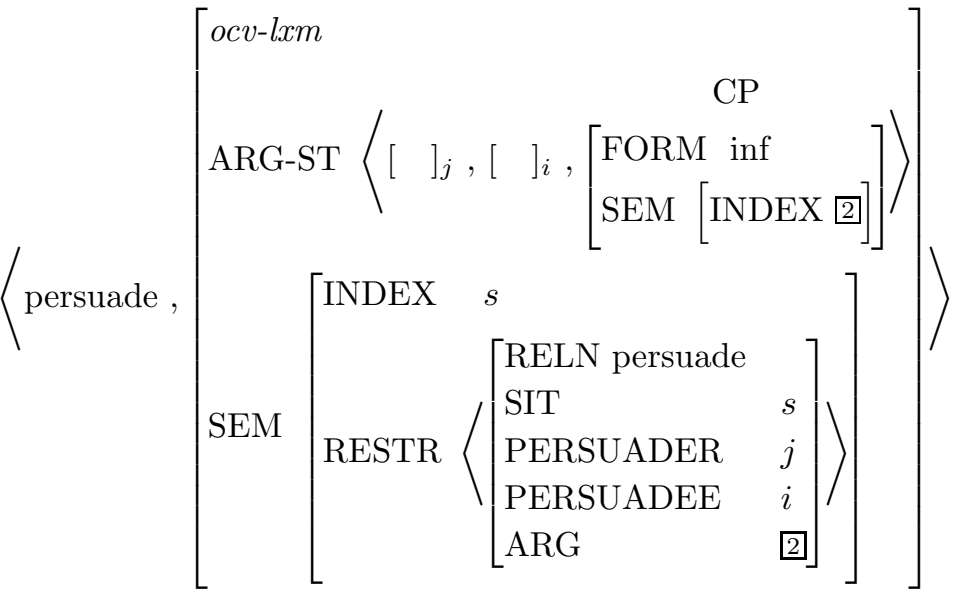

The words derived from these lexemes will then give rise to SDs like the following. 
$(35)$

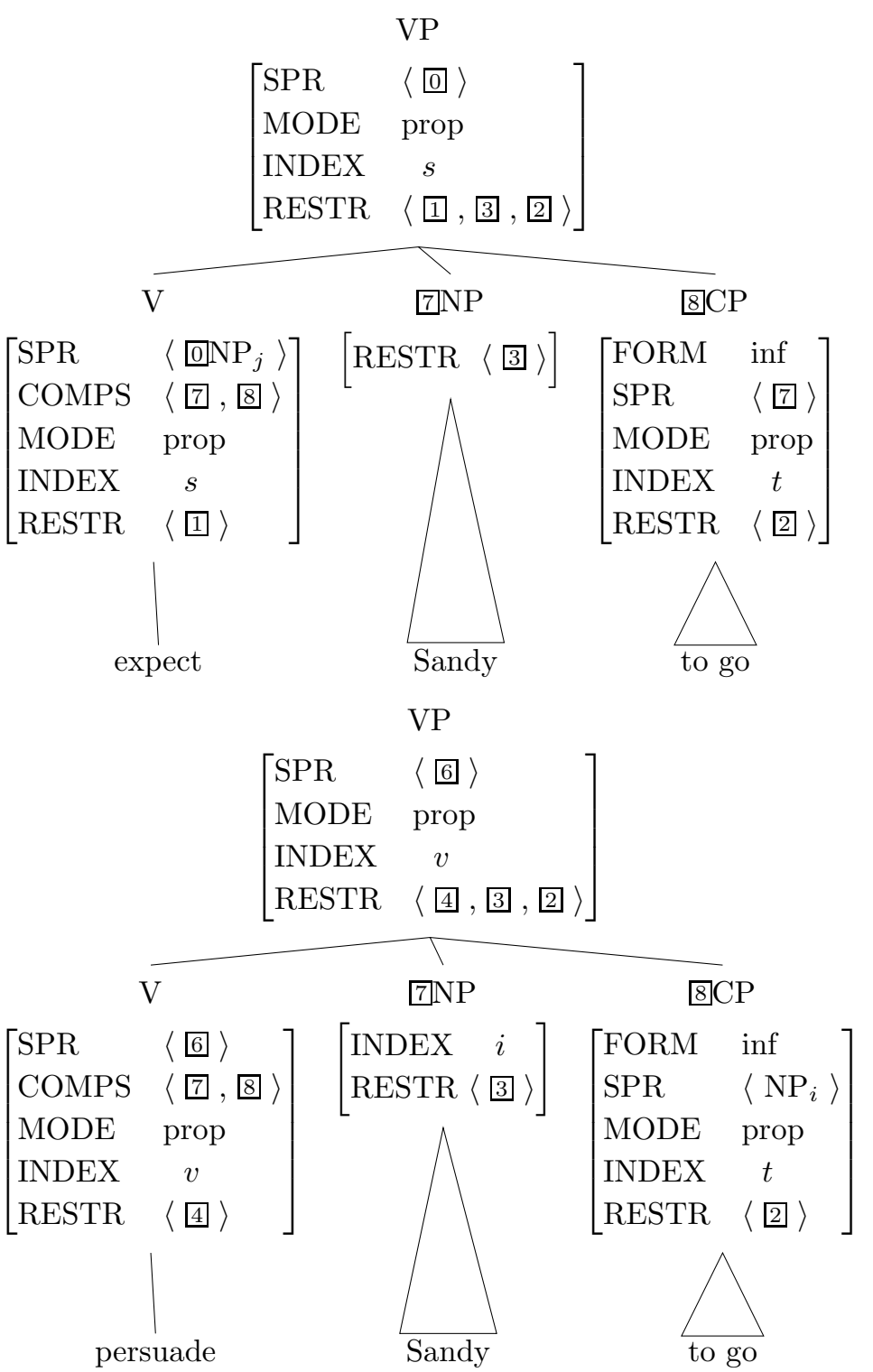

And the semantic analyses associated with these SDs are as shown in (37) and (38). 
292 / Syntactic Theory: A Formal Introduction

(37)

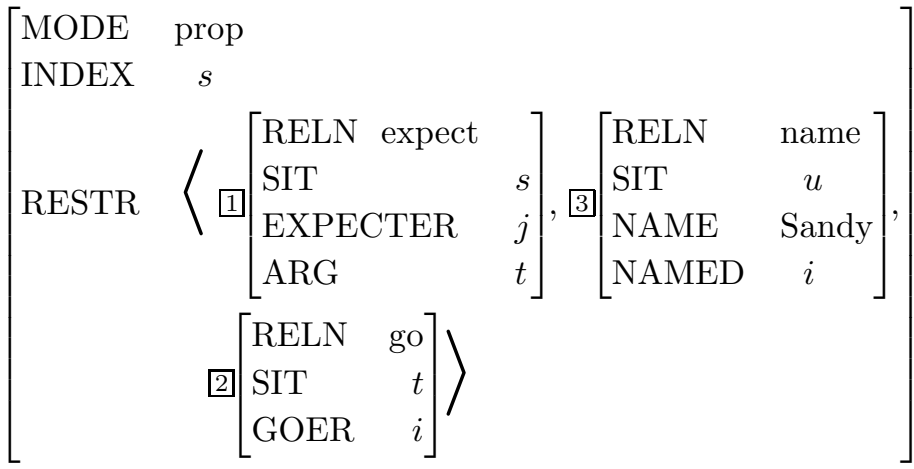

(38)

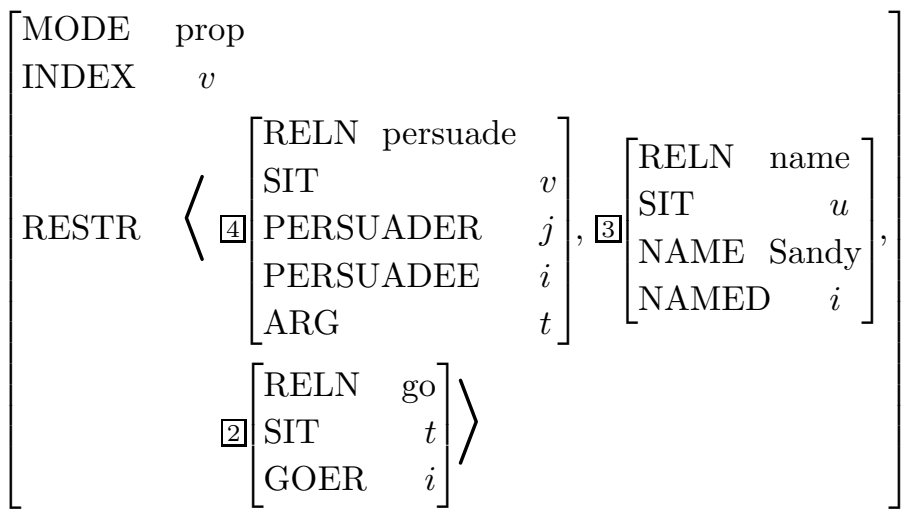

\. Problem 4: Expect vs. Persuade

We have just sketched an analysis of the verbs expect and persuade without providing justification for the fundamental distinction between the two types of lexeme we have posited. The purpose of this problem is to make you construct the arguments that underlie the proposed distinction between orv-lxm and ocv-lxm. ${ }^{10}$

A. Construct relevant examples with dummy there to support the proposed distinction.

B. Construct relevant examples with dummy it to support the proposed distinction.

${ }^{10}$ Again, make sure you ignore all irrelevant uses of these verbs, including cases of finite CP complements, e.g. persuade $N P$ that ... or expect that ... and anything else not directly relevant (I expect to go, I am expecting Kim, She is expecting, and so forth). 
C. Construct relevant examples to support the proposed distinction involving sentences containing parts of idioms.

D. Construct examples to support the proposed distinction involving paraphrase relations (or the lack thereof) between relevant pairs of sentences containing active and passive complements.

\section{Problem 5: Icelandic, Again}

As noted in an earlier problem, Icelandic has verbs that assign idiosyncratic cases to their subjects. Thus we get contrasts like the following (where other case markings on the subjects are unacceptable):

(i) Hun er vinsael.

She-NOM is popular

(ii) Hana vantar peninga.

Her-ACC lacks money

(iii) Henni batanadi veikin.

Her-DAT recovered-from the-disease

In infinitival constructions, two patterns are observed (again, other case markings on the subjects are unacceptable):

(iv) Eg vonast til ad vanta ekki peninga I-NOM hope for to lack not money

(v) Eg vonast til ad batnad veikin I-NOM hope for to recover-from the-disease

(vi) Hana virdist vanta peninga.

Her-ACC seems to-lack money

(vii) Henni virdist hafa batnad veikin. Her-DAT seems to-have recovered-from the-disease

Use these examples to argue for a distinction between control and raising verbs in Icelandic, and explain how our analysis of the distinction accounts for the two patterns.

\section{\1 Problem 6: There, There....}

In an earlier problem, you were asked to develop an analysis of verb agreement in sentences with there as the subject. Simplifying somewhat, finite forms of be that take there as their subject agree in number with the NP following be. This could be formalized in either of two ways: 
- the relevant lexical entries for is, are, was, and were could stipulate the value of the NUM feature of the second element of their ARGST list; or

- the entry for the lexeme be could stipulate that the NUM value on there was the same as the NUM value on the second element of the ARG-ST list (and this basic entry would then undergo the normal inflectional lexical rules).

Both analyses cover the data in simple there sentences.

A. Explain how each analysis covers the data in simple there sentences.

The two analyses make different predictions, however, with regard to the interaction of there with raising.

B. Discuss the relevance of the following data for choosing between the two competing analyses of there-sentences:

(i) There continues to be a bug in my program.

(ii) ${ }^{*}$ There continue to be a bug in my program.

\section{(1) Problem 7: Reflexives in Infinitival Complements}

In Problem 4 above, you justified our analysis of expect and persuade.

A. Does that analysis, together with the analysis of reflexive and pronoun binding we developed in Chapter 7 account for the following contrasts?

(i) We expect the doctor to examine us.

(ii)* We expect the doctor to examine ourselves.

(iii) We expect them to examine themselves.

(iv) ${ }^{*}$ We expect them ${ }_{i}$ to examine them . $_{\text {. }}$

(v) We persuaded the doctor to examine us.

(vi)* We persuaded the doctor to examine ourselves.

(vii) We persuaded them to examine themselves.

(viii) $^{*}$ We persuaded them ${ }_{i}$ to examine them ${ }_{i}$.

Explain clearly why or why not.

(B) Consider the following contrasts.

(i) They appeared to us to support themselves.

(ii) ${ }^{*}$ They $_{i}$ appeared to us to support them . $^{\text {. }}$

(iii) ${ }^{*}$ They appealed to us to support themselves.

(iv) They $_{i}$ appealed to us to support them . $_{\text {. }}$

Develop an analysis of appear and appeal (that fits with our binding theory) that explains these contrasts. Do not worry too much 
Infinitival Complements / 295

about the details of the type hierarchy your analysis will require; it is sufficient to give the lexical entries needed for the lexemes appeal and appear and to explain how these interact with binding theory and other aspects of our grammar to explain the relevant data.

\subsection{Further Reading}

The raising/control distinction was first introduced into the generative literature (but with different terminology) by Chomsky (1965) and Rosenbaum (1967). Other discussions of these phenomena include Jackendoff (1972), Postal (1974), Bach (1979), Bresnan (1982c), and Sag and Pollard (1991). 

13

\section{Auxiliary Verbs}

\subsection{Introduction}

In this chapter, we investigate the English auxiliary verb system. This is one of the most extensively analyzed (and frequently reanalyzed) empirical domains in the literature on generative syntax. Chomsky's transformational treatment of auxiliaries in Syntactic Structures was immensely influential; it galvanized the field in support of transformational grammar. In the intervening four decades, numerous alternative treatments have been advanced within a wide range of theoretical frameworks.

The auxiliary verb system is a particularly attractive domain for syntacticians because it involves a relatively small number of elements (basically, just a handful of verbs and the word not) which interact with each other in intricate and apparently complex ways. Moreover, though the English auxiliary system is quite language-specific (even closely related languages like Dutch and French have verbal systems that behave very differently), there are analogous elements in many other languages. Thus, this is a fertile domain for examining the interaction of universal grammatical principles with language-specific variation.

Cross-linguistically, the elements that are called 'auxiliaries' tend to share the following semantic and syntactic characteristics: (i) they express notions of time (past, present, future; continuation, completion), necessity, possibility, obligation, permission, negation, or questioning; and (ii) they occur in fixed positions in sentences, usually at or near the beginning or end. English auxiliaries are a special kind of verb, including what are called 'modals' (can, could, may, might, must, shall, should, will, would), and uses of be, do, and have as 'helping verbs'.

Our analysis treats auxiliaries as a special kind of subject raising verb, an idea originally proposed by J. R. Ross in the 1960s. We proceed from this basis to show how the special properties of auxiliaries with respect to such phenomena as negation and questioning can be handled 
in terms of syntactic features, and how the relevant generalizations about them can be expressed in terms of lexical rules.

\subsection{Basic Facts about Auxiliaries}

Consider the following data:

(1) Pat tap-danced.

(2) a. Pat can tap-dance.

b. * Pat can $\left\{\begin{array}{l}\text { tap-danced } \\ \text { tap-dancing }\end{array}\right\}$.

c. Pat is tap-dancing.

d. * Pat is $\left\{\begin{array}{l}\text { tap-dance } \\ \text { tap-danced }\end{array}\right\}$.

e. Pat has tap-danced.

f. * Pat has $\left\{\begin{array}{l}\text { tap-dance } \\ \text { tap-dancing }\end{array}\right\}$.

(3) a. Pat could have tap-danced.

b. Pat could be tap-dancing.

c. Pat has been tap-dancing.

d. Pat could have been tap-dancing.

e. *Pat has could tap-danced.

f. *Pat is having tap-danced.

g. *Pat could be having tap-danced.

(4) a. *Pat could will tap-dance.

b. *Pat has had tap-danced.

c. ${ }^{*}$ Pat is being tap-dancing.

These examples illustrate the following generalizations about auxiliary verbs: ${ }^{1}$

(5) a. Auxiliaries are optional.

b. Auxiliaries determine the FORM of the following verb.

c. Auxiliaries can co-occur with each other, but only in a fixed order.

d. Auxiliaries (of any given type) cannot iterate.

\footnotetext{
${ }^{1}$ In some dialects of English,certain sequences of modals, such as in Pat might could tap-dance are possible, apparently violating (5d). However, even in those dialects, such sequences are restricted to certain combinations. This suggests that the variation should be handled in terms of differences in individual lexical entries (e.g. in their lexical types or in their possible FORM values), rather than through a wholly different analysis of modals as a class. Investigating this dialect variation is beyond the scope of this book.
} 
We find in the literature two basic approaches to the analysis of English auxiliaries. The first, going back to Chomsky's original treatment, involves introducing a new (phrasal) category called AUX which dominates all auxliary verbs. AUX is expanded with a rule something like the following: ${ }^{2}$

(6) AUX $\rightarrow(\mathrm{M})$ (PERF) $(\mathrm{PROG})$.

This approach has the attractive feature that it straightforwardly captures the optionality, the ordering, and the non-iterability of auxiliaries - that is, the properties listed in (5a, c, d). On the other hand, it doesn't say anything about the FORM dependencies - so it needs augmentation if it is to rule out the starred alternatives in (2).

The other type of analysis treats auxiliaries as verbs that take VP complements. This has the immediate advantage that it provides the tools for restricting the FORM value of the head of the following VP. Its potential disadvantage is that the mechanisms expressing the ordering and iteration constraints are somewhat less direct.

The second approach fits much better with the grammar we have been developing. This is because the AUX constituent doesn't seem to have a head, so it isn't clear how to fit it in with our phrase structure rules or with our way of handling co-occurrence restrictions such as the FORM dependencies between auxiliaries. In what follows, we will pursue the VP complement analysis of auxiliary verbs. In fact, as will become clear below, this treatment can be incorporated into our grammar without assuming any new grammar rules - all the essential information will be in the lexical entries for auxiliary verbs.

\subsubsection{Lexical Entries for Auxiliary Verbs}

We have already seen one auxiliary verb's entry, namely, the verb be. We will use this as a model for the others and then return to the issues of ordering and iteration.

The entry for be that we originally used for the passive was generalized in Chapter 11 to cover other uses of be, including the progressive. The entry we gave for be (which was intended to cover its co-occurrence with both passive and progressive VP complements) was the following:

\footnotetext{
2 'M' in (6) stands for 'modal', 'PERF' for 'perfective', and 'PROG' for 'progressive'. The latter two are fairly standard terms for the uses of have and be under discussion.
} 
300 / Syntactic Theory: A Formal Introduction

(7)

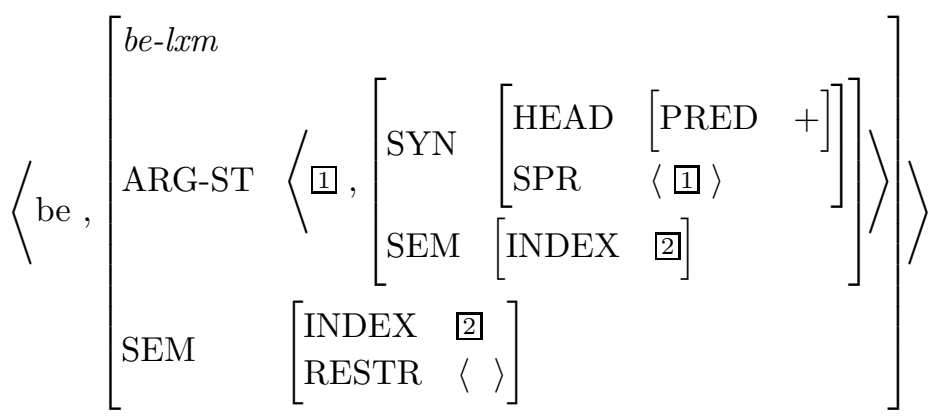

Recall that the specification [PRED +] distinguishes predicative phrases (certain APs, PPs, and NPs, as well as passive and present participial VPs) from other phrases. (See section 2 of Chapter 11.)

Note that (7) clearly bears a close resemblance to the type srv-lxm, which we introduced in Chapter 12. Thus instead of listing such shared properties in the lexical entries of be and the other auxiliary verbs, we posit a new subtype of srv-lxm, which we will call auxv-lxm.

In the analyses of negation, inversion, contraction, and ellipsis given below, we make use of a HEAD feature AUX to distinguish auxiliary verbs from all others. The constraint needed for the type auxv-lxm is thus minimally the following:

$$
\text { auxv-lxm: }[\mathrm{SYN}[\mathrm{HEAD}[\mathrm{AUX}+]]]]
$$

Once the type auxv-lxm is part of the lexical type hierarchy, the type be-lxm can then be eliminated in favor of a simpler analysis where be is assigned to the type auxv-lxm. This allows us to simplify the lexical entry of be, relying on inheritance to express further properties that be shares with other lexemes. Thus the lexical entry specified in (9) is enriched to (10) through constraint inheritance.

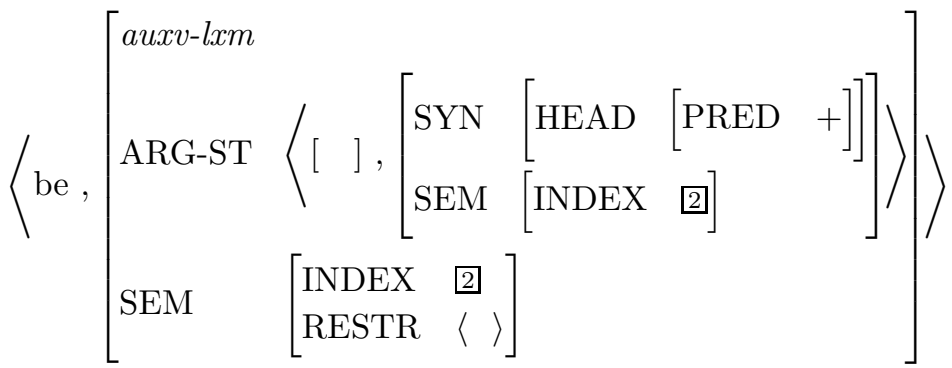




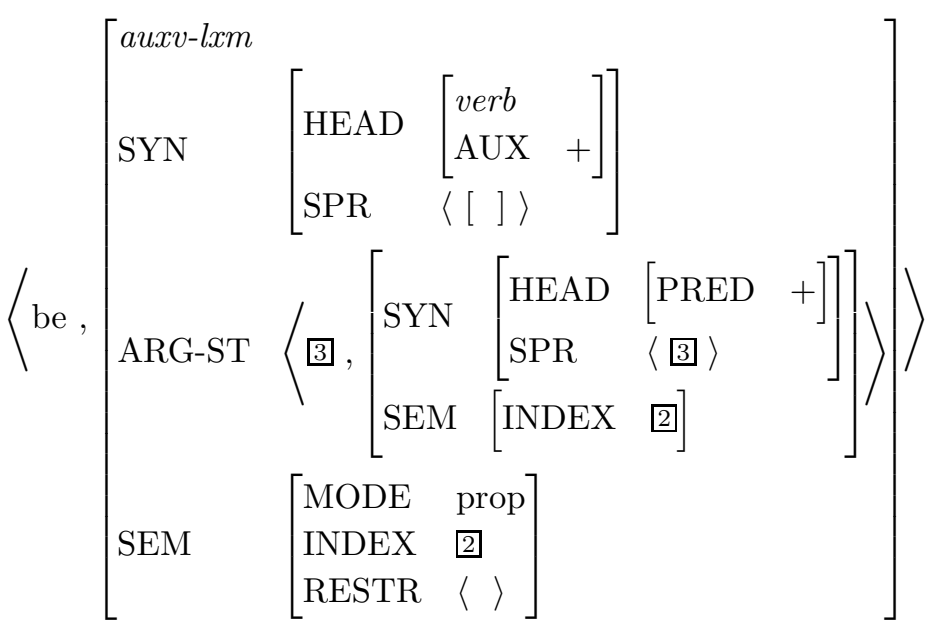

And the entry for auxiliary have will look something like this:

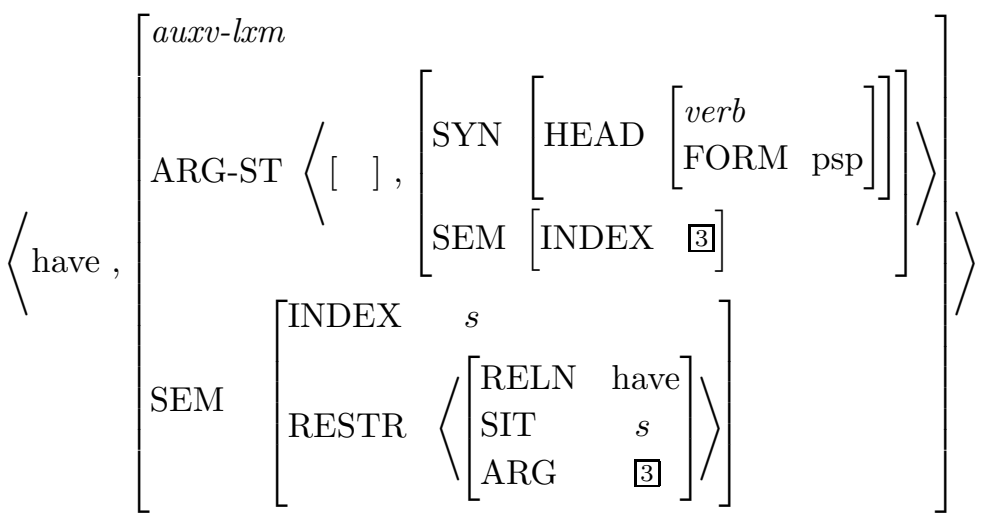

This entry differs from (9) in two essential ways: first, the complement (i.e. the second element of ARG-ST) must be a VP headed by a past participle (indicated with the specification [FORM psp]); second, the semantics of auxiliary have, unlike that of be, is not vacuous. The form of the complement and the meaning are, in fact, what distinguishes have from other auxiliary verbs in English. Again, no constraint needs to be stated in (11) to identify the first element of the ARG-ST list with the VP complement's SPR value, as this information is inherited from the supertype srv-lxm.

The modals have a peculiarity, illustrated by the following examples, that should be represented in our lexical entry.

(12) a. ${ }^{*}$ Pat hopes to can study syntax. 
b. * Sandy has musted study syntax.

These examples show that modals don't have entries with [FORM inf] or [FORM psp]. In fact, the only contexts in which modals can occur are ones where we normally expect finite verbs. Notice, by the way, that this restriction appears not to be semantically based, since appropriate forms of be able to (which is virtually synonymous with can) are fine in these contexts. So our entry for a modal could look like (13).

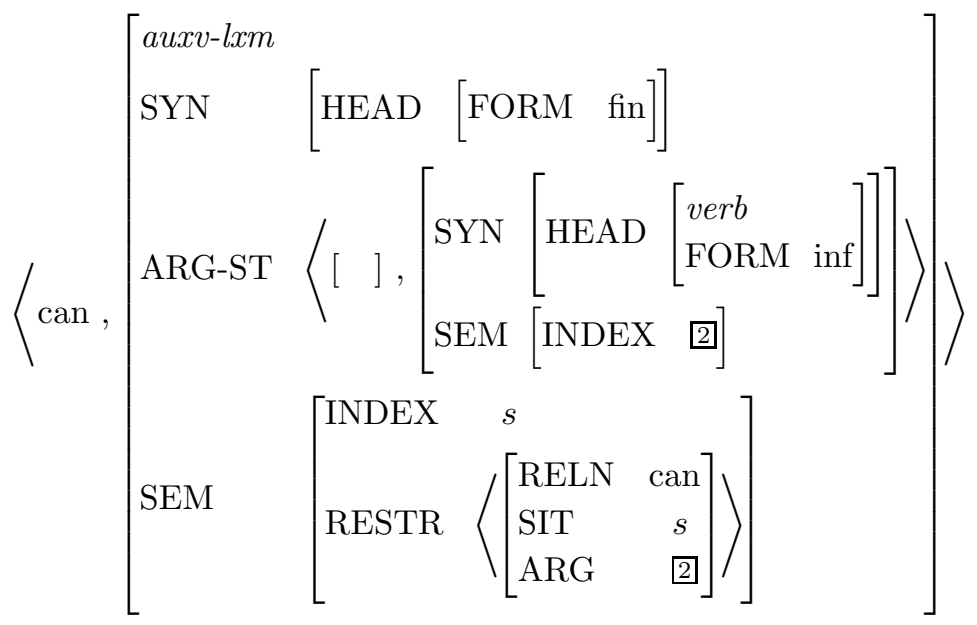

Notice that unlike most lexemes of type verb, (13) specifies a FORM value. The FORM values that we use for verbs (e.g. fin, psp) are, in most cases, introduced into lexical entries by inflectional rules like the PastTense Verb Lexical Rule or the Passive Lexical Rule. The stipulation that the lexeme can is [FORM fin] means that it cannot serve as the input to any lexical rule that introduces another FORM value, since the output FORM value would fail to unify.

\section{Problem 1: Modals and Imperatives}

Explain in detail why modals cannot occur in imperatives, as illustrated by the following:

(i)*Can solve all the problems! (cf. Be able to solve all the problems!)

Before we look at the ordering and iteration constraints on auxiliaries, notice that our analysis entails that auxiliaries are treated semantically like raising verbs. That is, they assign no semantic role to their subjects; rather, the subject of an auxiliary verb gets its seman- 
tic role - and various syntactic properties - from the complement of the auxiliary. Given this, our analysis makes predictions regarding the possibility of auxiliaries occurring in sentences with dummy subjects or idiom chunk subjects. Specifically, we correctly predict that sentences like the following are grammatical:

(14) a. There might be a unicorn in the garden.

b. There has been a unicorn in the garden.

c. It will annoy people that dogs are barking.

d. It is annoying people that dogs are barking.

e. Tabs have been kept on Pat by the FBI.

f. Tabs are being kept on Pat by the FBI.

We also predict that there is no difference in truth conditions between A doctor must have been examining Pat and Pat must have been being examined by a doctor. This also seems correct.

\subsubsection{Co-Occurrence Constraints on Auxiliaries}

Some of the facts we noticed earlier about the ordering among auxiliaries and the constraints on their iteration fall out naturally from our lexical entries. Others require some work.

- The fact that modals must come first in any string of auxiliaries follows from the fact that they only have finite entries. Since the complements to the auxiliaries have and be must have some FORM specification other than finite (namely, psp, prp, or pass), modals can't head complements to have or be.

- The fact that modals don't iterate also follows from their obligatory finiteness, together with the requirement that the head of the complement to a modal must be [FORM inf].

- The fact that perfective have can't follow progressive be can be seen as a manifestation of a wider generalization. Not all verbs can appear in the progressive. Specifically, verbs (often referred to as 'stative' verbs) whose semantics involves a state rather than an action or an activity, generally sound bad in the progressive:

(15) a. * Pat is owning a house.

b. ${ }^{*}$ Chris is knowing the answer.

This is pretty clearly a semantic restriction: making something progressive turns an action or activity into a state, namely, the state of that action or activity being in progress; for stative verbs such as own or know, it doesn't make sense to talk about the state being in progress. The perfective have is also used to denote states, namely, the state of completion for whatever its complement VP denotes. Since stative verbs don't have progressives, the perfective 
have doesn't either. Hence (16) is unacceptable for the same reason as (15).

$(16)^{*}$ Pat is having slept.

We will not attempt to formalize this restriction in our lexical entry, since it depends on dealing with aspects of semantics that go beyond the scope of this text.

- The same semantic restriction accounts for the failure of progressive be to iterate, since it, too, denotes a state, and hence can't be the complement to another occurrence of progressive be, ruling out (17). ${ }^{3}$

$(17)^{*}$ Chris is being sleeping.

Again, we'll leave the formalization of this to the semanticists.

- Finally, the failure of perfective have to iterate cannot be handled in just the same way as the last two restrictions, since stative verbs can occur in the perfective. We could stipulate that perfective have is an exception to the Past-Participle Lexical Rule. This would entail that it doesn't have a past participle entry, so it couldn't appear as the head of the VP complement to another occurrence of perfective have. Alternatively, we could try to find a semantic explanation, for example, that iterating perfective have would be redundant. For now, we will not choose between these analyses.

\subsection{The NICE Properties}

English auxiliary verbs differ from other verbs in (at least) four ways:

(18) a. NeGATion: They can be immediately followed by not as a way of negating the sentence.

b. INVERSION: They can precede the subject in questions.

c. CONTRACTION: They have contracted forms created with the suffix $n ' t$.

d. ELLIPSIS: Their complements can be omitted when the meaning of the missing complement can be reconstructed from the surrounding linguistic context.

\footnotetext{
${ }^{3}$ This is simplified in a number of respects. In particular, given the kind of semantic analysis we developed for inflected verbs in Chapter 8, the progressive participle (like other inflected forms) will be assigned a semantics with two predications on its RESTR list. The first is the predication that is lexemically specified and whose situation is embedded as an argument of the second predication, which introduces the progressive meaning. The semantic incompatiblity discussed in the text thus really arises from the fact that the situation of the progressive predication can't be the argument of another progressive predication. We will not work out the details of this analysis here, though they are straightforward.
} 
These are sometimes called the 'NICE' properties of auxiliaries. They are illustrated in the following examples. ${ }^{4}$

(19) a. Pat should not leave.

b. ${ }^{*}$ Pat raked not leaves.

(20) a. Has Pat left town?

b. * Left Pat town?

(21) a. They haven't cut the price.

b. *They cutn't the price.

c. ${ }^{*}$ They halven't the price.

(22) a. If anybody is spoiling the children, Pat is.

b. ${ }^{*}$ If anybody keeps spoiling the children, Pat keeps.

Our analysis of these differences will be purely lexical in nature. It will make use of a handful of features (including AUX) and a small set of lexical rules.

\subsection{Auxiliary Do}

Notice that the negative, interrogative, or elliptical counterparts to sentences with no auxiliary verb are usually expressed with the verb $d o$ :

(23) a. Pat raked leaves.

b. Pat did not rake leaves.

(24) a. Pat left town.

b. Did Pat leave town?

(25) a. They halved the price.

b. They didn't halve the price.

(26) If anybody keeps spoiling the children, Pat does.

So let's add $d o$ to our list of auxiliary verbs. To do so, we need to examine its properties. For example, what is the FORM of the head of its complement? Are there any other restrictions on the kind of complements it can take? Does it have any restrictions on the FORMS it can have?

The following examples illustrate properties of $d o$ that will have to be incorporated into the analysis.

(27) a. Pat does not eat garlic.

\footnotetext{
${ }^{4}$ In earlier stages of the English language, nonauxiliary verbs also exhibited certain of the NICE properties. In fact, this earlier state of affairs still survives today in certain fixed expressions, e.g. How goes it? or old sayings, such as Waste not, want not.
} 
306 / Syntactic Theory: A Formal Introduction

b. ${ }^{*}$ Pat does not $\left\{\begin{array}{l}\text { eats } \\ \text { eating } \\ \text { eaten }\end{array}\right\}$ garlic.

(28) a. Pat tried to take logic.

b. *Pat tried to don't take logic.

c. * Pat has done not take logic.

d. *Pat is doing not take logic.

(29) a. Does Pat like watching television?

b. * Does Pat be watching television?

c. ${ }^{*}$ Does Pat have watched television?

These examples show:

- The head of the complement of do must be [FORM inf].

- Auxiliary do itself only occurs in finite forms. ${ }^{5}$

- The head of the complement of $d o$ cannot be an auxiliary verb.

That is, do behaves just like the modals, but with the added restrictions that (i) its complement cannot be headed by an auxiliary verb and (ii) it makes no contribution to meaning, except to bear the tense information that corresponds to its finite morphology. This can be encoded straightforwardly into the lexical entry for the lexeme for auxiliary $d o$ :

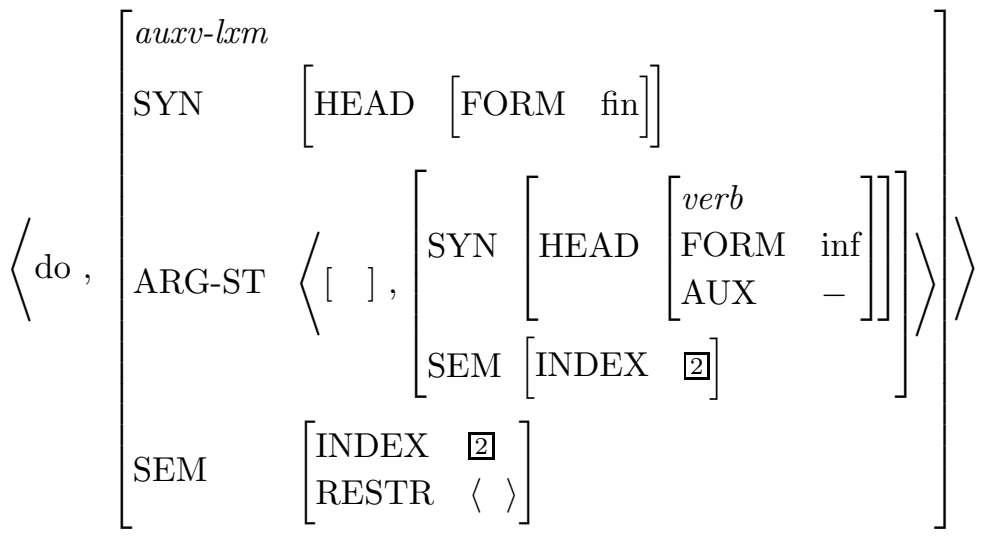

The semantics specified in (30) correctly guarantees that do makes no contribution to the semantics; and the [FORM fin] specification ensures that the only words derived from this root are finite forms. Of course,

\footnotetext{
${ }^{5}$ The do that is found in imperatives like Do sit down! is not the same do, as evidenced by the fact that it can co-occur with be, as in Do be quiet! (cf *Do they be quiet?).
} 
those words have nonempty RESTR values in their semantics, because the lexical rules that generate them add temporal restrictions.

The analysis of do as an auxv-lxm (and hence as a srv-lxm) also predicts that it will allow dummies and idiom chunks as subjects, given the right complements. It is actually tricky to construct such sentences, since the verbs that license dummies or idiom chunk subjects are very often forms of $b e$, and hence are [AUX +]. Since do doesn't allow [AUX +] complements, this requires an extra step in the construction of the relevant examples. The trick is to put a nonauxiliary raising verb in between, yielding sentences like (31).

(31) a. There did not continue to be riots in the park.

b. Does it continue to annoy people that dogs bark?

c. Close tabs don't seem to have been kept on anybody.

\subsection{Analyzing the NICE Properties}

Let us work out analyses within our theory for each of the NICE properties. This will involve one lexical rule per property, plus the addition of a few new features.

\subsubsection{Negation}

The word not can appear in a wide variety of contexts, but in this discussion, we will restrict our attention to sentence negation - that is, to cases in which it is the whole clause that is interpreted as being negated. Hence, we will not be dealing with non-sentence negation, that is, with uses of not like those in (32). ${ }^{6}$

(32) a. Not many arrows hit the target.

b. I try not to make trouble.

c. Pat must have not been listening.

We also exclude from our analysis the second occurrence of not in examples like (33), since this is negating only the VP, not the whole sentence.

(33) Kleptomaniacs can not NOT steal.

If both occurrences of not in (33) were instances of sentence negation, they would cancel each other out, and it would mean 'kleptomaniacs can steal'. While (33) clearly has this as an entailment, it actually asserts something stronger, namely, that kleptomaniacs MUST steal. This is the

\footnotetext{
${ }^{6}$ In a nutshell, our analysis of these is to treat not as a modifier that attaches to the left of phrases (other than finite verb phrases). Working this analysis out in detail would require some additional machinery that would take us too far afield, so we will not pursue it here.
} 
interpretation we get if we assume that the first not negates the whole sentence but the second one negates only steal.

The sentence in (33) shows that there can be only one sentential not per clause. To ensure this, we introduce a feature NEG. The negation rule will specify that its input must be [NEG -], and the output is $[\mathrm{NEG}+]$. This will prevent it from applying to its own output, thereby preventing any clause from having multiple sentential negations. ${ }^{7}$

The sort of data we do want to account for includes the following:

(34) a. Pat will not leave.

b. *Pat not will leave.

c. Pat has not left.

d. ${ }^{*}$ Pat not left.

e. Pat would not have left.

f. * Pat not has left.

g. Pat must not have been leaving.

The generalization about where sentential not can appear is that it must follow a finite auxiliary verb. We can formalize this by having a lexical rule that inserts not as a second argument of a finite auxiliary and requires the output to be [NEG + ].

\footnotetext{
${ }^{7} \mathrm{NEG}$ is declared as appropriate only for the part of speech verb. And by adding the default constraint [NEG / - ] to the type verb-lxm, we can ensure that the only verbs that are [NEG +] either are exceptionally so listed or else have undergone a lexical rule that makes them $[\mathrm{NEG}+]$.
} 
(35) Negation Lexical Rule

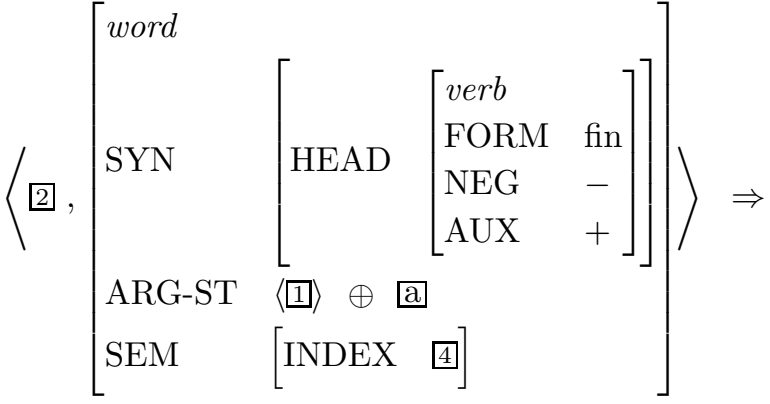

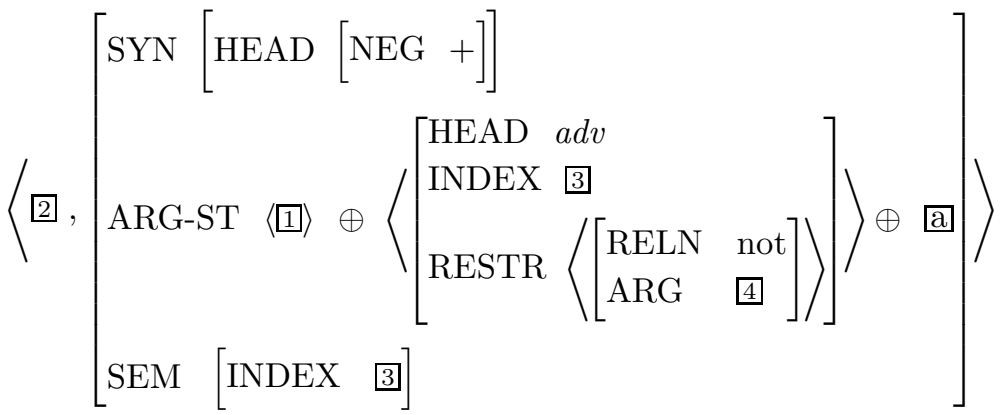

Note that this is the first lexical rule we have seen that creates new words from words already formed via application of lexical rules to lexemes. Other analyses are possible, but this is the most straightforward way of limiting the application of the rule to finite forms.

The rule in (35) has the intended effect because the word not is treated as an adverb, and it is the only adverb lexically specified as having 'not' as the value of RELN on its RESTRICTION list, as sketched in (36).

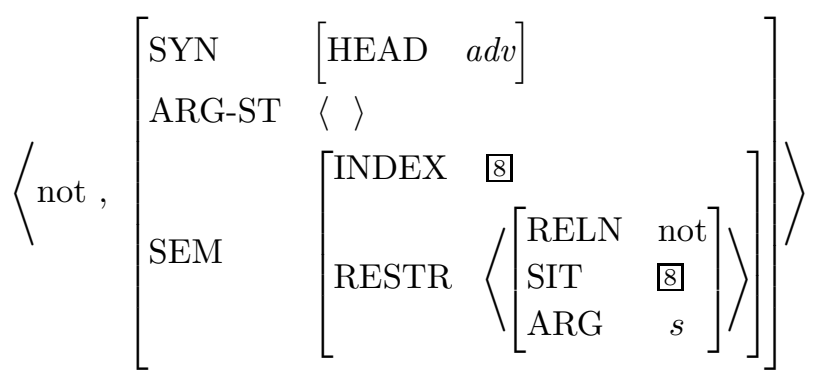

The effect of the rule in (35), then, is to permit not (and only not) to appear as an optional second argument of an auxiliary. And when this 
happens, the index of not (3 in (35)) is the INDEX of the auxiliary, and the semantic index of the rule input (4 in (35)) is the argument of the 'not' relation. This produces a negated semantics for a VP (and hence for a sentence), as shown in (37).

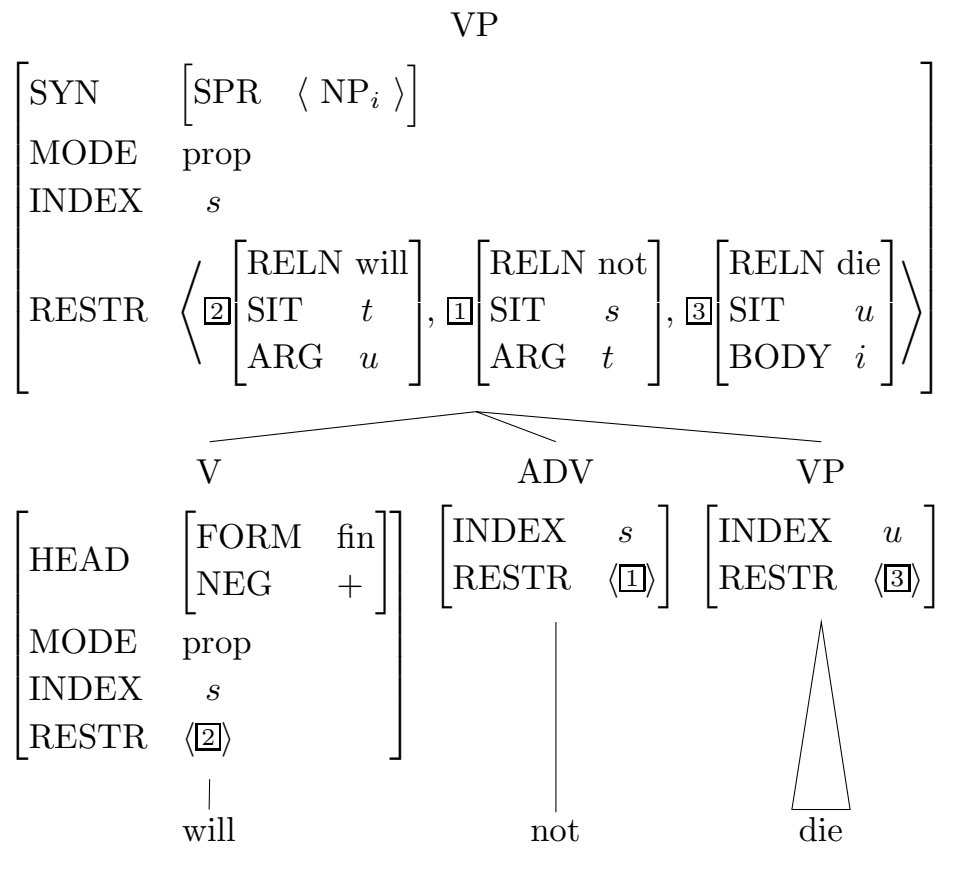

\section{\Problem 2: Negation and Do}

How do our rules and lexical entries so far account for the following contrast? Be precise.

(i) * Pat put not the ice cream in the freezer.

(ii) Pat did not put the ice cream in the freezer.

\subsubsection{Inversion}

In questions, a finite auxiliary verb precedes the subject. All the same restrictions that apply to auxiliaries in declaratives apply when they occur in this inverted order. Thus we have:

(38) a. Can Pat tap-dance? 
b. ${ }^{*}$ Can Pat $\left\{\begin{array}{l}\text { tap-danced } \\ \text { tap-dancing }\end{array}\right\}$ ?

c. Is Pat tap-dancing?

d. ${ }^{\text {Is Pat }}\left\{\begin{array}{l}\text { tap-dance } \\ \text { tap-danced }\end{array}\right\}$ ?

e. Has Pat tap-danced?

f. *Has Pat $\left\{\begin{array}{l}\text { tap-dance } \\ \text { tap-dancing }\end{array}\right\}$ ?

g. Could Pat be tap-dancing?

h. * Is Pat coulding tap-dance?

i. Could Pat have tap-danced?

j. *Has Pat could tap-dance?

k. Has Pat been tap-dancing?

1. *Is Pat having tap-danced?

m. Could Pat have been tap-dancing?

n. ${ }^{*}$ Could Pat be having tap-danced?

o. * Could Pat will tap-dance?

p. *Has Pat had tap-danced?

q. ${ }^{*}$ Is Pat being tap-dancing?

These data suggest that we want to maintain the relationship between the auxiliary verb and its complement even when the auxiliary precedes the subject. The immediate problem then is how to get the subject to appear on the right of the (finite) auxiliary instead of on the left. A simple approach is to treat the postauxiliary NP not as a specifier, but rather as its first complement. Recall that the Head-Complement Rule generates complements in the same order in which they are listed in the head's COMPS list. ${ }^{8}$ Hence, making the subject NP into the first complement of the lexical entry for the inverted auxiliary puts it just where we want it.

This can be easily achieved by a lexical rule. Since the inverted verb in yes-no questions is the first element in the sentence, the Inversion Lexical Rule must be formulated so that the SPR list of the output is empty and the output's semantic mode is 'ques(tion)', rather than 'prop'. ${ }^{9}$

\footnotetext{
${ }^{8} \mathrm{~A}$ more general approach to constituent ordering will ultimately be necessary, especially for languages with much freer word order than English. There are a number of interesting proposals in the literature, but they go beyond the scope of this text.

${ }^{9}$ Here we make the simplifying assumption that inverted sentences are always associated with interrogative semantics. In fact, inverted clauses appear in other kinds of constructions, e.g. the following:
}

(i) Never have I heard such a beautiful rendition! 
312 / Syntactic Theory: A Formal Introduction

(39) Inversion Lexical Rule

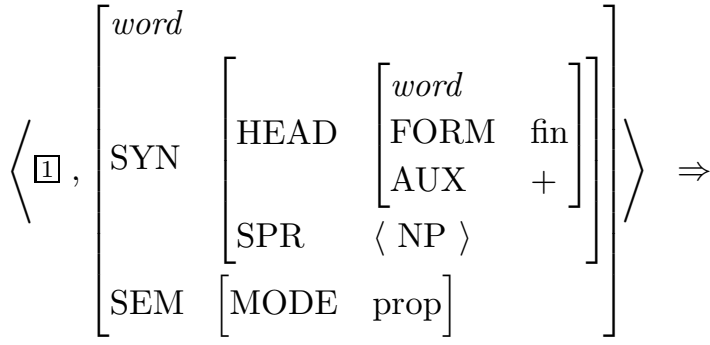

$$
\begin{aligned}
& \left\langle 1,\left[\begin{array}{lll}
\text { SYN } & {\left[\begin{array}{ll}
\text { HEAD } & {[\text { INV }+} \\
\text { SPR } & \langle\rangle
\end{array}\right]} \\
\text { SEM } & {[\text { MODE }}
\end{array}\right]\right\rangle
\end{aligned}
$$

The outputs of this rule are finite auxiliary verbs that take no specifier. Hence, in any well-formed lexical SD that these outputs give rise to, all members of the ARG-ST list will also appear on the COMPS list, as illustrated in (40).

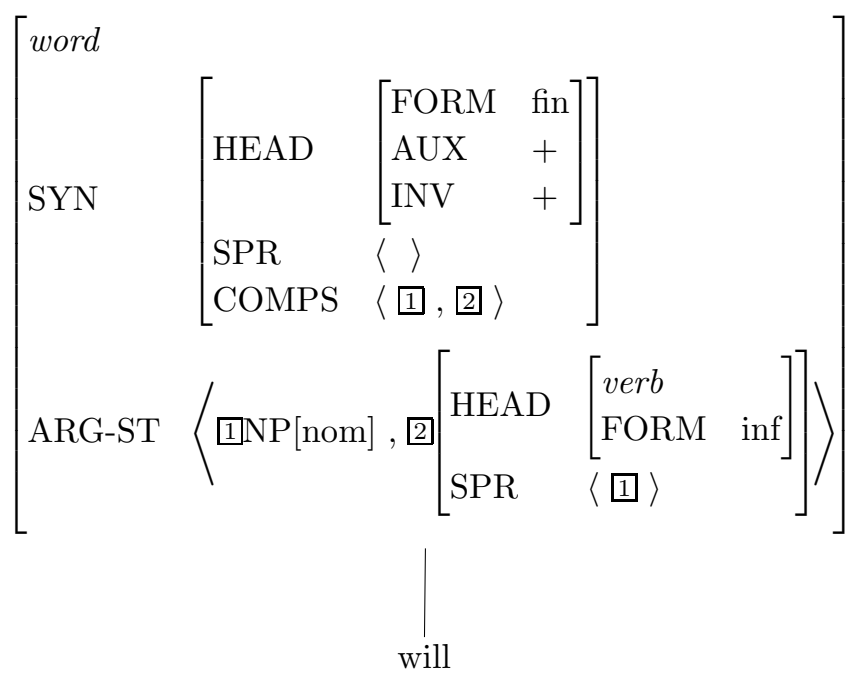

Otherwise, because the SPR value is the empty list, the ARP will not be satisfied. And because such a lexical SD has the first ARG-ST member on the COMPS list, that element will be realized as a complement, to

(ii) Had we known that beforehand, we would never have participated. 
the right of the lexical head in a phrasal SD constructed in accordance with the Head Complement Rule.

Recall that the lexical rules for finite forms presented in Chapter 8 specify constraints (about case and agreement features) on the first member of the ARG-ST list. Because the first element of the ARG-ST list is the first complement, all words formed from the outputs of this lexical rule will specify the appropriate CASE and AGR constraints not on the SPR value, but rather on the first COMPS member, as shown in (40). The lexical rules for finite verbal forms interact with the Inversion Lexical Rule in (39) to predict contrasts like the following:

(41) a. Can she tap-dance?

b. * Can her tap-dance?

c. Are we winning?

d. *Are us winning?

Similarly, for those auxiliaries that exhibit agreement with their subjects (namely, finite forms of be, have, and $d o$ ), the agreement in the inverted entries (i.e. the outputs) will be with the first complement. This correctly predicts data like the following:

(42) a. Is the dog barking?

b. * $\left\{\begin{array}{l}\text { Am } \\ \text { Are }\end{array}\right\}$ the dog barking?

c. Have you finished the assignment?

d. *Has you finished the assignment?

Moreover, since as we have seen, auxiliaries are raising verbs, their first argument is identical to the element on the SPR list of their VP argument. Hence, in the output (inverted) auxiliary's entry, the first complement will also function as the second complement's subject. We therefore predict data like the following:

(43) a. Will there be children in the audience?

b. ${ }^{*}$ Will there win the game?

c. Has it annoyed people that dogs bark?

d. Are tabs kept on all linguists by the FBI?

e. *Are tabs taken on all linguists by the FBI?

f. Was advantage taken of the opportunity by students?

g. *Was advantage kept of the opportunity by students?

h. Did it continue to annoy people that nobody listened?

i. * Did it try to annoy people that nobody listened?

In short, this rule creates lexical entries whose first complement has all the properties of a subject, except that it comes after the verb. 


\section{\) Problem 3: Inverted SDs}

Draw SDs for the following sentences. (You need not include all features on the nodes, but you should give a complete feature specification for the head of sentence. Otherwise, you can just concentrate on getting the tree geometry right, using standard abbreviations for node labels.)

(i) Did Pat put the ice cream in the freezer?

(ii) Is there a monster in Loch Ness?

\section{Problem 4: Inversion and Do}

How does our analysis account for the unacceptability of the following sentence (be precise!):

${ }^{*}$ Put Pat the ice cream in the freezer?

\section{(1) Problem 5: Negation and Inversion}

Our Negation and Inversion Lexical Rules can interact to allow us to generate negative questions. Which of the following sentences will be licensed by our rules so far?

(i) Has Pat not been sleeping?

(ii) Has not Pat been sleeping?

Explain your answer. Do the predictions of the grammar with respect to such sentences accord with your intuitions of well-formedness?

\subsubsection{Contraction}

Auxiliary verbs with the contracted negative suffix -n't exhibit a number of lexical idiosyncrasies. For example, we say won't, not * willn't. Others are don't, mustn't and shan't (though many speakers don't have any form of shall with -n't suffixed). (Don't be fooled by spelling: don't and mustn't are exceptional because of their pronunciation, which is not what would result from simply appending the sound 'nt' to do and must). There are also exceptions to the rule: *amn't and, for most speakers, *mayn't.

We will treat contraction like the inflectional lexical rules we presented earlier, which also allowed for idiosyncratic morphological exceptions; that is, we will posit a morphological function, $\mathrm{F}_{N E G}$, that relates inflected forms of auxiliaries to their negative contracted forms. The rule is then fairly straightforward: it applies to finite forms of auxiliaries, changing their morphology and semantics. The only other modification 
needed is to add the feature [NEG - ] to the input and [NEG +] to the output. ${ }^{10}$ The rule, then, is as follows:

(44) Contraction Lexical Rule
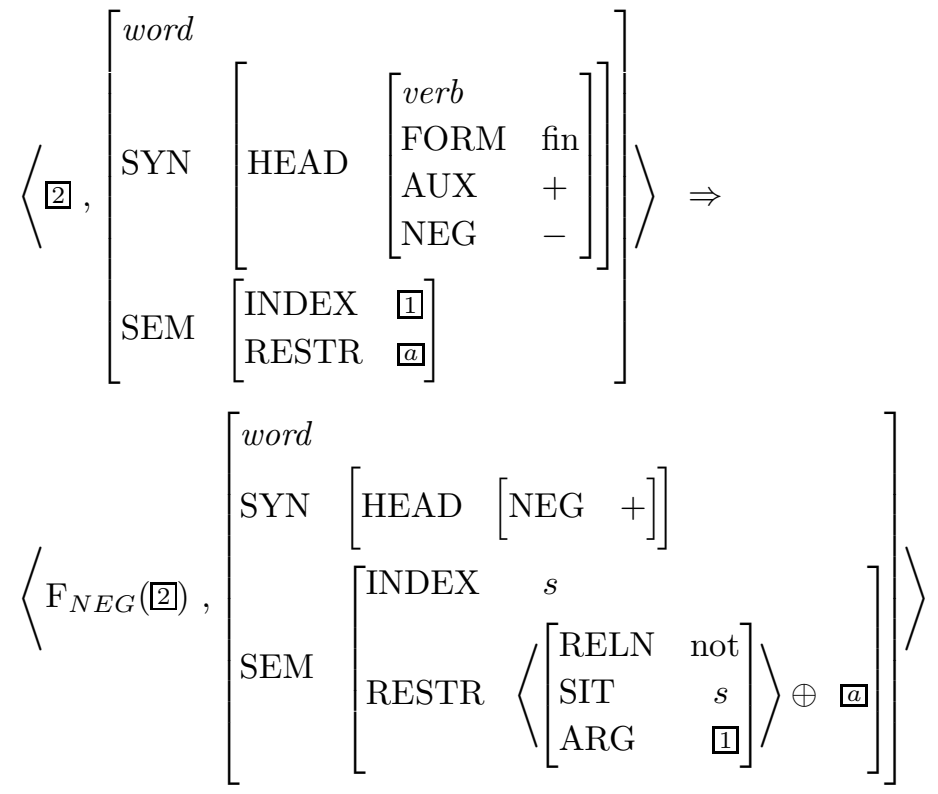

Problem 6: Contraction and Double Negation

As formulated, neither the Negation Lexical Rule nor the Contraction Lexical Rule can apply to the other one's output.

A. Explain why not.

B. Does the mutual exclusivity of these two lexical rules make the right empirical predictions? Provide data and an explanation to support your answer.

Problem 7: First-Person Aren't

In colloquial usage, examples like (i)-(iii) are common, although their uninverted counterparts, (iv)-(vi) are ungrammatical.

(i) Aren't I lucky?

${ }^{10}$ Note that this keeps the rule from applying to its own output, and hence automatically blocks forms like * can'tn't. 
(ii) Aren't I doing a good job?

(iii) Aren't I chosen?

(iv) *I aren't lucky.

(v) * I aren't doing a good job.

(vi) * I aren't chosen.

How could we account for this in our theory? [Hint: Assume that this aren't is a separate lexical entry from the one in examples like You aren't doing a good job. Then provide an explicit lexical entry for the aren't in (i)-(iii) and explain why it behaves as it does.]

\subsubsection{Ellipsis}

Ellipsis is a discourse phenomenon, in the sense that the interpretation of the missing constituent sometimes depends on something said in an earlier sentence - possibly even by another speaker, as in the following dialogue: ${ }^{11}$

(45) Speaker A: I haven't been reading the newspapers.

Speaker B: Well, I have.

Consequently, we will not try to provide an analysis of the semantic relation between an elliptical sentence and its antecedent. Instead, we will concentrate on the syntactic constraints on ellipsis.

We saw earlier that ellipsis could not immediately follow nonauxiliary verbs. The following example shows that the auxiliary verb immediately before an ellipsis site does not have to be finite.

(46) I haven't been following the debate on taxes, but I should have.

${ }^{11}$ This example also illustrates an argument for the phrase structure we assign to auxiliary constructions (with nested VPs), rather than the alternative with a node AUX containing all auxiliary verbs. On our analysis, what is missing from speaker B's utterance (namely, been reading the newspapers) forms a constituent (a VP). But under the AUX alternative, what is missing is part of the AUX, plus the following VP. In examples like (i), we see that all three of the possible ellipses correspond to a constituent in our analysis, but only one of them would be a constituent in the AUX analysis.

(i) Pat couldn't have been doing the reading, but Chris could (have (been (doing the reading))) 
In this respect, ellipsis differs from the previous three NICE properties we looked at, in which the auxiliary is always finite. Hence the lexical rule we formulate for ellipsis will not have to stipulate [FORM fin], though it will still require [AUX +].

There is one word, however, that can immediately precede an ellipsis site that we are not analyzing as a verb at all - namely, the complementizer to that is used to introduce infinitives.

(47) a. We asked them to open the window, and they tried to.

b. We hoped that the wine would improve with age, but it didn't seem to.

We can handle this if we leave open the part of speech of the input to the Ellipsis Lexical Rule, and say that infinitival to is [AUX +]. Then the only $[\mathrm{AUX}+]$ elements in the lexicon are auxiliary verbs and the complementizer to, so it is just these elements that can precede an elliptical VP.

The following formulation of the rule captures our syntactic observations:

(48) Ellipsis Lexical Rule

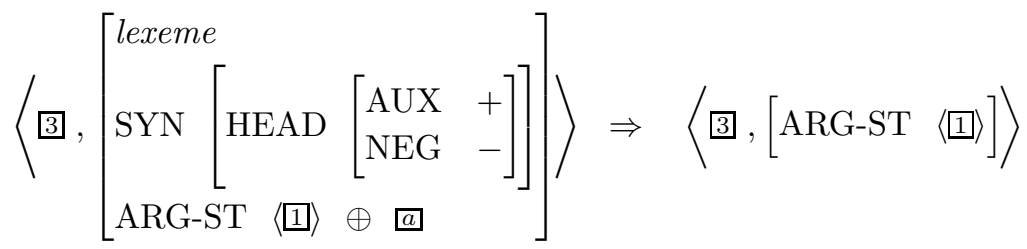

The outputs of this rule are entries whose ARG-ST no longer contains any of its arguments except the first one. Note that the semantics of such outputs is incomplete, and must be supplemented by appropriate material from the surrounding linguistic context. This analysis permits us to account for the fact that elliptical sentences behave in some ways as if they contained a constituent that is not in fact present. For example, nonreferential subjects are possible in elliptical sentences, so long as the missing complements are understood properly:

(49) a. We hoped it would be obvious that we were unhappy, and it was.

b. The students thought there would be a trick question on the exam, and there was.

The rule as formulated in (48) says nothing about the discourse properties of ellipsis. In particular, it does not specify that ellipsis is only possible in contexts where there is an antecedent phrase that provides the interpretation of the missing complement. It simply specifies that 
the lexicon includes lexical entries that can give rise to lexical SDs like the following:

$$
\begin{aligned}
& {\left[\begin{array}{lll}
\text { SYN } & {\left[\begin{array}{ll}
\text { HEAD } & {\left[\begin{array}{ll}
\text { FORM } & \text { fin } \\
\text { AUX } & +
\end{array}\right]} \\
\text { SPR } & \langle\text { G }\rangle \\
\text { COMPS } & \langle\rangle
\end{array}\right]} \\
\text { ARG-ST } & \langle\text { GNP[nom }]\rangle \\
& {\left[\begin{array}{lll}
\text { MODE } & \text { prop } \\
\text { INDEX } & s & \\
\text { SEM } & \left.\left[\begin{array}{ll}
\text { RELN } & \text { will } \\
\text { SIT } & s \\
\text { ARG } & t
\end{array}\right]\right\rangle
\end{array}\right]}
\end{array}\right.} \\
& \mid
\end{aligned}
$$

In SDs like these, $t$ - the argument of the 'will' relation - is not associated with any predication. Hence the meaning of any clause built from (50) is inherently incomplete. ${ }^{12}$

Problem 8: The Interaction of Ellipsis with Negation and Inversion

A final observation, independent of these last concerns, is that sentential not can occur in elliptical clauses:

(i) We wanted to taste the salad, but we could not.

(ii) They were asked to help the cook, but they did not.

(iii) You thought you were clearing the table, but you were not.

(iv) We thought that they had arrived, but they had not.

In addition, inversion is possible in elliptical clauses, as in the following sentences:

(v) [They will become famous.] Will they?

(vi) You thought you were helping them out. Were you?

${ }^{12}$ Presenting a fully worked out treatment of VP ellipsis is beyond the scope of this text. There is a rich literature on the subject that we cannot survey here. 
A. Does our grammar predict that examples like (i)-(iv) are well formed? Explain clearly why or why not.

B. Does our grammar predict that examples like (v)-(vi) are well formed? Explain clearly why or why not.

\subsection{Conclusion}

English auxiliaries comprise a richly structured system with numerous idiosyncratic properties. Our theory handles the complexities of the system straightforwardly. Auxiliary verbs are raising verbs that impose special conditions on their second argument. Auxiliary verbs exhibit some exceptional behaviors, such as the restriction of modals to [FORM fin]. And there are several lexical rules that are restricted to auxiliaries, which create specialized versions of the auxiliary verbs to account for the NICE properties.

In the next chapter, we examine some of the dialect variation in the behavior of English auxiliaries and discuss how our analysis can be modified to handle it.

\subsection{Further Reading}

As noted above, Chomsky (1957) is the seminal work on the English auxiliary system; Lasnik (1995) is an elementary (and updated) presentation of Chomsky's analysis. Among the most influential subsequent work is Ross (1969), McCawley (1971), Akmajian et al. (1979), Steele (1981), Gazdar et al. (1982), and Pollock (1989). The standard reference on negative contraction is Zwicky and Pullum (1983). The analysis presented here is based in part on Warner (1993), Kim (1995) and Kim and Sag (1995). 



\section{4}

\section{Variation in the English Auxiliary System}

\subsection{Introduction}

English auxiliaries constitute a particularly interesting syntactic system, involving a small set of lexical items that exhibit many intricate interactions and some fascinating idiosyncracies. This system is peculiar to English, though many other languages have elements with intriguingly parallel properties.

English auxiliaries have changed considerably over the last thousand years or so, and their evolution has been well documented, making them a natural domain for studying syntactic change. ${ }^{1}$ Change begins with variation, and the auxiliary system is also the locus of some fascinating differences among varieties of English. Variation is interesting in its own right, but studying it also helps us to ascertain which properties of our grammar we should formulate as or deduce from general principles, and which ones we should treat as essentially accidental.

In this chapter, we provide two examples of variation in English auxiliaries. The first example concerns the behavior of have, whose syntax is that of an auxiliary verb in some instances but not in others. The second example deals with a much studied phenomenon in the dialect known as African American Vernacular English (AAVE, for short). In both cases, we will explore how the variation might be handled within our theory. Our examples and discussion are intended only as samples of how data on variation that might be relevant to work on syntactic theory. Syntactic variation is a topic worthy of a textbook in its own right, and we make no pretense of doing it justice here.

\footnotetext{
${ }^{1}$ Discussion of the history of the English auxiliary system would take us too far afield, although it is a fascinating topic.
} 


\subsection{Auxiliary Behavior in the Main Verb Have}

Our first example of variation in the English auxiliary system is one that occurs in a number of varieties of English, and can even vary for individual speakers, depending on the formality of the context.

In some circumstances, certain uses of have as a main verb exhibit auxiliary-like behavior. For example, we have all heard usages like those in (1).

(1) a. Have you any idea of the time?

b. I haven't a clue.

c. They said we had a problem, and we have.

This is not the first time we have seen a verb that is not a 'helping verb' - that is, one that is the sole verb in its clause - exhibiting some of the NICE properties. According to our analyses, the lexeme be must belong to the type auxv-lxm: all finite forms of be (e.g. is, were) undergo the Negation, Inversion, and Contraction Lexical Rules, and all forms of be undergo the Ellipsis Lexical Rule. ${ }^{2}$

In our grammar, the feature [AUX +] (associated with all instances of the type auxv-lxm and with certain other elements, e.g. the complementizer to) is associated with a certain range of properties, notably (i) the NICE properties, and (ii) not being a possible complement to the auxiliary $d o$. In the dialects that allow main verb have to exhibit NICE properties, we can posit a lexical entry for have that takes an NP complement (rather than the VP complement of the auxiliary have), but which is still [AUX +]. That is, we posit an entry along the following lines:

\footnotetext{
${ }^{2}$ There are a few exceptions, which we will not attempt to deal with here. When be is used to denote activities over which the subject has clear voluntary control (meaning something close to 'act'), it can co-occur with the auxiliary $d o$, as in Bob Dole's statement to supporters during the 1996 presidential campaign:

(i) You're not gonna get that tax break if you don't be quiet. In addition, as first noted by Akmajian and Wasow (1974), being appears to be an exception to the Ellipsis Lexical Rule (for reasons that remain obscure):

(ii) They said we were being followed by a blue car, and we were (*being).
} 
Variation in the English Auxiliary System / 323

(2)

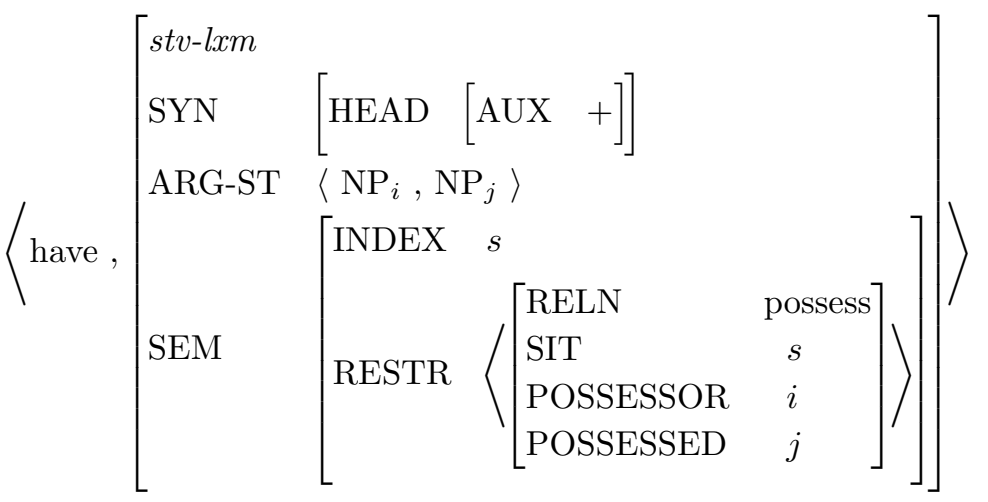

To the best of our knowledge, all dialects that allow examples like (1) also permit their counterparts in (3).

(3) a. Do you have any idea of the time?

b. I don't have a clue.

c. They said we had a problem, and we do.

This suggests that the entry in (2) is not quite right. Instead of stipulating $[$ AUX +$]$, it should allow either [AUX +$]$ or [AUX -$].^{3}$ This will permit the verb to occur where [AUX +] is required (i.e. in the lexical rules involved in the NICE phenomena) as well as where [AUX -] is required (in the complement of auxiliary $d o$ ). Thus, the dialect allowing the main verb have to behave like an auxiliary differs from the standard dialect only in the specification of this one feature.

The situation is actually a bit more complex than this. Not all uses of have exhibit the NICE properties, even in the dialects that accept (1). Some examples are given in (4)-(6).

(4) a. They had a fit.

b. ${ }^{*}$ They had not a fit.

c. *Had they a fit?

\footnotetext{
${ }^{3}$ The careful reader will note that we have been slightly evasive about what formal mechanism to use in allowing both values for AUX. The most parsimonious way to do this would be simply to underspecify the entry for this feature. Such an analysis would be consistent with everything in the text to this point. However, it would only work if verb-lxm had no default specification for the feature AUX, and this, in turn, would require that every nonauxiliary verb entry in the lexicon (that is, the overwhelming majority of verb entries) be individually marked [AUX - ]. It is natural, therefore, to put a default [AUX / - ] specification on the type verb-lxm, which is overridden by the specification on auxv-lxm. If we do this, we need some way for (2) to override the default and still allow both values for AUX. We could do this by modifying $(2)$ to say either [AUX $+\mid-]$ or $([$ AUX +$])$. Both of these options raise some technical questions for the formal theory of defaults in inheritance hierarchies, but these are beyond the scope of this text.
} 
d. *They hadn't a fit.

e. *I said they would have a fit, and they had.

(5) a. You have cancer.

b. *You have not cancer.

c.?*Have you cancer?

d. *You haven't cancer.

e.?*They said you had cancer, and you have.

(6) a. Every day at lunch, Lou had a martini.

b. * Every day at lunch, Lou had not a martini.

c. *Every day at lunch, had Lou a martini?

d. *Every day at lunch, if Fran had a martini, Lou had, too.

There seems to be a semantic difference between those uses of have that exhibit NICE properties in the dialect in question and those that do not. Roughly, have means 'possess' in the NICE uses, but it assumes more specialized meanings in (4)-(6). To capture the correlation of syntactic behavior with semantics, we need to postulate multiple lexical entries for have. In particular, the entry in (2) specifies that the semantic relation is possession. Other main verb entries for have specify different relations, and they are all [AUX -].

Although the postulation of multiple entries for have might seem a priori undesirable, the fact that it has so many meanings would require separate entries, even if the meanings were not correlated with syntactic differences.

\subsection{African American Vernacular English}

The next case of variation we will examine concerns a dialect we will refer to as African American Vernacular English (which is also known as Black English or African American English). We will treat AAVE as a homogeneous dialect, just as we have been assuming for simplicity that there is a homogeneous standard American English. There is, in fact, considerable variation within AAVE, but there are also many properties that are quite general to AAVE and legitimize treating it as one dialect. ${ }^{4}$

\footnotetext{
${ }^{4}$ No two humans speak exactly the same way, or share exactly the same intuitions about every sentence. Indeed, individual speakers change the way they speak over time and under different circumstances. Hence the concepts of 'language' and 'dialect' are idealizations, based on undefined notions of similarity. They are nevertheless indispensible starting points for linguistic analysis. Moreover, providing precise characterizations of similarities and differences across varieties of speech could provide a scientific foundation for some of the intuitions underlying the way people individuate languages.

Linguists place little stock in the language/dialect distinction. One might attempt to characterize two varieties of speech as dialects of the same language if they are
} 
Before delving into the data, we need to digress briefly, to clear up some common misconceptions about AAVE. In the autumn of 1996, as we were writing the first draft of the present chapter, the school board in Oakland, California passed a resolution calling for the use of 'Ebonics' (another name for AAVE, favored particularly by afrocentric activists who wish to deny that AAVE is a variety of English) as a tool in the teaching of standard English to African American children. This event unleashed a storm of public outrage across the country. Much of the criticism was directed at the unfortunate wording of the Oakland resolution, and some of it concerned specific classroom practices; neither of these is relevant to the present text. A great deal of the outcry, however, concerned the linguistic status of AAVE, revealing widespread misunderstanding about the nature of language and grammar, even among many highly educated and sophisticated commentators.

Eldridge Cleaver, the former Black Panther leader, wrote in the Los Angeles Times, 'When I was growing up, what is now euphemistically being called Ebonics was accurately called bad English.' This statement - like numerous others that appeared in the press - reveals the powerful influence of prescriptivism. It presupposes that some varieties of English are correct and that others are incorrect. But what is the basis for attributions of correctness or 'badness' to a language variety - or for that matter to a particular utterance? In this text, we have appealed to native speaker intuitions of well-formedness to determine what is grammatical and what is not. This is clearly not the standard of correctness Cleaver had in mind, nor is it the one that prescriptivists employ. Rather, prescriptivism singles out one linguistic variety as the standard, and labels deviations from that standard as incorrect. The choice of the standard is determined by a variety of historical, social, and political factors, and it can be quite different from the language people actually use on a day-to-day basis. There is no linguistic basis for elevating one language variety in this way.

No language or dialect is inherently 'good' or 'bad.' They can only be evaluated with respect to some objective. As linguists, we are interested in languages as natural phenomena. To say that one language is 'better'

mutually intelligible, but even this intuitive characterization is problematic. Thus varieties that we have other reasons to think of as distinct languages (e.g. Swedish and Danish) are to a very large extent mutually intelligible. Moreover the relation of mutual intelligibility is not transitive: given three varieties $\mathrm{A}, \mathrm{B}$, and $\mathrm{C}$, mutual intelligibility between $\mathrm{A}$ and $\mathrm{B}$ and between $\mathrm{B}$ and $\mathrm{C}$ does not guarantee that $\mathrm{A}$ and $\mathrm{C}$ are mutually intelligible. Thus one can talk at best about 'complexes' of language varieties (Hockett 1958) among which certain patterns of mutual intelligibility exist. Fortunately, these terminological issues do not need to be resolved in order for linguistic analysis to proceed. 


\section{6 / Syntactic Theory: A Formal Introduction}

than another per se makes no more sense to us than saying one atomic particle is better than another. ${ }^{5}$ For our purposes, AAVE is just as legitimate an object of study as any other language variety.

Another widespread and more serious misconception is exemplified in a column by Brent Staples in the New York Times, who described AAVE as 'broken English.' Similarly, in a column by The Washington Post's William Raspberry, AAVE is described as 'a language that has....no discernable rules.' Raspberry's piece recounts a fictional conversation with a taxicab driver, who says, 'you can say pretty much what you please [in Ebonics], as long as you're careful to throw in a lot of 'bes' and leave off final consonants.' If such claims were true, it would make linguistic investigation of AAVE trivial and pointless. But they are in fact patently false. Careful investigation of AAVE by linguists over the past three decades has shown that it has as much structure and system to it as any other linguistic variety. The differences between it and other varieties of English are quite systematic. One of those differences will be discussed briefly in this chapter, but see Rickford and Green (in press) for a much more detailed survey. ${ }^{6}$

\subsubsection{Missing Forms of Be}

Examples like (7), though ungrammatical in standard American English (SAE, for short), are well-formed sentences of AAVE.

(7) a. Chris at home.

b. We angry with you.

c. You a genius!

d. They askin for help.

SAE requires a form of the copula (that is, a form of be) in each of these sentences, but, as noted in Chapter 11, many other languages (e.g. Russian and Hungarian) are like AAVE in permitting analogues of (7) without a copula. In fact, Ferguson (1971) noted a number of similarities between the environments in which copulas may be absent across languages, including AAVE.

Labov (1995) presents a number of arguments for an analysis of examples like (7) that involves deletion of a form of be. More specifically,

\footnotetext{
${ }^{5}$ Of course, if the objective for evaluation of language varieties is getting ahead economically, then there can be little doubt that in contemporary American society, AAVE fares poorly (outside of some pockets of the entertainment industry). For this reason, virtually all American educators - including the Oakland School Board - recognize the need to teach standard English in the schools. Unfortunately, this point of agreement seems to have been lost in much of the discussion of 'Ebonics'.

${ }^{6}$ Our data on AAVE are taken largely from published sources, cited below. In addition, we are grateful to John Baugh, Erica Denham, Lisa Green, and Zakiyyah Langford for giving us their judgments on some examples.
} 
he claims that such 'zero copula' sentences involve an extension of SAE contraction: is and are reduce to 's and 're in all dialects of English, and AAVE carries the process one step further, deleting the remaining consonants. He concludes that 'the copula is present in the underlying grammar, and is an object of cognition' (p. 46).

This sort of deletion analysis does not fit comfortably with the surface-oriented, constraint-based, strongly lexicalist grammar we have been developing in this text. In Chapter 9, we described this conception of grammar as follows:

Our grammar....provides a....structure that is directly associated with the string of words that constitute each sentence. The ancillary structure that has to be computed....expresses information that is straightforwardly derivable from properties of the words in the string....There are no operations that destructively modify any representations.... We have localized most grammatical and semantic information within lexical entries.... [that] correspond directly to the words present in the sentence.

The only way to accommodate Labov's treatment of the zero copula within our theory would be to posit phonological or phonetic processes that are exempt from the conditions just quoted. But such a move would greatly weaken the force of our claims, at least in the absence of some principled method for demarcating the boundaries between syntax and morphology on the one hand, and phonetics and phonology on the other. If deletions can be freely reformulated as a phonetic processes, then a prohibition against syntactic deletion has no substance.

It would be interesting, therefore, to explore how the AAVE zero copula might be accounted for in the theory we have been developing, and to compare such an analysis to a deletion account.

In doing this, however, we do not wish to deny that there may be strong connections between contraction and the AAVE 'zero copula', in terms of their histories or in terms of the functions they serve (such as shortening utterances or signalling an informal style). This does not entail that the best synchronic description of the language must include a deletion rule. Indeed, we will see below that the nondeletion analysis we are led to has distinct advantages over a deletion account.

The most obvious nondeletion analysis would posit a lexical entry with no phonology, which is otherwise just like is and are: ${ }^{7}$

\footnotetext{
${ }^{7}$ Actually, (8) is not quite right, because it fails to exclude first-person singular subjects. That is, it allows examples like (i), which are ungrammatical in AAVE
}

(i) *I hungry. 
(8)

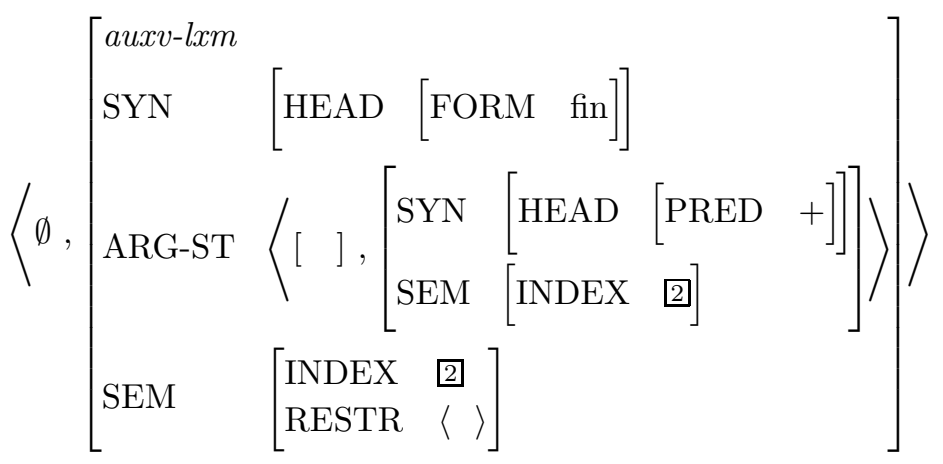

While a silent variant of is and are would be straightforward, it clearly violates the spirit of surface-oriented, constraint-based, strong lexicalism. Up to this point, we have avoided positing inaudible lexical entries for 'understood' elements (such as a silent you for the subject of imperatives). Analogues of such elements do show up in places in our feature structures (notably in valence features and ARG-ST lists), but their presence is always licensed by a particular grammar rule or word. Hence our grammar fits well with a model of sentence processing that is driven only by the words that are actually audible (or visible): the processing mechanism never needs to guess where in the string there might be silent words. This is a highly desirable design feature that we would not abandon lightly. In addition, as we will see below, there is ample independent evidence against the existence of an inaudible copula in AAVE.

\subsubsection{A Phrase Structure Rule Analysis}

An alternative analysis of the missing copula in AAVE is to allow the complements of be (whether they are VPs, NPs, PPs, or APs) to combine directly with NP subjects to make sentences. In fact, our analysis of be already requires that its second argument have a nonempty SPR list. Remember, the SPR of be has to be identical to the SPR of its complement, so the complement always must have something on its SPR list.

Hence, unless something is added specifically to exclude them, the following expressions are all generated by our grammar as phrases that are [SPR \langle\rangle$]$ and [COMPS \langle\rangle$]$, differing in their part of speech types:

To exclude examples like (i), the 'silent copula' analysis would have to be modified in some way. We will return to this restriction on the zero copula construction, providing an account that could be fairly straightforwardly adapted to the silent copula analysis under discussion here. 
(9) a. It wild.

b. You in trouble.

c. Leslie the boss.

d. Somebody coming to dinner.

e. Jean interviewed by a reporter.

These strings are not well-formed stand-alone sentences in SAE, because the initial symbol of our grammar is:

$$
\left[\begin{array}{ll}
\text { phrase } & \\
\text { SYN } & {\left[\begin{array}{ll}
\text { HEAD } & \left.\begin{array}{ll}
\text { verb } & \\
\text { FORM } & \text { fin }
\end{array}\right] \\
\text { SPR } & \langle\rangle
\end{array}\right]}
\end{array}\right]
$$

As noted earlier (Chapter 9), this says that a stand-alone sentence (or what linguists sometimes call a 'root sentence') must be headed by a verb in a finite form. None of the examples in (9) contains a finite verb, so they are clearly ruled out as SAE sentences. It is worth noting that there are constructions (which we will not analyze here) that permit strings similar to those in (9) to appear as subordinate clauses, for example:

$$
\text { With the cat }\left\{\begin{array}{l}
\text { away } \\
\text { in the kitchen } \\
\text { a prisoner } \\
\text { sleeping } \\
\text { locked up }
\end{array}\right\} \text {, the mice will play. }
$$

However, SAE does not permit the strings in (12) as independent sentences.

$$
\text { *The cat }\left\{\begin{array}{l}
\text { away } \\
\text { in the kitchen } \\
\text { a prisoner } \\
\text { sleeping } \\
\text { locked up }
\end{array}\right\} \text {. }
$$

Thus we want our grammar to generate strings like those in (9) as wellformed constituents in SAE but make their distribution quite restricted. In particular, they do not occur as root sentences because of the initial symbol (10). Similarly, they do not occur as complements of the complementizer that or of the verb think because both take complements headed by finite verbs.

Returning now to AAVE, we could try to account for examples like (7) by modifying the initial symbol, allowing a wider range of feature 
structures to be root sentences. In particular, we could say that AAVE allows another alternative in addition to (10), namely, (13).

$$
\left[\begin{array}{lll}
\text { HEAD } & {\left[\begin{array}{ll}
\text { pos } & \\
\text { PRED } & +
\end{array}\right]} \\
\text { VAL } & {\left[\begin{array}{ll}
\text { SPR } & \langle
\end{array}\right]}
\end{array}\right]
$$

However, this analysis is not tenable, for it incorrectly predicts that the missing copula is possible only in root sentences. ${ }^{8}$ But examples like (7) and (9) can easily be embedded; sentences like (14) are perfectly normal in AAVE.

(14) a. If you alone, watch out!

b. The man she lookin for ain't here.

Here the strings you alone and she lookin for are in positions in which $\mathrm{SAE}$ would require a clause headed by a finite verb (you are alone and she is looking for), but AAVE permits the copula to be missing. It seems that the grammars of SAE and AAVE differ in that, where the former permits a finite clause headed by is or are, the latter allows a clause without the copula.

We can capture this informal generalization more precisely by introducing a new phrase structure rule for AAVE, rather than an additional initial symbol. Specifically, we can handle the missing copula in AAVE with a grammar rule that says that a sequence of a nominative NP followed by another phrase can function as a finite clause. The following rule does just this:

\footnotetext{
${ }^{8}$ Ferguson (1971) notes that some languages allow the copula to be missing only in main clauses, suggesting that there may be languages with initial symbols like (13).
} 


$$
\text { Zero Copula Rule (AAVE) }
$$

$$
\begin{aligned}
& {\left[\begin{array}{l}
\text { SYN }\left[\begin{array}{ll}
\text { HEAD } & {\left[\begin{array}{ll}
\text { verb } & \\
\text { FORM } & \text { fin }
\end{array}\right]} \\
\text { SPR } & \langle>
\end{array}\right] \\
\text { SEM }\left[\begin{array}{ll}
\text { MODE } & \text { prop } \\
\text { INDEX } & 2
\end{array}\right]
\end{array}\right] \rightarrow} \\
& {\left[\begin{array}{ll}
\text { CASE } & \text { nom } \\
\text { AGR } & \text { non-1sing }
\end{array}\right]\left[\begin{array}{l}
\text { phrase } \\
\text { SYN }\left[\begin{array}{ll}
\text { HEAD } & {[\text { PRED }} \\
\text { SPR } & \langle\square\rangle
\end{array}\right] \\
\text { SEM }\left[\begin{array}{ll}
\text { INDEX } & 2
\end{array}\right]
\end{array}\right]}
\end{aligned}
$$

This rule says that wherever a finite clause is called for, AAVE allows a [PRED +] phrase preceded by its nominative subject. It also says that the subject cannot be first-person singular, that is, $I$ (see below). In addition, (15) stipulates the INDEX and MODE values on the left hand side, since the Semantic Inheritance Principle applies only to headed rules.

The Zero Copula Rule yields clauses that occur where finite clauses do, but which appear to be missing a verb. The resulting clauses will look as if they are missing a copula; and since the copula adds nothing semantically (except tense), they will also be interpreted as if they contained a copula. The effect of (15) is thus to license SDs like the following in AAVE: 


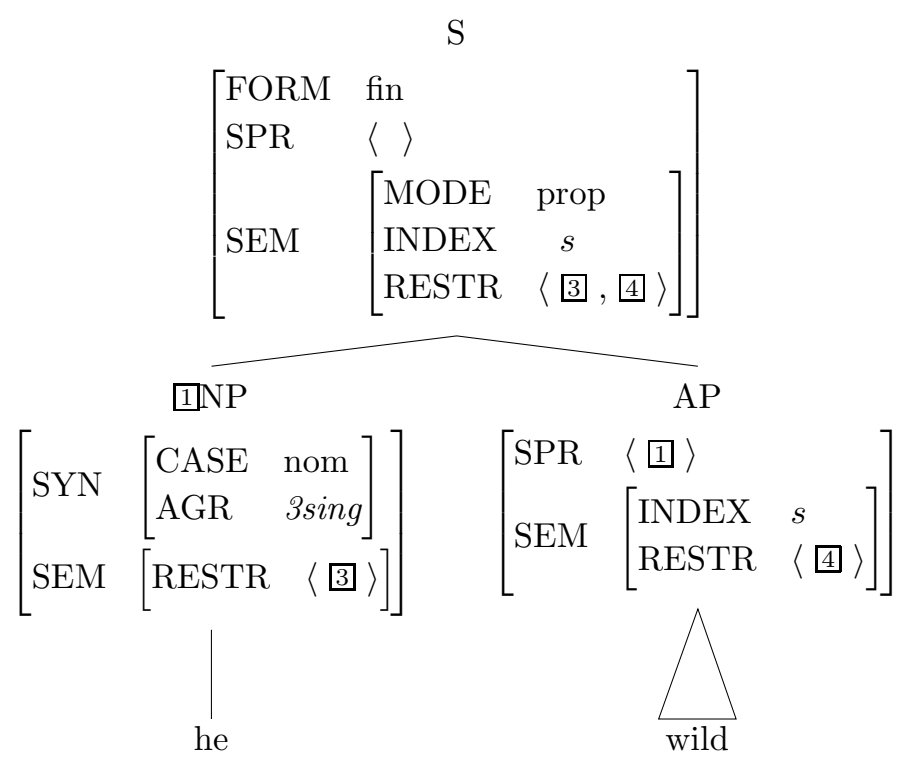

We return now to the specification [AGR non-1sing] on the NP in the Zero Copula Rule. This was added because the first-person singular pronoun I cannot appear as the subject in the zero copula construction in AAVE.

$$
\text { *I }\left\{\begin{array}{l}
\text { hungry } \\
\text { at home } \\
\text { the winner }
\end{array}\right\} \text {. }
$$

This appears to be a rather singular exception: other varieties of English with the zero copula construction (such as Jamaican Creole) do allow it with first-person singular subjects. It evidently requires a special stipulation on any available analysis of the phenomenon. ${ }^{9}$

The careful reader will have noticed, however non-1sing is one of the AGR types we introduced in Chapter 4 (footnote 10). To this point, however, we have distinguished only the two AGR subtypes 3sing and

\footnotetext{
${ }^{9}$ On Labov's deletion account, it is one of several counterexamples to the assertion (Labov (1995), p. 39) that 'Where other English dialects do permit contraction of the copula, AAVE permits deletion,' since examples like I'm hungry are impeccable in SAE. On the other hand, the deletion account offers some hope of providing a principled account of why the first-person singular exhibits this singular behavior, in terms of phonetic differences between $[\mathrm{z}]$ and $[\mathrm{r}]$, on the one hand, and $[\mathrm{m}]$ on the other. Phonetic differences between these segments might also provide an historical explanation of why, on our account, the synchronic Zero Copula Rule requires a stipulation blocking first-person singular subjects.
} 
non-3sing. In SAE, we would distinguish several subtypes of non-3sing, as illustrated in (18).

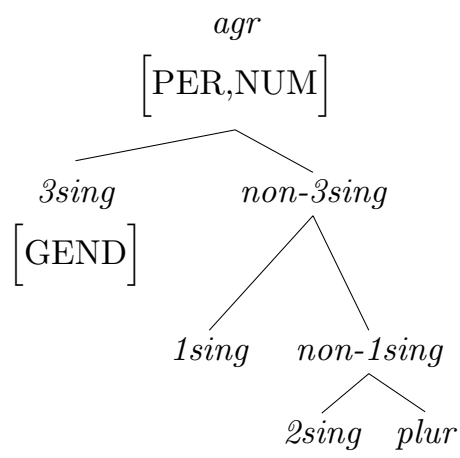

The type 1sing is the AGR value for the specifier of the verb am, and would also be used for handling the agreement between coindexed firstperson singular pronouns. Likewise, in SAE, non-1sing is the AGR value for the specifier of are; its subject can only be plural or second-person singular, as its two subtypes indicate.

This hierarchy of AGR types would give the wrong results for AAVE. In conjunction with our Zero Copula Rule, it would require the subject of zero copula sentences to be either plural or second-person singular. But, as we have seen (for example in (14b)), third-person singular subjects are possible in AAVE zero copula sentences. This suggests the following slightly different AGR hierarchy in AAVE:

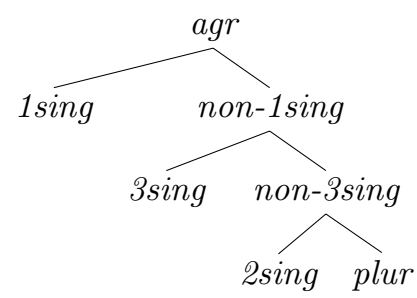

With the hierarchy in (19), the Zero Copula Rule gives us the right results, since it excludes only the first-person singular. Notice, however, that it has eliminated the high-level distinction between third-person singular and all other AGR types that we had in the SAE hierarchy in (18). This distinction is needed in SAE because almost all verbs take the suffix $-s$ in the present tense just in case their subject is third-person singular. Thus, the AAVE hierarchy that we posited in (19) would not be tenable for SAE. But it does work for AAVE, because AAVE verbs (other than be) do not mark agreement with their subjects. Present 
tense AAVE verbs have the same form as the infinitive, irrespective of the person and number of the subject.

(20) a. He see us.

b. Pat do good work.

c. Andy make a lot of money.

d. She have good luck.

This allows the AAVE lexicon to be slightly simpler than the SAE lexicon. Where SAE requires separate entries for present tense verbs with third-person singular subjects, AAVE can simply have one present tense entry. This is illustrated in (21)-(22).

(21) SAE:

$$
\begin{aligned}
& \left\langle\text { eats, }\left[\begin{array}{cc}
\text { HEAD } & {\left[\begin{array}{ll}
\text { FORM } & \text { fin }
\end{array}\right]} \\
\text { ARG-ST } & \left\langle\left[\begin{array}{cc}
\text { CASE } & \text { nom } \\
\text { AGR } & 3 \text { sing }
\end{array}\right], \text { NP }\right\rangle
\end{array}\right]\right\rangle \\
& \left\langle\text { eat, }\left[\begin{array}{ll}
\text { HEAD } & {\left[\begin{array}{ll}
\text { FORM } & \text { fin }
\end{array}\right]} \\
\text { ARG-ST } & \left\langle\left[\begin{array}{ll}
\text { CASE } & \text { nom } \\
\text { AGR } & \text { non-3sing }
\end{array}\right], \text { NP }\right\rangle
\end{array}\right]\right\rangle
\end{aligned}
$$

(22) AAVE:

$$
\left\langle\text { eat, }\left[\begin{array}{ll}
\text { HEAD } & {\left[\begin{array}{ll}
\text { FORM } & \text { fin }
\end{array}\right]} \\
\text { ARG-ST } & \left.\left\langle\begin{array}{cc}
\text { NP } \\
\text { CASE } & \text { nom }
\end{array}\right], \text { NP }\right\rangle
\end{array}\right]\right\rangle
$$

Because these forms are not differentiated in AAVE, it needs only one Present Tense Lexical Rule, in place of the two needed in SAE.

Thus, the Zero Copula Rule permits SDs for the zero copula sentences under discussion, without positing any deletion rules or unpronounced words. In order to exclude first-person singular subjects in AAVE zero copula sentences, we needed to revise the AGR type hierarchy we had posited for SAE. This was possible because of a morphological difference 
between the two dialects, namely, that only SAE marks the agreement with the subject on most present-tense verbs.

\subsubsection{Worksection: Predictions and Responses}

Two nondeletion analyses of examples like (7) have been introduced here: one involving a verb that goes unpronounced (see the lexical entry (8) above); and a second that treats the examples as truly verbless (see the rule in (15) above). These analyses make subtly different predictions. The problems in this section explore these differences.

\section{Problem 1: The Zero Copula and Ellipsis}

The analyses make different predictions about the behavior of zero copula sentences in elliptical constructions. Explain the differences and explain how the following AAVE data bear on the choice between the analyses.

(i) * They said he wild, and he.

(ii) ${ }^{*}$ They said we on tape, and we.

Problem 2: The Auxiliary Status of the Zero Copula

One response to the argument from the previous problem might be to claim that the silent variant of finite be should not be exactly as given in (8), but should be a nonauxiliary (i.e. [AUX -]) subject-raising verb instead. This would prevent it from undergoing the Ellipsis Lexical Rule, and would block the generation of (22). It would also prevent it from undergoing other lexical rules that are limited to [AUX +] verbs, which include the Inversion and Contraction lexical rules.

The evidence from Contraction seems to support such a treatment:

(ii) *We n’t gonna lose.

A. Explain how examples like (i) are ruled out by the phrase structure rule analysis of the missing copula.

However, if there is a silent copula that is [AUX -], it should also fail to undergo the Inversion lexical rule. Since the silent copula is inaudible, it is a bit tricky to determine its location. Hence, some ingenuity is required to test whether it inverts. The following data (common to SAE and AAVE) indicate that the material following the question word in what linguists call wh-questions must be an inverted clause - that is, a clause whose head verb has undergone the Inversion Lexical Rule.

(iii) Where am I going? vs. *Where I'm going?

(iv) Who were you talking to? vs. ${ }^{*}$ Who you were talking to? 
(v) What did they find? vs. *What they found there?

(vi) When can we see it? vs. ${ }^{*}$ When we can see it?

B. With this knowledge about $w h$-questions in mind, use the following data to argue (assuming an analysis that includes a silent copula) that that copula could not be [AUX -]:

(vii) Where he going?

(viii) Who you talking to?

C. Given the conclusion in (B), explain why the example in (i) is problematical for the silent copula analysis.

\subsubsection{Some Advantages of the Zero Copula Rule}

Methodological considerations led us to our analysis of the AAVE zero copula construction, rather than the sort of deletion account that is standardly assumed. There are, however, some empirical advantages to our treatment.

There are a number of environments in which forms of be occur in SAE and the corresponding AAVE sentences also require be. A deletion analysis has to explain in these cases why deletion does not occur; likewise, a silent verb analysis must account for differences in the distribution of the audible and inaudible variants of be. Labov (1995) gives a list of such environments. We will not go through them all in detail, but we note that our Zero Copula Rule, in conjunction with our analyses of other phenomena in this book (notably, ellipsis and long-distance dependencies) correctly accounts for the obligatoriness of be in all of the environments in question.

We will illustrate how our analysis rules out zero copulas in just three environments: negation, emphatics, and elliptical constructions.

Emphasis. When is or are is emphasized, as in Allah is god, the copula is obligatory. This is obvious on our analysis, since it would be impossible to emphasize what is not there. On a deletion or silent verb analysis, some mechanism is needed to prevent emphasis from falling on the verb (or to prevent deletion of an emphasized verb).

This is another case in which AAVE is like other languages that permit zero copulas. Ferguson (1971, p. 142) points out that, in such languages,

when emphasis is put on the semantic link, as in definitions and exclamatory pronouncements, a copula equivalent is used, either a special verb (e.g. 'stands', 'is found') or a pronoun (e.g. 'he', 'they'), 
or a verb 'to be' which is normally used in other tenses or in existential clauses.

Ellipsis. Constructions that allow the complement to be to be missing are incompatible with the zero copula construction. We saw one such construction in Chapter 13, where we provided an analysis that accounts for examples like (23):

(23) a. Pat isn't fond of dogs, but Chris is.

b. The children were making noise because the grownups were.

We introduced a lexical rule that allowed [AUX + ] elements to occur without their normally obligatory complements in appropriate contexts.

This sort of ellipsis is possible in AAVE, but not when the clause in question is a zero copula:

(24) a. Sandy ain't in the car, but Terry is.

b. * Sandy ain't in the car, but Terry.

Another type of ellipsis occurs in comparative sentences like the following:

(25) She is more intelligent than he is.

Again, the copula is obligatory in AAVE:

(26) * She (is) more intelligent than he.

In both kinds of elliptical constructions, a deletion analysis must block deletion of a form of be immediately preceding an ellipsis site. Likewise, a silent verb analysis needs to provide some mechanism to keep the inaudible variant of be from occurring just before ellipsis. Our analysis, however, requires no such mechanism. The Zero Copula Rule licenses a construction with a subject and a predicate. The elliptical examples we have been discussing have no predicate. Hence, they could not be licensed by the Zero Copula Rule.

Notice that the comparative construction does not always lack a predicate. In examples like (27), the clause following than has both a subject and a VP headed by a finite form of $b e$.

(27) The table is longer than it is wide.

In (27), it is wide is a finite clause (that is, S[FORM fin]). Hence, our analysis predicts that the Zero Copula Rule should be applicable, licensing (28) in AAVE:

(28) The table (is) longer than it wide.

Our consultants confirm that (28) is indeed acceptable in AAVE.

Wh-Questions. (29a) is grammatical, but its zero copula counterpart, (29b), is ungrammatical. 
(29) a. I wonder where he is.

b. ${ }^{*}$ I wonder where he.

This construction is an instance of the topic of the next chapter, what we are calling 'long-distance dependencies'. The word where in these examples is the complement of $i s$, although it is not in the usual position of a complement (following the head). In our analysis of such constructions, the information that the complement appears in a non-canonical position is encoded on the head verb. But our treatment of the zero copula construction employs a non-headed rule: there is no head verb. Lacking a crucial element needed to license the placement of the complement (where) at the front of its clause, an example like (29b) cannot be generated.

Summing up, our Zero Copula Rule (15) correctly requires a form of be to be present in a variety of environments where deletion or silent verb analyses might allow them. We hasten to add that sufficiently sophisticated versions of these other analyses might provide principled accounts of the same data. Labov's contraction-based account is one example, because most of the environments that require a form of be in AAVE also disallow contracted forms of be in SAE.

Our analysis has the further advantage of bringing out the commonalities between AAVE and other zero copula languages. In general, the data from these other languages do not support plausible phonological or phonetic approaches like Labov's for English. Because of this, one might accept Labov's account as part of an account of the historical evolution of the zero copula construction in AAVE. However, we are inclined to say that the construction has now been reanalyzed in syntactic terms, fitting into a well established cross-linguistic pattern. This would help to explain why the correlation between where contraction occurs in SAE and where be may be absent in AAVE is only partial.

\section{Problem 3: AAVE Invariant $B e$}

The use of be in environments where SAE would require a finite form is one of the best known features of AAVE. (This is what Raspberry was ridiculing in the column quoted earlier in the chapter). This socalled 'invariant $b e$ ' conveys a special meaning, usually characterized as habitual. Thus, for example, (i) means roughly that the students are usually or always in the hall when the bell rings.

(i) The students be in the hall when the bell ring. (from Green, 1993) The goal of this problem is to determine how this use of be should be analyzed in our theory. Specifically, provide answers to the following three questions, and give arguments for your answers: 
A. Should the 'invariant be' be analyzed as a distinct lexical entry, or can it be assimilated to one of the entries for be we have already posited? [Hint: Consider the semantics.]

B. Is the 'invariant be' a verb, and, if so, is it finite?

C. Is the 'invariant be' an auxiliary - that is, is it [AUX +]?

The following data should be helpful in answering B and C.

(ii) ${ }^{*}$ The students be not in the hall when the bell ring.

(iii) * Be the students in the hall when the bell ring?

(iv) ${ }^{*}$ The students ben't in the hall when the bell ring.

(v) * Bob be eating when the news come on, and Sue be, too.

(vi) Bob be eating when the news come on, and Sue do, too.

\subsection{Conclusion}

There is much more that could be said about variation within the English auxiliary system. Our superficial look at two cases of linguistic variation was meant only to demonstrate that the theory of grammar developed here can accommodate such variation naturally. In the case of the main verb have, careful analysis revealed a close link between its variable syntactic and semantic properties. In the case of AAVE, we were able to provide a grammar for the zero copula construction, without positing silent lexical entries or deletion rules. Moreover, this led us to notice the difference in the agreement properties of AAVE and SAE verbs, which allows the lexicon of AAVE to be somewhat simpler than that of SAE. Finally, we saw that our analysis of the AAVE zero copula construction captures its commonalities with verbless sentences in other languages.

\subsection{Further Reading}

Much of the pioneering work on AAVE was done by William Labov and his collaborators and students. Among the most important works are Labov, et al. (1968), Labov (1969, 1972), Baugh (1983), and Rickford and Green (in press). Labov (1995) is a clear presentation of a different analysis of some of the same phenomena discussed in this chapter. Labov's analysis is examined in detail in Wasow and Sag (forthcoming). An early discussion of the dialect variation with respect to the main verb have can be found in Chomsky (1975), which was written in 1955. 



\section{Long-Distance Dependencies}

\subsection{Introduction}

One of the principal tasks of a theory of grammar is to provide mechanisms that allow economical formulations of the sorts of co-occurrence restrictions that exist in natural languages. In earlier chapters, we developed techniques for analyzing such aspects of syntax as differences in the valence of particular verbs, agreement between subject and verb, agreement between determiner and head noun, and restrictions on the distribution of dummy NPs. All of these co-occurrence restrictions are quite local, in the sense that they involve limitations on what can occur together as elements of a single clause. We extended this locality slightly with our analysis of raising, which in effect permits the co-occurrence restrictions of one verb to be transmitted to a higher verb.

The present chapter introduces a new type of construction in which the locality of co-occurrence restrictions appears to be violated in a more radical way. In these cases, two elements (say, an NP and a verb) appear far from one another in a sentence, despite the existence of a syntactic dependency (such as case marking or agreement) between them. Handling these 'long distance dependencies' (or LDDs, as we will call them) will require several changes to our theory:

- a new feature,

- a reformulation of one of our principles,

- a new principle,

- a new phrase structure rule, and

- a new lexical rule.

\subsection{Some Data}

Our current grammar correctly rules out examples like the following:

(1) a. ${ }^{*}$ They handed to the baby. 
b. ${ }^{*}$ They handed the toy.

c. ${ }^{*}$ You have talked to.

d. ${ }^{*}$ The children discover.

Because the lexical entry for hand specifies that its COMPS list has both an object NP and a PP, (1a-b) are ruled out through the interaction of the lexicon, the headed grammar rules, the Argument Realization Principle, and the Valence Principle. Similarly, (1c-d) are ruled out because both the preposition to and the verb discover require an object $\mathrm{NP}$, which is absent from these examples.

So it's interesting to find that there are grammatical sentences that contain exactly the ungrammatical strings of words in (1). For example, there are questions containing $w h$-words ('wh-questions') such as following:

(2) a. What did they hand to the baby?

b. Who(m) did they hand the toy?

c. Who $(\mathrm{m})$ should you have talked to?

d. What will the children discover?

There are also NPs modified by RELATIVE CLAUSES which contain the same ungrammatical strings:

(3) a. The toy which they handed to the baby...

b. The baby that they handed the toy to...

c. The people who(m) you have talked to...

d. The presents that the children discover...

And finally there is a kind of sentence that is used for a certain sort of emphasis that is usually called a 'topicalized' sentence. In this kind of sentence, a topicalized element can be followed by one of those same ungrammatical word sequences in $(1):^{1}$

\footnotetext{
${ }^{1}$ When examples like (4) are first presented, some students claim that they find them unacceptable, but examination of actual usage indicates that topicalization is quite common, e.g. in examples like the following (from a cartoon):

(i) Me, you bring an empty food dish; him, you bring a leash.

The name 'topicalization' is actually rather misleading. To be sure, the fronted element refers to an entity whose role in the discourse is distinguished in some way, but that entity need not correspond to the 'topic of discussion' in any straightforward way, as (i) indicates.

To illustrate these points, consider a context like the following, where an example like (4b) seems quite natural:

(v) A: How did they deal with all the screaming kids?

B: They promised to take the twins to a movie; they tossed new baseball gloves at Pat and Chris; and the baby, they handed a toy.
} 
(4) a. That toy, they handed to the baby.

b. The baby, they handed a toy.

c. That kind of person, you have talked to (many times).

d. Presents that come from grandma, the children (always) discover.

In each of the examples in (2)-(4), there is a dependency between an 'extra' phrase or 'filler' at the beginning of a clause and a 'gap' somewhere within the clause.

In short, we see that elements which cannot normally be missing from a clause are allowed to be missing if there is an appropriate filler in the right place. Likewise, if there is a filler, then there must be a gap somewhere within the sentence that follows the filler:

(5) a. *What did Kim hand the toys to the baby?

b. ${ }^{*}$ The dolls that Kim handed the toys to the baby....

c. ${ }^{*}$ The dolls, Kim handed the toys to the baby.

In such constructions, the filler can be separated from the gap by extra clauses, as indicated in (6)-(8). To help readers identify the location of the gaps, we have marked them with an underlined space.

(6) a. What did you say they handed _ to the baby?

b. Who(m) did he claim that they handed the toy to _-?

c. Who(m) do you think you have talked to _ ?

d. What will he predict that the children discover _ ?

(7) a. The toy which we believe they handed _ to the baby...

b. The baby that I think they handed the toy to

c. The person who(m) everyone thinks you have talked to

d. The presents that it annoys me that the children discover _....

(8) a. That toy, I think they handed _ to the baby.

b. This baby, I know that they handed a toy to

c. That kind of person, you know you have talked to

d. Presents that come from grandma, I know that the children (always) discover

In fact, there can be multiple extra clauses intervening:

(9) What did you think Pat claimed I said they handed _ to the baby?

\subsection{Formulating the Problem}

We want to be able to build clauses with elements missing within them. But somehow we have to keep track of the fact that something is missing. Furthermore, as the following contrasts show, we need to keep track of just what is missing: 
(10) a. This, you can rely on.

b. ${ }^{*}$ This, you can rely.

c. ${ }^{*}$ On this, you can rely on.

d. On this, you can rely.

e. ${ }^{*}$ On this, you can trust.

(11) a. Him, you can rely on.

b. ${ }^{*} \mathrm{He}$, you can rely on.

(12) a. The twins, I can't tell the difference between.

b. *That couple, I can't tell the difference between.

We can think of this as an information problem. We have to make sure that the phrases within the sentence keep track of what's missing from them as they are built. This has to be done just right, so that sentences missing a phrase of type $X$ (no matter how deeply embedded the $X$-type gap may be) combine with a filler of type $X$, and that fillers are allowed only when there is a gap for them to fill (cf. (5)).

\subsection{The Feature GAP}

Suppose we use a feature, GAP, to encode the fact that a phrase is missing a certain kind of element. There are examples of clauses where more than one phrase is missing ${ }^{2}$, although a detailed analysis of them is beyond the scope of this text:

(13) a. Problems this involved, my friends on the East Coast are difficult to talk to _ about

b. ?That outrageous announcer on the radio, I couldn't figure out which picture of to print

Note that the two gaps in each of these sentences have distinct fillers. In (13a), for example, the filler for the first gap is my friends on the East Coast, and the filler for the second one is problems this involved. Such examples are rare in English and sound a bit awkward, but there are other languages (e.g. Polish) that allow multiple gaps more freely.

Given the existence of sentences with multiple gaps, we need a mechanism that can keep track of many missing elements. This suggests that the value of GAP is a list of feature structures, like the values of COMPS, SPR, and ARG-ST.

The intuitive significance of a phrase specified as, say, [GAP $\langle\mathrm{NP}\rangle$ ] is that it is missing exactly one NP. The trick will be to make GAP have the right values in the right places. What we want is to allow a transitive verb or preposition to build a VP or PP without ever combining with

\footnotetext{
${ }^{2} \mathrm{Or}$, as linguists sometimes say (though it is somewhat of an oxymoron): 'where more than one gap appears'.
} 
an object NP. Furthermore, we want to ensure that it is only when an $\mathrm{NP}$ is missing that the relevant phrase is specified as [GAP $\langle\mathrm{NP}\rangle]$ ], as illustrated in (14).

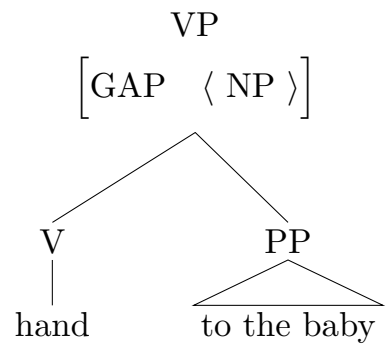

When nothing is missing, we want the relevant phrase to be [GAP \langle\rangle ], as in (15).

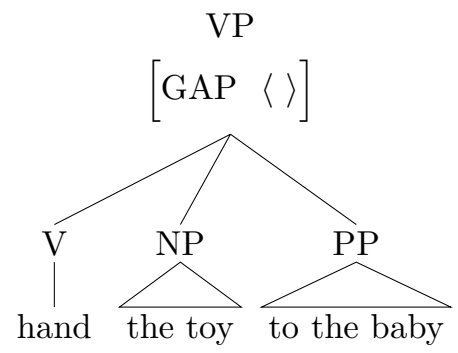

We will deal with this latest kind of 'missing element' as an instance of something that is present in argument structure but absent from the valence features. We could accomplish this by means of a lexical rule, but a more general solution is to modify the Argument Realization Principle. Our current version of the principle says that a word structure tree is well formed only if the valence lists (SPR and COMPS) add up to the argument structure (ARG-ST). We now want to allow for the possibility that some element or elements of ARG-ST are on neither the SPR list nor the COMPS list, but on the GAP list instead.

To make this modification precise, we will introduce a kind of subtraction operation on lists, which we will mark with the symbol $\ominus$. Intuitively, if $\mathrm{A}$ and $\mathrm{B}$ are lists, then $\mathrm{A} \ominus \mathrm{B}$ is a list that results from removing the elements of $\mathrm{B}$ from $\mathrm{A}$. A couple of caveats are in order here. First, we want $\mathrm{A} \ominus \mathrm{B}$ to be defined only when the elements of $\mathrm{B}$ all occur in A, and in the same order. So there are many pairs of lists for which this kind of list subtraction is undefined. This is unlike our form of list addition $(\oplus)$, which is defined for any pair of lists. Second, when A $\ominus \mathrm{B}$ is defined, it need not be unique. For example, if $\mathrm{A}=\langle\mathrm{NP}, \mathrm{PP}, \mathrm{NP}\rangle$ and $\mathrm{B}=\langle\mathrm{NP}\rangle$, then there are two possible values for $\mathrm{A} \ominus \mathrm{B}$, namely 
$\langle\mathrm{NP}, \mathrm{PP}\rangle$ and $\langle\mathrm{PP}, \mathrm{NP}\rangle$. We will interpret an equation like $\mathrm{A} \ominus \mathrm{B}=\mathrm{C}$ to mean that there is some value for $\mathrm{A} \ominus \mathrm{B}$ that is identical to $\mathrm{C}$.

With this new tool in hand, we can restate the Argument Realization Principle as indicated in following revised definition of lexical satisfaction:

(16) Lexical Satisfaction

A word structure:

F

satisfies a lexical entry $\langle\omega, \delta\rangle$ just in case:

1. $F$ satisfies $\delta$,

2. Argument Realization Principle

$F$ satisfies the following feature structure description:

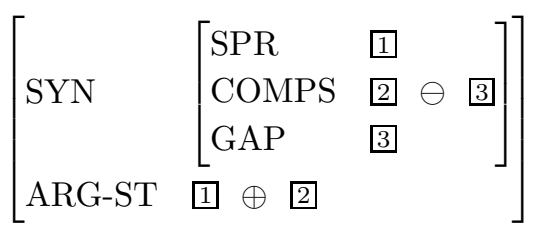

and

3. Case Constraint

Any NP in a noninitial position of $F$ 's ARG-ST list is [CASE acc].

Intuitively, clause 2 in (16) (the revised ARP) guarantees that any argument that could appear on a word's COMPS list can appear on its GAP list instead. (We will deal with gaps that correspond to subjects, rather than complements, in section 15.5.) Further, (16) guarantees that whenever an argument is missing, any co-occurrence restrictions the word imposes on that argument will be registered on the element that appears on the GAP list.

Because the result of list subtraction (as we have defined it, namely, $\ominus$ ) is not always unique, when we specify the ARG-ST in a verb's lexical entry without also specifying its SPR, COMPS, and GAP values, we are actually providing an underspecified lexical entry that can be satisfied by multiple word structures. Consider, for example, the lexical entry for the lexeme hand, as specified in (17). 


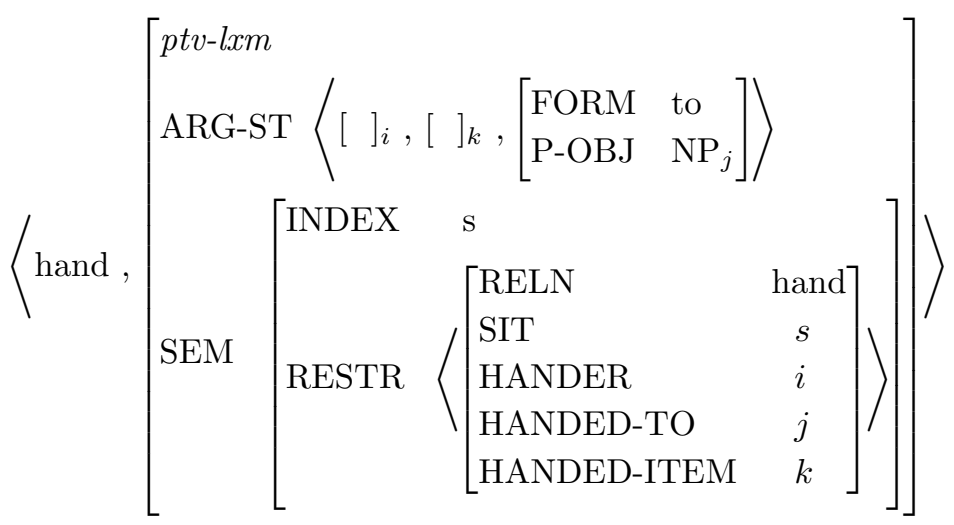

This can undergo the Non-3rd-Singular Verb Lexical Rule presented in Chapter 8, yielding the following lexical entry:

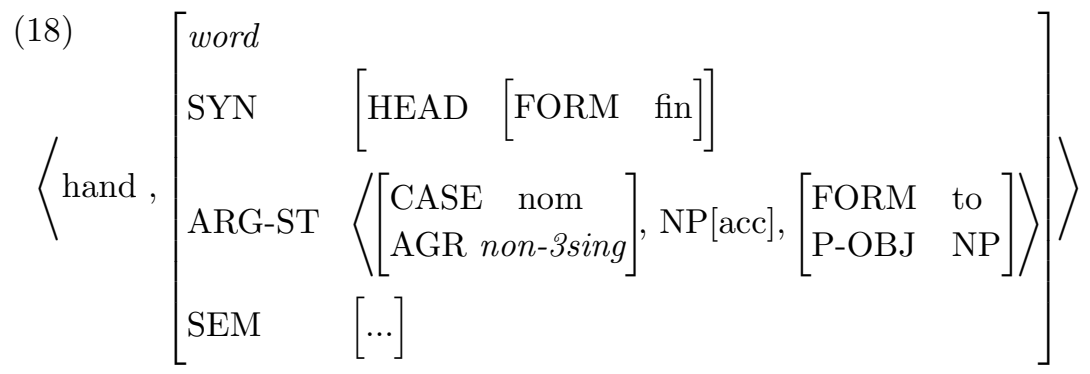

There are a number of word structures that this lexical entry can give rise to. They differ in their specifications for the features SPR, COMPS, and GAP. In particular, each of the following lexical SDs (omitting features not relevant to the present context) illustrates a distinct way that a word structure can satisfy the ARP: 
348 / Syntactic Theory: A Formal Introduction

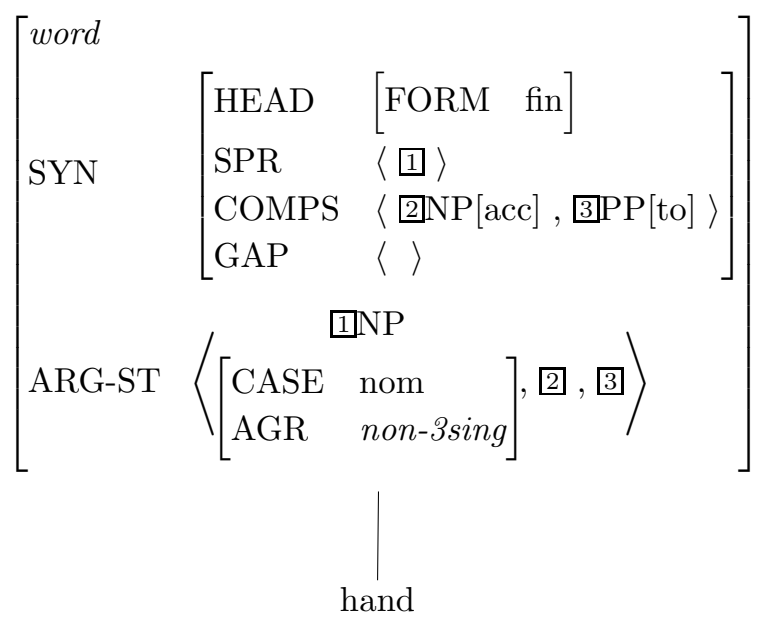

(20)

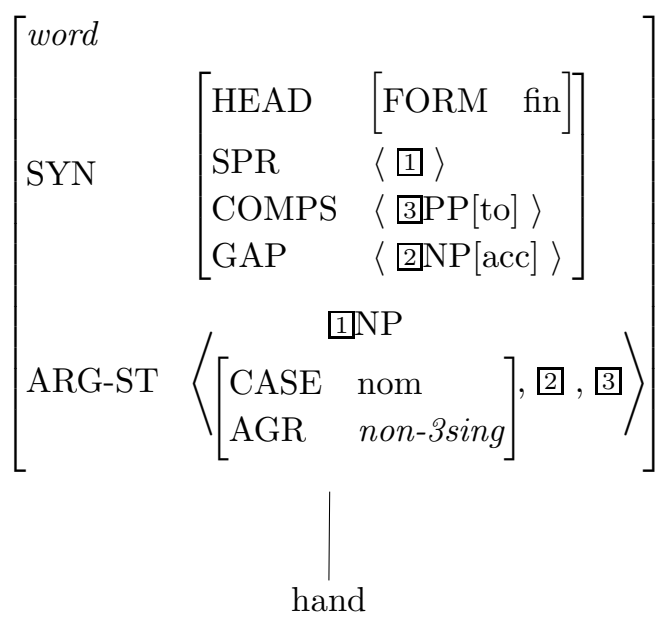


Long-Distance Dependencies / 349

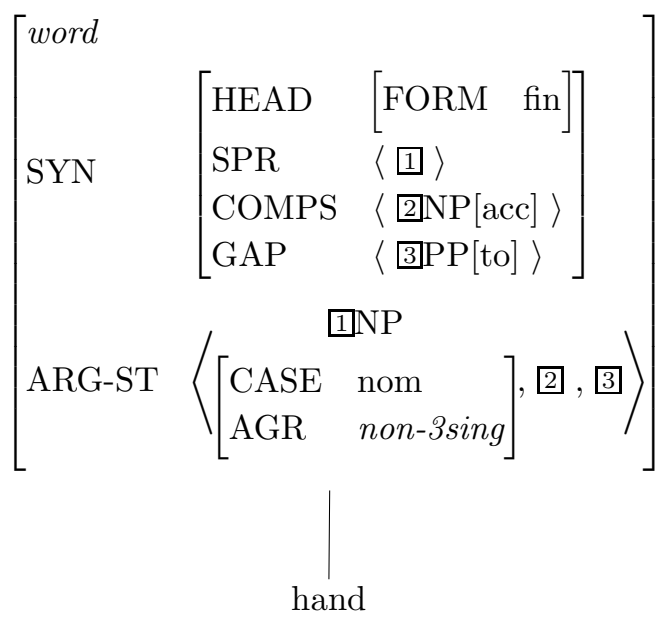

All of these are legitimate lexical SDs, and they serve as the bases for distinct phrase structure trees: (19) describes hand in sentences like (22a); (20) is the way hand appears in sentences like (22b); and (21) is the basis for our description of sentences like $(22 \mathrm{c}) .^{3}$

(22) a. You handed the toy to the baby.

b. What did you hand to the baby?

c. To whom did you hand the toy?

The prepositional lexeme in (23) will now give rise to the lexical SDs in (24) and (25) (again omitting what is not directly relevant).

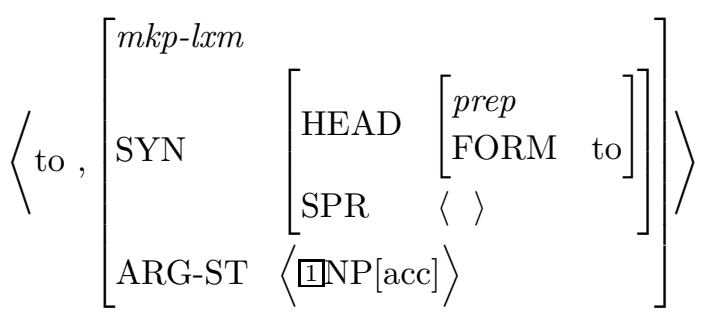

\footnotetext{
${ }^{3}$ The ARP also allows for a word structure in which both the NP and PP complements are in the GAP list, rather than the COMPS list. As noted above, however, consideration of examples with multiple gaps (which are relatively rare in English) goes beyond the scope of this text.
} 
350 / Syntactic Theory: A Formal Introduction

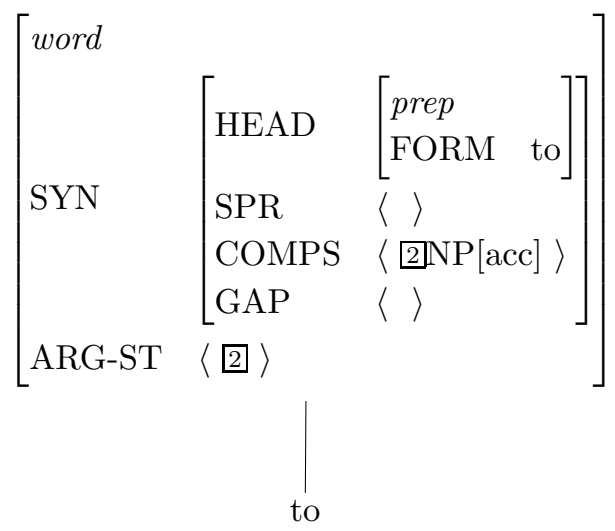

$(25)$

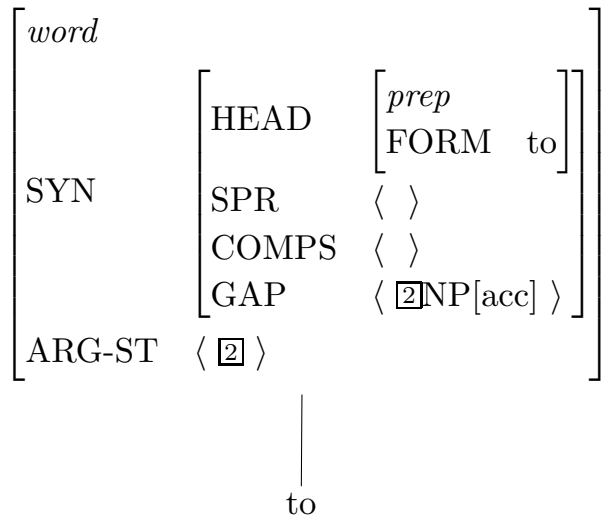

This last lexical SD is the one that allows for sentences like (26).

(26) Which baby did you hand the toy to?

The GAP feature tells us which of a word's arguments is missing. The Argument Realization Principle, as we have reformulated it, permits us to instantiate gaps freely (other than elements that must be on the SPR list). Now we need some way of passing the information in the GAP value up from words like those just illustrated so that the phrases that they head will register the fact that something is missing. In fact, we need to pass up ${ }^{4}$ the GAP information more generally, as we'll see in a moment, so we will adopt the more general principle in (27).

\footnotetext{
${ }^{4}$ The metaphor of passing information between nodes should not be taken too literally. What the principle in (27) does is similar to what the Head Feature Principle and Valence Principle do, namely, enforce a particular relationship between certain feature values in mothers and daughters in phrase structure trees. That is, it is simply part of our complex definition of well-formedness for phrase structure trees.
} 
(27) The GAP Principle

A well-formed phrase structure licensed by a headed rule other than the Head Filler Rule must satisfy the following SD:

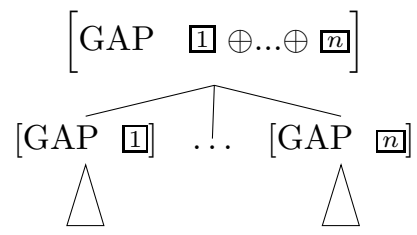

In other words, in a headed structure, the GAP values of all the daughters must add up to be the GAP value of the mother, unless the rule sanctioning the structure is the Head-Filler Rule (to which we turn directly).

The notion of lists 'adding up to' something is the same one we have employed before, namely the operation that we denote with the symbol ' $\oplus$ '. In most cases, most of the lists that are added up in this way are in fact empty, so that the addition is quite trivial. The purpose of this principle, once again, is to make sure that any information represented in the GAP value of a node will also be represented in that node's mother, but most constituents don't contain gaps. The effect of (27), then, given our lexical entries (and the word structures they sanction in virtue of our revision of the ARP), is illustrated in (28). 
352 / Syntactic Theory: A Formal Introduction

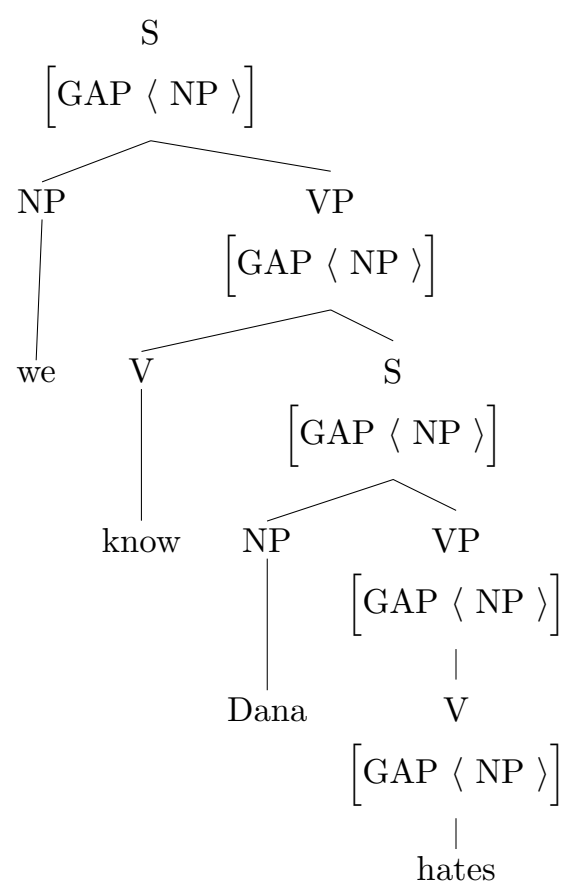

Note that we are suppressing empty GAP specifications on three nodes in (28). But it is true everywhere in (28) that the GAP values of the daughters add up to the mother's GAP value: $((\langle\rangle \oplus\langle N P\rangle)=\langle N P\rangle=$ $(\langle N P\rangle \oplus\langle\rangle))$.

Of course, we know Dana hates by itself is not a well-formed sentence. It must be combined with an NP to fill the gap corresponding to the object of hates. This requires a structure along the following lines: 
Long-Distance Dependencies / 353

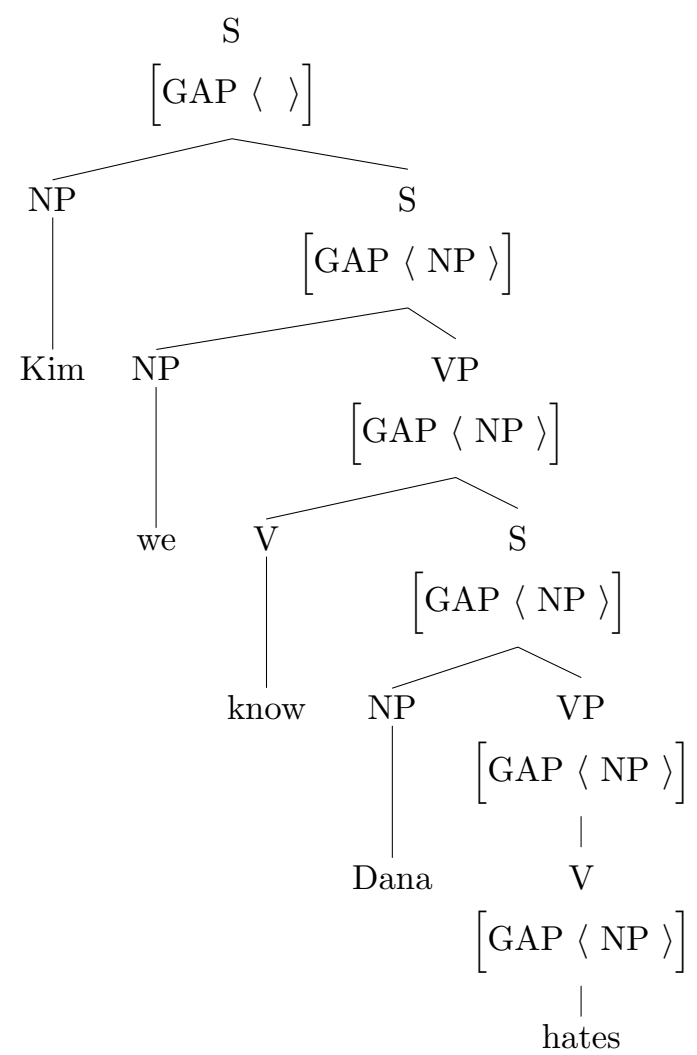

We have not yet accounted for the disappearance of the NP from the GAP value at the topmost node of (29). Intuitively, what is going on here is that the filler NP (Kim) is introduced, thus discharging the obligation encoded in the GAP value. To formalize this intuition, we introduce a new grammar rule.

(30) Head-Filler Rule

$$
\left[\begin{array}{ll}
\text { phrase } & \\
\text { GAP } & \langle\rangle
\end{array}\right] \rightarrow \text { 目 }\left[\begin{array}{ll}
\text { phrase } & \\
\text { GAP } & \langle\rangle
\end{array}\right] \mathrm{H}\left[\begin{array}{ll}
\text { phrase } & \\
\text { FORM } & \text { fin } \\
\text { SPR } & \langle\rangle \\
\text { GAP } & \langle\square\rangle
\end{array}\right]
$$

The GAP Principle (like the Valence Principle) allows the gap information to be propagated upward through tree structures. But because the Head-Filler Rule is not subject to the GAP Principle, it in effect terminates the upward propagation of a nonempty GAP specification. 
Rule (30) introduces the filler as its first daughter, specifying that it is identical to the value of the GAP feature in the second daughter, as shown in the highest local tree in (29). The mechanisms introduced to handle fillers and gaps guarantee that whenever a node of a tree structure is licensed by the Head Filler Rule, somewhere inside the finite $\mathrm{S}$ (the head daughter) there is exactly one word with a nonempty GAP list. The Head-Filler Rule is the only way we have of licensing the removal of elements from a GAP list. More precisely, this is our only way to license a node that is [GAP \langle\rangle$]$ itself but which dominates another node that has something on its GAP list. Consequently, the analysis presented introduces a filler if and only if there is a gap for it to fill.

We need one more modification in our grammar to ensure that all gaps ultimately get filled. We do this by revising our initial symbol to include a specification that it must have an empty GAP list. That is, our initial symbol is now the following:

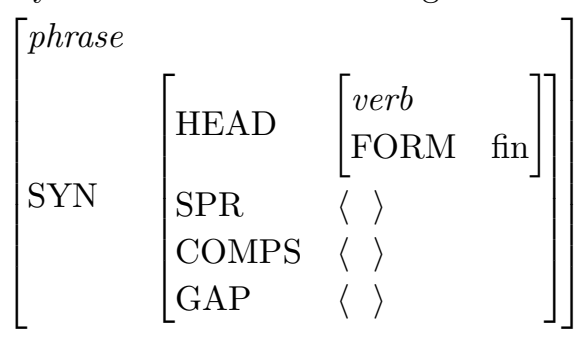

\section{Problem 1: An SD with a Gap}

Draw an SD for (8b). You should provide about the same level of formal detail as in (29).

\section{Problem 2: Blocking Filled Gaps}

Examples (i) and (ii) are well formed, but example (iii) is ungrammatical.

(i) Pat thinks that I rely on some sort of trick.

(ii) This mnemonic, Pat thinks that I rely on.

(iii) ${ }^{*}$ This mnemonic, Pat thinks that I rely on some sort of trick.

Explain in detail why the mechanisms that license (i) and (ii) do not also permit (iii). 
Long-Distance Dependencies / 355

\subsection{Subject Gaps}

We have covered only the basic cases of long-distance dependencies. There are many additional complexities. For example, we have not discussed cases in which the gaps are not complements, but rather subjects or modifiers. In addition, we have not discussed the distribution of whwords (such as who, what, which, etc.) in questions and relative clauses, nor the obligatory inverted order of subject and auxiliary verb in many wh-questions. There is a rich literature investigating these and many other questions associated with LDDs, but such matters are beyond the scope of this text. In this section we sketch the basics of an account of what is known as subject extraction - that is LDDs in which the gaps are in subject position.

Our present account does not yet deal with examples like (32).

(32) a. Which candidates do you think like raw oysters?

b. That candidate, I think likes raw oysters.

This is because it is a general property of verbs (analyzed in terms of a constraint inherited from the type verb-lxm) that they must have a singleton SPR list, rather than an empty one. That is, the ARP requires that the first member of a word's ARG-ST list (the verb's subject, in the present context) must also be on its SPR list. It follows that any elements that appear on a verb's GAP list must correspond to noninitial members of the ARG-ST list. Consequently, there is no way for a subject argument of a verb such as likes to appear on the GAP list. Hence we have no account (yet) of examples like (32).

Subject extraction is subject to special constraints in many languages. Hence it is not unreasonable for us to treat it in terms of a special rule, in this case, the following lexical rule:

(33) Subject Extraction Lexical Rule

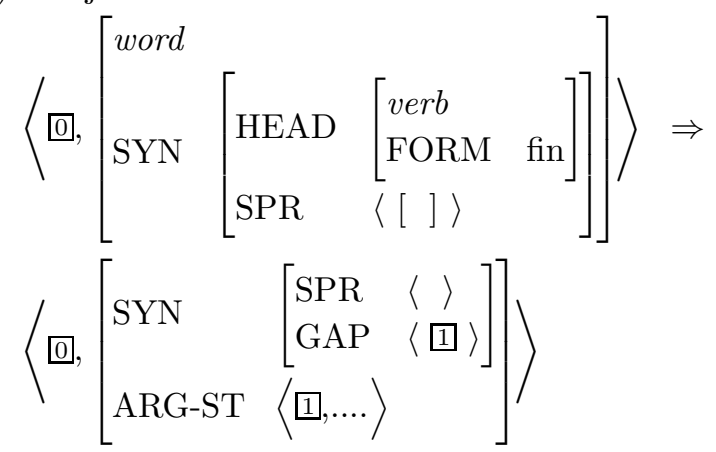

This rule maps any lexical entry for a finite verb form into a lexical entry 
with an empty SPR list and a GAP list containing an element identified with the first argument - the subject of the verb. Lexical entries that are outputs of this rule, like (34), give rise to lexical SDs like (35).

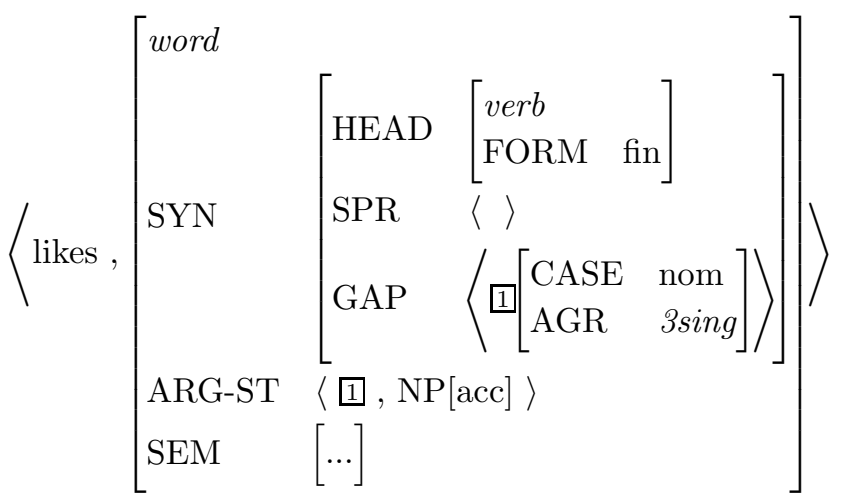

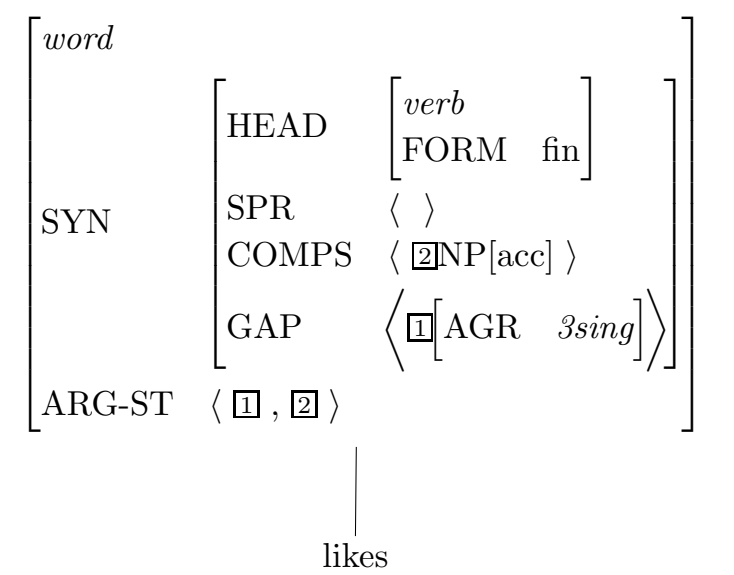

Note that the ARP is satisfied in (35): the SPR list is empty, and the rest of the ARG-ST list (i.e. the whole ARG-ST list) is in the appropriate relation with the list values of COMPS and GAP.

\section{Problem 3: Subject Gaps}

This problem is to make sure you understand how our analysis accounts for examples like (32).

(a) Sketch the lexical entry for likes that is the input to the Subject Extraction Lexical Rule.

(b) Sketch the entire SD for the sentence in (32b). Be sure to explain 
how our grammar ensures that all relevant feature specifications are as they are in your sketch.

(c) Does our analysis correctly predict the contrast between (32b) and (i)?

(i) ${ }^{*}$ Those candidates, I think likes raw oysters.

Explain why or why not.

Problem 4: Irish Complementizers

Consider the following example that shows the typical word order pattern of Modern Irish (data from McCloskey (1979)):

(i) Shil mé goN mbeadh sé ann thought I COMP would-be he there 'I thought that he would be there.' [M 2a]

Irish is a VSO language, one which we might describe in terms of a HeadSpecifier-Complement Rule that introduces both kinds of dependents as sisters of the lexical head.

A. Formulate such a rule and show the structure for sentence (i).

Now consider some further Irish data:

(ii) Dúirt mé gurL shı̨ mé goN mbeadh sé ann said I goN+past thought I COMP would-be he there 'I said that I thought that he would be there.' [M 3a]

(iii) an fear aL shil mé $a L$ bheadh ann the man COMP thought I COMP would-be _ there 'the man that I thought would be there' [M 2b]

(iv) an fear aL dúirt mé $a L$ shil mé $a L$ the man COMP said I COMP thought I COMP bheadh ann would-be _ there 'the man that I said I thought would be there' [M 3a]

(v) anfear aL shil goN mbeadh sé ann [the man $]_{j}$ COMP thought _ COMP would-be he ${ }_{j}$ there '[the man $]_{j}$ that thought he ${ }_{j}$ would be there' [M 4a]

(vi) an fear aL dúirt sé $a L$ shíl goN the man COMP said he COMP thought _ COMP mbeadh sé ann would-be he there 'the man that he said thought he would be there' [M 4c]

The complementizers $g o N$ and $a L$ are in complementary distribution. 
That is, wherever $g o N$ is possible in these examples, $a L$ is not, and vice versa. ${ }^{5}$ Assume that both these elements are heads of CPs similar to those headed by that complementizers in English. If we then make the further assumption that LDDs in Irish work much as they do in English, we have all the tools we need to analyze the contrasts in (i)-(vi).

B. Provide lexical entries for these two complementizers.

C. Show how your analysis successfully explains the distributional differences between the two complementizers.

\subsection{The Coordinate Structure Constraint}

One of the most discussed topics related to LDDs concerns restrictions on possible pairings of fillers and gaps. Although the position of filler and gap may be arbitrarily far apart, there are certain configurations that do not permit LDDs. Such configurations are known as 'islands' (a term due to Ross (1967)), and a major goal of syntactic research over the past three decades has been to understand where and why islands occur. In this section, we will look at one type of island and show how our grammar correctly predicts its existence and its properties.

The following examples illustrate what Ross called the 'Coordinate Structure Constraint':

(36) a. ${ }^{*}$ Here is the student that [the principal suspended [_ and Sandy]].

b. ${ }^{*}$ Here is the student that [the principal suspended [Sandy and []].

(37) a. * Here is the student that [the principal suspended _ and Sandy defended him].

b. ${ }^{*}$ Here is the student that [the student council passed new rules and the principal suspended _ ].

(38) a. *Apple bagels, I can assure you that [Leslie likes _ and Sandy hates cream cheese].

b. *Apple bagels, I can assure you that [Leslie likes cream cheese and Sandy hates _ ].

Translating Ross's transformation-based formulation of the constraint into the language of fillers and gaps that we have been using, it can be stated as follows:

\footnotetext{
${ }^{5}$ For the purposes of this problem, you should ignore the difference between gurL and $g o N$.
} 
(39) Coordinate Structure Constraint (CSC)

In a coordinate structure,

(a) no conjunct can be a gap,

(b) nor can a gap be contained in a conjunct if its filler is outside of that conjunct.

Ross also noticed a systematic class of exceptions to this constraint, illustrated by (40).

(40) a. This is the dancer that [we bought [a portrait of _ and two photos of _ ]].

b. Here is the student that [the principal suspended _ and the teacher defended _ _ .

c. Apple bagels, I can assure you that [Leslie likes _ and Sandy hates _ ].

To handle these, he appended an additional clause to the constraint, which we can formulate as follows:

(41) 'Across-the-Board' Exception (addendum to CSC):

... unless each conjunct has a gap paired with the same filler.

As presented, the Coordinate Structure Constraint seems quite arbitrary, and the Across-the-Board Exception is just an added complication. The descriptive generalizations of (39) and (41) ideally should not have to be stipulated. It would be preferable if they emerged as consequences of independently motivated aspects of the analyses of LDDs and coordination. And in fact, our accounts of LDDs and coordination interact to make precisely the right predictions in this domain, as we will now show.

Recall the grammar rule for coordination:

$\left[\begin{array}{ll}\mathrm{SYN} & 0 \\ \mathrm{IND} & s_{0}\end{array}\right] \rightarrow$

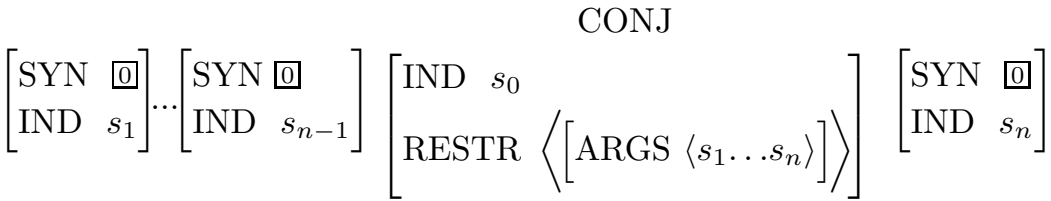

Intuitively, this allows any number of elements with the same SYN value to be conjoined. But GAP is part of SYN, so two phrases with different GAP values will have different $\mathrm{SYN}$ values. Consequently, two conjuncts in a coordinate structure cannot differ in their GAP value. If one has an empty GAP list and the other has a nonempty GAP list (as in (36)- 
(38)), then the structure is not licensed. The SYN values that should unify cannot, as shown in (42):

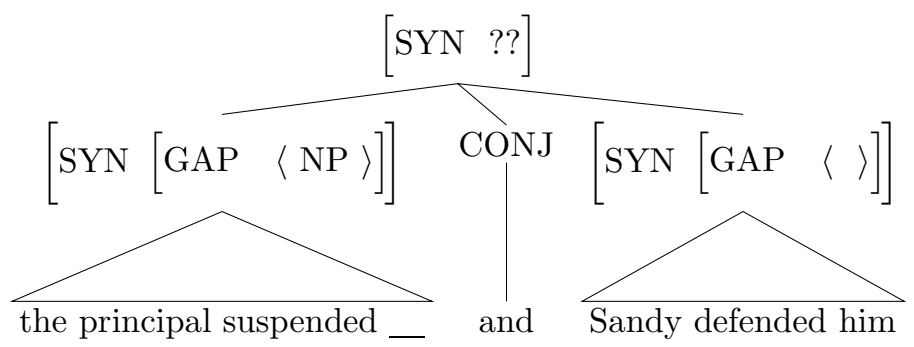

On the other hand, it is possible for conjuncts to have nonempty GAP lists if they are all nonempty and all share the same value. This is what is illustrated in (40), whose structure is as shown in (43):

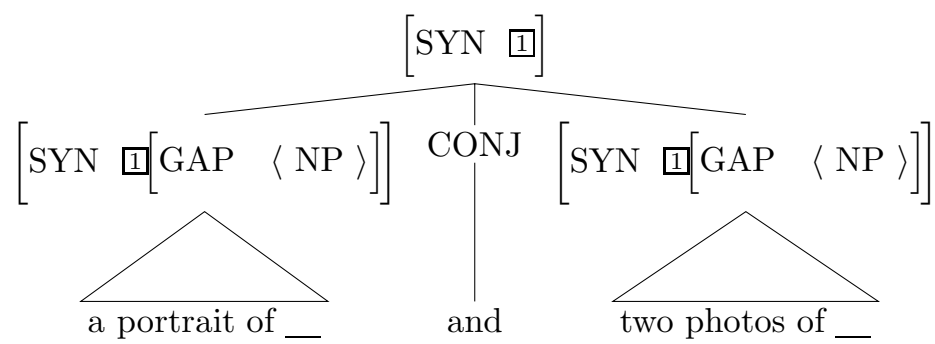

In short, both the CSC and the Across-the-Board exception to it fall out of our analysis as straightforward consequences.

We close this discussion with one final observation about LDDs and coordinate structures. There is an exception to (41), illustrated by (44):

$(44)^{*}$ Which rock legend would it be ridiculous to compare [[_] and [_]]?

Our statements of the generalizations in (39) and (41), like Ross's original formulations of them, would in fact permit (44), whose deviance should have a syntactic (rather than a semantic) explanation, it would appear, because the meaning of this putative sentence could certainly be expressed as (45):

(45) Which rock legend would it be ridiculous to compare _ with himself?

But our analysis correctly rules out any sentences in which a gap constitutes a full conjunct. This is because nonempty GAP values in the lexicon are licensed by the Argument Realization Principle. Hence our notion of a gap is an argument that fails to be realized in the syntax, not a phonetically empty phrase (or 'trace', as such phrases are often 
called). The difference is subtle, but the predictions are quite striking: our traceless analysis of gaps provides an immediate account of the deviance of (44) - and also of the examples in (36) - (38). The Coordinate Structure Constraint and its exceptions are thus explained in the analysis we have developed. Many alternative approaches - particularly those involving movement transformations to account for LDDs - have had to incorporate ad hoc stipulations to deal with this range of facts, or else have not accounted for them at all.

\subsection{Conclusion}

Deducing the Coordinate Structure Constraint and its exceptions from our analyses of coordination and LDDs is an elegant result, ${ }^{6}$ providing significant support for our general approach to syntax.

We will not examine other island constraints in this text. As with the Coordinate Structure Constraint, linguists have not been content to catalog the environments in which filler-gap pairings are impossible. Rather, a great deal of effort has gone into the search for explanations of syntactic islands, either in terms of the interaction of independently motivated elements of the theory (as in the example given above), or in terms of such factors as the architecture of the human language-processing mechanisms. This is a fertile area of research, in which definitive answers have not yet been found.

\subsection{Further Reading}

Ross (1967) is probably the most influential work to date on the topic of long-distance dependencies. Chomsky (1973, 1977, 1986b) developed one of the most influential approaches to analyzing these constructions, using transformations. Nontransformational treatments are presented by Zaenen (1983) and Gazdar (1981). The treatment presented here is unusual in not positing an empty category (a trace) in the position of the gap. Arguments for such a traceless analysis are discussed by Sag and Fodor (1994).

\footnotetext{
${ }^{6}$ Essentially this account was first developed by Gazdar (1981), within the theory of Generalized Phrase Structure Grammar.
} 



\section{6}

\section{From Rule to Sign}

\subsection{Taking Stock}

Let us examine the road we have travelled in the preceding chapters. We began with the inadequacy of simple context-free grammars, given their inability to express the notion of headedness, their inability to express cross-categorial generalizations, and the redundancy inherent in their descriptions of natural languages like English. We set out to modify CFGs - first by introducing the notion of feature structure, to model not only syntactic categories but also a variety of other constructs that became necessary as we developed our analyses (lexemes, words, agrcats, synsem-strucs, etc.). Central to our theory of feature structures has been the notion of a linguistic type, which we used both to declare which features are appropriate for particular kinds of linguistic entities and to classify linguistic entities (and the constraints they obey) in a hierarchical fashion.

After examining and analyzing a host of grammatical phenomena, however, we find that our system has not strayed very far from its CFG origins. We continue to specify well-formed tree structures in essentially the CFG fashion: we specify a lexicon plus a set of grammar rules, and we combine the information from these two sources in terms of a third, namely, the set of conditions that constitute our definition of well-formed tree structures. The lexicon is more elaborate than it would be in a simple CFG, with its hierarchical type structure and its lexical rules. Likewise, we have enriched the definition of phrasal satisfaction (part of our well-formedness definitions) to incorporate various general principles, such as the Head Feature Principle. The grammar rules, on the other hand, have become rather simple and schematic. Nevertheless, we have continued to divide the work of the grammar among these three components, each with its own formal character.

If we now step back from this picture of grammar, we can see things 


\section{4 / Syntactic Theory: A Formal Introduction}

from a new perspective. For example, we can now ask why phrases have no place in the hierarchy of linguistic entities. Surely they are linguistic entities just as much as words, lexemes, and synsem-strucs are. Yet they have an altogether different status in our current grammar: they are defined indirectly, in terms of the system of rules and principles that together characterize the well-formed phrase structure trees. As we shall see in this chapter, it is possible to reformulate our theory so that the grammar of phrases is not radically different from that of other linguistic objects. This reformulation (which represents the practice of much current research) has the formal advantage of providing a more uniform treatment of the lexicon and grammar rules, the descriptive advantage of allowing generalizations about phrases to be better expressed, and the explanatory advantage of fitting better with what we know about human sentence-processing, as discussed in Chapter 9.

\subsection{Words and Signs}

The renowned Swiss linguist Ferdinand de Saussure (1857-1913) conceived of language as a system of what he called 'signs'. A sign in Saussure's sense is nothing more than a conventional association of form with meaning. ${ }^{1}$ The sign-based conception of language is attractive in that it lets us put all linguistic expressions - words and phrases alike on an equal footing. Phrases like love, Kim loves Sandy, Go away, to continue to like anchovies, and so forth can all be viewed as associations of sound and meaning that are in accord with the constraints of English grammar, while l'amour, Kim aime Sandy, Allez-vous en, and continuer d'aimer les anchois are phrasal signs of French - different forms associated with the same meanings - and likewise for the corrsponding phrasal signs of Inuit, Tongan, or any other natural language.

Moreover, if we could reformulate all of our grammar as a set of constraints on signs, so that the English language would be just the set of signs that satisfied the constraints of English grammar, then we would have a picture of grammar that was completely true to the constraint-based architecture that we motivated in Chapter 9 on psychological grounds. On the sign-based conception of grammar (where, as we will see, lexical rules can be eliminated), the well-formed structures are just those that satisfy all the constraints of the theory. The well-formed structures must satisfy all the relevant type constraints, but they need not satisfy those constraints in any fixed order.

How, then, can we relate the grammar we have developed to the

\footnotetext{
${ }^{1}$ Or, as Saussure would have it, an association of a mental representation of sound with a mental representation of meaning.
} 
Saussurean conception? To begin with, our current notion of a lexical entry is very much like a sign, in that lexical entries specify a form and a meaning. Our lexical entries are richer than signs, though, in that they also specify syntactic information. Note that there has been a terminological tension throughout the preceding chapters about the term 'word'. We introduced the type word (a subtype of synsem-struc) and sometimes when we use the term 'word', that is what we mean. But on other occasions, we have used the term 'word' to refer to a lexical entry (i.e. a pair consisting of a form and a feature structure) whose feature structure is of type word, e.g. (1).

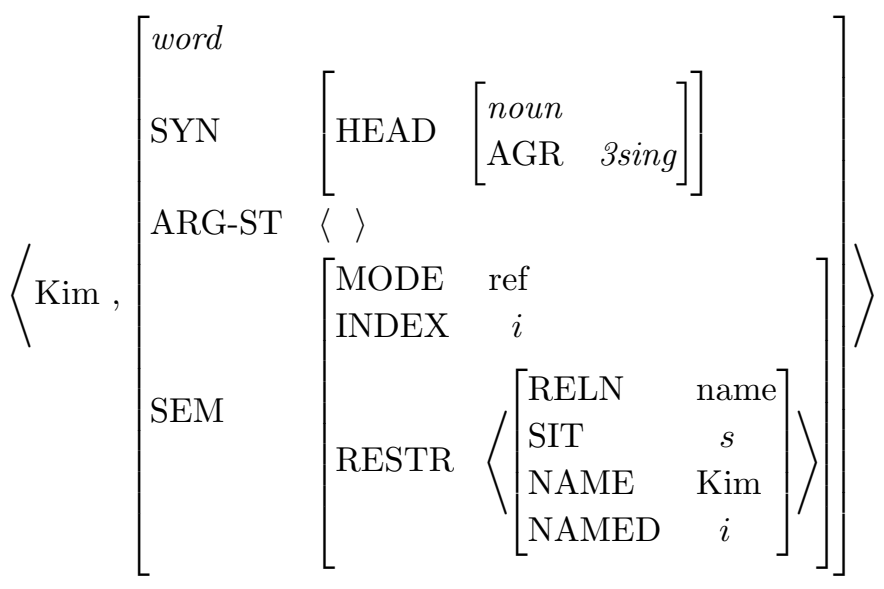

In our current grammar this word is the result of applying the Singular Noun Lexical Rule to the lexical entry whose first member is Kim and whose second member is a description of type pn-lxm.

It would be a small but significant move to revise our notion of word along Saussurean lines, thereby eliminating the terminological tension mentioned above. Suppose that we posited a type, word, whose features were PHON(OLOGY) and SEM(ANTICS). We could then model the word Kim as follows: 
366 / Syntactic Theory: A Formal Introduction

$$
\begin{aligned}
& \text { (2) [word } \\
& \text { PHON 〈Kim > } \\
& \left.\operatorname{SEM}\left[\begin{array}{ccc}
\text { MODE } & \text { ref } & \\
\text { INDEX } & i & \\
& \left.\quad\left[\begin{array}{ll}
\text { RELN } & \text { name } \\
\text { SIT } & s \\
\text { NAME } & \text { Kim } \\
\text { NAMED } & i
\end{array}\right]\right\rangle
\end{array}\right]\right\rangle
\end{aligned}
$$

This would be true to Saussure's conception, but it contains none of the SYNTAX or ARG-ST information that has been central to our analyses of a variety of complex grammatical phenomena. Indeed, Saussure is often faulted for failing to address syntactic issues.

But it requires only a small modification to include the syntactic information that is missing from (2). We could introduce a SYNTAX attribute parallel to SEMANTICS; alternatively we could make PHON and SYNSEM the only features appropriate for instances of the type word, making its SYNSEM values the synsem-strucs that have served us well in the analyses of the preceding chapters. The result would look something like (3).

(3)

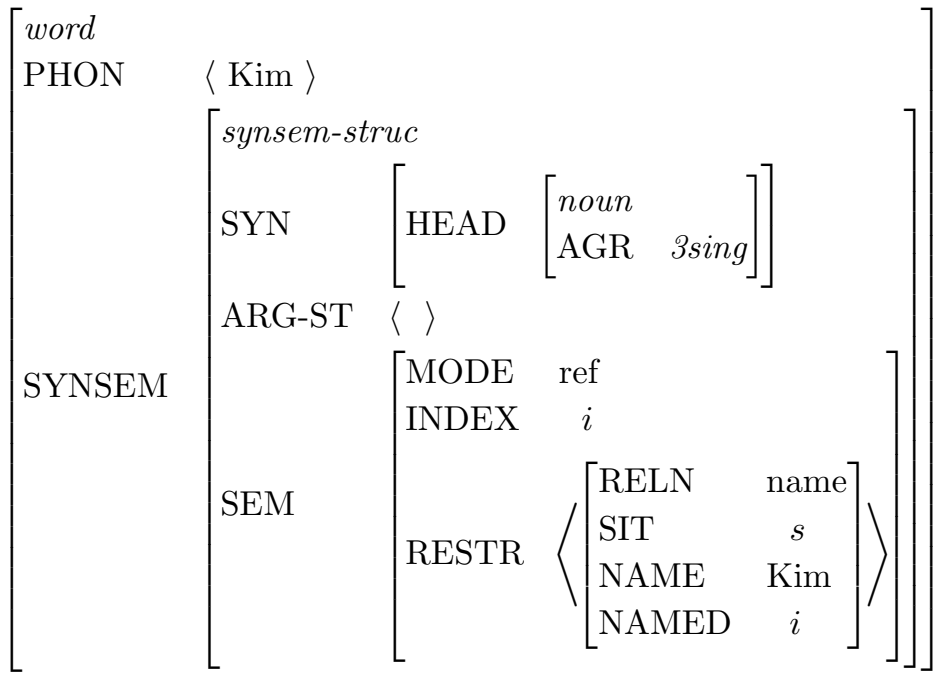

This is in essence the format in which the lexical analyses presented in this text have appeared in much of the published literature on sign-based grammar. 
Notice that the PHON value in (3) is presented as a list. This not only provides a uniform representation for words (recall our analysis of kick the bucket in Chapter 11), but also allows us to generalize the notion of sign to include phrases, where the PHON value will usually be a list of more than one form. Because feature structures like (3) are descriptions, they often omit certain grammatical specifications. And such descriptions are satisfied by a family of fully specified word structures - those objects of type word that are consistent with all the information that is specified. (See the discussion of satisfying descriptions in Chapter 3.)

Adopting a sign-based conception of grammar doesn't change our lexical analyses in any fundamental way; in fact, it enables us to improve them, as we will see. With the introduction of the notion of sign, though, we will want to revise our terminology a bit. For example, the type lexical-item, whose two immediate subtypes are word and lexeme (see Chapter 8 and the summary at the beginning of Chapter 9), is probably better recast as lexical-sign (lex-sign). The change is primarily terminological - constraint inheritance works just as before: an individual lexeme description (what we used to call a 'lexical entry for a lexeme') inherits constraints from the types that it is an instance of (with the possibility of overriding default constraints). The only substantive difference is that phonological information is now in the feature structures that participate in the inheritance.

The picture we have at the highest level of the grammar, then, is of signs subdivided according to the following type hierarchy.

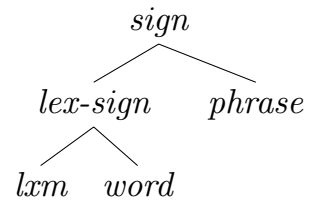

All signs are specified for the features PHON and SYNSEM; this will allow us to better reflect the common properties of words and phrases (while still drawing a type distinction between them). As we shall see below, phrases have additional features that are inappropriate for lexical signs.

In the next section, we explore the consequences of the sign-based architecture for the structure of the lexicon. In section 16.4, we sketch a sign-based conception of phrase, which will simplify certain aspects of our grammar and allow us to express cross-classifying generalizations about phrasal constructions. 


\subsection{The Organization of the Lexicon}

There are two distinct advantages to treating words as signs: (i) it allows us to express generalizations that cut across words and lexemes; and (ii) it provides a new, more elegant constraint-based alternative to lexical rules.

\subsubsection{Multiple Inheritance Hierarchies}

Recall that in Chapter 12, we introduced a distinction between raising and control elements. As we noted there, however, the distinction cuts across part-of-speech distinctions, for example, the distinction between verbs and adjectives. In fact, cross-cutting regularities are not at all uncommon in human languages, and our theory of grammar should be able to express such generalizations.

But in fact, the theory of lexical types we presented in Chapter 8 doesn't fare well in expressing cross-cutting generalizations. Because the hierarchy of lexemes is a tree, we posit distinct types (e.g. $a d j-l x m$ vs. verb-lxm) whenever a distinction must be made. But this means that if these types each have a subtype with a common property - for example, the type of subject-raising verb (continue) versus that of the subjectraising adjective (likely) - then we must state the constraint on subjectraising twice - once on each of the distinct types. Our 'single-mother' inheritance hierarchies thus fail to express certain generalizations that we find in natural language.

There is a solution to this problem. Suppose that we allow a type to be subdivided in two (or more) independent ways. That is, suppose we allow independent partitions of certain types into subtypes so that each maximal (leaf) type must have a supertype in each partition. Intuitively, the partitions correspond to distinct informational dimensions. For example, we can distinguish types based on part-of-speech distinctions from types based on distinctions of argument structure, thus allowing the contrast of, say, verb versus adjective to be independent of the contrast of intransitive versus transitive versus subject-raising, and so on.

This kind of classification is called a 'multiple inheritance hierarchy'. Such hierarchies are commonly used to organize multiple dimensions of information about objects in particular knowledge domains. Literary works, for example, might be organized in terms of types that form two independent hierarchical dimensions of classification, as shown in (5). 
(5)

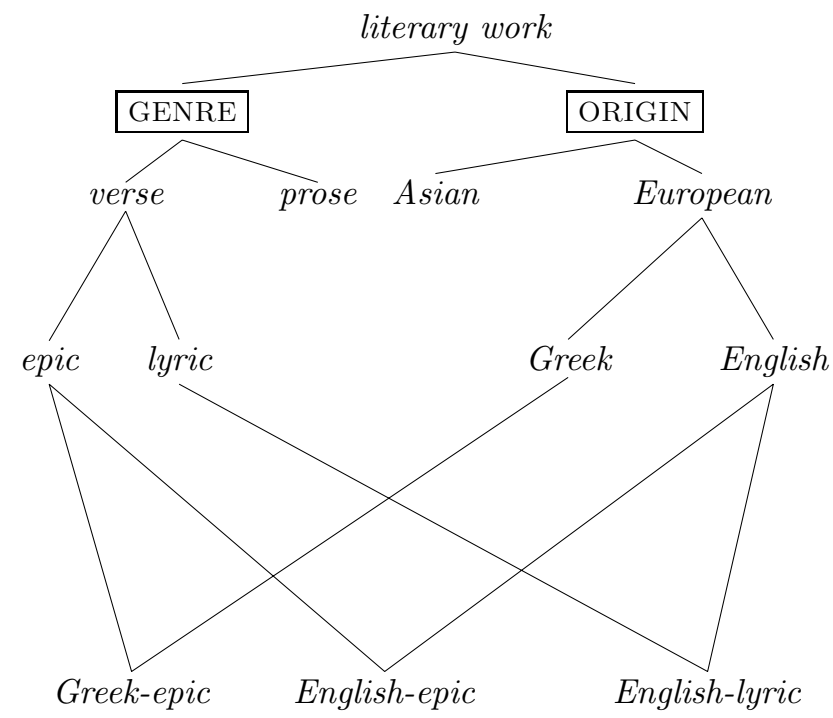

\section{The Odyssey Beowolf Ode to a Nightingale}

In (6), we illustrate one way our lexeme hierachy could be revised using multiple inheritance to express the cross-cutting generalizations our current hierarchy fails to capture.

(6)

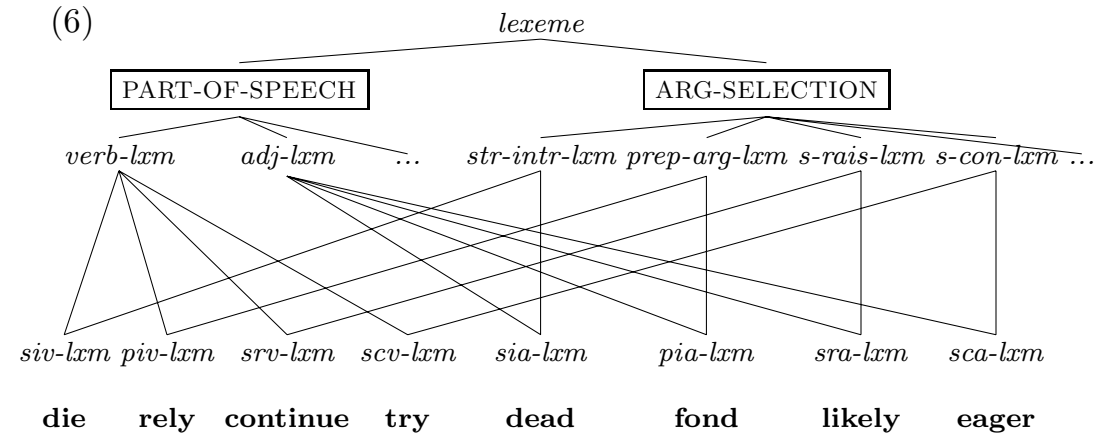

Several comments about this hierarchy are in order. First, we are using some new abbreviations for type names, so we have spelled out all of the names in $(7)$.

(7) a. str-intr-lxm: strict-intransitive-lexeme

b. prep-arg-lxm: PP-argument-lexeme

c. s-rais-lxm: subject-raising-lexeme

d. s-con-lxm: subject-control-lexeme 
370 / Syntactic Theory: A Formal Introduction
e. siv: strict-intransitive-verb-lexeme
f. piv: PP-intransitive-verb-lexeme
g. srv: subject-raising-verb-lexeme
h. scv: subject-control-verb-lexeme
i. sia: strict-intransitive-adjective-lexeme
j. pia: PP-intransitive-adjective-lexeme
k. sra: subject-raising-adjective-lexeme
1. sca: subject-control-adjective-lexeme

Second, the ARG-SELECTION types (str-intr-lxm, prep-arg-lxm, etc.) should be constrained so as to express the generalizations across ARG-ST values that are common to the particular verbal and adjectival subtypes. These constraints can be stated as in (8). ${ }^{2}$

(8) a
str-intran-lxm: $[\mathrm{SS} \quad[\mathrm{ARG-ST}\langle[]\rangle]]$
b. prep-arg-lxm: $[\mathrm{SS}[\mathrm{ARG-ST}\langle[], \mathrm{PP}\rangle]]$
c.

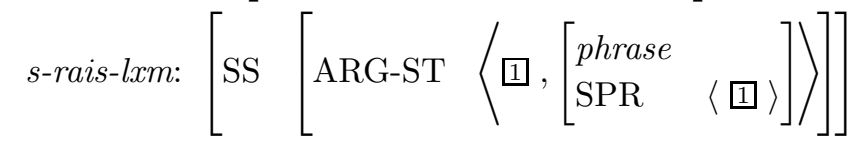
d. s-con-lxm: $\left[\mathrm{SS}\left[\mathrm{ARG-ST}\left\langle\mathrm{NP}_{i},\left[\begin{array}{ll}\text { phrase } & \\ \mathrm{SPR} & \left\langle\mathrm{NP}_{i}\right\rangle\end{array}\right]\right\rangle\right]\right]$

The PART-OF-SPEECH types can remain subject to the earlier constraints, namely:

(9) a.

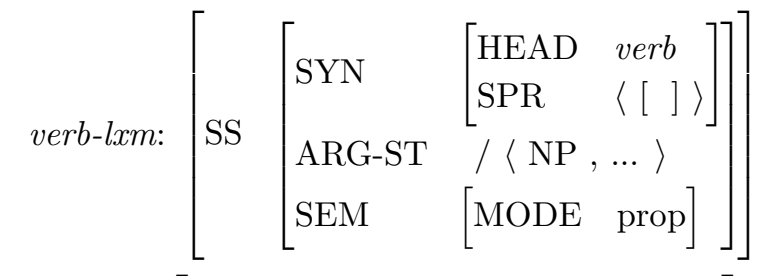

b.

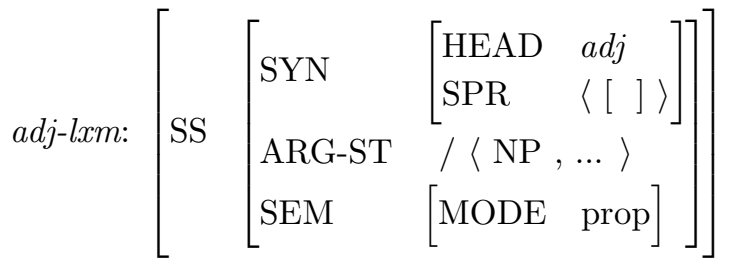

Given the similarity of these constraints, of course, we could further

\footnotetext{
${ }^{2}$ Here and throughout, we use 'SS' to abbreviate 'SYNSEM'.
} 
revise the system so that the ARG-ST and SEM specifications are associated with a common supertype.

Our purpose here is not to make a definitive proposal for the analysis of the English lexeme hierarchy, ${ }^{3}$ but rather to show that multiple inheritance hiearchies can be used to factor cross-cutting generalizations of words into succinct constraints that are associated with cross-classifying types.

\subsubsection{Lexical Rules as Word Types}

Let us now consider how lexical rules can be eliminated in favor of a hierachical system of types, given a sign-based architecture. In this text, we have presented the function of lexical rules as expanding the set of lexical entries. That is, we have treated them as mappings from lexical descriptions to lexical descriptions. We have seen three kinds of lexical rules: derivational rules that map lexemes to lexemes (e.g. the Extraposition Lexical Rule), inflectional rules mapping lexemes to words (e.g. the 3rd Singular Verb Lexical Rule), and other rules mapping words to words (e.g. the Negation Lexical Rule).

The work of all three kinds of lexical rule can be handled by types. Here we will focus on inflectional rules. Suppose that we declare a feature STEM as appropriate for the type word. If the value of STEM is of type lexeme, then we have a feature structure where a lexeme fits inside of a word. This is one way to build the word/lexeme relation into a single feature structure. We might further add the default constraints that the SYNSEM and PHON values of a word are identical to those of its STEM value. These constraints, illustrated in (10), will have the same effect as our convention that lexical rules preserve information that is not explicitly contradicted.

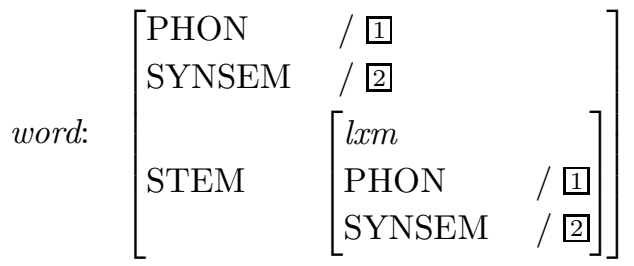

Each lexeme-to-word (inflectional) lexical rule will then correspond to a distinct subtype of word, with appropriate constraints added. For example, the Constant Lexeme Lexical Rule can be replaced by a subtype of word, constrained as shown in (11).

\footnotetext{
${ }^{3}$ Such a proposal might also involve separating the distinction between constant and inflecting lexemes into a separate dimension.
} 
(11) const-wd: [STEM const-lxm]

Note that the PHON and SYNSEM identifications are inherited from (10) by default.

If we replace lexical rules with a hierarchy of subtypes of word, we can not only express the same generalizations that our lexical rules used to express, but we can also group lexical rules into families, establishing intermediate types to express further generalizations, that is, those properties that families of lexical rules have in common. Suppose for example that we posit (for English) the three basic subtypes of word shown in (12) and associate them with the constraints shown in (13). ${ }^{4}$

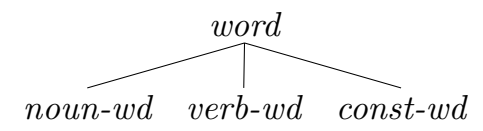
a. noun-wd: [STEM noun-lxm]
b. verb-wd: [STEM verb-lxm]
c. const-wd: [STEM const-lxm]

Note that these constraints interact with the default identities imposed on the type word by (10). For example, the HEAD value of a verb-wd will be of type verb.

More interestingly, we can establish the following hierarchy of the subtypes of verb-wd:

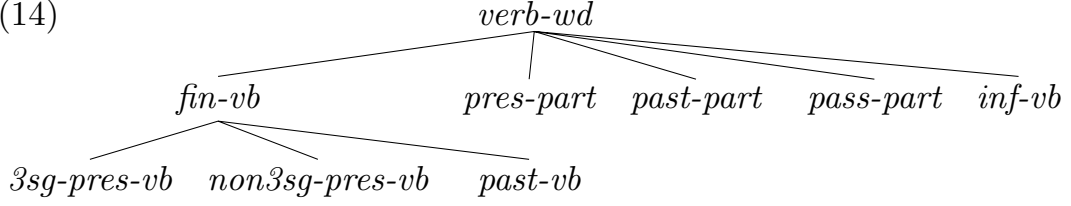

This enables us to pull out a common property of all finite verbal forms and express it as a single constraint on the intermediate level type fin-vb:

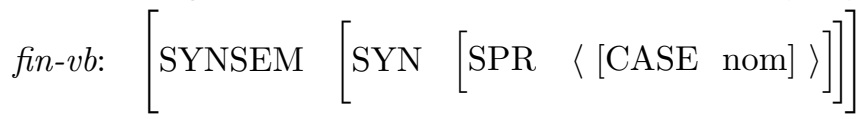

All remaining effects of the relevant lexical rules now become constraints on the subtypes in (14). The default constraint in (10) guarantees that whatever constraints are lexemically specified and not specifically contradicted by constraints on the word type will be inherited by the appropriate word.

\footnotetext{
${ }^{4}$ The lexeme types assumed here are just those we made use of in Chapter 8 .
} 
For example, the type 3sg-pres- $v b$ will be constrained as shown in $(16):^{5}$

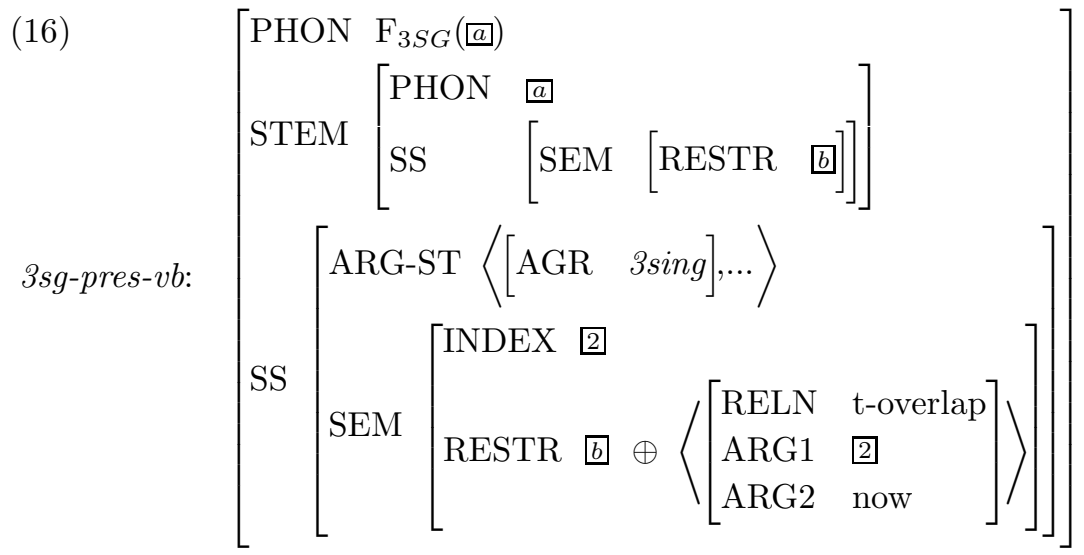

Hence, through inheritance of constraints on superordinate types, words such as walks will have a feature structure like the one in (17).

\footnotetext{
${ }^{5}$ The careful reader will notice that we have slightly modified our conception of morphological functions like $\mathrm{F}_{3 S G}$. Instead of mapping forms onto forms, they now map lists of forms onto lists of forms. In cases where the input list has more than one member, the relevant morphological change affects just the first member of the list; see Chapter 11, footnote 13.
} 
374 / Syntactic Theory: A Formal Introduction

(17)

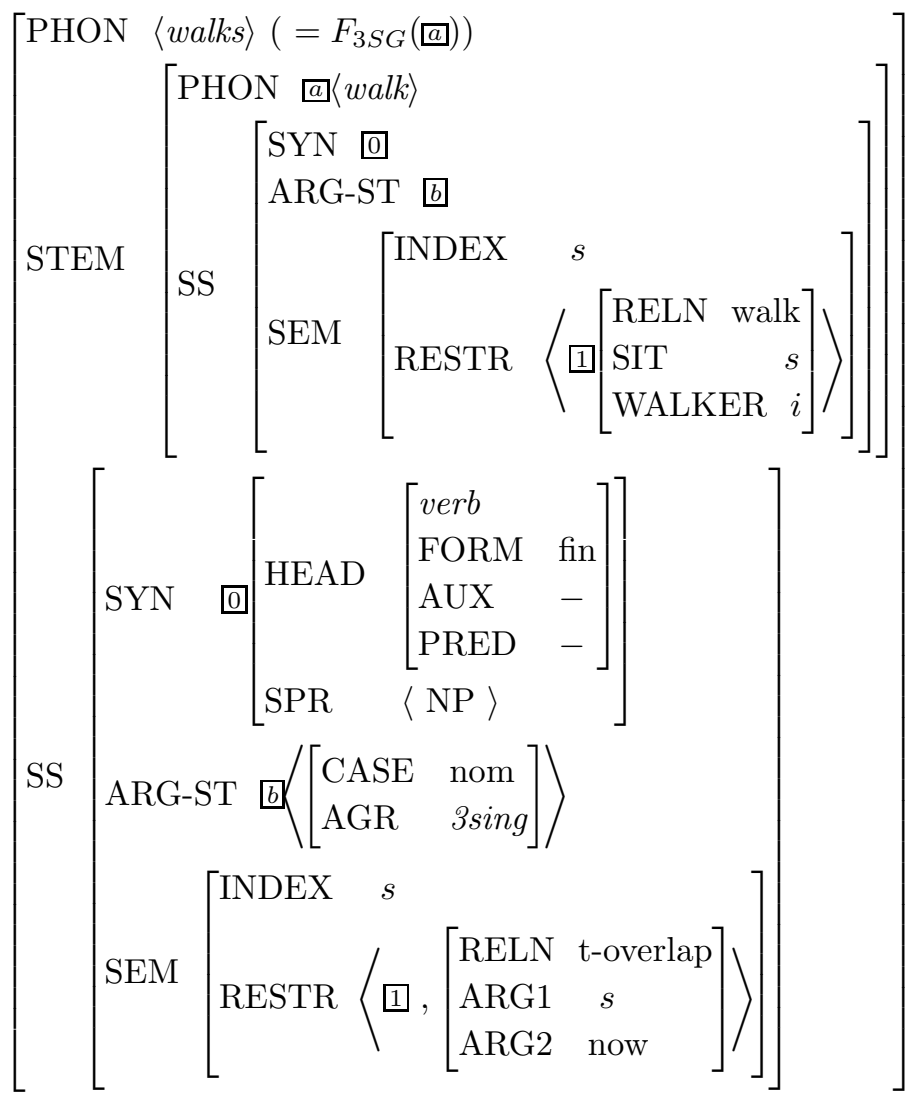

Note that the ARG-ST and SYN values of the word walks are unified with those of its STEM value. This is a consequence of the default unification in (10): the constraints in (16) specifically alter the SEM value, but the other parts of SYNSEM remain unified. ${ }^{6}$

What we have just done is to eliminate a construct from our theory: lexical rules that map lexical descriptions to other lexical descriptions. In place of these mappings, we have pressed into service the independently necessary notion of type hierarchy, which, as we have seen, allows us to express certain generalizations about words (common properties of lexical rules) that the earlier grammar relying on lexical rules was not able to express.

This new conception of lexical rules also makes possible a reformu-

\footnotetext{
${ }^{6}$ For a precise discussion of the approach to default unification that we make use of here, see Lascarides and Copestake (1998).
} 
lation of the Argument Realization Principle. This now becomes a nondefeasible constraint associated with the type word:

$$
\text { word: }\left[\text { SYNSEM }\left[\begin{array}{llll}
\text { SYN } & {\left[\begin{array}{lll}
\text { SPR } & \square & \\
\text { COMPS } & b & \ominus \\
\text { GAP } & \square & \square
\end{array}\right]} \\
\text { ARG-ST } & \square \oplus \square & & \square
\end{array}\right]\right]
$$

As a consequence of this constraint, the word walks obeys further specifications, as shown in (19).

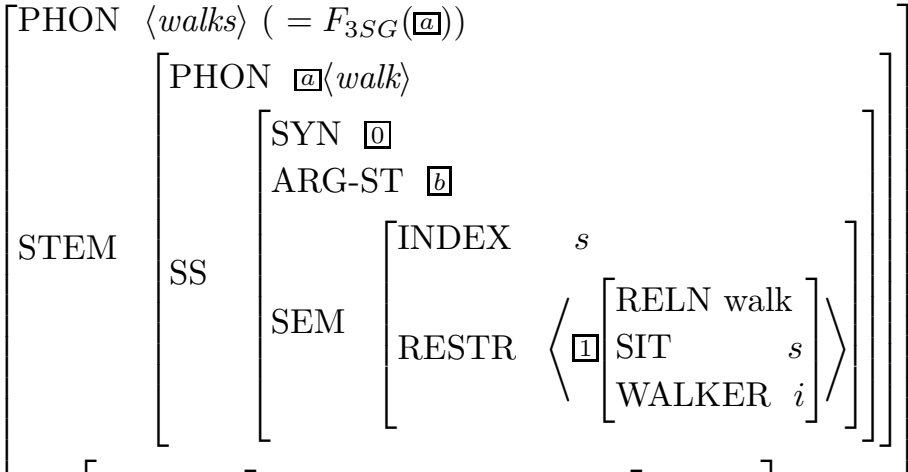

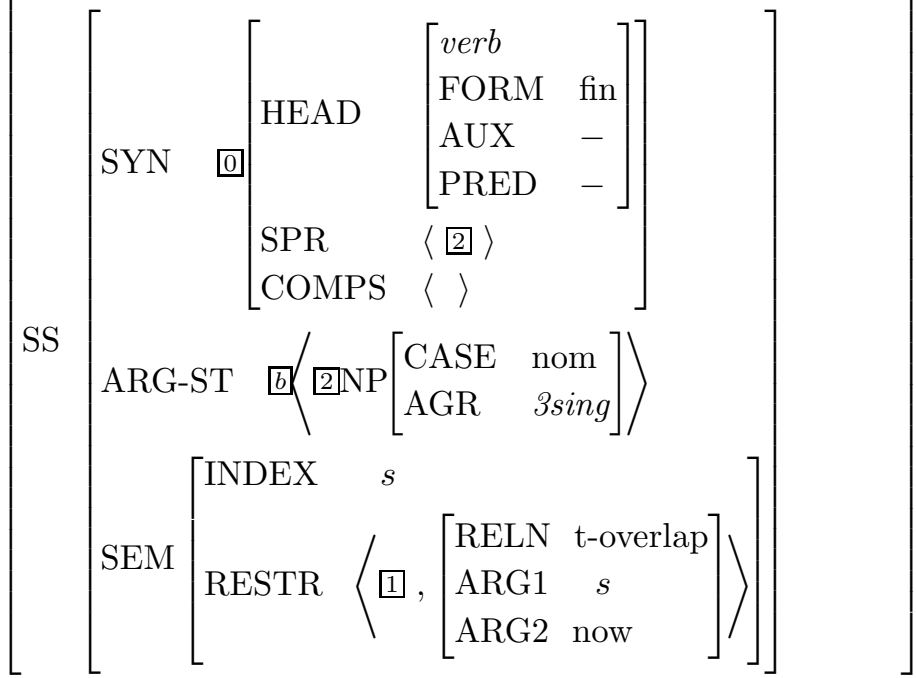

The final constraint we will discuss here in connection with lexical signs is the Case Constraint, which was formulated in Chapter 8 as (20), a constraint on word structures. 
(20) Case Constraint:

Any NP in a noninitial position of a word's ARG-ST list is [CASE acc].

Making this constraint more precise in terms of a sign-based architecture is an interesting challenge, as we have no direct way of expressing quantification (i.e. 'any NP [on a list]'). In approaching this problem, let us first propose a minor change to the hierarchy of part of speech types, so as to treat all nonnominal types as instances of a common type:

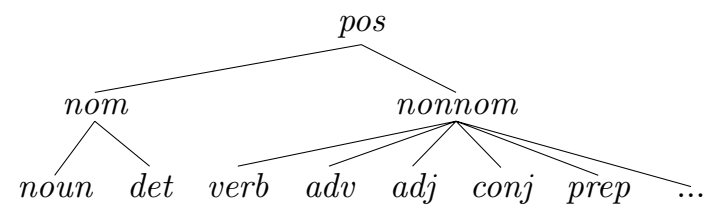

The introduction of the type nonnom(inal) will simplify the statement of constraints we consider in a moment.

To create a kind of list that can contain diverse kinds of synsemstrucs, except a nominative NP, we first need to consider more precisely what lists are. As noted briefly in Chapter 8, note 7, a list is commonly treated as a feature structure with the two features FIRST and REST. The empty list is a special (atomic) type of list that we will call elist. Thus in these terms, the lists that we have been writing as $\langle\mathrm{NP}\rangle$, $\langle\mathrm{NP}, \mathrm{PP}\rangle$, and $\langle\mathrm{NP}, \mathrm{NP}, \mathrm{PP}\rangle$ would be represented as $(22 \mathrm{a}, \mathrm{b}, \mathrm{c})$, respectively.

(22) a.

$$
\left[\begin{array}{ll}
\text { FIRST } & \text { NP } \\
\text { REST } & \text { elist }
\end{array}\right]
$$

b.

$\left[\begin{array}{lll}\text { FIRST } & \text { NP } & \\ \text { REST } & {\left[\begin{array}{ll}\text { FIRST } & \text { PP } \\ \text { REST } & \text { elist }\end{array}\right]}\end{array}\right]$

c.

$\left[\begin{array}{llll}\text { FIRST } & \text { NP } & & \\ \text { REST } & {\left[\begin{array}{lll}\text { FIRST } & \text { NP } & \\ & \text { REST } & {\left[\begin{array}{ll}\text { FIRST } & \text { PP } \\ \text { REST } & \text { elist }\end{array}\right]}\end{array}\right]}\end{array}\right.$

If we conceive of lists in this way, we can contemplate the idea of special types of list that are subject to specific constraints. In particular, suppose we hypothesize a type called comp(lement)-list, which is intuitively the kind of list that completes an ARG-ST list, and so is the 
kind of list that is an ARG-ST list's REST value. We could then divide comp-lists into three subtypes as follows:

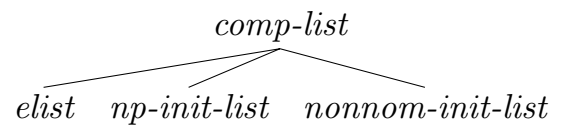

Intuitively, $n p$-init-list is the type of a list beginning with an NP (synsemstruc), and nonnom-init-list is the type of a list beginning with a synsemstruc whose HEAD value is (any subtype of) nonnom.

Part of the work previously done by the Case Constraint can now be accomplished by the following type constraints:

$$
\begin{array}{ll}
\text { a. } & \text { np-init-list: }\left[\begin{array}{lll}
\text { FIRST } & \text { NP }\left[\begin{array}{lll}
\text { CASE } & \text { acc }
\end{array}\right] \\
\text { REST } & \text { comp-list }
\end{array}\right] \\
\text { b. } & \text { nonnom-init-list: }
\end{array}\left[\begin{array}{lll}
\text { FIRST } & {\left[\begin{array}{lll}
\text { SYN } & {[\mathrm{HEAD}} & \text { nonnom }]
\end{array}\right]} \\
\text { REST } & \text { comp-list } &
\end{array}\right]
$$

From these type constraints, it follows that any NP on a comp-list must be [CASE acc].

Once we have a type like comp-list, we can specify what kinds of lists can be the value of a word's ARG-ST feature by defining subtypes of a new type, word-argument-structure-list (wd-as-list). This is illustrated in $(25){ }^{7}$

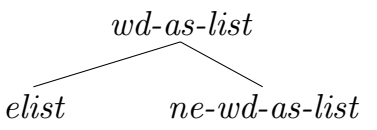

The new type nonempty-word-argument-structure-list (ne-wd-as-list) is now constrained as in (26):

$$
\text { ne-wd-as-list: } \quad[\mathrm{REST} \text { comp-list }]
$$

And because of this constraint, we can achieve the effect of the Case Constraint simply by specifying that a word's ARG-ST value must be of type wd-as-list, as in (27).

$$
\text { word: }[\text { SYNSEM }[\text { ARG-ST wd-as-list }]]
$$

A word's ARG-ST value, then, must either be empty or be a nonempty list of synsem-strucs whose noninitial NPs (if any) are all [CASE acc].

Note that this analysis leaves an initial NP on a word's ARG-ST list unspecified for CASE, unless some other type is subject to a further constraint on that NP. The constraint stated in (15), which requires that

\footnotetext{
${ }^{7}$ Note that some types, like elist, have many supertypes.
} 
the first argument of all finite verb forms by [CASE nom], is an example of such a type constraint.

We have now revised our treatment of both the Argument Realization Principle and the Case Constraint, both of which were formerly clauses in the definition of lexical satisfaction. The current formulation, by contrast, utilizes only type constraints, providing a purely constraintbased account of the grammar of lexical signs.

\section{Problem 1}

Explain why the first argument of nonfinite verb forms must be unspecified for CASE, rather than specified as [CASE acc]. [Hint: Consider raising constructions.]

\subsection{Phrasal Signs}

Thus far, we have recast our notions of word and lexeme in Saussurean terms by modelling his notion of 'sign' in terms of feature structures. There is ample reason to do the same for phrases. Reflect, for a moment, on the theory of phrases we have developed. All phrases share one property: they each have at least one daughter. In addition, there are different types of phrases: headed and nonheaded. Moreover, the headed phrases have many subvarieties (e.g. head-specifier phrase, headcomplement phrase), as do the nonheaded phrases (e.g. imperative clause and coordinate phrase). This is just the sort of classification and subclassification which, in the lexicon, led us to posit a hierarchy of types.

Further, the current architecture of our grammar treats grammar rules as a special kind of theoretical entity, and provides no way to capture properties that are shared by some, but not all, grammar rules. A bifurcation of grammar rules into headed and nonheaded rules is referred to in our definition of phrasal satisfaction, but in our current system, this distinction is simply stipulated in prose. We have not made use of the formal machinery introduced to handle analogous classifications in the lexicon. In short, the representational differences between grammar rules and lexical entries in the earlier chapters obscure the fundamental similarities among lexemes, words and phrases.

Capturing these similarities is desirable not just for the sake of making the theory formally more uniform; it also has analytic advantages. Some generalizations hold of certain kinds of phrases and not others. For example the Head Feature Principle obviously applies only to those phrases that have a head daughter, whereas the Semantic Composition- 
ality Principle holds of all phrases. In addition, our formulations of principles like the Valence Principle and the Gap Principle specifically excluded certain grammar rules from their domain. We had no technical machinery for doing this, other than naming particular rules that were to be excluded in the appropriate clauses of the definition of phrasal satisfaction. However, if phrases are treated as feature structures assigned to particular types and if those types constitute a type hierarchy, then we could state monotonic constraints that hold at different levels (e.g. that apply to all phrases, or else just to headed phrases), or we could use default constraints to express generalizations that hold over most, but not all kinds of phrases. Thus adopting the sign-based architecture for phrases allows us to exploit mechanisms we already employ in our grammar. We can define lexical and phrasal signs in a uniform way, eliminating the entire ancillary apparatus that we have introduced to define phrasal satisfaction.

What exactly do we mean by the 'sign-based architecture for phrases'? What we are after is a way of modelling a phrase like Kim walks as a kind of sign, that is, a feature structure which, like the lexical signs we have just presented, specificies values for the features PHON and SYNSEM. This is illustrated in (28).

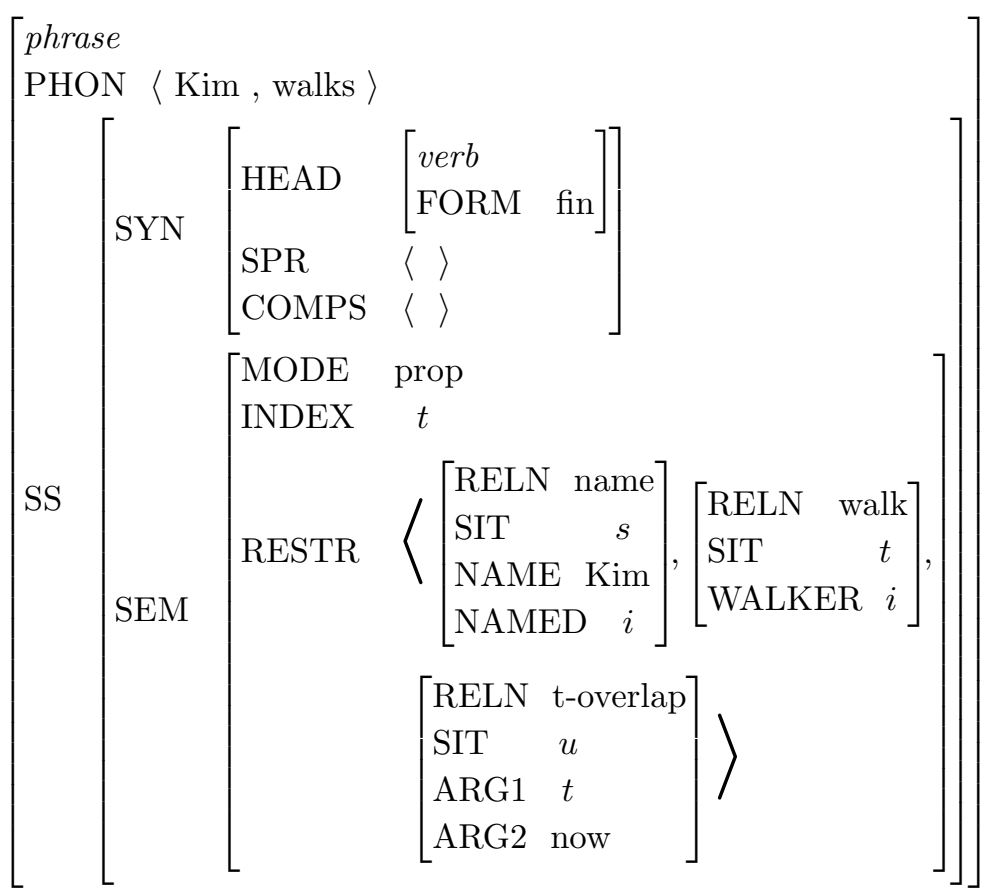


This unfamiliar representation explicitly embodies the Saussurean idea that the grammar of English defines a conventional association between the string of forms $\langle$ Kim, walks $\rangle$ and its meaning, which we have treated in terms of the proposition 'there is a walking situation $t$ in which someone named Kim is walking, where $t$ temporally overlaps the time of utterance'.

Our sign-based grammar must now specify which feature structures like (28) are well-formed English phrases and which are not. But to do so, we no longer need to formulate a definition of phrasal satisfaction. Rather, we need only specify our inventory of phrasal types as follows. ${ }^{8}$

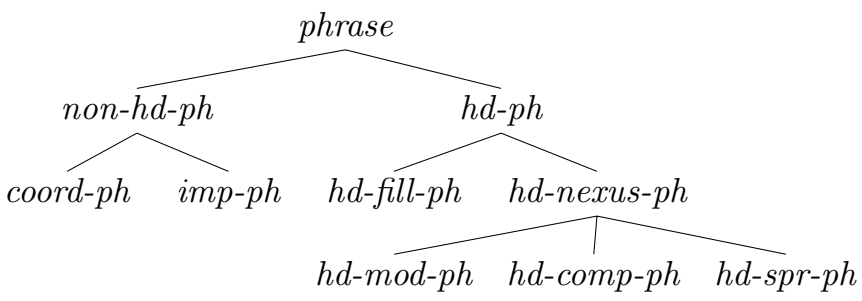

Each of the six phrasal types at the bottom of this hierarchy (i.e. those with no subtypes) corresponds to one of our old grammar rules. The types $h d-p h$ and non-hd-ph correspond to our old classification of rules as headed or nonheaded. The one truly novel element in this hierarchy is the type $h d$-nexus-ph, which includes as subtypes all the headed phrases except for the head-filler phrases. As we will see shortly, what the three subtypes of $h d$-nexus-ph have in common is that they are all subject to the GAP Principle.

Before proceeding, we need to specify (just as we have done for all types we have considered in earlier chapters) which features are appropriate for which types. This can be done as summarized in (30).

\footnotetext{
${ }^{8}$ Here we abbreviate as indicated: coord-ph coordinate-phrase hd-fill-ph head-filler-phrase hd-comp-ph head-complement-phrase hd-mod-ph head-modifier-phrase

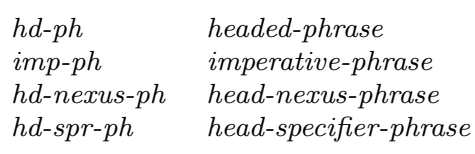


(30)

\begin{tabular}{|l|l|l|}
\hline TYPE & FEATURES/VALUE TYPES & IST \\
\hline sign & {$\left[\begin{array}{ll}\text { PHON } & \text { list }(\text { form }) \\
\text { SYNSEM } & \text { synsem-struc }\end{array}\right]$} & \\
\hline phrase & {$\left[\begin{array}{lll}\text { NHD-DTRS list }(\text { sign })\end{array}\right]$} & sign \\
\hline hd-ph & {$\left[\begin{array}{ll}\text { HD-DTR } & \text { sign }\end{array}\right]$} & phrase \\
\hline
\end{tabular}

According to the table in (30), the features PHON $^{9}$ and SYNSEM are appropriate for all signs, the feature NON-HEAD-DAUGHTERS (NHDDTRS) is appropriate for all phrases, and HEAD-DAUGHTER (HDDTR) is appropriate only for headed phrases.

This organization of types and features uses the features HD-DTR and NHD-DTRS for the embedding of one phrase within another. In so doing, we eliminate the need for a separate definition of phrasal satisfaction. But the feature structures will look even less familiar than the last one we considered. For example, the sentence Kim walks will be modelled by the feature structure described in (31):

\footnotetext{
${ }^{9}$ We have introduced the type form to classify all the possible shapes of lexemes or words. We will not explore the structure of PHON values in any further detail here.
} 
382 / Syntactic Theory: A Formal Introduction

(31)

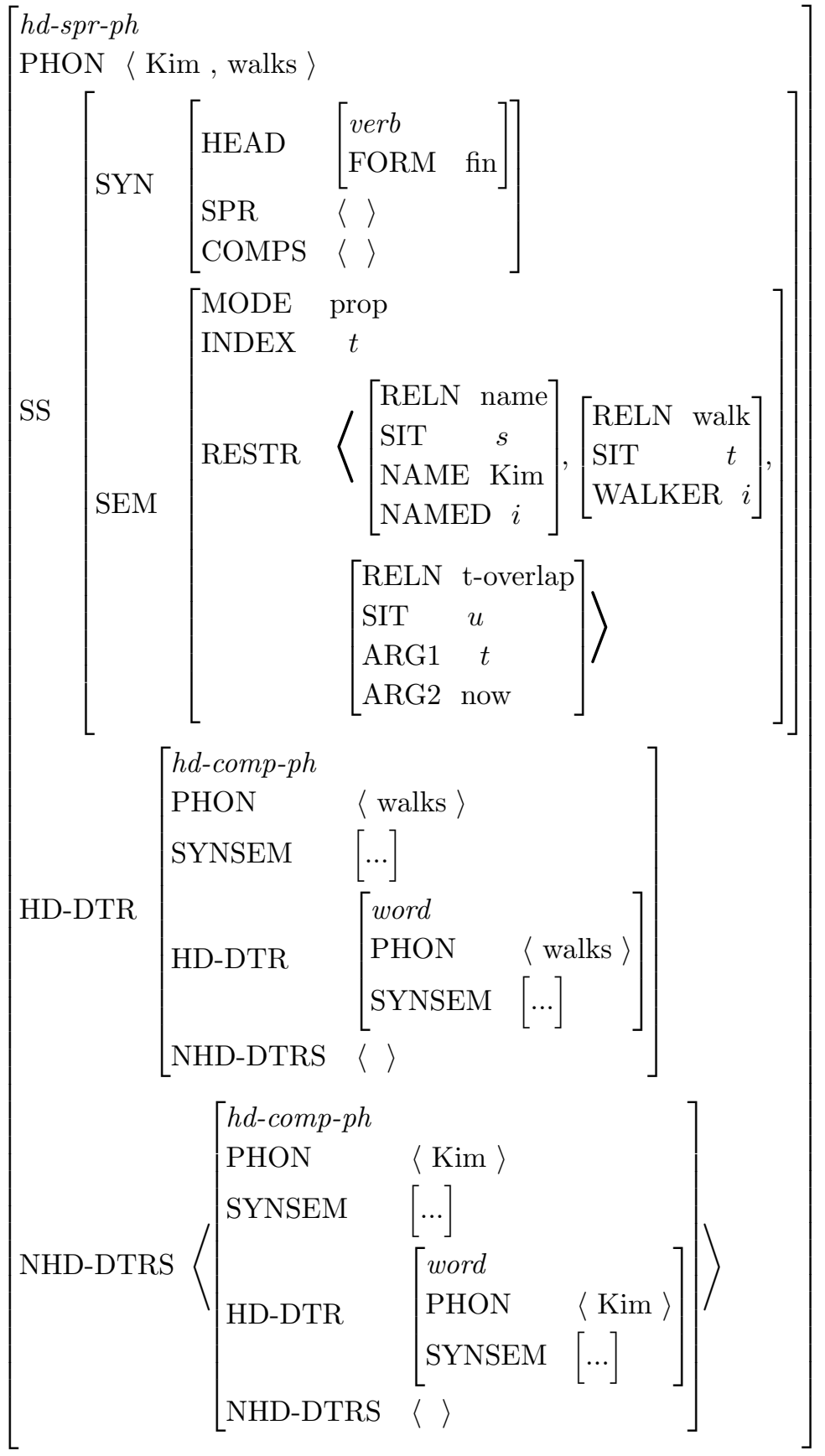


What has changed is that the embedding of one phrase (or phrase description) within another now grows from left to right, rather than topdown as in familiar tree descriptions. But a moment's reflection reveals that the same information is conveyed. The incomplete decription in (31) is true to the analyis in the text - the head of the S is the VP walks, whose head (and only) daughter is the $\mathrm{V}$ walks, and the subject of the $\mathrm{S}$ is the NP Kim, whose head (and only) daughter is the N Kim.

So how will our grammar be recast in this sign-based architecture? One aspect that will be revised is linear order. Recall that in Chapter 4 we hinted that our treatment of the ordering of constituents might not be adequate. Aside from that brief allusion, however, we have simply assumed that the sequencing of elements followed stipulations in the grammar rules or in the valence features of heads. The sign-based architecture allows us to factor linear precedence rules into a new kind of constraint that specifies the PHON values of larger phrases in terms of the PHON values of their immediate constituents. In simple cases, the PHON values of the daughters are simply added together to yield the PHON value of the mother. This is illustrated in (32), which shows the PHON values in the analysis of Kim loves Sandy. ${ }^{10}$

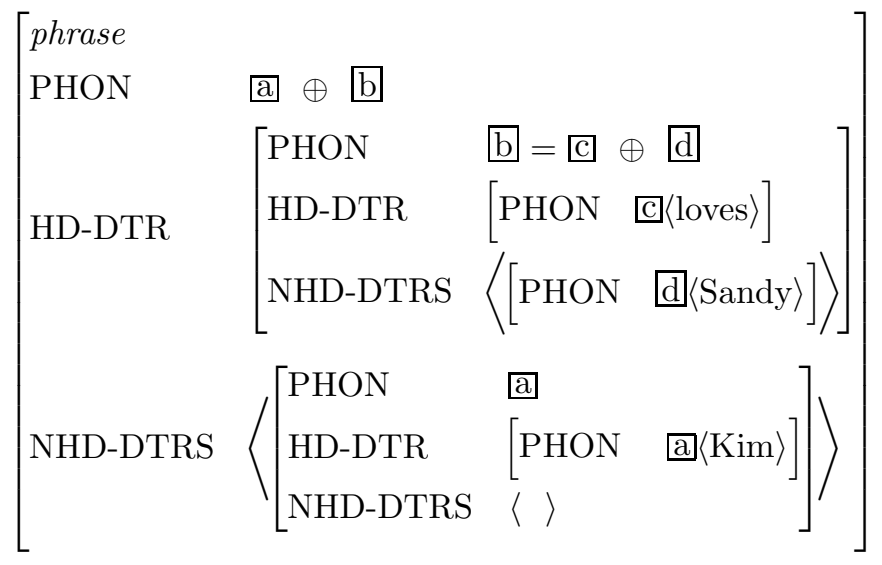

Recall that each grammar rule specifies certain constraints on the mother and daughter nodes. These constraints now become constraints on the various types of phrase - analogous to the constraints on lexical types. The Head-Complement Rule, Head-Specifier Rule and Head-Modifier

${ }^{10}$ It is possible formally to combine the PHON values of daughters using more complex operations than simple list addition. A number of languages exhibit complex word-order patterns suggesting that some such device is desirable. However, consideration of such matters is beyond the scope of this text. 
Rule, for example, correspond to the following constraints on phrasal types:

(33) a.

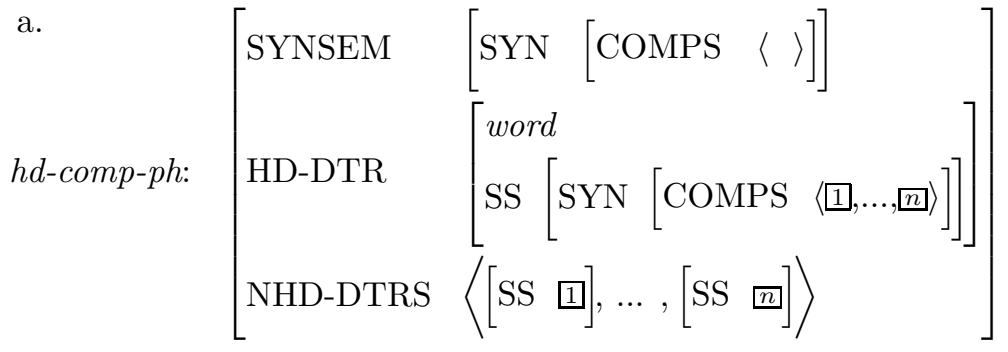

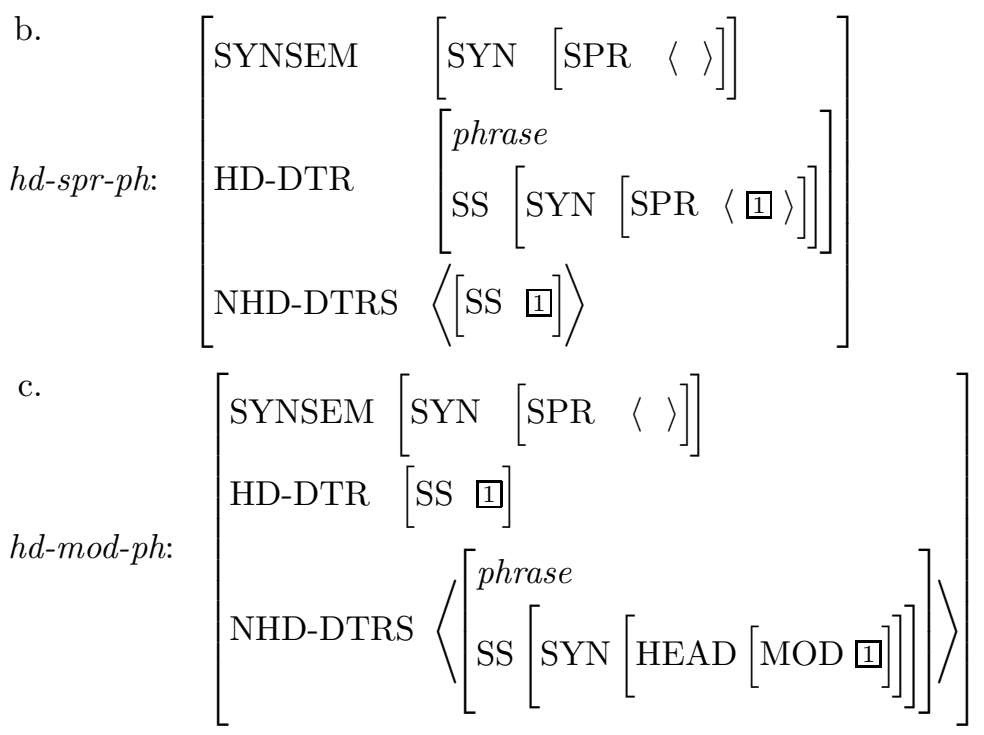

These constraints correspond directly to the constraints we wrote into our grammar rules. But because (i) the features HD-DTR and NHDDTRS introduce in their values feature structures of type phrase and (ii) these phrases themselves have HD-DTR and NHD-DTRS specifications, we effectively introduce recursion directly through the type system. The phrases that appear as daughters will themselves have daughters (obeying the appropriate constraints). Hence there is no limit on how many instances of each type of phrase can occur in a single phrase. This is exactly the effect we got in CFG by allowing recursive rules (or recursive instantiations of schematic rules) and requiring that every local subtree in a well-formed phrase structure satisfy some lexical entry or some grammar rule. But now that phrases are feature structures, the recursion is an automatic consequence of our type constraints. 
In addition, by making one daughter select for the SYNSEM value of another daughter (via COMPS, SPR, or MOD), we get the kind of locality restriction that CFG-like systems exhibit: because HD-DTR and NHD-DTRS are not features of synsem-strucs (i.e. are specified at the same level as SYNSEM), only local information can be selected for. That is, the values of COMPS, SPR, and MOD can only include SYNSEM values and hence will never be able to mention the HD-DTR or NHDDTRS of complements, specifiers, or modifiers. Consequently, under our current assumptions, we would be hard-pressed to write a grammar for a language where, for example, some verb selected for a complement that was an $\mathrm{S}$ whose VP head daughter contained an accusative NP. CFGs allow selection between sisters; our CFG-like system extended this notion of locality, allowing a kind of 'aunt' selection since the SPR value is passed up and discharged at a higher level of structure. The CFG-like system and its sign-based variant can describe a nonlocal dependency only if some further and otherwise unmotivated feature is introduced to carry the information about the accusative NP up to the VP and to the $\mathrm{S}$ it heads. Once the relevant information is encoded on the $\mathrm{S}$ node, it would become locally selectable. The predication of locality in this sense is exactly right: there are no human languages (as far as we know) where a verb selects for an $\mathrm{S}$ complement whose VP head daughter must contain an accusative NP.

What about our theory of headed phrases? The Head Feature Principle and the Valence Principle now become constraints on the type $h d-p h$. The new formulation of the HFP is given in (34).

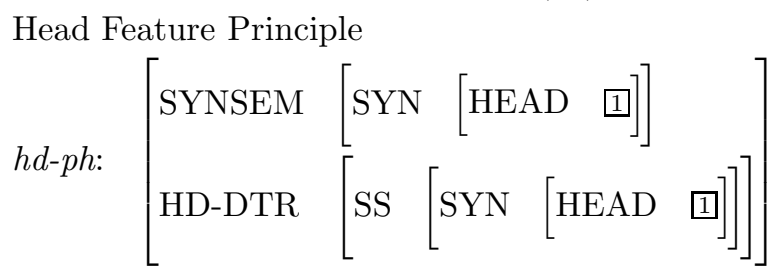

This constraint applies to all instances of the type $h d-p h$, that is, to instances of all four kinds of headed phrase.

The Valence Principle is now formulated as in (35). 
386 / Syntactic Theory: A Formal Introduction

$$
\begin{aligned}
& \text { Valence Principle } \\
& \left.h d-p h:\left[\begin{array}{lll}
\text { SYNSEM } & {\left[\text { SYN }\left[\begin{array}{ll}
\text { COMPS } & / 1 \\
\text { SPR } & / 2
\end{array}\right]\right]} \\
\text { HD-DTR } & {\left[\text { SS }\left[\text { SYN }\left[\begin{array}{lll}
\text { COMPS } & / 0 \\
\text { SPR } & / 2
\end{array}\right]\right]\right.}
\end{array}\right]\right]
\end{aligned}
$$

Note that this is a defeasible constraint (i.e. one that can be overridden), saying that a headed phrase's SPR and COMPS values are by default the same as those of the head daughter. The Valence Principle applies so long as no constraint on a subtype (e.g. hd-spr-ph or hd-comp-ph) specifically contradicts it.

The Semantic Inheritance Principle can be similarly reformulated to apply only to headed phrases:

$$
\begin{aligned}
& \text { Semantic Inheritance Principle } \\
& \left.h d-p h:\left[\begin{array}{llll}
\text { SYNSEM } & {[\text { SEM }} & {\left[\begin{array}{ll}
\text { MODE } & 1 \\
\text { INDEX } & 2
\end{array}\right]}
\end{array}\right]\right]
\end{aligned}
$$

Its effect remains exactly the same.

These three principles are now cast as type constraints, rather than as clauses in a definition of phrasal satisfaction. Because phrases are feature structures in our new conception of grammar, we achieve exactly the same result as before, but now our method of expressing lexical and phrasal constraints is uniform. The theory has gained considerable elegance and simplicity.

Consider now the GAP Principle, which we reformulate as the following nondefeasible constraint on the type $h d$-nexus-ph: 
(37) GAP Principle

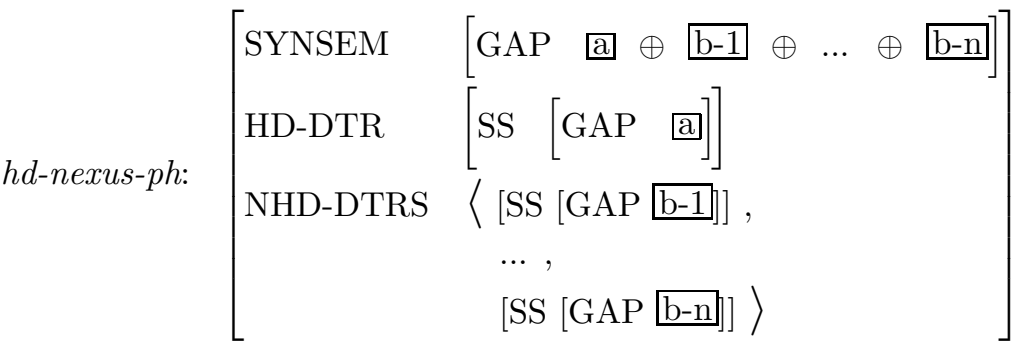

Because head-filler phrases are not a kind (subtype) of $h d$-nexus-ph, they do not obey this constraint. Rather, they are constrained as follows:

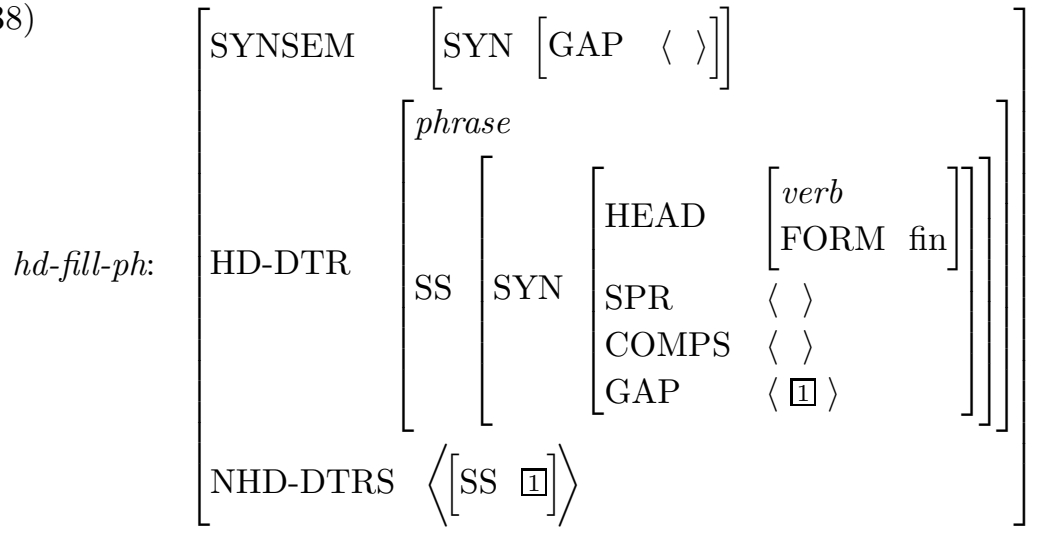

This type constraint corresponds to the feature specifications that we wrote into the Head-Filler Rule in Chapter 15. The two constraints just given guarantee that the topicalization construction is treated correctly. The head daughter of a head-filler phrase must have an empty SPR list, an empty COMPS list, and a nonempty GAP list. Hence it can be a head-specifier phrase, which in turn can have a head-complement phrase as its head daughter, as shown in (39). 
388 / Syntactic Theory: A Formal Introduction

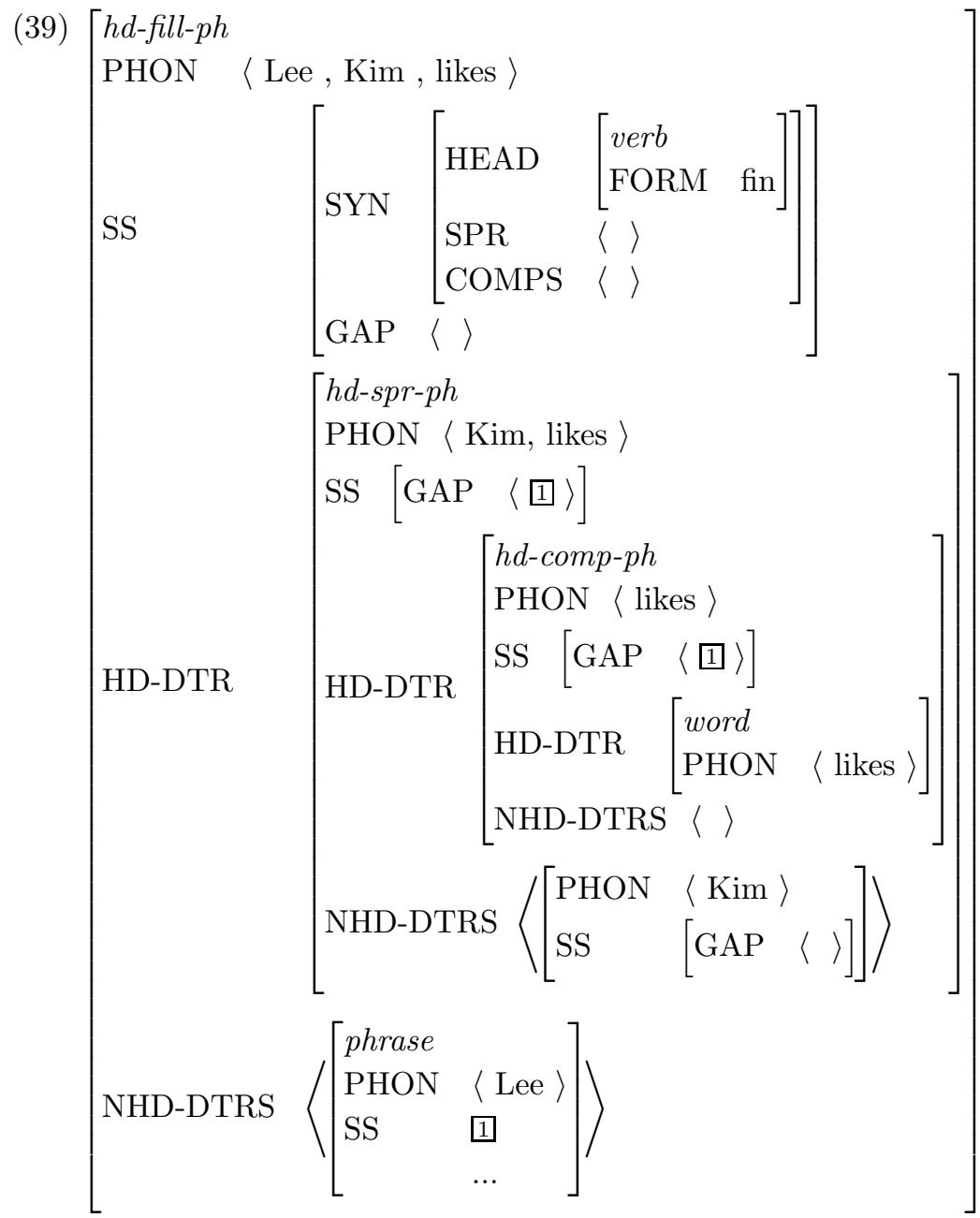

Our rules for unheaded constructions lend themselves to a similar reanalysis in terms of type constraints. The Imperative Rule corresponds to the type constraint in (40). 


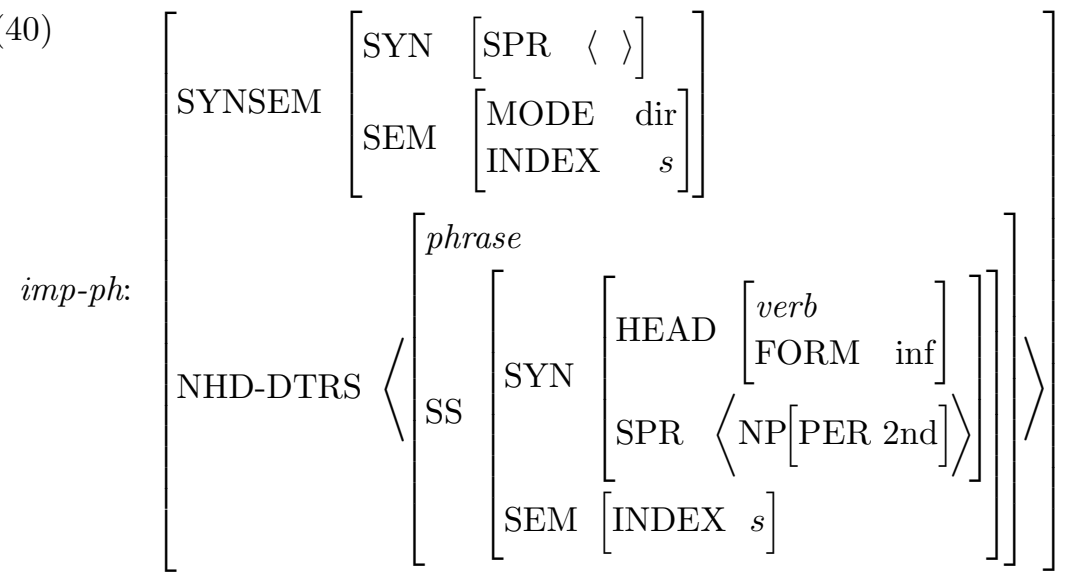

This constraint correctly requires imperative phrases to contain a nonhead daughter that is an infinitival VP whose unexpressed subject is second person.

Finally, the Coordination Rule, modified in Chapter 5, can also be recast in terms of a type constraint.

(41) coord-ph:

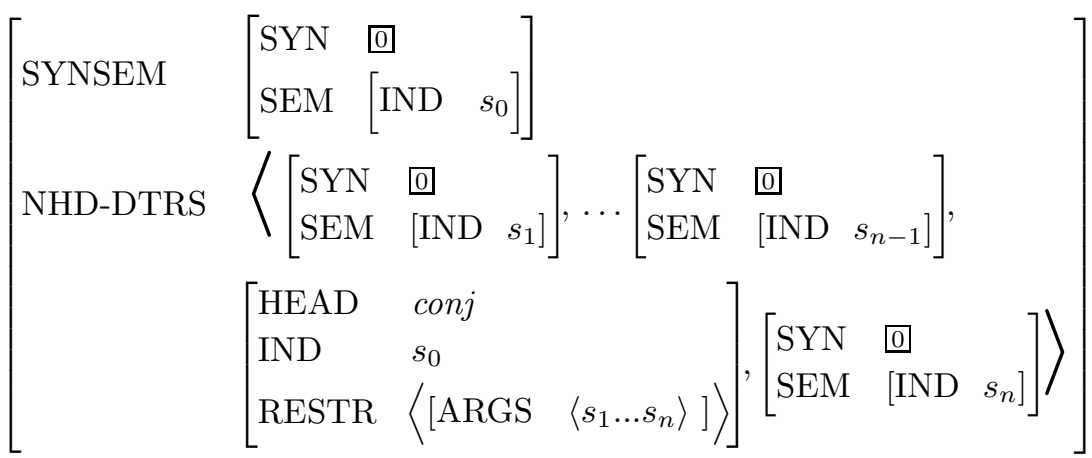

So we see that both nonheaded and headed phrases can be treated in terms of types and type constraints. The distinction between the two types of phrase is drawn precisely to restrict the domain of application of certain constraints, for example, the Head Feature Principle and the Valence Principle, so that they apply only to headed phrases. Other principles are stated as constraints on the type phrase, and hence apply to headed and nonheaded phrases alike. ${ }^{11}$

\footnotetext{
${ }^{11}$ Given the absence of any feature DAUGHTERS corresponding to all daughters
} 
Of course, we still must distinguish those phrases that can stand alone as complete (nonelliptical) utterances from all other kinds of phrases. That is, we still need something that corresponds to our earlier notion of an 'initial symbol' definition. Here we can posit a type called root and assume that all stand-alone phrases must be instances of it. Having declared such a type, we could constrain it as follows:

$$
\text { root: } \left.\left[\begin{array}{lll}
\text { phrase } & \text { SYNSEM } \left.\left[\begin{array}{lll}
\text { HEAD } & \text { verb } & \\
\text { FORM } & \text { fin }
\end{array}\right]\right] \\
\text { SPR } & \langle\rangle & \\
\text { COMPS } & \langle\rangle & \\
\text { GAP } & \langle\rangle &
\end{array}\right]\right]
$$

Finally, we must consider a further advantage of the sign-based architecture: it enables the expression of new generalizations about diverse types of phrases. Recall that in Chapter 15, we noted that topicalization was not the only example of a filler-gap dependency. Relative clauses (e.g. (the person) who Sandy thought that Pat visited) and wh-questions (e.g. Who did Sandy think that Pat visited?) are two other kinds of clauses that involve filler-gap dependencies. To put it differently, these are two subvarieties of head-filler construction. As further investigation of clauses and other kinds of constructions reveals, this same pattern of cross-cutting generalizations holds true throughout the grammar. ${ }^{12}$ There are many kinds of constructions in a language, yet they fall into groups that have many common properties.

To deal with this fundamental fact about languages, we can again exploit the notion of multiple inheritance. For example, consider the idea that phrases are partitioned into two dimensions: CLAUSALITY and HEADEDNESS. This could lead to the following (incomplete) hierarchy of phrases:

irrespective of their status as head, certain principles, e.g. the Semantic Compositionality Principle, either become somewhat cumbersome, or else must be narrowed so as to apply only to headed phrases.

${ }^{12}$ See Fillmore and Kay (forthcoming), Sag (1997), Ginzburg and Sag (forthcoming). 
(43) phrase

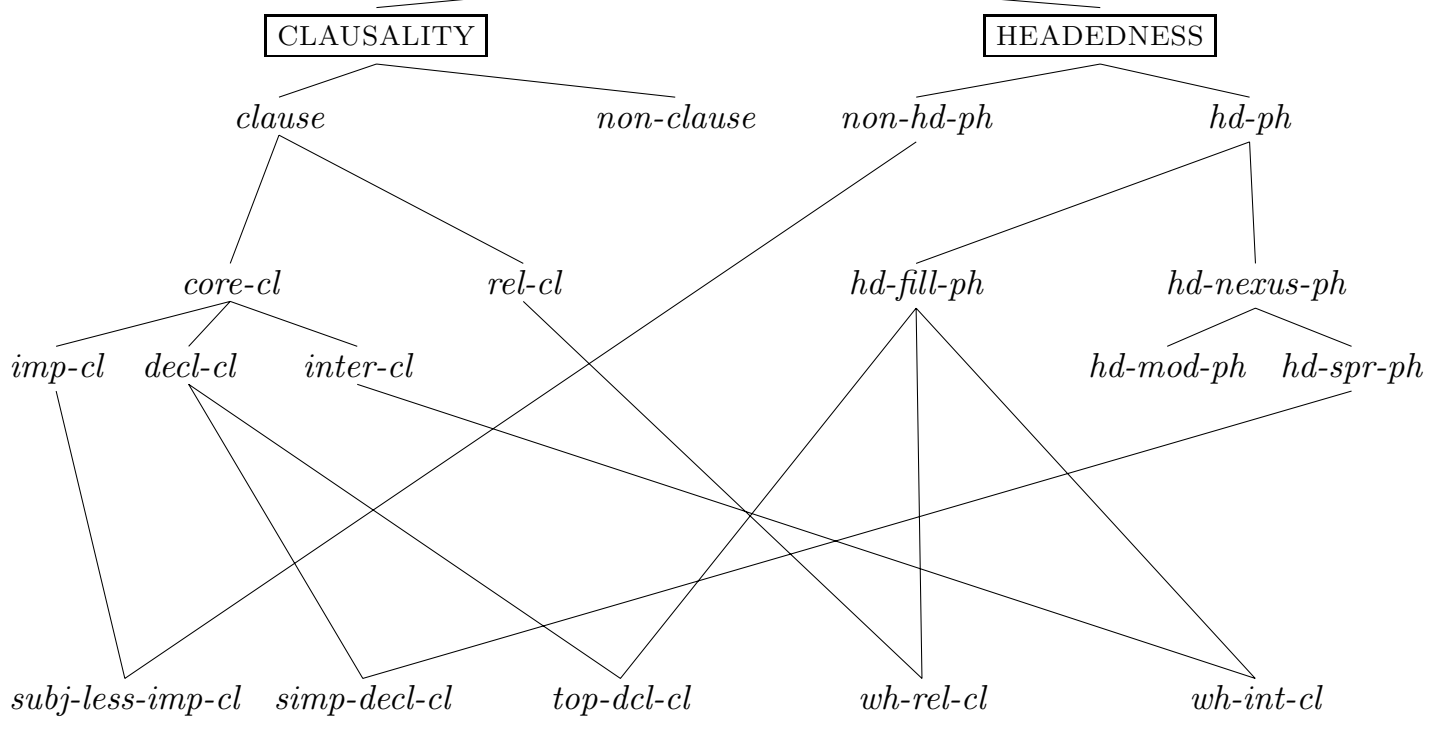

Go home! Kim left Lee, Kim likes which Pat read Who did Pat visit? 
Here we again use a number of new abbreviations:

(44) a. subj-less-imp-cl: subjectless-imperative clause

b. simp-decl-cl: simple-declarative-clause

c. top-decl-cl: topicalized-declarative-clause

d. wh-rel-cl: wh-relative-clause

e. wh-int-cl: wh-interrogative-clause

Once more, we appeal to the superordinate types to serve as the locus of constraints that cut across the various subtypes. The constraints on the type $h d-f i l l-p h$, for example, will cut across topicalization and the various wh-constructions, though each kind of head-filler construction will have properties of its own (e.g. the inversion that is characteristic of the main clause $w h$-questions). Working out the details of this factorization of grammatical constraints in terms of superordinate types is the object of ongoing inquiry in a variety of constraint-based grammatical frameworks, including HPSG, Construction Grammar, and Word (Dependency) Grammar (see Appendix B).

This completes our sketch of how we can modify our framework to embrace Saussure's conception of language as a system of signs. With the revisions to the theoretical architecture that we have outlined modelling both words and phrases as kinds of signs and using feature structures to model both these notions - a grammar becomes nothing more than a set of descriptions of typed objects. The language defined by the grammar is the set of fully resolved models - that is, the objects that satisfy the descriptions.

This view of grammar comports well with the psycholinguistic results discussed in Chapter 9, for the constructs of the grammar are now no longer clauses in a recursive definition of linguistic structures. Every construct in our theory is now a flexible unordered constraint, including lexical entries, grammar rules, and syntactic and semantic principles. We have retained the surface-oriented and strongly lexicalist character of our theory, which we argued for earlier. And our grammar is now even more strongly constraint-based than it was. We have removed the ordering among the lexical rules, the grammar rules, and the general principles that was implicit in their distinct formal statuses. Formulating all the essential components of our theory as constraints on (descriptions of) signs renders the theory compatible with a wide range of procedural implementations. Evidence like that cited in Chapter 9 suggests that distinct tasks or contexts might employ the same linguistic knowledge in divergent processes and orders. Hence the formal homogeneity introduced by means of the sign-based architecture has empirical advantages, in addition to its conceptual simplicity. 
From Rule to Sign / 393

\subsection{Conclusion}

This final chapter has suggested a major reconceptualization of the grammatical theory developed in the preceding fifteen chapters. We have not worked out all of the details here, but we have presented enough to indicate that recasting various theoretical constructs as signs would have a number of advantages, including the following:

- Parsimony Eliminating the diversity in the kinds of formal machinery used for lexical entries, grammar rules, phrase structures, and general principles of well-formedness makes the theory simpler and more elegant.

- Constructions By replacing phrase structure rules with an inheritance hierarchy of phrase types, we can capture generalizations across different kinds of constructions that we had to treat as accidental in our earlier theory. Indeed, the phrase types may provide a formal explication of the traditional notion of a 'grammatical construction' (see Goldberg (1995) and Sag (1997) for two recent discussions of this notion).

- Interfaces By including phonological, syntactic, and semantic information within the same data structures (feature structures), we clear the way for stating constraints that express the relationships among these. We have explored some of the syntax-semantics interface constraints in earlier chapters; the new architecture allows similar interactions between the phonology and the syntax and semantics, as is required in order to account for the interpretational effect of pitch accent, for example the distinct semantic/pragmatic effects of KIM loves Sandy, Kim loves SANDY, and Kim LOVES Sandy.

A natural extension would be to include in our feature structures some information about the pragmatics - that is, about appropriate contexts of use; and the sign-based architecture would allow us to state constraints on the interactions of pragmatics with semantics, syntax, and phonology.

- Process Independence By providing purely declarative descriptions of the relationships among different pieces of information, our grammar can be used in comprehension models, production models, or many kinds of computational applications.

Investigation of sign-based grammars is a robust area of current research. Our remarks in this chapter have only scratched the surface. Indeed, the same could be said on a more general level: syntactic theory 
is a multifaceted field of study that is rapidly changing; our introduction to it has been necessarily very partial and somewhat simplified.

It is impossible to predict with any confidence how the field of syntax will develop in the future. No doubt many of the specific analyses put forward in this text will eventually need to be revised - as has been the case with most specific analyses in the generative tradition over the past several decades. But each reanalysis deepens our understanding of the phenomena under investigation and lays the groundwork for more refined accounts.

Moreover, working through and comparing analyses of specific phenomena in considerable detail illustrates how syntactic theorizing is done, and hence how progress in the field comes about. Our primary goals in this text have been to introduce the reader to modes of argumentation and to teach the value of explicit formalization of hypotheses, the need to take semantics seriously in syntactic analysis, and the value of paying attention to processing considerations. If we have achieved these goals, then we have accomplished what we set out to do. 


\section{Appendix A: Summary of the Grammar}

\section{The Type Hierarchy}

The types that are used in the grammar we have developed in Chapters 3-15 are presented here in two distinct formats (as in the interim summary in Chapter 9). The first is a tree diagram indicating the hierarchical organization of all relevant types. The second is a list of particular types, with an indication of which features are appropriate for each, what type of value is appropriate for each such feature, and each type's immediate supertype (IST). 
396 / Syntactic Theory: A Formal Introduction

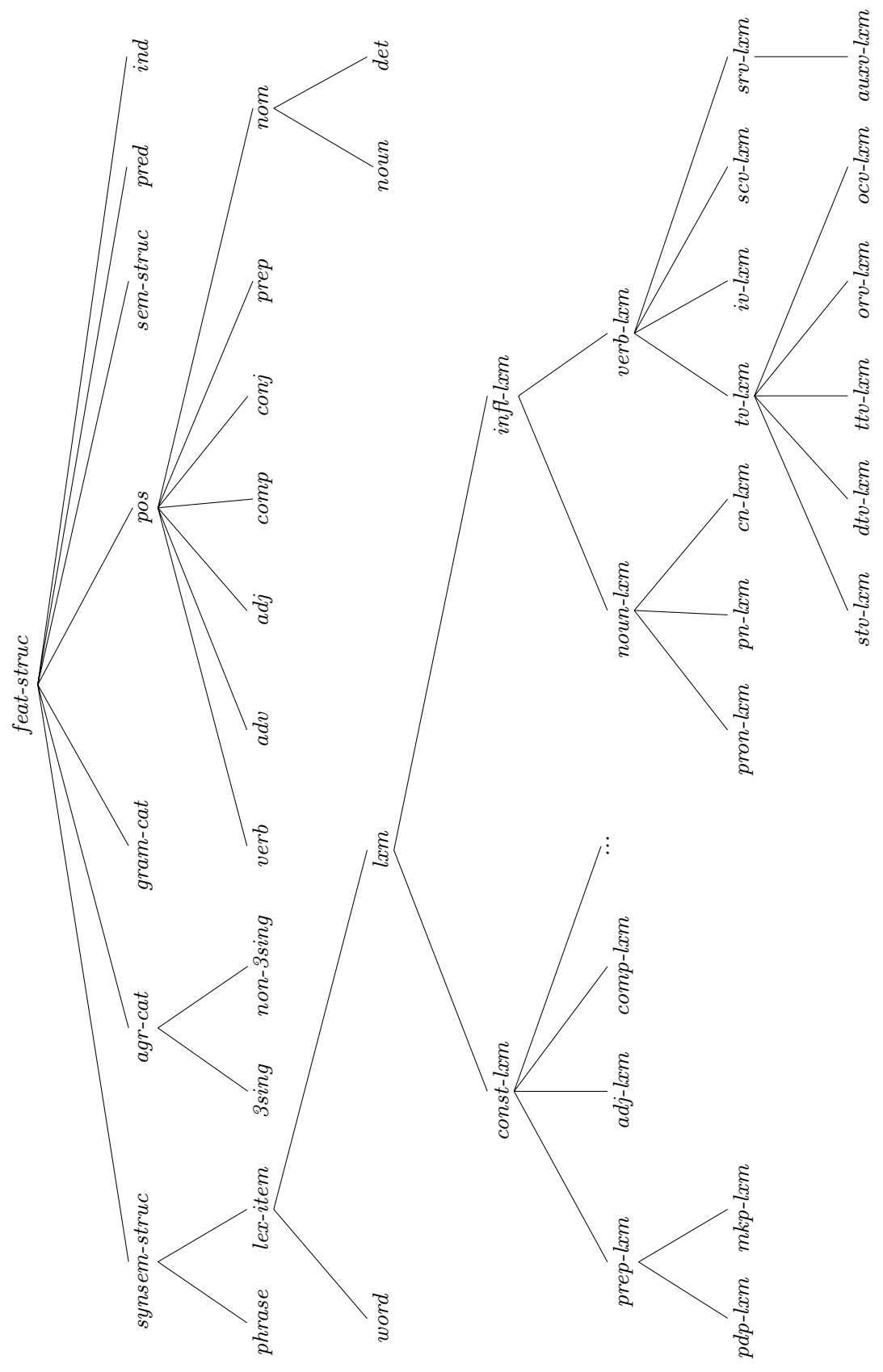




\begin{tabular}{|l|l|l|}
\hline \multicolumn{3}{|c|}{ SOME GENERAL TYPES } \\
\hline \hline TYPE & FEATURES/CONSTRAINTS & IST \\
\hline feat-struc & & feat-struc \\
\hline synsem-struc & {$\left[\begin{array}{ll}\mathrm{SYN} & \text { gram-cat } \\
\mathrm{SEM} & \text { sem-struc }\end{array}\right]$} & \\
\hline phrase & & synsem-struc \\
\hline lex-item & {$[$ ARG-ST list(synsem-struc) $]$} & synsem-struc \\
\hline word & {$\left[\begin{array}{ll}\text { lexeme } \\
\text { lex-item }\end{array}\right.$} & lex-item \\
\hline
\end{tabular}

\begin{tabular}{|c|c|c|c|c|}
\hline \multicolumn{5}{|c|}{ LEXEME TYPES } \\
\hline TYPE & $\overline{\mathrm{CONS}}$ & $\overline{\text { RAINTS }}$ & & 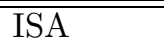 \\
\hline $\begin{array}{l}\text { infl-lxm, } \\
\text { const-lxm }\end{array}$ & & & & lexeme \\
\hline prep-lxm & {$[\mathrm{SYN}$} & HEAD & $r e p]]$ & const-lxm \\
\hline$p d p-l x m$ & {$\left[\begin{array}{l}\mathrm{SYN} \\
\mathrm{ARG}\end{array}\right.$} & $\begin{array}{l}{[\mathrm{HEAD}} \\
\mathrm{SPR} \\
\mathrm{T}<\mathrm{NP}\end{array}$ & 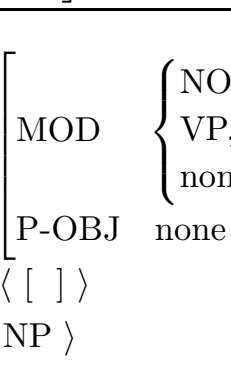 & prep-lxm \\
\hline$m k p-l x m$ & {$\left[\begin{array}{l}\mathrm{SYN} \\
\mathrm{ARG}\end{array}\right.$} & 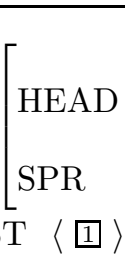 & $\begin{array}{l}{\left[\begin{array}{ll}\text { MOD } & \text { none } \\
\text { P-OBJ } & 1\end{array}\right]} \\
\langle\rangle\end{array}$ & prep-lxm \\
\hline
\end{tabular}


398 / Syntactic Theory: A Formal Introduction

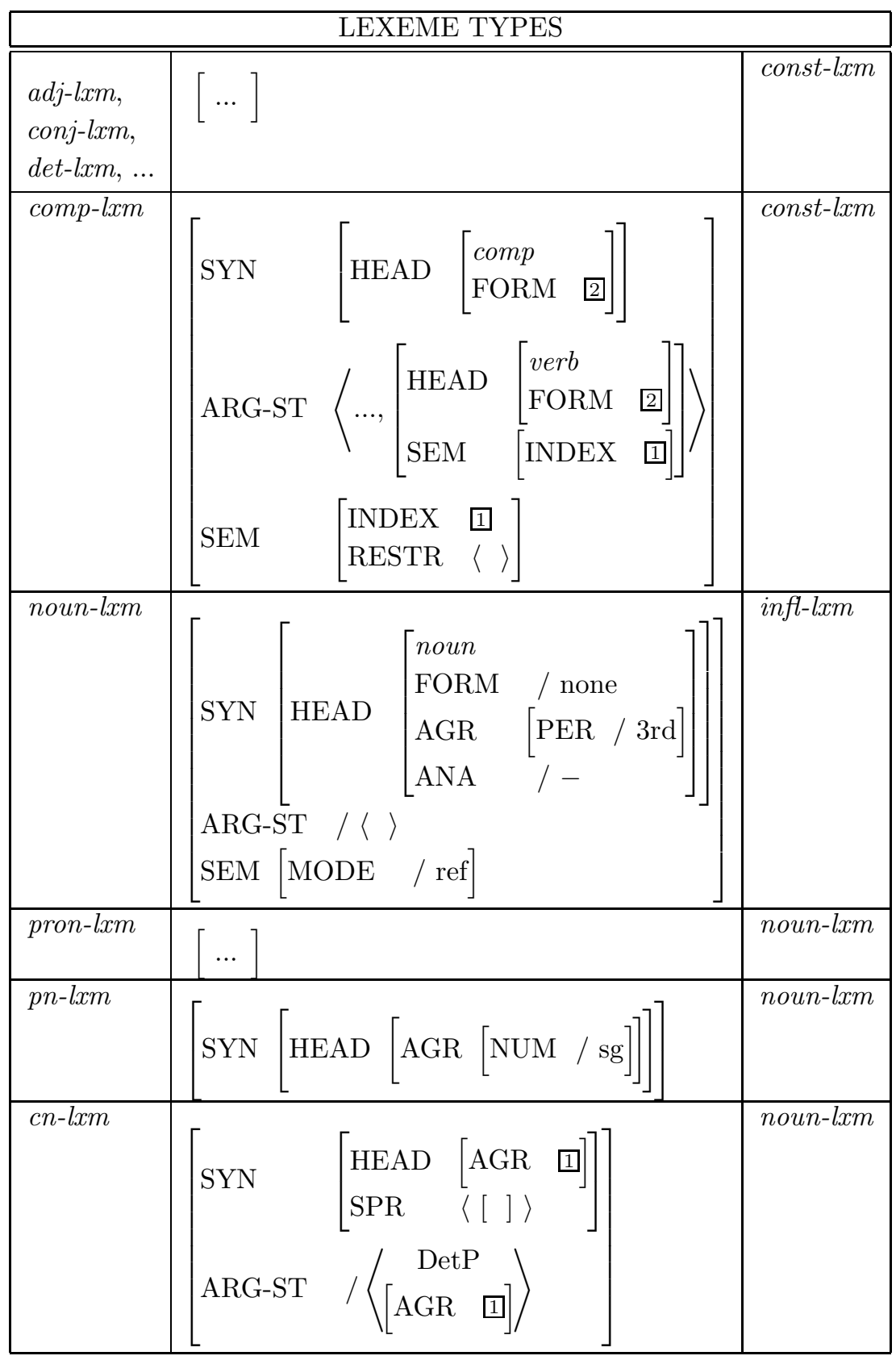




\begin{tabular}{|c|c|c|}
\hline \multicolumn{3}{|c|}{ LEXEME TYPES } \\
\hline TYPE & CONSTRAINTS & $\overline{\mathrm{ISA}}$ \\
\hline verb-lxm & {$\left[\begin{array}{llll}\text { SYN } & {\left[\begin{array}{lll}\text { HEAD } & \left.\begin{array}{ll}\text { verb } & \\
\text { AUX } & /- \\
\text { NEG } & /-\end{array}\right] \\
\text { SPR } & \langle[]\rangle & \end{array}\right]} \\
\text { ARG-ST } & /\langle\text { NP, .. }\rangle \\
\text { SEM } & {[\text { MODE }} & \text { prop }]\end{array}\right.$} & infl-lxm \\
\hline$i v-l x m$ & ARG-ST $\langle[]\rangle]$ & verb-lxm \\
\hline piv-lxm & ARG-ST $\langle[], \mathrm{PP}\rangle]$ & $i v-l x m$ \\
\hline tv-lxm & ARG-ST $\langle[], \mathrm{NP}, \ldots\rangle]$ & verb-lxm \\
\hline stv-lxm & ARG-ST $\langle[],[]\rangle]$ & $t v-l x m$ \\
\hline dtv-lxm & ARG-ST $\langle[],[], \mathrm{NP}\rangle]$ & $t v-l x m$ \\
\hline ptv-lxm & ARG-ST $\langle[],[], \mathrm{PP}\rangle]$ & tv-lxm \\
\hline srv-lxm & $\left.{ }^{-} \mathrm{ARG}-\mathrm{ST}\left\langle\square,\left[\begin{array}{ll}\operatorname{SPR} & \langle\square\rangle\end{array}\right]\right\rangle\right]$ & verb-lxm \\
\hline scv-lxm & $\left.\operatorname{ARG}-\mathrm{ST}\left\langle\mathrm{NP}_{i},\left[\begin{array}{ll}\mathrm{SPR} & \left\langle\mathrm{NP}_{i}\right\rangle\end{array}\right]\right\rangle\right]$ & verb-lxm \\
\hline orv-lxm & 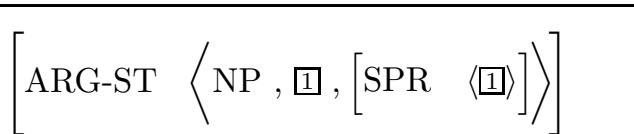 & $t v-l x m$ \\
\hline ocv-lxm & 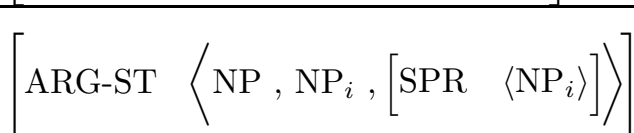 & $t v-l x m$ \\
\hline auxv-lxm & $\left.\left.\left[\begin{array}{llll}\mathrm{SYN} & {[\mathrm{HEAD}} & {[\mathrm{AUX}} & +\end{array}\right]\right]\right]$ & srv-lxm \\
\hline
\end{tabular}


400 / Syntactic Theory: A Formal Introduction

\begin{tabular}{|c|c|c|}
\hline \multicolumn{3}{|c|}{ OTHER GRAMMATICAL TYPES } \\
\hline$\overline{\text { TYPE }}$ & "FEATURES/CONSTRAINTS & 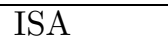 \\
\hline gram-cat & {$\left[\begin{array}{ll}\text { HEAD } & \text { pos } \\
\text { COMPS } & \text { list }(\text { synsem-struc }) \\
\text { SPR } & \text { list }(\text { synsem-struc }) \\
\text { GAP } & \text { list }(\text { synsem-struc })\end{array}\right]$} & feat-struc \\
\hline pos & $\begin{array}{ll}\text { FORM } & \{\text { fin, to }, \ldots\} \\
\text { PRED } & \{+,-\}\end{array}$ & feat-struc \\
\hline verb & $\left.\begin{array}{ll}\operatorname{AUX} & \{+,-\} \\
\mathrm{NEG} & \{+,-\}\end{array}\right]$ & pos \\
\hline prep & $\left.\begin{array}{ll}{[\text { P-OBJ }} & \{\text { synsem-struc }, \text { none }\} \\
\text { MOD } & \{\text { NOM }, \mathrm{VP}, \text { none }, . . .\end{array}\right\}$ & pos \\
\hline nominal & AGR $\quad$ agr-cat $]$ & pos \\
\hline noun & {$\left[\begin{array}{ll}\text { CASE } & \{\text { nom, acc }\} \\
\text { ANA } & \{+,--\}\end{array}\right]$} & nominal \\
\hline det & $\operatorname{COUNT}\{+,-\}]$ & nominal \\
\hline$a d v$ & $\operatorname{MOD}\{\mathrm{VP}$, none,...$\}]$ & pos \\
\hline$a d j$ & {$[\operatorname{MOD}\{$ NOM, none $\}]$} & $\overline{p o s}$ \\
\hline conj & & pos \\
\hline
\end{tabular}




\begin{tabular}{|c|c|c|}
\hline \multicolumn{3}{|c|}{ OTHER GRAMMATICAL TYPES } \\
\hline TYPE & FEATURES/CONSTRAINTS & $\overline{\mathrm{ISA}}$ \\
\hline agr-cat & $\begin{array}{ll}\text { PER } & \{\text { 1st, 2nd, 3rd }\} \\
\text { NUM } & \{\mathrm{sg}, \mathrm{pl}\}\end{array}$ & feat-struc \\
\hline $3 \operatorname{sing}$ & {$\left[\begin{array}{ll}\text { PER } & \text { 3rd } \\
\text { NUM } & \text { sg } \\
\text { GEND } & \{\text { masc, fem, neut }\}\end{array}\right]$} & agr-cat \\
\hline non-3sing & & $a g r$-cat \\
\hline sem-struc & $\begin{array}{ll}\text { MODE } & \{\text { prop, ques, dir, ref }\} \\
\text { INDEX } & \{\text { index }, \text { none }\} \\
\text { RESTR } & \operatorname{list}(\text { predication })\end{array}$ & feat-struc \\
\hline predication & $\underset{\ldots}{\operatorname{RELN}}\{$ love,walk,...\}\}] & feat-struc \\
\hline index & & feat-struc \\
\hline
\end{tabular}

\section{The Basic Lexicon}

Here are some sample lexical entries that are part of the basic lexicon. '...' means 'this is something we haven't dealt with but a complete grammar would have to'.

\section{Nouns}

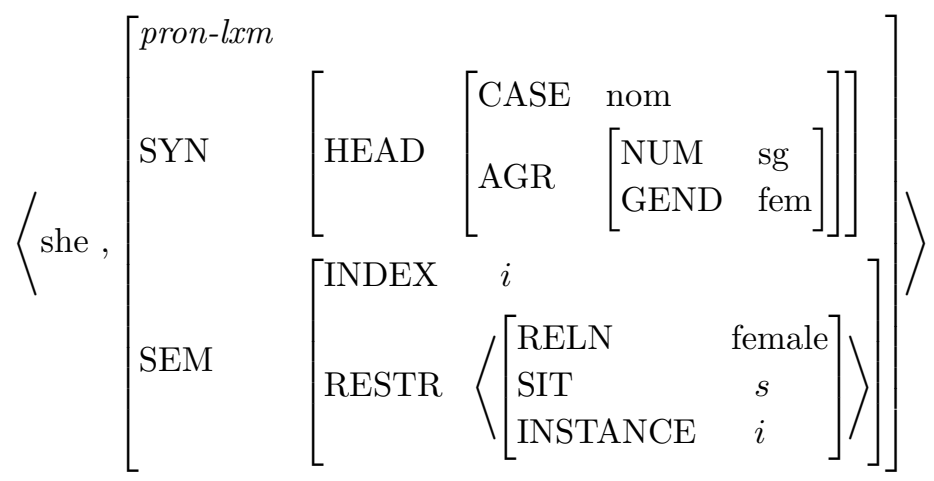


402 / Syntactic Theory: A Formal Introduction

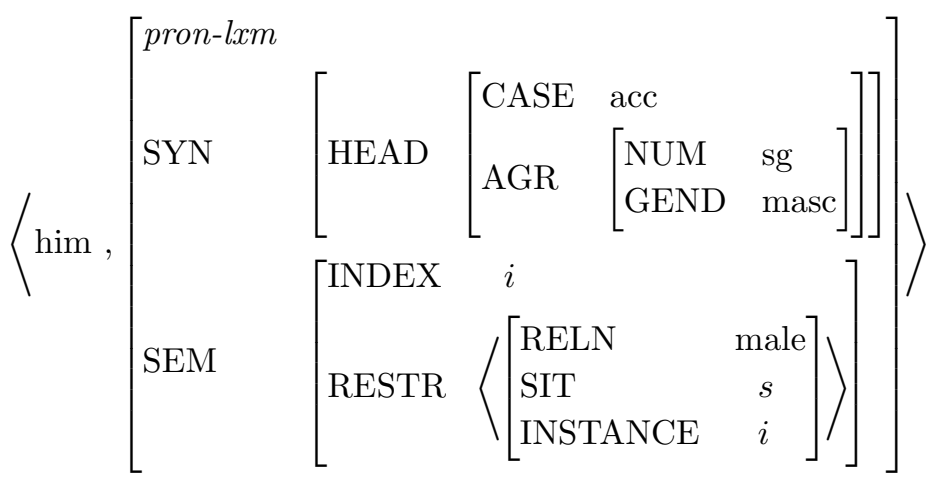

(47)

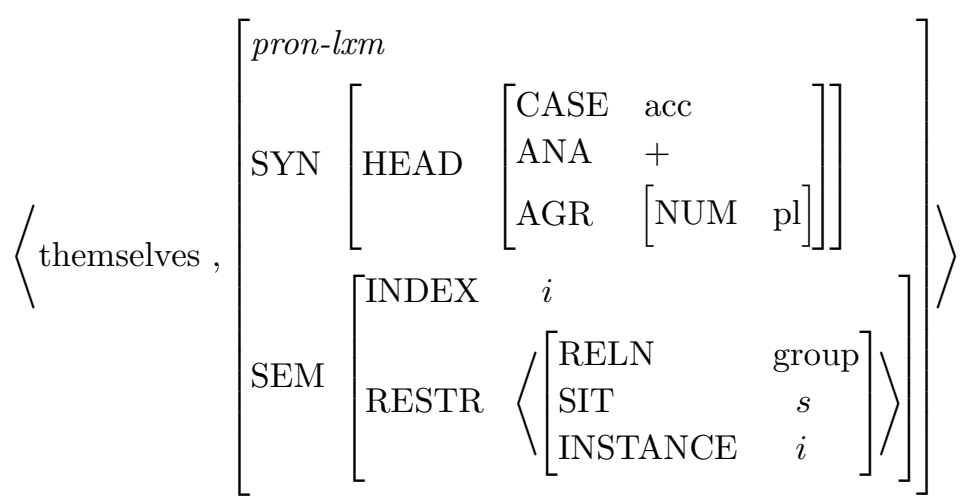

(48)

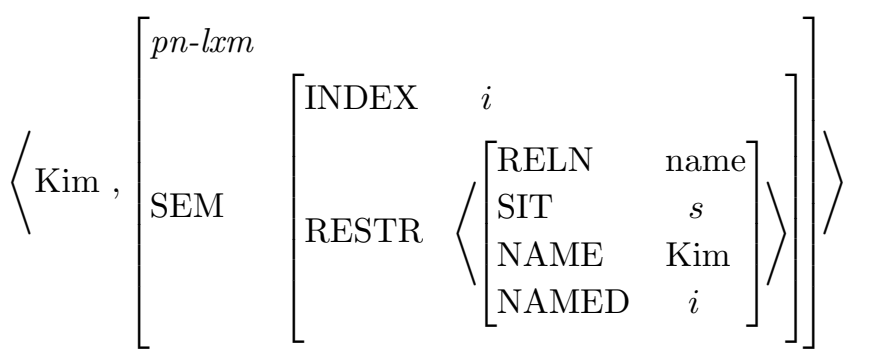

(49)

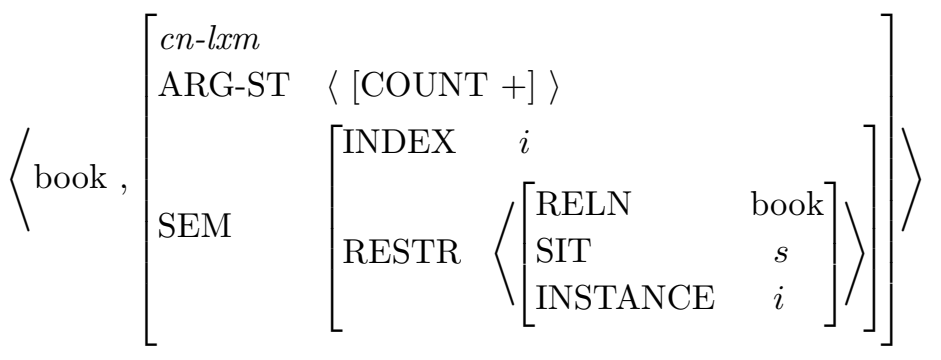


(50)

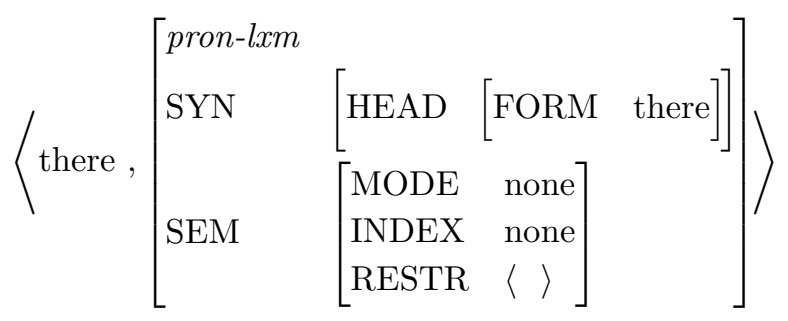
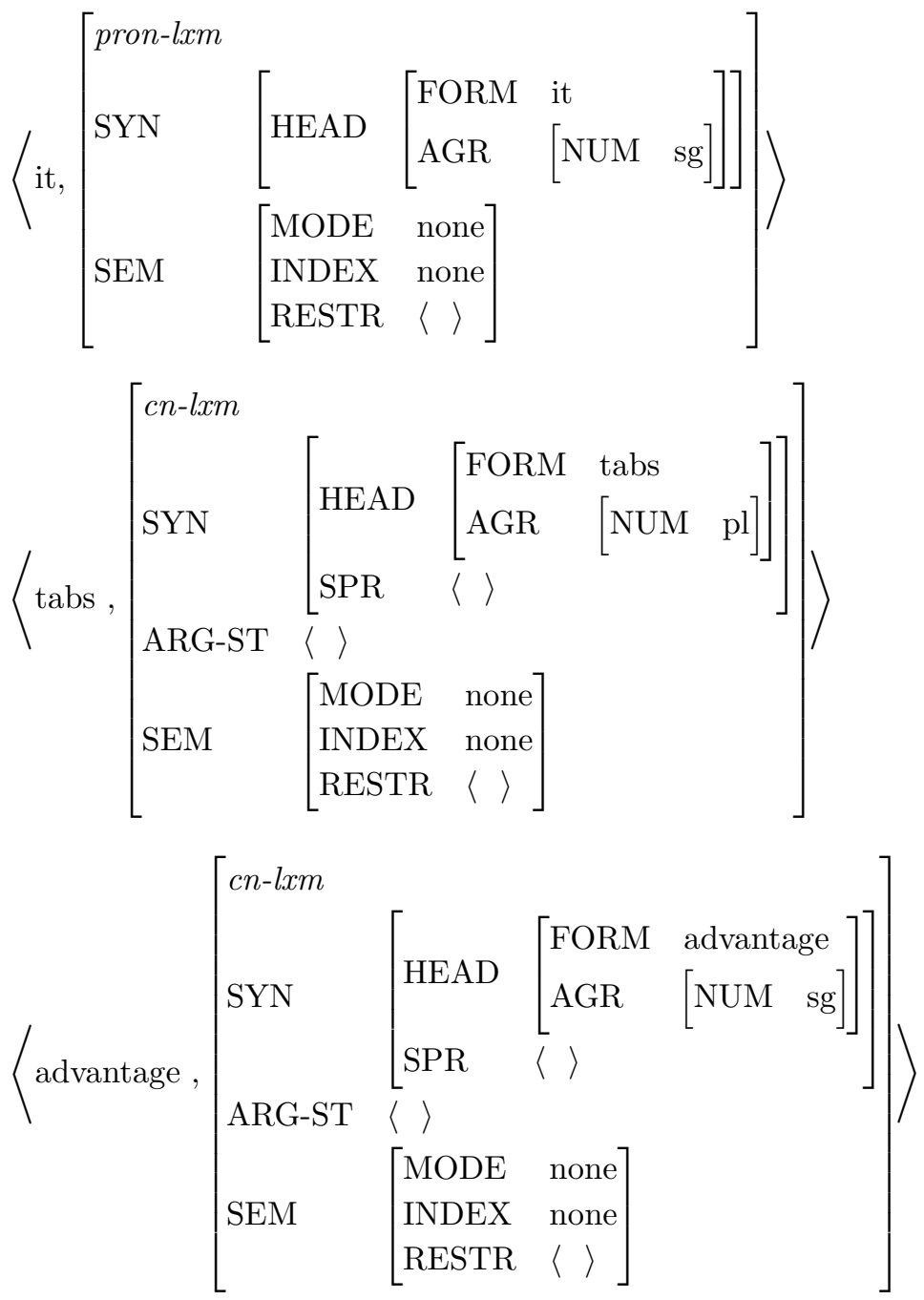
404 / Syntactic Theory: A Formal Introduction

Verbs

(54)

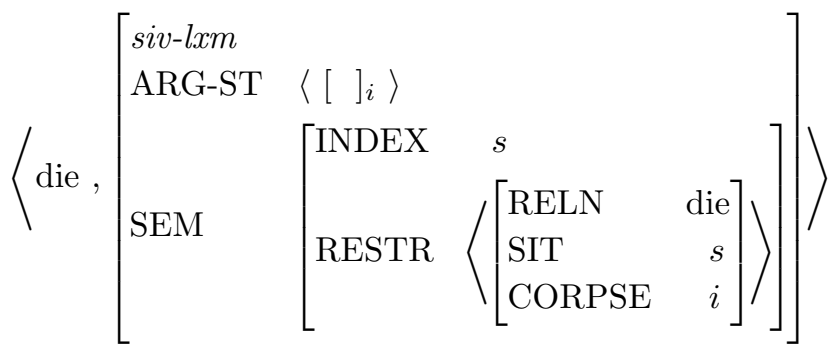

(55)

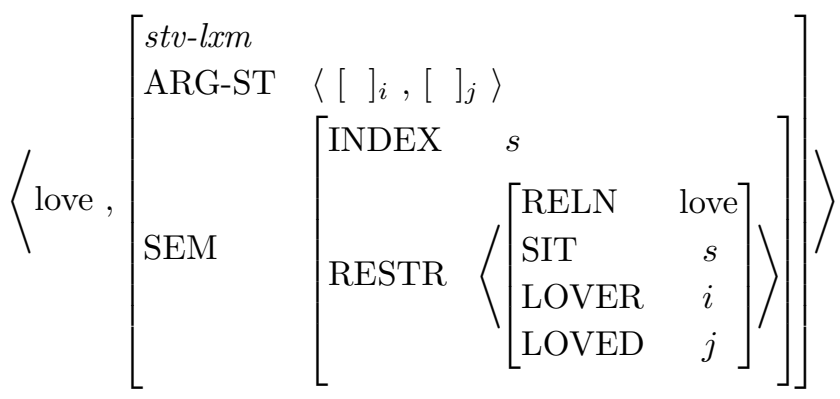

(56)

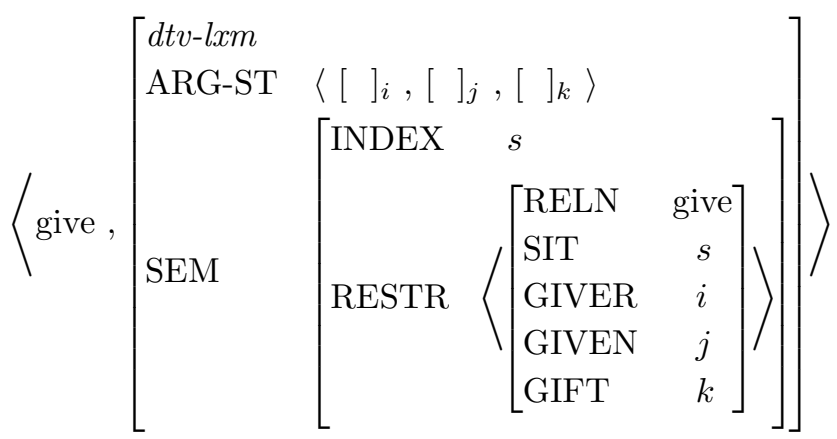

(57)

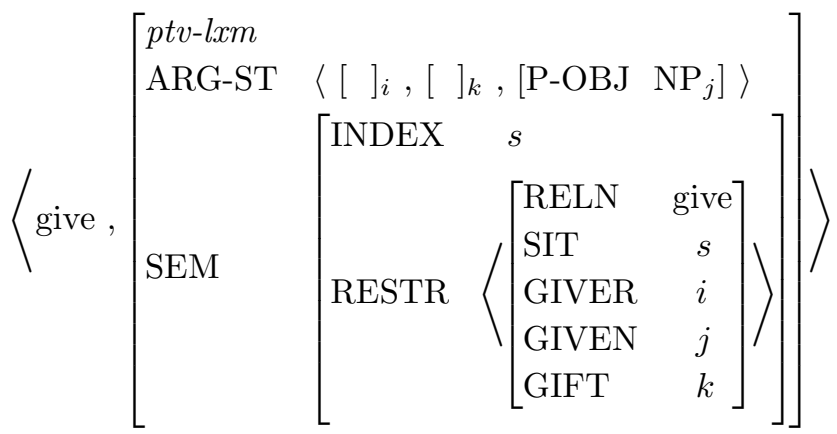


Appendix A / 405

(58)

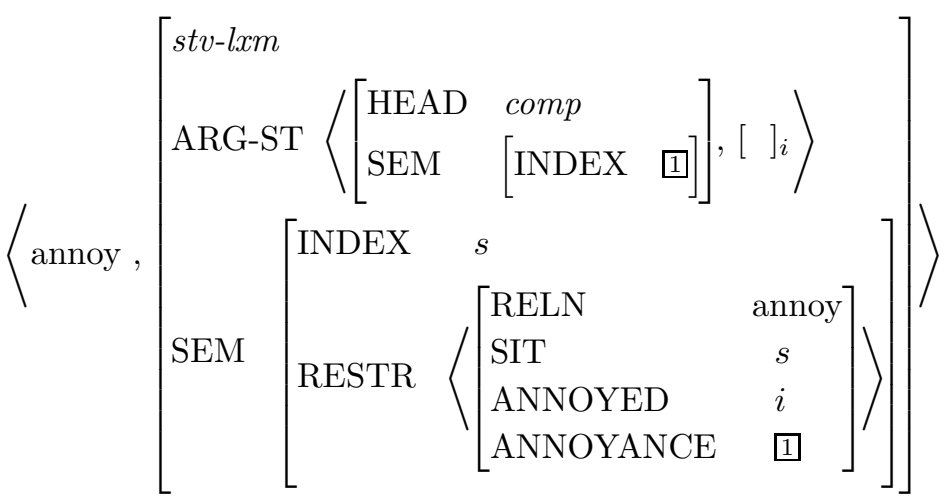

(59)

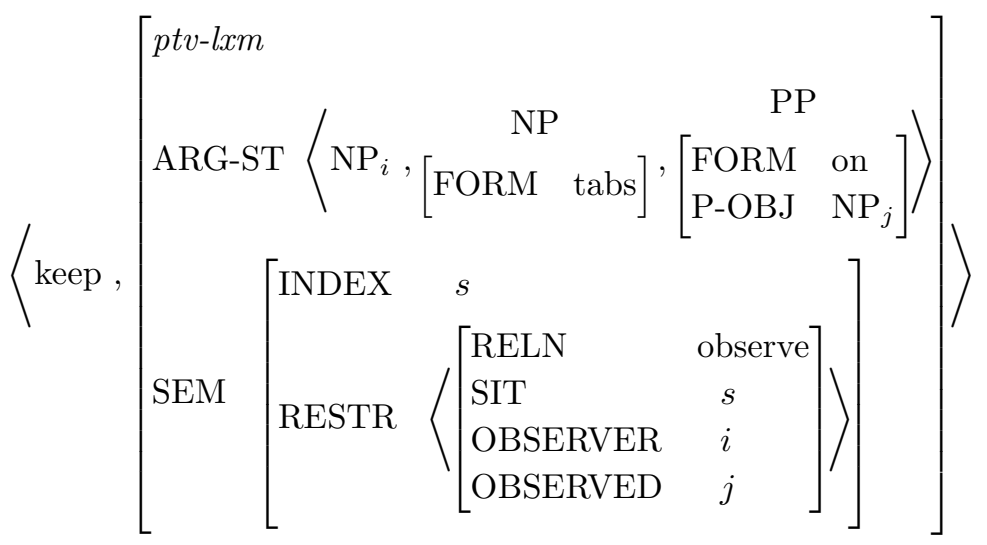

(60)

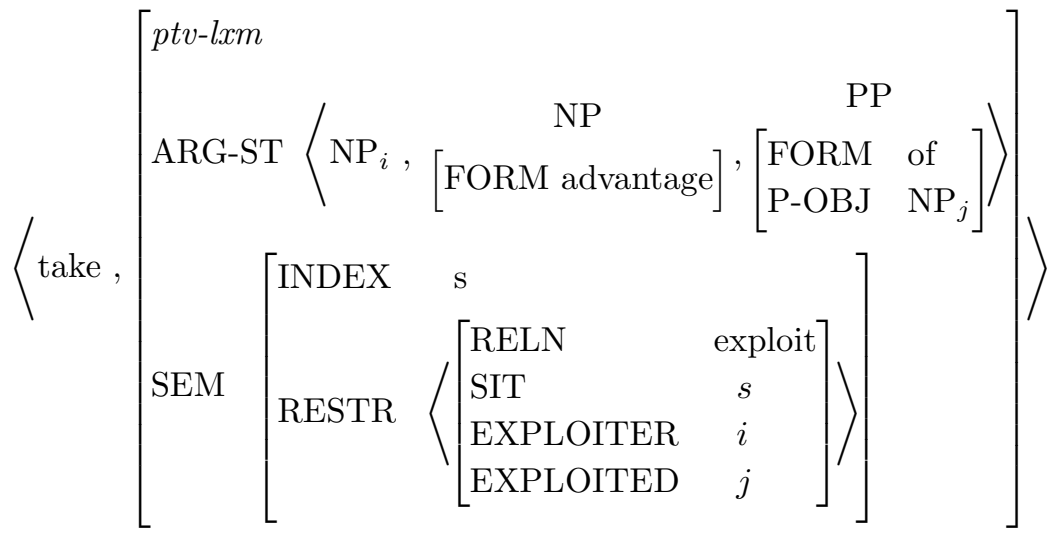


406 / Syntactic Theory: A Formal Introduction

(61)

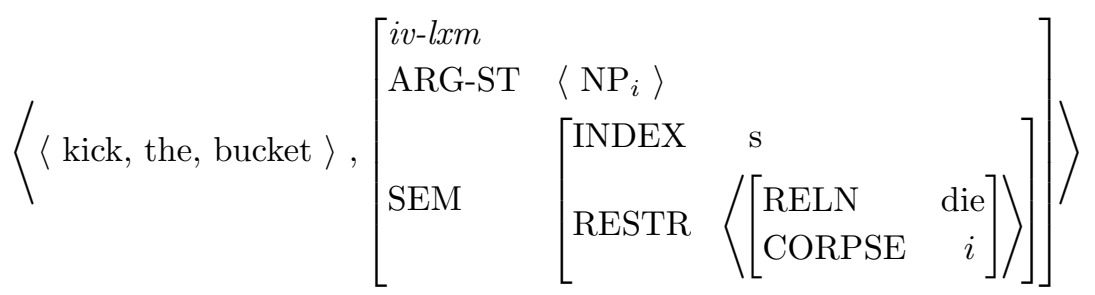

(62)

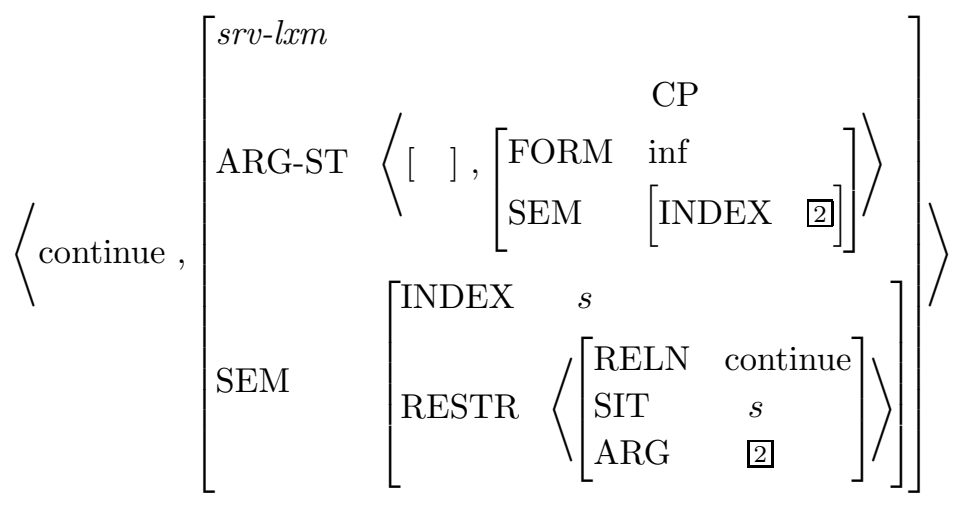

(63)

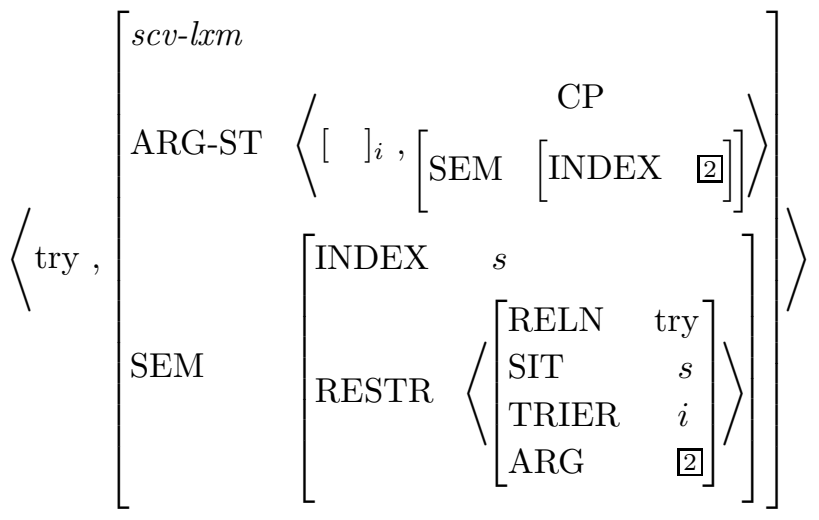


Appendix A / 407

(64) a

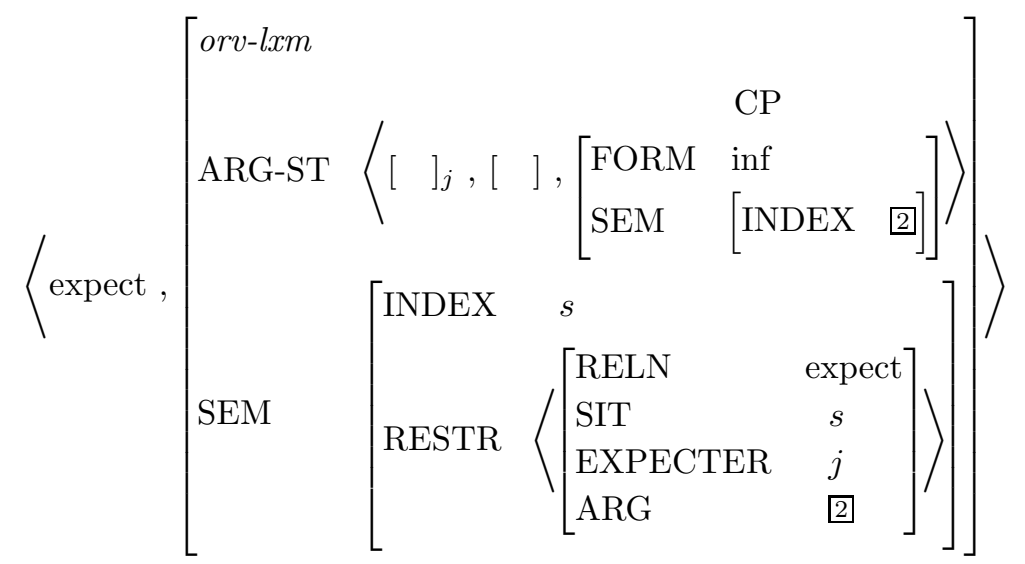

b.

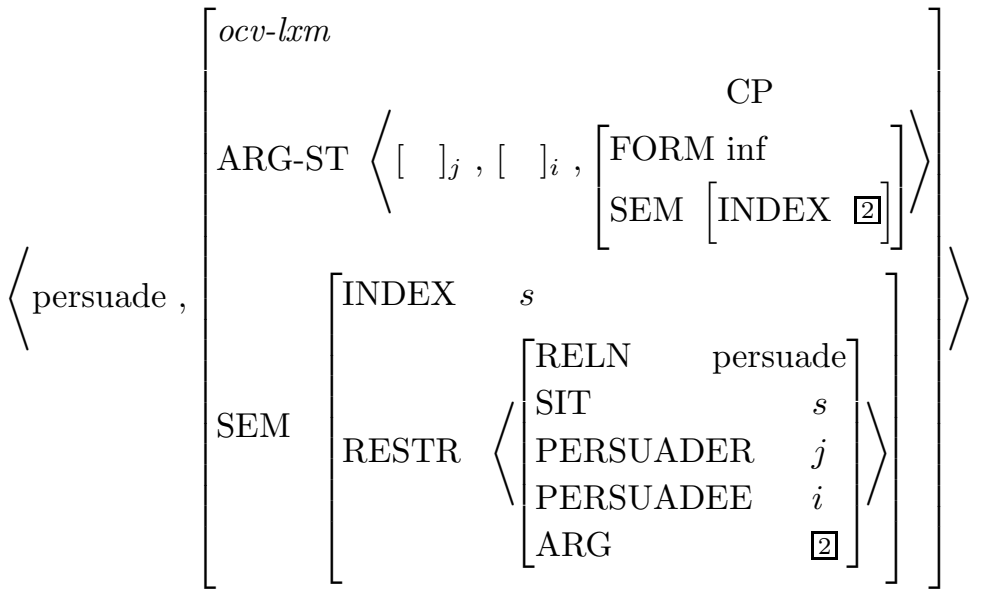


408 / Syntactic Theory: A Formal Introduction

(65)

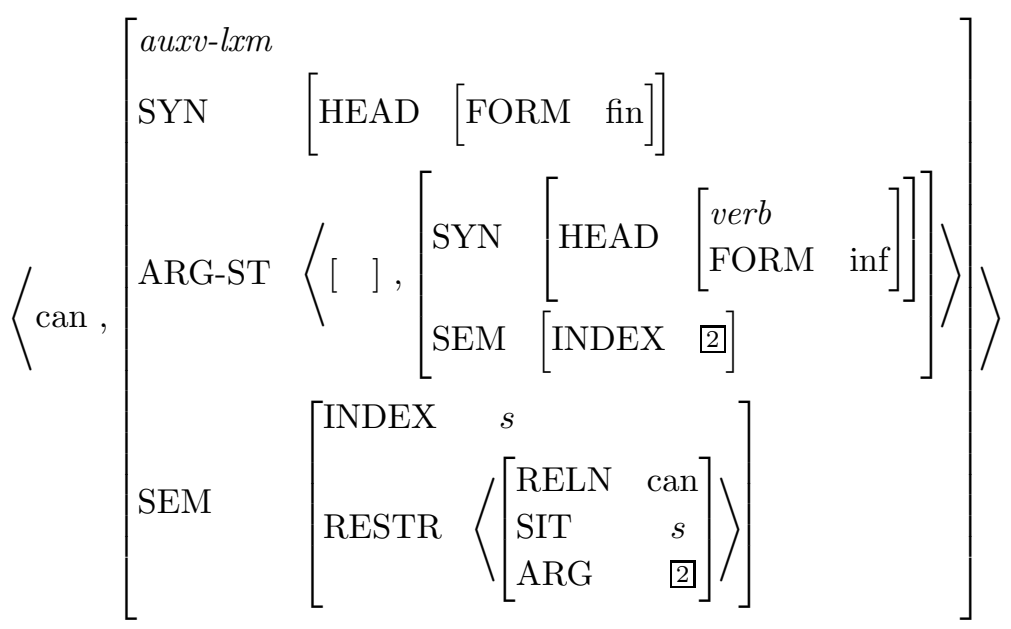

(66)

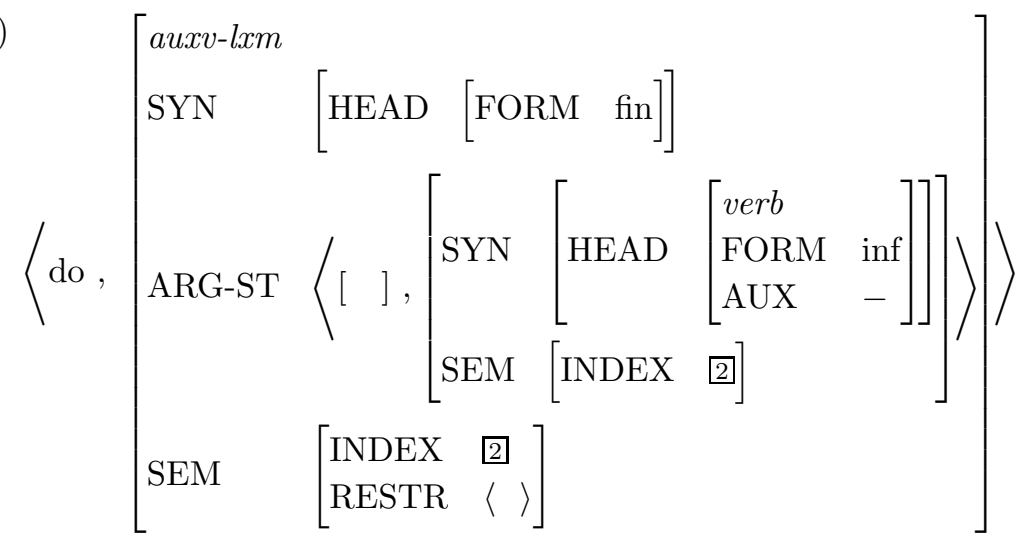

(67)

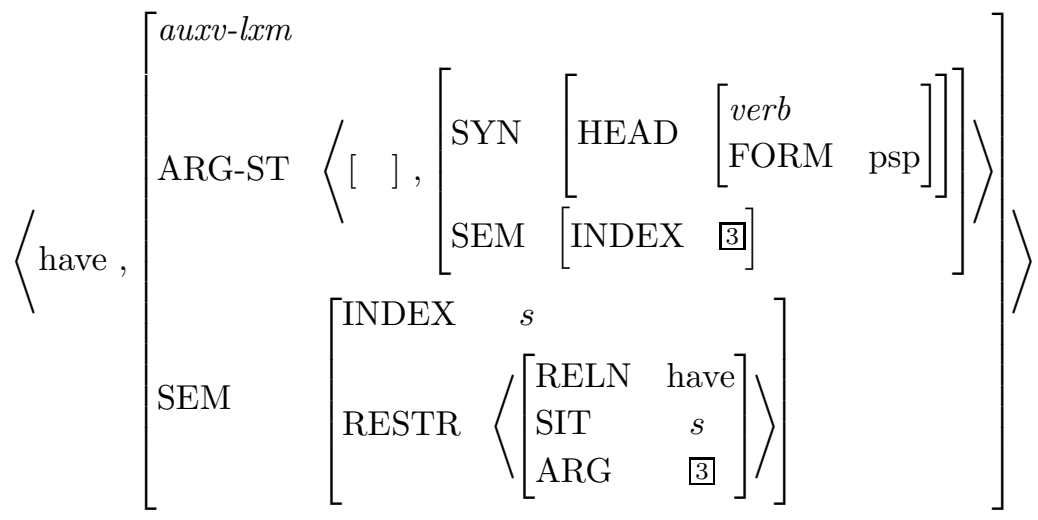


(68)

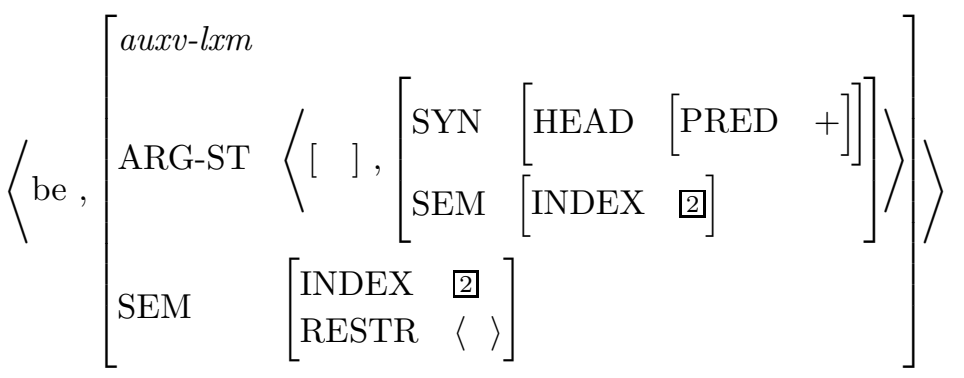

(69)

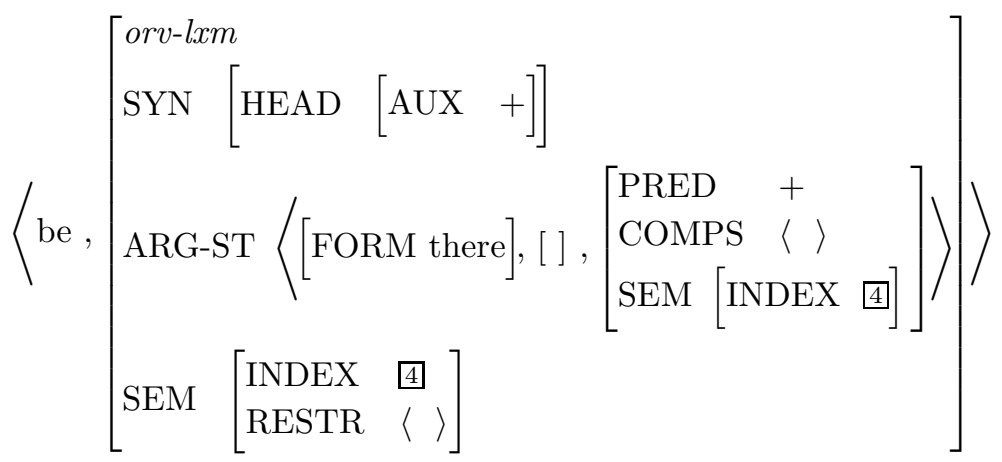

Miscellaneous

(70)

$$
\left\langle\text { the },\left[\begin{array}{ll}
\text { det-lxm } & \\
\text { ARG-ST } & \langle\rangle \\
\text { SEM } & {[. . .]}
\end{array}\right]\right\rangle
$$

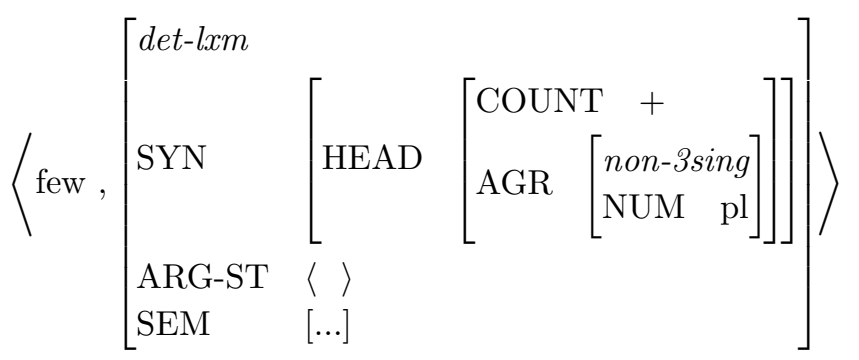

(72)

$$
\left\langle\text { 's },\left[\begin{array}{ll}
\text { det-lxm } & \\
\text { ARG-ST } & \langle\mathrm{NP}\rangle \\
\text { SEM } & {[\ldots]}
\end{array}\right]\right\rangle
$$

(73) $\left\langle\right.$ to,$\left.\left[\begin{array}{c}m k p-l x m \\ \cdots\end{array}\right]\right\rangle$ 
410 / Syntactic Theory: A Formal Introduction

(74)

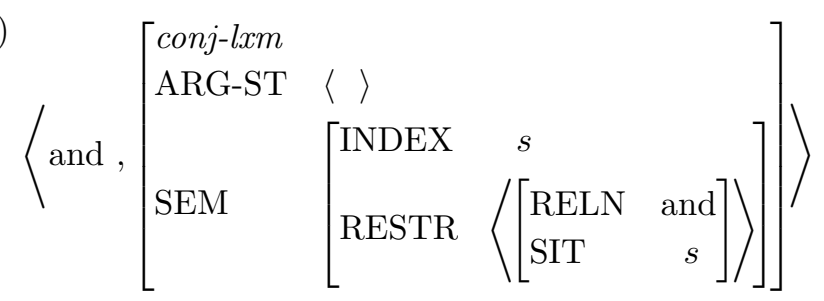

(75)

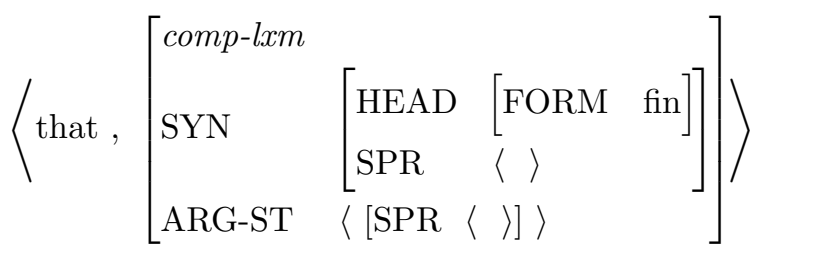

(76)

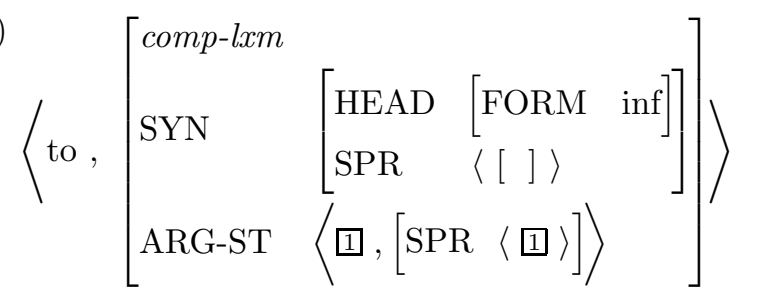

(77)

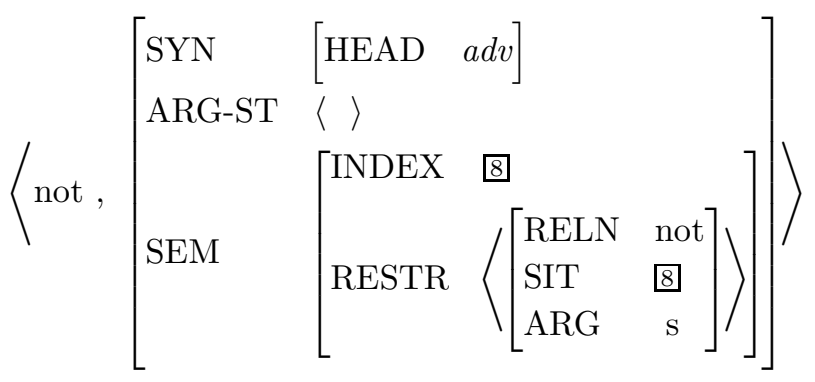




\section{The Grammar Rules}

- Head-Complement Rule

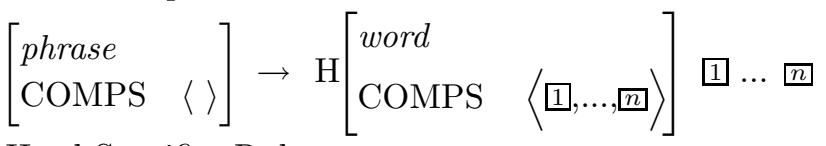

- Head-Specifier Rule

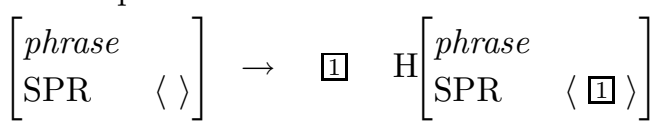

- Head-Modifier Rule

$$
[\text { phrase }] \rightarrow \mathrm{H}[1 \text { phrase }] \quad\left[\begin{array}{ll}
\text { phrase } & \\
\mathrm{MOD} & 1
\end{array}\right]
$$

- Coordination Rule

$$
\left[\begin{array}{ll}
\mathrm{SYN} & 0 \\
\mathrm{IND} & s_{0}
\end{array}\right] \rightarrow
$$

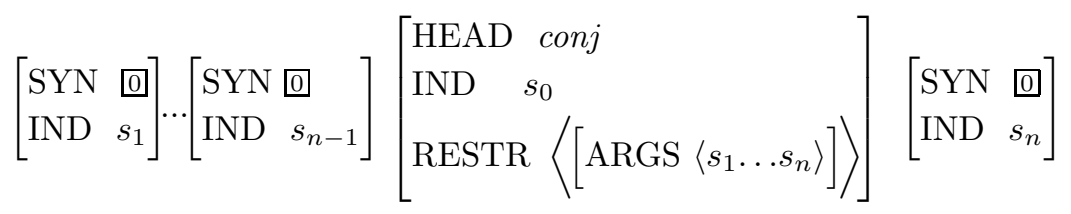

- Imperative Rule

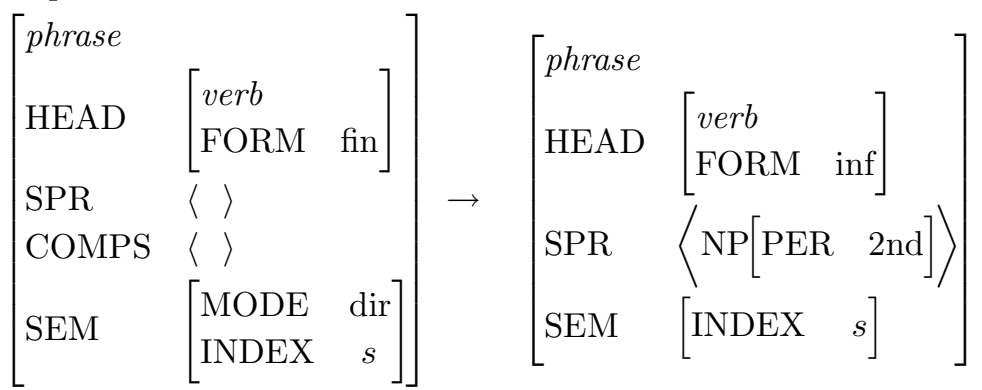

- Head-Filler Rule:

$$
\left[\begin{array}{ll}
\text { phrase } & \\
\text { GAP } & \langle\rangle
\end{array}\right] \rightarrow \text { 1 }\left[\begin{array}{ll}
\text { phrase } & \\
\text { GAP } & \langle\rangle
\end{array}\right] \mathrm{H}\left[\begin{array}{ll}
\text { phrase } & \\
\text { FORM } & \text { fin } \\
\text { SPR } & \langle\rangle \\
\text { GAP } & \langle 1\rangle
\end{array}\right]
$$


412 / Syntactic Theory: A Formal Introduction

\section{Well-Formedness Definitions}

In the following definitions, we take for granted the notions of resolved feature structure and tree structure, as described in Chapter 6 (See also the earlier summary in Chapter 9).

(78) Well-Formed Tree Structure

$\Phi$ is a well-formed tree structure just in case every local subtree in $\Phi$ satisfies some lexical entry $\eta$ or some grammar rule $\rho$.

(79) Lexical Satisfaction

A word structure:<smiles>F[Te]</smiles>

satisfies a lexical entry $\langle\omega, \delta\rangle$ just in case:

1. $F$ is of type word and $F$ satisfies $\delta$,

2. Argument Realization Principle

$F$ satisfies the following feature structure description:

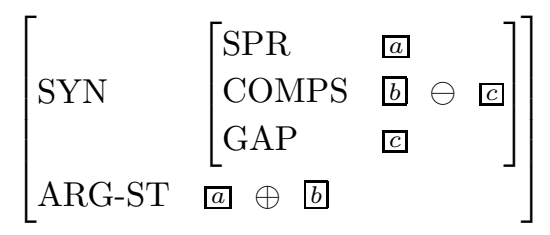

and

3. Case Constraint

Any NP in a noninitial position of F's ARG-ST list is [CASE acc]. 
(80) Phrasal Satisfaction

A local subtree $\Phi=$

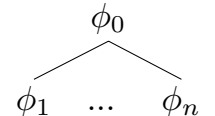

satisfies a grammar rule $\rho=\delta_{0} \rightarrow \delta_{1} \ldots \delta_{n}$ just in case: $^{1}$

1. The sequence $\left\langle\phi_{0}, \phi_{1}, \ldots \phi_{n}\right\rangle$ satisfies the description $\left\langle\delta_{0}, \delta_{1}, \ldots \delta_{n}\right\rangle$

2. $\Phi$ satisfies the Semantic Compositionality Principle, the Binding Theory, and the Anaphoric Agreement Principle,

3. if $\rho$ is a headed rule, then $\Phi$ satisfies the Head Feature Principle and the Semantic Inheritance Principle,

4. if $\rho$ is a headed rule other than the Head-Complement Rule, then $\Phi$ satisfies Part A of the Valence Principle,

5. if $\rho$ is a headed rule other than the Head-Specifier Rule, then $\Phi$ satisfies Part B of the Valence Principle, and

6. if $\rho$ is a headed rule other than the Head-Filler Rule, then $\Phi$ satisfies the GAP Principle.

(81) Semantic Compositionality Principle

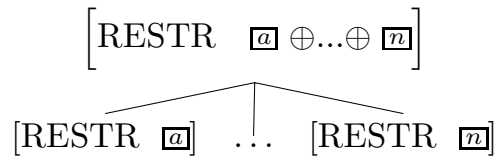

(82) The Binding Theory

Principle A: An [ANA +] synsem-struc must be outranked by a coindexed synsem-struc.

Principle B: An [ANA -] synsem-struc must not be outranked by a coindexed synsem-struc.

(83) Anaphoric Agreement Principle

Coindexed elements agree (i.e. share a single AGR value).

\footnotetext{
${ }^{4}$ Note that clause (1) here must speak of a sequence of resolved feature structures satisfying a sequence description. This is because of identities that must hold across members of the sequence, e.g. those required by the rules themselves or by the principles given below.
} 
414 / Syntactic Theory: A Formal Introduction

(84) Head Feature Principle

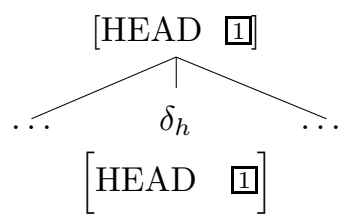

(85) Semantic Inheritance Principle

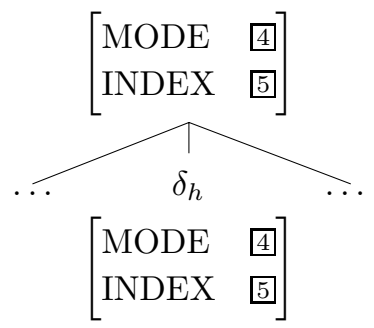

(86) Valence Principle

A

[COMPS b

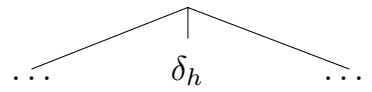

[COMPS

B

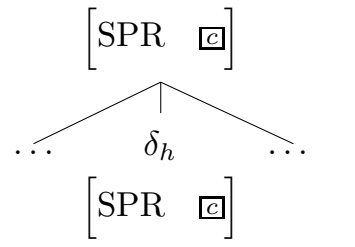

(87) The GAP Principle

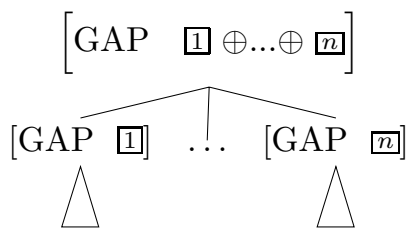


(88) Initial Symbol Definition

A CFG must include a statement of which category (or categories) can start a tree derivation. The analogue of this 'initial symbol' definition in our grammar is a constraint guaranteeing that for a phrase to stand alone, its synsem-struc must satisfy the following description:

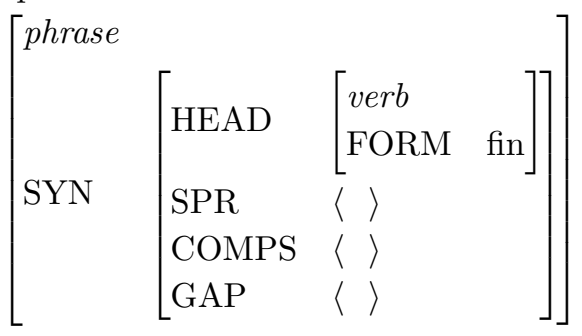

\section{Lexical Rules}

(89) Singular Noun Lexical Rule

$\langle[,[$ noun-lxm $]\rangle \Rightarrow$

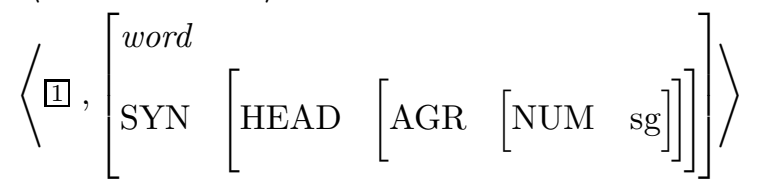

(90) Plural Noun Lexical Rule

$$
\begin{aligned}
& \left\langle\square,\left[\begin{array}{ll}
\text { noun-lxm } \\
\text { ARG-ST } & \langle[\text { COUNT }+]\rangle
\end{array}\right]\right\rangle \Rightarrow
\end{aligned}
$$

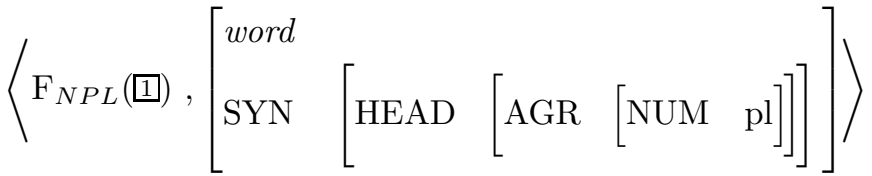


416 / Syntactic Theory: A Formal Introduction

(91) 3rd-Singular Verb Lexical Rule

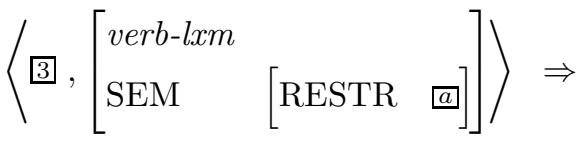

$$
\begin{aligned}
& \left\langle\mathrm{F}_{3 S G}(\text { (3) }),\left[\begin{array}{lll}
\text { SYN } & {\left[\begin{array}{lll}
\text { HEAD } & {[\text { FORM }} & \text { fin }
\end{array}\right]} \\
\text { ARG-ST } & \left\langle\left[\begin{array}{ll}
\text { CASE } & \text { nom } \\
\text { AGR } & 3 s i n g
\end{array}\right], \ldots\right\rangle
\end{array}\right]\right.
\end{aligned}
$$

(92) Non-3rd-Singular Verb Lexical Rule

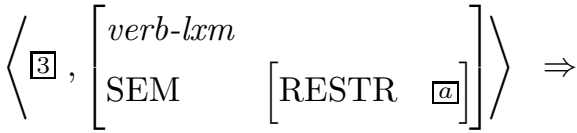

$$
\begin{aligned}
& \left\langle 3,\left[\begin{array}{ccc}
\text { SYN } & {\left[\begin{array}{ll}
\text { HEAD } & {\left[\begin{array}{ll}
\text { FORM } & \text { fin }
\end{array}\right]}
\end{array}\right]} \\
\text { ARG-ST } & \left\langle\left[\begin{array}{lll}
\text { CASE } & \text { nom } \\
\text { AGR } & \text { non-3sing }
\end{array}\right], \ldots .\right\rangle
\end{array}\right]\right\rangle
\end{aligned}
$$


(93) Past-Tense Verb Lexical Rule

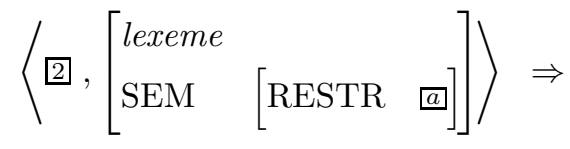

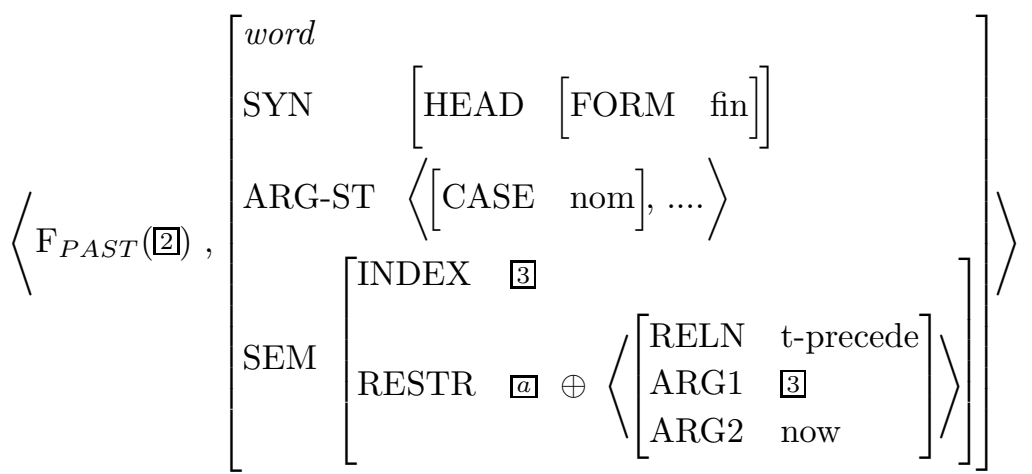

(94) Present Participle Lexical Rule

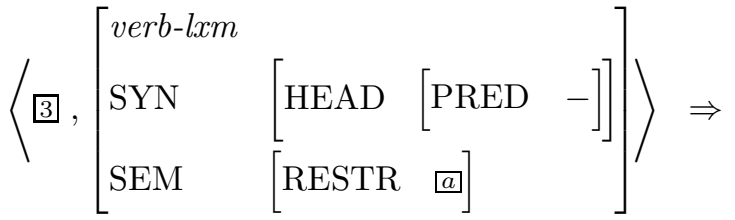

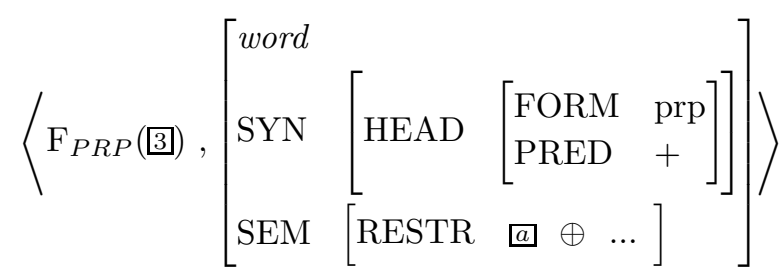

(95) Past Participle Lexical Rule

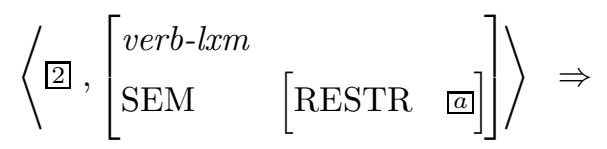

$$
\begin{aligned}
& \left.\left\langle\mathrm{F}_{P S P}(2),\left[\begin{array}{llll}
\text { word } & & & \\
\mathrm{SYN} & {[\mathrm{HEAD}} & {[\mathrm{FORM}} & \mathrm{psp}
\end{array}\right]\right]\right\rangle
\end{aligned}
$$


418 / Syntactic Theory: A Formal Introduction

(96) Infinitival Lexical Rule

$$
\begin{aligned}
& \left\langle 2,\left[\begin{array}{ll}
\text { verb-lxm } & \\
\text { SEM } & {\left[\begin{array}{ll}
\text { RESTR } & a
\end{array}\right]}
\end{array}\right]\right\rangle \Rightarrow
\end{aligned}
$$

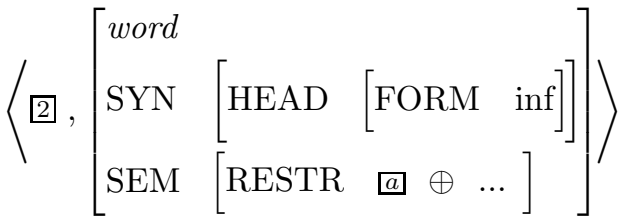

(97) Passive Lexical Rule

$$
\begin{aligned}
& \left\langle\square,\left[\begin{array}{llll}
t v-l x m & & & \\
\text { ARG-ST } & \left\langle\mathrm{NP}_{i}\right\rangle & \oplus & \mathbf{a}
\end{array}\right]\right\rangle \Rightarrow
\end{aligned}
$$

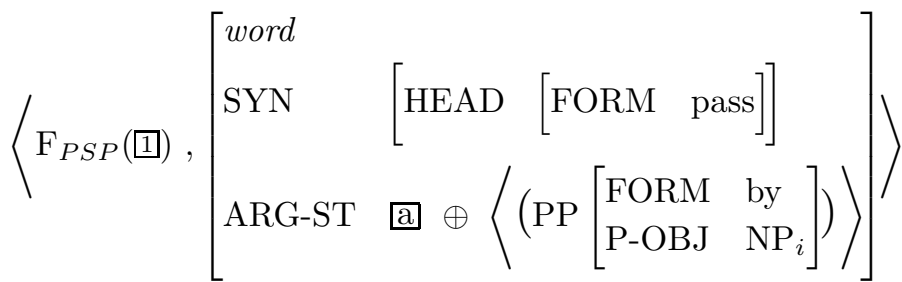

(98) Extraposition Lexical Rule:

$$
\begin{aligned}
& \left\langle 1,\left[\begin{array}{l}
l x m \\
\text { ARG-ST } \left.\mathrm{a},\left\langle\left[\begin{array}{ll}
\text { HEAD } & \text { comp }
\end{array}\right]\right\rangle \oplus \mathrm{b}\right]
\end{array}\right]\right\rangle \Rightarrow
\end{aligned}
$$

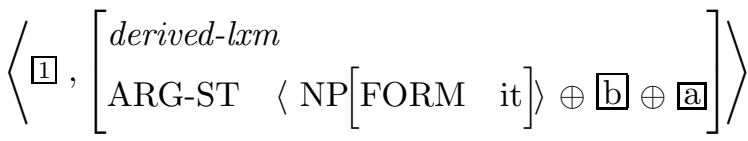


(99) Negation Lexical Rule

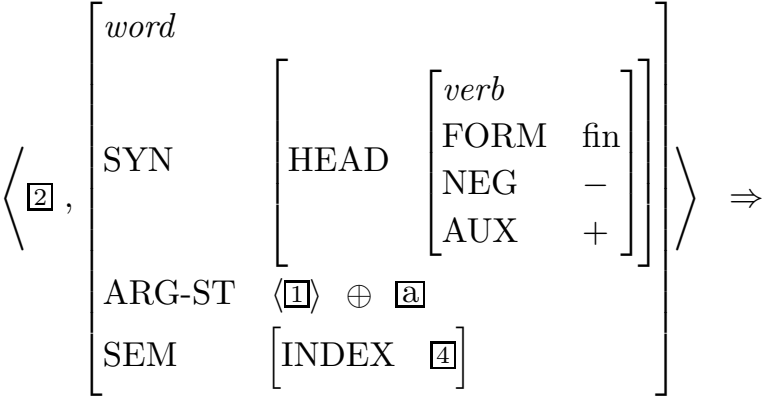

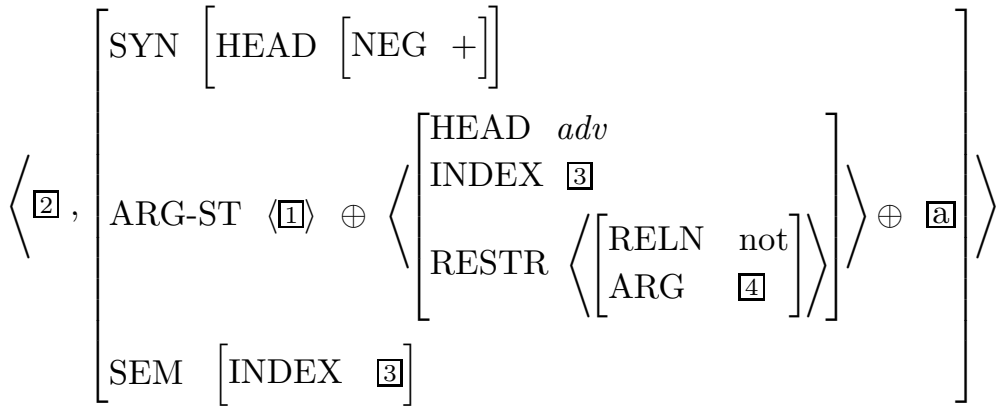

(100) Inversion Lexical Rule

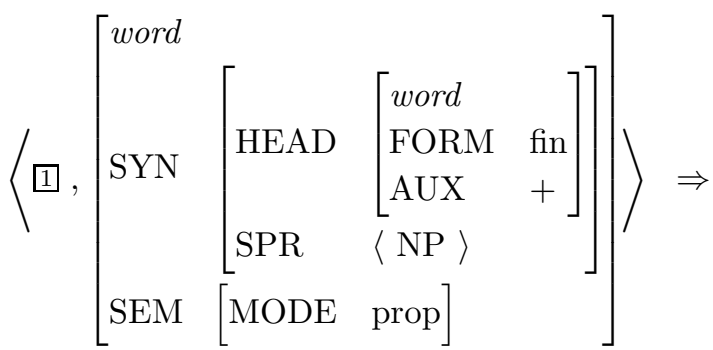

$$
\begin{aligned}
& \left\langle\square,\left[\begin{array}{ll}
\text { SYN } & {\left[\begin{array}{ll}
\text { HEAD } & {[\mathrm{INV}+} \\
\text { SPR } & \langle\rangle
\end{array}\right]} \\
\text { SEM } & {\left[\begin{array}{ll}
\text { MODE } & \text { ques }
\end{array}\right]}
\end{array}\right]\right\rangle
\end{aligned}
$$


420 / Syntactic Theory: A Formal Introduction

(101) Contraction Lexical Rule

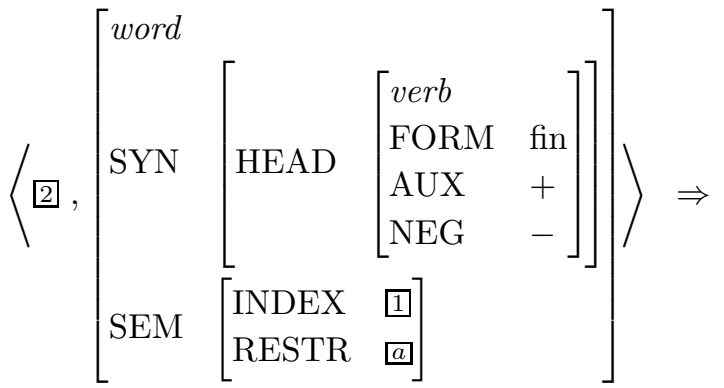

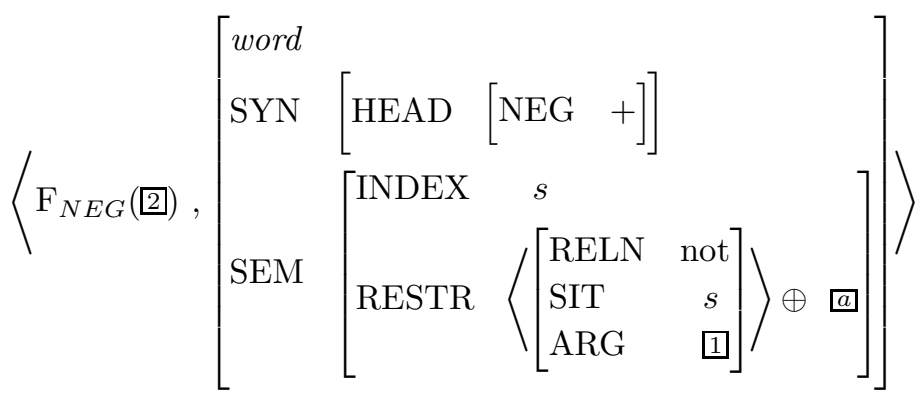

(102) Ellipsis Lexical Rule

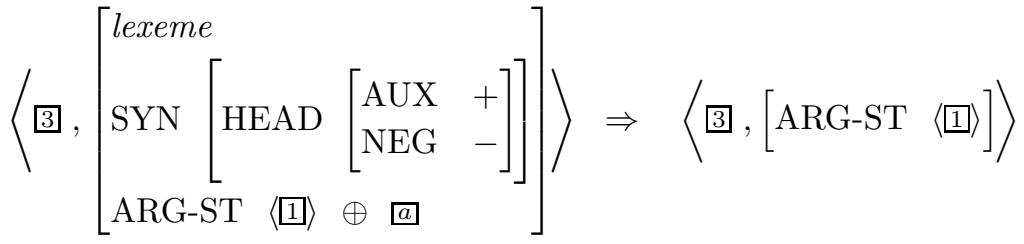

(103) Subject Extraction Lexical Rule

$$
\begin{aligned}
& \left\langle 0,\left[\begin{array}{ll}
\text { word } \\
\text { SYN }
\end{array}\left[\begin{array}{lll}
\text { HEAD } & \left.\begin{array}{ll}
\text { verb } \\
\text { FORM }
\end{array}\right] \\
\text { SPR } & \langle[]\rangle
\end{array}\right]\right]\right\rangle \Rightarrow \\
& \left\langle\square,\left[\begin{array}{ll}
\operatorname{SYN} & {\left[\begin{array}{ll}
\operatorname{SPR} & \langle\rangle \\
\operatorname{GAP} & \langle 1\rangle
\end{array}\right]} \\
\mathrm{ARG}-\mathrm{ST} & \langle 1, \ldots\rangle
\end{array}\right]\right\rangle
\end{aligned}
$$


(104) Constant Lexeme Lexical Rule

$\langle 1,[$ const-lxm $]\rangle \Rightarrow\langle 1,[$ word $]\rangle$

(105) Agent Nominalization Lexical Rule

$$
\begin{aligned}
& \left\langle 2,\left[\begin{array}{ll}
\text { verb-lxm } & \\
\text { ARG-ST } & \left\langle\mathrm{NP}_{i}(, \text { 1]NP })\right\rangle \\
\mathrm{SEM} & {\left[\begin{array}{ll}
\operatorname{INDEX} & s
\end{array}\right]}
\end{array}\right]\right\rangle \Rightarrow
\end{aligned}
$$

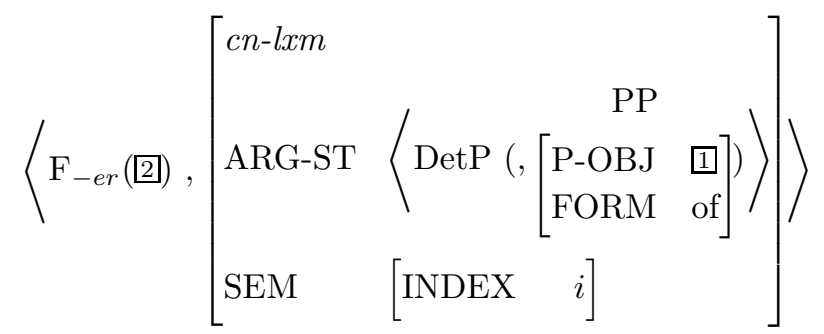





\section{Appendix B Generative Grammar: an historical overview}

As noted in Chapter 2, the theory of grammar developed in this text is most closely related to the framework known as 'Head-driven Phrase Structure Grammar', or HPSG. HPSG is one of a number of frameworks for syntactic analysis that have been developed within the Chomskyan paradigm, broadly conceived. The intellectual tradition it represents is eclectic in its orientation, synthesizing ideas from several approaches to the study of language. To clarify these connections, we provide here a brief history of generative grammar, including a survey of its major branches, ${ }^{1}$ with notes on their relevant contribution or influence to the ideas presented within this text.

The basic concept of generative grammar is simply a system of rules that defines in a formally precise way (i.e. 'generates') a set of sequences (strings over some vocabulary of words or 'formatives') that represent the well-formed sentences of a given language. Thus both of the systems considered in Chapter 2 (the regular expression (finite-state) and context-free phrase structure grammars) are generative grammars, as is the grammar summarized in Appendix A.

Generative syntax began in the 1950s when Noam Chomsky and others he influenced developed and formalized a theory of grammar based on the notion of 'transformation'. ${ }^{2}$ The architecture of a transformational generative grammar defines sentence well-formedness indirectly:

\footnotetext{
${ }^{1}$ The dates given in parentheses are roughly the periods during which a substantial number of researchers have been or are still active in developing the theory.

${ }^{2} \mathrm{~A}$ somewhat different though related concept of transformation can be found in the work of Zellig Harris, beginning in the 1950s. A number of relevant papers are collected in Harris 1970.
} 


\section{4 / Syntactic Theory: A Formal Introduction}

first, base (or 'underlying' or 'kernel') structures are generated via a system of phrase structure rules; ${ }^{3}$ and then transformational rules apply successively to map these phrase structures into other phrase structures. The sentences of the language, then, are just those that can be derived by applying transformational rules to the base structures according to a particular regime, e.g. a regime of ordered transformations, at least some of which are obligatory. A transformational derivation thus involves a sequence of phrase structures (or structural descriptions), the first of which is a base structure and the last of which is a phrase structure (usually called a 'surface structure') whose word string corresponds to a sentence of the language:
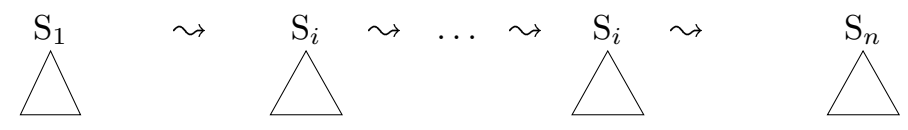

BASE

Transformational generative grammar (which has dominated the mainstream of syntactic theory from the 1960s through the 1990s) has changed significantly over the years, in ways we sketch below. Yet, despite considerable evolution within this framework, the notion of transformational derivation has been present in one guise or another in virtually every formulation of transformational grammar. ${ }^{4}$ Similarly, other commonalities remain in the practice of transformational grammarians, such as the treatment of sublexical entities (e.g. inflectional affixes) as independent syntactic elements, that is, as syntactic primitives on a par with words. ${ }^{5}$

In contrast to the transformational tradition, there is another approach to generative grammar, equally committed (if not more so) to the original goal of developing precisely formulated grammars. This tradition has two distinctive properties:

\footnotetext{
${ }^{3}$ In some versions of this approach, lexical insertion into the structures generated by the CFG is accomplished by a separate specialized mechanism.

${ }^{4}$ There are exceptions, though, e.g. Koster 1987 and Brody 1995. Chomsky has always maintained that it is 'not easy' to provide empirical (or theory-independent) evidence that would lead one to prefer a transformational theory over simpler alternatives. Despite this repeated claim (Chomsky (1981: 90f; 1995: 223f)), Chomsky has included transformational operations in every version of grammatical theory he has developed since the 1950s.

${ }^{5}$ Hence Chomsky's introduction of the term (syntactic) 'formative' to encompass stems, noninflecting words, and inflectional affixes.
} 
(i) Constraint-Based Architecture: Grammars are based on the notion of constraint satisfaction, rather than transformational derivation.

(ii) Strict Lexicalism: Words, formed in accordance with an independent lexical theory (or 'module'), are the atoms of the syntax. Their internal structure is invisible to syntactic constraints.

These two design properties together form the basis of the 'constraintbased lexicalist' (CBL) approach to generative grammar. In CBL approaches, surface structures are generated directly, though ancillary kinds of syntactic representation may be cogenerated (see below).

The principle of strict lexicalism has its origin in the pioneering work of Chomsky (1970), who challenged previous attempts to derive nominalizations (e.g. the enemy's destruction of the city) from clauses (e.g. the enemy destroyed the city) via syntactic transformations. In the midand late 1970s, many other alternatives to transformational analyses were developed. There are two particularly significant developments in this period. The first is Bresnan's 'Realistic' Transformational Grammar (widely circulated in unpublished form; a version was published as Bresnan (1978)), which for the first time provided a cogent treatment of numerous phenomena (e.g. passivization) in lexical rather than transformational terms. Bresnan's dramatic first step inspired a number of people, notably Brame (1979) and Gazdar (1981) [first drafted in 1980], to take the further step of purging transformations from syntactic theory altogether. Second, the emergence of the framework of Montague Grammar provided new techniques for characterizing meanings directly in terms of surface structure, thereby eventually eliminating any semantic motivation for syntactic transformations. In many versions of transformational grammar, active and passive sentences were derived from a common underlying structure, leading to the (controversial) suggestion that many aspects of meaning are preserved by transformational derivations. With the advent of more sophisticated methods of semantic analysis, distinct surface structures could be assigned formally distinct but equivalent semantic interpretations, thus accounting for the semantics in a principled fashion without appeal to transformations.

'Realistic' Transformational Grammar and Montague Grammar together set the stage for the emergence of fully nontransformational generative frameworks in the late 1970s and early 1980s. Most notable among these are Lexical-Functional Grammar, Generalized Phrase Structure Grammar, Categorial Grammar, and Dependency Grammar, each of which we summarize below. The subsequent history of CBL generative grammar witnessed not only considerable development in each of 


\section{6 / Syntactic Theory: A Formal Introduction}

these frameworks, but also the introduction of such new approaches as Construction Grammar. Of immediate relevance also is the evolution of Generalized Phrase Structure Grammar, through the integration of ideas from various other frameworks, into the framework of HPSG, from which are directly drawn many analyses and the general orientation of the present text.

The remainder of this appendix briefly sketches a number of the most influential theories of grammar developed over the past forty years. We do not pretend that this is a comprehensive listing of approaches to syntactic description that have been explored in that period; our main purpose here is to situate the theory developed in this text in a wider intellectual landscape, indicating the many conceptual strands that have contributed to our approach. At the same time, we hope to convey a general sense of the historical development of generative grammar and its various schools.

\section{Transformational Grammar}

Early Transformational Grammar (1955-1964) An early version of transformational generative grammar was presented in Chomsky's 1957 book, Syntactic Structures. The analyses presented there and in other transformational works of the period included explicit formal statements of rules intended to license all and only the well-formed sentences of the language under discussion (usually English). This emphasis on the precise formulation of hypotheses is perhaps the greatest influence of early transformational grammar on the approach presented here.

As noted above, a key claim of transformational grammar (in all its versions) is that an empirically adequate grammar requires that sentences be associated not with a single tree structure, but with a sequence of trees, each related to the next by a transformation. The initial trees in Chomsky's Syntactic Structures theory were to be generated by a CFG. For example, passive sentences (such as The cat was chased by the $d o g$ ) were derived from the same underlying structures as their active counterparts (The dog chased the cat) by means of a passivization transformation that permuted the order of the two NPs and inserted the words be and by in the appropriate places.

The most celebrated analysis in this theory is its treatment of the English auxiliary system (roughly, the material covered in Chapter 13 of this text). Chomsky (1957) proposed that tense was, in the underlying syntactic structure, a 'formative' separate from the verb on which it ultimately appears. A movement transformation was posited to account for inversion in questions (deriving, e.g. Is the sun shining? from 
the same underlying structure as The sun is shining); and an insertion transformation placed not in the appropriate position for sentence negation. Both these transformations in some instances have the effect of stranding tense - that is, leaving it in a position not adjacent to any verb. For these cases, Chomsky posited a transformation to insert do as a carrier of tense. Several other uses of auxiliary do (e.g. in ellipsis) were also treated on this view as instances of tense stranding. This unified account of apparently disparate uses of $d o$, together with the formal explicitness of the presentation, won many converts to transformational grammar.

The Standard Theory (1965-1970) Katz and Postal (1964) and Chomsky (1965) introduced a number of major changes into transformational grammar, and Chomsky dubbed the resulting theory 'the standard theory'. It differed from early transformational grammar in several ways, some rather technical. Among the important innovations of this theory were the use of recursive phrase structure rules (allowing for the elimination of transformations that combined multiple trees into one) and the introduction of syntactic features to account for subcategorization (valence).

Perhaps the most important conceptual change was the addition of a semantic component to the theory of transformational grammar. In this theory, the initial tree in each sentence's derivation, known as its 'deep structure', transparently represented all the information necessary for semantic interpretation. In particular, it was claimed that there is a simple mapping between the semantic roles played by arguments to a verb (intuitively, who did what to whom) and the deep structure grammatical relations (subject, object, etc.). In the final tree of the derivation (the surface structure), the words and phrases were arranged as the sentence would actually be pronounced. On this theory, then, transformations were thought to be the primary link between sound and meaning in natural language.

The standard theory had great intuitive appeal and attracted much attention from neighboring disciplines. In particular, many philosophers were attracted by the idea that deep structures might provide something very much like the 'logical form' of sentences needed for precise analysis of their role in inference. Likewise, psychologists hoped that the transformational derivations were a first approximation to the mental processes involved in the production and comprehension of utterances. Initial experiments gave credibility to this idea, in that they showed a correlation between the psychological complexity of a sentence and the number of transformations posited in its derivation. Further research 
on this idea (known as the 'derivational theory of complexity') failed to support it, however, and by the early 70s it had been largely abandoned (Fodor, Bever and Garrett (1974)).

Most contemporary grammatical theories have preserved the most important innovations of the standard theory, namely, syntactic features, recursive phrase structure, and some sort of semantic component. On the other hand, no current theory maintains the centrality of transformations in mediating between sound and meaning.

Generative Semantics (1967-1974) Generative Semantics was the first major challenge to Chomsky's views within the generative paradigm; its leading figures included George Lakoff, James McCawley, Paul Postal, and John ('Haj') Ross. They carried the central idea of the standard theory to its logical conclusion, claiming that deep structures should themselves be viewed as representations of meaning, and denying that syntactic and semantic rules should be considered distinct components of a grammar. That is, on the generative semantics view, something was considered a possible input to the transformational rules just in case it represented a proposition that made sense. Hence all languages could be derived from the same underlying source, differing only in how the underlying representations get transformed into sounds.

The underlying trees of generative semantics were far larger and more elaborate than those of the standard theory (though the inventory of grammatical categories was much reduced). Virtually all the work involved in describing the relationships between form and meaning in language was done in this theory by transformations, though these rules were rarely formulated explicitly.

Generative semantics enjoyed wide currency for a few years and served as the vehicle for the exploration of a wide range of fascinating phenomena in many languages. Although the theory itself had a short life span (for reasons that have been debated by historians of linguistics), many of the constructions first discovered by generative semanticists continue to figure prominently in theoretical discussions. Moreover, some recent analyses have borne striking resemblances to earlier generative semantics proposals; see Harris (1993) and Huck and Goldsmith (1995) for discussion.

The Extended Standard Theory (1967-1980) Unlike the generative semanticists, Chomsky and some others (notably, Ray Jackendoff) quickly abandoned the idea that pairs of sentences with identical deep structures must be synonymous. In particular, they argued that transformations that reordered quantified NPs could change the scopes of the quantifiers (e.g. Many people read few books was claimed to have 
a range of interpretations different from Few books are read by many people). Hence they claimed that structures other than deep structures must play a role in semantic interpretation.

Instead of the complex underlying trees and elaborate transformational derivations of generative semantics, the framework that Chomsky dubbed the 'Extended Standard Theory' (EST) posited a relatively impoverished theory of transformations; instead, it enriched other components of the theory to carry much of the descriptive burden. In addition to the new types of semantic rules alluded to above, schematization over phrase structure rules and an enriched conception of the lexicon - including lexical rules - were introduced. These innovations have been carried over into much contemporary work, including the theory developed in this text. The approach of EST led to a highly 'modularized' theory of grammar, with a variety of distinct types of mechanisms to account for different kinds of empirical phenomena.

EST also saw the introduction of 'empty categories' - that is, elements that occupy positions in a tree but which have no phonetic realization. These included a type of null pronoun used in control constructions (e.g. the subject of leave in We tried to leave) and 'traces' of elements that have been moved.

A central concern of EST and much subsequent work has been to constrain the power of the theory - that is, to restrict the class of grammars that the theory makes available. The primary rationale for seeking such constraints has been to account for the possibility of language acquisition, which (as noted in Chapters 1 and 9) Chomsky regards as the central question for linguistics.

Government and Binding Theory (1980 to the present) Government and Binding theory, or GB, as it is generally called, was first laid out in Chomsky's (1981) book, Lectures on Government and Binding. ${ }^{6}$ It develops the modular style of EST, dividing the theory of grammar into a set of subtheories, each with its own set of universal principles. Although GB still uses transformational derivations to analyze sentences, it reduces the transformational component to a single rule (referred to as 'Move $\alpha$ '), which can move anything anywhere. The idea is that general principles will filter out most derivations, preventing the massive overgeneration that might be expected.

Elaborating on earlier work in EST, GB analyses posit a rich array of empty categories. Binding theory, which has been a major topic of research within GB, is applied not only to overt pronouns, but also to empty categories. Movement leaves behind traces (a kind of empty cat-

\footnotetext{
${ }^{6}$ For an introductory presentation, see Haegeman (1994).
} 


\section{0 / Syntactic Theory: A Formal Introduction}

egory), which are bound by the moved element. Binding theory thus relates constraints on movement to possible pronoun-antecedent relations. Since movement is used to deal with a wide range of phenomena (including filler-gap dependencies, the active-passive relation, raising, extraposition, and auxiliary inversion), linking all of these to the binding principles yields a richly interconnected system.

The primary focus in GB has been the development of a theory of universal grammar. GB claims that many of the principles that make up the theory of grammar are parameterized, in the sense that they vary within a narrow range. (Another name for this approach to syntax is 'Principles and Parameters'). Learning a language, on this view, consists of fixing a small set of parameters (plus learning vocabulary). That is, GB claims that all languages are essentially alike, with only a few restricted parameters of possible variation. Working out the details of this ambitious program has been the most active area of syntactic research since the early 1980s.

The literature within this framework is massive, and it represents a much wider range of analyses than any of the other theories listed here, though these analyses are seldom formulated with a precision comparable to that assumed in this text and hence are often difficult to evaluate. However, they tend to share certain noteworthy characteristics, including the following:

- Highly articulated phrase structures (linguistically significant distinctions and relations are encoded into tree configurations);

- Use of movement (that is, the transformation Move $\alpha$ );

- Extensive use of empty categories;

- A rich set of universal principles, some of which are parameterized;

- Avoidance of language-particular rules (properties specific to a language are to be expressed in terms of values of universally available parameters);

- Deductive structure (small changes in a grammar should have farreaching consequences for the language, so that stipulation is minimized).

The theory we have presented here has been influenced by GB in a number of ways. These include very general goals, such as striving for deductive structure in the theory. They also include more specific design features, such as the general form of the Binding Theory (though not the detailed statement of the binding principles). Finally, there are specific points of our grammar that were first proposed within GB analyses, such as treating complementizers as heads that could take sentences as their complements. 
The Minimalist Program As of this writing, the most recent descendant of GB is the 'Minimalist Program'. As its name implies, MP is a program for research, rather than a theory of syntax.

MP explores the idea that instead of generating sentences directly, what grammars do is pick out the best expressions from some candidate set. MP grew out of Government and Binding theory, but it represents a significant departure from earlier work in that framework. Its goal is to explain linguistic structure in terms of intuitively natural 'economy conditions' on grammars and their operations. Analyses are most highly valued if they minimize the amount of structure and the length of derivations posited. In his seminal work on MP, Chomsky (1995) lays out some principles that embody this general idea. These include 'GREED', which says that a constituent will move only to satisfy its own licensing requirements, and 'PROCRASTINATE', which says that movement will take place as late as possible. More recent work has sought to localize such effects, applying economy constraints at each step of a transformational derivation. This conception of grammar, in which the properties of competing transformational derivations are crucial in determining sentence well-formedness, ${ }^{7}$ represents a radical departure from the original goals and methodology of generative grammar and has no direct connection with the theoretical orientation of the present work. For an extensive comparison of MP with CBL approaches in general, see Johnson and Lappin (in press).

The work of filling in the details of MP is still in the early stages, but it has stimulated a great deal of interest within the field. For an elementary exposition of MP, see Radford (1997); also useful is Webelhuth, ed. (1995).

\section{Constraint-Based Lexicalist Grammar}

Categorial Grammar (1974 to the present) Categorial Grammar (CG) has a long history dating back to the 1930s, but it was developed primarily by mathematical logicians before the early 1970s. It came to the attention of linguists when the logician Richard Montague used it as the syntactic framework to go with his new approach to analyzing natural language semantics. ${ }^{8}$

The central idea of CG is that an enriched conception of grammatical

\footnotetext{
${ }^{7}$ Generative semanticists in the early 1970s briefly discussed the need for what were then termed 'transderivational constraints', but the idea was not pursued for long.

${ }^{8}$ Montague's intensional logic and his precise framework for studying the relation between expressions and their meaning had a considerable influence on work in linguistics. His famous remark (Montague, 1970:373) that 'there is in my opinion no important theoretical difference between natural languages and the artificial lan-
} 
categories can eliminate the need for many of the constructs (e.g. transformations) found in other theories of grammar. CG makes no formal distinction between lexical and nonlexical categories. Hence, for example, an intransitive verb like sleeps is treated as belonging to the same category as a phrase consisting of a transitive verb plus a direct object, such as gets some rest. Categories are defined in terms of their members' potential for combining with other constituents (and thus CG is often seen as a variety of Dependency Grammar - q.v.). So, for example, verb phrases (and intransitive verbs) can be characterized as those elements which, when combined with a noun phrase on their left, form sentences; one notation for this is $\mathrm{NP} \backslash \mathrm{S}$. A transitive verb like gets belongs to the category of elements that take an NP on their right to form a NP $\backslash \mathrm{S}$; this can be written (NP $\backslash \mathrm{S}) / \mathrm{NP}$. A categorial grammar consists simply of a lexicon together with a few simple rules for how categories can be combined.

The primary attractions of CG have always been its conceptual simplicity and the fact that it is well suited to the formulation of tightly linked syntactic and semantic analyses. It also provides particularly elegant and appealing accounts of coordination. For a general introduction to categorial grammars, see Wood (1993) and Carpenter (1997). Steedman (1996) summarizes a tradition within CG that makes use of logical 'combinators'. For the competing 'type-logical' approach, see Morrill (1994) and Moortgat (1997).

The influence of CG on the theory developed in this text is quite clear. The valence features of HPSG do much the same work as complex category labels do in CG. The nodes of trees in HPSG are labeled with feature structures that contain all the information in CG category labels (plus quite a bit more). Our grammar rules cancel elements off our valence lists in a way analogous to the combinatory rules of CG. Hence many CG analyses can be translated fairly straightforwardly into HPSG.

Construction Grammar (1988 to the present) Construction Grammar $(\mathrm{CnG})$ represents a related yet interestingly different perspective from other CBL frameworks. Linguistic objects (sentences, phrases, and words), viewed formally, are taken to be constituent structure trees whose nodes are occupied by feature structures. The feature structures contain additional syntactic as well as semantic and phonological information. The constructions that license linguistic objects are partial descriptions of such feature structure trees, much as in the presentation of this text.

guages of logicians' is sometimes referred to (following Bach 1989) as Montague's Hypothesis. 
A construction grammar is a set of constructions that can be unified to create the set of sentences of a language (and no sentence objects not in the language). A CnG feature-structure tree can be translated into either an HPSG feature structure of type sign, or the analogue of this in Lexical Functional Grammar (q.v.) (insofar as the detailed kinds of information contained in the particular implementations of these theories at hand are comparable). ${ }^{9}$

There are two principle substantive motivations for $\mathrm{CnG}$. The first is to account for grammatically determined, non-truth-conditional aspects of meaning - including such 'pragmatic' factors as conventional implicature and presupposition - in the formal representations of sentences and of the grammatical constructions that license them. The second is to account for the full range of idiomatic and semi-idiomatic constructions that occur in every language. Although every generative approach to the grammar of natural languages is interested in full coverage of the facts of every language, as well as in the extraction of intralanguage and interlanguage generalizations - the latter usually considered to constitute the stuff of universal grammar - varying approaches differ in their relative emphasis on the full coverage of language facts versus the development of a parsimonious theory of universal grammar. CnG falls at the end of this scale concerned primarily with empirical coverage. For more on CnG, see Fillmore and Kay (forthcoming), Fillmore et al. (1988), and Goldberg (1995).

Dependency Grammar (1959 to the present) Work on transformational grammar rests on two crucial (but controversial) assumptions about sentence structure: that it is organized hierarchically into 'phrases' (hence 'phrase structure'), and that grammatical relations such as 'subject' and 'object' are redundant. The assumption of phrase structure is a distinctively American contribution to linguistics, having been suggested by Leonard Bloomfield (1933). Bloomfield suggested that sentences should be analysed by a process of segmentation and classification: segment the sentence into its main parts, classify these parts, then repeat the process for each part, and so on until the parts are 'morphemes', the indivisible atoms of grammar. Thus Cool students write short essays divides into the noun phrase cool students plus the verb phrase write short essays, which in turn divides into the verb write plus short essays, and so on. This contrasts with the European tradition (which dates back to classical Greece) in which the focus is on individual words and their

\footnotetext{
${ }^{9}$ Recent developments in HPSG have reduced many of the analytic differences between CnG and HPSG. See Sag (1997) and the discussion of phrase hierarchies in Chapter 16.
} 
relationships - for example, cool is an 'attributive modifier' of students, and students is the subject of write.

The attraction of phrase structure analysis is its formal clarity, which is revealed by the familiar phrase structure trees. Various linguists (mainly European) have attempted to develop the traditional approach in the same way, with the emphasis on the relationships among words rather than on the groupings of words. One of the characteristics of these relationships is that the words concerned are generally not equal, in that one serves to modify the meaning of the other; so cool students denote certain students, and students writing essays denotes a kind of writing. The relationships are called 'dependencies', with the modifying word depending on the modified (so cool depends on students, and students on write), and the approach is called 'dependency grammar' (DG) to contrast it with phrase structure grammar.

There are several ways to represent DG analyses diagramatically, including a system that has been widely used in American schools since the nineteenth century which is often called simply 'sentence diagramming'. The first real attempt to build a theory of DG analysis was Tesnière (1959), but since then developments in PSG theory have been parallelled in DG theories. One of these which is particularly close in other respects to HPSG is 'Word Grammar' (Hudson 1984, 1990, 1998). In some respects, HPSG bridges the gap between DG and PSG, for in HPSG all the parts of a phrase depend directly on its head word - the phrase is 'head-driven', just as in DG. On the other hand, in HPSG the dependent parts are themselves phrases with their own internal structure consisting of a head word and its dependents.

Generalized Phrase Structure Grammar (1979-1987) Generalized Phrase Structure Grammar, or GPSG, as it was known, was initiated by Gerald Gazdar in a pair of papers (Gazdar 1981, 1982) that attracted the attention of numerous researchers in the field of syntax. The theory was further developed by him and a number of colleagues in the early 1980s and was codified in the 1985 book, Generalized Phrase Structure Grammar (Gazdar et al. 1985), which provides a detailed exposition of the theory.

The central idea of GPSG is that standard context-free phrase structure grammars can be enhanced in ways that do not enrich their generative capacity, but which do make them suitable for the description of natural language syntax. The implicit claim of work in GPSG was that the tenable arguments against CFG as a theory of syntax were arguments about efficiency or elegance of notation, and not about coverage in principle. 
Among the important ideas that originated in GPSG are the separation of CFG rules into (i) rules of immediate dominance ('ID rules'), which specify only which phrases can appear as daughters in a local syntactic tree, and (ii) rules of linear precedence ('LP rules'), which specify general constraints determining the order of daughters in any local tree. This factorization of the two functions of traditional CFG rules is preserved in HPSG, though we have not employed it in the formulation of grammar rules in this text.

A second idea stemming from work in GPSG is the treatment of long-distance dependency constructions, including filler-gap constructions (such as topicalization, wh-questions, and relative clauses). The GPSG treatment of these phenomena involved locally encoding the absence of a given constituent via a feature specification. The remarkable result of the transformationless GPSG analysis of filler-gap dependencies was that it succeeded where transformational theories had failed, namely in deriving the coordinate structure constraint and its 'acrossthe-board' exceptions (see Chapter 15). This feature-based analysis of filler-gap dependencies is preserved in HPSG, and we have carried it over virtually intact to the current text.

Head-Driven Phrase Structure Grammar (1984 to the present) HPSG evolved directly from attempts to modify GPSG in the interdisciplinary environment of Stanford's Center for the Study of Language and Information (CSLI), the site of several experimental computational systems for language processing. From its inception, HPSG has been developed as a conscious effort to synthesize ideas from a variety of perspectives, including those of Situation Semantics (which originated at CSLI at about the same time as HPSG), data type theory, and a variety of other linguistic frameworks under development in the early and mid1980s. The name 'Head-driven Phrase Structure Grammar' was chosen to reflect the increasingly recognized importance (as compared with, say, GPSG) of information encoded in the lexical heads of syntactic phrases. Dependency relations are lexically encoded, as they are in Dependency Grammar (q.v.), Categorial Grammar (q.v.) and LFG (q.v.). The theoretical aspects of HPSG have been developed in considerable detail in two books (Pollard and Sag 1987, 1994) and a number of major articles.

Some of the key ideas of work in HPSG are: (1) a sign-based architecture (see Chapter 16); (2) the organization of linguistic information via types, type hierarchies, and constraint inheritance; (3) the projection of phrases via general principles from rich lexical information; (4) the organization of such lexical information via a system of lexical types; and (5) the factorization of phrasal properties into construction-specific 
and more general constraints. These properties have all been discussed at various places in this text.

Since the inception of HPSG, researchers have been involved with its computational implementations. From 1980 until 1991, Hewlett-Packard Laboratories in Palo Alto, California supported one such project, which involved the authors of this text and a number of colleagues and students. It was with this project that many of us learned for the first time how far the rhetoric of theoretical linguistics can be from the reality of working grammars. At the time of this writing, implementations of HPSG and HPSG-like grammars are being developed at numerous universities and industrial research laboratories around the world, including sites in Canada, the United States, western and eastern Europe, Japan, Korea, Taiwan, and Australia.

Lexical Functional Grammar (1979 to the present) The theory of Lexical Functional Grammar, commonly referred to as 'LFG' (Bresnan (1982); Dalrymple et al. (1995); Bresnan (to appear)) shares with Relational Grammar (q.v.) the idea that relational concepts like 'subject' are of central importance and cannot be defined in terms of tree structures. But it also treats phrase structure as an essential part of grammatical description and has focussed on the development of a universal theory of how constituent structures are associated with grammatical relations.

In LFG, each phrase is associated with multiple structures of distinct types, with each structure expressing a different sort of information about the phrase. The two representations that have been the center of attention in most LFG literature are: the 'functional structure', which expresses the relational information that is analogous in certain respects to our ARG-ST and in other respects to the valence features SPR and COMPS; and the 'constituent structure', which is a tree diagram very similar to the surface structures of the Standard Theory. General principles and construction-specific constraints define the possible pairings of functional and constituent structures. In addition, LFG recognizes a number of further levels of representation. Perhaps most notable among these are $\sigma$-structure, which represents linguistically significant aspects of meaning, and a-structure, which serves to link syntactic arguments with aspects of their meanings. Thus the analogue of the HPSG sign presented in Chapter 16 is a tuple of LFG structures, possibly the fourtuple consisting of a sentence's c-structure, f-structure, a-structure, and $\sigma$-structure.

There are no transformations in LFG. Much of the descriptive work done in earlier theories by transformations is handled by an enriched lexicon, an idea pioneered by LFG researchers. For example, the active- 
passive relation in English is treated as a lexical relation between two forms of verbs. In early LFG, this was codified in terms of lexical rules similar to those presented in this text. Subsequent work has sought to develop a more abstract conception of lexical relations in terms of 'lexical mapping theory'. LMT provides for constraints on the relation between f-structures and a-structures, that is, constraints associated with particular arguments that partially determine their grammatical function. It also contains mechanisms whereby arguments can be supressed in the course of lexical derivation. In LFG, information from lexical entries and phrasal annotations is unified to produce the functional structures of complex expressions.

As the above description makes clear, LFG and HPSG bear many resemblances. In particular, HPSG has been able to incorporate many insights from work in LFG, most notably: the significant use of lexical analyses, well-developed dependency-based analyses of numerous phenomena (rather than accounts based on constituent structure), and the general constraint-based approach to grammatical description. There are crucial differences between LFG and HPSG as well (e.g. the use of types and type-based inheritance, which plays no role in the LFG literature, and HPSG's use of defaults). The differences in practice between the HPSG and LFG communities often lead to rather different analyses of the same phenomena; yet these analyses are often compatible with either framework. For an overview of current developments in LFG, see Bresnan (forthcoming).

\section{Two Other Grammatical Frameworks}

Relational Grammar (1974 to the present) We can now return to the second controversial claim of PSG, namely, that grammatical relations are redundant. In early theories of generative grammar, transformations were defined in terms of structural properties of tree diagrams. To the extent that traditional notions like 'subject' and 'direct object' were employed in these theories, they were regarded simply as shorthand for relations between linguistic elements definable in terms of the geometry of trees. Relational grammar (RG), developed by Paul Postal, David Perlmutter, David Johnson and others, adopts primitives that are conceptually very close to the traditional relational notions of subject, direct object, and indirect object. In this respect there is a strong affinity between RG and Dependency Grammar (q.v.). The grammatical rules of RG are formulated in relational terms, replacing the earlier formulations based on tree configurations. For example, the passive rule is 
stated in terms of promoting the direct object to subject, rather than as a structural rearrangement of NPs.

This approach allows rules to be given very general formulations that apply across languages. The characterization of passivization as promotion of the object does not depend on whether subjecthood and objecthood are indicated by word order or by other means, such as case marking on the nouns or some marking on the verb.

Although the influence of RG on the theory presented here may not be obvious, it is real. The notions of 'specifier' and 'complement' employed in this text are generalizations of 'subject' and 'object'. Languages use different grammatical devices to mark these relations (word order, case marking, agreement, etc.), and so a theory whose primitives are too closely linked to these devices would be unable to express crosslinguistic similarities. A number of contemporary theories, including LFG (q.v.) and HPSG, have adopted this central insight of RG.

The RG framework was applied to the description of a much wider variety of languages than were earlier generative theories (which tended to concentrate on the familiar European languages, East Asian languages, and a few others). Various results of work in this framework are anthologized in Perlmutter, ed. (1983) and Postal and Joseph, eds. (1990). 'Arc Pair Grammar' (Johnson and Postal (1980)) is an axiomatization and elaboration of many of the central ideas of Relational Grammar. The graph-theoretic foundations of HPSG are essentially the same as those first developed for Arc Pair Grammar by Johnson and Postal.

Optimality Theory Optimality Theory, or OT was first developed as a phonological framework (Prince and Smolensky (forthcoming)), and has recently been adapted to syntactic analysis (see Barbosa et al. (1998)). OT posits a universal set of defeasible constraints. The grammar of a language consists of a ranking of the constraints. Determining whether a given string of words is a well-formed sentence involves comparing it with other candidate expressions of the same proposition. The candidate whose highest-ranking constraint violation is lower than any other candidate's is grammatical. For example, if constraint $A$ outranks constraint $B$, which outranks constraint $C$, and if candidate sentence 1 violates $A$ whereas candidate sentence 2 violates $B$ and $C$, then sentence 2 is preferred over sentence 1 , and sentence 1 is ungrammatical. If no other candidate sentence wins such a competition against sentence 2, then sentence 2 is licensed by the grammar.

The idea of constraints that can be violated is also incorporated in the theory presented in this book, since default constraints specified in type hierarchies can be overridden. Moreover, a hierarchy of types with 
defeasible constraints defines a partial ordering on those constraints, with those introduced lower in the hierarchy taking precedence over those introduced at higher levels. Although there are substantive differences, certain central properties of OT can also be found in inheritance hierarchies with default constraints. ${ }^{10}$

OT follows much earlier work in generative grammar in positing rich systems of universal grammar. However, the idea that determinations of well-formedness necessarily involve comparing structures or derivations is a break with past views, as we already noted in discussing the Minimalist Program (q.v.). Another common characteristic of MP and OT is the use of defeasible constraints. As noted above, such constraint mechanisms of various sorts have been proposed from time to time within some theories, including the theory presented in this book. This is not surprising, since idiosyncratic exceptions to general patterns are commonplace in natural languages. Defeasible constraint mechanisms are now accepted fairly widely in various theories of syntax. It remains to be seen whether a similar consensus will arise concerning the idea of defining well-formedness in terms of the outcome of some sort of competition.

\footnotetext{
${ }^{10}$ Such hierarchies are explored in some detail in the artificial intelligence literature of the 1970s.
} 



\section{Glossary}

This glossary contains linguistic terms that either play a direct role in or are presupposed by this book. For further information, there are a number of dictionaries of linguistics, including Crystal (1985) and Trask (1993).

AAVE In informal speech, many African Americans use a distinctive variety of English known as African American Vernacular English, or AAVE for short. Also known as Black English, African American English, and Ebonics, both the phonology and aspects of the syntax of AAVE have been extensively studied, largely by sociolinguists.

active A verb form or clause that is not in the passive is referred to as active. See also passive; voice.

affix An affix is a morphological element added to a stem to form another stem or a word. Two common types of affix are prefixes (e.g. re-, as in reread; out-, as in outperform) and suffixes (-ed, as in visited; -s, as in visits. Many languages exhibit other types of affix as well, including infixes (an element inserted into a stem) and circumfixes (e.g. a pair of elements wrapped around a stem).

agreement In many languages, the forms of certain elements can vary to indicate such properties as person [q.v.], number [q.v.], animacy, gender [q.v.], etc. Often, these variations are marked with affixes. Some grammatical relationships between pairs of linguistic elements require that they agree on these properties. In English, for example, present tense verbs are marked to indicate whether their subjects are third-person singular (with the suffix $-s$ ), and nouns indicate plurality (also with a suffix $-s$ ). The systematic covariation of the forms of the subject and verb is called 'subjectverb agreement.' Similarly, third-person pronouns must agree with 
their antecedents in person, number, and gender. See also inflection.

anaphor See anaphora.

anaphora Certain expressions depend for their interpretation on their association with some other element in the discourse (usually earlier). Paradigm examples are pronouns like he, her, and itself; other examples include do so and verb phrase ellipsis. 'Anaphora' is the term for the relationship between such elements and their antecedents. The term 'anaphor' is sometimes used for all anaphoric elements and is sometimes reserved for only certain kinds (primarily reflexives [q.v.] and reciprocals [q.v.]). See also antecedent; binding; ellipsis.

antecedent This term is used for a linguistic element that is the basis for interpreting some anaphoric element occuring (typically later) in the sentence or discourse. In particular, pronouns are often described as referring back to their antecedents (or to the referents of their antecedents). See also anaphora.

argument (or syntactic argument) This is a general term for any phrase that is selected for by a lexical head, such as a complement or specifier. This usage derives from the semantic term 'argument', which refers to a component of a proposition that must occur with a given predicate or relation. For example, the meaning of the verb wash has two semantic arguments (the washer and the washee) that correspond to the two syntactic arguments of the verb wash (the subject and the object) - as in Alex washed the car. The simplest examples of (syntactic) arguments are noun phrases, but prepositional phrases and subordinate clauses can also function as arguments. See also complement; specifier; subcategorization; valence; argument structure.

(argument)-marking preposition English prepositions serve two distinct functions. In some cases, a preposition is used to indicate the role its object NP plays within the semantic structure of some predicate. In other cases, the preposition itself functions as a predicate, and its object is one of its arguments. In this text, the first kind of use is called an 'argument-marking preposition,' or just a 'marking preposition.' An example is the preposition on in They rely on us. We call the second kind of preposition 'predicational,' illustrated by the use of on in They are on the porch.

argument structure In the theory developed in this text, the phrases that serve as arguments of a given head are listed in the value of a feature called ARGUMENT-STRUCTURE. This term is also 
sometimes used in a less technical sense to designate the semantic relations between a head [q.v.] and its arguments. See also argument.

aspect Many languages have special grammatical elements for locating in time the situations referred to. Among the temporal notions often expressed are whether situations are in process or completed and whether they occur repeatedly. These notions are often called 'aspect,' and words or affixes whose function is to express aspect are called 'aspectual markers.' See also perfective, progressive.

aspectual marker See aspect.

auxiliary This term refers to elements found in many languages that share the following semantic and syntactic characteristics: (i) they express such notions as time (past, present, future; continuation, completion), necessity, possibility, obligation, permission, negation, or questioning; and (ii) they occur in fixed positions in sentences, usually at or near the beginning or end. English auxiliaries are a special kind of verb. It is the auxiliary verb that is inverted with the subject in yes/no questions (e.g. Did she fall?) and that carries the negative suffix in contractions [q.v.] (e.g. can't, won't).

binding Pronouns are often said to be 'bound' by their antecedents [q.v.], and the term 'binding' is used to refer to the relationship between pronouns and their antecedents. The study of the principles governing pronominal anaphora [q.v.] is called 'binding theory.' See also reciprocal; reflexive.

case Certain words - particularly nouns and pronouns - can appear in different forms depending on their relationship to other elements in the sentence in which they appear. In English, for example, personal pronouns exhibit nominative case (e.g. I, they) or accusative case (e.g. me, them), depending on whether they are subjects or objects. In many languages, case is the primary way of indicating the roles of the noun phrases in the clause - that is, who did what to whom. Among the names of cases commonly used across languages are 'nominative,' 'accusative,' 'dative,' 'genitive,' 'ergative,' and 'absolutive.' See also inflection.

clause A clause is a phrase that includes a predicate and all of its arguments and modifiers. The term is sometimes limited to phrases headed by a verb.

common noun Nouns are often divided into two kinds: proper and common. Proper nouns are names, denoting individual things by themselves, and do not normally take determiners, complements, or modifiers. Common nouns stand for kinds of things and take 
determiners [q.v.], modifiers [q.v.], and (sometimes) complements [q.v.]. In English orthography, proper nouns are conventionally capitalized, but common nouns are not.

competence In a number of works, Chomsky has distinguished between the (largely unconscious) knowledge of language that people have and how they put that knowledge to use in speech and writing. The former is called 'competence'; the latter 'performance.' The study of linguistic competence abstracts away from such factors as memory limitations, disfluencies, and speech errors. Work in generative grammar [q.v.] has concentrated largely on developing models of competence, though there has been much discussion of how such models relate to what is known about performance.

complement The lexical head [q.v.] of a phrase characteristically selects which arguments co-occur with it, and some of these are referred to as 'complements.' When the phrase's head is a verb, the complements include what are traditionally called direct and indirect objects, as well as some prepositional phrases and subordinate clauses [q.v.]. Subjects - and determiners of NPs - are arguments that are not complements, but specifiers. Complements occur as sisters to the lexical head in syntactic structure and, in English, follow the head. For example, a verb such as hit takes one complement, namely, an NP (e.g. hit the ball); rely takes a PP complement (e.g. rely on Sandy). A preposition such as in also takes a single NP complement (e.g. in the box). Some nouns can also take complements, such as picture which takes an optional PP complement (e.g. picture of Kim). See also argument; specifier.

complementizer Words whose sole function is to introduce certain kinds of complements are known as 'complementizers.' This label is normally reserved for elements introducing clausal complements headed by a verb. In English, the use of that to introduce subordinate clauses [q.v.] (as in It is remarkable that ice floats) is the clearest example of a complementizer. In this text, the to that introduces VPs (as in Everybody wants to win) is also analyzed as a complementizer. See also complement.

conjunction (conjunct) Traditional grammarians use the term 'conjunction' to refer to words that connect two linguistic units in some way. In this text, we use it only for what are traditionally called 'coordinate conjunctions,' that is, words connecting elements that are, intuitively, of equal status. In English, the paradigmatic coordinate conjunctions are and and or, though but and nor can also function in this way. The individual words or phrases that have 
been conjoined can be referred to as conjuncts. See also coordination.

constituent The term 'constituent' is used by linguists as a near synonym for 'phrase,' meaning a part of a sentence that functions syntactically as a single unit. The difference is that 'constituent' is usually limited to phrases that are proper parts of larger expressions.

context-free grammar (CFG) A context-free grammar is a particular type of formal system that has proved very useful in the precise characterization of computer languages and also serves as the starting point for much work in syntactic theory. CFGs consist of an initial symbol [q.v.], a finite lexicon with words classified into grammatical categories [q.v.], and a finite collection of rules of the form $\mathrm{A} \rightarrow \omega$, where $\mathrm{A}$ is a single symbol (representing a type of phrase), and $\omega$ is a finite string of lexical and/or phrasal categories.

contraction Reduced forms of words are sometimes combined with other words (that would typically occur adjacent to the reduced words) to form a new word; these are referred to as 'contractions.' English examples include combinations of a finite auxiliary [q.v.] verb with a reduced form of not to produce such words as isn't and can't, as well as simple contraction of finite auxiliaries, e.g. They're arriving tomorrow and Kim's here.

control Some complements have no overt specifier, but are interpreted as if they had subjects with the same reference as (i.e. coindexed with) another complement to the same predicate. For example, in both Pat tries to be on time and We urged Pat to be on time the individual Pat is understood as the person who is meant to be on time. This relationship (between two noun phrases, the second typically an unexpressed subject) is referred to as 'control'; in this case with the NP Pat being the 'controller' of the unexpressed subject of the infinitival phrase. Control predicates are not to be confused with raising [q.v.] predicates (like continue and expect), one of whose arguments actually is simultaneously an argument of another complement. A number of syntactic and semantic diagnostics distinguish these two types of predicates. See also raising.

coordination This term refers to the linking of two words or phrases of equivalent syntactic status (i.e. neither conjoined element is subordinate to the other). An example of a coordinated clause is Kim cooked the food and Sandy washed the dishes. See also conjunction. 
copula This term is used by traditional grammarians to refer to verbs with little semantic content, which serve to link a subject with a predicate. In English, the copula is be and its various inflections.

coreference (or) coreferential Two referring expressions that refer to the same entity are called 'coreferential,' and the relationship between them is called 'coreference.' See also anaphora.

count noun Nouns are divided into two subclasses known as 'count' and 'mass' according to which determiners they can combine with. Count nouns co-occur with $a(n)$, several, few, etc; mass nouns cooccur with much and can occur in the singular with no determiner. This distinction is correlated with a semantic distinction: mass nouns usually are used to refer to substances and count nouns to (sets of) entities. A portion of a substance (e.g. helium) is still the same substance, whereas a portion of an entitiy (e.g. a bicycle) is not usually an entity of the same kind. This correlation is not perfect, however, as evidenced by the mass noun furniture and by minimal pairs like cabbage (which can be either count or mass) vs. lettuce (which, for many speakers, must be mass).

declarative/interrogative/imperative These are terms used in the classification of sentence types. Declarative sentences are used to make a statement (or - equivalently for our purposes - to assert the truth of a proposition), as in The mayor is reading a book. Interrogative sentences are used to ask questions, as in What are they doing? Imperative sentences are used to give orders (or to issue 'directives'), as in Read a book!

defeasible A constraint is said to be 'defeasible' if it can be overridden - that is, if it allows for the existence of exceptions.

demonstrative Expressions used for referring through direct indication (often accompanied by pointing) are called 'demonstratives.' The best examples in English are this, that, these, and those.

descriptive grammar See prescriptive grammar.

determiner The sorts of specifiers [q.v.] that nouns take are called 'determiners.' These include articles ( $a$, the, etc.), quantifiers [q.v.] (some, every, many, two, etc.), and possessives [q.v.] (my, Kim's, etc.). See also specifier.

discourse This term refers to units of language longer than a sentence - for example, dialogues or paragraphs.

distribution Linguists use this term to refer to the set of total environments - or contexts - in which some linguistic unit can occur.

ditransitive verb Verbs that take two NP objects are called 'ditransitive.' The standard example is give, in examples like The teacher 
gave the students an exam. See also intransitive verb; transitive verb; valence.

dummy Words that evidently have no meaning and serve only to fill some grammatical function are sometimes called 'dummies.' The paradigm examples in English are the there that occurs in existential sentences (e.g. There is a seat available and the it of extraposition [q.v.] (e.g. It is fortunate that you have a seat). Other terms used for these are 'expletives' and 'pleonastic' elements.

ellipsis Ellipsis means 'leaving out' or 'omitting': in certain contexts, parts of a sentence can be omitted if their interpretation is reconstructable. An example is the following case of verb phrase ellipsis, where the bracketed material may be left out:

(107) Pat won't taste the soup, but Chris will [taste the soup].

See also anaphora.

existential be/existential there English has a special construction for expressing existence, involving the dummy there as subject and forms of the verb be. These are called 'existential.' See also dummy.

extraposition Predicates that can take complementizer [q.v.] phrases (i.e. that-clauses or to-infinitive phrases) as subjects can also occur with a dummy it as subject and the CP as the last complement. The latter construction is called 'extraposition.' The term is also sometimes used for expressions in which a complement or modifier is separated from its head by intervening material, as in $A$ review appeared of Lee's latest book. See also dummy.

feature structure A standard way of representing linguistic information is in terms of complexes of features and values. A feature can be thought of as a dimension along which different linguistic entities (such as words, phrases, or sentences) may differ, and values identify locations on those dimensions. A feature-value pair models a property of an expression that distinguishes it in a linguistically interesting way from some other expressions. For example, the feature PERSON in English has three possible values, namely '1st,' '2nd,' and '3rd.' It is a property of the word you that it is second person, and we represent that with the feature-value pair [PERSON 2nd]. A feature structure can thus be treated as a set of such feature-value pairs, in which no feature is paired with more than one value. Values of features in our theory may themselves be feature structures, or even lists of feature structures. A variant of this idea formalizes feature structures as directed graphs, where the arcs are labelled by feature names. Feature structure 
descriptions are standardly given in terms of matrices, listing feature names paired with their values, also known as 'feature specifications.' See also inheritance hierarchy; type.

finite-state grammar finite-state grammars are a type of formal system sometimes used to describe certain rather simple artificial languages. They are mathematically equivalent to regular expressions. See also context-free grammar; regular expression.

finite verb $\mathrm{A}$ finite verb is one that is marked for tense [q.v.] (present or past, in English).

gap See long-distance dependency.

gender The nouns in many languages divide into classes, differing in their patterns of inflection and agreement. In a number of languages (e.g. French and German), these noun classes are referred to as 'genders,' because nouns used to refer to males or females (of any species) are generally (though not invariably) grammatically masculine or feminine, respectively. In English, gender is marked grammatically only on third-person singular pronouns (he, she, and $i t$, ) and is virtually always predictable from the actual gender of the referent.

generative grammar Chomsky introduced this term based on the idea that a grammar is a formal system for generating the sentences of a language. The term is now used somewhat ambiguously, either to mean work in the Chomskyan tradition (fairly broadly conceived), or to mean an explicit system of rules, principles, and/or constraints that characterizes all and only the well-formed sentences of a language.

grammatical category Words and phrases can be classified in various ways, any of which can be called a 'grammatical category.' The term is usually used to refer to parts of speech [q.v.], such as noun, verb, etc., as well as types of phrases, such as noun phrase, verb phrase, and sentence. But it can also be used for less traditional classifications, such as the various types of lexemes [q.v.] presented in this text.

head The constituent [q.v.] of a phrase that is grammatically the most important constituent of that phrase is called the 'head' of the phrase. The head usually determines the category of the phrase, as well as many of its other properties. Thus noun phrases have nouns as heads, verb phrases have verbs as heads, etc. The term is used ambiguously to refer to the word that functions as head of the phrase and any subphrase containing that word. For example, 
in the destruction of the city, both destruction and destruction of the city can be called heads of the phrase.

idiom Some combinations of words have interpretations that are not fully predictable from the meanings that those same words have in other contexts. These are known as 'idioms.' English examples include take advantage to mean (roughly) 'exploit,' keep tabs on for 'monitor,' and kick the bucket for 'die.' Parts of an idiom are sometimes called 'idiom chunks,' e.g. advantage in take advantage. Idiom chunks play a central role in one of the diagnostics for distinguishing raising [q.v.] predicates from control [q.v.] predicates.

imperative See declarative

infinitive A certain class of nonfinite verbs is referred to as 'infinitives.' English infinitives are uninflected, but in many other languages, there are special infinitive affixes. Use of the term in English grammar is sometimes limited to verbs preceded by 'to,' but we also use it elsewhere, e.g. for verbs following modals [q.v.].

inflection Languages often add affixes to words to mark the syntactic function or relationships of the word in the sentence. For example, present tense verbs in English are usually inflected with the suffix $-s$ when the subject is third person singular, and past tense verbs are inflected with -ed. The term may also be used to refer to the affix itself. Among the common uses of inflectional affixes are to indicate tense [q.v.], agreement [q.v.], number [q.v.] (singular or plural), and case [q.v.].

inheritance hierarchy The elements of some domains of study can naturally be organized into classes, based on shared properties. Some classes can be further subdivided into subclasses, with additional shared properties. The organization of such domains can be thought of as a hierarchy, with the most inclusive class (encompassing the entire domain) at the top, and the individual members of the domain at the bottom. In between are the various classes of interest, with classes above their subclasses. The properties associated with particular classes are inherited by their subclasses, and ultimately by their individual members. Domains organized in this way are referred to as 'inheritance hierarchies.' In linguistics, inheritance hierarchies have been used to organize lexical information, among other things. See also type.

initial symbol Grammars characterize languages. But languages can be conceived of in a variety of ways: as consisting of sentences, of phrases, of any expressions that can serve as stand-alone utterances, etc. A formal theory of grammar must include a specifica- 
tion of which of the expressions it characterizes are to be regarded as those that constitute the language. The initial symbols of a formal theory are precise statements of what is to count as an element of the language. In this book, the initial symbol definition specifies conditions that phrases must satisfy if they can stand alone, i.e. be used in isolation to communicate a message.

interrogative See declarative.

intonation This term is used to refer to the patterns of pitch in speech. intransitive verb A verb that does not take any NP objects is referred to as 'intransitive.' A standard example in English is die. See also ditransitive verb; transitive verb; valence.

inversion Grammarians use this term fairly generally to refer to any construction in which two elements appear with their typical ordering reversed. In this text, it is used in particular for sentences (mostly questions) in which a finite auxiliary [q.v.] verb precedes the subject, as in Are you sleeping?

island constraint While long-distance dependencies can, in principle, stretch over arbitrary distances, there are some pairings of filler and gap positions that are not possible. For example, a gap inside a CP subject cannot, in general, be paired with a filler outside that $\mathrm{CP}$, as in ${ }^{*}$ Which candidate did [that I voted for __] bother you. The constraints on possible filler-gap pairings are known as 'island constraints.' See also long-distance dependency.

Kleene star It is useful in the formal representation of languages (both natural and artificial) to allow certain patterns to be repeated any finite number of times (including zero). The standard notation for this is a superscripted asterisk, known as the 'Kleene star' (after the mathematician Stephen Kleene). For example, $a b^{*} c$ is shorthand for the infinite set of strings: $a c, a b c, a b b c, a b b b c, \ldots .$. 'Kleene plus,' denoted by a superscripted plus sign, means any nonzero number of repetitions. See also regular expression.

lexeme The term 'word' is used ambiguously to mean either a particular form, such as sees, or a set of related forms such as see, sees, saw, seen, and seeing. To avoid this ambiguity, linguists sometimes posit an abstract entity called a 'lexeme' that gives rise to a family of related words. See also word.

lexicalism Lexicalism often refers to the doctrine that (1) the internal structure of words is independent of how words are put together to make sentences, and (2) words are the atoms of syntactic combination. For example, in a lexicalist theory, bound morphemes (inflectional affixes that must be attached to a word) 
are not treated as independent syntactic elements, as they are in most (non-lexicalist) versions of Transformational Grammar (see Appendix B). Theories of grammar also differ in their organization and in where they locate syntactic information. Some theories (e.g. Generalized Phrase Structure Grammar) have rich systems of rules and relatively impoverished lexical entries. Others (e.g. Categorial Grammar or Lexical Functional Grammar) have highly structured lexical entries and a small number of very general rule schemata. 'Lexicalism' is sometimes also used to distinguish the latter sort of theory.

lexicon The list of all words [q.v.] (or lexemes [q.v.]) of a language is called its 'lexicon.' The lexicon is the repository of all idiosyncratic information about particular words, including syntactic, semantic, and phonological information. In some theories of grammar, the lexicon can also contain a great deal more systematic information, organized by a type hierarchy [q.v.] and/or lexical rules.

long-distance dependency Certain constructions, including wh-questions, relative clauses, and topicalization, permit an element in one position to fill the grammatical role associated with another position. The two positions can be arbitrarily far apart. For example, in Which student did the principal say that the teacher thought was responsible? the NP which student functions as the subject of was responsible, although they are separated by most of the sentence. Such constructions are called 'long-distance dependencies' (LDDs). Elements like which student in the above example are called 'fillers,' and the position normally associated with the filler's role (in this case, immediately preceding was responsible) is called the 'gap.' See also island constraints.

main clause See root sentence.

modal The English verbs can, could, may, might, must, shall, should, will, and would, along with their negated forms (can't, etc.) are referred to as 'modals' or 'modal verbs.' They share the following properties: they function only as finite verbs [q.v.]; they exhibit auxiliary behavior (negation, inversion, contraction, and ellipsis); they take infinitival [q.v.] VP complements; and they show no agreement [q.v.] (i.e. no third-person singular $-s$ suffix). Some other languages have similar syntactically distinctive classes of words expressing necessity, possibility, obligation, and permission; these are also known as modals. See also auxiliary.

mass noun See count noun. 
modifier Most phrases consist of a head [q.v.], together with that head's arguments [q.v.]. Semantically, the head typically denotes either a situation or an individual, and the arguments denote essential associated entities. In addition, phrases may contain modifiers, which serve to place further restrictions on the situation or individual picked out by the phrase as a whole. Modifiers can take a wide variety of syntactic forms, including adjectives and adjective phrases, adverbs and adverbial phrases, prepositional phrases, and modifying clauses (such as relative clauses). See also argument structure.

morphology This term refers ambiguously to the study of word structure - how words are put together out of stems and affixes - or to word structure itself.

negation Languages include devices for reversing or contradicting the meaning or truth conditions of expressions, a semantic effect known as 'negation.' In English, the paradigmatic element expressing negation is the word not.

nominalization Nominalizations are nouns constructed out of words of other categories, usually through affixation. An example is destruction, derived from the verb destroy through the affixation of -tion (together with some other modifications). The term 'nominalization' is also used to refer to a process of turning verbs and adjectives into nouns.

number Most English nouns take different forms depending on whether they refer to single entities or multiple entities, e.g. $d o g / d o g s$, man/men. Similarly, present tense [q.v.] verbs with third-person subjects have different forms depending on whether the subjects are singular or plural. The term 'number' is used for such distinctions. Some languages also mark number on other types of words, e.g. adjectives may be marked for the number of the noun they modify. There are also languages that make finer number distinctions than just singular vs. plural, notably languages that have special 'dual' forms for expressions referring to sets with exactly two members.

orthography This term refers to written representations of language. For example, the plural of the noun doe and the present tense form of the verb do that goes with a third-person singular subject share the same orthography (namely, 'does'), although their pronunciations (and almost everything else about them) are different.

paradigm Certain words have multiple inflectional forms. For example, verbs in English typically change their form depending on whether 
they are past or present tense, and their present-tense forms depend on the person and number of the subject. They also have a variety of nonfinite forms. The full array of inflectional forms of a word is known as its 'paradigm.' See also inflection.

parsing This term refers to the process of assigning a structural description to a sentence. Many computer systems designed to process natural language include components for parsing, and much psycholinguistic research is concerned with discovering what (if any) parsing strategies humans use in language comprehension.

part of speech This is the traditional term for lexical categories (i.e. categories of words), based on a combination of semantic and distributional criteria. Among the standard parts of speech are noun, verb, adjective, preposition, adverb, and conjunction. See also grammatical category.

participle Certain nonfinite verbs - usually ones that share some properties with adjectives - are referred to as 'participles.' English has three types of participles: present participles, which end in -ing and usually follow some form of be; past participles, which usually end in -ed or -en and follow some form of have; and passive participles, which look exactly like past participles but indicate the passive voice [q.v.]. The three participles of eat are illustrated in the following sentences:

a. Termites are eating the house.

b. Termites have eaten the house.

c. The house was eaten by termites.

passive Many languages have a construction in which the grammatical subject of a verb plays the same semantic role that the object plays when the verb in question appears elsewhere (in active [q.v.] forms). The term 'passive' is used to refer both to this construction, and to the verb whose arguments' roles are at issue. In English, the passive form of the verb looks exactly like the past participle and is usually preceded by a form of $b e$; a prepositional phrase headed by by is also common, and is used for marking what would be the subject if the verb were not passive. An example is The dog was attacked (by wombats). See also participle; voice.

perfective Many languages have special verb forms or constructions used to indicate that the event denoted by the verb is completed. These are referred to as 'perfective' (or just 'perfect') in aspect. The English perfective involves the combination of have with a past participle [q.v.], as in The dog has eaten the cake. See also aspect. 
performance See competence.

person Many languages distinguish grammatically among expressions referring to the speaker, to the hearer, and to third parties. This is called the expression of 'person.' Reference to the speaker or a set including the speaker is called 'first person'; reference to (sets including) the adressee(s) is called 'second person'; and everything else is called 'third person.' Person distinctions are clearest with pronouns, since these are the most common forms used to refer to the speaker and hearer. But in some languages nouns also show person marking, and verbs and adjectives may then agree with the noun in person.

phonetics Phonetics is the study of the acoustic or articulatory properties of speech sounds.

phonology Phonology is the study of the sound systems of languages, i.e. the systematic grammatical patterns in the distribution [q.v.] of speech sounds.

possessive Many languages have grammatical mechanisms for indicating a relation of possession between the referents of two NPs. When one noun or NP is marked as the possessor of another, this marking is referred to as the 'possessive.' In English, the possessive is marked by 's attached at the end of the noun phrase functioning as the 'possessor.'

pragmatics The information conveyed by a linguistic expression in a particular instance of use is typically much more than just its literal (or 'linguistic') meaning. The study of how linguistic meaning contributes more generally to communication is called (linguistic) 'pragmatics.'

predicational preposition See (argument)-marking preposition.

prescriptive grammar Much of traditional work in grammar is concerned with setting norms - that is, dictating that some usages are 'incorrect' and hence to be avoided. Modern linguists refer to this as 'prescriptive grammar' (or just 'prescriptivism') and argue that it has no scientific basis. Instead, they advocate describing languages as they are ('descriptive grammar') and seeking scientific explanations for what they find.

productive A relationship between two linguistic forms is said to be 'productive' if it generalizes to novel forms. For example, the use of the suffix -ing to mark the present participle form of a verb is productive, since it gets applied to new coinages (as in faxing). Productivity is usually thought of as a matter of degree, with ex- 
ceptionless relationships counting as more productive than those with exceptions.

progressive Special verb forms or constructions used to indicate that the event denoted by the verb is in progress are referred to as 'progressive' aspect. The English progressive involves combination of be with a present participle [q.v.], as in The dog is eating the cake. see also aspect.

proper noun See common noun.

quantifier Words or phrases used to restrict the number or amount of some referent are called 'quantifiers.' In English, these include such expressions as all, each, some, many, few, two, more than half, etc.

raising Some predicates take one more syntactic argument than semantic argument. In these cases, the extra syntactic argument functions as the subject of another complement and must obey any special co-occurrence restrictions imposed by that complement. These predicates are called 'raising' predicates. Raising is exemplified by the sentences Pat continues to be on time and We expected Pat to be on time. In these examples, Pat, though a syntactic argument of seem and expect, is semantically an argument only of be on time. A semantically empty dummy [q.v.] is possible with raising predicates, where it would not be possible in the corresponding positions with control predicates: There continued/*tried to be demonstrations on campus. See also control.

reciprocal A reciprocal pronoun is one that expresses a mutual relationship, such as the English pronoun each other. See also anaphora; reflexive.

referent This term is used for the entity (e.g. a person, object, notion, or situation) that is denoted by (a use of) a linguistic expression.

reflexive Many languages use special forms of pronouns when the subject and object refer to the same individual or individuals, e.g. the English forms ending in -self or -selves. These are called 'reflexives' or 'reflexive pronouns.' It is common for these pronouns also to be acceptable in some other environments, but those environments differ from language to language. See also anaphora; binding; reciprocals.

regular expression It is possible to characterize the well-formed expressions of some simple formal languages by means of a few abbreviatory devices. One system that has proved very useful in some contexts involves templates, made up of words and/or categories of words, together with parentheses (to indicate optionality), a dis- 
junction symbol (to indicate alternatives), and Kleene star [q.v.] (and/or Kleene plus), to indicate arbitrary numbers of repetitions of a sequence. Such templates are called 'regular expressions.' See also finite-state grammar.

relative clause These are clauses that are used to modify nouns or noun phrases. A relative clause characteristically contains either a gap or a pronoun understood to be coreferential with the noun or NP they modify.

root sentence The traditional distinction between main clause and subordinate clause is motivated in part by the fact that certain phenomena seem to be restricted to main clauses, e.g. the inversion of finite auxiliaries [q.v.] in English questions (compare: Will I win? vs. I wonder whether I will win). Consequently, some version of this distinction has been maintained in most formal theories of grammar. The term 'root sentence' is sometimes used for main clauses, or, more technically, a phrase of category $\mathrm{S}$ that is not dominated by anything else. See also subordinate clause.

saturated In the system of grammar developed in this book, a saturated phrase is one that is specified as [SPR < rangle] (and [COMPS < rangle]). The intuition behind this is that headed phrases can be thought of as being generated bottom-up, starting from the lexical head, via a regime of cancelling elements from the head's valence specifications. For example, a verb combines first with however many complements are on its COMPS list to build a VP (a verbal phrase that is [COMPS < rangle] but [SPR $\langle$ Prangle]); the resulting (SPR-)unsaturated phrase then combines with the subject NP to build a saturated phrase, i.e. an S.

semantics Semantics is the branch of linguistics concerned with the study of linguistic meaning. Linguists also use the locution 'the semantics of' some expression as a way of talking about the literal interpretation of that expression. Not all information that is conveyed by the utterance of an expression is part of its semantics, but the line between literal meaning and what is conveyed can be hard to draw. At a minimum, the semantics of a (declarative) sentence is usually taken to include a specification of the conditions under which it would be true. See also pragmatics.

specifier We use the term 'specifier' to cover subjects of clauses, determiners of noun phrases, and certain other constituents that are neither heads of the phrases they appear in nor complements to 
the heads. In English, the specifier of a phrase precedes its head [q.v.] and complements [q.v.]. See also determiner.

structural description (SD) In this text, phrases are modeled by phrase structures - trees consisting of subtrees whose nodes are fully specified feature structures. Phrase structures are informally described by means of 'structural descriptions,' which share the same tree configuration as the phrase structures themselves, but can leave some features underspecified. Likewise, a word is modeled by a word structure (tree), consisting of a fully specified feature structure that exhaustively dominates the word. Partial descriptions of word structures (lexical SDs) are also structural descriptions. See also feature structure.

subcategorization Lexical heads differ according to how many and what types of things they must combine with in order to make complete phrases. Each grammatical category [q.v.] (that is, part of speech [q.v.]) can be divided into subcategories, based on the valence, or combinatory potential, of the particular words. When we talk of the subcategorization of a verb (or other type of head), we mean the restrictions on which sorts of phrases it can combine with. Another common locution is to say that a given verb 'subcategorizes for' a certain phrase, meaning that it combines with such a phrase. See also valence

subordinate clause A subordinate clause is one that is dependent on, and usually a constituent [q.v.] of, another clause [q.v.]. An example of a subordinate clause is when Kim went in Sandy came when Kim went. See also root sentence.

tense Finite verbs come in different forms depending on the time they denote; these forms are called 'tenses.' English has present and past tense, exemplified by the present tense forms walk and walks, and by the past tense form walked. Some languages also have future tenses, but English uses other means (e.g. the modal [q.v.] will) to express future time. See also aspect; finite verb.

transitive verb Verbs that take an NP object are called 'transitive.' The term can also be used for other parts of speech that can take objects, e.g. prepositions. It is sometimes taken to encompass both simple transitive verbs (that is, those taking a single object) and ditransitive verbs. A standard example of a transitive verb is hit. See also ditransitive verb; intransitive verb; valence.

type Elements of any collection can be sorted into types, based on similarities of properties. In the theory presented in this text, linguistic entities (e.g. words and phrases) are described by means 
of feature structures [q.v.]. Particular features are appropriate only to certain types of entities, and constraints on possible featurevalue pairings are also associated with some types. The types of linguistic entities are arranged in an inheritance hierarchy. The type hierarchy is especially important for capturing regularities in the lexicon. See also feature structure; inheritance hierarchy; lexicon.

unification The operation of unification merges two feature structure descriptions into a description that contains all the information in both. Two feature structure descriptions can unify so long as the information in them is consistent - that is, so long as there is no feature for which they have conflicting values. The unification simply consists of all of the features and values specified in the two feature structure descriptions. If description $\mathrm{D}_{1}$ is satisfied by a family of feature structures $F_{1}$ and description $D_{2}$ is satisfied by a family of feature structures $\mathrm{F}_{2}$, then the unification of $\mathrm{D}_{1}$ and $\mathrm{D}_{2}$ (written $\mathrm{D}_{1} \sqcup D_{2}$ ) is satisfied by all feature structures in the intersection of $\mathrm{F}_{1}$ and $\mathrm{F}_{2}$.

universal grammar Many linguists claim that there is a great deal in common among the grammars of the world's languages. Most advocates of this position believe that the commonalities exist because linguistic structure is largely determined by human biology. The term 'universal grammar' is used to mean three subtly different things: (i) what is common to the world's languages; (ii) linguists' representations of these commonalities; and (iii) the biological structures that are claimed to underlie the common features.

unsaturated See saturated.

valence This term is used (by analogy with the chemical term) to refer to the combinatoric potential of words and phrases. In this text, the VALENCE features are those that specify this kind of information. Specifically, the VALENCE features SPR and COMPS for the verb put specify that it requires a subject NP, an object NP, and a PP in order to form a clause. See also argument; argument structure; ditransitive verb; intransitive verb; transitive verb.

voice This term refers to the way the semantic arguments of the verb are expressed grammatically. The term is used in English primarily to distinguish active voice and passive voice, but some other languages have far richer systems of voice. See also active; passive. 
word This term is used in many different ways. In this text, a word is a particular form whose lexical entry is derived from a lexeme by some inflectional rule. See also lexeme, lexicon. 



\section{References}

Akmajian, Adrian, Susan Steele, and Thomas Wasow. 1979 The Category AUX in Universal Grammar. Linguistic Inquiry 10: 1-64.

Akmajian, Adrian and Thomas Wasow (1974) The Constituent Structure of VP and AUX and the Position of the Verb BE. sl Linguistic Analysis 1: 205-245.

Andrews, Avery (1982). The Representation of Case in Modern Icelandic. In Bresnan (1982a).

Bach, Emmon (1979) Control in Montague Grammar. Linguistic Inquiry 10: $515-31$.

Bach, Emmon (1989) Informal Lectures on Formal Semantics. Albany: SUNY Press.

Barbosa, Pilar, Danny Fox, Paul Hagstrom, Martha McGinnis, and David Pesetsky, eds. (1998) Is the Best Good Enough? Optimality and Competition in Syntax. Cambridge, Mass.: MIT Press.

Bar-Hillel, Yehoshua and Eliyahu Shamir (1960) Finite-State Languages: Formal Representations and Adequacy Problems. The Bulletin of the Research Council of Israel 8F, 155-166. Reprinted in Y. BarHillel, Language and Information: Selected Essays on Their Theory and Application (1964). Reading, Mass.: Addison-Wesley.

Barlow, Michael and Charles Ferguson, eds. (1988) Agreement in Natural Language: Approaches, Theories, Descriptions. Stanford: CSLI Publications. 
462 / Syntactic Theory: A Formal Introduction

Barwise, Jon and John Etchemendy (1989) Semantics. In M. I. Posner (ed.), Foundations of Cognitive Science. Cambridge, Mass.: MIT Press.

Bates, Elizabeth and Brian MacWhinney (1989) Functionalism and the Competition Model. In B. MacWhinney and E. Bates (eds.), The Cross-linguistic Study of Sentence Processing. Cambridge: Cambridge University Press.

Baugh, John (1983) Black Street Speech: Its History, Structure, and Survival. Austin: University of Texas Press.

Bear, John (1981). Gaps as Syntactic Features. Technical Report. Center for Cognitive Science - University of Texas at Austin.

Bever, Thomas (1970) The Cognitive Basis for Linguistic Structures. In J. R. Hayes (ed.) Cognition and the Development of Language. New York: Wiley.

Bloomfield, Leonard (1933) Language, New York: H. Holt and Company.

Brame, Michael K., (1979) Essays Toward Realistic Syntax. Seattle: Noit Amrofer.

Bresnan, Joan, (1978) A Realistic Transformational Grammar. In M. Halle, J. Bresnan, and G. A. Miller (eds.), Linguistic Theory and Psychological Reality. Cambridge, Mass.: MIT Press.

Bresnan, Joan, ed. (1982a) The Mental Representation of Grammatical Relations. Cambridge, Mass.: MIT Press.

Bresnan, Joan (1982b) The Passive in Lexical Theory. In Bresnan (1982a).

Bresnan, Joan (1982c) Control and Complementation. In Bresnan (1982a).

Bresnan, Joan (1995) Linear Order, Syntactic Rank, and Empty Categories: On Weak Crossover. In M. Dalrymple, et al. (eds.), Formal Issues in Lexical-Functional Grammar. Stanford: CSLI Publications. 
Bresnan, Joan (forthcoming) Lexical Functional Syntax. Oxford and Cambridge, Mass.: Basil Blackwell.

Briscoe, Edward, Ann Copestake, and Valeria de Paiva, eds. (1993) Inheritance, Defaults, and the Lexicon. Cambridge: Cambridge University Press.

Brody, Michael. 1995. Lexico-Logical Form: a radically minimalist theory. Cambridge, Mass.: MIT Press.

Burzio, Luigi (1986) Italian Syntax. Dordrecht: Reidel.

Carpenter, Bob (1992) The Logic of Typed Feature Structures: with Applications to Unification Grammars, Logic Programs, and Constraint Resolution. Cambridge: Cambridge University Press.

Carpenter, Bob (1997) Type-Logical Semantics. Cambridge, Mass.: MIT Press.

Chierchia, Gennaro and Sally McConnell-Ginet (1990) Meaning and Grammar : An Introduction to Semantics. Cambridge, Mass.: MIT Press.

Chomsky, Noam (1957) Syntactic Structures. The Hague: Mouton.

Chomsky, Noam (1959) Review of B. F. Skinner's Verbal Behavior. Language 35: 26-58. Reprinted in J. A. Fodor and J. J. Katz (eds.), The Structure of Language: Readings in the Philosophy of Language. Englewood Cliffs, N.J.: Prentice-Hall (1964).

Chomsky, Noam (1963) Formal Properties of Grammars. In R.D. Luce, R. Bush, and E. Galanter (eds.), Handbook of Mathematical Psychology, Volume II. New York: Wiley.

Chomsky, Noam (1965) Aspects of the Theory of Syntax. Cambridge, Mass.: MIT Press.

Chomsky, Noam (1970) Remarks on Nominalization. In R. A. Jacobs and P. S. Rosenbaum (eds.), Readings in English Transformational Grammar. Waltham, Mass.: Ginn-Blaisdell. 
464 / Syntactic Theory: A Formal Introduction

Chomsky, Noam (1972) Language and Mind, enlarged edition. New York: Harcourt, Brace, Jovanovich.

Chomsky, Noam (1973) Conditions on Transformations. In Stephen Anderson and Paul Kiparsky (eds.), A Festschrift for Morris Halle. New York: Holt, Rinehart and Winston.

Chomsky, Noam (1975) The Logical Structure of Linguistic Theory. Chicago: University of Chicago Press.

Chomsky, Noam (1977) On Wh-Movement. In Peter Culicover, Adrian Akmajian, and Thomas Wasow (eds.), Formal Syntax. New York: Academic Press.

Chomsky, Noam (1980) Rules and Representations. New York: Columbia University Press.

Chomsky, Noam (1981) Lectures on Government and Binding. Dordrecht: Foris.

Chomsky, Noam (1986a) Knowledge of Language: Its Nature, Origin, and Use. New York: Praeger.

Chomsky, Noam (1986b) Barriers. Cambridge, Mass.: MIT Press.

Chomsky, Noam (1995) The Minimalist Program. Cambridge, Mass.: MIT Press.

Crain, Steven and Mark Steedman (1985) On Not Being Led Up the Garden Path: The Use of Context by the Psychological Syntax Processor. In David R. Dowty, Lauri Karttunen, and Arnold M. Zwicky (eds.), Natural Language Processing. Cambridge: Cambridge University Press.

Dalrymple, Mary (1993) The Syntax of Anaphoric Binding. Stanford, California: CSLI Publications.

Dalrymple, Mary, Ronald M. Kaplan, John T. Maxwell III, and Annie Zaenen (eds.) (1995). Formal Issues in Lexical-Functional Grammar. Stanford: CSLI Publications. 
Davidson, Donald (1980) Essays on Actions and Events. Oxford: Clarendon Press; New York: Oxford University Press.

Davis, Anthony (1996) Lexical Semantics and Linking in the Hierarchical Lexicon. unpublished Ph. D. dissertation. Stanford University.

Dowty, David, Robert Wall, and Stanley Peters (1981) Introduction to Montague Semantics. Dordrecht: D. Reidel.

Emonds, Joseph (1975) A Transformational Approach to Syntax. New York: Academic Press.

Fasold, Ralph W. (1972) Tense Marking in Black English. Washington: Center for Applied Linguistics.

Fasold, Ralph W. and Walt Wolfram (1970) Some Linguistic Features of Negro Dialect. In Ralph W. Fasold and Roger W. Shuy (eds.), Teaching Standard English in the Inner City. Washington, D.C.: Center for Applied Linguistics.

Ferguson, Charles (1971) Absence of Copula and the Notion of Simplicity: A Study of Normal Speech, Baby Talk, Foreigner Talk, and Pidgins. In Dell Hymes (ed.), Pidginization and Creolization of Languages. New York: Cambridge University Press.

Fillmore, Charles J., Paul Kay and M.C. O'Connor (1988) Regularity and idiomaticity in grammatical constructions: the case of let alone. Language. 64 (3): 501-538.

Fillmore, Charles, J., and Paul Kay (forthcoming) Construction Grammar. Stanford: CSLI Publications.

Flickinger, Daniel, Carl Pollard, and Thomas Wasow (1985) Structure Sharing in Lexical Representation. In Proceedings of the 23rd Annual Meeting of the Association for Computational Linguistics. Morristown, N.J.: Association for Computational Linguistics.

Fodor, Janet D. (1995) Comprehending Sentence Structure. In Lila R. Gleitman and Mark Liberman (eds.), Language: An Invitation to Cognitive Science, Volume 1, second edition. Cambridge, Mass: MIT Press. 
466 / Syntactic Theory: A Formal Introduction

Fodor, Jerry A., Thomas Bever, and Merrill Garrett (1974) The Psychology of Language. New York: McGraw-Hill.

Fraser, Bruce (1970) Idioms within a Transformational Grammar. Foundations of Language 6: 22-42.

Frege, Gottlob (1892) On Sense and Reference. Zeitschrift für Philosophie und philosophische Kritik 100: 25-50. Translation (under the title 'On Sense and Meaning') appears in Geach and Black (1980).

Gamut, L. T. F. (1991) Logic, Language, and Meaning. Chicago: University of Chicago Press.

Garrett, Merrill (1990) Sentence Processing. In Daniel Osherson and Howard Lasnik (eds.), Language: An Invitation to Cognitive Science, Volume 1, first edition. Cambridge, Mass.: MIT Press.

Gazdar, Gerald (1981) Unbounded Dependencies and Coordinate Structure. Linguistic Inquiry 12: 155-184.

Gazdar, Gerald (1982) Phrase Structure Grammar. In P. Jacobson and G. K. Pullum (eds.), The Nature of Syntactic Representation. Dordrecht: Reidel.

Gazdar, Gerald, Ewan Klein, Geoffrey K. Pullum, and Ivan A. Sag (1985) Generalized Phrase Structure Grammar. Cambridge, Mass.: Harvard University Press and Oxford: Basil Blackwell.

Gazdar, Gerald and Geoffrey K. Pullum (1981) Subcategorization, Constitutent Order, and the Notion 'Head'. In Michael Moortgat, Harry van der Hulst, and T. Hoekstra (eds.), The Scope of Lexical Rules. Dordrecht: Foris.

Gazdar, Gerald, Geoffrey K. Pullum, and Ivan A. Sag (1982) Auxiliaries and Related Phenomena in a Restrictive Theory of Grammar. Language 58: 591-638.

Geach, Peter, and Max Black, eds. (1980) Translations from the Philosophical Writings of Gottlob Frege, third edition. Oxford: Basil Blackwell. 
Ginzburg, Jonathan, and Ivan A. Sag (forthcoming) English Interrogative Constructions. Stanford: CSLI Publications.

Gleitman, Lila R. and Mark Liberman, eds. (1995) Language, Volume I of Daniel Osherson (ed.) An Invitation to Cognitive Science, Second Edition. Cambridge, Mass.: MIT Press.

Goldberg, Adele E. (1995) A Construction Grammar Approach to Argument Structure. Chicago: University of Chicago Press.

Green, Lisa (1993) Topics in African American English: The Verb System Analysis. unpublished Ph. D. dissertation. U. Massachusetts at Amherst.

Greenbaum, Sidney (1996) The Oxford English Grammar. Oxford: Oxford University Press.

Grice, H. Paul (1989) Studies in the Way of Words. Cambridge, Mass.: Harvard University Press.

Haegeman, Liliane (1994) Introduction to Government and Binding Theory, second edition. Oxford and Cambridge, Mass.: Basil Blackwell.

Harman, Gilbert (1963). Generative Grammar without Transformation Rules: A Defense of Phrase Structure. Language 39: 597-616.

Harris, Randy Allen (1993) The Linguistic Wars. Oxford: Oxford University Press.

Harris, Zellig S. (1970) Papers in structural and transformational linguistics. Dordrecht: Reidel

Hockett, Charles Francis (1958) A Course In Modern Linguistics. New York: Macmillan.

Hopcroft, John E. and Jeffrey D. Ullman (1979) Introduction to Automata Theory, Languages, and Computation. Reading, Mass.: AddisonWesley.

Huck, Geoffrey J. and John A. Goldsmith (1995) Ideology and Linguistic Theory. London and New York: Routledge. 
468 / Syntactic Theory: A Formal Introduction

Hudson, Richard. 1984. Word Grammar. Oxford: Blackwell.

Hudson, Richard. 1990. English Word Grammar. Oxford: Blackwell.

Hudson, Richard. 1998. Word Grammar. For Agel, V. et al. (eds.), Dependency and Valency. An International Handbook of Contemporarary Research. Berlin: Walter de Gruyter.

Jackendoff, Ray (1972) Semantic Interpretation in Generative Grammar. Cambridge, Mass.: MIT Press.

Jackendoff, Ray (1975) Morphological and Semantic Regularities in the Lexicon. Language 51: 639-671.

Jackendoff, Ray (1994) Patterns in the Mind. New York: Basic Books.

Johnson, David (1977) On Relational Constraints on Grammars. In Peter Cole and Jerrold Sadock (eds.), Syntax and Semantics, Volume 8, Grammatical Relations. New York: Academic Press.

Johnson, David and Shalom Lappin (in press) Local Constraints vs. Economy. Stanford: CSLI Publications.

Johnson, David and Paul Postal (1980) Arc Pair Grammar. Princeton: Princeton University Press.

Kaplan, Ronald M. (1975) Transient Processing Load in Relative Clauses. unpublished Ph. D. dissertation. Harvard University.

Katz, Jerrold J. and Paul M. Postal (1964) An Integrated Theory of Linguistic Descriptions. Cambridge, Mass.: MIT Press.

Katz, Jerrold J. and Paul M. Postal (1991) Realism versus Conceptualism in Linguistics, Linguistics and Philosophy 14: 515-554.

Kay, Martin (1979) Functional Grammar. In Chiarello, C. (ed.), Proceedings of the Fifth Annual Meeting of the Berkeley Linguistic Society.

Kim, Jong-Bok (1995) The Grammar of Negation. unpublished Ph. D. dissertation. Stanford University. 
Kim, Jong-Bok and Ivan A. Sag (1995) The Parametric Variation of English and French Negation. In Proceedings of the West Coast Conference on Formal Linguistics. Stanford: CSLI Publications.

King, Paul J. (1989). A Logical Formalism for Head-Driven Phrase Structure Grammar. Ph. D. dissertation. University of Manchester.

Koster, Jan. (1987). Domains and Dynasties, the Radical Autonomy of Syntax. Dordrecht: Foris.

Labov, William (1969) Contraction, Deletion, and Inherent Variability of the English Copula. Language 45: 715-762.

Labov, William (1972) Language in the Inner City: Studies in the Black English Vernacular. Philadelphia: University of Pennsylvania Press.

Labov, William (1995) The Case of the Missing Copula: The Interpretation of Zeroes in African-Amerircan English. In Lila R. Gleitman and Mark Liberman (eds.), Language, volume I of Daniel Osherson (ed.), An Invitation to Cognitive Science, second edition, pp. 25-54. Cambridge, Mass.: MIT Press.

Labov, William, Paul Cohen, Clarance Robins, and John Lewis (1968) A Study of the Nonstandard English of Negro and Puerto Rican Speakers in New York City. Final Report, Cooperative Research Project No. 3288, United States Office of Education.

Lascarides, Alex, Edward Briscoe, Nicholas Asher, and Ann Copestake (1996) Order Independent and Persistent Typed Default Unification. Linguistics and Philosophy 19.1: 1-89.

Lascarides, Alex, and Ann Copestake (1998) Default Representation in Constraint-Based Frameworks. unpublished manuscript.

Lasnik, Howard (1976) Remarks on Coreference. Linguistic Analysis 2: 1-22. Reprinted in Lasnik (1989).

Lasnik, Howard (1989) Essays on Anaphora. Dordrecht: Kluwer.

Lasnik, Howard (1995) The Forms of Sentences. In Lila R. Gleitman and Mark Liberman (eds.), Language, volume I of Daniel Osherson (ed.), An Invitation to Cognitive Science, second edition, pp. 25-54. Cambridge, Mass.: MIT Press. 
470 / Syntactic Theory: A Formal Introduction

Levin, Beth (1993) English Verb Classes and Alternations: A Preliminary Investigation. Chicago: University of Chicago Press.

MacDonald, Maryellen C., Neal J. Pearlmutter, and Mark S. Seidenberg (1994) The Lexical Nature of Syntactic Ambiguity Resolution. Psychological Review 101(4).

Manzini, M. Rita (1983) On Control and Control Theory. Linguistic Inquiry 14: 421-446.

Matthews, Peter (1993) Grammatical Theory in the United States: from Bloomfield to Chomsky. Cambridge: Cambridge University Press.

McCawley, James D. (1971) Tense and Time Reference in English. In C.J. Fillmore and D.T. Langendoen (eds.), Studies in Linguistic Semantics. New York: Holt, Rinehart, and Winston.

McCloskey, James (1979) Transformational Syntax and Model Theoretic Semantics. Dordrecht: Reidel.

Milsark, Gary (1977) Towards an Explanation of Certain Peculiarities of the Existential Construction in English. Linguistic Analysis 3: $1-31$.

Montague, Richard (1970) Universal grammar. Theoria 36: 373-398. Reprinted in Richard Thomason, (ed.), Formal Philosophy. New Haven: Yale University Press (1974).

Moortgat, Michael (1997) Categorial Type Logics. In Johan van Benthem and Alice ter Meulen (eds.), Handbook of Logic and Language. Amsterdam: Elsevier and Cambridge, Mass.: MIT Press. Pp. 93-177.

Morrill, Glynn (1994) Type Logical Grammar. Dordrecht: Kluwer.

Newmeyer, Frederick J. (1986) Linguistic Theory in America, Second Edition. London: Academic Press.

Nunberg, Geoffrey (1983) The Grammar Wars. The Atlantic Monthly $256.6,31-58$. 
Nunberg, Geoffrey, Ivan A. Sag, and Thomas Wasow (1994) Idioms. Language 70: 491-538.

Partee, Barbara H. (1995) Lexical Semantics and Compositionality. In Gleitman and Liberman (1995).

Partee, Barbara H., Alice ter Meulen, and Robert Wall (1990) Mathematical Methods in Linguistics. Dordrecht: Kluwer.

Pearlmutter, Neal J. and Maryellen MacDonald (1992) Plausibility and Syntactic Ambiguity Resolution. In Proceedings of the 14th Annual Conference on Cognitive Science, 498-503. Hillsdale, N.J.: Erlbaum.

Pedersen, Holger. (1959) The Discovery of Language: Linguistic Science in the Nineteenth Century. Translated by John Webster Spargo. Bloomington: Indiana University Press.

Perlmutter, David, ed. (1983) Studies in Relational Grammar 1. Chicago: University of Chicago Press.

Perlmutter, David and Paul Postal (1977) Toward a Universal Characterization of Passivization. In Proceedings of the 3rd Annual Meeting of the Berkeley Linguistics Society. Berkeley: University of California, Berkeley. Reprinted in Perlmutter (1983).

Perlmutter, David and Scott Soames (1979) Syntactic Argumentation and the Structure of English. Berkeley: University of California Press.

Pinker, Steven (1994) The Language Instinct. New York: Morrow.

Pollard, Carl and Ivan A. Sag (1987). Information-Based Syntax and Semantics, Volume 1: Fundamentals. CSLI Lecture Note Series No. 13. Stanford University: CSLI Publications. Distributed by University of Chicago Press.

Pollard, Carl and Ivan A. Sag (1992) Anaphors in English and the Scope of Binding Theory. Linguistic Inquiry 23: 261-303.

Pollard, Carl and Ivan A. Sag (1994) Head-Driven Phrase Structure Grammar. Chicago: University of Chicago Press. 
472 / Syntactic Theory: A Formal Introduction

Pollock, Jean-Yves (1989) Verb Movement, Universal Grammar, and the Structure of IP. Linguistic Inquiry 20: 365-424.

Postal, Paul (1967) Constituent Structure: A Study of Contemporary Models of Syntactic Description. Bloomington: Research Center for the Language Sciences, Indiana University.

Postal, Paul (1974) On Raising. Cambridge, Mass.: MIT Press.

Postal, Paul (1986) Studies of Passive Clauses. Albany: SUNY Press.

Postal, Paul and Brian Joseph, eds. (1990) Studies in Relational Grammar 3. Chicago: University of Chicago Press.

Postal, Paul and Geoffrey K. Pullum (1988) Expletive Noun Phrases in Subcategorized Positions. Linguistic Inquiry 19: 635-670.

Prince, Alan and Paul Smolensky (forthcoming) Optimality Theory: Constraint Interaction in Generative Grammar. Cambridge, Mass.: MIT Press.

Pullum, Geoffrey K. and Gerald Gazdar (1982) Natural Languages and Context-Free Languages. Linguistics and Philosophy 4: 471-504.

Quirk, Randoph, Sidney Greenbaum, Geoffrey Leech, and Jan Svartvik (1972) A Grammar of Contemporary English. London and New York: Longman.

Quirk, Randoph, Sidney Greenbaum, Geoffrey Leech, and Jan Svartvik (1985) A Comprehensive Grammar of the English Language. London and New York: Longman.

Radford, Andrew (1997) Syntactic Theory and the Structure of English: a minimalist approach. New York and Cambridge: Cambridge University Press.

Rickford, John R. and Lisa Green (in press) African American Vernacular English in Its Context. Cambridge: Cambridge University Press.

Robins, R. H. (1967) A Short History of Linguistics. Bloomington: University of Indiana Press. 
Rosenbaum, Peter (1967) The Grammar of English Predicate Complement Constructions. Cambridge, Mass.: MIT Press.

Ross, John R. (1967) Constraints on Variables in Syntax. Ph. D. dissertation. MIT. (Published as Infinite Syntax!, Norwood, N.J.: Ablex, 1986).

Ross, John R. (1969) Auxiliaries as Main Verbs. In W. Todd (ed.), Studies in Philosophical Linguistics 1. Evanston, Ill.: Great Expectations Press.

Ruwet, Nicolas (1991) Syntax and Human Experience. Edited and translated by J. Goldsmith. Chicago: University of Chicago Press.

Sag, Ivan A. (1997) English Relative Clause Constructions. Journal of Linguistics 33.2: 431-484.

Sag, Ivan A., and Janet D. Fodor (1994) Extraction Without Traces. In Proceedings of the Thirteenth Annual Meeting of the West Coast Conference on Formal Linguistics. Stanford: CSLI Publications.

Sag, Ivan A. and Carl Pollard (1991) An Integrated Theory of Complement Control. Language 67: 63-113.

de Saussure, Ferdinand (1916) Cours de Linguistique Générale. Paris: Payot. (Translated as Course in General Linguistics. New York: McGraw-Hill, 1959.)

Savitch, Walter J., Emmon Bach, William Marsh, and Gila Safran-Naveh (1987) The Formal Complexity of Natural Language. Dordrecht: D. Reidel.

Schütze, Hinrich (1996) Ambiguity Resolution in Language Learning. CSLI Lecture Notes No. 71. Stanford: CSLI Publications.

Shieber, Stuart (1986) An Introduction to Unification-Based Approaches to Grammar CSLI Lecture Notes No. 4. Stanford: CSLI Publications.

Sells, Peter (1985) Lectures on Contemporary Syntactic Theories. Stanford: CSLI Publications. 
474 / Syntactic Theory: A Formal Introduction

Skinner, B. F. (1957) Verbal Behavior. New York: Appleton-CenturyCrofts.

Smith, Henry (1996) Restrictiveness in Case Theory. Cambridge: Cambridge University Press.

Steedman, Mark (1996) Surface Structure and Interpretation. Cambridge, Mass.: MIT Press.

Steele, Susan (1981) An Encyclopedia of AUX. Cambridge, Mass.: MIT Press.

de Swart, Henriëtte (1998) Introduction to Natural Language Semantics. Stanford: CSLI Publications.

Tabossi, P., M. J. Spivey-Knowlton, K. McRae, and M. K. Tanenhaus (1994) Semantic Effects on Syntactic Ambiguity Resolution: Evidence for a Constraint-Based Resolution Process. In C. Umilta and M. Moscovitch (eds.), Attention and Performance XV. Hillsdale, N.J.: Erlbaum.

Tanenhaus, Michael K. and John C. Trueswell (1995) Sentence Comprehension. In J. Miller and P. Eimas (eds.) Handbook of Perception and Cognition: Volume 11. London: Academic Press.

Tesnière, Lucien (1959) Elements de Syntaxe Structurale. Paris: C. Klincksieck.

Tomasello, Michael (1992) The Social Bases of Language Acquisition. Social Development 1: 67-87.

Trask, Robert Lawrence (1993) A Dictionary of Grammatical Terms in Linguistics. London and New York: Routledge.

Trueswell, John C., Michael K. Tanenhaus, and Susan M. Garnsey (1992) Semantic Influences on Parsing: Use of Thematic Role Information in Syntactic Disambiguation. Journal of Memory and Language 33: $285-318$.

Trueswell, John C., Michael K. Tanenhaus, and Christopher Kello (1993) Verb-Specific Constraints in Sentence Processing: Separating Effects of Lexical Preference from Garden-Paths. Journal of Experimental Psychology: Learning, Memory, and Cognition 19: 528-553. 
Warner, Anthony (1993) English Auxiliaries: Structure and History. Cambridge; New York : Cambridge University Press.

Wasow, Thomas (1977) Transformations and the Lexicon. In P. Culicover, A. Akmajian, and T. Wasow (eds.) Formal Syntax. New York: Academic Press.

Wasow, Thomas (1989) Grammatical Theory. In M. I. Posner (ed.), Foundations of Cognitive Science. Cambridge, Mass.: MIT Press.

Wasow, Thomas and Ivan A. Sag (in preparation) Verbless Sentences in AAVE.

Webelhuth, Gert, ed. (1995) Government and Binding Theory and the Minimalist Program. Oxford: Basil Blackwell.

Wilkes-Gibbs, Deanna (1986) Collaborative Proccesses of Language Use in Conversation. Ph. D. dissertation. Stanford University.

Wood, Mary (1993) Categorial Grammars. Linguistic Theory Guides. London and New York: Routledge.

Zaenen, Annie (1983) On Syntactic Binding. Linguistic Inquiry 14: 469504.

Zwicky, Arnold and Geoffrey K. Pullum (1983) Cliticiziation vs. Inflection: English $n ' t$. Language 59: 502-13. 